\title{
Selection and use of DNA aptamers for applications within the central nervous system
}

\author{
by \\ Erin Marie McConnell
}

A thesis submitted to the Faculty of Graduate and Postdoctoral Affairs in partial fulfillment of the requirements for the degree of

Doctor of Philosophy

in

Chemistry

Carleton University

Ottawa, Ontario

(C) 2016

Erin Marie McConnell 


\subsection{Abstract}

Derived by an iterative in vitro selection process termed Systematic Evolution of Ligands by EXponential enrichment ( $\underline{\text { SELEX)}}$, aptamers are short single-stranded oligonucleotides that bind to their cognate targets with high affinity and selectivity. Generally, aptamers have been widely used in biological and pre-clinical medical applications. A comprehensive analysis of aptamer selection data maintained in the Aptamer Database identified factors that should be carefully considered upon the design of selection experiments to optimize success. These findings should be applied to selections for nervous system related targets to improve the quality of selected aptamers. Specifically, the nervous system presents an especially interesting field of investigation. For example the aptamer target thrombin is a protein involved in the coagulation cascade and has important relevancy for stroke. The development of an aptamer-based $\mathrm{pH}$-driven DNA nanomachine (pHAST) for the specific catch-and-release of thrombin is described. This work is an important example of how existing aptamers could be incorporated into a nanodevice to add specific functionality for applications within the nervous system. Dopamine is a small molecule neurotransmitter implicated in mental illness and neurodegenerative disease. The ability of dopamine-binding aptamers to attenuate perseveration and locomotor behaviour associated with dopamine over-activity was shown in live rodents. Finally, an aptamer-gold nanoparticle based sensor was developed based on a dopamine binding aptamer, DopaA20min, identified from a novel selection. 


\subsection{Acknowledgments}

To the people who share my DNA, Maria DeRosa - the person who taught me everything I know about DNA, and the people who stood by my side while I complained about DNA, every single day,

Thank you. 


\subsection{Table of Contents}

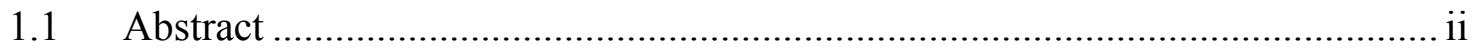

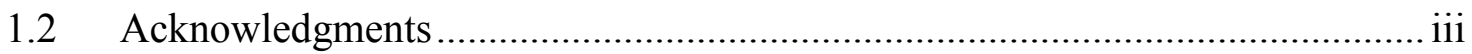

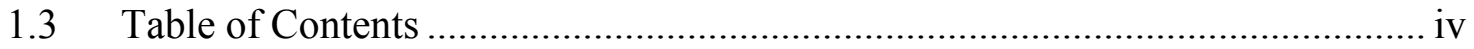

1.4 List of Abbreviations............................................................................. xiv

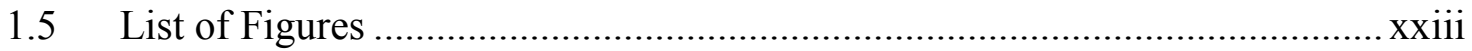

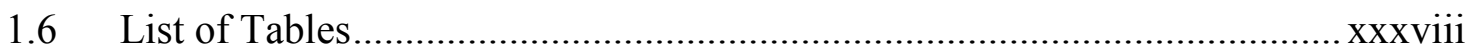

1 An introduction to aptamers, the selection of aptamers by Systematic Evolution of Ligands by EXponential enrichment (SELEX), and a comprehensive review of the use of aptamer-based technology in the central nervous system................................................. 1

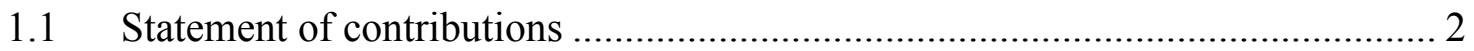

1.2 Resulting publications ........................................................................ 2

1.3 Introduction ......................................................................................... 3

1.3.1 A brief history of functional nucleic acids............................................... 3

1.3.2 Naturally occurring and in vitro selected aptamers .................................... 3

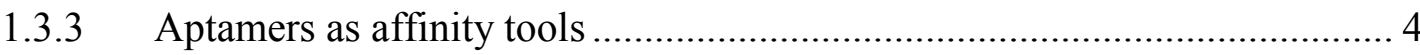

1.3.4 Systematic Evolution of Ligands by EXponential enrichment (SELEX)..... 8

1.3.5 In vivo applicability and utility of aptamers ............................................... 9

1.3.6 Aptamers as potential tools for the central nervous system......................... 10

1.4 Highlights of aptamer based research for CNS targets .................................... 12

1.4.1 Neurotransmitter and neurotoxin targets ................................................ 13

1.4.1.1 Dopamine and Schizophrenia ..................................................................... 13

1.4.1.1.1 Dopamine binding aptamer (DBA) based detection assays.......................... 13

1.4.1.1.2 The efficacy of a dopamine binding aptamer in a models of Schizophrenia and cocaine-induced hyperlocomotion ................................................................... 13

1.4.1.2 Aptamers for other neurotransmitters ............................................................ 14

1.4.1.3 Aptamers for the detection of botulinum neurotoxin......................................... 14

1.4.1.4 Aptamers for other neurotoxins .................................................................. 15

1.4.2 Demyelinating disease and spinal cord injury ……................................... 16

1.4.2.1 Multiple Sclerosis, myelin and myelin related targets ....................................... 16

1.4.2.2 Aptamers for the treatment of spinal cord injuries............................................. 17

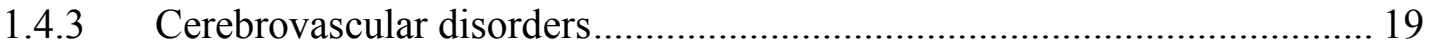




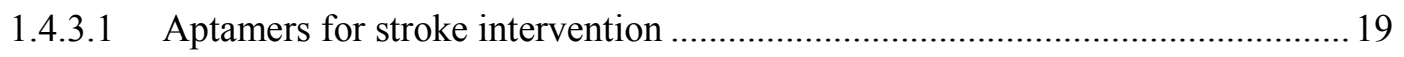

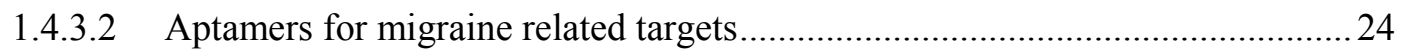

1.4.4 Protein aggregation related pathologies................................................... 26

1.4.4.1 Aptamers for targets related to Alzheimer's disease ...........................................26

1.4.4.2 Slow off-rate modified aptamers (SOMAmers) for the identification of AD

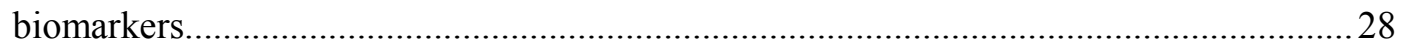

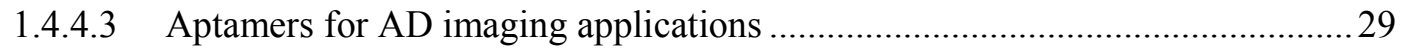

1.4.4.4 Aptamers for Parkinson's disease related targets............................................. 31

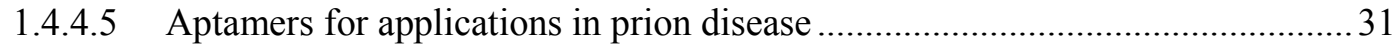

1.4.5 Aptamers for brain cancer (Glioblastoma and Glioma) related targets ...... 33

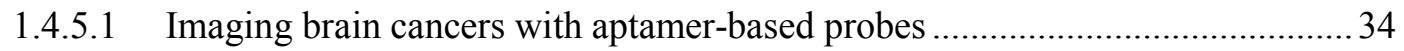

1.4.5.2 The use of aptamers to initiate cell death in brain cancer .................................. 36

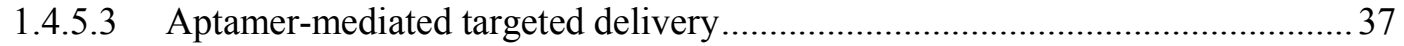

1.4.6 Aptamer-based regulation of receptor function ..................................... 39

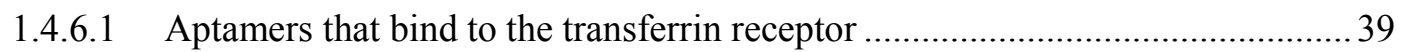

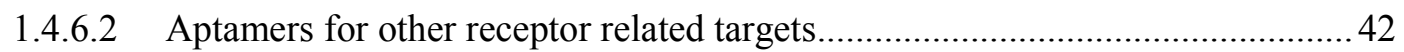

1.4.6.2.1 $\alpha$-amino-3-hydroxy-5methyl-4-isoxazole propionic acid (AMPA) receptors

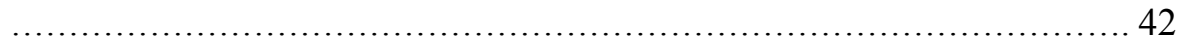

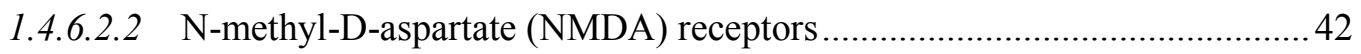

1.4.6.2.3 Nicotinic acetylcholine (nAChR) receptors............................................... 43

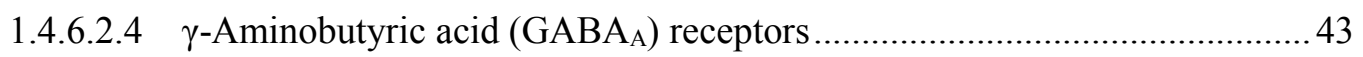

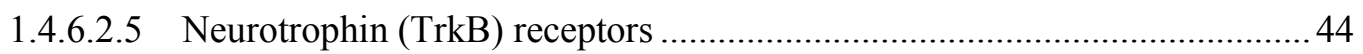

1.4.7 Interneuronal signaling controlled by aptamers...................................... 44

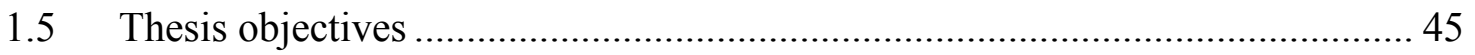

2 A comprehensive analysis of SELEX and aptamer data from the Aptamer Base.... 47

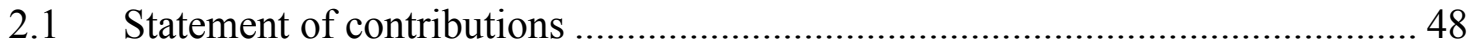

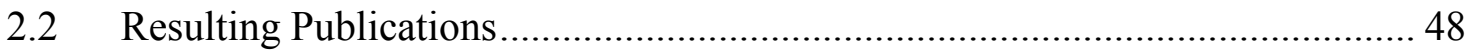

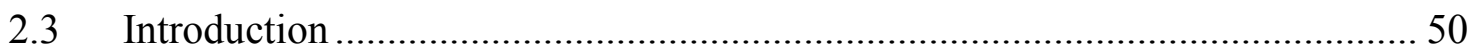

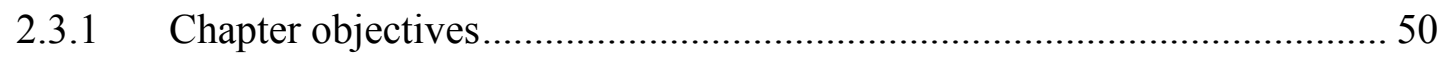

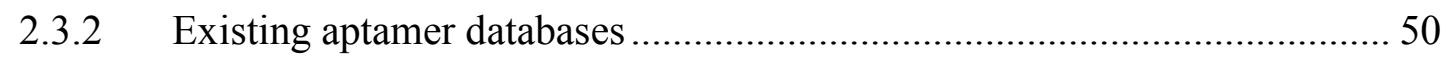

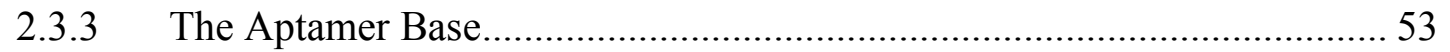

2.3.3.1 Freebase as a host platform for the Aptamer Base .............................................54

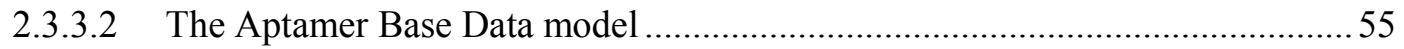




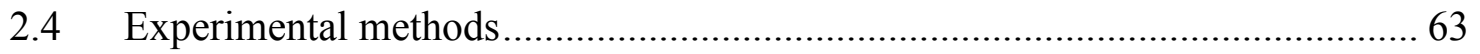

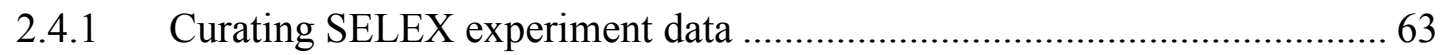

2.4.2 Accessing and querying data from the Aptamer Base ................................ 64

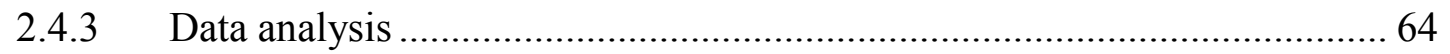

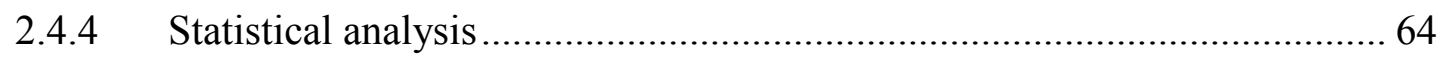

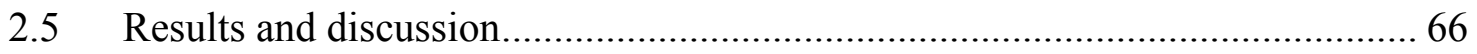

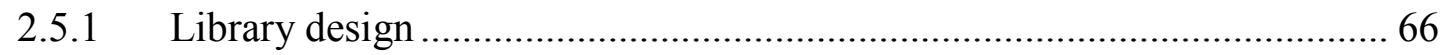

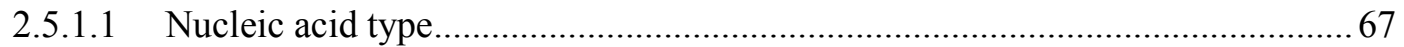

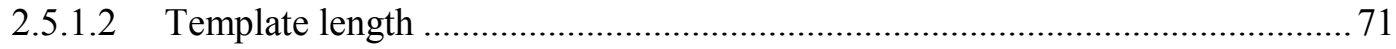

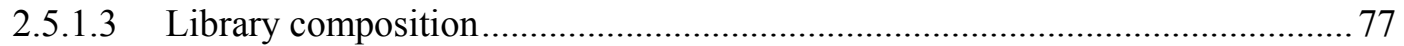

2.5.2 Aptamer target ................................................................................. 88

2.5.3 Selection conditions ............................................................................ 92

2.5.3.1 Metal cation concentration........................................................................... 93

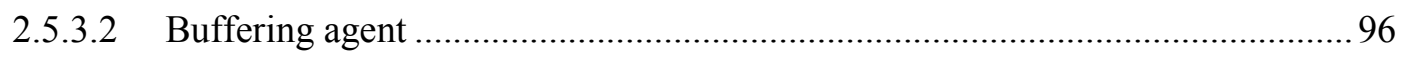

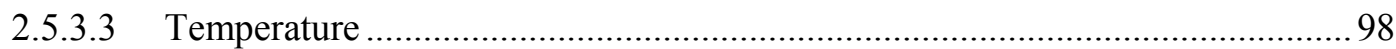

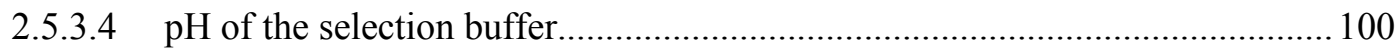

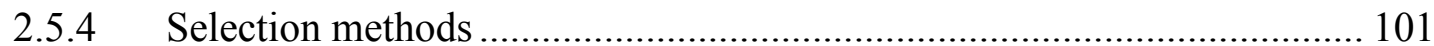

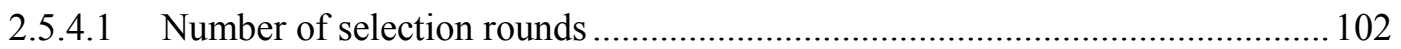

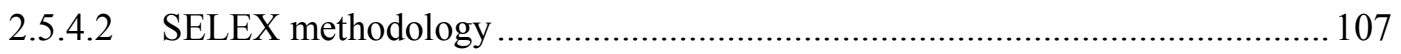

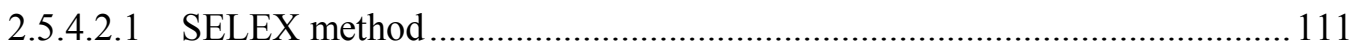

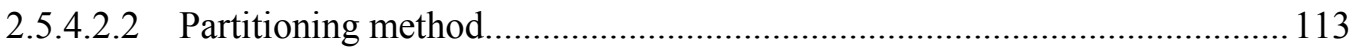

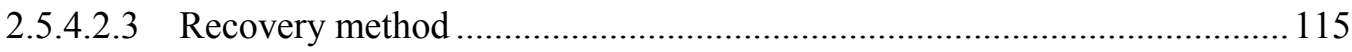

2.5.5 Affinity testing methodology …………………................................ 117

2.5.5.1 Buffering agent used in affinity experiment …………………….................. 117

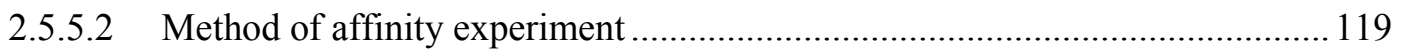

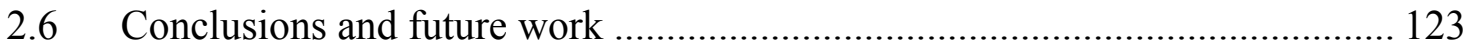

3 pHAST (pH-Driven Aptamer Switch for Thrombin) Catch-and-Release of Target

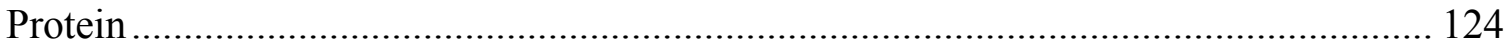

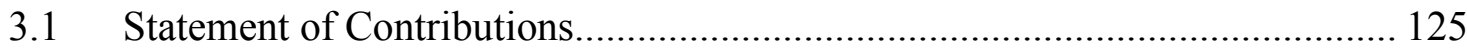

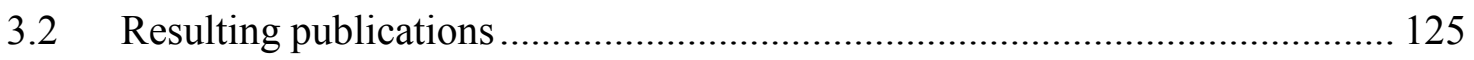

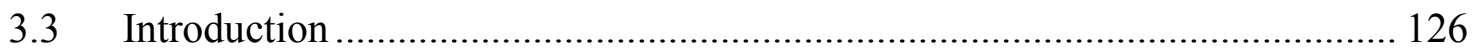

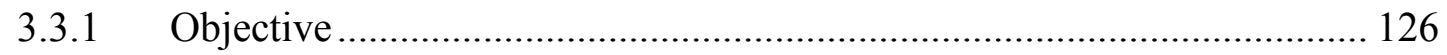

3.3.2 $\mathrm{pH}$ and the Central Nervous System.................................................... 126 


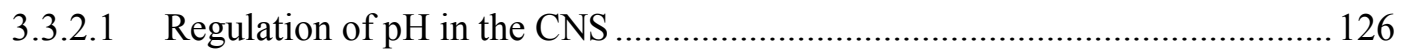

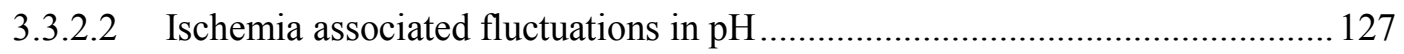

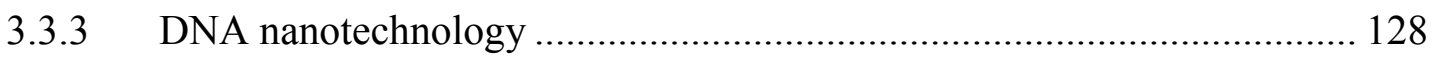

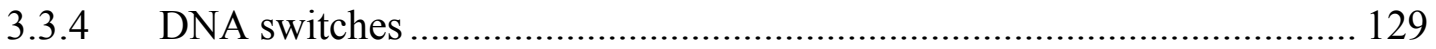

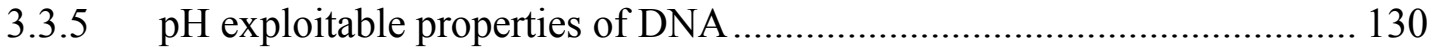

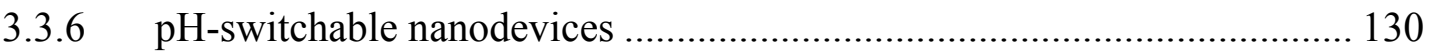

3.3.7 The incorporation of aptamer technology into functional nanomachines 131

3.3.8 DNA switches based on the $\mathrm{A}^{+}($anti $) \cdot \mathrm{G}($ syn $)$ mispair............................ 132

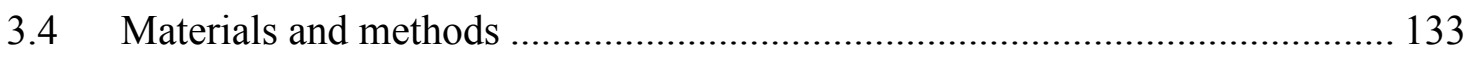

3.4.1 Reproduction from publication notice ................................................ 133

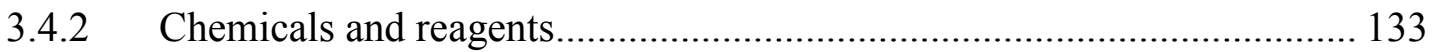

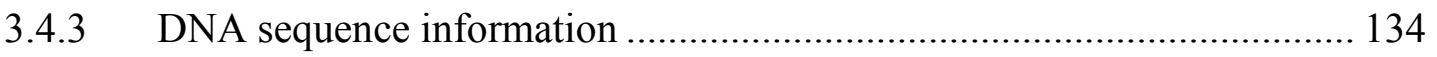

3.4.4 Structural Analysis by Melting Temperature determination ................... 135

3.4.5 Circular dichroism (near U/V) analyses of DNA samples...................... 136

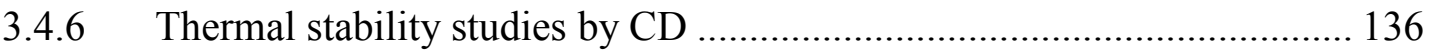

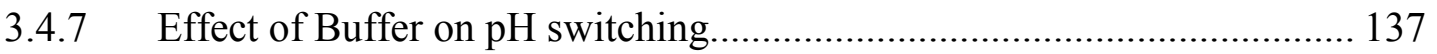

3.4.8 Ratiometric analysis of pHAST conformational change due to cyclic

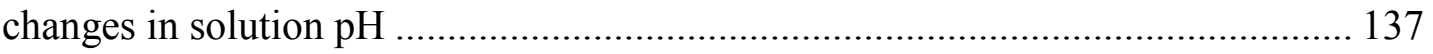

3.4.9 Conformational sensitivity to incremental change in solution $\mathrm{pH}$............ 138

3.4.10 Cy3-TBA and Cy3-pHAST binding to thrombin measured by

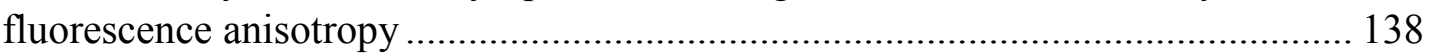

3.4.11 Catch-and-release of target protein by non-denaturing EMSA............ 139

3.4.12 Fluorescence anisotropy of Cy3-pHAST binding to thrombin over

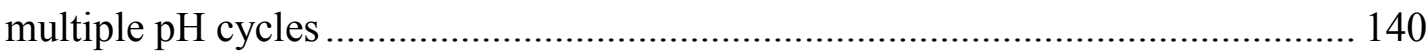

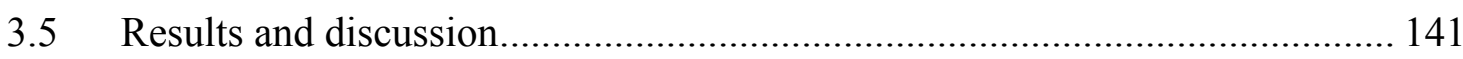

3.5.1 Design of the $\mathrm{pH}$-driven, aptamer-based unimolecular nanomachine

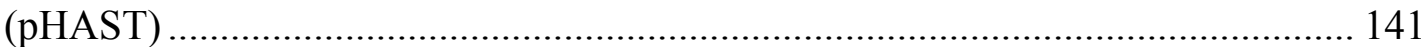

3.5.2 Evaluation of TBA and pHAST stability and structural transition by circular

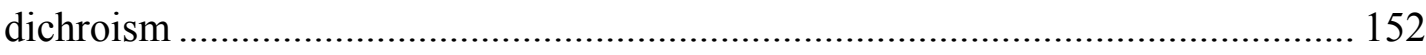

3.5.3 Determination of conformational switching efficiency ......................... 156

3.5.4 Evaluation of pHAST binding to thrombin ......................................... 164

3.5.5 Catch-and-Release of thrombin by Cy3-pHAST ................................... 168

3.6 Conclusions and future work ................................................................... 173 
4 Intra-Accumbens Injection of a Dopamine Aptamer Abates MK-801-Induced Cognitive Dysfunction in a model of Schizophrenia................................................ 174

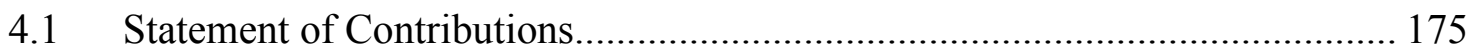

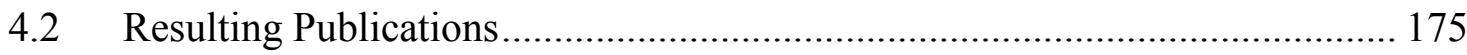

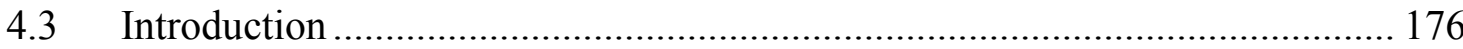

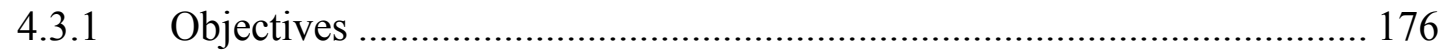

4.3.2 Schizophrenia - the dopamine hypothesis ....................................... 176

4.3.3 The ventral tegmental-nucleus accumbens reward circuit, NMDA receptors,

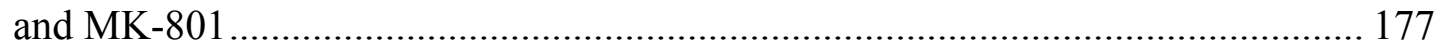

4.3.4 Justification for the MK-801 model of perseveration............................ 179

4.3.5 Dopamine-binding aptamers (DBAs) ............................................... 181

4.3.6 The dopamine-binding aptamer hypothesis ......................................... 182

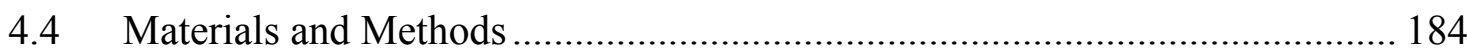

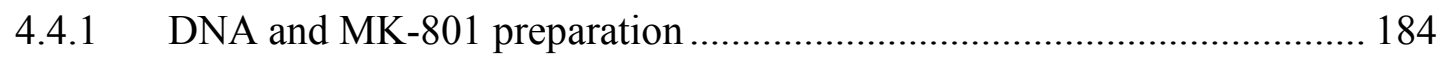

4.4.2 Ethics Statement............................................................................... 185

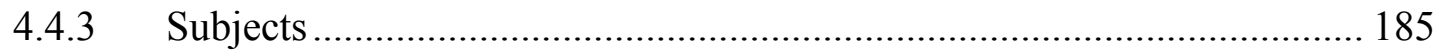

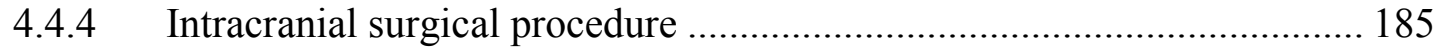

4.4.5 Behavioural testing: Operant Conditioning Procedure ............................ 186

4.4.6 Behavioural testing: Locomotor testing.............................................. 187

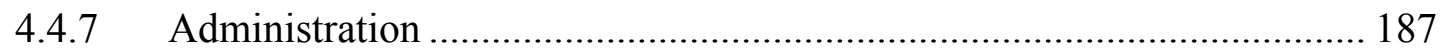

4.4.7.1 Central - intra accumbens injections ........................................................... 187

4.4.7.2 Peripheral - subcutaneous injections ......................................................... 188

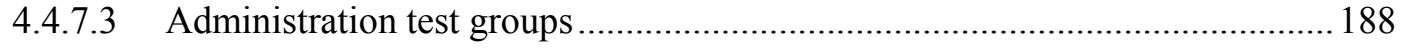

4.4.8 Confirmation of cannula placement ................................................. 188

4.4.9 Histology and Immunohistochemisty .................................................. 189

4.4.9.1 Preparation of samples for Immunohistochemistry ........................................ 189

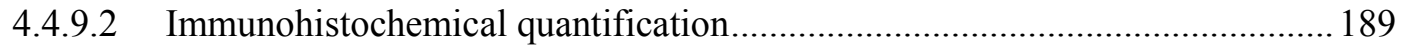

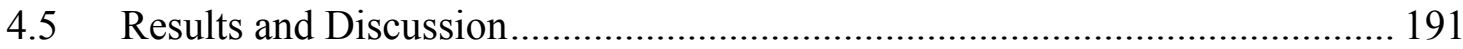

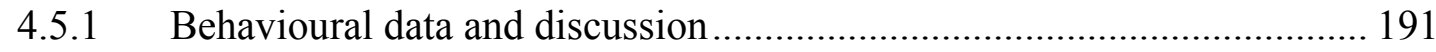

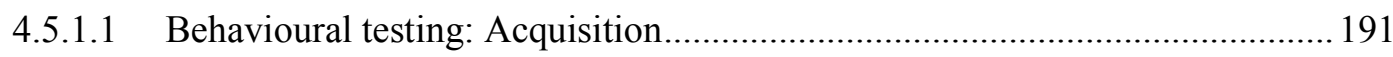

4.5.1.2 The efficacy of the DBA was tested in an MK-801 induced model of

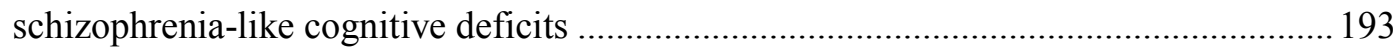

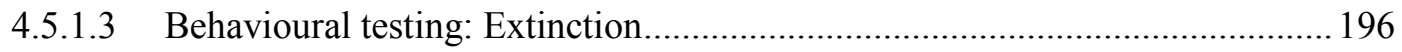




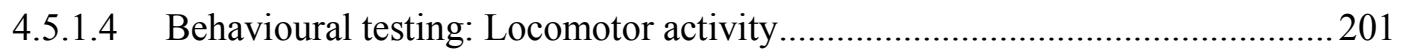

4.5.1.5 Behavioural effects as a result of the DBA's affinity for dopamine................ 203

4.5.2 Histology and Immunohistochemistry data and discussion...................... 205

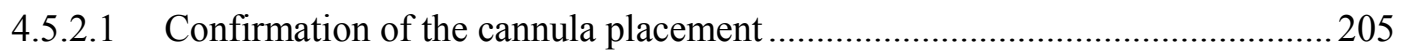

4.5.2.2 Immunohistochemical analysis of aptamer treated animals............................207

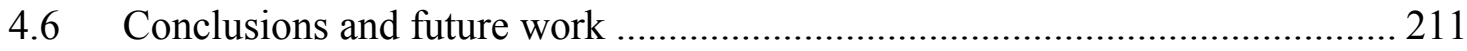

5 In vivo use of a multi-DNA aptamer-based payload/targeting system to study dopamine dysregulation in in the central nervous system ....................................... 212

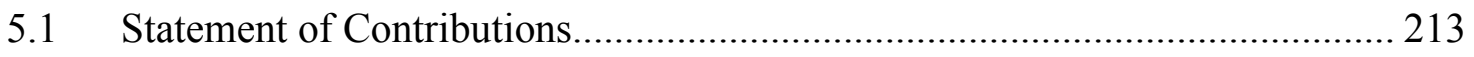

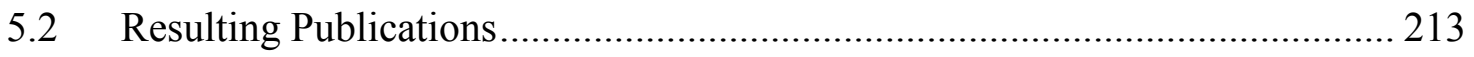

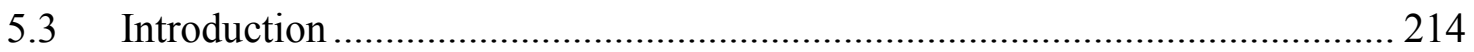

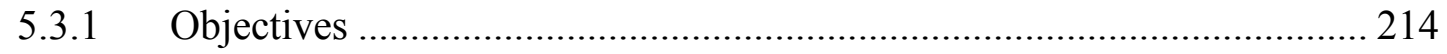

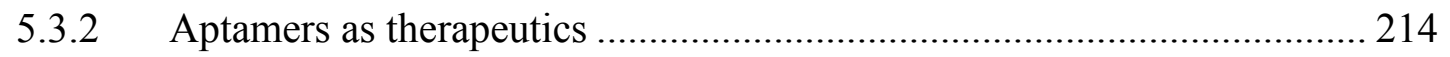

5.3.3 Intra-accumbens injection of a dopamine binding aptamer abates MK-801induced cognitive dysfunction in a model of Schizophrenia.................................. 215

5.3.4 The transferrin receptor aptamer and transferrin receptor mediated

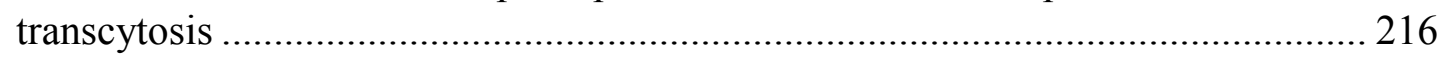

5.3.5 Design of the "Molecular Trojan Horse" inspired dopamine binding aptamer loaded-transferrin receptor aptamer modified liposomes (DAL-TRAM) . 217

5.3.6 Cocaine induced hyperlocomotion as a model for addiction................... 218

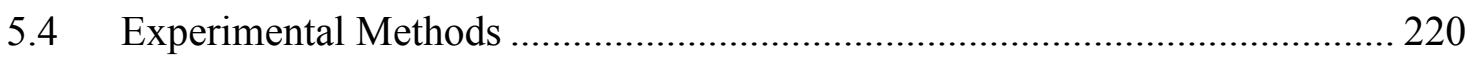

5.4.1 Chemicals and reagents - liposome and cocaine solution preparation..... 220

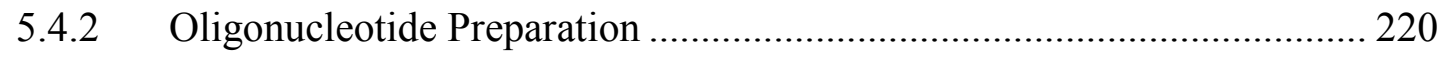

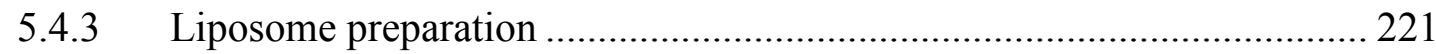

5.4.4 Transmission Electron Microscopy (TEM) imaging .............................. 223

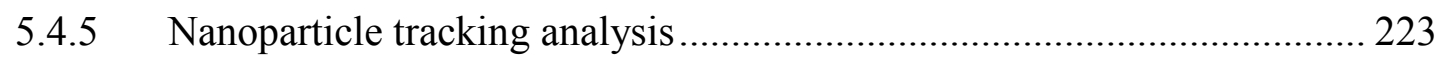

5.4.6 Fluorescent detection of DAL-TRAM in the Brain............................... 224

5.4.7 RT-qPCR detection of DBA delivered to the brain by DAL-TRAM ....... 225

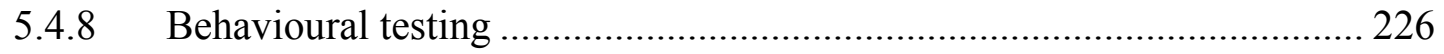

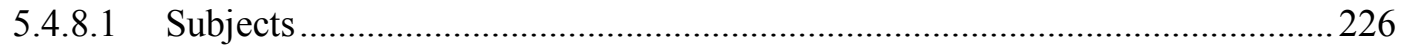

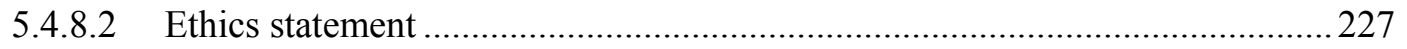

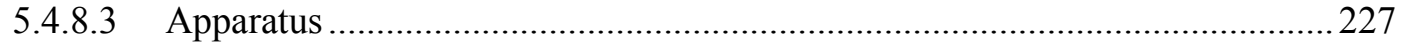


5.4.8.4 Experiment I and II: Examining the efficiency of DAL-TRAM in reducing cocaine-induced hyperlocomotion and the specificity of the DAL-TRAM components 227

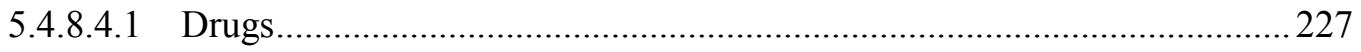

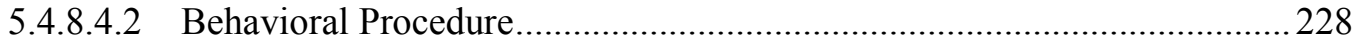

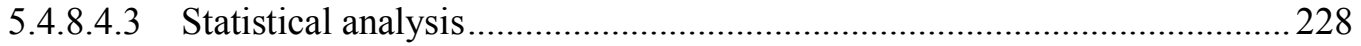

5.4.8.5 Experiment III A: Chronic administration of DAL-TRAM in saline treated

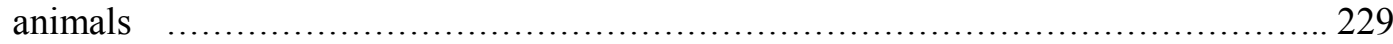

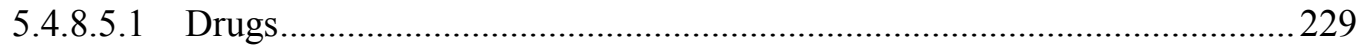

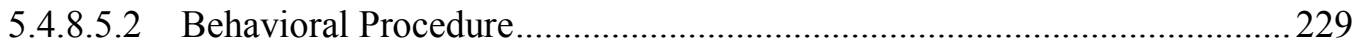

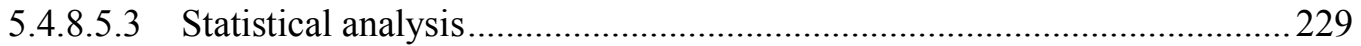

5.4.9 Experiment III B: Fluorescent histochemical staining by Fluoro-Jade B. 230

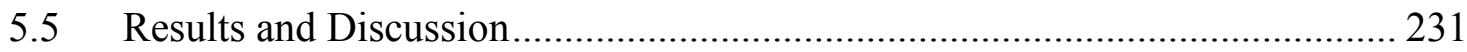

5.5.1 Design and synthesis of dopamine aptamer-loaded, transferrin receptor aptamer-modified (DAL-TRAM) liposomes................................................... 231

5.5.2 Detection of DAL-TRAM in the brain by fluorescent microscopy......... 242

5.5.3 Identification of systemically delivered DBA in brain tissue through amplification by RT-qPCR.

5.5.4 Behavioural and histochemical assessment of systemically administered DAL-TRAM

5.5.4.1 Experiment I: Examining the efficiency of DAL-TRAM in reducing cocaineinduced hyperlocomotion.

5.5.4.2 5.4.4.2 Experiment II: Variations of the multi-aptamer payload/targeting system (DAL-TRAM) were examined to determine the specific role of each component of the system

5.5.5 Experiment III: The effect of chronic administration of DAL-TRAM was examined by behavioural and histochemical methods

5.5.5.1 Experiment III A: Chronic administration of DAL-TRAM in saline treated

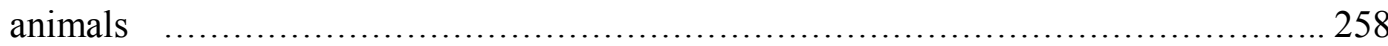

5.5.5.2 Experiment III B: Fluorescent histochemical investigation of neuronal

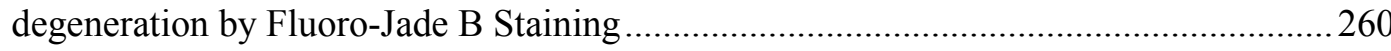

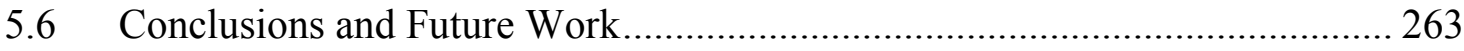

6 The selection of dopamine-binding aptamers for central nervous system related

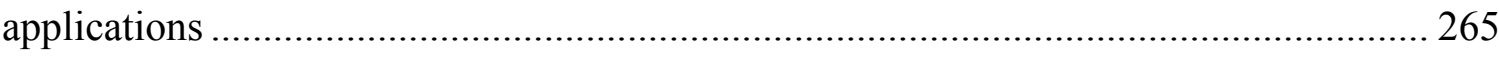

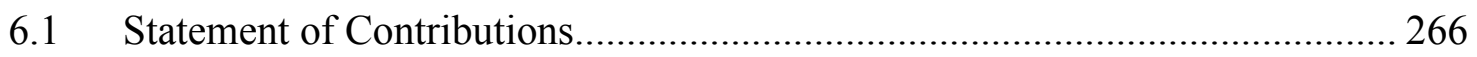

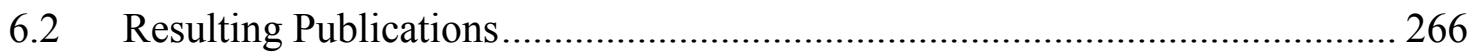

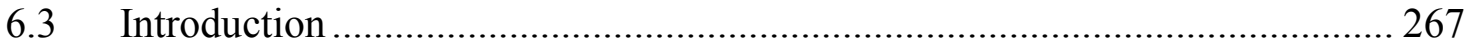




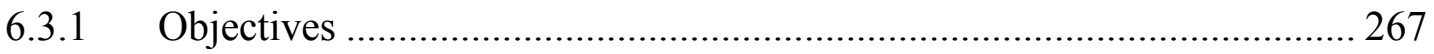

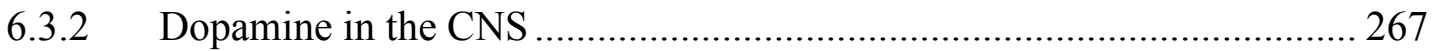

6.3.3 Existing dopamine binding aptamers (DBAs) ......................................... 267

6.3.4 Dopamine binding aptamer (DBA) based detection assays...................... 269

6.3.5 Development of an aptamer-gold nanoparticle (Apt-AuNP) based sensor for

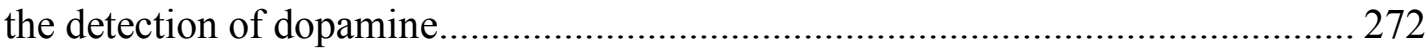

6.3.6 Expanding the functionality of DBA from simple detection.................... 274

6.3.7 Justification for the selection of novel dopamine binding aptamers......... 274

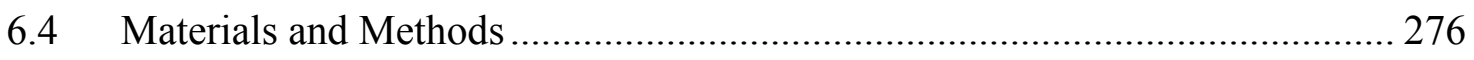

6.4.1 Selection of a DNA aptamer that binds to dopamine ............................... 276

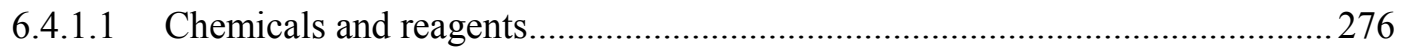

6.4.1.2 Oligonucleotide library preparation ............................................................. 276

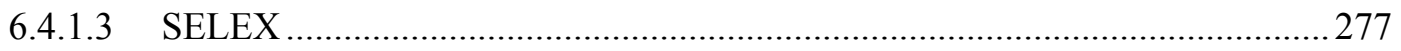

6.4.1.4 Amplification of the DNA library by polymerase chain reaction (PCR)........2278

6.4.1.5 Single-stranded DNA library purification by polyacrylamide gel electrophoresis

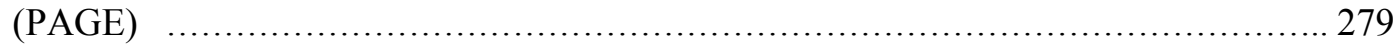

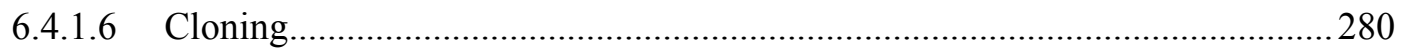

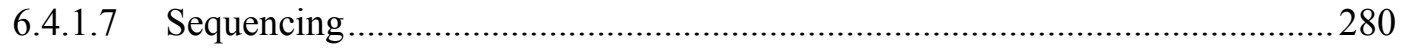

6.4.1.8 Screening of aptamer candidates by fluorescence anisotropy...........................281

6.4.1.9 Identification of minimal aptamers from DopaA20 ........................................2 281

6.4.2 Determination of aptamer stability by thermal denaturation .................... 281

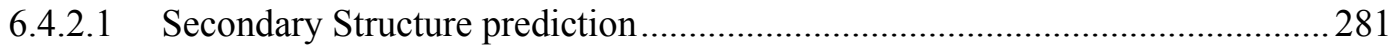

6.4.2.2 Investigation of secondary structure by thermal denaturation ..........................228

6.4.3 Determination of the affinity of DopaA20min for dopamine .................... 282

6.4.4 Development of an Aptamer-AuNP sensor for the detection of dopamine

6.4.4.1 Chemicals and reagents....................................................................... 284

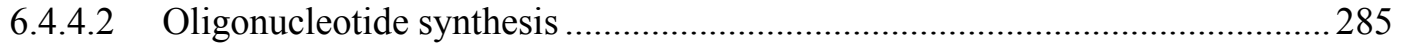

6.4.4.3 Gold nanoparticle (AuNP) synthesis........................................................... 285

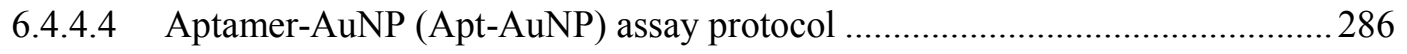

6.4.4.5 Determination of the apparent $\mathrm{K}_{\mathrm{D}}$ from the Apt-AuNP assay ........................ 287

6.4.4.6 Transmission electron microscopy (TEM) imaging ........................................ 288

6.4.5 Investigation of the efficacy of DopaA20 $\mathrm{min}$ in reducing cocaine-induced hyperlocomotion animal model ............................................................................ 288 


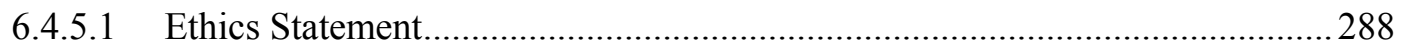

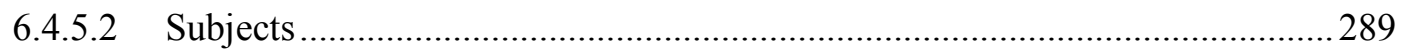

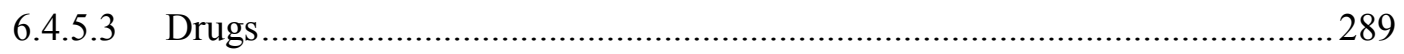

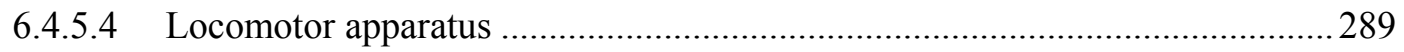

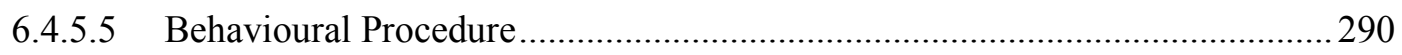

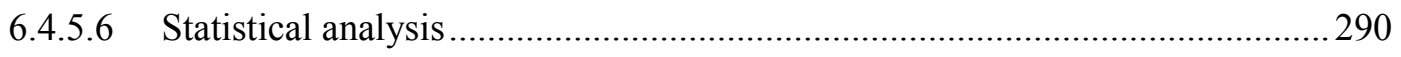

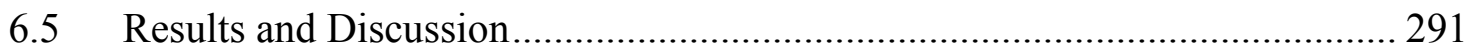

6.5.1 Selection of DNA aptamers for dopamine ............................................ 291

6.5.2 Physical characterization of DopaA20min .......................................... 292

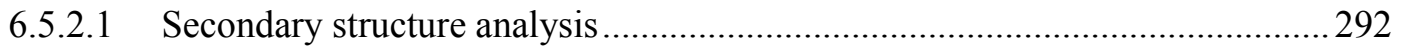

6.5.3 Evaluation of the affinity of DopaA20min for dopamine....................... 298

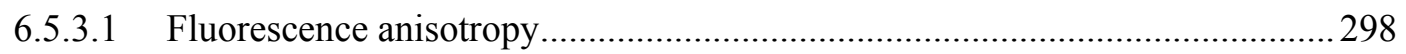

6.5.4 Development of an aptamer-gold nanoparticle (Apt-AuNP) based sensor for

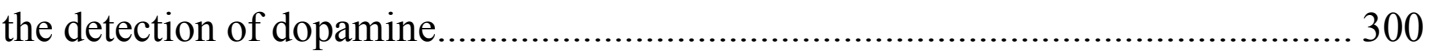

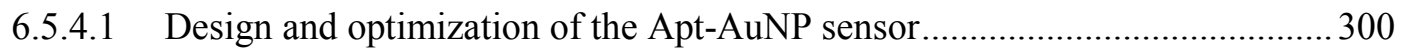

6.5.4.2 Determination of the linear dynamic range and detection limit of dopamine of

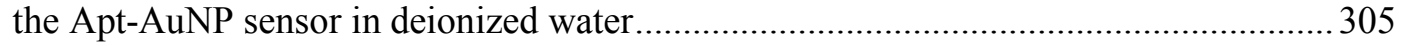

6.5.4.3 Characterization of the affinity of DopaA20min for dopamine by the Apt-AuNP

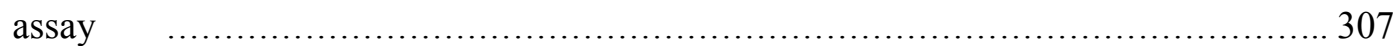

6.5.4.4 The selectivity of DopaA20min was determined by Apt-AuNP assay .............307

6.5.4.5 Detection of dopamine in artificial cerebral spinal fluid ................................... 311

6.5.5 Investigation of DopaA20min in a model of cocaine induced

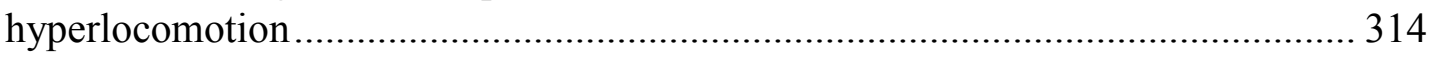

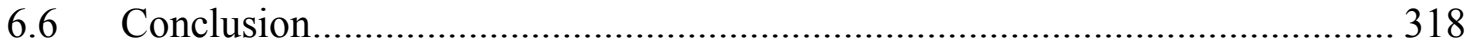

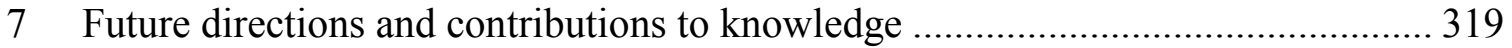

7.1 The use of aptamers for nervous system related applications ....................... 320

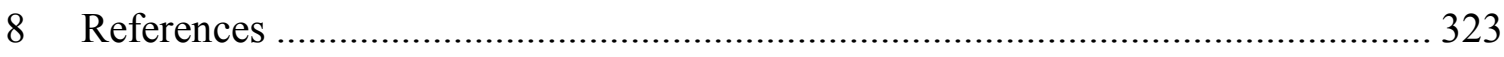

9 Appendix 2.1: Additional information for Chapter 3 "A comprehensive analysis of

SELEX and aptamer data from the Aptamer Base"'.................................................. 349

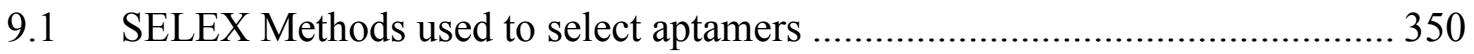

9.2 Partitioning methods used in SELEX experiments .................................. 351

9.3 Recovery methods used in SELEX experiments...................................... 352

9.4 Affinity methods used to determine the dissociation constant of selected

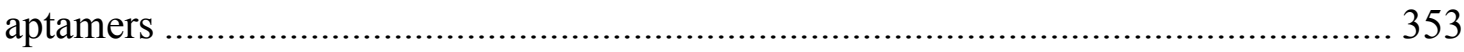


9.5 The Freebase Aptamer Base User Guide .................................................. 356

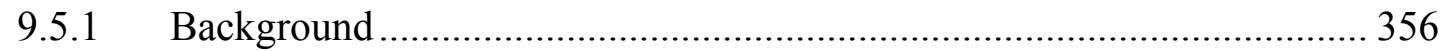

9.5.2 Modeling of Minimal Aptamers ........................................................... 360

9.5.3 Extracting Information from scientific literature ................................. 362

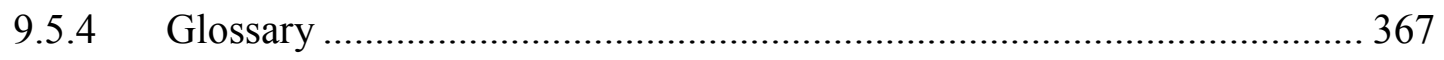

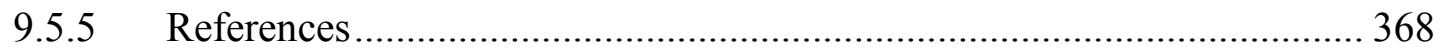




\subsection{List of Abbreviations}

A: adenine

AA: ascorbic acid

$\mathrm{A} \beta$ : beta amyloid peptide

AC: affinity chromatography

ACSF: artificial cerebrospinal fluid

AD: Alzheimer's disease

Ad5: adenovirus serotype-5

AE: affinity electrophoresis

AMPA: $\alpha$-amino-3-hydroxy-5methyl-4-isoxazole propionic acid

ANOVA: Analysis of variance

Ap-PTX-NP: aptamer-paclitaxel loaded-nanoparticles

Apt: aptamer

Apt-AuNP: aptamer-gold nanoparticle

Apt-MNC: aptamer-magnetic nanocrystals

ATP: adenosine triphosphate

AuNP: gold nanoparticle

BBB: blood-brain barrier 
BCSFB: blood-cerebrospinal fluid barrier

BoNT: Botulinum Neurotoxin

C: cytosine

CAA: cerebral amyloid angiopathy

CAR: Coxsackie and adenovirus receptor

CBA: cell binding assay

CD: circular dichroism

CE: capillary electrophoresis

CSF: cerebrospinal fluid

CGRP: Calcitonin gene-related peptide

ChEBI: Chemical Entities of Biological Interest

CJD: Creutzfeldt-Jacob disease

CNS: central nervous system

Comp: complement

Cy: Cyanine

DA: dopamine

DAL: Dopamine binding Aptamer Loaded liposome 
DAL-TRAM: Dopamine binding Aptamer Loaded-Transferrin Receptor Aptamer Modified liposomes

DA20m-TRAM: DopaA20min aptamer loaded-Transferrin Receptor Aptamer Modified liposomes

DBA: dopamine-binding aptamer

DDAB: Dimethyldioctadecylammonium (Bromide Salt)

DNA: deoxyribonucleic acid

DOI: digital object identifier

DRG: dorsal root ganglia

DR: detection/dynamic range

dsDNA: double-stranded DNA

DSPE-PEG 2000: 1,2-distearoyl-sn-glycero-3-phosphoethanolamine-N-[amino(polyethylene glycol)-2000] (ammonium salt)

DSPE-PEG 2000-malemide: 1,2-distearoyl-sn-glycero-3-phosphoethanolamine-N-

[maleimide(polyethylene glycol)-2000] (ammonium salt)

EDTA: Ethylenediaminetetraacetic acid

EGRFvIII: Epidermal growth factor receptor variant III

EGFRwt: Epidermal growth factor receptor wild-type

ELAA: enzyme-linked aptamer assay 
EMSA: electrophoretic mobility shift assay

ESI-MS: electrospray ionization mass spectrometry

FA: fluorescence anisotropy

FBA: filter binding assay

FC: flow cytometry

FP: fluorescence polarizations

FRET: Förster resonance energy transfer

G: guanine

G-quad: G-quadruplex

GABAA: $_{\gamma}$-Aminobutyric acid

HD: Huntington's disease

HEPES: 2-[4-(2-hydroxyethyl)piperazin-1-yl]ethanesulfonic acid

HPLC: high performance liquid chromatography

HTP: high throughput

HTPS: high throughput sequencing

i.p.: intraperitoneal

IAA: immunoaffinity assay

IL-6R: human interleukin-6 receptor 
ITC: isothermal titration calorimetry

$\mathrm{K}_{\mathrm{D}}$ : dissociation constant

KCE: kinetic capillary electrophoresis

L-DOPA : L-3,4-dihydroxyphenylalanine

LDL: low-density lipoprotein

Liss Rhod PE: 1,2-dipalmitoyl-sn-glycero-3-phosphoethanolamine-N-(lissamine rhodamine B sulfonyl) (ammonium salt)

LOD: limit of detection

LRP-1: LDL family receptor-related protein-1

LSD: least significant difference

MAG: myelin-associated glycoprotein

MAPK: Mitogen-activated protein kinase

MDI: myelin-derived inhibitor

MfNPs: multifunctional nanoparticles

MIPs: molecularly imprinted polymers

miRNA: microRNA

MK: MK-801

MRI: magnetic resonance imaging 
MS: multiple sclerosis

nAChR : nicotinic acetylcholine receptors

NAc: nucleus accumbens

NE: norepinephrine

NgR: Nogo-66 receptor

NMDA: N-methyl-D-aspartate

nt: nucleotide

NTA: nanoparticle tracking analysis

OMgp: oligodendrocyte myelin glycoprotein

PAGE: polyacrylamide gel electrophoresis

PBS: phosphate buffered saline

PC: partial complement

PCR: polymerase chain reaction

PD: Parkinson's disease

PDT: photodynamic therapy

PEG: polyethylene glycol

PET: positron emission tomography

pHAST: pH-driven Aptamer Switch for Thrombin 
PLGA: poly(lactic-co-glycolic acid)

PMID: Pubmed Identification

POPC: 1-palmitoyl-2-oleoyl-sn-glycero-3-phosphocholine

$\operatorname{PrP}^{\mathrm{C}}$ : normal cellular form prion protein

$\operatorname{PrP}^{\mathrm{Sc}}$ : abnormal, pathological form prion protein (Sc: scrapie)

pTH: phosphorylated tyrosine hydroxylase

RNA: ribonucleic acid

RO: random oligonucleotide

ROL-TRAM: Random Oligonucleotide loaded- Transferrin Receptor Aptamer Modified liposomes

RT-qPCR: real-time quantitative polymerase chain reaction

SCB: SELEX column buffer

SD: standard deviation

SELEX: Systematic Evolution of Ligands by EXponential enrichment

SEM: standard error of the mean

SNLPs: siRNA-containing stable nucleic acid lipid particles

SOMAmers: slow off-rate modified aptamers

SPR: surface plasmon resonance 
ssDNA: single-stranded DNA

ST: serotonin

Sub-TRAM: Substituted dopamine binding aptamer control loaded-Transferrin Receptor Aptamer Modified liposomes

$\mathrm{T}$ : thymine

TBA: Thrombin binding aptamer

TEM: transmission electron microscopy

TEMED: Tetramethylethylenediamine

TIC: tumor initiating cells

TH: tyrosine hydroxylase

$\mathrm{T}_{\mathrm{m}}$ : melting temperature

TMEV: Theiler's encephalomyelitis virus

TPA: tissue-type plasminogen activator

TfRs: transferrin receptor

TRA: transferrin receptor aptamer

TRAM: Transferrin Receptor Aptamer Modified liposomes

Tris: tris(hydroxymethyl) aminomethane

TSEs: Transmissible spongiform encephalopathies 
HSD: honest significant difference

Tyr: tyramine

$\mathrm{U}$ : uracil

UV: ultraviolet

VCAM-1: vascular cell adhesion molecule-1

VEGFR2: vascular endothelial growth factor receptor 2

VTA: ventral tegmental area

vWF: von Willebrand Factor 


\subsection{List of Figures}

Figure 1.1: Systematic Evolution of Ligands by EXponential enrichment (SELEX) is used to select aptamers with high affinity and selectivity for target molecules. Coloured lines represent aptamer candidates, grey stars represent the target molecule. (1) Initial library; inset - library template. (2) Selection target. (3) Library and target incubation. (4) Partitioning. (5) Amplification. (6) Enriched library. Typically 8-12 selection rounds are required to produce high quality aptamers.

Figure 1.2: Aptamer treatment induces recovery of neurite outgrowth in dorsal root ganglia (DRG). In control groups, neurons (200-500/group) were treated with buffer, a random oligonucleotide (RO RNA), myelin-derived inhibitors (MDIs), MDIs + RO RNA, or MDIs + soluble NgR. The experimental groups were treated with MDIs and one of five different aptamers (Clone 40 shown). $100 \mathrm{nM}$ of each MDI (Nogo-A,MAG and OMgp) was added (300 nM total concentration). Aptamers were added at $10 \mu \mathrm{M}$. Non-specific anti-beta III tubulin antibody was used to stain DRGs. The average neurite length was normalized by comparing groups to the buffer alone control. Scale bar $200 \mu \mathrm{m}$. Figure reproduced with permission under Creative Commons licensing from Wang, Y.; Khaing, Z. Z.; Li, N.; Hall, B.; Schmidt, C. E.; Ellington, A. D. PLoS One 2010, 5 (3), e9726.

Figure 1.3: Aptamer -antidote dependent fibrin clotting (A) and recovery of rotarod latency post stroke (B). In A, when the aptamer was bound to Factor IXa (in the absence of the antidote) it was able to act as an anticoagulator. When the antidote was present, the aptamer preferentially bound to the antidote (the aptamer's complementary oligonucleotide) allowing coagulation to occur. Shown in B, aptamer treated animals $(n=12)$ experienced significantly greater neurological functional recovery than those treated with an inactivated oligonucleotide control $(\mathrm{n}=12)$. Statistical analysis by repeated measures ANOVA with Fisher partial least squares difference post hoc analysis revealed a significant difference between groups $\mathrm{p}<0.05)$. Figure $3 \mathrm{~B}$ was produced from data presented in Blake, C. M.; Wang, H.; Laskowitz, D. T.; Sullenger, B. A. Oligonucleotides 2011, 21 (1), 11-19.

Figure 1.4: In vivo 2-photon microscopy was used to image plaques in the cortex (a) and amyloid (b) in the vasculature from an 18-month-old amyloid precursor protein (APP)/presenilin-1 (PS1) transgenic mouse that had received a topical application of the fluorescent aptamer probe 1 hour before imaging. Aptamer localization is shown in green and vasculature was visualized with Texas red-labelled dextran. Scale bars: $20 \mu \mathrm{m}$. CAA: cerebral amyloid angiopathy. Figure reproduced from Farrar et al., 2013 in accordance with the PLOS Creative Commons Attribution (CC BY) licence. ${ }^{167}$

Figure 1.5: Effect of paclitaxel (Taxol $\left.{ }^{\circledR}\right)$, Paclitaxel loaded nanoparticles (PTX-NP) and aptamermodified paclitaxel loaded nanoparticles (Ap-PTX-NP) on glioma xenograft ( $n=8$ nude mice). A) Tumor xenografts were removed from sacrificed animals at the study endpoint. B) The average tumor volume at the study endpoint for each group is shown. C) The average tumor weight at the study endpoint for each group is shown. Significant difference between the Ap-PTX-NP and Taxol groups as well as the PTX-NP and Taxol group are indicated by $* \mathrm{p}<0.05$, and $* * * \mathrm{p}<0.001$ respectively. The significant difference between the Ap-STX-NP and PTX-NP groups is shown by \# $\mathrm{p}<0.05$. "Reprinted from Biomarterials, 32 , Guo, J., Gao, X., Su, L., Xia, H., Gu, G., Pang, Z., Jiang, X., Yao, L., Chen, J., Chen, H., Aptamer 
functionalized PEG-PLGA nanoparticles for enhanced anti-glioma drug delivery, 8010-8020., Copyright (2011), with permission from Elsevier. ${ }^{234}$

Figure 2.1: General schematic of data relationships in the Aptamer Base. Topics and types are contained in light green coloured boxes. The unique identifiers for the RNA dopamine aptamer selection are shown in the dark green boxes. ${ }^{280}$

Figure 2.2: Additional SELEX experiment conditions. Topics are contained in light green coloured boxes. The RNA aptamer for dopamine example is continued. ${ }^{280}$ The unique identifiers for each topic is shown in dark green where applicable.

Figure 2.3: General schematic describing the aptamer and target components of the interaction topic (light green). Specific identifiers from the RNA aptamer for dopamine are shown in dark green boxes. ${ }^{280}$

Figure 2.4: General schematic of the relationship between affinity experiment and dissociation constant (shown in light green). Specific experimental identifiers for the RNA aptamer for dopamine are shown in dark green boxes. ${ }^{280}$

Figure 2.5: The relationship between a minimal aptamer and its ancestral sequence is represented generally (light green boxes). The specific identifiers for the minimal aptamer identified from the ancestral RNA aptamer for dopamine are shown in dark green boxes. ${ }^{280}$

Figure 2.6: DNA vs. RNA selections by year (1990-2013). A) The number of selection experiments performed using DNA (pink) vs. RNA (turquoise) templates by year. B) The total percentage of DNA versus RNA based selections for the entire period, pre-2007 and 2008-2013. Figure reprinted with permission of Springer from McKeague, M.; McConnell, E. M.; Cruz-Toledo, J.; Bernard, E. D.; Pach, A.; Mastronardi, E.; Zhang, X.; Beking, M.; Francis, T.; Giamberardino, A.; Cabecinha, A.; Ruscito, A.; Aranda-Rodriguez, R.; Dumontier, M.; DeRosa, M. C. Analysis of in vitro selection parameters. J. Mol. Evol. 2015, 81 (5), 150. Copyright Springer 2015.

Figure 2.7: Affinity of DNA (pink) versus RNA (turquoise) aptamers. The distribution of the highest affinity aptamers reported, represented by the $\log _{10}\left(\mathrm{~K}_{\mathrm{D}}\right)$. Figure reprinted with permission of Springer from McKeague, M.; McConnell, E. M.; Cruz-Toledo, J.; Bernard, E. D.; Pach, A.; Mastronardi, E.; Zhang, X.; Beking, M.; Francis, T.; Giamberardino, A.; Cabecinha, A.; Ruscito, A.; Aranda-Rodriguez, R.; Dumontier, M.; DeRosa, M. C. Analysis of in vitro selection parameters. J. Mol. Evol. 2015, 81 (5), 150. Copyright Springer 2015.

Figure 2.8: Distribution of sequence length for DNA (pink) versus RNA (turquoise) based selection experiments. An overlay of the distribution of the random region (of the pool template) length is shown. Sequence length is represented by number of nucleotides (nt). Figure reprinted with permission of Springer from McKeague, M.; McConnell, E. M.; Cruz-Toledo, J.; Bernard, E. D.; Pach, A.; Mastronardi, E.; Zhang, X.; Beking, M.; Francis, T.; Giamberardino, A.; Cabecinha, A.; Ruscito, A.; ArandaRodriguez, R.; Dumontier, M.; DeRosa, M. C. Analysis of in vitro selection parameters. J. Mol. Evol. 2015, 81 (5), 150. Copyright Springer 2015 
Figure 2.9: Relationship between random region length and sequence space. A) As the length (N) of the template random region increases, the size of the sequence space $\left(4^{\mathrm{N}}\right)$ increases as well. B) However, as the length of the template random region increases, due to the practical experimental limitations, the fraction of the sequence space that is sampled decreases.

Figure 2.10: Linear regression analysis of template length vs. affinity $\left(\log _{10}\left(K_{D}\right)\right)$. The best affinity aptamers from selection experiments between the years 1990-2013 are shown. Figure reprinted with permission of Springer from McKeague, M.; McConnell, E. M.; Cruz-Toledo, J.; Bernard, E. D.; Pach, A.; Mastronardi, E.; Zhang, X.; Beking, M.; Francis, T.; Giamberardino, A.; Cabecinha, A.; Ruscito, A.; Aranda-Rodriguez, R.; Dumontier, M.; DeRosa, M. C. Analysis of in vitro selection parameters. J. Mol. Evol. 2015, 81 (5), 150. Copyright Springer 2015.

Figure 2.11: Base frequencies of DNA (pink) versus RNA (turquoise) aptamers selected between the years 1990-2012. Figure reprinted with permission of Springer from McKeague, M.; McConnell, E. M.; Cruz-Toledo, J.; Bernard, E. D.; Pach, A.; Mastronardi, E.; Zhang, X.; Beking, M.; Francis, T.; Giamberardino, A.; Cabecinha, A.; Ruscito, A.; Aranda-Rodriguez, R.; Dumontier, M.; DeRosa, M. C. Analysis of in vitro selection parameters. J. Mol. Evol. 2015, 81 (5), 150. Copyright Springer 2015....... 78

Figure 2.12: Linear regression analysis of affinity versus $\%$ base content for RNA aptamers selected between the years of 1990-2012. The \%G (orange) and \%C (purple) contents (top) and \%A (green) and $\% \mathrm{U}$ (red) contents (bottom) are shown. Figure reprinted with permission of Springer from McKeague, M.; McConnell, E. M.; Cruz-Toledo, J.; Bernard, E. D.; Pach, A.; Mastronardi, E.; Zhang, X.; Beking, M.; Francis, T.; Giamberardino, A.; Cabecinha, A.; Ruscito, A.; Aranda-Rodriguez, R.; Dumontier, M.; DeRosa, M. C. Analysis of in vitro selection parameters. J. Mol. Evol. 2015, 81 (5), 150. Copyright Springer 2015.

Figure 2.13: Linear regression analysis of affinity versus \% base content of DNA aptamers selected between the years 1990-2012. The \%G (orange) and \%C (purple) contents (Top) and \%A (green) and \% (red) contents (bottom) are shown. Figure reprinted with permission of Springer from McKeague, M.; McConnell, E. M.; Cruz-Toledo, J.; Bernard, E. D.; Pach, A.; Mastronardi, E.; Zhang, X.; Beking, M.; Francis, T.; Giamberardino, A.; Cabecinha, A.; Ruscito, A.; Aranda-Rodriguez, R.; Dumontier, M.; DeRosa, M. C. Analysis of in vitro selection parameters. J. Mol. Evol. 2015, 81 (5), 150. Copyright Springer 2015.

Figure 2.14: \%G content of DNA aptamers for small molecules (left) compared to protein (right) targets. Linear regression analysis was performed on data from the years 1990-2012. Figure reprinted with permission of Springer from McKeague, M.; McConnell, E. M.; Cruz-Toledo, J.; Bernard, E. D.; Pach, A.; Mastronardi, E.; Zhang, X.; Beking, M.; Francis, T.; Giamberardino, A.; Cabecinha, A.; Ruscito, A.; Aranda-Rodriguez, R.; Dumontier, M.; DeRosa, M. C. Analysis of in vitro selection parameters. J. Mol. Evol. 2015, 81 (5), 150. Copyright Springer 2015.

Figure 2.15: Combined \%GC (left) and \%AT (right) content for DNA. Linear regression analysis was performed on data collected between the years of 1990-2012. Figure reprinted with permission of Springer from McKeague, M.; McConnell, E. M.; Cruz-Toledo, J.; Bernard, E. D.; Pach, A.; Mastronardi, E.; Zhang, X.; Beking, M.; Francis, T.; Giamberardino, A.; Cabecinha, A.; Ruscito, A.; Aranda- 
Rodriguez, R.; Dumontier, M.; DeRosa, M. C. Analysis of in vitro selection parameters. J. Mol. Evol. 2015, 81 (5), 150. Copyright Springer 2015.

Figure 2.16: Combined \%GC (left) and \%AT (right) content for RNA. Linear regression analysis was performed on data collected between the years of 1990-2012. Figure reprinted with permission of Springer from McKeague, M.; McConnell, E. M.; Cruz-Toledo, J.; Bernard, E. D.; Pach, A.; Mastronardi, E.; Zhang, X.; Beking, M.; Francis, T.; Giamberardino, A.; Cabecinha, A.; Ruscito, A.; ArandaRodriguez, R.; Dumontier, M.; DeRosa, M. C. Analysis of in vitro selection parameters. J. Mol. Evol. 2015, 81 (5), 150. Copyright Springer 2015.

Figure 2.17: Linear regression analysis of the $\% \mathrm{GC}$ content for the best aptamer versus aptamer affinity $\left(\log _{10}\left(K_{D}\right)\right)$. Figure reprinted with permission of Springer from McKeague, M.; McConnell, E. M.; CruzToledo, J.; Bernard, E. D.; Pach, A.; Mastronardi, E.; Zhang, X.; Beking, M.; Francis, T.; Giamberardino, A.; Cabecinha, A.; Ruscito, A.; Aranda-Rodriguez, R.; Dumontier, M.; DeRosa, M. C. Analysis of in vitro selection parameters. J. Mol. Evol. 2015, 81 (5), 150. Copyright Springer 2015. 86

Figure 2.18: Distribution of \%GC content of all aptamers (1990-2012). Figure reprinted with permission of Springer from McKeague, M.; McConnell, E. M.; Cruz-Toledo, J.; Bernard, E. D.; Pach, A.; Mastronardi, E.; Zhang, X.; Beking, M.; Francis, T.; Giamberardino, A.; Cabecinha, A.; Ruscito, A.; Aranda-Rodriguez, R.; Dumontier, M.; DeRosa, M. C. Analysis of in vitro selection parameters. J. Mol. Evol. 2015, 81 (5), 150. Copyright Springer 2015.

Figure 2.19: A) Percentage of aptamers selected sorted by target type. B) Occurrence of target type for aptamer selections. Data for both figures encompasses selections from 1990-2013. Figure reprinted with permission of Springer from McKeague, M.; McConnell, E. M.; Cruz-Toledo, J.; Bernard, E. D.; Pach, A.; Mastronardi, E.; Zhang, X.; Beking, M.; Francis, T.; Giamberardino, A.; Cabecinha, A.; Ruscito, A.; Aranda-Rodriguez, R.; Dumontier, M.; DeRosa, M. C. Analysis of in vitro selection parameters. J. Mol. Evol. 2015, 81 (5), 150. Copyright Springer 2015.

Figure 2.20: Mean affinity $\left(\log _{10}\left(\mathrm{~K}_{\mathrm{D}}\right)\right)$ of selected aptamers for different target types. Error bars represent standard deviation. Figure reprinted with permission of Springer from McKeague, M.; McConnell, E. M.; Cruz-Toledo, J.; Bernard, E. D.; Pach, A.; Mastronardi, E.; Zhang, X.; Beking, M.; Francis, T.; Giamberardino, A.; Cabecinha, A.; Ruscito, A.; Aranda-Rodriguez, R.; Dumontier, M.; DeRosa, M. C. Analysis of in vitro selection parameters. J. Mol. Evol. 2015, 81 (5), 150. Copyright Springer 2015.

Figure 2.21: Boxplot of the mean affinity $\left(\log _{10}\left(K_{D}\right)\right)$ of common buffering agents. Error represents standard deviation. Figure reprinted with permission of Springer from McKeague, M.; McConnell, E. M.; Cruz-Toledo, J.; Bernard, E. D.; Pach, A.; Mastronardi, E.; Zhang, X.; Beking, M.; Francis, T.; Giamberardino, A.; Cabecinha, A.; Ruscito, A.; Aranda-Rodriguez, R.; Dumontier, M.; DeRosa, M. C. Analysis of in vitro selection parameters. J. Mol. Evol. 2015, 81 (5), 150. Copyright Springer 2015....... 97

Figure 2.22: Boxplot of mean affinity $\left(\log _{10}\left(\mathrm{~K}_{\mathrm{D}}\right)\right)$ based on selection temperature. Figure reprinted with permission of Springer from McKeague, M.; McConnell, E. M.; Cruz-Toledo, J.; Bernard, E. D.; Pach, A.; Mastronardi, E.; Zhang, X.; Beking, M.; Francis, T.; Giamberardino, A.; Cabecinha, A.; Ruscito, A.; 
Aranda-Rodriguez, R.; Dumontier, M.; DeRosa, M. C. Analysis of in vitro selection parameters. J. Mol. Evol. 2015, 81 (5), 150. Copyright Springer 2015.

Figure 2.23: Linear regression analysis of the relationship between affinity $\left(\log _{10}\left(\mathrm{~K}_{\mathrm{D}}\right)\right)$ and $\mathrm{pH}$. Figure reprinted with permission of Springer from McKeague, M.; McConnell, E. M.; Cruz-Toledo, J.; Bernard, E. D.; Pach, A.; Mastronardi, E.; Zhang, X.; Beking, M.; Francis, T.; Giamberardino, A.; Cabecinha, A.; Ruscito, A.; Aranda-Rodriguez, R.; Dumontier, M.; DeRosa, M. C. Analysis of in vitro selection parameters. J. Mol. Evol. 2015, 81 (5), 150. Copyright Springer 2015.

Figure 2.24: The distribution of frequency of SELEX experiments using a particular number of selection rounds is shown. Selection experiments were further divided by nucleic acid type; DNA (pink) and RNA (turquoise). Figure reprinted with permission of Springer from McKeague, M.; McConnell, E. M.; CruzToledo, J.; Bernard, E. D.; Pach, A.; Mastronardi, E.; Zhang, X.; Beking, M.; Francis, T.; Giamberardino, A.; Cabecinha, A.; Ruscito, A.; Aranda-Rodriguez, R.; Dumontier, M.; DeRosa, M. C. Analysis of in vitro selection parameters. J. Mol. Evol. 2015, 81 (5), 150. Copyright Springer 2015. 102

Figure 2.25: Linear regression analysis of number of selection rounds compared to aptamer affinity $\left(\log _{10}\left(K_{D}\right)\right)$. Figure reprinted with permission of Springer from McKeague, M.; McConnell, E. M.; CruzToledo, J.; Bernard, E. D.; Pach, A.; Mastronardi, E.; Zhang, X.; Beking, M.; Francis, T.; Giamberardino, A.; Cabecinha, A.; Ruscito, A.; Aranda-Rodriguez, R.; Dumontier, M.; DeRosa, M. C. Analysis of in vitro selection parameters. J. Mol. Evol. 2015, 81 (5), 150. Copyright Springer 2015. 104

Figure 2.26: Representative minimum (green) and maximum (red) affinity domains in the sequence space of a selection library. 105

Figure 2.27: Comparison of SELEX method frequency by target type. Figure reprinted with permission of Springer from McKeague, M.; McConnell, E. M.; Cruz-Toledo, J.; Bernard, E. D.; Pach, A.; Mastronardi, E.; Zhang, X.; Beking, M.; Francis, T.; Giamberardino, A.; Cabecinha, A.; Ruscito, A.; Aranda-Rodriguez, R.; Dumontier, M.; DeRosa, M. C. Analysis of in vitro selection parameters. J. Mol. Evol. 2015, 81 (5), 150. Copyright Springer 2015.

Figure 2.28: Comparison of partitioning method frequency by target type. Figure reprinted with permission of Springer from McKeague, M.; McConnell, E. M.; Cruz-Toledo, J.; Bernard, E. D.; Pach, A.; Mastronardi, E.; Zhang, X.; Beking, M.; Francis, T.; Giamberardino, A.; Cabecinha, A.; Ruscito, A.; Aranda-Rodriguez, R.; Dumontier, M.; DeRosa, M. C. Analysis of in vitro selection parameters. J. Mol. Evol. 2015, 81 (5), 150. Copyright Springer 2015.

Figure 2.29: Comparison of recovery method frequency by target type. Figure reprinted with permission of Springer from McKeague, M.; McConnell, E. M.; Cruz-Toledo, J.; Bernard, E. D.; Pach, A.; Mastronardi, E.; Zhang, X.; Beking, M.; Francis, T.; Giamberardino, A.; Cabecinha, A.; Ruscito, A.; Aranda-Rodriguez, R.; Dumontier, M.; DeRosa, M. C. Analysis of in vitro selection parameters. J. Mol. Evol. 2015, 81 (5), 150. Copyright Springer 2015.

Figure 2.30: Boxplot of affinity $\left(\log _{10}\left(K_{D}\right)\right)$ by SELEX method. Figure reprinted with permission of Springer from McKeague, M.; McConnell, E. M.; Cruz-Toledo, J.; Bernard, E. D.; Pach, A.; Mastronardi, E.; Zhang, X.; Beking, M.; Francis, T.; Giamberardino, A.; Cabecinha, A.; Ruscito, A.; Aranda- 
Rodriguez, R.; Dumontier, M.; DeRosa, M. C. Analysis of in vitro selection parameters. J. Mol. Evol. 2015, 81 (5), 150. Copyright Springer 2015.

Figure 2.31: Boxplot of affinity by partitioning round. Figure reprinted with permission of Springer from McKeague, M.; McConnell, E. M.; Cruz-Toledo, J.; Bernard, E. D.; Pach, A.; Mastronardi, E.; Zhang, X.; Beking, M.; Francis, T.; Giamberardino, A.; Cabecinha, A.; Ruscito, A.; Aranda-Rodriguez, R.; Dumontier, M.; DeRosa, M. C. Analysis of in vitro selection parameters. J. Mol. Evol. 2015, 81 (5), 150. Copyright Springer 2015 114

Figure 2.32: Boxplot of affinity by recovery method. Figure reprinted with permission of Springer from McKeague, M.; McConnell, E. M.; Cruz-Toledo, J.; Bernard, E. D.; Pach, A.; Mastronardi, E.; Zhang, X.; Beking, M.; Francis, T.; Giamberardino, A.; Cabecinha, A.; Ruscito, A.; Aranda-Rodriguez, R.; Dumontier, M.; DeRosa, M. C. Analysis of in vitro selection parameters. J. Mol. Evol. 2015, 81 (5), 150. Copyright Springer 2015. .116

Figure 2.33: Box plot comparison of mean affinity $\left(\log _{10}\left(K_{D}\right)\right)$ of the buffering agent used in affinity experiments. Figure reprinted with permission of Springer from McKeague, M.; McConnell, E. M.; CruzToledo, J.; Bernard, E. D.; Pach, A.; Mastronardi, E.; Zhang, X.; Beking, M.; Francis, T.; Giamberardino, A.; Cabecinha, A.; Ruscito, A.; Aranda-Rodriguez, R.; Dumontier, M.; DeRosa, M. C. Analysis of in vitro selection parameters. J. Mol. Evol. 2015, 81 (5), 150. Copyright Springer 2015. 118

Figure 2.34: Comparison of the mean affinity between different affinity characterization methods. Abbreviations in parentheses: Affinity chromatography (AC), affinity electrophoresis (AE), capillary electrophoresis (CE), cell binding assay (CBA), dialysis, filter binding assay (FBA), flow cytometry (FC), fluorescence, fluorescence polarization (FP), immunoaffinity assay (IAA), isothermal titration calorimetry (ITC), kinetic capillary electrophoresis (KCE), not described, surface plasmon resonance (SPR) and, UVVisible Spectroscopy. Figure reprinted with permission of Springer from McKeague, M.; McConnell, E. M.; Cruz-Toledo, J.; Bernard, E. D.; Pach, A.; Mastronardi, E.; Zhang, X.; Beking, M.; Francis, T.; Giamberardino, A.; Cabecinha, A.; Ruscito, A.; Aranda-Rodriguez, R.; Dumontier, M.; DeRosa, M. C. Analysis of in vitro selection parameters. J. Mol. Evol. 2015, 81 (5), 150. Copyright Springer 2015..... 119

Figure 2.35: Aptamer characterization workflow. Figure reproduced with permission from the American Chemical Society. McKeague, M.; De Girolamo, A.; Valenzano, S.; Pascale, M.; Ruscito, A.; Velu, R.; Frost, N. R.; Hill, K.; Smith, M.; McConnell, E. M.; DeRosa, M. C. Comprehensive analytical comparison of strategies used for small molecules aptamer evaluation. Anal. Chem. 2015, 87 (17), 8608. Copyright 2015 American Chemical Society.

Figure 3.1: The $\mathrm{A}^{+}$(anti)•G(syn) mispair (right) that forms only in acidic conditions could be used to develop $\mathrm{pH}$-driven nanoswitches from aptamer sequences that form G-quadruplex secondary structures from multiple G-quartets (left). Reprinted in part with permission from McConnell, EM., Bolzon, R., Mezin, P., Frahm, G., Johnston, M and DeRosa, MC. pHAST (pH-Driven Aptamer Switch for Thrombin) Catch-and-Release of Target Protein. 2016, 27: 1493-1499. Bioconjugate Chemistry. Copyright 2016 American Chemical Society. 132

Figure 3.2: Schematic representation of pHAST. The catch-and-release of the target, thrombin, is regulated by the $\mathrm{pH}$-driven confirmation of $\mathrm{pHAST}$. The $\mathrm{Cy} 3 / \mathrm{Cy} 5-\mathrm{pHAST}$ was designed so that at neutral 
$\mathrm{pH}$ it exists in the G-quad conformation and the thrombin binding domain is capable of binding to thrombin. Upon acidification, the Cy3/Cy5-pHAST experiences a conformational change such that the Gquadruplex is disrupted and the hairpin forms. This conformational change blocks the thrombin binding dopamine and as a result the affinity of the pHAST towards thrombin is eliminated. Dashed blue bars indicate G-quadruplex formation and red bars indicate $A^{+}$(anti) $G($ syn) base pairs. Reprinted with permission from McConnell, EM., Bolzon, R., Mezin, P., Frahm, G., Johnston, M and DeRosa, MC. pHAST (pH-Driven Aptamer Switch for Thrombin) Catch-and-Release of Target Protein. 2016, 27: 14931499. Bioconjugate Chemistry. Copyright 2016 American Chemical Society.

Figure 3.3: The location of inserted $\mathrm{A}^{+}(\mathrm{anti}) \cdot \mathrm{G}(\mathrm{syn})$ base pairs in each partially complementary sequence (PC) with respect to the TBA and its fully complementary sequence (Comp) are shown. Abbreviations: TBA (thrombin binding aptamer), Comp (TBA complement). Sequences were designed with terminal (PC1), internal (PC2) or both terminal and internal (PC3) $A^{+}$(anti) $\cdot \mathrm{G}(\mathrm{syn})$ base pairs. A 5'-CTCT-3' linker was used to create the unimolecular pHAST from TBA+PC3. Reprinted with permission from McConnell, EM., Bolzon, R., Mezin, P., Frahm, G., Johnston, M and DeRosa, MC. pHAST (pH-Driven Aptamer Switch for Thrombin) Catch-and-Release of Target Protein. 2016, 27: 1493-1499. Bioconjugate Chemistry. Copyright 2016 American Chemical Society.....

Figure 3.4: Tm profiles of TBA were obtained by UV-Vis thermal denaturation. The absorbance was measured for the TBA in both neutral ( $\mathrm{pH} 7.5)$ and acidic (pH 5.0) conditions at either $260 \mathrm{~nm}$ or $295 \mathrm{~nm}$.

Figure 3.5: Tm profiles of TBA+COMP were obtained by UV-Vis thermal denaturation. The absorbance was measured for the TBA+COMP in both neutral ( $\mathrm{pH} 7.5)$ and acidic ( $\mathrm{pH}$ 5.0) conditions at either 260 $\mathrm{nm}$ or $295 \mathrm{~nm}$.

Figure 3.6: $\mathrm{Tm}$ profiles of $\mathrm{TBA}+\mathrm{PC} 1$ were obtained by UV-Vis thermal denaturation. The absorbance was measured for the TBA+PC1 in both neutral ( $\mathrm{pH}$ 7.5) and acidic $(\mathrm{pH} \mathrm{5.0)}$ conditions at either $260 \mathrm{~nm}$ or $295 \mathrm{~nm}$.

Figure 3.7: Tm profiles of TBA $+\mathrm{PC} 2$ were obtained by $\mathrm{UV}-\mathrm{V}$ is thermal denaturation. The absorbance was measured for the TBA+PC2 in both neutral ( $\mathrm{pH} 7.5)$ and acidic (pH 5.0) conditions at either $260 \mathrm{~nm}$ or $295 \mathrm{~nm}$.

Figure 3.8: Tm profiles of TBA $+\mathrm{PC} 3$ were obtained by UV-Vis thermal denaturation. The absorbance was measured for the TBA+PC3 in both neutral ( $\mathrm{pH} 7.5)$ and acidic (pH 5.0) conditions at either $260 \mathrm{~nm}$ or $295 \mathrm{~nm}$.

Figure 3.9: The stability of TBA at neutral and acidic $\mathrm{pH}$ and the stability of $\mathrm{pHAST}$ at neutral $\mathrm{pH}$ was confirmed by circular dichroism. (A) CD spectra of TBA $(2.5 \mu \mathrm{M})$ in either $0.1 \mathrm{M}$ sodium phosphate buffer $(140 \mathrm{mM} \mathrm{NaCl}, 0.5 \mathrm{mM} \mathrm{KCl}, \mathrm{pH} 7.5)$ or $0.1 \mathrm{M}$ sodium acetate buffer $(140 \mathrm{mM} \mathrm{NaCl}, 0.5 \mathrm{mM} \mathrm{KCl}$, $\mathrm{pH} 5.0)$ at $20^{\circ} \mathrm{C}$. Each of the spectrum shown represent the average of 5 scans of a single sample. (B) Relative $\left(\mathrm{F}_{(\mathrm{obs})}\right)$ ellipticity of TBA $(2.5 \mu \mathrm{M})$ in either $0.1 \mathrm{M}$ sodium phosphate buffer $(140 \mathrm{mM} \mathrm{NaCl}, 0.5$ $\mathrm{mM} \mathrm{KCl}, \mathrm{pH} 7.5)$ or $0.1 \mathrm{M}$ sodium acetate buffer $(140 \mathrm{mM} \mathrm{NaCl}, 0.5 \mathrm{mM} \mathrm{KCl}, \mathrm{pH} 5.0)$ as a function of temperature at $294 \mathrm{~nm}$ as monitored by far-UV circular dichroism with a Jasco 815 spectropolarimeter. Reprinted with permission from McConnell, EM., Bolzon, R., Mezin, P., Frahm, G., Johnston, M and 
DeRosa, MC. pHAST (pH-Driven Aptamer Switch for Thrombin) Catch-and-Release of Target Protein. 2016, 27: 1493-1499. Bioconjugate Chemistry. Copyright 2016 American Chemical Society. 152

Figure 3.10: The CD spectra of pHAST $(2.5 \mu \mathrm{M})$ at $\mathrm{pHs}$ varying from 8.0-4.5. The significant structural change between $\mathrm{pH}$ values 5.5 and 5.0 is denoted by solid and dashed lines before and after the structural transition respectively. The average of 5 scans of a single sample was used to produce each of the spectra shown. Depending on the $\mathrm{pH}$, the $\mathrm{pHAST}$ was prepared in one of three different buffers adjusted to the appropriate $\mathrm{pH}$ value. The buffers were as follows; $10 \mathrm{mM}$ Tris- $\mathrm{HCl}, 0.5 \mathrm{mM} \mathrm{KCl,} \mathrm{pH} 8.0 ; 10 \mathrm{mM}$ Tris$\mathrm{HCl}, 0.5 \mathrm{mM} \mathrm{KCl}, \mathrm{pH} 7.5 ; 10 \mathrm{mM}$ Tris-HCl, $0.5 \mathrm{mM} \mathrm{KCl}, \mathrm{pH} 7.0 ; 10 \mathrm{mM}$ sodium phosphate, $0.5 \mathrm{mM} \mathrm{KCl}$, pH $6.5 ; 10 \mathrm{mM}$ sodium phosphate, $0.5 \mathrm{mM} \mathrm{KCl}, \mathrm{pH} 6.0 ; 10 \mathrm{mM}$ sodium acetate, $0.5 \mathrm{mM} \mathrm{KCl}, \mathrm{pH} 5.5$; $10 \mathrm{mM}$ sodium acetate, $0.5 \mathrm{mM} \mathrm{KCl}, \mathrm{pH} 5.0 ; 10 \mathrm{mM}$ sodium acetate, $0.5 \mathrm{mM} \mathrm{KCl}, \mathrm{pH} 4.5$. Reprinted with permission from McConnell, EM., Bolzon, R., Mezin, P., Frahm, G., Johnston, M and DeRosa, MC. pHAST (pH-Driven Aptamer Switch for Thrombin) Catch-and-Release of Target Protein. 2016, 27: 14931499. Bioconjugate Chemistry. Copyright 2016 American Chemical Society. 154

Figure 3.11: Fluorescence spectra of the Cy3/Cy5-pHAST $(0.4 \mu \mathrm{M})$ in neutral vs acidic conditions. The solution $\mathrm{pH}$ was changed from neutral to acidic, $\mathrm{Cy} 3 / \mathrm{Cy} 5$-pHAST underwent a conformational change from the G-quadruplex conformation to the $\mathrm{A}^{+}($anti) $\bullet \mathrm{G}(\mathrm{syn})$ base pair stabilized hairpin. FRET, which most efficiently occurred when the $\mathrm{Cy} 3 / \mathrm{Cy} 5$-pHAST was in the hairpin conformation, was used to monitor the conformation of $\mathrm{Cy} 3 / \mathrm{Cy} 5$-pHAST. The fluorescence intensity values of each of the cyanine dyes were not corrected for the $\mathrm{pH}$ effect on their respective quantum yields. Reprinted with permission from McConnell, EM., Bolzon, R., Mezin, P., Frahm, G., Johnston, M and DeRosa, MC. pHAST (pHDriven Aptamer Switch for Thrombin) Catch-and-Release of Target Protein. 2016, 27: 1493-1499. Bioconjugate Chemistry. Copyright 2016 American Chemical Society.

Figure 3.12: The effect of $\mathrm{pH}$ cycling on the conformation of the $\mathrm{Cy} 3 / \mathrm{Cy} 5-\mathrm{pHAST}$ was monitored over 10 switches in each buffer condition. Each buffer contained $140 \mathrm{mM} \mathrm{NaCl}$ and either $0.0 \mathrm{mM} \mathrm{KCl}, 0.5$ $\mathrm{mM} \mathrm{KCl}$ or $5.0 \mathrm{mM} \mathrm{KCl}$. The fluorescence intensity ratio of Cy 3 emission over Cy 5 emission was indicative of conformation. At low ratio intensities the Cy3/Cy5-pHAST was in the hairpin conformation. At high ratio intensities the Cy3/Cy5-pHAST was in the G-quad conformation. The fluorescence intensity values of each of the cyanine dyes were not corrected for the $\mathrm{pH}$ effect on their respective quantum yields. Reprinted with permission from McConnell, EM., Bolzon, R., Mezin, P., Frahm, G., Johnston, M and DeRosa, MC. pHAST (pH-Driven Aptamer Switch for Thrombin) Catch-and-Release of Target Protein. 2016, 27: 1493-1499. Bioconjugate Chemistry. Copyright 2016 American Chemical Society.. 158

Figure 3.13: Reversible conformational change was shown by extended $\mathrm{pH}$ cycling of the $\mathrm{Cy} 3 / \mathrm{Cy} 5-$ pHAST in neutral and acidic $\mathrm{pH}$. The ratio of fluorescence emission intensity (cps) of the Cy3/Cy5 peaks was measured over 69 alternating $\mathrm{pH}$ conditions. A cycle consisted of a neutral and acidic $\mathrm{pH}$ condition. Cycles 1-6 and 27-35 are shown, cycles 7-26 are represented by a break in the $\mathrm{x}$-axis. When the Cy3/Cy5 ratio was high ( $\mathrm{pH} 7)$, the pHAST was in the G-quadruplex conformation. When the Cy3/Cy5 ratio was low (pH 5), the pHAST was in the hairpin conformation. The fluorescence intensity values of each of the cyanine dyes were not corrected for the $\mathrm{pH}$ effect on their respective quantum yields. Reprinted with permission from McConnell, EM., Bolzon, R., Mezin, P., Frahm, G., Johnston, M and DeRosa, MC. pHAST (pH-Driven Aptamer Switch for Thrombin) Catch-and-Release of Target Protein. 2016, 27: 14931499. Bioconjugate Chemistry. Copyright 2016 American Chemical Society. 
Figure 3.14: Continuous $\mathrm{pH}$ cycling over multiple switches led to a sequential decrease in fluorescence emission intensity. This trend is illustrated for all $\mathrm{pH} 7$ (red) and $\mathrm{pH} 5$ (blue) conditions. Traces are named based on the "pH_cycle number". Buffer: $1 \mathrm{mM}$ phosphate buffer with $140 \mathrm{mM} \mathrm{NaCl}$ and $0.5 \mathrm{mM}$ $\mathrm{KCl}$.

Figure 3.15: A) pH switching ability is maintained at each buffer condition, following desalting. Samples (from trial 1) from each buffer condition were desalted post $\mathrm{pH}$ cycling then subjected to an additional 5 switches. Note: the inconsistency observed for the second $\mathrm{pH}=7$ value was likely caused by an inaccurate $\mathrm{pH}$ measurement. B) Representative profiles from the sample tested in trial 1 of Figure 3.12. (1 mM phosphate buffer, $140 \mathrm{mM} \mathrm{NaCl}$ and $5 \mathrm{mM} \mathrm{KCl}$ ) before and after desalting are shown. Switches 1-7 and 1-5 respectively are depicted. The fluorescence intensity values of each of the cyanine dyes were not corrected for the $\mathrm{pH}$ effect on their respective quantum yields.

Figure 3.16: Thrombin binding to $\mathrm{Cy} 3-\mathrm{pHAST}$ was disrupted between $\mathrm{pH} 6.5$ and $\mathrm{pH}$ 6.0. Cy3-pHAST, in the G-quad conformation, binding to thrombin is shown by the fluorescence (black) in the top bands of lanes 3-6. Unbound Cy3-pHAST, in the hairpin conformation is shown by the fluorescence in the bottom bands in lane 7-11. Lanes: 1. Cy3-pHAST with $0.0 \mu \mathrm{M}$ thrombin at $\mathrm{pH}$ 7. Lanes 3-11 contained $1.0 \mu \mathrm{M}$ Cy3-pHAST incubated with $2.5 \mu \mathrm{M}$ thrombin at $\mathrm{pH}$ 8.0, 7.5, 7.0, 6.5, 6.0, 5.5, 5.0, 4.5, and 4.0 respectively. Reprinted with permission from McConnell, EM., Bolzon, R., Mezin, P., Frahm, G., Johnston, $\mathrm{M}$ and DeRosa, MC. pHAST (pH-Driven Aptamer Switch for Thrombin) Catch-and-Release of Target Protein. 2016, 27: 1493-1499. Bioconjugate Chemistry. Copyright 2016 American Chemical Society.

Figure 3.17: The binding of the Cy3-TBA and Cy3-pHAST to thrombin over a range of thrombin concentrations was evaluated by fluorescence anisotropy. Error bars represent standard deviation. The apparent $\mathrm{K}_{\mathrm{D}} \mathrm{S}$ were; Cy3-TBA (pH 7): $0.45 \pm 0.01 \mu \mathrm{M}$, Сy3-TBA (pH 5): $0.46 \pm 0.02 \mu \mathrm{M}$, Cy3-pHAST (pH 7): $0.63 \pm 0.18 \mu \mathrm{M}, \mathrm{Cy} 3-\mathrm{pHAST}$ (pH 5): $1.08 \pm 0.18 \mu \mathrm{M}$. For each sample, the apparent $\mathrm{K}_{\mathrm{D}}$ values $\left(\mathrm{K}_{\mathrm{D}} \pm\right.$ Standard Error) were derived from the fit of the average of the closest two data points at each concentration. Reprinted with permission from McConnell, EM., Bolzon, R., Mezin, P., Frahm, G., Johnston, M and DeRosa, MC. pHAST (pH-Driven Aptamer Switch for Thrombin) Catch-and-Release of Target Protein. 2016, 27: 1493-1499. Bioconjugate Chemistry. Copyright 2016 American Chemical Society.

Figure 3.18: Representative gels of full concentration range of thrombin with $\mathrm{Cy} 3$-pHAST at $\mathrm{pH} 7.0$ (lanes $\mathrm{C} 7.0$ and 1-8) and $\mathrm{pH} 5.0$ (lanes $\mathrm{C} 5.0$ and 9-14) where $\mathrm{C}$ is the control at a given $\mathrm{pH}$. Cy3-pHAST $(1.0 \mu \mathrm{M})$ was incubated with varying concentrations of thrombin; $0.0 \mu \mathrm{M}$ thrombin (lanes C7.0, C5.0), $5.0 \mu \mathrm{M}$ thrombin (lanes 1,9$), 2.5 \mu \mathrm{M}$ (lanes 2, 10), $0.50 \mu \mathrm{M}$ (lanes 3, 11), $0.25 \mu \mathrm{M}$ (lane 4), $50 \mathrm{nM}$ (lanes 5, 12), $25 \mathrm{nM}$ (lane 6), $5.0 \mathrm{nM}$ (lanes 7,13), and 0.5 nM (lanes 8,14).

Figure 3.19: $\mathrm{pH}$ cycling in the presence of thrombin shows that the Cy3-pHAST can predictably catchand-release thrombin based on solution $\mathrm{pH}$. Lanes: 1: $1.0 \mu \mathrm{M} \mathrm{Cy3-pHAST}$ control ( $0.0 \mu \mathrm{M}$ thrombin); 4, 6, 8, 10: $1.0 \mu \mathrm{M} \mathrm{Cy3-pHASTwith} 2.5 \mu \mathrm{M}$ thrombin at $\mathrm{pH} 7$ and 5,7,9,11: $1.0 \mu \mathrm{M} \mathrm{Cy3-pHAST}$ with 2.5 $\mu \mathrm{M}$ thrombin at $\mathrm{pH}$ 5. Reprinted with permission from McConnell, EM., Bolzon, R., Mezin, P., Frahm, G., Johnston, M and DeRosa, MC. pHAST (pH-Driven Aptamer Switch for Thrombin) Catch-andRelease of Target Protein. 2016, 27: 1493-1499. Bioconjugate Chemistry. Copyright 2016 American Chemical Society. 
Figure 3.20: Top) The fluorescence anisotropy was measured Cy3-pHAST with thrombin $(0.5 \mu \mathrm{M})$ in 1 $\mathrm{mM}$ sodium phosphate buffer $(140 \mathrm{mM} \mathrm{NaCl}, 0.5 \mathrm{mM} \mathrm{KCl})$ during $\mathrm{pH}$ cycling. The large difference in size due to target binding, resulted in different anisotropy values under neutral ( $\mathrm{pH} 7$ ) versus acidic ( $\mathrm{pH} 5)$ conditions. Error bars represent the standard deviation between three trials. Bottom) The fluorescence anisotropy the Cy3-pHAST in $1 \mathrm{mM}$ sodium phosphate buffer $(140 \mathrm{mM} \mathrm{NaCl}, 0.5 \mathrm{mM} \mathrm{KCl})$ was measured while the $\mathrm{pH}$ was cycled to determine the effect of $\mathrm{pH}$-dependent structure on anisotropy values. The change in anisotropy in the absence of thrombin is shown. The change of conformation of the pHAST from G-quadruplex to hairpin resulted in a small change in the measured anisotropy. Error bars represent the standard deviation between three trials. Reprinted with permission from McConnell, EM., Bolzon, R., Mezin, P., Frahm, G., Johnston, M and DeRosa, MC. pHAST (pH-Driven Aptamer Switch for Thrombin) Catch-and-Release of Target Protein. 2016, 27: 1493-1499. Bioconjugate Chemistry. Copyright 2016 American Chemical Society.

Figure 4.1: Schematic representation of the ventral tegmental area-nucleus accumbens (VTA-NAc) reward circuit. The major dopaminergic (green), glutamatergic (red) and GABAergic (blue) projections between the VTA and NAc in the rodent brain are shown. Other brain structures shown for reference include; the medial prefrontal cortex ( $\mathrm{mPFC}$ ), the striatum, the hippocampus (Hipp), the amygdala (Amy), the lateral dorsal tegmentum (LDTg), the rostromedial tegmentum (RTMg), the lateral habenula $(\mathrm{LHb})$ and the lateral hypothalamus (LH). Reprinted by permission from Macmillan Publishers Ltd: Nature Reviews Neuroscience, (Russo, SJ., and Nestler, EJ. The brain reward circuitry in mood disorder. 2013. Nature Reviews Neuroscience. 14:609-625) $)^{383}$ copyright 2013.

Figure 4.2: Illustration of the operant conditioning chamber. During the acquisition phase the animal is trained to press the lever twice, which shuts off the house light, changes the panel light from red to green and results in the delivery of a chocolate

Figure 4.3: During the acquisition phase, the cumulative correct lever presses $/ 5 \mathrm{~min}$ interval over the 30 min session, by day reached a maximum between days 4 and 5 . Groups were randomly assigned to be pretreated with intra-accumbens vehicle ( $0 \mathrm{nM} / \mathrm{MK} ; \mathrm{n}=7)$, aptamer $(200 \mathrm{nM} / \mathrm{MK} ; \mathrm{n}=7)$, random oligonucleotide control (Random/MK; $n=7)$, saline control ( $0 \mathrm{nM} / \mathrm{MK} ; \mathrm{n}=5)$ and aptamer/saline control $(200 \mathrm{nM} /$ Saline; $\mathrm{n}=5)$. Numbers in parenthesis = animals per group. Data expressed as mean $\pm \mathrm{SEM} \ldots 191$

Figure 4.4: Summary of mean correct lever presses by day during the acquisition phase. Groups were randomly assigned to be pretreated with intra-accumbens vehicle $(0 \mathrm{nM} / \mathrm{MK} ; \mathrm{n}=7)$, aptamer (200 $\mathrm{nM} / \mathrm{MK} ; \mathrm{n}=7)$, random oligonucleotide control (Random/MK; $n=7)$, saline control $(0 \mathrm{nM} / \mathrm{MK} ; \mathrm{n}=5)$ and aptamer/saline control ( $200 \mathrm{nM} /$ Saline; $\mathrm{n}=5)$. Two-way ANOVA (day by group) revealed a significant effect of day $(\mathrm{F}(4,16)=94.44, \mathrm{p}<0.001)$, and no group differences $(\mathrm{F}(4,26)=1.57)$ or interaction $(\mathrm{f}(16,104)=1.27) . \mathrm{p}<0.05$; numbers in parenthesis $=$ animals per group. Data expressed as mean $\pm \mathrm{SEM}$.

Figure 4.5: Dopamine (purple) release from monoaminergic neurons (MANTs) is mediated by direct glutamatergic (Glu) and indirect glutamatergic/GABAergic (GABA) neuronal pathways. A balance between the accelerator and brake pathways regulates dopamine (DA) and other monoaminergic neurotransmitter release. Typically (left) the brake pathway predominates to regulate normal dopamine release. Failure (right) of these pathways, for example by MK-801 (black) induced hypoglutamatergia, 
results in over activation of MANTs, and therefore increased dopamine release in terminal projection sites, such as the nucleus accumbens (NAcb). ${ }^{406,407}$

Figure 4.6: Cumulative correct lever presses during the extinction phase ( $30 \mathrm{~min}$ session). Groups pretreated with intra-accumbens vehicle ( $0 \mathrm{nM} / \mathrm{MK} ; \mathrm{n}=7)$, aptamer $(200 \mathrm{nM} / \mathrm{MK} ; \mathrm{n}=7)$, random oligonucleotide control (Random/MK; $n=7)$, saline control ( $0 \mathrm{nM} / \mathrm{MK} ; \mathrm{n}=5)$ and aptamer/saline control ( $200 \mathrm{nM} /$ Saline; $\mathrm{n}=5$ ). Two-way, repeated measures ANOVA (group by $5 \mathrm{~min}$ time interval) revealed a significant main effects of group $(F(4,26)=3.84, \mathrm{p}<0.05)$ and interval $(\mathrm{F}(5,20)=56.74, \mathrm{p}<0.001)$. Significant interactions between interval and group were observed $(\mathrm{F}(20,130)=6.47, \mathrm{p}<0.001)$. *, $\mathrm{p}<0.05$. Numbers in parenthesis $=$ animals per group. Data expressed as mean $\pm \mathrm{SEM}$.

Figure 4.7: Cumulative incorrect lever pressing during the extinction phase. Groups pretreated with intraaccumbens vehicle $(0 \mathrm{nM} / \mathrm{MK} ; \mathrm{n}=7)$, aptamer $(200 \mathrm{nM} / \mathrm{MK} ; \mathrm{n}=7)$, random oligonucleotide control (Random/MK; $\mathrm{n}=7$ ), saline control ( $0 \mathrm{nM} / \mathrm{MK} ; \mathrm{n}=5)$ and aptamer/saline control (200 nM/Saline; $\mathrm{n}=5)$. A two-way ANOVA (group by 5-min interval) revealed no significant main effect. Numbers in parenthesis $=$ animals per group. Data expressed as mean \pm SEM.

Figure 4.8: Nose pokes during the extinction session as an indication of reward seeking behaviour. Groups pretreated with intra-accumbens vehicle ( $0 \mathrm{nM} / \mathrm{MK} ; \mathrm{n}=7)$, aptamer $(200 \mathrm{nM} / \mathrm{MK} ; \mathrm{n}=7)$, random oligonucleotide control (Random/MK; $n=7)$, saline control ( $0 \mathrm{nM} / \mathrm{MK} ; \mathrm{n}=5)$ and aptamer/saline control $(200 \mathrm{nM} /$ Saline; $\mathrm{n}=5)$. A two-way ANOVA revealed a significant main group effect $(\mathrm{F}(4,26)=6.10$, $\mathrm{p}<0.05)$. Numbers in parenthesis $=$ animals per group. Data expressed as mean $\pm \mathrm{SEM}$.

Figure 4.9: Intra-accumbens injection of the DBA did not significantly affect motor behaviour. The locomotor activity of a subset of animals was measured in an elevated cross maze (A) over a 30 min session. Animals were placed in the center of the maze (denoted by $\mathrm{C}$ in Figure $10 \mathrm{~A}$ ) and their locomotor activity was measured as the total distance (meters) travelled (B), the average speed (meters/sec; C) and the total arm entries (D). Groups were pretreated with intra-accumbens vehicle $(0 \mathrm{nM} / \mathrm{MK} ; \mathrm{n}=5)$, aptamer (200 nM/MK; $n=5)$, random oligonucleotide control (Random/MK; $n=5)$, vehicle/saline control (0 $\mathrm{nM} / \mathrm{MK} ; \mathrm{n}=5)$ and aptamer/saline control (200 nM/Saline; $n=4)$. Number in parenthesis is number of animals per group. Data is presented as mean \pm SEM. $(*, p<0.05)$. 202

Figure 4.10: Confirmation of cannula placement in the nucleus accumbens of aptamer treated and control animals (reproduced with permission from Dan Madularu's M.Sc. thesis (Carleton University, 2010)). A) Coronal section of a rat brain. The location of the nucleus accumbens is shown in the small square. B-D) The placement of cannula and injector (indicated by orange arrows) in three different animals pre-treated with aptamer is shown. Staining of the brain coronal sections (4X) was done with cresyl violet. E) 10X magnification of cresyl violet stained brain coronal sections showing placement of cannula and injector. The nucleus accumbens core and shell are delimitated by the green dashed line. Abbreviations: nucleus accumbens (NAcc) and anterior commissure (ACA)

Figure 4.11: Representative images of reduced pTH staining in the nucleus accumbens of aptamer-treated rats shown by histological analysis. A) The ratio of phosphorylated tyrosine hydroxylase (pTH) to tyrosine hydroxylase $(\mathrm{TH})$ in the nucleus accumbens shell region was quantified. The ratio was significantly lower in the $200 \mathrm{nM} / \mathrm{MK}$ group $(* * \mathrm{p}<0.01)$. B) TH staining in the nucleus accumbens shell region was quantified and similar levels were observed between the $0 \mathrm{nM} / / \mathrm{MK}$ and $200 \mathrm{nM} / \mathrm{MK}$ groups. 
C) pTH quantification in the nucleus accumbens shell region revealed lower levels of staining in the 200 $\mathrm{nM} / \mathrm{MK}$ group than the $0 \mathrm{nM} / \mathrm{MK}$ group $\left({ }^{* *} \mathrm{p}<0.01\right)$. The sample size (n) was $3 /$ group. Scale bars represent $500 \mu \mathrm{m}$. The magnification shown at the top of the $0 \mathrm{nM}$ (Tris) images was the same for the 200 $\mathrm{nM}$ images. In the top panel the data is represented as mean $\pm \mathrm{SEM}$

Figure 5.1: Schematic representation of the mechanism of action of the DBA in hyperdopaminergic conditions. A) Under normal conditions, dopamine (purple) is released from the pre-synaptic neuron into the synaptic cleft and is free to bind to post-synaptic dopamine receptors (green and yellow) and presynaptic dopamine autoreceptors (blue) and transporters (orange). B) Cocaine (red) increases the synaptic concentration of dopamine which intensifies signal transduction (grey). C) The DBA (black hairpin) binds to excess dopamine, normalizing signal transduction.

Figure 5.2: A) Schematic of the multi-DNA aptamer payload/targeting system. The lipid vesicle (blue sphere) is loaded with payload aptamer (black ribbons), and surface modified with a rhodamine-label (pink stars) and transferrin receptor aptamer (TRA: blue ribbons). B) Representative transmission electron microscope image of aptamer loaded-TRAM liposomes.

Figure 5.3: Schematic representation of the synthesis of DAL-TRAM and control variations of the multiDNA aptamer payload/targeting system. 236

Figure 5.4: Encapsulation of the DNA payload was confirmed by non-denaturing PAGE analysis. Lanes: Loading dye (1), DNA (control oligo) (5), after extrusion (7), after dialysis 1 (9), after nuclease digestion (11), after dialysis 2 (13) and after lysis (15). DNA was imaged by ethidium bromide staining. 237

Figure 5.5: The homogeneity of the DAL-TRAM was characterized by transmission electron microscopy (TEM). TEM images of the liposome targeting system at $0.2 \mu \mathrm{m}$ (left) and $100 \mathrm{~nm}$ (right) scale. Images were obtained at $120 \mathrm{kV}$.

Figure 5.6: TEM was used to determine the measurement (A) and size distribution histogram (B) of the liposome targeting system. TEM images (A) were obtained at $120 \mathrm{kV}$. 240

Figure 5.7: Stability of the liposome formulation over time was assessed by TEM (left) and by nanoparticle tracking analysis (right) after 5 and 2 months respectively. The TEM image was obtained at $200 \mathrm{kV}$.

Figure 5.8: Fluorescence microscopy was used to determine the distribution of rhodamine fluorescence in a coronal section ( $35 \mu \mathrm{m}$ thickness) of the nucleus accumbens. Mice were injected with a solution containing TRA-positive liposomes (DAL-TRAM: rhodamine (red) labelled) and TRA-negative liposomes (DAL: NBD (green) labelled) then euthanized 10 min later. Digital fluorescence images were obtained at 20X magnification. The scale bar for the DAL and overlay images are as indicated in DALTRAM image. 242

Figure 5.9: Specific amplification by RT-qPCR of DBA delivered to the brain tissue by DAL-TRAM in saline treated animals compared to a non-template control (NTC). The amplification curves of the DBA from animals 29 and 31 are shown in (A). Amplification of the desired product was confirmed by gel 
electrophoresis (B). Lanes from left to right: 50 bp DNA ladder (1), animal 29 (2), non-template control (NTC) (3) and animal 31 (4)....

Figure 5.10: Representative amplification (A) and melt curves (B) for the RT-qPCR product of the DBA delivered to the brain via DAL-TRAM after oligonucleotide isolation from nucleus accumbens tissue. 245

Figure 5.11: Representative amplification (A) and melt (B) curves of the Sub sequence from nucleus accumbens tissue after the systemic injection of Sub-TRAM are shown.

Figure 5.12: Horizontal locomotor activity of animals treated with $10 \mathrm{mg} / \mathrm{mL}$ cocaine. There was a significant decrease in locomotor behaviour of the DAL-TRAM treated animals compared to animals treated with TRAM. The horizontal locomotor activities over a 30 min session for the following groups; DAL-TRAM $+10 \mathrm{mg} / \mathrm{mL}$ cocaine $(\mathrm{n}=8), \mathrm{TRAM}+10 \mathrm{mg} / \mathrm{mL}$ cocaine $(\mathrm{n}=8)$, and DAL-TRAM + saline $(\mathrm{n}=5)$ are shown. Error bars represent "mean \pm SEM".

Figure 5.13: Horizontal locomotor activity of animals treated with $5 \mathrm{mg} / \mathrm{mL}$ cocaine. A significant decrease in locomotor behaviour of the DAL-TRAM treated animals compared to animals treated with TRAM was observed. The horizontal locomotor activities over a $30 \mathrm{~min}$ session of animals from the following groups; DAL-TRAM $+5 \mathrm{mg} / \mathrm{mL}$ cocaine $(\mathrm{n}=8)$, TRAM $+5 \mathrm{mg} / \mathrm{mL}$ cocaine $(\mathrm{n}=8)$, and DALTRAM+saline $(n=8)$ are shown. Error bars represent "mean \pm SEM".

Figure 5.14: Horizontal locomotor activity of animals treated with $1 \mathrm{mg} / \mathrm{mL}$ cocaine. No significant effect on locomotor behaviour after treatment with either DAL-TRAM or TRAM was observed. The horizontal locomotor activities over a $30 \mathrm{~min}$ session of animals from the following groups; DALTRAM $+1 \mathrm{mg} / \mathrm{mL}$ cocaine $(\mathrm{n}=7)$, TRAM $+1 \mathrm{mg} / \mathrm{mL}$ cocaine $(\mathrm{n}=7)$, and DAL-TRAM + saline $(\mathrm{n}=5)$ are shown. Error bars represent "mean \pm SEM".

Figure 5.15: Total horizontal locomotor activity of DAL-TRAM and control treated animals over a 30 min session. A significant effect of group $(\mathrm{F}(6,46)=22.71, \mathrm{p}<0.001)$ was revealed by On-Way ANOVA analysis. Post-hoc analyses by Fisher's LSD revealed significant differences between treatment conditions as indicated $(* \mathrm{p}<0.05)$. Groups sizes were as follows; Sub-TRAM $+10 \mathrm{mg} / \mathrm{mL}$ cocaine $(\mathrm{n}=8)$, DALTRAM $+10 \mathrm{mg} / \mathrm{mL}$ cocaine $(\mathrm{n}=8)$, DAL-TRAM $+5 \mathrm{mg} / \mathrm{mL}$ cocaine $(\mathrm{n}=8)$, DAL-TRAM $+1 \mathrm{mg} / \mathrm{mL}$ cocaine $(n=7)$, DAL-TRAM + saline $(n=8)$, TRAM + saline $(n=8)$ and saline + saline $(n=6)$. Error bars represent "mean + SEM".

Figure 5.16: The efficacy of multiple control variations of the multi-DNA aptamer payload/targeting system in decreasing cocaine $(10 \mathrm{mg} / \mathrm{mL})$ induced hyperlocomotion was examined. The total horizontal activity over a 30 min session is shown. A significant group effect $(F(7,54)=14.22, p<0.001)$ was revealed through analysis by a One-way ANOVA. Fisher's LSD post-hoc analyses revealed a significant difference between groups $(* \mathrm{p}<0.05)$. Examined groups were as follows; saline + saline $(n=6)$, TRAM+saline $(\mathrm{n}=8)$, DAL-TRAM + saline $(\mathrm{n}=8)$, DAL-TRAM $+10 \mathrm{mg} / \mathrm{mL}$ cocaine $(\mathrm{n}=8), \mathrm{DAL}+10$ $\mathrm{mg} / \mathrm{mL}$ cocaine $(\mathrm{n}=9)$, ROL-TRAM $+10 \mathrm{mg} / \mathrm{mL}$ cocaine $(\mathrm{n}=7)$, TRAM $+10 \mathrm{mg} / \mathrm{mL}$ cocaine $(\mathrm{n}=8)$, and Sub-TRAM $+10 \mathrm{mg} / \mathrm{mL}$ cocaine $(\mathrm{n}=8)$. Error bars represent "mean + SEM"

Figure 5.17: A) No significant effect on locomotor activity was observed after chronic administration of either DAL-TRAM or TRAM. The horizontal locomotor activity of the following groups was monitored 
over a 30 min session once a day for 6 days; DAL-TRAM ( $\mathrm{n}=5)$, TRAM ( $\mathrm{n}=5)$, and no injections ( $\mathrm{n}=5)$. Horizontal activity is divided into $5 \mathrm{~min}$ bins (shown on the $\mathrm{x}$-axis) for each $30 \mathrm{~min}$ session. Error bars represent "mean $\pm \mathrm{SEM".} \mathrm{B)} \mathrm{Total} \mathrm{horizontal} \mathrm{activity} \mathrm{recorded} \mathrm{during} \mathrm{the} \mathrm{final} 30$ min session of the experiment (day 6) is shown. One-way ANOVA revealed no significant group effect $(\mathrm{F}(3,24)=0.949$, $\mathrm{p}=0.433)$. The following groups were examined; TRAM $(\mathrm{n}=5)$, DAL-TRAM $(\mathrm{n}=5)$ and no injection $(\mathrm{n}=5)$. Error bars represent "mean + SEM".

Figure 5.18: No indication of neuronal degeneration in animals that had been chronically treated with DAL-TRAM was revealed by Fluoro-Jade B staining. (A) Regions of neural tissue damage are indicated by white arrows. Magnification is at 20X.

Figure 6.1: Schematic representation of the DopaA20min aptamer-AuNP based sensor. A) When no aptamer (apt)-target binding occurs, the test solution remains red upon addition of $\mathrm{NaCl}$. B) When apttarget binding occurs, the addition of $\mathrm{NaCl}$ led to a noticeable colour change of the test solution from red to blue. DNA (aptamer or random control) is represented by grey lines, the target is yellow, AuNP are red, and aggregated AuNP are shown as blue.

Figure 6.2: The secondary structure of DopaA20min was predicted by RNAstructure Fold. ${ }^{493}$ The colourcoded index represents the probability of the base pair in percent. 293

Figure 6.3: Base composition of DopaA20min .294

Figure 6.4: Melting temperature (Tm) analysis of DopaA20min in the presence (blue) and absence (red) of dopamine. Data points represent the average of either two (DopaA20min + DA) or three (DopaA20min) temperature ramps. The fit of the average values was predicted by SigmaPlot 10 and is represented by a solid line.....

Figure 6.5: The apparent dissociation constant of DopaA20min was determined by fluorescence anisotropy. The dopamine concentration was held constant $\left(1.0 \times 10^{-7} \mathrm{~mol} / \mathrm{L}\right)$ and the concentration of the aptamer was varied. Anisotropy values were measured with an excitation and emission wavelength of 285 $\mathrm{nm}$ and $317 \mathrm{~nm}$ respectively. Data points represent a single trial and the corresponding fit.

Figure 6.6: Representative images of the aptamer-AuNP assay before and after the addition of $\mathrm{NaCl}$. Top panel: before the addition of $\mathrm{NaCl}$, all of the samples (with and without dopamine) look similarly red. Bottom panel: following $\mathrm{NaCl}$ addition, the presence of higher dopamine concentrations resulted in more significant aggregation. Visually aggregation was represented by a colour change from red to blue. M: $\mathrm{mol} / \mathrm{L}$

Figure 6.7: Representative UV-Visible absorbance spectra of the DopaA20min aptamer-AuNP based sensor in response to various dopamine concentrations ranging from $1.92 \times 10^{-5} \mathrm{M}$ to $1.92 \times 10^{-10} \mathrm{M}$. The "DA: control (no DA)" was run with the appropriate amount of aptamer but water instead of dopamine (DA). The "DA: Control (no DA or DNA)" was run with deionized water in place of DA and DNA. Spectra were corrected to the DA: Control (no DNA) absorbance at $450 \mathrm{~nm}$.

Figure 6.8: Transmission electron microscopy images of the aptamer-AuNP sensor in the absence (A) and presence $(B, C)$ of dopamine. A) When no dopamine (DA) is present, the DopaA20min aptamer 
remained nonspecifically interacting with the surface of the AuNP, thereby having a protective effect against salt-induced aggregation. B) When DA was present, the aptamer specifically bound to DA, leaving the unprotected AuNP susceptible to salt induced aggregation.

Figure 6.9: A) The average ( $\mathrm{n}=3$ ) absorbance ratios at $660 / 524 \mathrm{~nm}$ of the DopaA20min aptamer-AuNP sensor in response to varying DA (blue), and methionine (green) concentrations. The response of random DNA (red) to increasing DA concentrations was also investigated. Error bars represent "mean \pm SD" of $\mathrm{n}=2$ or 3 . B) The linear dynamic range of the sensor in response to DA was determined to be $1.92 \times 10^{-8}$ $\mathrm{mol} / \mathrm{L}$ to $1.92 \times 10^{-6} \mathrm{~mol} / \mathrm{L}$. The limit of detection $(3 \sigma)$ was $9.98 \times 10^{-9} \mathrm{~mol} / \mathrm{L}$

Figure 6.10: A) The AuNP assay was used to determine the specificity of the DopaA20min aptamer. Error represents "mean $\pm \mathrm{SD}$ " of $\mathrm{n}=2$. B) At the target concentration investigated $\left(1.92 \times 10^{-6} \mathrm{~mol} / \mathrm{L}\right)$, only NE elicited a noticeable degree of aggregation. C) The following targets were investigated due to their structural similarity to dopamine (DA); norepinephrine (NE), ascorbic acid (AA), tyramine (Tyr) and serotonin (ST). Red circles represent a single difference of the addition or subtraction of an hydroxyl group compared to DA

Figure 6.11: The response of the Aptamer-AuNP sensor to increasing concentrations of norepinephrine (NE) was evaluated. A) Increasing the second incubation time from $10 \mathrm{~min}$ (brown; $\mathrm{n}=2$ ) to $85 \mathrm{~min}$ (orange; $\mathrm{n}=1$ ) produced a more consistent response curve. B) Compared to the Aptamer-AuNP sensor response to increasing concentrations of dopamine (DA; blue, $n=3)$, the sensor's response to NE after an 85 min incubation (dark orange; $n=1$ ) was less sensitive even with a longer incubation period. Error bars represent "mean \pm SD".

Figure 6.12: Detection of dopamine (DA) in 100X diluted artificial cerebral spinal fluid (ACSF) by aptamer (blue; $n=3$ ) AuNP assay. As a control the response of random DNA (red; $n=2$ ) in the presence of increasing DA was monitored. Error bars represent "mean \pm SD".

Figure 6.13: Cumulative horizontal locomotor activity of DA20m-TRAM compared to controls over an entire 30 min session. One-way ANOVA analysis revealed a significant effect of group $(\mathrm{F}(4,34)=20.12$, $\mathrm{p}<0.001)$. Significant differences between treatment conditions were revealed by Post-hoc analysis by Fisher's LSD. Significance is indicated by $(* p<0.05)$. Group sizes were as follows; Sub-TRAM +10 $\mathrm{mg} / \mathrm{mL}$ cocaine $(\mathrm{n}=7)$, DA20m-TRAM $+10 \mathrm{mg} / \mathrm{mL}$ cocaine $(\mathrm{n}=8)$, DAL-TRAM $+10 \mathrm{mg} / \mathrm{mL}$ cocaine $(\mathrm{n}=8)$, DA20m-TRAM + saline $(\mathrm{n}=9)$ and DAL-TRAM + saline $(\mathrm{n}=8)$. Error bars represent "mean + SEM". 


\subsection{List of Tables}

Table 1.1: Characteristics of antibodies, aptamers, binding proteins and MIPs. Partially reproduced and modified with permission from Portland Press, Ltd from Ruigrok, VJB., Levisson, M., Eppink, MH., Smidt, H., and Van der Oost, J. Alternative affinity tools: more attractive than antibodies. Biochem J. 2011, 436: 1-13. Copyright Portland Press, Ltd. 2011.

Table 1.2: A comparison of the advantages and limitations of aptamers and antibodies 7

Table 2.1: Correlation between template length and aptamer dissociation constant $\left(\mathrm{K}_{\mathrm{D}}\right)$ by nucleic acid type and target type. Table reproduced with permission of Springer from McKeague, M.; McConnell, E. M.; Cruz-Toledo, J.; Bernard, E. D.; Pach, A.; Mastronardi, E.; Zhang, X.; Beking, M.; Francis, T.; Giamberardino, A.; Cabecinha, A.; Ruscito, A.; Aranda-Rodriguez, R.; Dumontier, M.; DeRosa, M. C. Analysis of in vitro selection parameters. J. Mol. Evol. 2015, 81 (5), 150. Copyright Springer 2015....... 75

Table 2.2: Summary of results from the pairwise Mann-Whitney-U test. Each target type was compared to small molecules. Significance was described at $(\alpha=0.05)$ and is indicated by an *. Table reproduced with permission of Springer from McKeague, M.; McConnell, E. M.; Cruz-Toledo, J.; Bernard, E. D.; Pach, A.; Mastronardi, E.; Zhang, X.; Beking, M.; Francis, T.; Giamberardino, A.; Cabecinha, A.; Ruscito, A.; Aranda-Rodriguez, R.; Dumontier, M.; DeRosa, M. C. Analysis of in vitro selection parameters. J. Mol. Evol. 2015, 81 (5), 150. Copyright Springer 2015.

Table 2.3: The correlation (represented by the correlation coefficient, $\mathrm{R}$ ) and slope of the linear regression trendline $(\mathrm{m})$ of $\log 10$ (cation concentration) and $\log _{10}\left(\mathrm{~K}_{\mathrm{D}}\right)$ for individual cations. Table reproduced with permission of Springer from McKeague, M.; McConnell, E. M.; Cruz-Toledo, J.; Bernard, E. D.; Pach, A.; Mastronardi, E.; Zhang, X.; Beking, M.; Francis, T.; Giamberardino, A.; Cabecinha, A.; Ruscito, A.; Aranda-Rodriguez, R.; Dumontier, M.; DeRosa, M. C. Analysis of in vitro selection parameters. J. Mol. Evol. 2015, 81 (5), 150. Copyright Springer 2015.

Table 3.1: DNA sequences of the oligonucleotides used in this study. Table reprinted with permission from McConnell, EM., Bolzon, R., Mezin, P., Frahm, G., Johnston, M and DeRosa, MC. pHAST (pHDriven Aptamer Switch for Thrombin) Catch-and-Release of Target Protein. 2016, 27: 1493-1499. Bioconjugate Chemistry. Copyright 2016 American Chemical Society.

Table 3.2: The melting temperature ( $\left.T_{m}\right)$ of sequences described in Table 3.1 were obtained by UV-Vis spectroscopy. Analysis of the $\mathrm{T}_{\mathrm{m}}$ and absorbance profiles at $295 \mathrm{~nm}$ showed that TBA+PC1 showed mostly duplex conformation at both $\mathrm{pH}$ conditions, and TBA $+\mathrm{PC} 2$ revealed that mostly G-quad conformation existed at both $\mathrm{pH}$ conditions. The absorbance spectra of TBA+PC3 exhibited G-quad character at neutral $\mathrm{pH}$ and duplex character at acidic $\mathrm{pH}$.

Table 3.3: Thermal denaturation was used to determine the melting temperature of the pHAST in various $\mathrm{KCl}$ concentrations. The Tm of the pHAST were consistent with values for TBA+PC3 described in Table 3.2 . 
Table 5.1: Sequences of oligonucleotides

Table 5.2: Forward and reverse primers for RT-qPCR amplification of the DBA and Sub aptamer sequences

Table 5.3: Aptamer sequences used in the multi-aptamer targeting/payload system design .232

Table 5.4: Aptamer and, multi-aptamer payload/targeting system and control variation abbreviations.. 233

Table 6.1: RNA DBA ${ }^{280}$ based dopamine detection assays 269

Table 6.2: Sensors developed based on both the RNA ${ }^{280}$ and $\mathrm{DNA}^{284}$ dopamine-binding aptamers (DBAs)

Table 6.3: Dopamine detection assays based on the DNA dopamine-binding aptamer ${ }^{284}$ 270

Table 6.4: Possible G-Quadruplexes predicted by QGRS Mapper 295

Table 6.5: Possible G-Quadruplexes predicted by imGQfinder 295

Table 9.1: SELEX methods used to select aptamers 350

Table 9.2: Partitioning methods used in SELEX experiments 351

Table 9.3: Recovery methods used in SELEX experiments 352

Table 9.4: Affinity methods used to determine the dissociation constant of selected aptamers .353 
1 An introduction to aptamers, the selection of aptamers by Systematic Evolution of Ligands by EXponential enrichment (SELEX), and a comprehensive review of the use of aptamer-based technology in the central nervous system 


\subsection{Statement of contributions}

The introduction chapter was based on a review written by EM McConnell, Matthew R. Holahan and Maria C. DeRosa. Additionally, the chapter contains updated literature to June 2016.

\subsection{Resulting publications}

This chapter is based mainly on the review "Aptamers as promising molecular recognition elements for diagnostics and therapeutics in the central nervous system" published in Nucleic Acid Therapeutics (McConnell, E.M; Holahan, M.R; DeRosa, M.C; Nucleic Acid Ther. 2014 24(6):388-404). ${ }^{1}$ 


\subsection{Introduction}

\subsubsection{A brief history of functional nucleic acids}

Historically, DNA and RNA were described in the context of the Central Dogma of Molecular Biology which, simply stated, defines the biological role of DNA as coding for RNA and subsequently the role of RNA as coding for proteins. ${ }^{2}$ Further, nucleic acids, particularly DNA, were understood to be non-functional as compared to proteins and were mostly responsible for information storage by means of the genetic code. ${ }^{3}$ In contrast to this belief, excellent work in the field of functional nucleic acids revealed that DNA behaves much more similarly to proteins than was originally believed. ${ }^{4,5}$ Though nucleic acids, which are made up of five different naturally occurring nucleotides (A,C,G,T, and U), lack chemical diversity compared to proteins (with as many as 20 different basic amino acids), like proteins, DNA and RNA can fold into complex 3dimensional structures capable of binding diverse target types. ${ }^{4,5}$ A particularly interesting class of functional nucleic acids are called aptamers.

\subsubsection{Naturally occurring and in vitro selected aptamers}

Aptamers are small, synthetic single-stranded nucleic acid polymers that bind with high affinity and selectivity to target molecules. Aptamers have been selected for targets ranging from small molecules to whole cells. ${ }^{6-8}$ Often described as 'natural aptamers', riboswitches are located in mRNA. These oligonucleotide sequences contain an highly conserved aptamer domain that binds small molecules with high specificity and affinity and an expression platform domain. ${ }^{9}$ Upon target molecule binding, a structural transition of the aptamer domain is stabilized. ${ }^{9}$ This change in secondary structure of the 
riboswitch either exposes or blocks the expression platform domain thereby regulating expression of the gene. ${ }^{9}$ Examples of known riboswitch-binding molecules include: adenine, guanine, flavin mononucleotide, thiamine, glycine, lysine, and Sadenosylmethionine. ${ }^{10-19}$ Artificial riboswitches, in which an aptamer domain was inserted into a transcript, have also been designed which were capable of regulating gene expression. ${ }^{20-25}$ Compared to in vitro selected aptamers, riboswitches tend to have longer binding motifs and higher affinities for their cognate targets. ${ }^{9}$

\subsubsection{Aptamers as affinity tools}

Aptamers belong to a class of molecules characterized by their affinity for specific target molecules. ${ }^{26}$ These affinity tools are used for detection, purification and removal, diagnostics, therapeutics, theranostics and smart material applications. ${ }^{26-28}$ The most commonly used, and most well characterized affinity tools are antibodies. ${ }^{26}$ Antibodies are biologically derived proteins that bind to a specific target with high affinity and specificity. They can be either monoclonal (one binding epitope) or polyclonal (multiple binding epitopes) depending on how they were produced. Aptamers, engineered binding proteins, and molecularly imprinted polymers (MIPs) have all emerged as potential alternatives to antibodies for affinity based applications. ${ }^{26,29,30}$ Engineered binding proteins are non-antibody proteins that have been modified into binding proteins. Based on the molecular composition of antibodies, engineered binding proteins are isolated from protein variant libraries based on the typical structure of antibodies. ${ }^{26,30}$ Molecularly imprinted polymers are synthetic scaffolds that can recognize a single target molecule or a group of structurally and/or chemically similar 
molecules. ${ }^{26,29}$ The characteristics of these affinity tools are compared in Table 1.1..$^{8,26,31,32}$ Though aptamers are often described as functionally similar to antibodies, aptamers have multiple advantages over antibodies (Table 1.2). ${ }^{26,28,33}$ 
Table 1.1: Characteristics of antibodies, aptamers, binding proteins and MIPs. Partially reproduced and modified with permission from Portland Press, Ltd from Ruigrok, VJB., Levisson, M., Eppink, MH., Smidt, H., and Van der Oost, J. Alternative affinity tools: more attractive than antibodies. Biochem J. 2011, 436: 113. Copyright Portland Press, Ltd. 2011.

\begin{tabular}{|c|c|c|c|c|}
\hline & Antibodies & Aptamers & $\begin{array}{l}\text { Binding } \\
\text { Proteins }\end{array}$ & MIPs \\
\hline $\begin{array}{l}\text { Selection } \\
\text { method }\end{array}$ & In vivo & In vitro & In vitro & In vitro \\
\hline $\begin{array}{l}\text { Production } \\
\text { source }\end{array}$ & $\begin{array}{l}\text { Animal or } \\
\text { recombinant }\end{array}$ & Synthetic & Recombinant & Synthetic \\
\hline $\begin{array}{l}\text { Typical Size } \\
\text { (kDa) }\end{array}$ & $\sim 150-160$ & $<30$ & $\sim 5-20$ & N/A \\
\hline $\begin{array}{l}\text { Target } \\
\text { molecules }\end{array}$ & $\begin{array}{l}\text { Mostly } \\
\text { immunogenic } \\
\text { macromolecules }\end{array}$ & $\begin{array}{l}\text { Small } \\
\text { molecules to } \\
\text { cells including } \\
\text { toxins }\end{array}$ & $\begin{array}{l}\text { Macromolecules } \\
\text { and low- } \\
\text { molecular-mass } \\
\text { molecules }\end{array}$ & $\begin{array}{l}\text { Mostly low- } \\
\text { molecular-mass } \\
\text { molecules }\end{array}$ \\
\hline $\begin{array}{l}\text { Post-selection } \\
\text { modifications }\end{array}$ & Feasible & $\begin{array}{l}\text { Multiple } \\
\text { options for } \\
\text { modification of } \\
\text { backbone, } \\
\text { sugar and base } \\
\text { at terminal or } \\
\text { internal } \\
\text { positions }\end{array}$ & Possible & $\begin{array}{l}\text { Achievable } \\
\text { when } \\
\text { considered } \\
\text { during } \\
\text { imprinting }\end{array}$ \\
\hline Stability & Weeks at $4^{\circ} \mathrm{C}$ & $\begin{array}{l}\text { RNA: months } \\
\text { at }-80^{\circ} \mathrm{C} \text { and } \\
\text { DNA: years at } \\
\text { room } \\
\text { temperature }\end{array}$ & Variable & $\begin{array}{l}\text { Years at room } \\
\text { temperature }\end{array}$ \\
\hline $\begin{array}{l}\text { Binding site } \\
\text { variation }\end{array}$ & $\begin{array}{l}\text { Monoclonal: } \\
\text { homogeneous } \\
\text { and polyclonal: } \\
\text { heterogeneous }\end{array}$ & Homogenous & Homogenous & $\begin{array}{l}\text { Heterogeneous } \\
\text { or } \\
\text { homogeneous } \\
\text { depending on } \\
\text { imprinting } \\
\text { method }\end{array}$ \\
\hline $\begin{array}{l}\text { Application } \\
\text { conditions }\end{array}$ & Physiological & $\begin{array}{l}\text { Both } \\
\text { physiological }\end{array}$ & $\begin{array}{l}\text { Both } \\
\text { physiological }\end{array}$ & $\begin{array}{l}\text { Mainly organic } \\
\text { solvents. Some }\end{array}$ \\
\hline
\end{tabular}




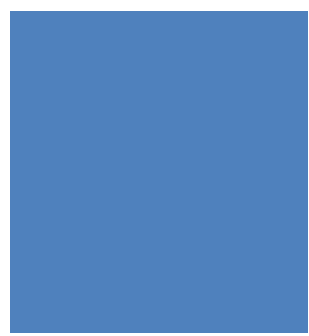

and aqueous

non-

physiological.

and non-

use in aqueous

Some use in

physiological

solutions.

organic

solvents.

Table 1.2: A comparison of the advantages and limitations of aptamers and antibodies

\begin{tabular}{|c|c|c|}
\hline \multicolumn{2}{|c|}{ Aptamers } & Antibodies \\
\hline Advantages & 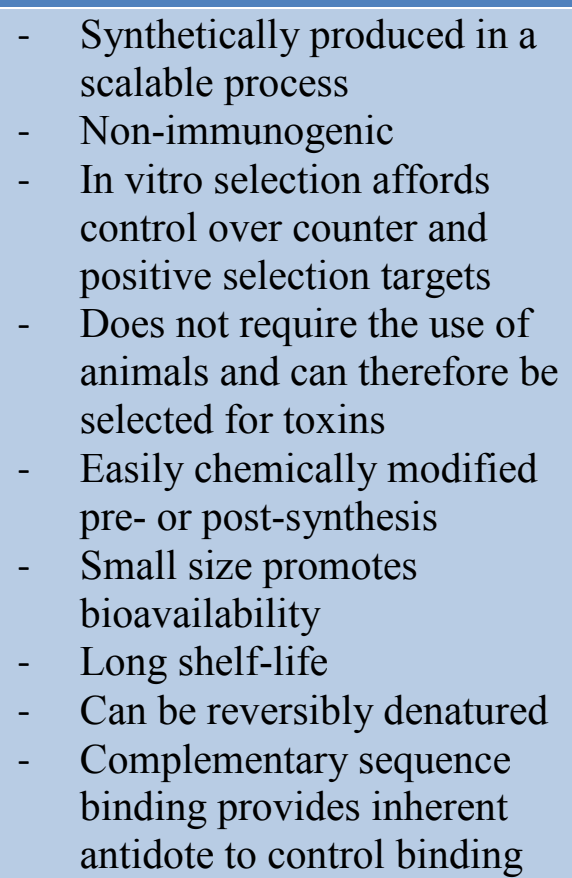 & $\begin{array}{l}\text { - } \begin{array}{l}\text { Not susceptible to nuclease } \\
\text { degradation }\end{array} \\
\text { - } \quad \text { Large size prevents renal } \\
\text { clearance } \\
\text { - } \quad \text { Typically have very high } \\
\text { affinity (at least nanomolar) } \\
\text { and specificity for their } \\
\text { cognate targets }^{26}\end{array}$ \\
\hline Limitations & 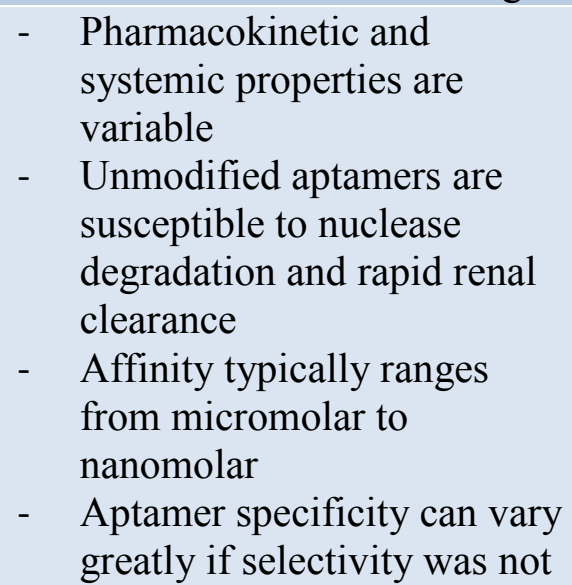 & $\begin{array}{ll}\text { - } & \text { Tend to be immunogenic } \\
\text { - } & \text { Large size limits } \\
\text { bioavailability } \\
\text { - } & \text { Biological production limits } \\
\text { scalability and selection } \\
\text { against counter targets } \\
\text { - } \quad \text { Limited shelf-life } \\
\text { - } \quad \text { Can be irreversibly } \\
\text { denatured } \\
\text { - Chemical modification by } \\
\text { attachment chemistry can } \\
\text { lead to reduced activity }\end{array}$ \\
\hline
\end{tabular}


controlled for during

selection

\subsubsection{Systematic Evolution of Ligands by EXponential enrichment (SELEX)}

Aptamers are selected by an in vitro process termed Systematic Evolution of

Ligands by EXponential enrichment ( $\underline{\text { SELEX }} .{ }^{34-36}$ Briefly summarized in Figure 1.1, the general process begins with the incubation of a pool of $10^{14}-10^{16}$ randomized oligonucleotide molecules (1) with the target molecule (2). From this incubation reaction (3), sequences with an affinity for the target (5) are partitioned from sequences that either weakly interact or do not bind to the target molecule (4). The relatively small pool of oligonucleotides identified as target binders (5) is then amplified to create an enriched library (6) which is then reintroduced to the target molecule and the iterative process begins again. The number of selection rounds required to obtain high affinity aptamers depends on a combination of factors but is mostly dependent on the selection method and the target type. Since the first in vitro selection methods were described, the SELEX process has evolved in many ways for a variety of target types and applications. ${ }^{8}$ Typically the selection template (Figure 1.1: inset) was described as having a randomized region (green) of variable length $(\mathrm{N})$ that was flanked by two known primer binding regions (red). Diversification in the process has shown success using templates with more than one random region, as well as templates designed for a particular structural bias or function. ${ }^{37}$ The different types and specific adaptations of SELEX have been reviewed elsewhere and are described in Chapter 2 and the accompanying Appendix $2.1 .{ }^{38}$ 


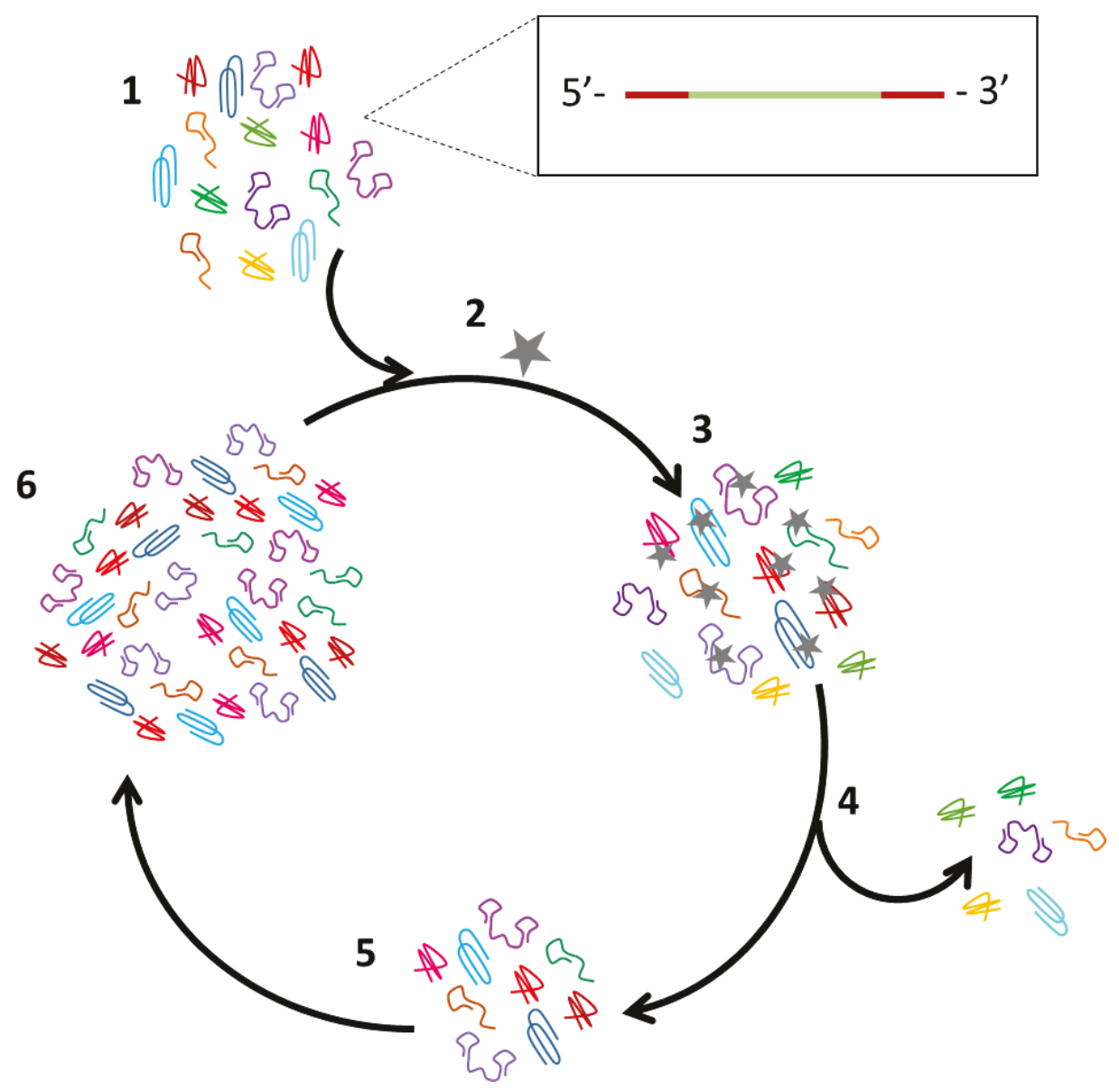

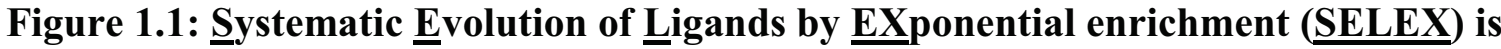
used to select aptamers with high affinity and selectivity for target molecules. Coloured lines represent aptamer candidates, grey stars represent the target molecule. (1) Initial library; inset - library template. (2) Selection target. (3) Library and target incubation. (4) Partitioning. (5) Amplification. (6) Enriched library. Typically 8-12 selection rounds are required to produce high quality aptamers.

\subsubsection{In vivo applicability and utility of aptamers}

The in vivo applicability and utility of aptamers is limited by the factors described in Table 1.2. One of the biggest challenges is the limited in vivo stability and circulation times of unmodified nucleic acids. There are several strategies that minimize nucleic acid degradation including modifications to the bases and/or backbone, the addition of 5'- and

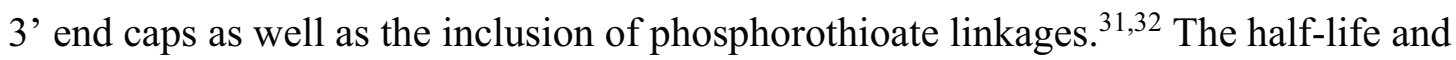


blood clearance of aptamers in vivo can also be improved by increasing the molecular weight of the aptamer. Another strategy to increase molecular weight and in some cases add functionality is to create an aptamer conjugate. One of the simplest and most effective conjugation strategies is the addition of polyethylene glycol (PEG). ${ }^{39,40}$ Additionally, the blood circulation time could be tuned according to the modification used, allowing the aptamer properties to be tailored for specific applications. Antibodies have been designed in this way. For example, antibodies have been conjugated to imaging probes to improve specific targeting, but the relatively long blood residence times of antibodies have led to toxicity and decreased imaging quality. Aptamers could be used as an alternative to antibodies in instances where a relatively long blood residence time is disadvantageous, such as cases of dose-related toxicity and improving

in vivo imaging. ${ }^{41}$ In most cases, modifications can be made to the aptamer, application design or delivery system to overcome these obstacles. ${ }^{42}$ The chemical modification chosen should be carefully considered however, as any modification to the aptamer sequence may have diminishing or even deleterious effects on aptamer affinity and specificity.

\subsubsection{Aptamers as potential tools for the central nervous system}

Central nervous system (CNS) related disorders are estimated to affect 3.8 million individuals in Canada alone. ${ }^{43}$ Additionally, the age distribution of Canada's population is shifting which will eventually result in an increase of the 65 year and older demographic from $15 \%$ to $23 \%$ by $2031 .{ }^{43}$ Given the socioeconomic burden of CNS disorders, the current limitations of treatment strategies and the increasing prevalence of 
onset with age, developing novel diagnostic and treatment strategies is essential. A great deal of work has focused on the potential of aptamers in medical fields. For example, aptamers have been selected and applied in the fields of diagnostics, imaging, targeted delivery, and therapeutics. ${ }^{39,44} 46$ Although the central nervous system presents an interesting and diverse application base, aptamer research for CNS related targets and applications is relatively constrained, likely due to the structural complexity of the biological system.

The CNS presents interesting challenges and limitations for aptamer research that are unparalleled in other biological systems. The blood-cerebrospinal fluid barrier (BCSFB) and blood-brain barrier (BBB) function to protect the brain and spinal cord from changes in the plasma content and regulate permeability. ${ }^{47,48}$ Of particular relevance, the $\mathrm{BBB}$ and $\mathrm{BCSFB}$ regulate the transfer of drugs into and out of the brain. Structurally, the BCSFB and the BBB are composted mainly of choroid plexus epithelial and cerebrovascular endothelial cells respectively. ${ }^{47,49}$ The rate and efficiency of drug transport into the brain depends on the interplay of the pharmacokinetics and pharmacodynamics of the drug. These properties, and eventually the rate and efficiency of drug transfer into the brain are determined by the physical and chemical characteristics of the drug (molecular weight ( $<400 \mathrm{Da})$, charge, polarity, lipophilicity, target affinity, etc.) as well as biological system parameters (cerebral blood flow, blood/tissue $\mathrm{pH}$, barrier permeability, etc.). ${ }^{49,50}$ Since aptamers are large, polar, negatively charged molecules, brain permeability was expected to be low. In 2004, Healy et al., presented evidence that the intravenously injected aptamer and aptamer conjugates they studied were not distributed to the brain and therefore did not cross the brain barriers. ${ }^{40}$ Later, 
Perschbacher et al., (2015) used quantitative PCR to show that some of the intraperitoneally injected aptamers they studied did circumvent the brain barriers and were detected in brain tissue, suggesting that some aptamers can cross the brain barriers. ${ }^{51}$ Differences in the sequence and secondary structure of the aptamers as well as the sensitivity of the detection techniques could explain the difference in detection. Interestingly, unmodified RNA aptamers that were capable of traversing the brain barriers were selected by Cheng et al. (2013). ${ }^{50}$ Further investigation is required to determine the exact mechanism by which the aptamers penetrate the brain barriers, but aptamer entry into the brain endothelia cells and parenchyma was observed. The authors hypothesized that since paracellular aqueous or transcellular lipophilic mechanisms were unlikely due to the physical properties of aptamers, the most likely entry method was either cellular/receptor-mediated uptake or pinocytosis. ${ }^{50}$ Currently, aptamer-based technologies are typically delivered to the brain by either the incorporation or encapsulation into a delivery vehicle such as liposomes, nanoparticles, micelles and dendrimers. ${ }^{48,52}$ The development of brain-penetrating aptamers expands the functionality and viability of delivery options. Conjugation of these aptamers to delivery vehicles or preparation of aptamer conjugates could prove useful in adding specificity or decreasing the complexity of CNS-targeting technologies. ${ }^{39,53}$

\subsection{Highlights of aptamer based research for CNS targets}

Aptamer research for CNS related targets has focused on a limited number of targets and pathologies. The remainder of the chapter will highlight aptamer research for neurotransmitter and neurotoxin targets, demyelinating disease and spinal cord injury, 
cerebrovascular disorders, protein aggregation related pathologies (Alzheimer's, Parkinson's and prion disease), brain cancer (glioblastomas and gliomas), receptor function regulation, and investigating interneuronal signaling.

\subsubsection{Neurotransmitter and neurotoxin targets}

\subsubsection{Dopamine and Schizophrenia}

\subsection{Dopamine binding aptamer (DBA) based detection assays}

Ranging from small molecules to proteins, neurotransmitters are chemical messengers that are released from presynaptic neuronal membranes and exert their effect by acting on post-synaptic neuronal membranes. ${ }^{54}$ The majority of selections and characterization of aptamers for neurotransmitters have focused on the small molecule dopamine (DA). These dopamine-binding aptamers (DBAs), and the sensors that have been developed based on them are discussed in detail in Chapter 6.

\subsection{The efficacy of a dopamine binding aptamer in a models of Schizophrenia and cocaine-induced hyperlocomotion}

Dopamine related dysfunction has long since been associated with schizophrenia and addiction. The ability of the DNA DBA was investigated in an animal model of schizophrenia. This study is discussed in detail in Chapter 4. Further, the ability of the DNA homolog DBA to reduce cocaine-induced hyperlocomotion was examined (See Chapter 5). 


\subsubsection{Aptamers for other neurotransmitters}

For other neurotransmitter targets, aptamer research has focused mainly on novel selections and some sensor development. Selections have been performed for aptamers that bind to acetylcholine, adenosine, brain natriuretic peptide, recombinant antineuroexcitation peptide and neuropeptide $Y .{ }^{55-61}$ The development of aptamers for neurotransmitter targets is especially important as aptamers could be used to develop technology to enable researchers to garner more information about neurotransmitter function and receptor transmission as well as investigate potential therapeutic strategies. In fact, a DNA aptamer selected for the target adenine was used to monitor the release of adenine compounds in real time by anchoring the aptamer to the cell surface of brain astrocytes. $^{62}$

\subsubsection{Aptamers for the detection of botulinum neurotoxin}

Botulinum neurotoxin (BoNT), one of the most devastating neurotoxins known, is classified as a Category A bioterrorism agent by the United States Centers for Disease Control. ${ }^{63}$ The ability to detect BoNT has been the focus of many studies which have been reviewed previously. ${ }^{64}$ As such, BoNT has been one of the most popular targets for neurotoxin related aptamer studies. The selection of DNA aptamers for BoNT additionally focused on optimizing generally applicable selection methods to obtain high affinity aptamers in minimal time with a small amount of target. ${ }^{65,66}$ Additionally, several aptamer based sensors have been developed for the detection of botulinum. ${ }^{67-72}$ Useful therapeutics may be developed from RNA aptamers that were selected to inhibit the toxicological mechanism of BoNT. ${ }^{73}$ Though BoNT is most commonly associated with 
botulism, it has been shown to have some therapeutic efficacy in other CNS related disorders such as diabetic neuropathy, migraine headaches and multiple sclerosis. Therefore, aptamers selected for BoNT may prove useful in the development or improvement of technology for multiple disorders and diseases. Additionally, these aptamers could have potential applications within the fields of food safety and bioterrorism. $^{64}$

\subsubsection{Aptamers for other neurotoxins}

Neurotoxin targets are an exception compared to the other aptamer targets discussed in this chapter in that they often act on the peripheral nervous system and sometimes result in death before any central nervous system related symptoms can manifest. ${ }^{63}$ The majority of non-botulinum neurotoxin targets investigated are those derived from algal and marine sources. Aptamers have been selected and investigated for targets in this group including: Anatoxin-a, brevetoxin-2, gonyautoxin 1-4, and saxitoxin. ${ }^{74-77}$ These targets are of particular interest because paralytic shellfish poisoning poses economic and public health burdens on costal populations worldwide. ${ }^{78,79}$ Another class of neurotoxin that has been investigated are neurotoxins contained in snake venom such as $\beta$-Bungarotoxin and Phospholipase $\mathrm{A}_{2}{ }^{80,81}$ Current estimates suggest that 5 million people a year are bitten by a snake. ${ }^{82}$ Aptamers offer a safer, more stable and more practical alternative to traditional antivenoms. ${ }^{57}$ Finally, second to botulinum, the most work for a single neurotoxin target has been done for ricin. Multiple selections have been performed to identify both DNA and RNA aptamers for ricin. ${ }^{83-86}$ These aptamers have been used extensively in sensors for the detection of ricin 
and inhibition of ricin activity. ${ }^{71,72,87-93}$ The binding interactions and conformation have also been thoroughly characterized ${ }^{94-98}$ Additionally, the stability of an RNA aptamer for ricin was enhanced by the incorporation of locked nucleic acids into the sequence. ${ }^{99,100}$

\subsubsection{Demyelinating disease and spinal cord injury}

\subsubsection{Multiple Sclerosis, myelin and myelin related targets}

Demyelinating diseases such as Multiple Sclerosis (MS) are inflammatory neurological diseases characterized by the degeneration of axon-insulating myelin. ${ }^{101}$ Clinically, demyelination presents as fatigue, as well as impairments of vision, cognition and gait. Aptamers that inhibit the biological function of their target molecule have been selected for targets related to MS that include; interleukin-23, midkine, 2',3'-cyclic nucleotide 3'-phosphodiesterase and interleukin-17A. ${ }^{102-105}$ DNA aptamers were selected to bind to the murine CD200R1 receptor and were shown to act as signaling molecules in the absence of the endogenous receptor target, CD200. ${ }^{106}$ The authors proposed that this aptamer could offer a nonsteroidal alternative to typical anti-inflammatory drugs. ${ }^{106}$ RNA aptamers have been selected for anti-myelin basic protein autoantibodies, the presence of which have been shown to be elevated in early on-set MS as well as autism. ${ }^{107-109}$ The authors presented preliminary work towards the development of a diagnostic laboratory test based on anti-MBP autoantibody detection by aptamer binding. ${ }^{107}$ This is an important step as currently a differential diagnosis of MS is based on clinical and paraclinical data and no diagnostic laboratory test exists. ${ }^{110}$ In another study, Rozenblum et al., (2014) selected DNA aptamers for myelin basic protein that outperformed commercially available anti-MBP antibodies in complex matrix as well as in an enzyme- 
linked immunosorbent assay. ${ }^{111}$ The aptamer identified may eventually lead to the development of an aptamer-based immune-protective therapeutic if binding of the aptamer to MBP can prevent immune-reactive T-cell activation. ${ }^{111}$

\subsubsection{Aptamers for the treatment of spinal cord injuries}

Injury to the spinal cord results in local and diffuse damage to the primary and surrounding tissue respectively. In response to injury, the pathophysiological response leads to physical axonal disruption, the expression of axon growth inhibitors, glial scarring and myelin degradation. ${ }^{112}$ For this reason, myelin is also a target of interest in spinal cord injury. Nastasijevic et al., (2012) selected a DNA aptamer that binds to myelin. ${ }^{101}$ The effect of an aptamer-biotin conjugate on remyelination of the spinal cord was examined in Theiler's encephalomyelitis virus (TMEV)-infected mice exhibiting MS-like lesions. ${ }^{101}$ At 27-weeks post TMEV-infection, mice were treated (i.p.) with aptamer conjugate solution $(0.5 \mathrm{nmol})$ twice weekly for 5 weeks. Significant remyelination (35\%) was observed in the spinal cords of TMEV-infected mice that had been treated with aptamer compared to non-binding control aptamers $(4-9 \%) \cdot{ }^{101}$ Further analysis revealed that the aptamer undergoes an ion-dependent conformational change from an anti-parallel G-quadruplex in the absence of potassium to a parallel Gquadruplex under physiological conditions. ${ }^{113}$ Additionally, the in vivo distribution of the aptamer and aptamer-biotin conjugate was evaluated by quantitative PCR. ${ }^{114}$ The aptamer and aptamer-biotin conjugate were present in the liver, spleen, kidney and blood. Interestingly, the aptamer and aptamer-biotin conjugate were also detected in the spinal cord and brain. 
In another study, an RNA aptamer was selected for the Nogo-66 receptor (NgR).

The $\mathrm{NgR}$ is known to be involved in the regulation of axon regeneration. ${ }^{115}$ The aptamer was investigated to determine its ability to competitively bind to NgR over myelinderived inhibitors such as myelin-associated glycoprotein (MAG), Nogo-A and oligodendrocyte myelin glycoprotein (OMgp). The aptamer was able to out compete the myelin-derived inhibitors and promote neurite outgrowth. When the axons of cells had been trimmed, the myelin-derived inhibitors decreased neurite outgrowth (Figure 1.2).

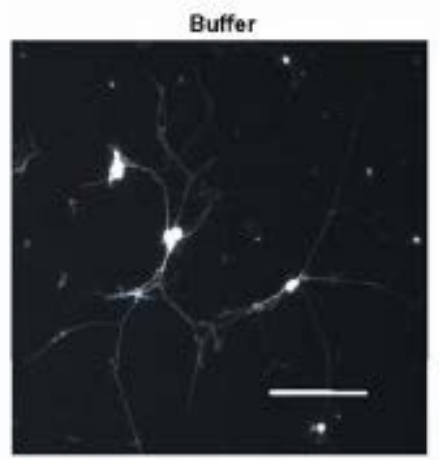

Myelin-Derived Inhibitors + R0 RNA

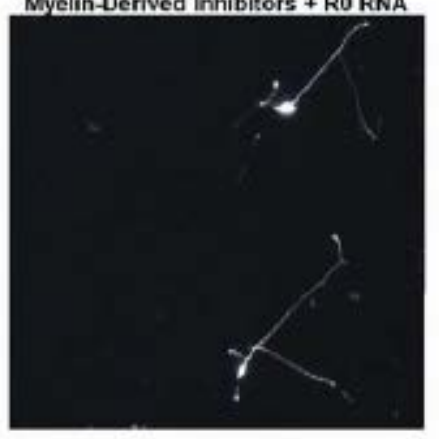

Ro RNA (Random Sequences)

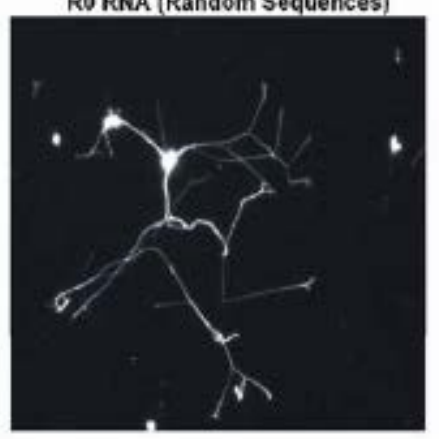

Myelin-Derived inhibitors + Soluble NgR

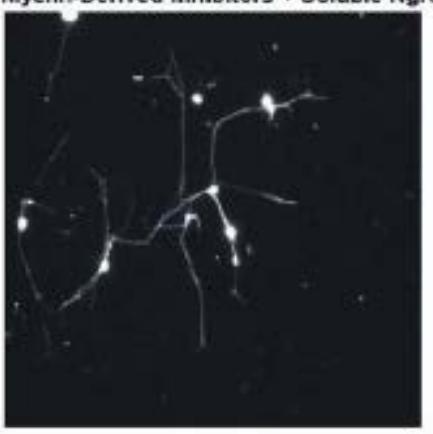

Dyelin-Derived inhibitors

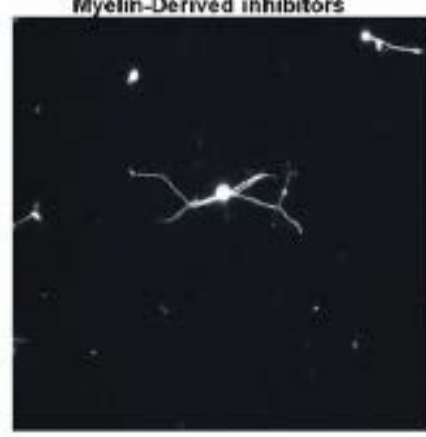

Myelin-Derived inhibitors + Clone 40

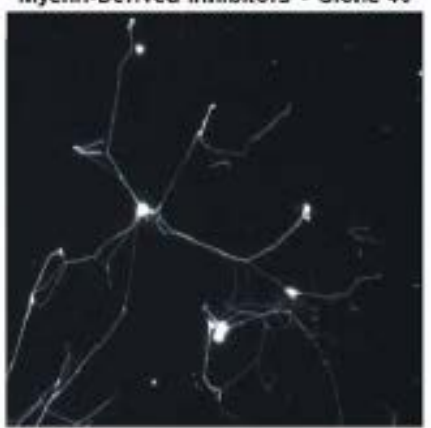

Figure 1.2: Aptamer treatment induces recovery of neurite outgrowth in dorsal root ganglia (DRG). In control groups, neurons (200-500/group) were treated with buffer, a random oligonucleotide (RO RNA), myelin-derived inhibitors (MDIs), MDIs + RO RNA, or MDIs + soluble NgR. The experimental groups were treated with MDIs and one of five different aptamers (Clone 40 shown). $100 \mathrm{nM}$ of each MDI (Nogo-A,MAG and OMgp) was added ( $300 \mathrm{nM}$ total concentration). Aptamers were added at $10 \mu \mathrm{M}$. Non-specific anti-beta III tubulin antibody was used to stain DRGs. The average neurite length was normalized by comparing groups to the buffer alone control. Scale bar $200 \mu \mathrm{m}$. Figure reproduced with permission under 


\section{Creative Commons licensing from Wang, Y.; Khaing, Z. Z.; Li, N.; Hall, B.;}

Schmidt, C. E.; Ellington, A. D. PLoS One 2010, 5 (3), e9726.

When the cells were treated with aptamer however, neurite outgrowth was increased significantly leading to $80-90 \%$ growth recovery compared to the buffer control whereas the inhibitor and inhibitor/random oligonucleotide treated controls only showed $\sim 50 \%$ recovery. This important work validates the potential of aptamers to be used to treat spinal cord injury but more generally as neuromodulators.

\subsubsection{Cerebrovascular disorders}

\subsubsection{Aptamers for stroke intervention}

Many of the diagnostic and therapeutic effects of anticoagulant and antithrombotic aptamers were investigated in models of cardiovascular disease, including but not limited to ischemic stroke. ${ }^{116}$ Several of these aptamers, which bind to the targets thrombin, vWF, factor IX or factor XII, have been investigated in clinical trials. ${ }^{116}$ The excellent work done by many aptamer research groups to investigate the regulation of blood coagulation outside the CNS was recently reviewed. ${ }^{117,118}$ Affecting one in six people worldwide, the most common acquired disability is stroke. ${ }^{119}$ In both ischemic and hemorrhagic strokes, normal blood flow is interrupted which leads to cell death. Without intervention, stroke can have disastrous effects leading to lack of functional recovery and death. Therapeutic strategies tend to focus on treatment with antithrombotics and antiplatelet factors to improve reperfusion. ${ }^{120}$ Recently, significant progress has been presented utilizing aptamer technology for stroke intervention. That progress is highlighted in the following section. 
Currently, tissue-type plasminogen activator (TPA) is the only treatment for cerebral ischemic stroke approved by the United States Food and Drug Administration. ${ }^{121}$ TPA works to increase reperfusion by promoting the destruction of intravascular clots. However, TPA is rarely used as it needs to be administered relatively quickly after symptom onset and has an associated risk of uncontrollable intracranial hemorrhage. ${ }^{120}$ Evidence suggests that the interaction of TPA with low-density lipoprotein (LDL) family receptors is advantageous to vasculature but detrimental to the cerebral parenchyma during ischemic stroke. ${ }^{121}$ Aptamers were selected to bind to TPA under conditions where LDL family receptor-related protein-1 (LRP-1) were inhibited and fibrinolytic activity of TPA was maintatined. ${ }^{121}$ Two aptamers were identified that were able to efficiently modulate LRP-1 function while minimally effecting clot lysis in cell culture. Additionally, receptor-associated protein (an LDL family receptor ligand) sensitive endocytosis was inhibited. Therefore, coadministration of a TPA binding aptamer and TPA may improve TPA treatment by aptamer-mediated restriction of the site of TPA action to the vasculature.

Aptamers were selected for factor IXa, an enzyme involved in the blood coagulation cascade, and their antithrombotic effect was investigated in a murine model of ischemic stroke. ${ }^{120,122}$ Specifically, the effect of the aptamer on anticoagulation, thrombin generation, inflammation and neurological deficit was explored. ${ }^{120}$ To determine the effect of the aptamer on anticoagulation, clotting time was assessed after treatment with the aptamer. The observed clotting times of animals treated with the aptamer was significantly increased compared to controls. When animals were further treated with the aptamer antidote (the aptamer complement), the clotting time observed 
was similar to that seen in controls. This is represented schematically in Figure 1.3 A.

The rotarod test, a vestibulomotor test during which the period of time an animal is able to stay on a rotating rod without falling off, was used to measure neurological function.

In animals that were treated with the aptamer, the amount of time spent on the rotarod (rotarod latency) was significantly increased compared to controls (Figure 1.3 B). This is an indication that aptamer treatment in some way minimized stroke associated motor dysfunction.

One of the biggest challenges associated with current stroke interventions such as tissue-type plasminogen activator (TPA) is associated risk of uncontrollable intracranial hemorrhage. ${ }^{120}$ An advantage of aptamers is that inherent to their design, their complementary sequence affords an antidote.
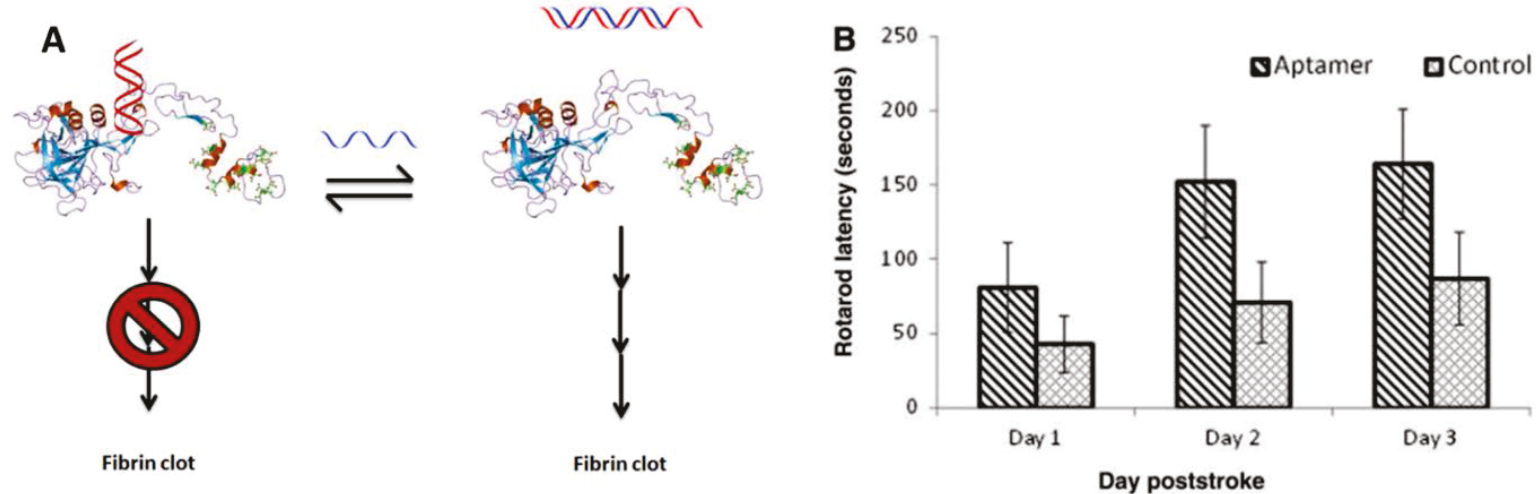

Figure 1.3: Aptamer -antidote dependent fibrin clotting (A) and recovery of rotarod latency post stroke (B). In A, when the aptamer was bound to Factor IXa (in the absence of the antidote) it was able to act as an anticoagulator. When the antidote was present, the aptamer preferentially bound to the antidote (the aptamer's complementary oligonucleotide) allowing coagulation to occur. Shown in B, aptamer treated animals $(n=12)$ experienced significantly greater neurological functional recovery than those treated with an inactivated oligonucleotide control $(n=12)$. Statistical analysis by repeated measures ANOVA with Fisher partial least squares difference post hoc analysis revealed a significant difference between groups 
p<0.05). Figure 3 B was produced from data presented in Blake, C. M.; Wang, H.; Laskowitz, D. T.; Sullenger, B. A. Oligonucleotides 2011, 21 (1), 11-19.

Blake et al., (2011) examined the efficacy of the aptamer antidote administration in reversing anticoagulatory-related hemorrhage in a model of subarachnoid hemorrhage. ${ }^{120}$ The authors found that the hemorrhage size observed in the aptamer/antidote group was significantly decreased compared to those observed in the aptamer/saline treated group. When the Factor IXa aptamer was administered without the antidote, a 71\% increased mortality rate was observed compared to animals that had received an inactivated control $(0 \%)$. As expected, the aptamer prevented coagulation, however to the detriment of the animals. Importantly, a significant decrease in mortality (33\%) was observed when the aptamer/antidote was administered. This work was the first example of a reversible aptamer-based therapeutic for the treatment of ischemic stroke but also serves as an important example of the potential of aptamer/antidote based treatment strategies to be used to add an extra element of control in otherwise risky treatments. Unfortunately, a clinical trial examining the ability of aptamer/antidote treatment to reduce ischemic events without increasing bleeding post percutaneous coronary intervention was recently terminated due to severe allergic reaction to the aptamer/antidote formulation. ${ }^{123}$ Additionally, from the preliminary data collected, there was insufficient evidence to suggest that the aptamer/antidote formulation reduced ischemic events or bleeding compared to the direct thrombin inhibitor (bivalirundin) control.

Another target investigated was von Willebrand (vWF) factor which is involved in thrombosis. Platelet aggregation is in part mediated by the interaction between vWF 
and platelet glycoprotein $\mathrm{Ib}^{124} \mathrm{vWF}$ is of particular interest because targeting it could allow for the inhibition of the prothrombotic function of vWF without disrupting the coagulation system. ${ }^{125}$ Therefore decreasing the risk of treatment complications such as intracerebral hemorrhage. ${ }^{125}$ The efficacy of an aptamer (ARC1779) selected to bind to vWF on cerebral embolization was examined in a randomized clinical trial. ${ }^{124-126}$ In this study, patients undergoing carotid endarterectomy for carotid stenosis were monitored for post-operative stroke, as risk associated with the procedure. ${ }^{125}$ Injections of ARC1779 were given to patients before, during and after surgery. A reduction in cerebral embolism was observed in patients that had received the aptamer treatments.

Finally, it is important to mention the work done investigating thrombin binding aptamers. Thrombin is a protease involved in the coagulation cascade. ${ }^{127}$ Fibrinogen is cleaved into fibrin by thrombin at the site of vascular injury which results in clot formation. ${ }^{127}$ Several aptamers have been selected that bind to thrombin isoforms, including human $\alpha$-thrombin. ${ }^{128-130}$ The aptamer-thrombin binding interaction has been an important relationship in aptamer research. It is the most commonly used model systems used in the design of proof-of-concept systems. In fact, approximately $20 \%$ of the aptamer-related research papers published between 1990 and 2013 made use of one or both of the thrombin aptamers. ${ }^{127}$ Deng et al., (2014) recently reviewed the development of over one hundred thrombin binding aptamer based analytical techniques with a focus on affinity separation, homogenous assays and electrochemical aptasensors. Chapter 3 describes the incorporation of a thrombin binding aptamer into a pH-driven Aptamer Switch for Thrombin (pHAST), which may provide valuable insight into $\mathrm{pH}$ changes during ischemic stroke. 
Additionally, work has been done to investigate the effect of creating multiaptamer conjugates or bivalent aptamers on clotting. In one example, two thrombin aptamers were tethered together by a poly A tail and showed a 5-fold increase in thrombin catalytic efficiency compared to the $\sim 2$-fold increase observed for either

thrombin binding aptamer alone. ${ }^{131}$ In another example, aptamers for factor Xa and prothrombin were linked together and were shown to increase clotting time more so than either aptamer alone. ${ }^{132}$ When the fully complementary antidote oligonucleotide was introduced the anticoagulatory effect was fully reversed. Interestingly, when either single aptamer antidote was added, the clotting time was reduced to that of the active aptamer alone.

\subsubsection{Aptamers for migraine related targets}

The term migraine encompasses a class of neurological disorders associated with disrupted neurovascular function. Calcitonin gene-related peptide (CGRP) is a neuropeptide that is a potent vasodilator and is associated with regulation of meningeal blood flow. ${ }^{133}$ Elevated plasma levels of CGRP have been linked to migraines and headaches. ${ }^{134}$ Further, infusion of CGRP was shown to lead to migraine-like attacks in individuals who had a history of migraines. ${ }^{135}$ The results of several phase I and II clinical trials support the causative role of CGRP in headaches as CGRP receptor antagonists reduced migraine pain. ${ }^{136}$ Two selections have been performed to select RNA Spiegelmers that bind to CGRP. ${ }^{133,136}$ Spiegelmers are the mirror-image aptamers. Spiegelmers are synthesized as L-configuration oligonucleotides, as opposed to the naturally occurring D-configuration. ${ }^{137}$ Since the L-configuration is non-naturally 
occurring, the configuration of Spiegelmers inherently affords biostability due to the stereoselective nature of enzymes such as nucleases. ${ }^{137}$ Spiegelmers are selected through a process in which the naturally occurring D-configuration aptamer against the mirrorimage of a chiral target is identified through traditional SELEX. The aptamer sequence is then synthesized as an L-oligonucleotide to produce a Spiegerlmer. The NOX-C89 aptamer described by Vater et al., (2003) was tested in several models. When NOX-C89 was applied topically to exposed dura mater of rats, a dose-dependent inhibition of blood flow was observed. ${ }^{138}$ Additionally, NOX-C89 treatment led to a reduction of CGRP release. ${ }^{138}$ The in vivo effect of the NOX-C 89 was compared to that of a monoclonal CGRP antibody in a rat closed cranial window model. ${ }^{139}$ Animals were pre-treated with either PEGylated NOX-C89 or the CGRP antibody and then received CGRP treatment. CGRP treatment induced dilatation of the dural and pial arteries. PEGylated NOX-C89 inhibited vasodilation of the dural and pial arteries to levels that were not significantly different than baseline measurements compared to the CGRP antibody which inhibited vasodilation of the dural artery, but not as low as baseline values. There was no significant difference in pial artery dilatation by CGRP before or after CGRP antibody administration. However, neither the PEGylated NOX-C89 or CGRP antibody were able to inhibit dilatation resulting from electrical stimulation, likely because neither the NOXC89 nor the CGRP antibody were able to cross the blood-brain barrier efficiently. ${ }^{139}$ Unfortunately, the NOX-C89 only showed moderate cross-reactivity with human CGRP and so clinical development was halted. ${ }^{136}$ Recently, Hoehlig et al., (2015) selected a Spiegelmer (NOX-L41) that binds to human and rat CGRP with high affinity and selectivity. ${ }^{136}$ Inhibition of plasma protein extravasation has been used as a model to 
select non-vasoconstrictive treatments for migraine. The authors showed that NOX-L41 was released from blood vessels of the dura mater, and was able to interact with CGRP and inhibit neurogenic meningeal plasma protein extravasation for a minimum of $18 \mathrm{~h}$ post injection. These studies are important as they show the potential of using aptamers to treat migraines but also the potential of Spiegelmer technology.

\subsubsection{Protein aggregation related pathologies}

\subsubsection{Aptamers for targets related to Alzheimer's disease}

Aggregation of beta amyloid peptide $(\mathrm{A} \beta)$ and hyperphosphorylated tau protein leads to the formation of amyloid plaques and neurofibrillary tangles, the presence of which is characteristic of the neuropathology of Alzheimer's disease. ${ }^{140}$ Patients with Alzheimer's disease present clinically with a deterioration of cognitive functioning and dementia. ${ }^{140}$ Physiologically, these symptoms are caused by microscopic and macroscopic changes that occur due to the abnormal aggregation of $A \beta$ peptide and hyperphosphorylated tau. ${ }^{140}$

The use of aptamers as potential tools to mitigate AD has been previously reviewed. ${ }^{141,142}$ With the intention of understanding and preventing protein aggregation, the majority of work done has focused on developing aptamers that selectively bind to either the monomeric or oligomeric forms of the $A \beta$ peptide. Only one selection focused on selecting aptamers that proved successful in degrading tau aggregates. ${ }^{143}$ Out of the selections done, there has been limited success in identifying aptamers capable of selectively binding either the monomeric or oligomeric target. $\mathrm{A} \beta$ peptide rapidly forms insoluble aggregates which has made selecting aptamers that bind to either the 
monomeric or oligomeric forms of $A \beta$ peptide over forms particularly difficult. Instead, the described aptamers tended to selectively bind to the fibrillary forms (A $\beta_{1-40}$ or $A \beta_{1-}$ 42). ${ }^{144,145}$ An important exception was work done by Takahashi et al., (2009) in which RNA aptamers were selected that selectively bound to monomeric A $\beta$ peptide. ${ }^{146}$ The ability of these monomeric $A \beta$ peptide binding aptamers to inhibit $A \beta$ aggregation and fibrillization was evaluated in an enzyme-linked immunosorbent assay and transmission electron imaging. Two aptamers, $\mathrm{N} 2$ and $\mathrm{E} 2$ were shown to reduce $\mathrm{A} \beta$ aggregation and fibrillization. Expanding on this work, the N2 aptamer was conjugated to poly(lactic-coglycolic acid) [PLGA]-coated curcumin encapsulated nanoparticles. ${ }^{147}$ The judicious choice of curcumin nanoparticles was used as it could have potentially amplified the effect as curcumin, has been shown to inhibit $\mathrm{A} \beta$ aggregate formation. ${ }^{148}$ Dynamic light scattering and scanning electron microscopy were used to evaluate the interaction between the aptamer modified nanoparticles and the protein aggregation. Though the degree of aggregation observed with the aptamer modified nanoparticles was similar to that observed for the nanoparticle control, the authors proposed that the aptamer modified nanoparticles could be used to specifically deliver curcumin to excess peripheral amyloid. Since marked reductions in peripheral amyloid have been associated with decreased brain amyloid, this may prove to be a useful strategy for treating $\mathrm{AD} .{ }^{147}$

Though the identification of aptamers that bind to amyloid fibrils holds potential for the detection and elucidation of amyloid related pathologies, several research groups have focused on alternative indirect approaches to preventing $A \beta$ aggregation. ${ }^{149}$ For example, RNA aptamers have been selected that bind to an epitope within the cytoplasmic domain of $\beta$-secretase (BACE1), a protease involved in initiating $\mathrm{A} \beta .{ }^{150}$ 
Recently, another selection against BACE1 identified DNA aptamers that were capable of binging to BACE1 with high affinity and specificity that when evaluated in an AD cell model, had an inhibitory effect on BACE1 activity. ${ }^{151}$ In another example, the role of nucleolin (a protein) in microglial associated phagocytosis of $A \beta_{1-40}$ and $A \beta_{1-42}$ was investigated using a nucleolin-binding DNA aptamer (known as AGRO100 or AS1411) developed by non-SELEX. ${ }^{152,153}$ Interestingly, AS1411 was successful in inhibiting microglial-associated phagocytosis of monomeric and fibril $\mathrm{A} \beta_{1-42}$ but not $\mathrm{A} \beta_{1-40}$.

Current strategies to diagnose AD include the identification and quantification of $\mathrm{AD}$ related biomarkers in blood and cerebrospinal fluid. Therefore existing aptamers may be successfully applied in diagnostic applications and there exists novel biomarker targets for which aptamers could be selected. ${ }^{154-156}$ Recently, the development of an $\alpha-1$ antitrypsin aptamer-antibody sandwich assay for the detection of $\alpha-1$ antitrypsin, a known biomarker for $\mathrm{AD}$, in serum was described that was able to detect $\alpha-1$ antitrypsin at clinically relevant levels. ${ }^{157-159}$

\subsubsection{Slow off-rate modified aptamers (SOMAmers) for the identification of AD biomarkers}

SOMAmers (slow off-rate modified aptamers) are a subclass of aptamers that hold significant potential for studying CNS-related disorders and diseases. ${ }^{160}$ SOMAscan is a SOMAmer-based capture array technology that allows for the identification of SOMAmers for protein biomarkers from biological samples. ${ }^{161}$ The technology, that requires less than $100 \mu \mathrm{L}$ of biological sample has been used to investigate 49000 samples from clinical studies, some of which focused on the identification of CNS- 
related biomarkers. ${ }^{162,163}$ SOMAscan technology has been used to discover blood-based protein biomarkers that could be useful in diagnosis and staging AD. ${ }^{158}$ SOMAmers have also been identified that bind to and inhibit interleukin-6 (relevant to brain cancer research), and nerve growth factor. ${ }^{164,165}$ Additionally, a SOMAmer array was developed which facilitated the identification of biomarkers of aging, which may provide clinical relevancy for AD and Parkinson's disease. ${ }^{166}$

\subsubsection{Aptamers for $\mathrm{AD}$ imaging applications}

Due to the relative ease of synthesis, chemical modification and in vivo stability, aptamers are excellent candidates for imaging applications within the CNS, for example diagnostic screening in AD. Recently, a multifluorescein-labelled RNA aptamer based probe was developed to detect and visualize $A \beta$ oligomers and amyloid plaques (Figure 1.4) in an in vivo mouse model of $\mathrm{AD} .{ }^{145,167}$ Construction of an imaging window by craniotomy allowed the aptamer probe to be applied topically to the surface of the brain and for multiphoton images to be obtained. Imaging with the fluorescent aptamer probe (green) 1 hour post application (Figure 1.4) showed the presence of amyloid plaques in the cerebral cortex and surrounding vasculature (red). 


\section{Plaque}

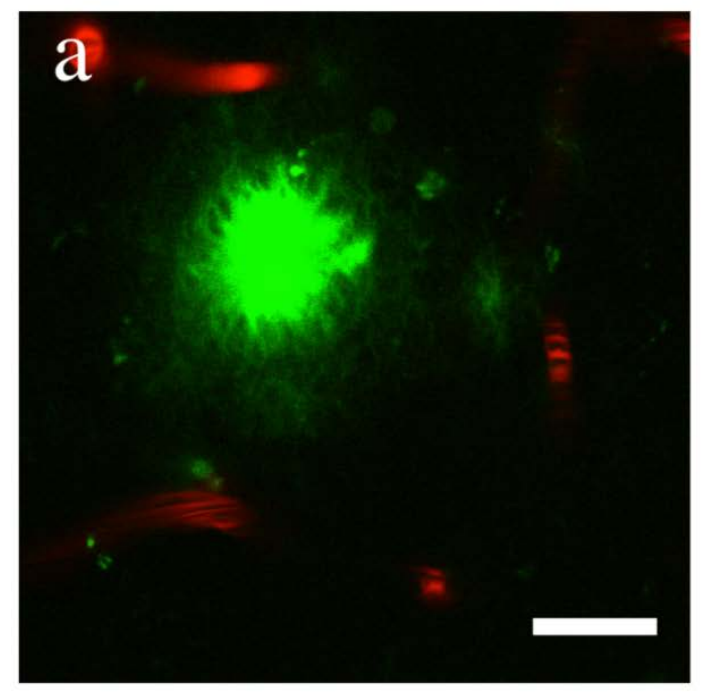

CAA

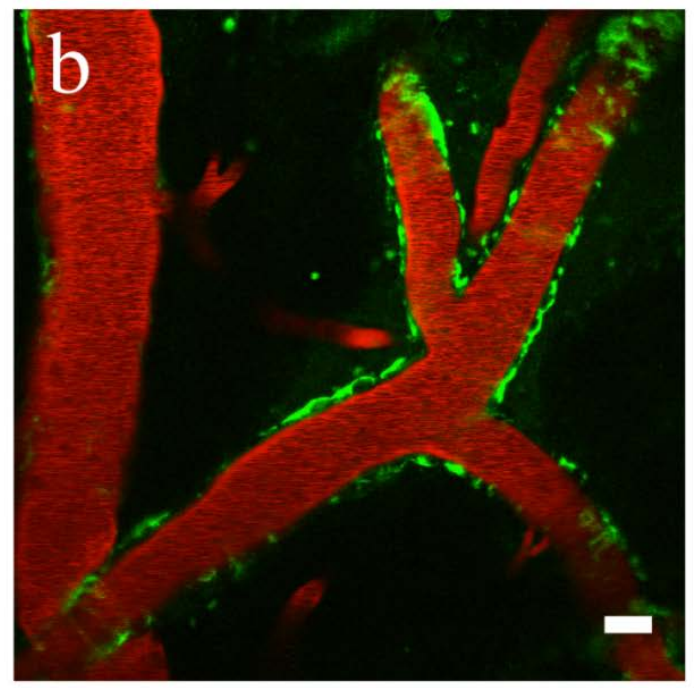

Figure 1.4: In vivo 2-photon microscopy was used to image plaques in the cortex (a) and amyloid (b) in the vasculature from an 18-month-old amyloid precursor protein (APP)/presenilin-1 (PS1) transgenic mouse that had received a topical application of the fluorescent aptamer probe 1 hour before imaging. Aptamer localization is shown in green and vasculature was visualized with Texas red-labelled dextran. Scale bars: $20 \mu \mathrm{m}$. CAA: cerebral amyloid angiopathy. Figure reproduced from Farrar et al., 2013 in accordance with the PLOS Creative Commons Attribution (CC BY) licence. $^{167}$

After a 24 hour period, another set of images were obtain which showed that the stability of the aptamer in vivo was at least 24 hours. Over this time, the amyloid plaque detection efficiency decreased from $96.0 \%$ at 1 hour to $77.8 \%$ after 24 hours. Simao et al., 2015 selected DNA aptamers for vascular cell adhesion molecule-1 (VCAM-1), a molecule that during inflammation is overexpressed by activated cerebrovasculature. ${ }^{168}$ From these aptamers an imaging probe was developed that was shown to identify $\mathrm{A} \beta$ plaque associated inflammation in the cerebellum of an AD mouse model. 


\subsubsection{Aptamers for Parkinson's disease related targets}

Characterized clinically by resting tremor, rigidity, postural instability, and bradykinesia, Parkinson's disease (PD) is a progressive neurodegenerative disease. ${ }^{169} \mathrm{On}$ the neuronal level, the pathology of PD is characterized by degeneration of dopaminergic neurons in the substantia nigra pars compacta that leads to a marked decrease in dopamine levels. ${ }^{169}$ In addition to decreased dopamine, the presence of cytoplasmic Lewy bodies (which are also implicated in Lewy body dementia) that are made up of ubiquitin and $\alpha$-synuclein aggregates. Evidence suggests that $\alpha$-synuclein aggregation is concentration dependent, and so research efforts have focused on selecting DNA aptamers that bind to the monomer and oligomer forms of $\alpha$-synuclein. ${ }^{169-171}$ Previously a DNA aptamer was selected that bound to both the monomer and oligomer forms of $\alpha$ synuclein but showed no affinity for $A \beta_{1-42 .}{ }^{170}$ More recently, a DNA aptamer was selected that showed affinity for both the $\alpha$-synuclein oligomer (but not the monomer or fibril) as well as the $\mathrm{A} \beta$ oligomer. ${ }^{171}$ In this case, binding was probably conformation specific; both proteins exhibit a $\beta$-sheet conformation. ${ }^{170,171}$ Just like the AD example, these aptamers could be used to improve existing imaging techniques such as magnetic resonance imaging (MRI) and positron emission tomography as well as to draw new insight into the pathophysiologic role of $\alpha$-synuclein in PD

\subsubsection{Aptamers for applications in prion disease}

Transmissible spongiform encephalopathies (TSEs) also known as prion diseases are neurodegenerative diseases that fatally affect humans and other mammals. ${ }^{172}$ Several prion diseases including sporadically occurring Creutzfeldt-Jacob disease (CJD), variant 
CJD (which occurs due to the consumption of contaminated meat product), genetic fatal familial insomnia, genetic Gerstmann-Sträussler-Scheinker syndrome, acquired kuru and the most well-known bovine spongiform encephalopathy ("mad-cow" disease) have been identified to date. ${ }^{173,174}$ It is not entirely understood in what way prions exert their toxic effect within the CNS, but it is known that the conversion of the normal form of the protein $\left(\mathrm{PrP}^{\mathrm{C}}\right)$ to the insoluble and proteinase K-resistant, pathological form of the protein $\left(\mathrm{PrP}^{\mathrm{Sc}}\right.$; where the $\mathrm{Sc}$ refers to scrapie) results in cellular death. ${ }^{175}$ The pathological form of the protein exponentially accumulates in the brain because the conversion of the normal form to the disease-state isoform is self-propogating. ${ }^{173}$

As with other protein aggregation pathologies, treatment strategies for prion disease have focused mainly on either preventing aggregation or removing existing aggregates. Aptamer work has been largely focused on selecting aptamers that bind to either the native form or disease-state isoform of the prion protein as well as developing detection methods that are highly sensitive and selective. ${ }^{141,173,174,176}$ Many selection experiments have been performed to identify aptamers that target prion isoforms. ${ }^{174,177-179}$ Work has been done to characterize the G-quadruplex secondary structure and binding sites of some of these aptamers to prion protein. ${ }^{178-183}$ Interestingly, the sequence and structure of the prion protein is highly conserved across species and therefore many of the selected aptamers exhibited affinity for the protein from multiple species sources. ${ }^{184}$ Recently, aptamer work with prion targets has focused on the development of aptamerbased sensors for the highly sensitive and selective detection of prion isoforms. ${ }^{185-205}$ Important work has also been done using the $\mathrm{PrP}^{\mathrm{C}}$-binding aptamers described to 
understand not only the pathological conversion of $\mathrm{PrP}^{\mathrm{C}}$ but also for understanding endocytic pathways. ${ }^{206,207}$ Additionally, an RNA aptamer was shown to reduce the disease-state isoform of the prion protein in a cellular assay. ${ }^{183}$ This work has important implications in studying self-propagation in TSEs but may also be widely applicable to understanding other protein-aggregation related neurodegenerative disease.

\subsubsection{Aptamers for brain cancer (Glioblastoma and Glioma) related targets}

Much of the early aptamer research for brain cancer targets focused on understanding the pathology of the specific cancers glioblastoma and glioma as well as developing aptamer-based therapeutics. ${ }^{208-212}$ Several reviews have discussed the progress made in these studies. ${ }^{141,176,213}$ Tenascin-C, an extracellular matrix protein that is overexpressed during tumor growth has been the interest of multiple studies. ${ }^{210,211}$ Interestingly, the structure of the Tenascin-C aptamer selected by Hicke et al., (2001) was compared to the structure of miRNAs over expressed in gliomas. ${ }^{211,214}$ From these data, the authors proposed that beyond their known function in gene regulation, miRNAs may also behave like aptamers to regulate the activity of specific proteins. ${ }^{214}$ Other work has focused on selecting aptamers for known biomarkers, identifying novel biomarkers for brain cancer and selecting aptamers that bind specifically to glioblastoma cell lines over other cancer cell lines. ${ }^{215-218}$ Further, aptamers that bind to glioblastoma tumor initiating cells (TICs) were identified and shown to be internalized, providing potential for aptamer-based targeted therapeutics. ${ }^{219}$ 


\subsubsection{Imaging brain cancers with aptamer-based probes}

Several aptamers have been used to develop imaging probes for brain cancer targets and are summarized well in Delač et al., 2015. ${ }^{212}$ A tenascin-C aptamer was either radiolabeled or fluorescently labelled and used to image glioblastoma tumor xenografts in live mice. ${ }^{41,211}$ Both imaging and biodistribution studies were performed and these studies revealed that the aptamer was efficiently taken into the cell within 10 mins and also accumulated in tumor tissue. The general applicability of this probe was shown by examining different radiometal chelators. Depending on the chelator used, the biodistribution and clearance were affected but aptamer-target binding functionality remained intact. Therefore the imaging probe could be tailored to the application by the judicious choice of metal chelator. Importantly, due to the expression of tenascin-C in other tissues, the tenascin-C aptamer could also be used to study breast, colon, lung, lymphoma, melanoma, prostate, and sarcoma carcinomas. ${ }^{41}$ Another important imaging example involved the development of an imaging probe based on an RNA aptamer for human matrix metalloprotease 9, a protein whose overexpression is known to contribute to tumor metastasis. ${ }^{220}$ This versatile imaging probe was used to image eight different brain tumor types in ex vivo brain tissue slices. Recently, aptamers were selected by CellSELEX for the biomarker Epidermal growth factor receptor variant III (EGRFvIII) and developed into molecular imaging probes to detect EGRFvIII in mouse xenografts. ${ }^{218}$

Some progress has been made using aptamers to image brain cancers in human tissue. For example, an imaging probe was developed from aptamers selected to bind to glioma cell lines that selectively identified glioma tissue compared to non-cancerous cells and tissues. ${ }^{221}$ Most recently, DNA aptamers were selected that bind to gliosarcoma, a 
highly invasive variant of glioblastoma multiforme. ${ }^{222}$ One of the aptamers selected by Cell-SELEX was modified into a molecular imaging probe and was successfully used to image gliosarcoma tissue from clinical samples. It is important to develop aptamer-based imaging probes as they may provide earlier detection, more accurate tumor grading, and more distinct tumor margin identification.

The development of novel brain tumor diagnostics and therapeutics based on multifunctional nanoparticles (MfNPs) holds great promise. These MfNPs can have magnetic, optic or thermal properties which could be used to design imaging probes, therapeutics and theranostics. ${ }^{223,224}$ Increasing attention has been paid to the development of aptamer-conjugated nanoparticles for applications in imaging and targeted delivery. Quantum dots are an excellent example of a nanoparticle that holds potential for application design. In particular, if the toxicity of quantum dots in vivo could be controlled by aptamer-conjugated quantum dots, they may serve as a dually functional alternative to traditional radiolabels, metal chelates, or fluorescence imaging methods. ${ }^{225}$ For example, dendrimer-modified CdSe quantum dots with aptamer conjugated to the surface of the nanoparticle were developed to specifically bind to U251 glioblastoma cells in vitro. ${ }^{226}$

Nanoparticles with magnetic properties have also been investigated. An aptamer for vascular endothelial growth factor receptor 2 (VEGFR2) was conjugated to magnetic nanocyrstals (Apt-MNC) to develop a contrast agent which was used for specific magnetic resonance (MR) imaging. ${ }^{227}$ VEGRF2 was a particularly interesting target for MR imaging because glioblastomas are highly vascularized. ${ }^{227}$ In an in vivo model of 
glioblastoma, the aptamer-based specific contrast agent, Apt-MNC was shown to enhance the MRI signal intensity two times over the nanocrystal control. Additionally, the AptMNC was non-toxic and biocompatible; advantageous properties compared to quantum dot based strategies. ${ }^{227}$ In another example, a "nanosurgeon" was developed based on aptamer-conjugated magnetic nanoparticles. ${ }^{228}$ The nanosurgeon, controlled by applying an 1-Hz rotational magnetic field, was able to selectively detach $\mathrm{U} 251$ glioblastoma cells from a culture surface and induce cell death in in vitro studies. ${ }^{228}$ If this technique were paired with MR imaging, it may allow for the removal of tumor cells from previously inaccessible and inoperable locations.

\subsubsection{The use of aptamers to initiate cell death in brain cancer}

Several studies have looked at the ability of aptamers to cause cell death during the initial phases of tumor growth. Epidermal growth factor receptor variant III (EGFRvIII) is a protein that is involved in brain tumor cell growth, differentiation and survival and is uniquely expressed in glioblastoma. ${ }^{229}$ In normally maturing cells, EGFRvIII is glycosylated, the surface expression of which is required for cell growth. ${ }^{229}$ Aptamers were selected that bound to newly synthesized unglycosylated EGFRvIII before post-translational glycosylation could occur. ${ }^{229}$ The interaction of unglycosylated EGFRvIII with aptamer reduced EGFRvIII cell surface expression by $90 \%$ and subsequently a 5-fold increase in apoptotic cells. In another study, aptamers were selected to inhibit human wild-type EGFR (EGFRwt) ${ }^{230}$. It was demonstrated that upon binding, the aptamer (CL4) inhibited the autophosporylation of EGFRvIII as well as downstream 
signaling pathways such that the migration, invasion and proliferation of EGFRvIIIexpressing glioblastoma cell lines were affected.

A significant challenge in brain tumor treatment is the specific delivery to and therapeutic accumulation within target tissue. One strategy to overcome these barriers has involved the use of photodynamic therapy (PDT). ${ }^{231}$ In PDT, reactive oxygen species are generated in situ by the irradiation of light sensitive drugs termed photosensitizers. ${ }^{231}$ These reactive oxygen species are used to treat tumors by initiating cell death. ${ }^{231}$ An aptamer based PDT probe was designed by conjugating chlorin e6 (a photosensitizer molecule) to the human interleukin-6 receptor (IL-6R +) aptamer. ${ }^{232}$ Previously, human IL-6R was shown to be over expressed in glioma tissue. ${ }^{233}$ Rapid, selective uptake was observed when the aptamer-based PDT probe was incubated with IL-6R + cells. $^{232}$ Activation of the aptamer based PDT probe by light irradiation lead to selective cell death. ${ }^{232}$ This method is particularly interesting as PDT probes could by developed by changing the aptamer component to an aptamer for other targets of interest.

\subsubsection{Aptamer-mediated targeted delivery}

The delivery of CNS-specific chemotherapeutics could be improved by the development of aptamer-conjugated drug delivery vehicles. In fact, chemotherapeutic agents have been specifically delivered to U87 tumor spheroids and C6 glioma

xenografts. ${ }^{234,235}$ In one study, PEG-PLGA nanoparticles that had the nucleolin-binding aptamer, AS1411, conjugated to the surface (Ap-PTX-NP) were loaded with the drug paclitaxel. ${ }^{234}$ In treated animals, Ap-PTX-NP accumulated in C6 glioma xenograft tissue 
which led to decreased tumor size, and significantly decreased volume and weight

(Figure 1.5). Further, Ap-PTX-NP animals showed increased survival.
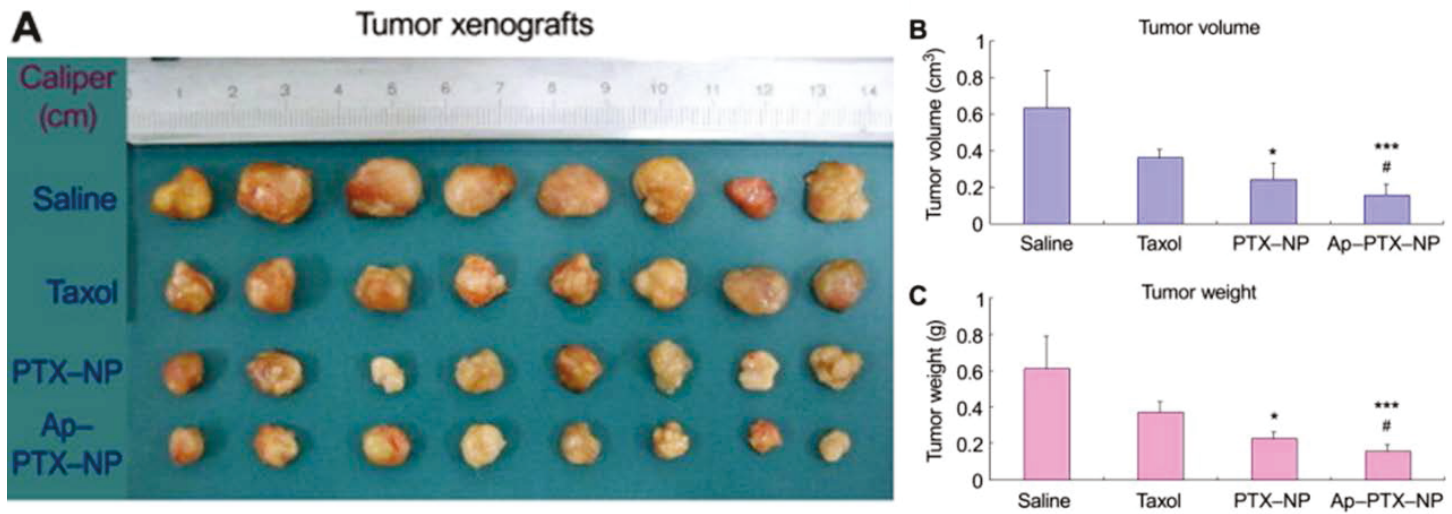

Figure 1.5: Effect of paclitaxel (Taxol $\left.{ }^{\circledR}\right)$, Paclitaxel loaded nanoparticles (PTX-NP) and aptamer-modified paclitaxel loaded nanoparticles (Ap-PTX-NP) on glioma xenograft ( $n=8$ nude mice). A) Tumor xenografts were removed from sacrificed animals at the study endpoint. B) The average tumor volume at the study endpoint for each group is shown. C) The average tumor weight at the study endpoint for each group is shown. Significant difference between the Ap-PTX-NP and Taxol groups as well as the PTX-NP and Taxol group are indicated by $* \mathbf{p}<0.05$, and *** $p<0.001$ respectively. The significant difference between the Ap-STX-NP and PTXNP groups is shown by \# $p<0.05$. "Reprinted from Biomarterials, 32, Guo, J., Gao, X., Su, L., Xia, H., Gu, G., Pang, Z., Jiang, X., Yao, L., Chen, J., Chen, H., Aptamer functionalized PEG-PLGA nanoparticles for enhanced anti-glioma drug delivery, 8010-8020., Copyright (2011), with permission from Elsevier. ${ }^{234}$

In another example, a DNA aptamer selected to bind to the U87 glioma cell line was used conjugated to the surface of PEG-poly-(E-caprolactone) nanoparticles loaded with docetaxel. ${ }^{215,235}$ The aptamer-conjugated nanoparticles led to tumor tissue accumulation, induced apoptosis and inhibited tumor growth in in vitro studies.

Additionally, aptamer-conjugated nanoparticle treated glioblastoma-bearing mice showed increased survival time compared to controls. Work has also been done to investigate the potential of aptamer-based delivery akin to gene-viral therapy. The adenovirus serotype-5 (Ad5), a vector that infects cells by interacting with adenovirus receptor (CAR) and 
coxsackie was investigated in glioblastoma cells. ${ }^{236}$ To overcome the limitation of low or even absent expression of CAR in associated cells, either AS1411 (for nucleolin) or GBI10 (for tenascin-C) aptamers were conjugated to Ad5 to add specificity to the targeting system. ${ }^{236}$ Data from this study suggested that the aptamer modified adenovirus would be an effective mechanism for cancer gene therapy. Compared to controls, the transduction efficiency of AS1411-Ad5 and GBI-10-Ad5 was approximately 4.1-fold and 5.2-fold respectively.

Delivery of therapeutics across the BBB and blood-brain tumor barrier remains one of the greatest challenges in the successful treatment of brain tumors. ${ }^{237}$ Recently, a cascade targeting system that first was able to transverse the BBB and then mediate the specific delivery of the drug delivery system to the tumor tissue was developed to target glioma. ${ }^{238}$ In this work, the BBB was targeted by a 12 -amino-acid peptide (TGN) that had previously been shown to interact with the BBB. ${ }^{239}$ To target the tumor, the AS1411 aptamer was used. The design of the nanoparticle-based, aptamer-modified drug delivery system (AsTNPs) allowed for the specific delivery of docetaxel to glioma tumor tissue. Furthermore, the AsTNPs were shown to penetrate the tumor core, which led to increased survival time.

\subsubsection{Aptamer-based regulation of receptor function}

\subsubsection{Aptamers that bind to the transferrin receptor}

The ability of receptors to mediate transport across a cellular barrier is often exploited in targeted delivery. A particularly interesting set of receptor target for aptamer-based delivery strategies are the family of transferrin receptors (TfRs), which are 
known to be involved in transcytosis across the BBB. TfRs are also a relevant target for BBB applications since TfRs highly expressed on endothelial cells. In fact, DNA (GS24) and RNA (FB4) aptamers have been selected to bind to mouse TfRs and were shown to be internalized into mouse fibroblasts (Ltk- cells) ${ }^{53}$ Further, the aptamer binding site is different than the transferrin-binding site, thus the normal cellular function was not disrupted upon aptamer binding. The interaction of GS24 with murine TfR was further investigated, which led to the rational design of a truncated minimal aptamer that exhibited increased affinity compared to the parent sequence. ${ }^{240}$ Finally, a DNA complex (GS24- NF-KB) was prepared through the conjugation of the GS24 TfR aptamer to a NF$\kappa B$ DNA decoy. ${ }^{241}$ The role of the TfR aptamer was to deliver the NF- $\kappa B$ DNA decoy to the brain endothelial cells so that the NF- $\mathrm{kB}$ DNA decoy could modulate the gene expression of NF- $\mathrm{KB}$, a protein implicated in inflammation associated with ischemic stroke and other neuro-inflammatory diseases. ${ }^{241}$ The GS24- NF-kB successfully delivered the NF- $\mathrm{kB}$ DNA decoy across the murine BBB model (bEND5 cell line) and an inhibited cerebral vascular inflammatory response was observed.

A TfR-binding aptamer was also used as the targeting moiety in an aptamerconjugated PEG-PLA micelle to deliver flurbiprofen across the BBB via receptor mediated transcytosis. ${ }^{53,242}$ Flurbiprofen, an anti-inflammatory drug, has been investigated in phase-3 clinical trials to treat $\mathrm{AD}$, but had limited success due to the low solubility and bioavailability of the drug itself. ${ }^{242}$ In this study, flurbiprofen was successfully delivered across a murine model of the BBB (bEND5 cells) via TfR aptamer-modified flurbiprofen loaded micelles. Preliminary analysis of the aptamerconjugated micelles in phosphate buffered saline $(\mathrm{pH} 7)$ revealed that flurbiprofen was 
rapidly released within the first 2 hours and continued to be released for up to 12 hours. Additionally, the aptamer-conjugated micelles were stable in serum and exerted no toxic effects on bEND5 cells. Compared to non-aptamer modified micelles, the in vitro delivery efficacy of flurbiprofen into bEND5 cells from TfR aptamer-conjugated micelles was $\sim 40 \%$ greater.

Given the inherent specificity properties of aptamers, for a TfR binding aptamer to be useful in human applications, the selection of aptamers capable of binding to the human TfR was necessary. Though it is not impossible that an aptamer selected for a mouse variant may exhibit cross-reactivity for a human variant, the slight differences between the molecular composition and structure of the different receptor variants may eliminate binding. For these reasons, an RNA aptamer for the human transferrin receptor was selected and demonstrated to be readily internalized into cells. ${ }^{243}$ Moreover, the human TfR aptamer was conjugated to the surface of siRNA-containing stable nucleic acid lipid particles (TfR-SNLPs). Compared to non-aptamer controls, the TfR-SNLPs showed increased uptake and targeted gene-knockdown in tissue culture. An imaging probe was also developed from the human TfR aptamer that outperformed the anti-TfR monoclonal antibody control. ${ }^{244}$ Aptamers that bind to TfRs present a widely applicable method to facilitate the specific delivery of imaging probes, diagnostics and therapeutics to the brain. Ultimately, the use of TfRs binding aptamers may facilitate novel insight into many CNS pathologies. 


\subsubsection{Aptamers for other receptor related targets}

\subsubsection{1 a-amino-3-hydroxy-5methyl-4-isoxazole propionic acid (AMPA) receptors}

In addition to the multiple delivery applications described above, aptamers that bind to CNS related receptors could be used therapeutically. An attractive target for therapeutic aptamer intervention are AMPA ( $\alpha$-amino-3-hydroxy-5methyl-4-isoxazole propionic acid) receptors, which have been implicated in addiction and neurodegenerative disease. ${ }^{245}$ Aptamers have been selected for multiple subunits of the AMPA receptor. These aptamers were successful in inhibiting receptor function, exhibited neuroprotective effects, inhibited phosphorylation and were useful in understanding the role of phosphorylation in spatial memory and synaptic plasticity. ${ }^{245-}$ 249

\subsection{N-methyl-D-aspartate (NMDA) receptors}

Another receptor involved in glutamate mediated synaptic transmission is the Nmethyl-D-aspartate (NMDA) receptor. ${ }^{250}$ Over-activation of NMDA receptors leads to excitotoxicity which has been associated with PD, schizophrenia, and neuropathic pain. ${ }^{250}$ Further, evidence suggests a role of NMDA receptors in cerebral ischemia, AD, dementia associated with PD, HD, and learning and memory. ${ }^{250}$ Aptamers have been selected that bind to GluN2A-containing NMDA receptors.. ${ }^{250}$ Due to the various roles of glutamate receptors in CNS related maladies, these aptamers could be used to develop tools and interventions for multiple glutamate receptor-related disorders. 


\subsection{Nicotinic acetylcholine $(\mathrm{n} \mathrm{AChR})$ receptors}

Upon activation, nicotinic acetylcholine receptors (nAChR) facilitate the movement of cations through receptor channels. ${ }^{251}$ The flow of ions through these channels results in a voltage difference across the membrane which results in signal transmission between nerve cells. ${ }^{251} \mathrm{nAChR}$ are widely expressed in brain tissue, and have been implicated in psychiatric, neuromuscular and neurological disorders. ${ }^{252}$ Drugs of abuse, such as phencyclidine and cocaine are noncompetitive inhibitors of nAChRs. MK-801, an anticonvulsant, binds to the same site as cocaine but can also inhibit nAChR function. This makes nAChRs an interesting aptamer target as aptamers that bind to and inhibit receptor function may offer potential as a therapeutic for certain drugs of abuse such as cocaine. Previously, two classes of aptamers were selected to bind to nAChRs. ${ }^{251,253-256}$ The first class inhibited receptor function upon binding by locking the receptor in the closed channel conformation. The second class was able to bind to both the open and closed channel conformations and was also able to alleviate the inhibition of receptor function due to cocaine and MK-801 binding.

\subsubsection{4 $\gamma$-Aminobutyric acid $\left(G A B A_{A}\right)$ receptors}

Inhibitory neurotransmission in the CNS is mediated by the $\mathrm{GABA}_{\mathrm{A}}$ receptor. The importance of proper functioning of the $\mathrm{GABA}_{\mathrm{A}}$ receptor is illustrated in epilepsy where the affinity of GABA for the receptor as well as channel opening and closing is compromised. Two classes of aptamers that bind to membrane-bound $\mathrm{GABA}_{\mathrm{A}}$ were selected. ${ }^{257}$ The first class of aptamers was able to inhibit receptor function. Whereas, the second class of aptamers was able to alleviate antiepileptic-mediated receptor inhibition. 
Studies with these aptamers may be helpful in developing new antiepileptic drugs and provide valuable insight into the malfunction of the $\mathrm{GABA}_{\mathrm{A}}$ receptor in epilepsy. ${ }^{257}$

\subsection{Neurotrophin $($ TrkB) receptors}

The neurotrophin receptor (TrkB) has been implicated in cell signaling, neuronal function and plasticity. ${ }^{258}$ In fact, epilepsy can be induced by over-activation of the TrkB receptor. ${ }^{258}$ Additionally, the abnormal function of TrkB has been associated with neurodegenerative diseases such as AD and Huntington's Disease (HD). ${ }^{258}$ Aptamers have been selected that bind to the extracellular domain of TrkB and were studied in a seizure model. ${ }^{258}$ One aptamer, C4-3 acted as a partial agonist and exhibited neuroprotective effects when examined in cortical neurons.

\subsubsection{Interneuronal signaling controlled by aptamers}

To understand the complex mechanism of intracellular signaling, a common approach is to use potent inhibitors that specifically hinder single components of interneuronal signaling cascades. ${ }^{259}$ As an alternative approach to the commonly investigated small molecule inhibitors, RNA aptamers were selected that were able to bind to and inhibit Erk1/2, a Mitogen-Activated Protein Kinase (MAPK) ${ }^{259}$ Erk1/2 is important in the molecular mechanism of learning and memory due to its role in synaptic plasticity ${ }^{260}$ In this proof-of-concept study, the authors demonstrated the utility of aptamers to investigate intracellular signaling. Specifically this study revealed that aptamer-mediated inhibition of Erk1/2 stopped MAPK-dependent plasticity. Further, the aptamer was delivered to single hippocampal neuron cells via the patch-clamp pipette technique, which allowed for the analysis of aptamer-mediated inhibition and signal 
transduction on the single cell level. Depending only on the availability of an aptamer for the target of interest, the approach described could be easily applied to study the specific role of target molecules in other signaling cascades.

\subsection{Thesis objectives}

The general objective of the work described in the following chapters of this thesis were to improve aptamer selections, to select better dopamine binding aptamers and to use existing aptamers in novel applications.

Chapter 2 "A comprehensive analysis of SELEX and aptamer data from the Aptamer Base" describes the analysis of selection experiment data. Several experimental parameters including the selection library composition, the selection method, the selection conditions, and the affinity method were examined for their effect on the affinity of selected aptamers. Important conclusions drawn from this work suggest that contrary to the existing literature bias, the choice of either DNA or RNA leads to equally successful selection experiments. Additionally, trends emerged that seemed to suggest that out of the selection conditions considered, target type had the greatest effect on the success of aptamer selections.

The work presented in Chapter 3 "pHAST (pH-Driven Aptamer Switch for Thrombin) Catch-and-Release of Target Protein" focused on the development of a pHdriven DNA nanomachine based on a thrombin binding aptamer and the $\mathrm{pH}$-dependent $\mathrm{A}^{+}($anti $) \cdot \mathrm{G}(s y n)$ base pair. Fluorescence spectroscopy, thermal denaturation, circular dichroism, electrophoretic gel mobility assay, and fluorescence anisotropy were used to characterize $\mathrm{pH}$-dependent structural transition and aptamer-target binding. 
Chapter 4 "Intra-Accumbens Injection of a Dopamine Aptamer Abates MK-801Induced Cognitive Dysfunction in a model of Schizophrenia" describes the use of a dopamine binding aptamer to attenuate perseveration. In this work, the aptamer was directly injected into the nucleus accumbens of mice treated with MK-801. The aptamer led to a significant decrease in perseveration compared to non-aptamer treated controls. This was the first example where an aptamer was injected directly into the brain of live rodents and an aptamer-dependent effect on behaviour was observed.

Building on the work presented in Chapter 4, Chapter 5 "In vivo use of a multiDNA aptamer-based payload/targeting system to study dopamine dysregulation in the central nervous system" focused on the development of an aptamer-mediated transport vehicle to deliver dopamine-binding aptamer to the brain of live rodents. In this work, dopamine binding aptamer loaded liposomes were surface modified with transferrin receptor aptamer (DAL-TRAM). Following i.p. injection of DAL-TRAM, a reduction in cocaine-induced hyperlocomotion compared to controls was observed. RT-qPCR was performed to confirm the delivery of dopamine binding aptamer to the brain.

Finally, Chapter 6 "The selection of dopamine-binding aptamers for central nervous system related applications" described the selection and characterization of a novel dopamine binding aptamer, DopaA20min. The new dopamine binding aptamer exhibited better affinity and comparable selectivity to existing dopamine binding aptamers. From DopaA20min, a colourimetric aptamer-gold nanoparticle based sensor was developed. Additionally, the delivery of DopaA20min to the brain by aptamerloaded TRAM led to a marked decrease in cocaine induced hyperlocomotion. 
2 A comprehensive analysis of SELEX and aptamer data from the Aptamer Base 


\subsection{Statement of contributions}

The Aptamer Base was conceptualized by Maria C. DeRosa, Maureen McKeague, and EM McConnell from the Department of Chemistry as well as Michel Dumontier, and Jose Cruz-Toledo from the Department of Biology. Web pages for the Aptamer Base and aptamerbase.semanticscience.org were developed by J Cruz-Toledo. The data to be extracted and curated from published SELEX experiments were decided upon after extensive meetings with MC DeRosa, M McKeague, EM McConnell, J Cruz-Toledo, and the curation team. SELEX experiment information was curated by Michael Beking, Elyse Bernard, Ashley Cabecinha, Amanda Foster, Tariq Francis, Amanda Giamberardino, Emily Mastronardi, EM McConnell, Annamaria Ruscito, and Xueru Zhang under the supervision of M McKeague and J Cruz-Toledo. Additionally the following individuals should be acknowledged for the insightful discussions, contributions, advice and suggestions for the design and maintenance of the database as well as the organization and statistical analysis of the data: Rocio Aranda-Rodriguez, Amal Awad, Alison Callahan, Matthew Chan, Ben Dorion, Phuong Que Ho, Prestin Liu, Dr. Heather ShanksMcElroy and Dr. Alexander Wahba.

\subsection{Resulting Publications}

This work was published in Database (2012) and the Journal of Molecular Evolution (2015). The Database manuscript was prepared by M McKeague, J CruzToledo, M Dumontier and MC DeRosa (Cruz-Toledo, J., McKeague, M., Zhang, X.,

Giamberardino, A., McConnell, E., Francis, T., DeRosa M.C., and Dumontier, M. Aptamer base: a collaborative knowledge base to describe aptamers and SELEX 
experiments. Database. 2012, Vol. 2012 Article ID bas006). ${ }^{261}$ The Journal of Molecular Evolution paper was an invited manuscript and was prepared by M McKeague, EM McConnell, MC DeRosa, and M Dumontier. M McKeague and EM McConnell contributed equally as co-first author. (McKeague, M., McConnell, E.M., Cruz-Toledo, J., Bernard, E., Foster, A., Mastronardi, E., Zhang, Z., Beking, M., Francis, T., Giamberardino, A., Cabecinha, A., Ruscito, A., Aranda-Rodriguez, R., Dumontier, M., and DeRosa, M.C. Analysis of in vitro aptamer selection parameters. Journal of Molecular Evolution, 2015, 81(5): 150-161). ${ }^{8}$ 


\subsection{Introduction}

\subsubsection{Chapter objectives}

The objectives of this work were two-fold. The first was to create, curate and maintain the Aptamer Database, a central repository that could be externally accessed and curated by aptamer researchers. The second was to extract and analyze existing aptamer selection data for trends that may generally improve the efficiency and success of aptamer selections.

\subsubsection{Existing aptamer databases}

The interactions between aptamers and their targets are complex and diverse. By some combination of hydrogen bonding, $\pi-\pi$ stacking, electrostatic interactions and hydrophobic interactions, aptamers interact with their cognate target. ${ }^{262}$ Since aptamer targets range from small molecules to whole cells there is no set of rules that governs the rational design of an aptamer for a particular target. ${ }^{8}$ As such, the computational resources that can be applied to the problem of aptamer discovery are limited. Typically, aptamers are identified by the SELEX method (described in Chapter 1), an in vitro combinatorial screening approach in which aptamers for a specific target are identified from a pool of random oligonucleotides after multiple selection rounds. Few examples of aptamer derivation by non-SELEX methods exist. ${ }^{263-266}$ The SELEX method, adaptations and optimizations have been extensively reviewed elsewhere. ${ }^{161,267-273}$

Before the rational design of aptamers becomes a reality, it is necessary to establish an easily accessible, public-domain repository where information about SELEX experiments and aptamers could be stored. A comprehensive, up-to-date database would 
enable researchers to draw meaningful conclusions from trends in existing data in order to streamline the aptamer selection process and potentially facilitate the design of aptamers from scratch. Currently, several databases exist which store various pieces of aptamer related information.

The Aptamer Database contained comprehensive sequence information for aptamers and unnatural ribozymes, but has since been reported as no longer maintained (9/2014). ${ }^{274}$ The Aptamer Database evolved into the Aptagen Apta-Index ${ }^{\mathrm{TM}}$ (Aptamer Database) which allows users to search for existing aptamers by antigen/target category, aptamer chemistry (which includes DNA, RNA, peptide, chimeric and various modifications), aptazyme restrictions, affinity $\left(\mathrm{K}_{\mathrm{D}}\right)$, and/or keyword. A search of the Apta-Index ${ }^{\mathrm{TM}}$ by "All” target types, "All” aptamer chemistries, and no aptazyme restrictions returns 505 results. For each result the aptamer chemistry, target, antigen/target category, affinity $\left(\mathrm{K}_{\mathrm{D}}\right)$, binding conditions/buffer, sequence, length, molecular weight, extinction coefficient, GC content, nmoles/OD260, and $\mu \mathrm{g} / \mathrm{OD} 260$ is listed. Additionally, an MFOLD predicted secondary structure, the reference to the original article and aptamers for related aptamer targets are shown.

The SELEX_DB was developed to determine whether selected aptamers for protein targets could be used to identify novel protein-binding sites that may shed light on genome mutation related disease by furthering single nucleotide polymorphism analysis. ${ }^{275}$ Another goal of this research was to determine whether the binding domains evolved by in vitro selection for a particular target would be homologous to those occurring naturally. Information about the aim of the experiment, type of experiment, 
experimental design and materials, experimental method and conclusions made by the author were entered into the database. Cross-validation of existing data allowed for sequence analysis and comparison to both naturally occurring genomic sites and other in vitro selected data. Currently, the SELEX_DB is available at the following link: http://wwwmgs.bionet.nsc.ru/mgs/systems/selex/.

In a typical SELEX experiment, fewer than 100 sequence candidates are identified and their binding affinity analyzed. ${ }^{276}$ The advent of high-throughput sequencing technology has allowed for significantly expanded data sets. Current methods have allowed for the technology to advance from processing thousands of sequences to the analysis of billions of sequences. The high-throughput (HTP) SELEX database (HTPSELEX) was developed to provide users access to primary experimental data (template, selection rounds, etc.) and sequencing data from transcription factor related SELEX experiments as well as derived data such as transcription factor binding sites. ${ }^{277}$ The database was developed to serve as a repository for HTP SELEX experiments allowing researcher access to primary data, to improve selection methodology and to aid in the development of better binding site models for transcription factors. The HTPSELEX database is still active and contains information for over 25 different transcription factors. It can be accessed at http://ccg.vital-it.ch/htpselex/.

The RiboaptDB was developed to store information about artificial ribozymes and apatmers. ${ }^{278}$ Like the aforementioned databases, the RiboaptDB sought to address the lack of information available for in vitro selected synthetic functional nucleic acids in general sequence databases such as GenBank, the European Molecular Biology 
Laboratory and the DNA Data Bank of Japan. At the time of its introduction, the RiboaptDB was the most comprehensive aptamer database in existence. Both experimental (template type, experimental conditions, target type and non-canonical base pairs) and publication (citation) information was entered for 423 publications culminating in over 4212 sequences; 3842 aptamers and 370 artificial ribozymes. Currently, the RiboaptDB is not active.

There are several challenges associated with maintaining an active database. Oftentimes highly trained personnel are required to identify and curate literature as it becomes available. Data entry is further complicated by inconsistent and incomplete information from published experiments. Further as technology evolves, the information that is useful to glean predictive knowledge from changes. For these reasons, it was necessary to develop a central, open-access repository that could be accessed and updated by aptamer researchers and where access was not limited to the team of researchers or research group that developed the database. To address this problem, the Aptamer Base was developed.

\subsubsection{The Aptamer Base}

The Aptamer Base was developed to provide a central, open-access repository for SELEX experiment information. ${ }^{279}$ The Aptamer Base contains information from 492 SELEX experiments published between 1990 and 2013. For each SELEX experiment, comprehensive details about the selection condition, selection methods, affinity experiments, aptamers, target, and the affinity of the aptamer-target interaction were 
entered. From the almost 500 publications, 2334 unique aptamer sequences described as having affinity for 569 unique targets were described.

\subsubsection{Freebase as a host platform for the Aptamer Base}

Freebase was a community-curated, openly licensed database located at http://www.freebase.com. It contains information on millions of topics sub-divided into hundreds of categories. The user-friendly interface allows non-programmers to enter data in a structured way so that it could be categorized (into databases) and semantically connected to produce meaningful, coherent relationships between individual facts. Technically, Freebase stores data as a series of nodes and relationships between data are established by linking the appropriate nodes. This non-hierarchical data structure allows users to extract complex data sets. In essence, the way data are structured and maintained in Freebase allows users to ask complex questions about the relationship between data sets. For example, in a typical hierarchical structure, one could search by specific keyword(s) such as "aptamer" but with the non-hierarchical structure Freebase uses, users can ask "what are all of the aptamers that bind to small molecule targets". The Aptamer Base (http://www.freebase.com/base/aptamer) was established as a collection of data linking SELEX experiment and aptamer data expertly curated from published literature that could be queried to ask complex questions about aptamers and their physical properties, SELEX experiments, aptamer-target interactions, affinity experiments and aptamer-target affinity. Freebase is currently available as read-only as the data are permanently transferred to Wikidata, a free linked database for the storage of structured 
data. Once the data becomes publically available, users should be able to access the structured data for analysis purposes.

\subsubsection{The Aptamer Base Data model}

To describe the organization of the data model upon which the Aptamer Base was established it is necessary to define the following terms; base, topic, type. The term base refers to a collection of topics. The Aptamer Base specifically contains information on aptamers and the molecular interactions which result in a dissociation constant. A topic is a collection of data. In this case, topics represent specific objects such as an aptamer, which has characteristics that can be described by types. Types are used to specify the properties of a topic. For instance, an aptamer (topic) could be either DNA or RNA (type).

Generally the information entered into the database for each SELEX experiment is described in Figure 2.1. The SELEX experiment topic is created and is described to have an interaction as an experimental outcome. The interaction represents every aptamer that has an associated dissociation constant. The number of interactions is dependent on the number of aptamers that have associated dissociation constants described, therefore if the experiment describes 3 aptamers, each with their own dissociation constant, 3 pairwise-type interactions are created. If a group of aptamers are described by a single dissociation constant, or a range of values, this group of aptamers would be typed as a collective interaction. Affinity experiments are used to confirm the interactions of the SELEX experiment. The experimental outcome of an affinity experiment is a dissociation constant, a numeric value that describes the affinity of a given interaction. 


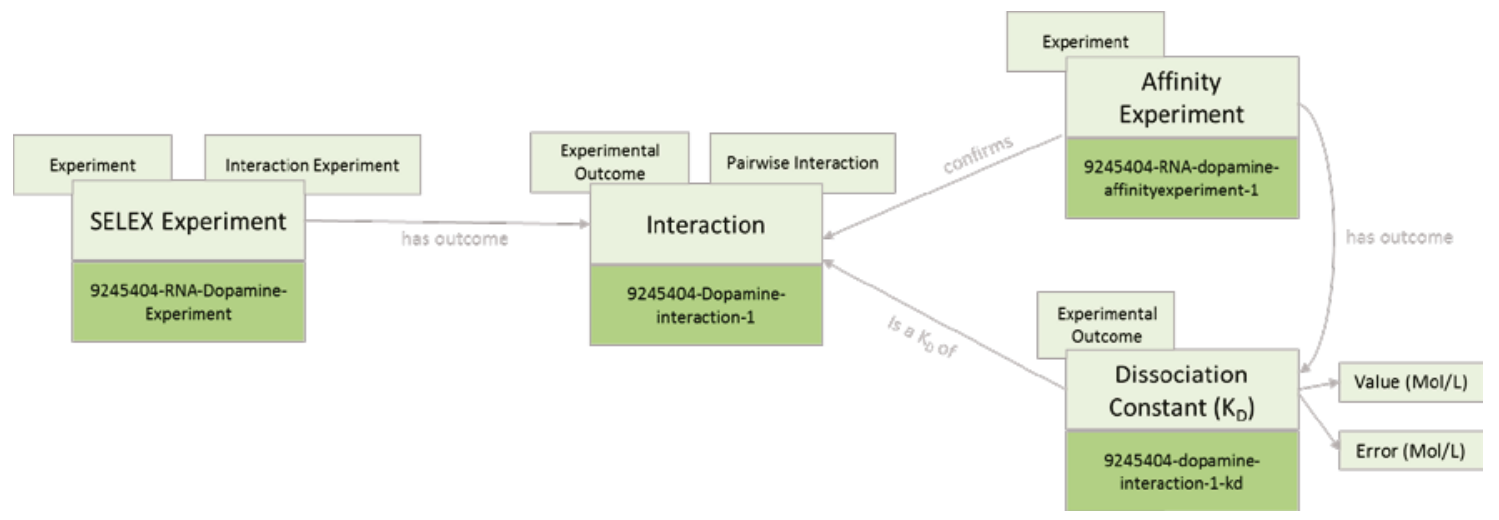

Figure 2.1: General schematic of data relationships in the Aptamer Base. Topics and types are contained in light green coloured boxes. The unique identifiers for the RNA dopamine aptamer selection are shown in the dark green boxes. ${ }^{280}$

When available, a number of additional details were added to describe the SELEX experiment. These details generally described the methods used during the selection and provided the unique identifier. When details were not available, curators selected "not described" or "not required" from drop down menus, entered n/a if text was required and/or - 1 if a value was required. Additional experimental details are summarized in Figure 2.2. In most cases, the unique identifiers for each SELEX experiment were derived from the PubMed ID (PMID). When the PMID was not available the digital object identifier (DOI) was used instead. The SELEX method, the experimental method used to select aptamers for a particular SELEX experiment, was selected from a drop down menu. The different methods are described in Appendix 2.1. Additionally, the method by which binding nucleic acids are separated from non-binding nucleic acids is described as the partitioning method and the technique used to recover nucleic acids from the aptamer-target complex is described as the recovery method were included. The partitioning methods and recovery methods are further described in Appendix 2.1. 
The selection conditions were entered under a separate topic linked to the SELEX experiment. Researchers can gain insight from trends in selection conditions for the design of future selections. The selection conditions entered into the database were as follows; the number of selection rounds, the template sequence, template length, the total length of the random region (which was cumulative if the template contained more than one random region), and whether or not a template bias existed. Template bias refers to the intentional bias towards one or more nucleotides $(\mathrm{A}, \mathrm{C}, \mathrm{G}$ or $\mathrm{T} / \mathrm{U})$ in the random region. For example, if a researcher wanted to bias their selection towards selecting aptamers able to form a G-quadruplex secondary structure, a higher proportion of G would have been used in the synthesis of the pool resulting in a G-rich template bias.

The physical properties of aptamers are significantly impacted by their environment. For this reason, details of the selection buffer were entered for each SELEX experiment. The buffering agent used (see Appendix 2.1), the solution $\mathrm{pH}$, the ionic strength and the selection temperature $\left({ }^{\circ} \mathrm{C}\right)$ were included. 


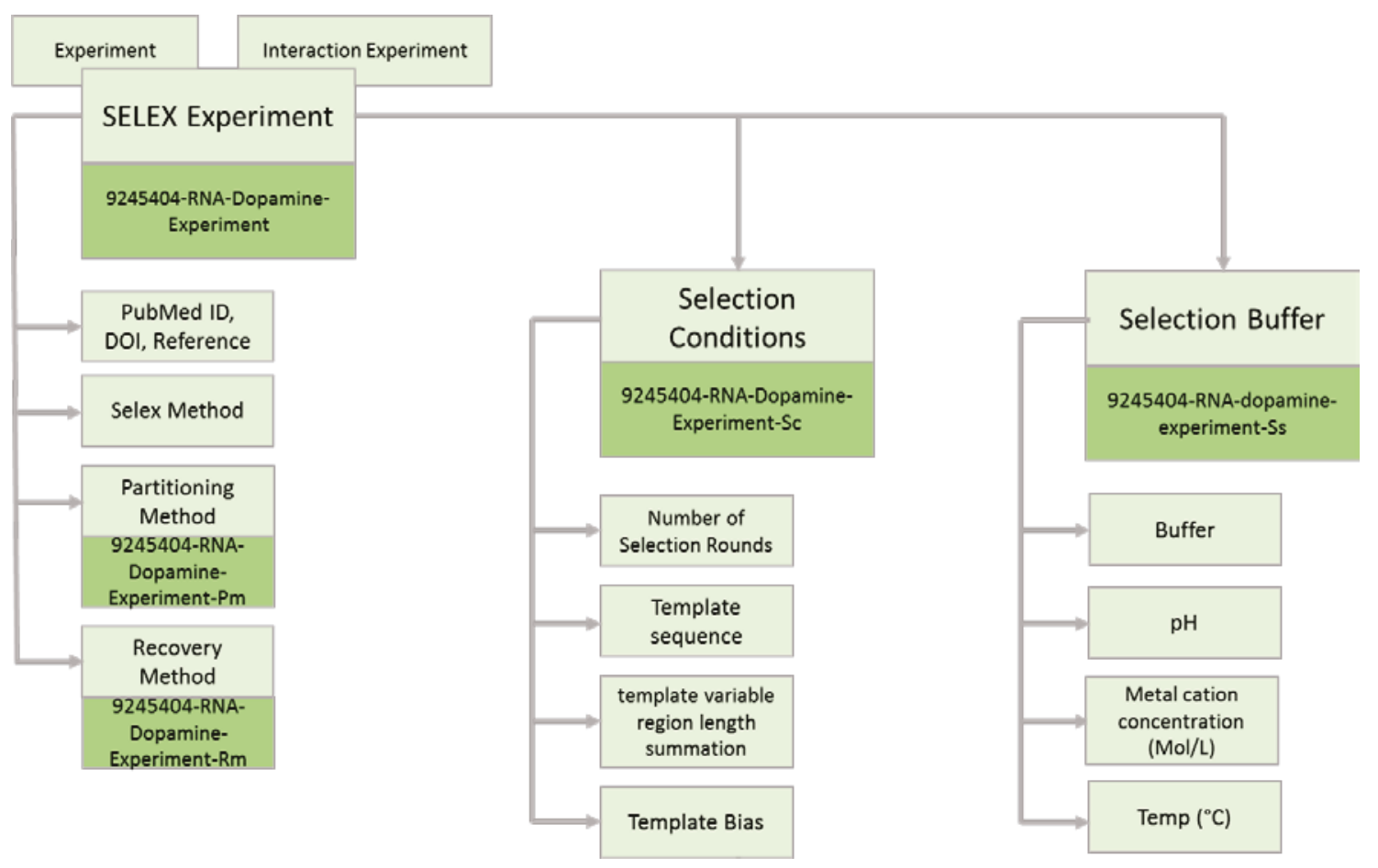

Figure 2.2: Additional SELEX experiment conditions. Topics are contained in light green coloured boxes. The RNA aptamer for dopamine example is continued. ${ }^{280}$ The unique identifiers for each topic is shown in dark green where applicable.

The interactions that stem from each SELEX experiment describe the respective aptamer and its cognate target and links each aptamer to the affinity experiment that produces a dissociation constant to describe the affinity of the aptamer and its cognate target. The detailed relationship between the aptamer and its cognate target is described in Figure 2.3. An interaction is typed as an experimental outcome of a SELEX experiment. Each interaction has interactors as participants. In this example, since the interaction is a pair-wise interaction there are two interactors, one is the aptamer and one is the cognate target dopamine. Collective interactions describe a single aptamer target interactor and multiple aptamer interactors that are all confirmed by the same affinity experiment and have the same dissociation constant. 


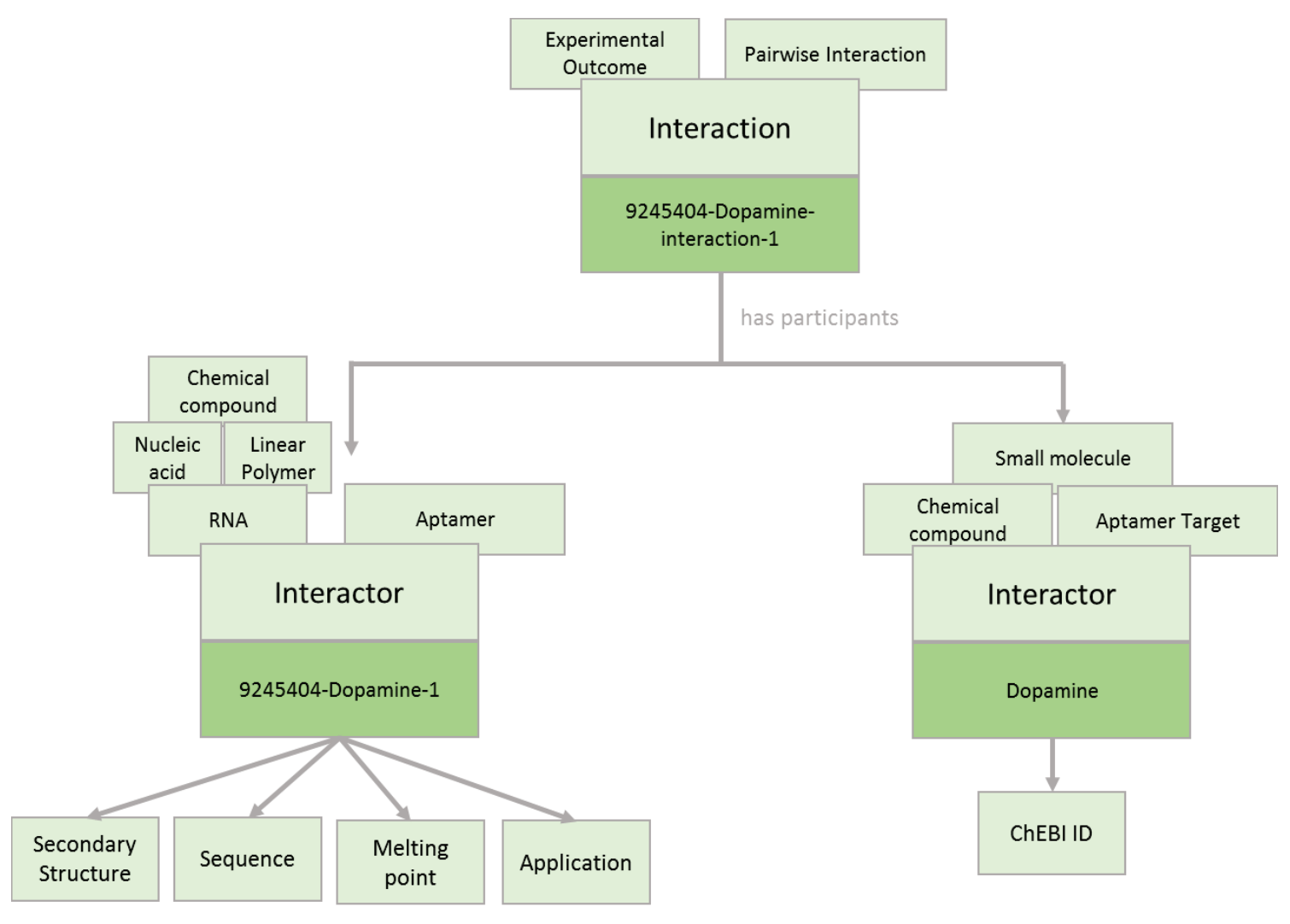

Figure 2.3: General schematic describing the aptamer and target components of the interaction topic (light green). Specific identifiers from the RNA aptamer for dopamine are shown in dark green boxes. ${ }^{280}$

By assigning multiple 'types' to each of the interactors the aptamer and target are able to link extra information to the SELEX experiment. The target is categorized as a chemical compound and as such has an associated Chemical Entities of Biological Interest (ChEBI) identification number. Additionally, the target interactor is typed as a 'small molecule' and as an 'aptamer target'. The aptamer is categorized by a number of different types to describe the physical properties of the nature of the aptamer; in this example the aptamer is an RNA aptamer, but is also a linear polymer, nucleic acid and more generally a chemical compound. In addition, specific details about that unique aptamer are included; information about secondary structure based on mutational analysis and 
experimental structure analysis, the unique sequence, the melting point and a link to any existing applications.

The relationship between the interactors in an interaction is confirmed by an affinity experiment. An affinity experiment has the experimental outcome dissociation constant $\left(\mathrm{K}_{\mathrm{D}}\right)$. Information about the method used to test the affinity of the aptamer to its cognate target (affinity method) and the conditions under which binding was investigated (affinity conditions) are outline in Figure 2.4. Affinity methods are described in Appendix 2.1.

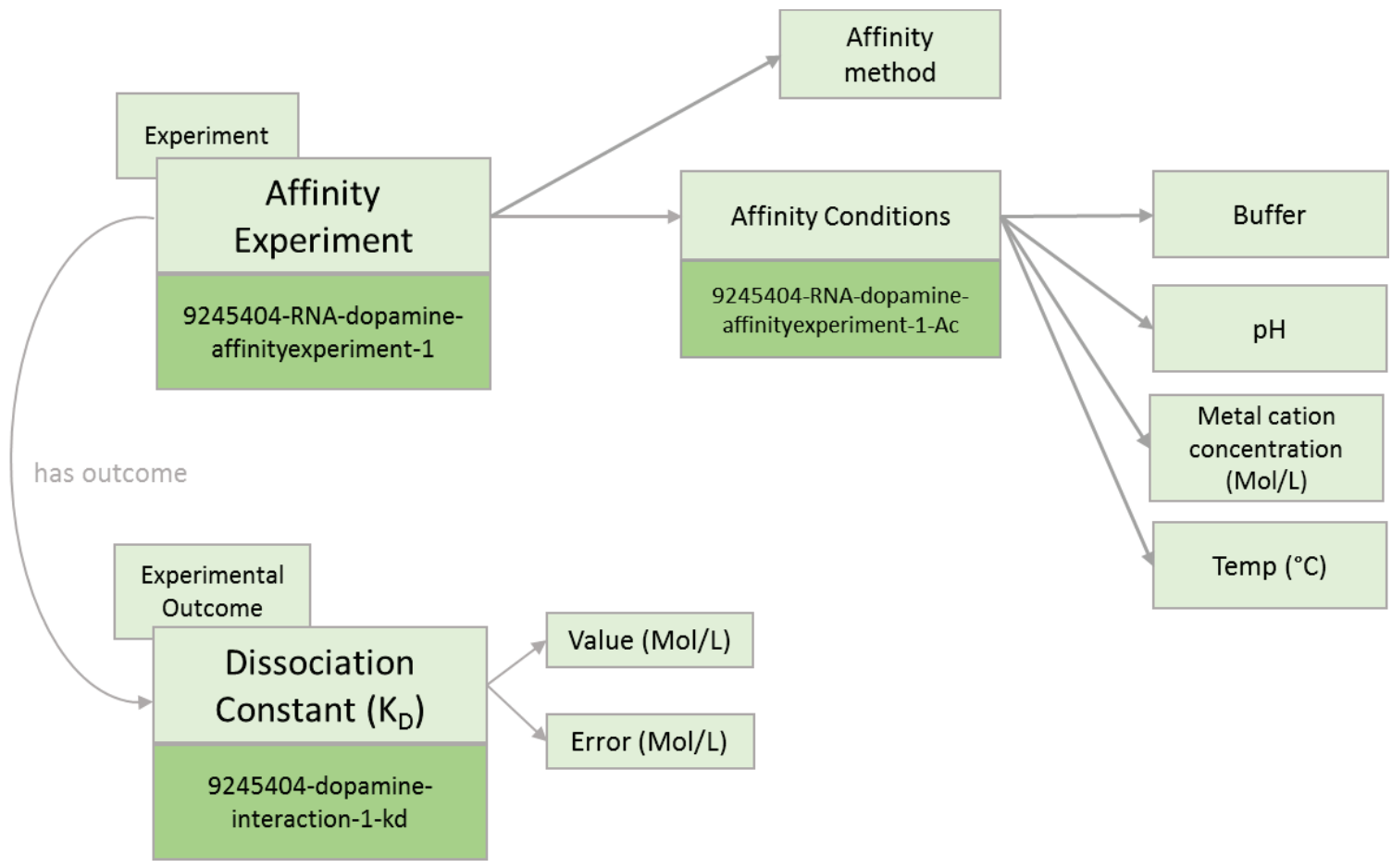

Figure 2.4: General schematic of the relationship between affinity experiment and dissociation constant (shown in light green). Specific experimental identifiers for the RNA aptamer for dopamine are shown in dark green boxes. ${ }^{280}$

In older literature, the affinity between aptamers and their cognate targets was often investigated by a single method. As aptamer technology evolved it became evident that 
often times the affinity as evaluated in one testing paradigm was not reflective of the affinity displayed in a different testing method. For this reason, researchers started to evaluate aptamer affinity by multiple affinity methods. The data model intrinsically accommodates the linking of multiple affinity experiments to a particular interactions and so multiple $\mathrm{K}_{\mathrm{D}}$ determined by multiple methods could be linked to a specific aptamer.

Due to the decreased cost and relative ease of synthesis of shorter oligonucleotides, researchers often seek to decrease the original aptamer's size by eliminating non-essential nucleobases while maintaining equivalent or better binding affinity. This process results in what is called a minimal aptamer. The Aptamer Base allows minimal aptamers to be linked to their ancestral aptamers as illustrated in Figure 2.5. 


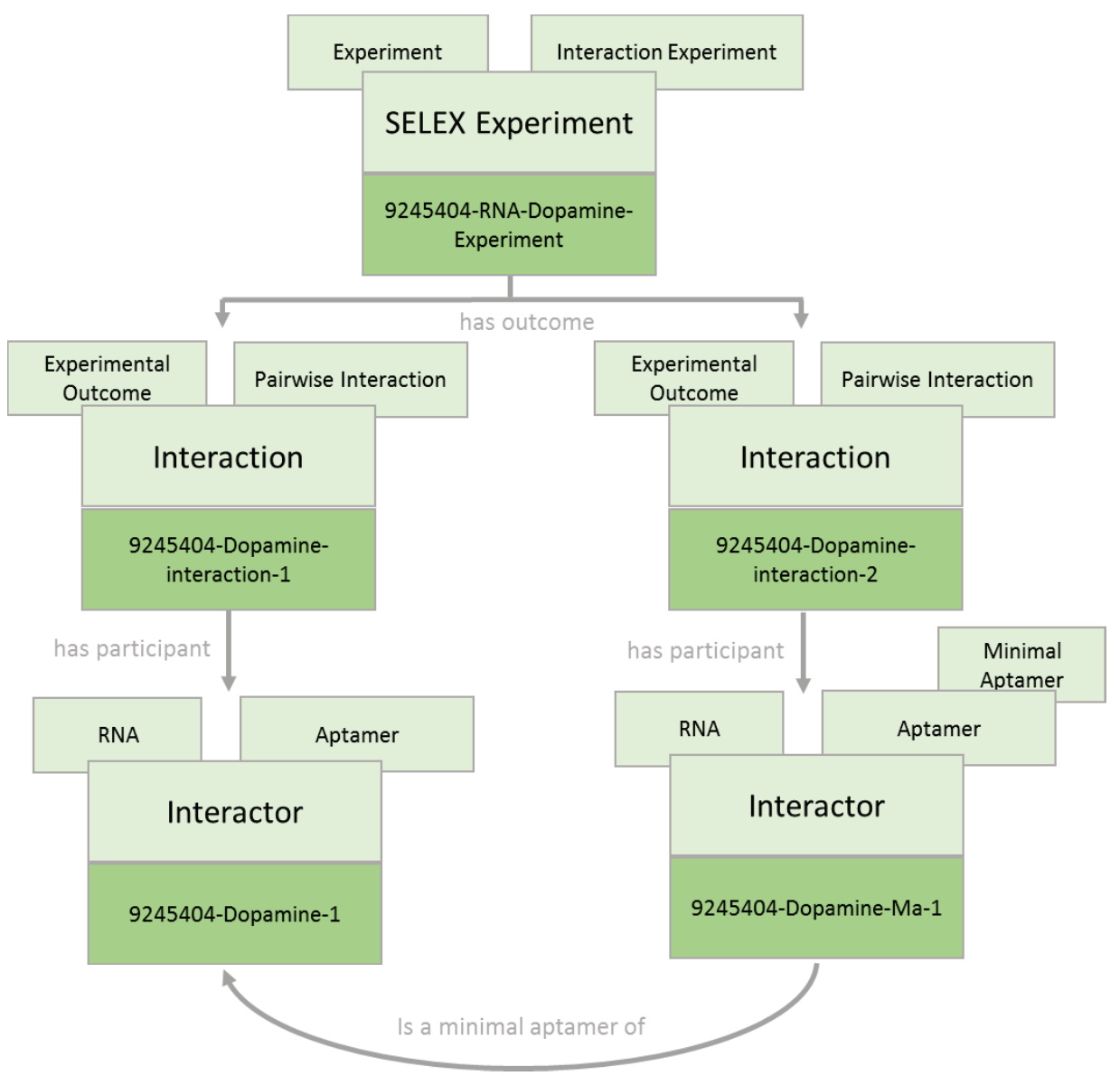

Figure 2.5: The relationship between a minimal aptamer and its ancestral sequence is represented generally (light green boxes). The specific identifiers for the minimal aptamer identified from the ancestral RNA aptamer for dopamine are shown in dark green boxes. ${ }^{280}$

To describe a minimal aptamer, a separate interaction is created. In this example, the ancestral aptamer is described by interaction 1 and the minimal aptamer is described by interaction 2. In both cases the interactions would have both an aptamer and aptamer target interactors as participants. The aptamer target interactors have been removed from the diagram for simplicity. For both the aptamer and the minimal aptamer the interaction and then interactor as set up in the same way. By typing the second interaction as a 
'minimal aptamer' the relationship between the derivative minimal aptamer and the ancestral aptamer is established.

\subsection{Experimental methods}

\subsubsection{Curating SELEX experiment data}

Criteria for a SELEX experiment to be entered into the Aptamer Base stated that for an aptamer-target interaction to be properly described it must have been associated with a dissociation constant. PubMed was used to search published literature using the keywords 'SELEX' or 'aptamer'. Articles that contained these terms in either the abstract or title were identified as potential SELEX experiments. These articles were manually screened by a team of expert graduate students with specific experience in aptamer and SELEX research. Articles that contained a unique SELEX experiment and described aptamers and associated dissociation constants were analyzed and the necessary information was extracted for database entry. Articles that did not contain a SELEX experiment, described a new application for an existing aptamer, further characterized or modified existing aptamers, review papers and those describing the selection of peptide aptamers or ribozymes were excluded.

To ensure consistent and accurate data entry a user guide was prepared and curators were given formalized training. The Freebase Aptamer Base User Guide was prepared by J Cruz-Toledo, M McKeague, M Chan, and M Dumontier and is reprinted in Appendix 2. Originally, curators entered data directly into Freebase. A comprehensive naming system was developed in which the PMID was used as a unique identifier for 
each SELEX experiment. This is shown throughout section 2.3.3.2 in the form of the RNA aptamer for dopamine example and is detailed in Appendix 2.

\subsubsection{Accessing and querying data from the Aptamer Base}

The Metaweb Query Language in conjunction with Freebase's application programming interface (API) was used to access the data from the Aptamer Base. This method allows users to query complex questions. The Aptamer Base was queried by $\mathbf{J}$ Cruz-Toledo using a suite of java 1.6 programs developed to implement Google's Java API clients (http://code.google.com/p/google-api-java-client/).

\subsubsection{Data analysis}

For every SELEX experiment entered into the Aptamer Base, one or multiple aptamer sequences with an associated dissociation constant were reported. Typically, a selection will yield multiple sequences with a range of reported dissociation constants that describe the affinity of a sequence for its cognate target. Unless otherwise noted, for the purposes of investigating affinity-dependent trends, only the aptamer with the best affinity (lowest dissociation constant) was considered. Some exceptions where it was appropriate to include all data for aptamer sequences existed.

\subsubsection{Statistical analysis}

A number of statistical tests were conducted to examine the significance of the presented data. When necessary, the equality of variance was evaluated by either Levene's or Barlett's test. The mean $\log _{10}\left(K_{D}\right)$ or the mean rank of the $\log _{10}\left(K_{D}\right)$ were examined using the parametric One-Way ANOVA or non-parametric Kruskal-Wallis Rank-Sum test respectively. In the cases where a significant difference between groups 
was revealed, those differences were examined using either the Tukey's honest significant difference (HSD) or the Mann-Whitney U test. Linear regression was used to identify correlational relationships between the affinity $\left(\log _{10}\left(K_{D}\right)\right)$ and the variable of interest (nucleic acid type, temperature, $\mathrm{pH}$, etc.). 


\subsection{Results and discussion}

The architecture of the Aptamer Base allows researchers to ask complex questions about the relationship between multiple parameters. As a preliminary analysis, the purpose of this work was to investigate the relationship between affinity and multiple user-defined parameters. Since a high affinity aptamer is often the desired outcome of a successful selection, affinity-based trends were investigated.

\subsubsection{Library design}

The library is the starting point of aptamer selections. The first important decision is the type of nucleic acid employed. DNA affords increased stability but RNA may offer more diverse secondary structure. ${ }^{32}$ Typically, the nucleic acid based template contains a random region of variable lengths flanked by known primer regions. There are a few notable exceptions where templates were designed to reduce the potential influence of the primer regions by decreasing the size of primer regions to completely eliminating the primer region from the template. ${ }^{281}$ When designing the template there are two major considerations. The first is that the template is long enough to provide sequences able to form stable, complex secondary structures. ${ }^{282}$ The second consideration is that the template is short enough that a greater proportion of the theoretical sequence space can be accessed during the selection. ${ }^{283}$ Shorter templates are also desirable due to practical reasons such as ease and cost of synthesis. Finally, the researcher must consider the nucleobase composition of the library. The composition can be controlled to bias the selection towards a particular base. For instance, a G-rich library may yield aptamers more likely to form stable G-quadruplex secondary structures. 


\subsubsection{Nucleic acid type}

To begin examining whether or not the nucleic acid type used in a selection experiment had any influence on the resulting aptamer affinity, trends in type of nucleic acid (DNA or RNA) used as the template in selection experiments over the last 23 years was investigated and are shown in Figure 2.6.

A)

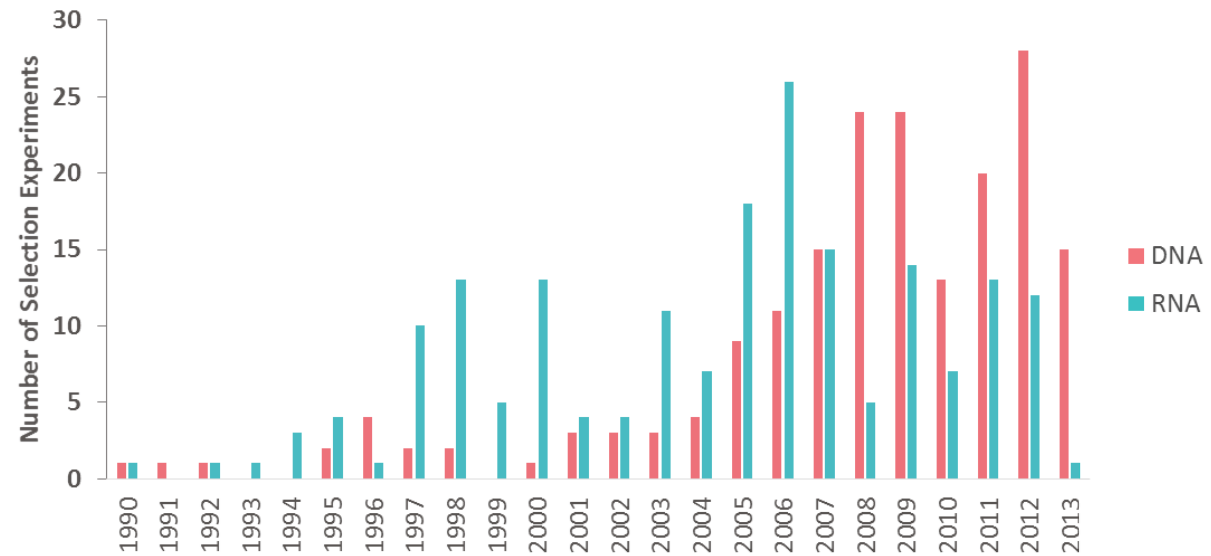

B)
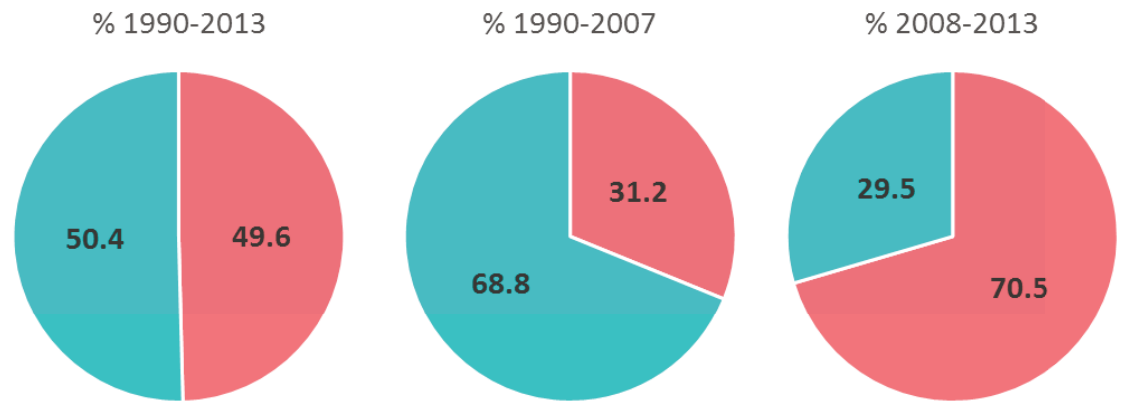

Figure 2.6: DNA vs. RNA selections by year (1990-2013). A) The number of selection experiments performed using DNA (pink) vs. RNA (turquoise) templates by year. B) The total percentage of DNA versus RNA based selections for the entire period, pre2007 and 2008-2013. Figure reprinted with permission of Springer from McKeague, M.; McConnell, E. M.; Cruz-Toledo, J.; Bernard, E. D.; Pach, A.; Mastronardi, E.; Zhang, X.; Beking, M.; Francis, T.; Giamberardino, A.; Cabecinha, A.; Ruscito, A.; 


\section{Aranda-Rodriguez, R.; Dumontier, M.; DeRosa, M. C. Analysis of in vitro selection parameters. J. Mol. Evol. 2015, 81 (5), 150. Copyright Springer 2015.}

There was a clear bias toward the use of RNA (turquoise) templates prior to the year of 2007 and then the frequency of use started to decrease. The use of DNA (pink) templates was relatively steady until the publication year 2004 and then started to quickly increase. This trend was further investigated by comparing the percentage of DNA and RNA based selections from 1990-2007 and then from 2008 to 2013 (shown in Figure 2.6 B). When all years are considered the distribution of DNA vs. RNA templates is $\sim 50 \%$ for each. However, there was a drastic shift observed when the percentage of DNA vs. RNA templates used was compared as date range based subpopulations. Prior to $2007, \sim 70 \%$ of selection templates were RNA. In the following years, RNA template use decreased to only $30 \%$ and DNA template use was $\sim 70 \%$.

Early aptamer selections were almost exclusively based on RNA templates due to the belief that RNA, compared to DNA, could fold into much more stable complex secondary structures. ${ }^{3}$ The base composition of DNA and RNA vary by the inclusion of either thymine or uracil respectively. Additionally, DNA and RNA differ by the absence or presence of a 2'-hydroxyl on the sugar of the backbone. ${ }^{32,284}$ When the 2'-hydroxyl is present in RNA, the resulting double helical structure is A-form. ${ }^{285}$ In DNA, where the 2'-hydroxyl is absent, the resulting double helical structure is typically B-form. ${ }^{284,285}$ These differences in structure result in differences in the physical properties of the nucleic acid molecule including the groove width (major and minor), the rise per base pair, the average axial diameter and the twist angle of the structure. ${ }^{284}$ RNA is able to form more stable secondary structures than single-stranded DNA, however, DNA is less 
susceptible to nuclease degradation. ${ }^{286,287}$ Given the increased stability against nucleases of DNA over RNA due to the lack of 2'-hydroxyl group in the deoxyribose sugar of the DNA backbone, DNA was investigated for its potential in aptamer selections. Soon after the initial aptamer studies, several groups reported the in vitro selection of DNA aptamers that had high affinity for their cognate targets. ${ }^{288}$ Further, experimental evidence suggested that DNA aptamers were in fact capable of forming complex secondary and tertiary structures comparable to RNA. ${ }^{284,288}$ However, because of the inherent structural differences, RNA and DNA with comparable sequences often times neither form comparable secondary structures nor maintain their similar functionalities, though some notable exceptions exist. ${ }^{60,284,289,290}$ One such example is the DNA homolog of the RNA aptamer for the neurotransmitter dopamine. ${ }^{284}$ In this case, the RNA aptamer sequence was converted into DNA. The resulting DNA aptamer has a similar predicted secondary structure as the RNA aptamer and also maintained its affinity for the target, dopamine. More commonly, examples where separate DNA and RNA selections lead to comparable affinities exist. For example both DNA and RNA aptamers for tetracycline, ATP, and kanamycin that have similar affinities for their targets have been selected. ${ }^{291}$ As the experimental evidence to support DNA aptamers emerged the frequency of DNA aptamer templates increased.

With sufficient evidence that both DNA and RNA offer viable templates for aptamer selection, further investigation concentrated on whether either nucleic acid type led to increased affinity compared to the other. Figure 2.7 shows the histograms of DNA vs. RNA sorted based on the number of observations (count) vs the $\log _{10}\left(K_{D}\right)$. Values 
were sorted into a bin based on their second decimal place. For example, an observation was assigned to the -8 bin if it ranged from a $\log _{10}\left(K_{D}\right)$ of -7.50 to -8.49 .

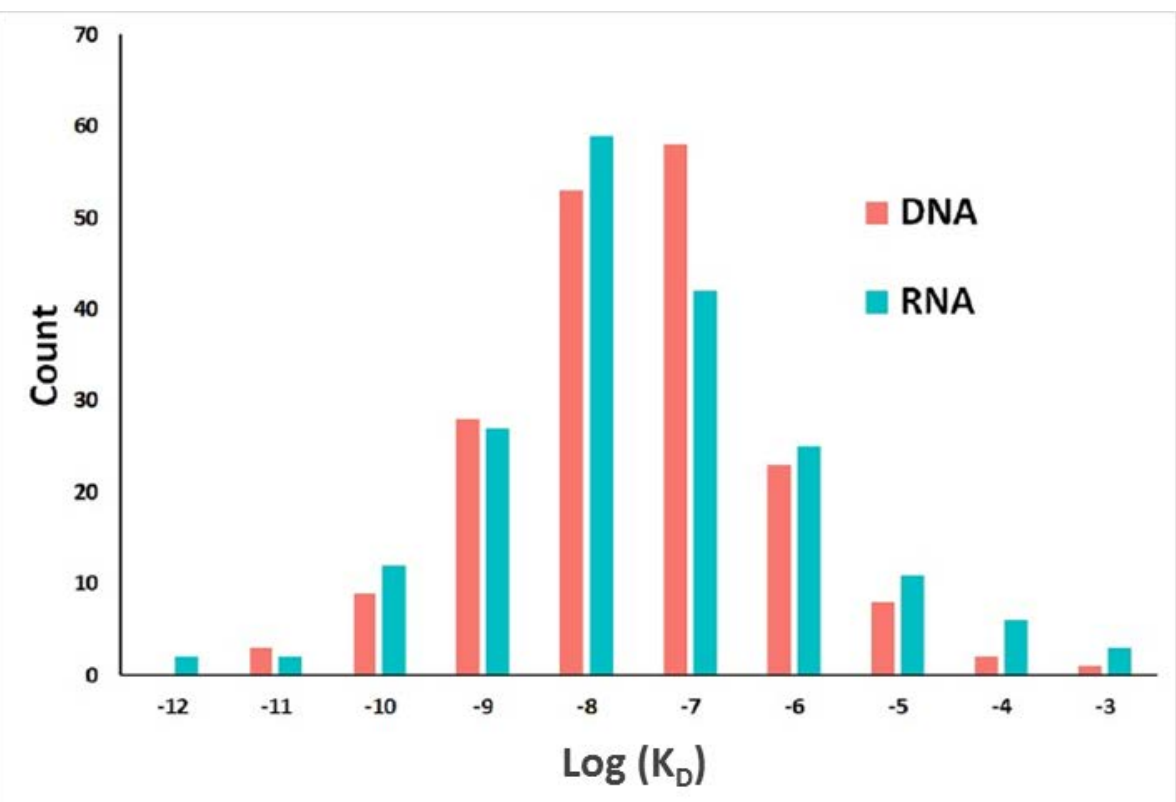

Figure 2.7: Affinity of DNA (pink) versus RNA (turquoise) aptamers. The distribution of the highest affinity aptamers reported, represented by the $\log _{10}\left(K_{D}\right)$. Figure reprinted with permission of Springer from McKeague, M.; McConnell, E. M.; Cruz-Toledo, J.; Bernard, E. D.; Pach, A.; Mastronardi, E.; Zhang, X.; Beking, M.; Francis, T.; Giamberardino, A.; Cabecinha, A.; Ruscito, A.; Aranda-Rodriguez, R.; Dumontier, M.; DeRosa, M. C. Analysis of in vitro selection parameters. J. Mol. Evol. 2015, 81 (5), 150. Copyright Springer 2015.

A t-test was run to determine whether there was a significant difference between the mean $\log _{10}\left(K_{D}\right)$ of DNA ( $\left.n=185\right)$ vs. RNA $(n=189)$ aptamers. The mean $\log _{10}\left(K_{D}\right)$ was $7.48+/-2.58$ and $-7.54+/-1.56$ for RNA and DNA respectively. There was no significant difference $(p=0.69)$ observed between the mean $\log _{10}\left(K_{D}\right)$, suggesting neither DNA nor RNA lead to increased affinity compared to the other nucleic acid type.

Several strategies have been developed to compensate for the instability of RNA based on the presence of the 2'-hydroxyl of the ribose sugar in the RNA backbone. 
Examples such as modified nucleotides and modifications to the backbone have also been incorporated in DNA based selections to improve chemical stability and protect against nuclease degradation. The incorporation of modified nucleotides into SELEX methods was reviewed previously. ${ }^{292,293}$ As more selections are performed with modified nucleotides it would be interesting to compare the affinity of the aptamers selected by these methods and see if these modifications lead to nucleic acid type specific trends.

\subsubsection{Template length}

Aptamers have been successfully selected using templates with random regions ranging in size from as small as 22 nucleotides to as long as 228 nucleotides. ${ }^{294,295}$ Though the random region length (length of $\mathrm{N}$ ) of a template has generally been arbitrarily chosen, the most common random region length ranges from N40 to N70. ${ }^{296}$ The distribution of library length of DNA vs RNA templates is shown in Figure 2.8. There is no bias in the

distribution observed in template length for either DNA or RNA where both nucleic acid types had libraries ranging in size from 10-150 nucleotides with some notable exceptions at $160-170$ for RNA and $270-280$ for DNA. 


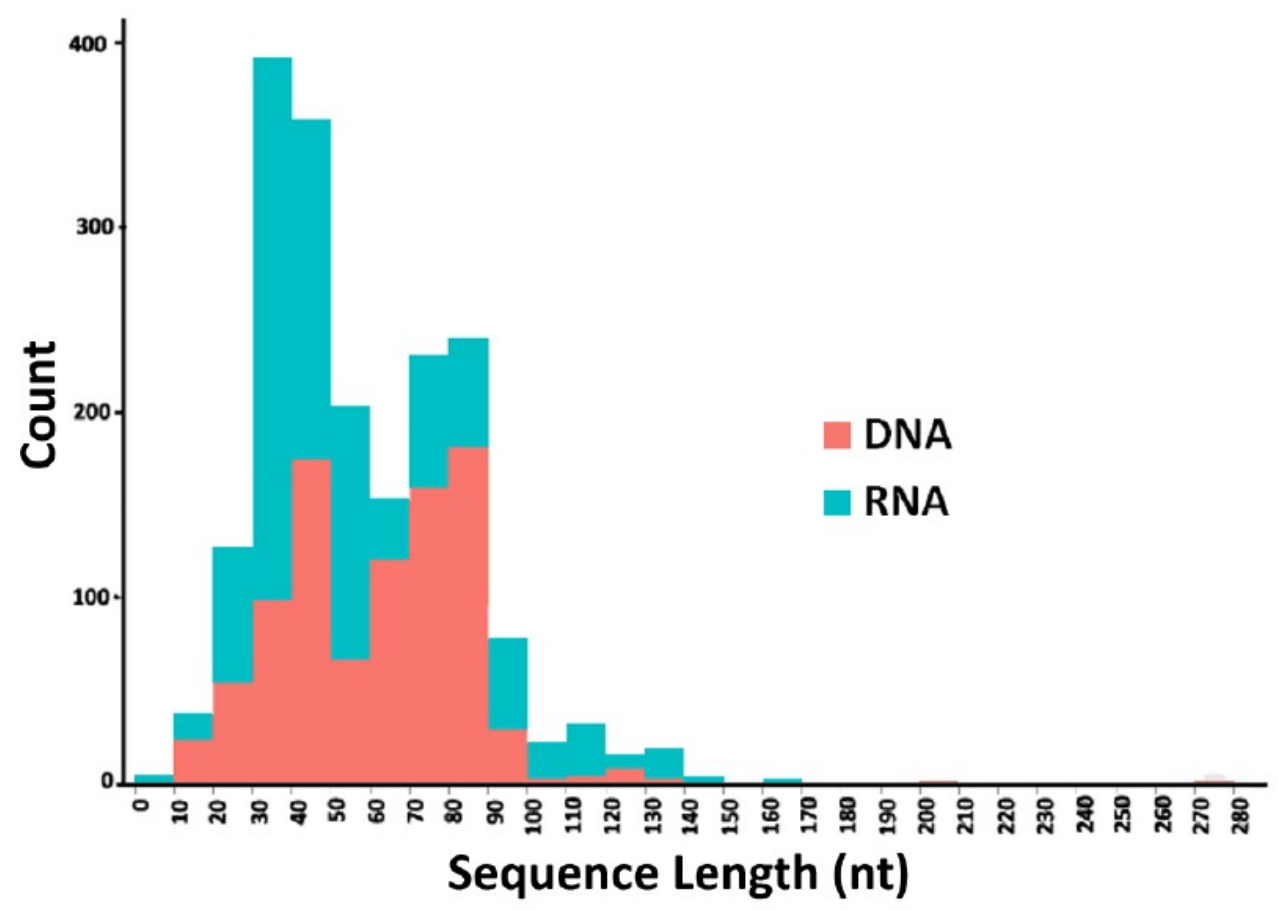

Figure 2.8: Distribution of sequence length for DNA (pink) versus RNA (turquoise) based selection experiments. An overlay of the distribution of the random region (of the pool template) length is shown. Sequence length is represented by number of nucleotides (nt). Figure reprinted with permission of Springer from McKeague, M.; McConnell, E. M.; Cruz-Toledo, J.; Bernard, E. D.; Pach, A.; Mastronardi, E.; Zhang, X.; Beking, M.; Francis, T.; Giamberardino, A.; Cabecinha, A.; Ruscito, A.; Aranda-Rodriguez, R.; Dumontier, M.; DeRosa, M. C. Analysis of in vitro selection parameters. J. Mol. Evol. 2015, 81 (5), 150. Copyright Springer 2015.

Template length is an important variable to consider because the length of the random region is related to the sequence space. Most selections start with $\sim 200$ pmol of DNA which translates to $\sim 10^{14}-10^{16}$ molecules depending on the size of the template. Since traditional templates were designed with 4 different nucleobases, theoretically given a random region, $\mathrm{N}$, each pool can have $4^{\mathrm{N}}$ potentially unique molecules. This is referred to as the sequence space. ${ }^{296}$ The relationship between template length and sequence space is shown in Figure 2.9. As an example to contrast the disparity between 
the fraction of sequence space sampled for shorter versus longer random region lengths, consider templates with $\mathrm{N}=20$ and $\mathrm{N}=70$. The sequence space for $\mathrm{N}=20$ is $10^{12}$ permutations compared to the sequence space for $\mathrm{N}=70$ which is $10^{42}$ permutations. Assuming a starting library with $10^{14}$ molecules available, the fraction of sequence space $\left(10^{14} / 4^{\mathrm{N}}\right)$ sampled is 91 and $10^{-29}$ for $\mathrm{N}=20$ and $\mathrm{N}=70$ respectively. In this case, the library derived from the template with $\mathrm{N}=20$ could have multiple representation of each unique sequence permutation, however, the pool derived from the $\mathrm{N}=70$ random region length would represent only $10^{-27 \%}$ of possible sequence permutations.
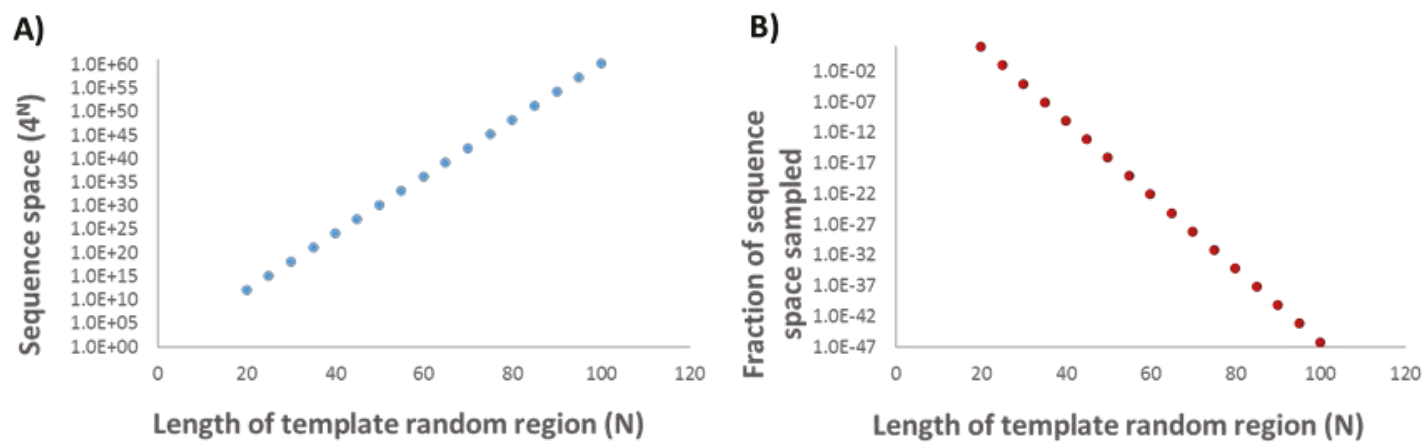

Figure 2.9: Relationship between random region length and sequence space. A) As the length $(N)$ of the template random region increases, the size of the sequence space $\left(4^{\mathrm{N}}\right)$ increases as well. B) However, as the length of the template random region increases, due to the practical experimental limitations, the fraction of the sequence space that is sampled decreases.

Very little research has been done to examine the influence of the random region length of the success of in vitro selection experiments. To examine the relationship between template length and affinity, the template length (number of nucleotides) was plotted against the $\log _{10}\left(K_{D}\right)$, shown in Figure 2.10. 


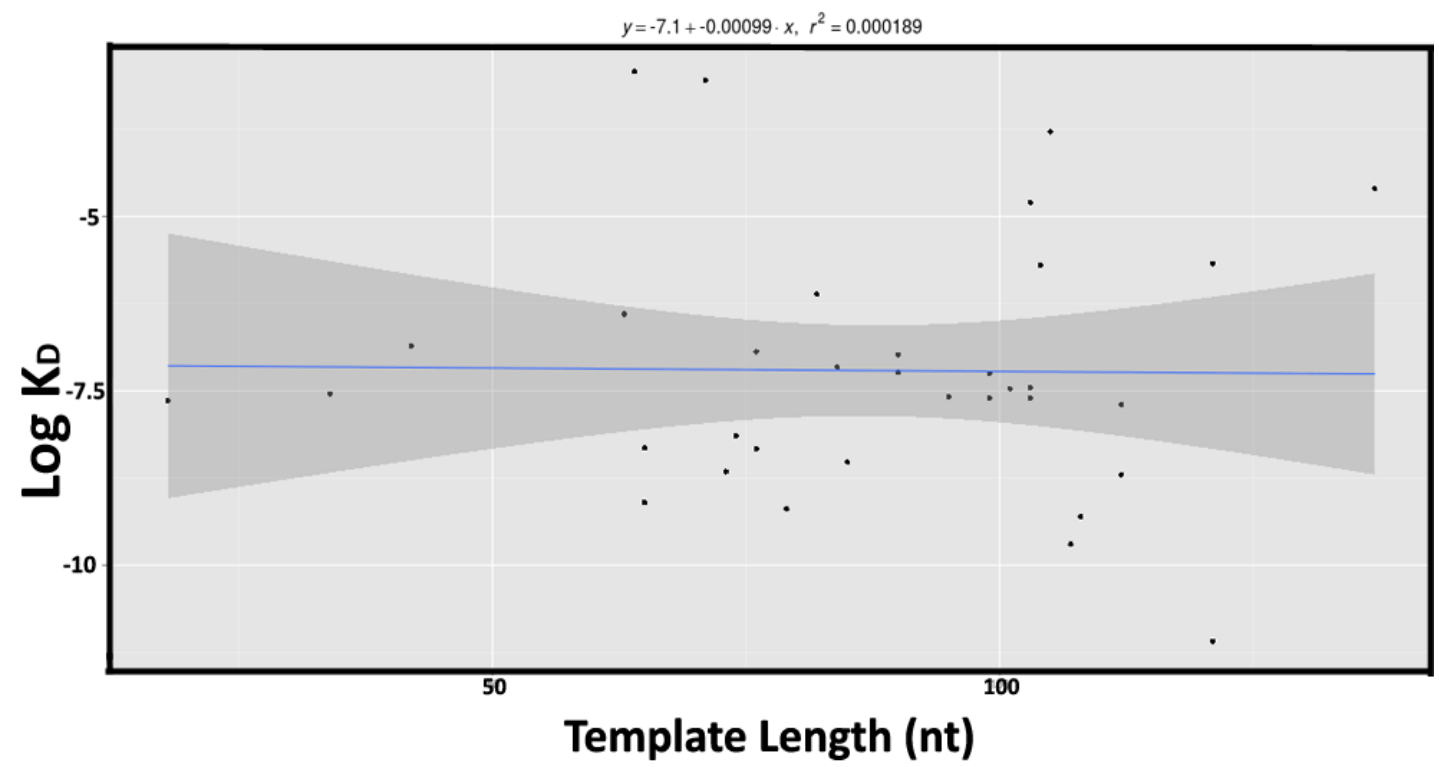

Figure 2.10: Linear regression analysis of template length vs. affinity $\left(\log _{10}\left(K_{D}\right)\right)$. The best affinity aptamers from selection experiments between the years 1990-2013 are shown. Figure reprinted with permission of Springer from McKeague, M.; McConnell, E. M.; Cruz-Toledo, J.; Bernard, E. D.; Pach, A.; Mastronardi, E.; Zhang, X.; Beking, M.; Francis, T.; Giamberardino, A.; Cabecinha, A.; Ruscito, A.; Aranda-Rodriguez, R.; Dumontier, M.; DeRosa, M. C. Analysis of in vitro selection parameters. J. Mol. Evol. 2015, 81 (5), 150. Copyright Springer 2015.

There was no significant correlation $(\mathrm{R}=0.01)$ observed between template length and affinity. This result is not surprising considering it encompasses template lengths for both nucleic acid types and all aptamer targets. Given the diversity in the physical and chemical properties of aptamer targets, it is likely that an appropriate template length for one nucleic acid type or target type would not be appropriate for another. Legiewicz et al., (2005) selected aptamers for isoleucine from an RNA library with random regions of variable length from 16-90 nucleotides. ${ }^{297}$ Their work showed that random regions of length 50 or 70 were optimal for their target, a small molecule. Recall the discussion of riboswitches in section 1.3.2. The small molecule-binding aptamer domains within riboswitches tend to have lengths ranging in size from $\sim 70-170$ nucleotides. ${ }^{298}$ This 
precedence suggests that smaller target types may require longer, more structurally complex templates than that which is required for larger molecules. Smaller template lengths may still be appropriate for other target types, for instance proteins, that are much larger and in most cases more chemically complex than small molecules.

Evidence to merit an investigation of this hypothesis is shown in Figure 2.8.

Interestingly, for both nucleic acid types there seems to be a bimodal distribution centered on 40 and 80 nucleotide lengths. To investigate this hypothesis, the relationship between template length and affinity $\left(\log _{10}\left(\mathrm{~K}_{\mathrm{D}}\right)\right)$ between both nucleic acid type and target type was examined. The results are summarized in Table 2.1.

Table 2.1: Correlation between template length and aptamer dissociation constant (KD) by nucleic acid type and target type. Table reproduced with permission of Springer from McKeague, M.; McConnell, E. M.; Cruz-Toledo, J.; Bernard, E. D.; Pach, A.; Mastronardi, E.; Zhang, X.; Beking, M.; Francis, T.; Giamberardino, A.; Cabecinha, A.; Ruscito, A.; Aranda-Rodriguez, R.; Dumontier, M.; DeRosa, M. C. Analysis of in vitro selection parameters. J. Mol. Evol. 2015, 81 (5), 150. Copyright Springer 2015.

\begin{tabular}{|c|c|c|c|c|}
\hline Nucleic acid type & $\begin{array}{l}\text { Slope of linear } \\
\text { regression trendline }\end{array}$ & $\mathbf{r}^{2}$ & $\mathbf{R}$ & $\begin{array}{l}\text { Significant at } \\
\mathbf{R}=0.50\end{array}$ \\
\hline RNA & 0.012 & 0.035 & 0.19 & No \\
\hline DNA & 0.008 & 0.068 & 0.26 & No \\
\hline \multicolumn{5}{|l|}{ Target type } \\
\hline Peptide & 0.041 & 0.252 & 0.50 & Yes \\
\hline Virus & 0.028 & 0.188 & 0.43 & No \\
\hline Carbohydrate & -0.053 & 0.248 & 0.50 & Yes \\
\hline
\end{tabular}

Consistent with the results shown in Figure 2.10, there were no significant correlations observed between the template length and affinity for DNA compared to RNA aptamers. 
Interestingly, though not significant, there was a relatively stronger correlation between template length and affinity for DNA $(\mathrm{R}=0.26)$ than for $\mathrm{RNA}(\mathrm{R}=0.19)$. More interesting trends started to emerge when the relationship between template length and affinity between target types was examined. Significant positive and negative correlations were observed for peptide and carbohydrate targets $(\mathrm{R}=0.50)$ respectively, and the positive correlation for virus targets was approaching significance $(\mathrm{R}=0.43)$. These results suggest that there is a correlation between affinity and template length for different target types. These results can be interpreted to mean that for peptide and virus targets, a smaller template length would lead to better affinity aptamers. On the contrary, for carbohydrate targets, longer templates would be required to produce higher affinity aptamers. Though it is not clear what may cause these trends to exist, further experimental analysis may provide insight into the effect that size, structure, and specific functional groups on the target molecule have on the preference of a particular template length. It is also important to consider that perhaps the specific sequence and the resultant secondary structure has a more important influence on binding affinity than the template length. Until more conclusive evidence is presented, the variable of template length could be controlled for by employing a selection pool with multiple template lengths. Work has been done where the functionality of deoxyribozymes was shown to be dependent on template length. Selections for deoxyribozymes with shorter templates led to increased activity over those derived from selections with longer template lengths. ${ }^{296}$ 


\subsubsection{Library composition}

One of the structural properties that affects the final functionality and structure of an aptamer is the base composition of the sequence. By extension, the base composition of the template library is a parameter than can be controlled to manipulate selection outcomes. Some work has been done to examine the effect of introducing template bias for the purpose of favouring one structure over another. ${ }^{32,37,299-302}$ For example, RNA libraries were designed to contain G-quartet forming domains. ${ }^{301,302}$ The G-quadruplex is a common secondary structure, in fact several G-quadruplex forming aptamers have been explored for use in various therapeutic and sensor applications. ${ }^{303-305}$

Previously the Ellington group examined the base composition of RNA aptamers and ribozymes of a data set from 1990-2004. A slight statistical skew towards higher G and $\mathrm{C}$ base compositions was revealed for RNA aptamers and ribozymes compared to the expected equal distribution of truly random sequences. ${ }^{274}$ To examine whether this trend was also observed with DNA aptamers, a subset of data for DNA and RNA aptamers from 1990-2012 was analyzed. The base frequencies (calculated with respect to the sequence length) for DNA and RNA aptamers are shown in Figure 2.11. 


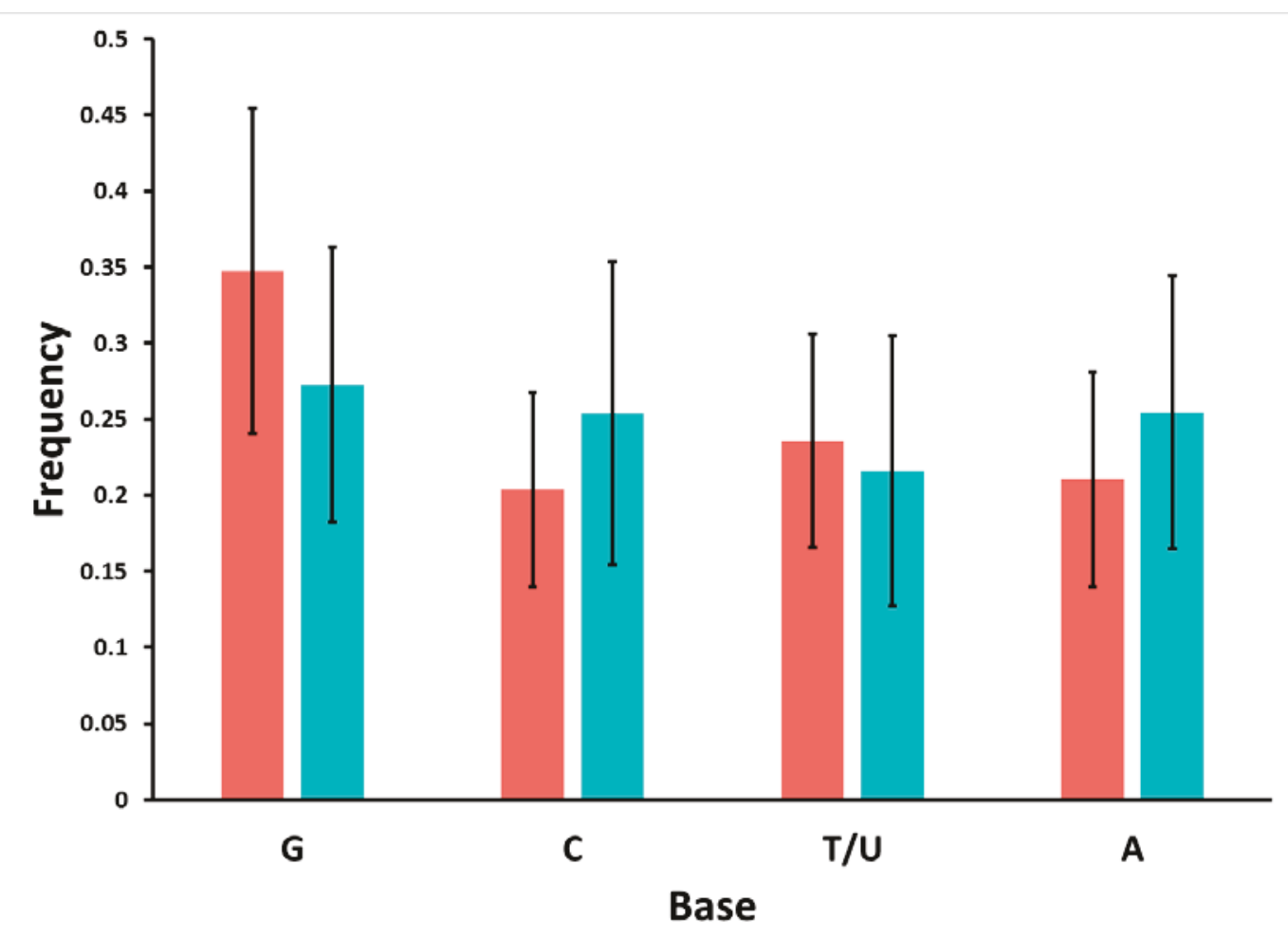

Figure 2.11: Base frequencies of DNA (pink) versus RNA (turquoise) aptamers selected between the years 1990-2012. Figure reprinted with permission of Springer from McKeague, M.; McConnell, E. M.; Cruz-Toledo, J.; Bernard, E. D.; Pach, A.; Mastronardi, E.; Zhang, X.; Beking, M.; Francis, T.; Giamberardino, A.; Cabecinha, A.; Ruscito, A.; Aranda-Rodriguez, R.; Dumontier, M.; DeRosa, M. C. Analysis of in vitro selection parameters. J. Mol. Evol. 2015, 81 (5), 150. Copyright Springer 2015.

Classical aptamer selection templates contain a single stretch of a completely randomized region flanked by known primer regions and so synthetic methods should yield sequences with an equal frequency base composition. Given four standard bases, a base frequency above 0.25 is considered elevated. Our data were consistent with the analysis done by the Ellington group; elevated $\mathrm{G}$ and $\mathrm{C}$ content was observed for RNA sequences.

Interestingly, G content was even more elevated in DNA sequences. To further investigate the importance of base composition, correlational analysis was performed to examine the relationship between individual percent base content and aptamer affinity. 
The linear regression analysis for RNA and DNA are shown in Figure 2.12 and Figure 2.13 respectively.
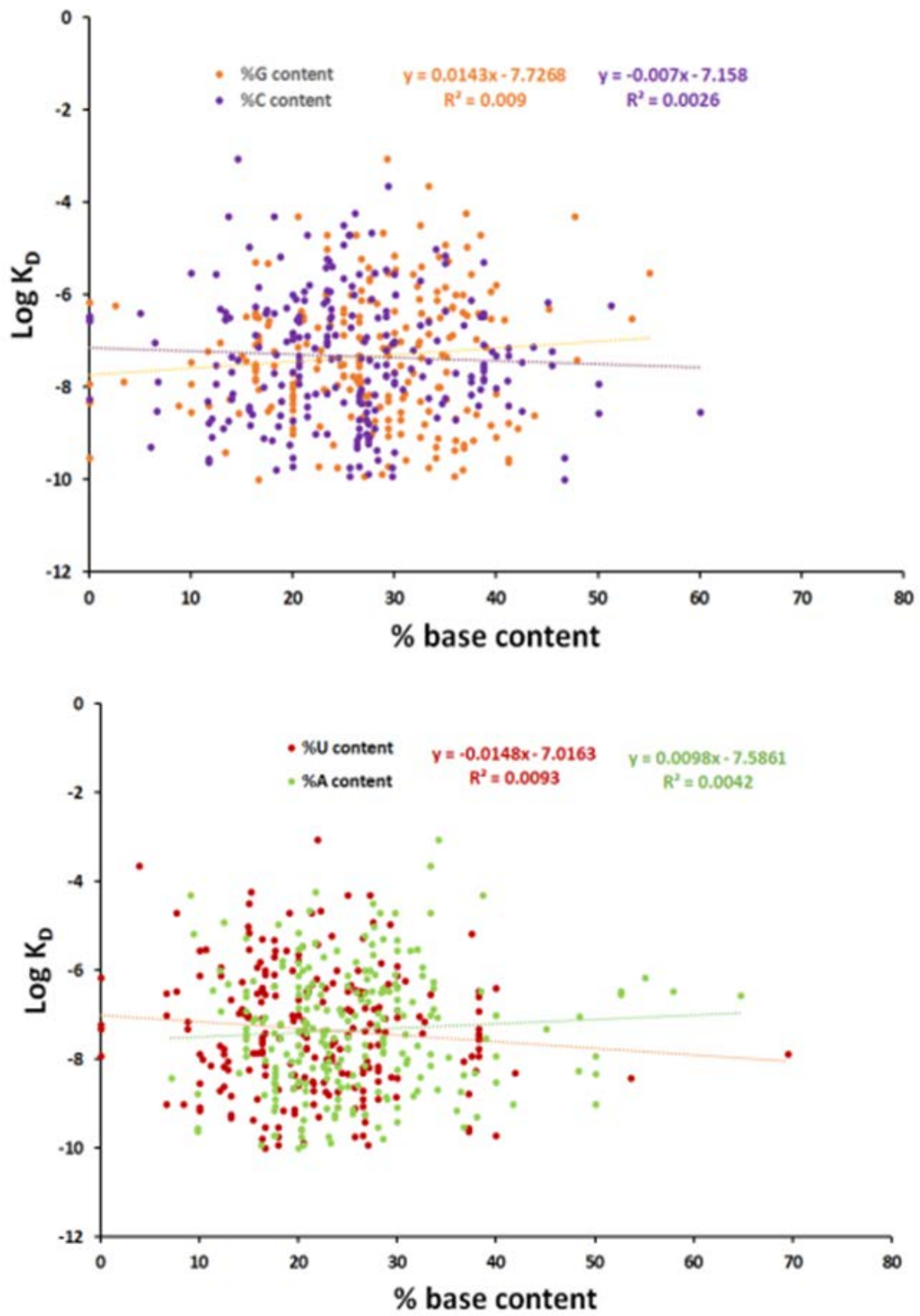

Figure 2.12: Linear regression analysis of affinity versus \% base content for RNA aptamers selected between the years of 1990-2012. The \%G (orange) and \%C (purple) contents (top) and \% $\mathrm{A}$ (green) and \% $\mathrm{U}$ (red) contents (bottom) are shown. Figure reprinted with permission of Springer from McKeague, M.; McConnell, E. 
M.; Cruz-Toledo, J.; Bernard, E. D.; Pach, A.; Mastronardi, E.; Zhang, X.; Beking, M.; Francis, T.; Giamberardino, A.; Cabecinha, A.; Ruscito, A.; Aranda-Rodriguez, R.; Dumontier, M.; DeRosa, M. C. Analysis of in vitro selection parameters. J. Mol. Evol. 2015, 81 (5), 150. Copyright Springer 2015.
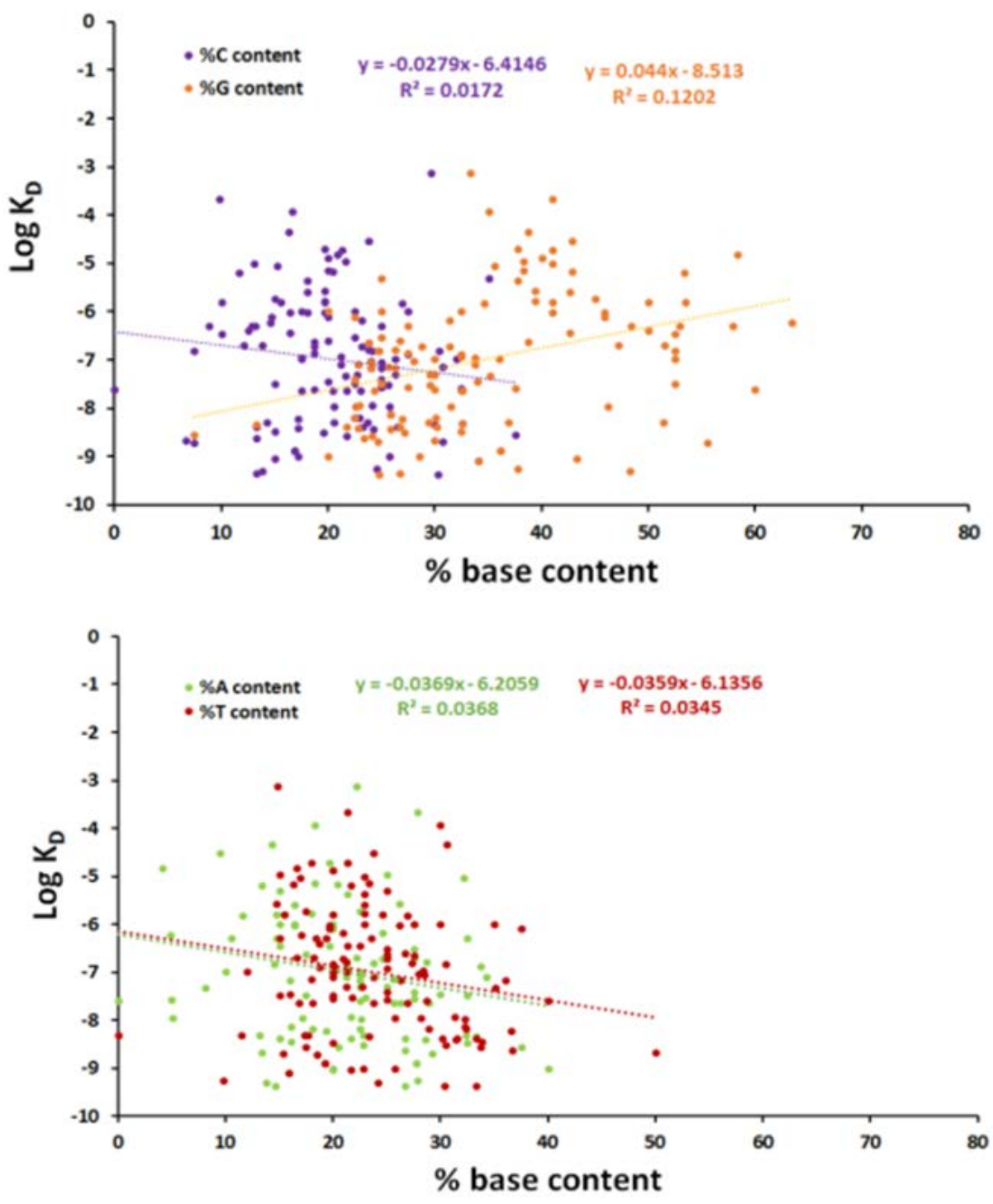

Figure 2.13: Linear regression analysis of affinity versus \% base content of DNA aptamers selected between the years 1990-2012. The \% (orange) and \%C (purple) contents (Top) and \%A (green) and \% T (red) contents (bottom) are shown. Figure reprinted with permission of Springer from McKeague, M.; McConnell, E. M.; Cruz-Toledo, J.; Bernard, E. D.; Pach, A.; Mastronardi, E.; Zhang, X.; Beking, M.; Francis, T.; Giamberardino, A.; Cabecinha, A.; Ruscito, A.; Aranda-Rodriguez, R.; 


\section{Dumontier, M.; DeRosa, M. C. Analysis of in vitro selection parameters. J. Mol. Evol. 2015, 81 (5), 150. Copyright Springer 2015.}

There were no significant correlations revealed, however the directionality of the linear regression trendline was examined to determine if any preliminary trends existed suggesting a bias toward a particular base resulted in increased aptamer affinity. Very weak positive correlations were observed for the percent $G(R=0.09)$ and percent $A$ $(\mathrm{R}=0.06)$ content of RNA aptamers which suggests that RNA aptamers with lower $\mathrm{G}$ and A percent contents actually led to higher affinity aptamers. Interestingly, RNA aptamers with higher percent base contents for $\mathrm{C}$ and $\mathrm{U}(\mathrm{R}=0.05$ and 0.10 respectively) led to better affinities. DNA aptamers that had higher $\mathrm{C}(\mathrm{R}=0.13), \mathrm{A}(\mathrm{R}=0.19)$ and $\mathrm{T}(\mathrm{R}=0.19)$ percent base contents tended to have lower affinities (higher $\mathrm{K}_{\mathrm{DS}}$ ) than DNA aptamers where the percent base content approached near random percent composition. Interestingly for DNA aptamers, though the frequency of G was high, linear regression analysis revealed that sequences with higher percent $\mathrm{G}$ content actually tended to exhibit lower affinity that DNA aptamers with lower $\mathrm{G}$ content $(\mathrm{R}=0.35)$. Given the commonness of G-quadruplexes as a secondary structure the correlation between percent $\mathrm{G}$ content and aptamer affinity may seem surprising; however there are several factors that could have skewed the trend. For instance, these data contain affinity information for all target types. Since target types vary greatly in size and physical properties it is likely that a bias towards one base may be effective in increasing affinity for one target type but disadvantageous for another target type. As a preliminary investigation, the percent $\mathrm{G}$ content of DNA aptamers for different target types (small molecules and proteins) versus 
affinity of a subset of data from 1990-2012 was examined by linear regression. The results are shown in Figure 2.14.
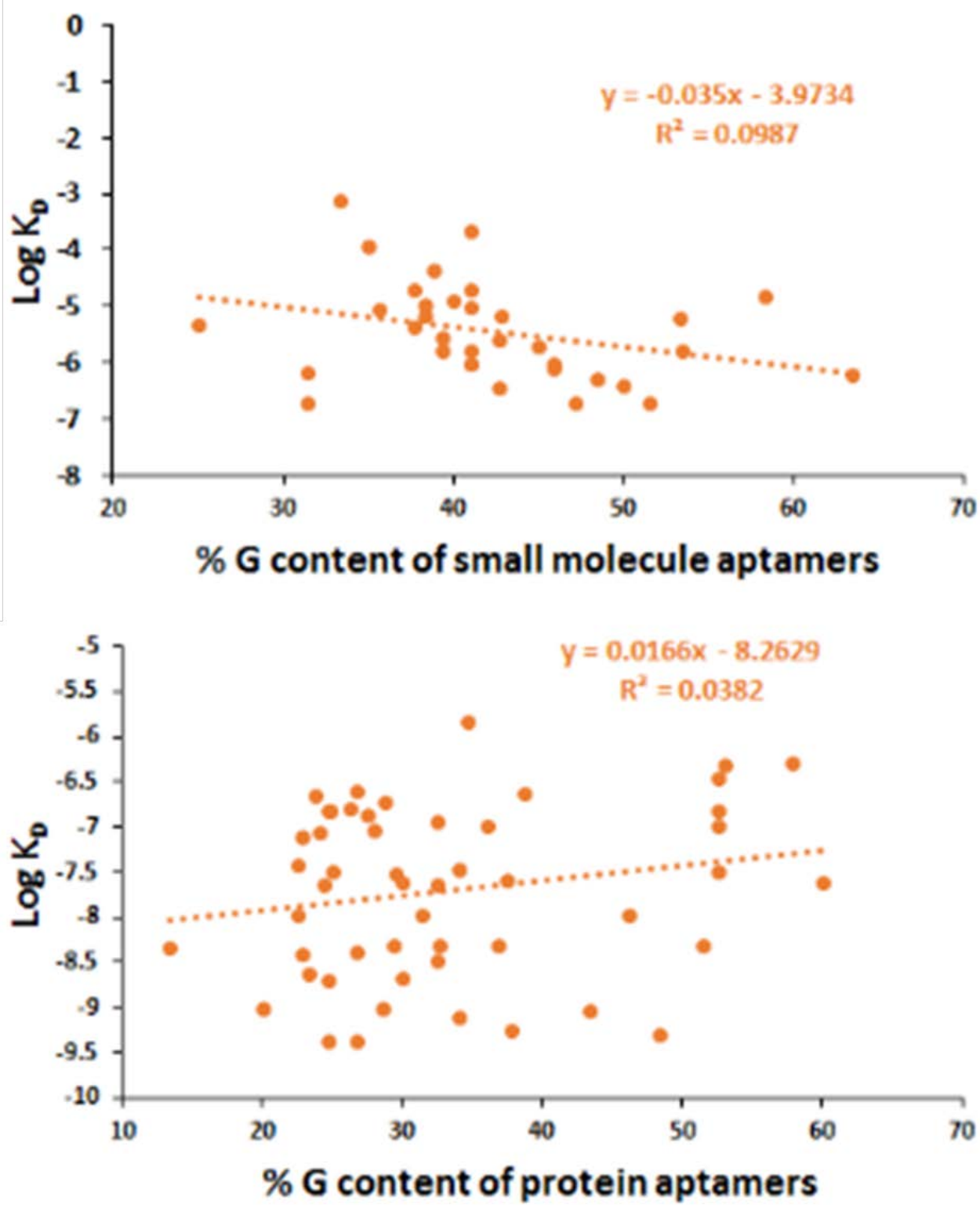

Figure 2.14: \%G content of DNA aptamers for small molecules (left) compared to protein (right) targets. Linear regression analysis was performed on data from the years 1990-2012. Figure reprinted with permission of Springer from McKeague, M.; McConnell, E. M.; Cruz-Toledo, J.; Bernard, E. D.; Pach, A.; Mastronardi, E.; Zhang, X.; Beking, M.; Francis, T.; Giamberardino, A.; Cabecinha, A.; Ruscito, A.; 


\section{Aranda-Rodriguez, R.; Dumontier, M.; DeRosa, M. C. Analysis of in vitro selection parameters. J. Mol. Evol. 2015, 81 (5), 150. Copyright Springer 2015.}

As predicted, the trends in percent $\mathrm{G}$ content versus affinity for aptamers selected for small molecule versus protein targets were different. Aptamers selected for small molecule targets had increased affinity with increased percent $G$ content $(R=0.31)$. Conversely, the affinity of aptamers selected for protein targets decreased with increasing percent $\mathrm{G}$ content $(\mathrm{R}=0.20)$.

Another factor to consider with respect to base composition is stability of potential base pairs. The stability of the aptamer secondary structure is due largely to the stability of the base pairing. For this reason the relationship between percent GC base content and percent AT base content compared to aptamer affinity was examined for a subset of data from 1990-2012. The results for DNA and RNA are shown in Figure 2.15 and Figure 2.16 respectively. 

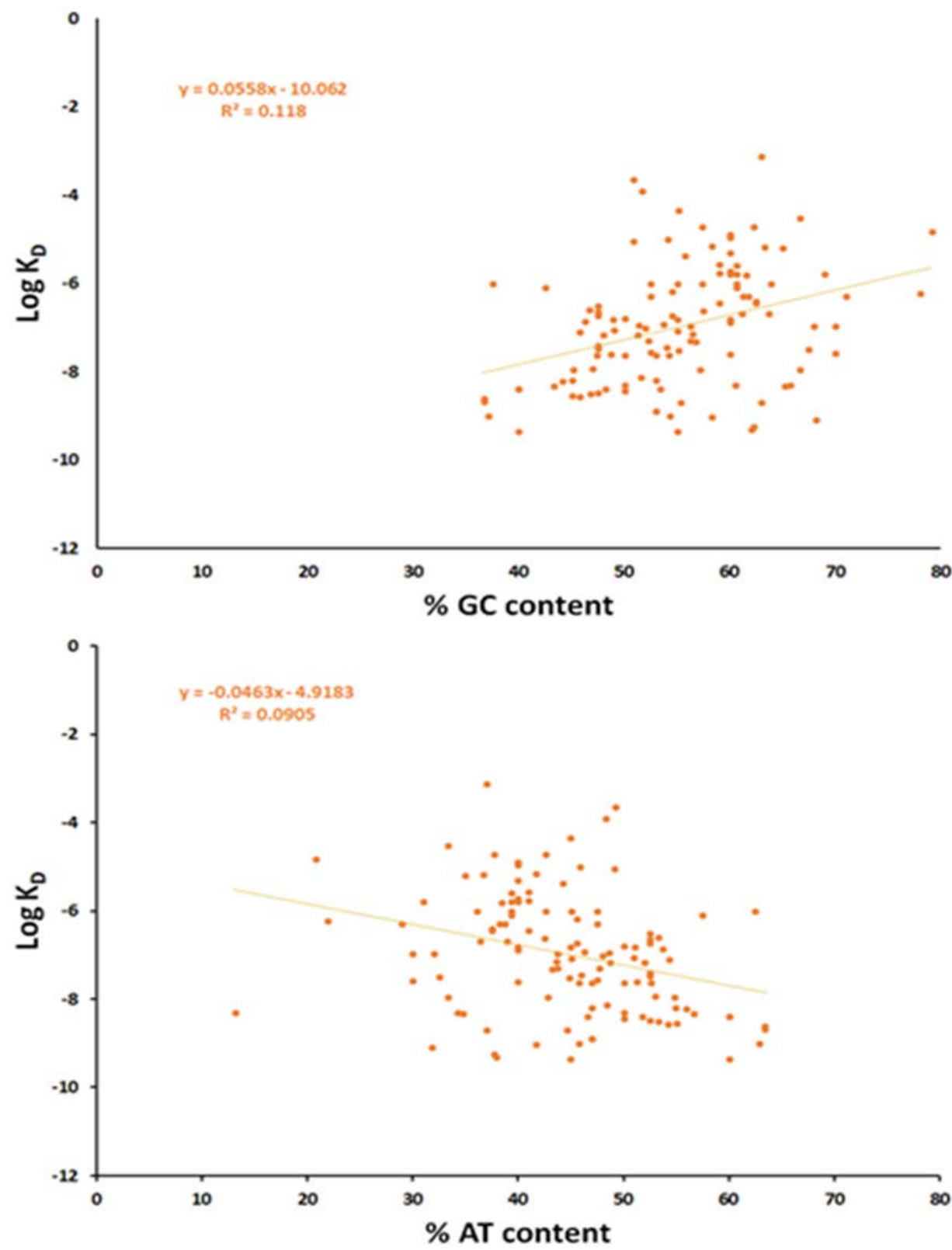

Figure 2.15: Combined \%GC (left) and \%AT (right) content for DNA. Linear regression analysis was performed on data collected between the years of 1990-2012. Figure reprinted with permission of Springer from McKeague, M.; McConnell, E. M.; Cruz-Toledo, J.; Bernard, E. D.; Pach, A.; Mastronardi, E.; Zhang, X.; Beking, M.; Francis, T.; Giamberardino, A.; Cabecinha, A.; Ruscito, A.; Aranda-Rodriguez, R.; Dumontier, M.; DeRosa, M. C. Analysis of in vitro selection parameters. J. Mol. Evol. 2015, 81 (5), 150. Copyright Springer 2015. 

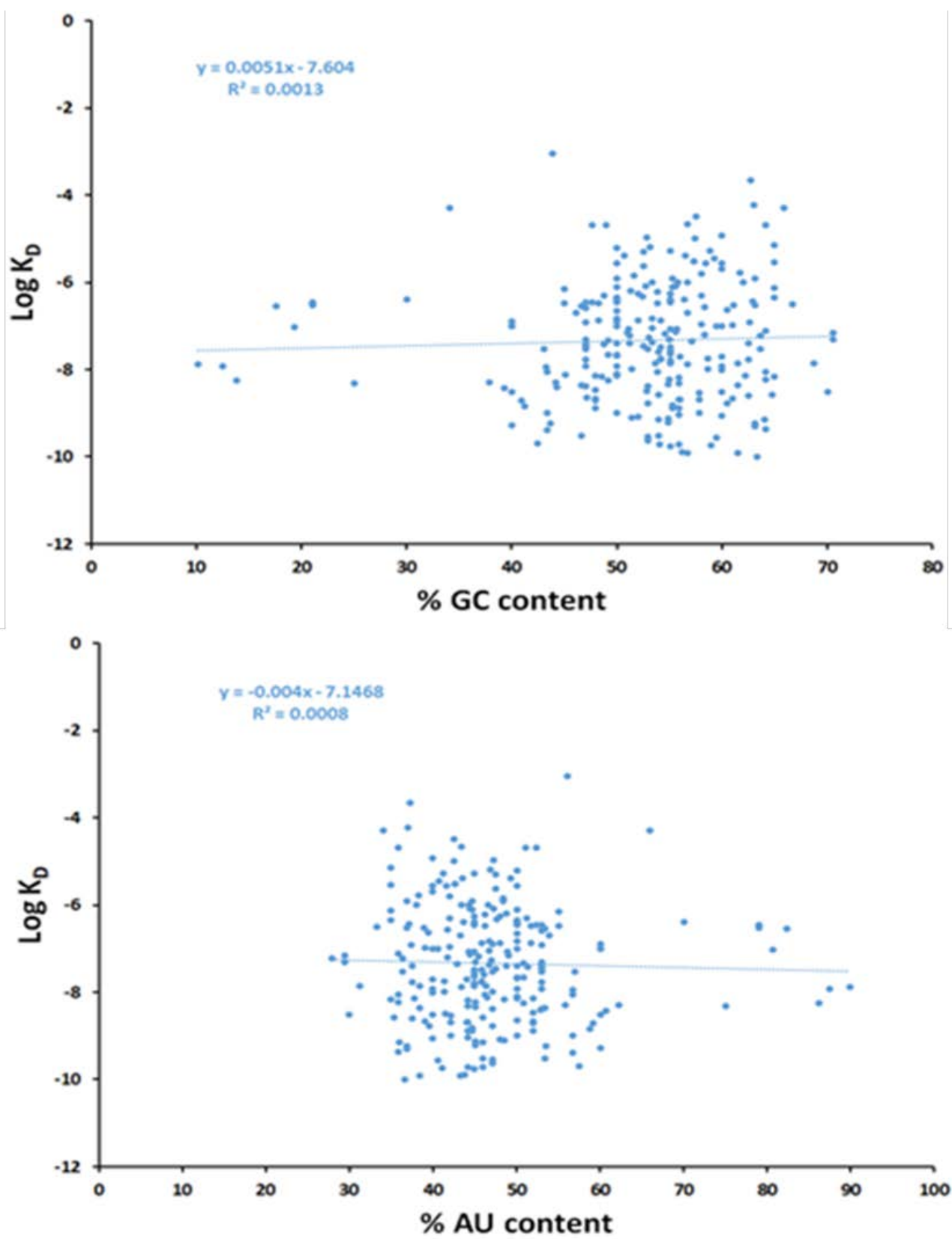

Figure 2.16: Combined \%GC (left) and \%AT (right) content for RNA. Linear regression analysis was performed on data collected between the years of 1990-2012. Figure reprinted with permission of Springer from McKeague, M.; McConnell, E. M.; Cruz-Toledo, J.; Bernard, E. D.; Pach, A.; Mastronardi, E.; Zhang, X.; Beking, M.; Francis, T.; Giamberardino, A.; Cabecinha, A.; Ruscito, A.; Aranda-Rodriguez, R.; Dumontier, M.; DeRosa, M. C. Analysis of in vitro selection parameters. J. Mol. Evol. 2015, 81 (5), 150. Copyright Springer 2015. 
From the distribution of percent GC and AT content it is clear that GC content is often elevated in both DNA and RNA aptamers with the majority of the distribution falling between $40 \%$ and $70 \%$, whereas AT content ranges from $30 \%$ to $60 \%$. The trends were more pronounced in DNA than RNA however, no significant correlations were observed. For both DNA and RNA, percent GC content versus aptamer affinity showed a slight positive correlation ( $\mathrm{R}=0.34$ and $\mathrm{R}=0.04$ respectively) suggesting that higher affinity aptamers contain lower percent GC content. On the contrary, for both DNA and RNA percent AT content versus aptamer affinity showed weakly negative correlations $(\mathrm{R}=0.30$ and $\mathrm{R}=0.02$ respectively), suggesting higher AT content leads to better affinity aptamers.

The percent GC content compared to the aptamer affinity from a sample of the best aptamer candidates per selection (Figure 2.17) was analyzed to determine if more significant trends would emerge if only the best binders were analyzed.

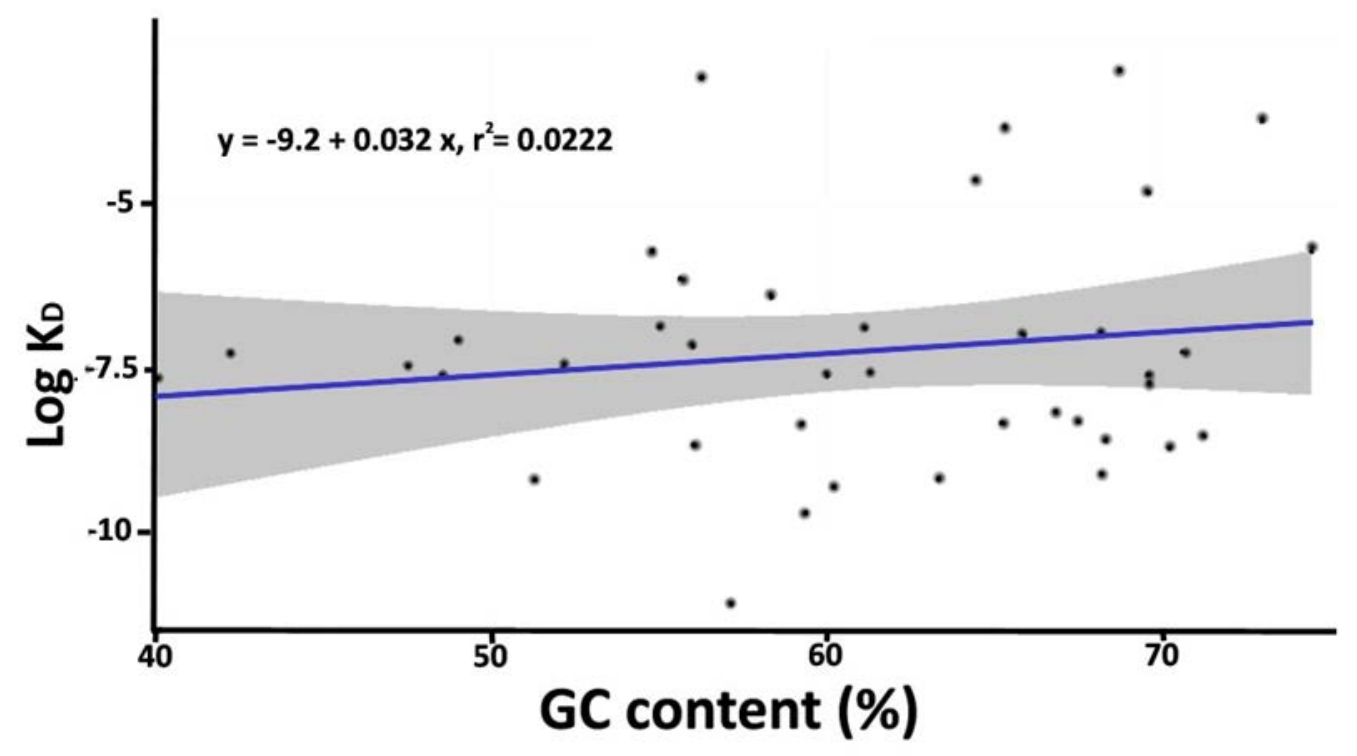

Figure 2.17: Linear regression analysis of the \%GC content for the best aptamer versus aptamer affinity $\left(\log _{10}\left(K_{D}\right)\right)$. Figure reprinted with permission of Springer 
from McKeague, M.; McConnell, E. M.; Cruz-Toledo, J.; Bernard, E. D.; Pach, A.; Mastronardi, E.; Zhang, X.; Beking, M.; Francis, T.; Giamberardino, A.;

Cabecinha, A.; Ruscito, A.; Aranda-Rodriguez, R.; Dumontier, M.; DeRosa, M. C. Analysis of in vitro selection parameters. J. Mol. Evol. 2015, 81 (5), 150. Copyright Springer 2015.

There was no significant correlation between the GC content of the best aptamer and aptamer affinity revealed $(\mathrm{R}=0.15)$.

Despite the surprising results that increased GC content does not lead to increased aptamer affinity our data did confirm the trend in the literature that aptamers tend to be GC rich (Figure 2.18).

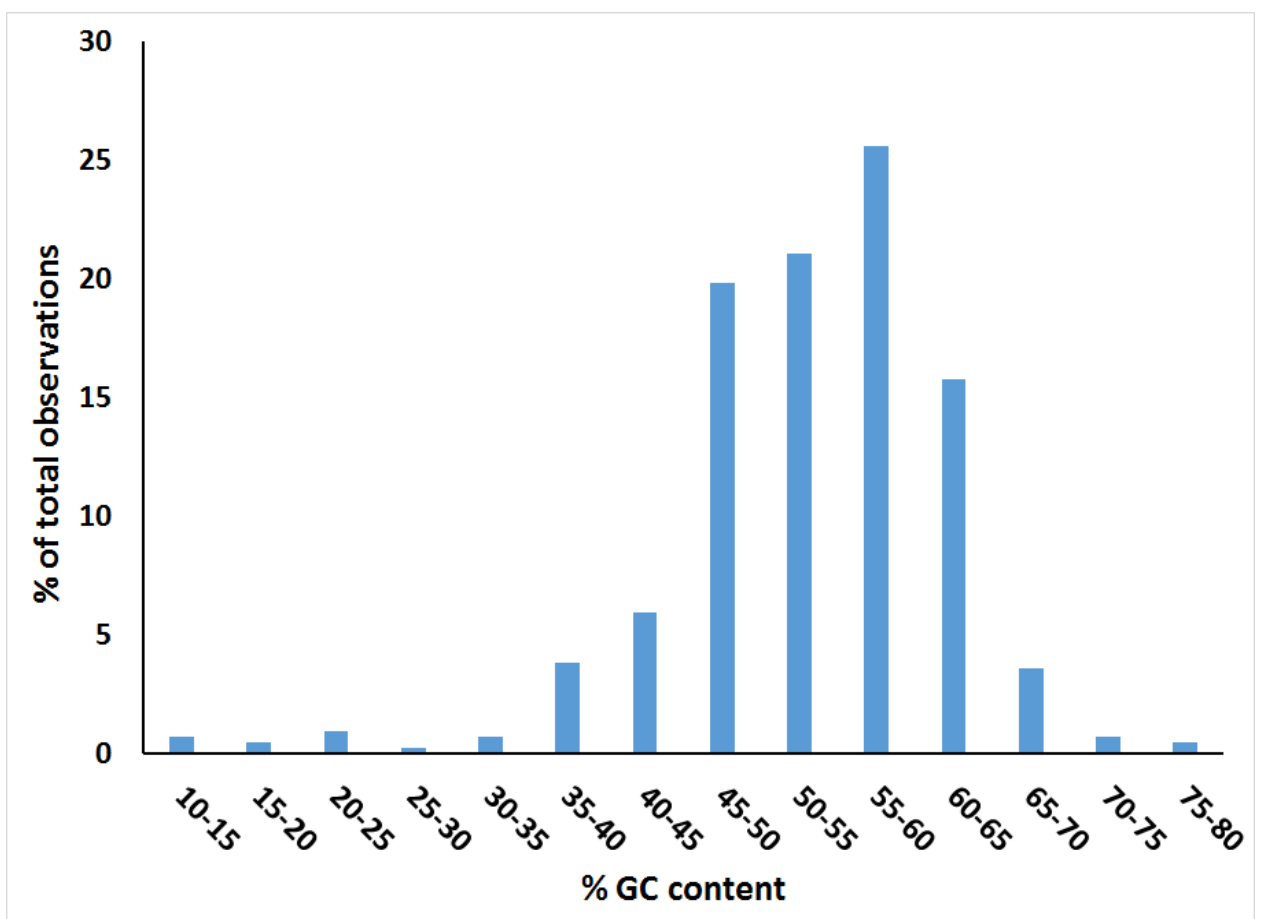

Figure 2.18: Distribution of \%GC content of all aptamers (1990-2012). Figure reprinted with permission of Springer from McKeague, M.; McConnell, E. M.; Cruz-Toledo, J.; Bernard, E. D.; Pach, A.; Mastronardi, E.; Zhang, X.; Beking, M.; Francis, T.; Giamberardino, A.; Cabecinha, A.; Ruscito, A.; Aranda-Rodriguez, R.; Dumontier, M.; DeRosa, M. C. Analysis of in vitro selection parameters. J. Mol. Evol. 2015, 81 (5), 150. Copyright Springer 2015. 
Of the aptamers examined, the highest population had a $50-55 \% \mathrm{GC}$ content. Interestingly, when the best aptamer candidates were examined by $\% \mathrm{GC}$ content distribution, the peak percent GC content shifted to $65-70 \%$, suggesting that for some reason, the percent GC content of the best aptamer candidates is enriched compared to all aptamer candidates. Further analysis will be required to determine why this may be the case. An important consideration that may skew this trend is the proportionate overabundance of G-quadruplex containing aptamers. The presence of this common secondary motif would explain an increased percent $\mathrm{G}$ and by extension percent GC content. Further analysis should examine whether the observed trends is skewed by the presence of G-quadruplexes or if G-content is also elevated in secondary structures distinct from G-quadruplexes. Interesting trends may also emerge from future work examining the relationship between the library composition (base composition, structural bias) and aptamer affinity. Additionally, analysis at the level of different target types may yield more interesting and significant trends.

\subsubsection{Aptamer target}

Aptamers have been selected for a diverse set of target types, therefore useful knowledge may be elucidated from the analysis of the relationship between target type and aptamer affinity. The occurrence of aptamers selected for various target types between the years 1990-2013 is displayed in Figure 2.19. 

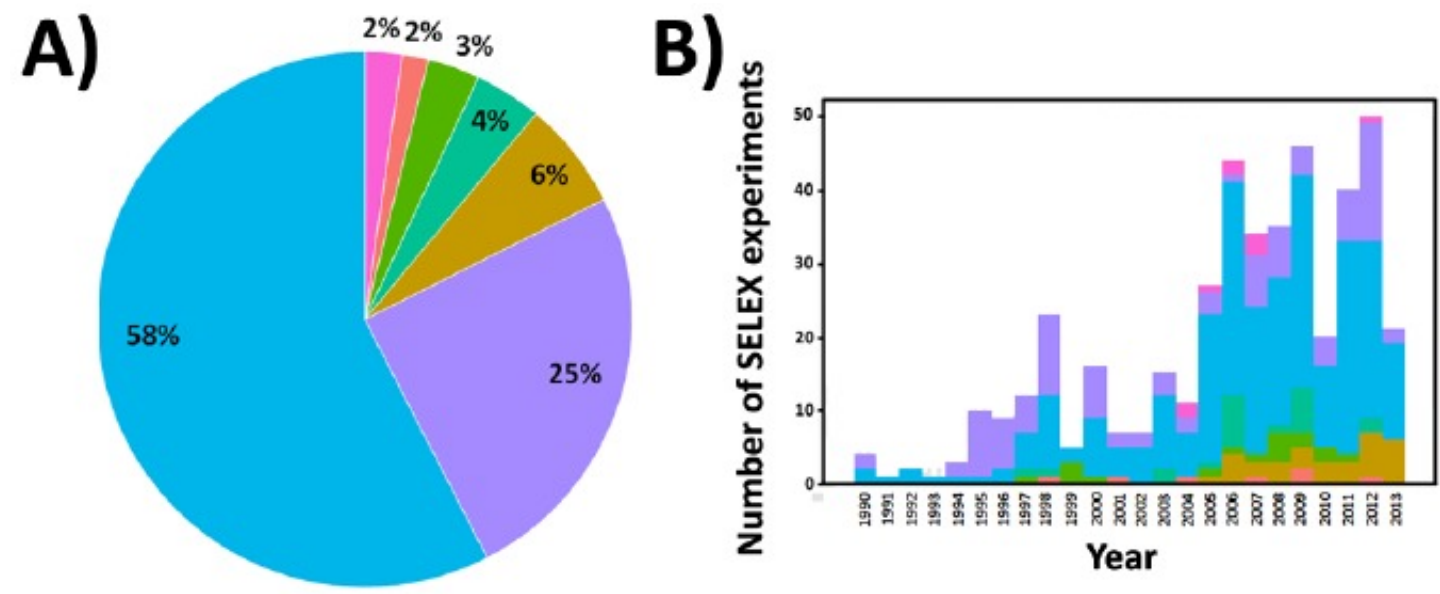

\section{Virus $\backsim$ Carbohydrate $₫$ Nucleic Acid $\approx$ Peptide Cell $₫$ Small Molecule $\square$ Protein}

Figure 2.19: A) Percentage of aptamers selected sorted by target type. B) Occurrence of target type for aptamer selections. Data for both figures encompasses selections from 1990-2013. Figure reprinted with permission of Springer from McKeague, M.; McConnell, E. M.; Cruz-Toledo, J.; Bernard, E. D.; Pach, A.; Mastronardi, E.; Zhang, X.; Beking, M.; Francis, T.; Giamberardino, A.; Cabecinha, A.; Ruscito, A.; Aranda-Rodriguez, R.; Dumontier, M.; DeRosa, M. C. Analysis of in vitro selection parameters. J. Mol. Evol. 2015, 81 (5), 150. Copyright Springer 2015.

From the data shown in Figure 2.19 A, it is evident that the majority of selections from 1990-2013 were for protein targets, followed by the second most common target, small molecules. As selection methods have evolved, the number of target types has increased.

Figure 2.19 B, a stacked bar graph of target type by year shows the diversification of target type over time; for example, in 1990 selections were for either protein or small molecule targets, by 2013 targets had expanded to include viruses and whole cells. The motivation to further analyze the relationship between affinity and target type stemmed from the observation that there was a stronger correlation between the library template length and the dissociation constant for different target types, as observed between 
peptide and carbohydrate targets in Table 2.1. Work was also done by Carothers et al. (2010) that provided an experimental example of another target dependent relationship. ${ }^{306}$ Carothers et al., (2010) examined the relationship between aptamer affinity and the structure and size of the aptamer target. ${ }^{306}$ Their work revealed that aptamer affinity was in fact proportional to the molecular weight of the target such that aptamers selected for larger targets had lower dissociation constant values.

For these reasons, the affinity of aptamers selected for the target types shown in Figure 2.19 were examined to determine if there was a significant difference between each target type. The mean $\log _{10}\left(\mathrm{~K}_{\mathrm{D}}\right)$ for each target type is presented in Figure 2.20. Statistical analysis by the Kruskal-Wallis test of the data revealed a significant difference in the mean $\log _{10}\left(\mathrm{~K}_{\mathrm{D}}\right)$ between at least two different target types $(\mathrm{p}<<0.05)$. Post-hoc analysis by pairwise Mann-Whitney-U test confirmed Carothers et al.'s findings. The mean rank of mean $\log _{10}\left(\mathrm{~K}_{\mathrm{D}}\right)$ of the aptamers selected for small molecule targets was significantly lower than all other target groups (results in Table 2.2) suggesting that the affinities of aptamers selected for small molecules may be lower than the other target types examined. 


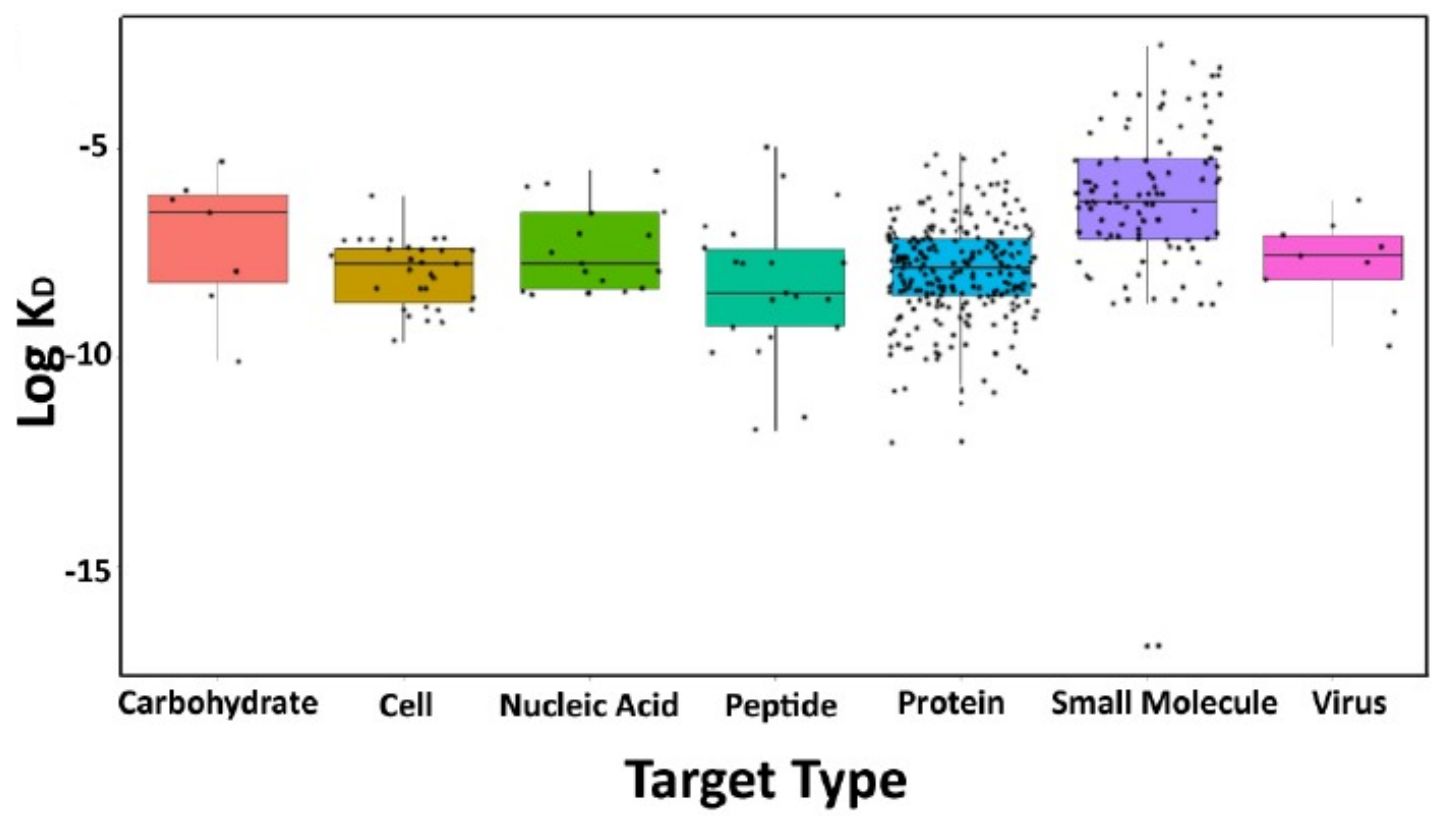

Figure 2.20: Mean affinity $\left(\log _{10}\left(K_{D}\right)\right)$ of selected aptamers for different target types. Error bars represent standard deviation. Figure reprinted with permission of Springer from McKeague, M.; McConnell, E. M.; Cruz-Toledo, J.; Bernard, E. D.; Pach, A.; Mastronardi, E.; Zhang, X.; Beking, M.; Francis, T.; Giamberardino, A.; Cabecinha, A.; Ruscito, A.; Aranda-Rodriguez, R.; Dumontier, M.; DeRosa, M. C. Analysis of in vitro selection parameters. J. Mol. Evol. 2015, 81 (5), 150. Copyright Springer 2015. 
Table 2.2: Summary of results from the pairwise Mann-Whitney-U test. Each target type was compared to small molecules. Significance was described at $(\alpha=0.05)$ and is indicated by an *. Table reproduced with permission of Springer from McKeague, M.; McConnell, E. M.; Cruz-Toledo, J.; Bernard, E. D.; Pach, A.; Mastronardi, E.; Zhang, X.; Beking, M.; Francis, T.; Giamberardino, A.; Cabecinha, A.; Ruscito, A.; Aranda-Rodriguez, R.; Dumontier, M.; DeRosa, M. C. Analysis of in vitro selection parameters. J. Mol. Evol. 2015, 81 (5), 150. Copyright Springer 2015.

\begin{tabular}{|l|c|c|}
\multicolumn{1}{|c|}{ Pairwise comparison } & $\begin{array}{c}\text { p-value } \\
(\boldsymbol{\alpha}=\mathbf{0 . 0 5})\end{array}$ & Sample size (n) \\
\hline Small Molecule and Cell & $4.10 \mathrm{E}-08^{*}$ & 103 vs 31 \\
\hline $\begin{array}{l}\text { Small Molecule and Nucleic } \\
\text { Acid }\end{array}$ & $2.40 \mathrm{E}-02^{*}$ & 103 vs 17 \\
\hline Small Molecule and Peptide & $6.10 \mathrm{E}-05^{*}$ & 103 vs 21 \\
\hline Small Molecule and Protein & $<2 \mathrm{e}-16^{*}$ & 103 vs 255 \\
\hline Small Molecule and Virus & $6.80 \mathrm{E}-02$ & 103 vs 9 \\
\hline
\end{tabular}

A large discrepancy between the sample sizes of each group existed and therefore may have skewed the results. As such, additional data will be required before more accurate trends can be reported.

\subsubsection{Selection conditions}

There are multiple examples from the literature of selection conditions influencing the affinity of an aptamer and therefore the relationship between selection conditions (metal cation concentration, buffering agent of selection buffer, $\mathrm{pH}$ and temperature) and the affinity of aptamers was examined. These data are presented to complement existing aptamer-specific experimental data. There are select sets of aptamers that are typically used in proof of concept studies and as such are well characterized. Comparative examples of the effect of altering a specific selection 
condition on the affinity of an aptamer were drawn from studies investigating these aptamers. $^{306-309}$

\subsubsection{Metal cation concentration}

The secondary structure and stability, and by extension the affinity of selected aptamers can be affected by the presence and concentration of metal cations. Since nucleic acids have a negative backbone, increasing the concentration of metal cations can results in shielding of the negative charge which therefore neutralizes the backbone and eliminates potential electrostatic interaction sites for target binding. ${ }^{307}$ In a similar way, the charge of the target may be affected by metal cation concentration. Additionally some targets, such as proteins, may exhibit metal cation concentration dependent aggregation. Both of these may lead to decreased aptamer-target affinity. The secondary structure of aptamers can also be affected by variations in ion content and concentration. For instance, an ion-dependent secondary structural transition may occur where an active aptamer conformation is able to bind a target in the presence of a particular metal cation, but affinity is substantially diminished or even eliminated due to the ion-dependent structural transition of the aptamer to an inactive non-binding conformation. There are in fact sensor designs that utilize this property of aptamers. ${ }^{310}$

The relationship between $\mathrm{Mg}^{2+}, \mathrm{Na}^{+}$, and $\mathrm{K}^{+}$and the $\mathrm{K}_{\mathrm{D}}$ were independently investigated to determine whether there was a global trend between metal cation concentration of the selection buffer and the affinity of selected aptamers. The role of metal cation concentration on affinity between different types of nucleic acids as well as 
different target types was examined by plotting the Log10(cation concentration) against the $\log _{10}\left(\mathrm{~K}_{\mathrm{D}}\right)$. The results of the linear regression analysis are summarized in Table 2.3.

Table 2.3: The correlation (represented by the correlation coefficient, $R$ ) and slope of the linear regression trendline $(\mathrm{m})$ of $\log 10$ (cation concentration) and $\log _{10}\left(K_{D}\right)$ for individual cations. Table reproduced with permission of Springer from McKeague, M.; McConnell, E. M.; Cruz-Toledo, J.; Bernard, E. D.; Pach, A.; Mastronardi, E.; Zhang, X.; Beking, M.; Francis, T.; Giamberardino, A.; Cabecinha, A.; Ruscito, A.; Aranda-Rodriguez, R.; Dumontier, M.; DeRosa, M. C. Analysis of in vitro selection parameters. J. Mol. Evol. 2015, 81 (5), 150. Copyright Springer 2015.

\begin{tabular}{ll|l|l|}
\multicolumn{1}{|c}{ Nucleic acid type } & \multicolumn{1}{c}{$\mathrm{Mg}^{2+}$} & \multicolumn{1}{c}{$\mathrm{Na}^{+}$} & \multicolumn{1}{c}{$\mathrm{K}^{+}$} \\
\hline RNA & $\mathrm{R}=0.38 ; \mathrm{m}=1.5$ & $\mathrm{R}=0.48 ; \mathrm{m}=2.8$ & $\mathrm{R}=0.21 ; \mathrm{m}=0.34$ \\
\hline DNA & $\mathrm{R}=0.21 ; \mathrm{m}=0.66$ & $\mathrm{R}=0.06 ; \mathrm{m}=0.25$ & $\mathrm{R}=0.06 ; \mathrm{m}=0.12$ \\
\hline Target Type & & & \\
\hline Small Molecule & $\mathrm{R}=0.43 ; \mathrm{m}=1.5$ & $\mathrm{R}=0.46 ; \mathrm{m}=2.2$ & $\mathrm{R}=0.39 ; \mathrm{m}=0.62$ \\
\hline Virus & $\mathrm{R}=0.88^{*} ; \mathrm{m}=2.5$ & $\mathrm{R}=1.00^{*} ; \mathrm{m}=-3.7$ & $(\mathrm{n} / \mathrm{a})$ \\
\hline Peptide & $\mathrm{R}=0.58^{*} ; \mathrm{m}=2.2$ & $(\mathrm{n} / \mathrm{a})$ & $(\mathrm{n} / \mathrm{a})$ \\
\hline Carbohydrate & $(\mathrm{n} / \mathrm{a})$ & $\mathrm{R}=0.38 ; \mathrm{m}=4.1$ & $(\mathrm{n} / \mathrm{a})$
\end{tabular}

* indicates significant correlation (significance at $\mathrm{R} \geq 0.50$ )

Linear regression analysis revealed that there was no significant correlation between the metal cation concentration of the selection buffer and the affinity for different nucleic acids types. There were however some interesting preliminary trends in the data that warrant further investigation when more data are available. In general a stronger correlation existed between the metal cation concentration and affinity of RNA than for DNA. Furthermore, upon comparison between metal cations of the RNA group, a stronger correlation existed for the concentration of $\mathrm{Na}^{+}$and affinity than for either $\mathrm{Mg}^{2+}$ or $\mathrm{K}^{+}$. Upon examination of the DNA group, a very weak correlation was observed 
between metal cation concentration and affinity for $\mathrm{Mg}^{2+}$ however almost no correlation was observed for either $\mathrm{Na}^{+}$or $\mathrm{K}^{+}$. In all cases, the correlations were positive, suggesting that higher affinity aptamers were selected in the presence of lower metal cation concentrations.

Aptamer-target binding is dependent on the intermolecular interactions that exist between the two components of the aptamer-target complex. The potential intermolecular interactions depend largely on the target type. Different target types are characterized by different physical properties such as size, polarity, charge, hydrophobicity, and chemical composition. The effect of these different properties on aptamer-target interactions has been examined experimentally by Carothers et al. (2010). ${ }^{306}$ Though data were limited, to begin to investigate these trends linear regression analysis was used to determine whether or not a correlation existed between metal cation concentration and the affinity of different target types (Table 2.3).

In general, stronger correlations between the aptamer affinity and metal cation concentration for different target types than for different nucleic acid types were observed. Generally, these data can be interpreted to suggest that researchers should choose metal cation concentrations for their selection buffer with more consideration for the target type than the nucleic acid type. Upon examination of the target type data there were no significant correlations revealed between aptamer affinity and metal cation concentrations for small molecule targets. Interestingly, significant positive correlations were revealed between aptamer affinity and $\mathrm{Mg}^{2+}$ concentration for both peptide and 
virus target types. Selections for these target types may yield better affinity aptamers if lower concentrations of $\mathrm{Mg}^{2+}$ cations are used in the selection buffer.

The trends revealed from this data analysis are consistent with experimental evidence from studies using model aptamers. One such aptamer is the thrombin binding aptamer. Hianik et al., (2007) showed that increasing $\mathrm{Na}^{+}$concentrations led to a decrease in the sensitivity of their human $\alpha$-thrombin aptamer-based sensor. ${ }^{307}$ Another commonly used aptamer is the ATP-binding aptamer. Deng et a., (2001) examined the effect of $\mathrm{Na}^{+}$ and $\mathrm{Mg}^{+}$concentration on analyte retention. ${ }^{308}$ They found that analyte retention was decreased with increasing $\mathrm{Na}^{+}$concentration and that analyte retention was increased with increasing $\mathrm{Mg}^{2+}$ concentration. As a final example, Neves et al., (2010) examined the cocaine aptamer. ${ }^{309}$ This aptamer, commonly used in bipartite aptamer studies and structure switching experiments, was shown to have the highest affinity for its target in the absence of $\mathrm{Na}^{+}$cation. Furthermore, there was a considerable decrease in aptamer affinity in the presence of $\mathrm{Na}^{+}$.

\subsubsection{Buffering agent}

The buffering agent used in selection buffers seems to be largely based on userpreference; however some evidence exists to suggest that the buffering agent used may affect the final aptamer affinity. The effect of buffering agent on the retention and resolution of multiple targets by the ATP aptamer from an affinity chromatography

column was examined. ${ }^{308}$ It was shown that using phosphate buffered solutions instead of tris(hydroxymethyl) aminomethane (Tris) actually led to better resolution between targets. The mean $\log _{10}\left(\mathrm{~K}_{\mathrm{D}}\right)$ of the most common buffering agents were compared to 
determine whether or not there was a significant difference between buffering agents (Figure 2.21). There was a significant difference in the mean rank of the affinity for different buffering agents (Kruskal-Wallis test; $\mathrm{p}=0.025$ ). As the most commonly reported buffering agent, post-hoc analysis was used to compare Tris buffered saline ( $\sim 50 \%$ of selections) to both HEPES ( 25\%) and phosphate buffered saline (PBS: $\sim 21 \%)$. The Mann-Whitney $\mathrm{U}$ test was used to compare the mean rank of the $\log _{10}\left(\mathrm{~K}_{\mathrm{D}}\right)$ of Tris buffered saline compared to either HEPES or PBS. Though there was a significant difference between Tris buffered saline and PBS $(\mathrm{p}=0.041)$, the difference between Tris and HEPES was not significant ( $\mathrm{p}>0.05)$.

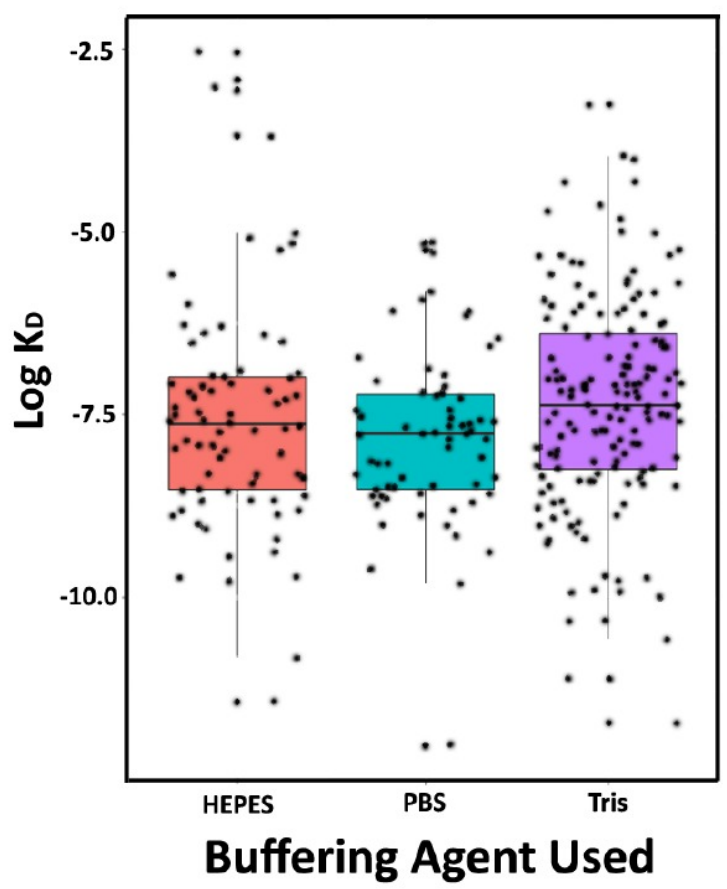

Figure 2.21: Boxplot of the mean affinity $\left(\log _{10}\left(K_{D}\right)\right)$ of common buffering agents. Error represents standard deviation. Figure reprinted with permission of Springer from McKeague, M.; McConnell, E. M.; Cruz-Toledo, J.; Bernard, E. D.; Pach, A.; Mastronardi, E.; Zhang, X.; Beking, M.; Francis, T.; Giamberardino, A.; Cabecinha, A.; Ruscito, A.; Aranda-Rodriguez, R.; Dumontier, M.; DeRosa, M. C. 


\section{Analysis of in vitro selection parameters. J. Mol. Evol. 2015, 81 (5), 150. Copyright Springer 2015.}

Due to incomplete selection condition reporting, approximately $5 \%$ of buffering agents were not described. The percentage of SELEX experiments lacking selection condition details is a great hindrance to thorough data analysis. It is important that the community reports their experimental details in sufficient detail for external researchers to be able to reproduce the selection method. ${ }^{267}$ As more data becomes available further analysis may reveal more significant buffering agent based affinity trends.

\subsubsection{Temperature}

Selection experiment temperature preference typically corresponds to target type and future application. For instance, targets of biological relevance could be selected at $37^{\circ} \mathrm{C}$ to mimic physiological conditions. Selection experiments performed at $4^{\circ} \mathrm{C}$ facilitate reduced nonspecific oligonucleotide uptake by cells. ${ }^{271}$ Data were analysed for selection experiments done at $4^{\circ} \mathrm{C}, 25^{\circ} \mathrm{C}$ (room temperature) or $37^{\circ} \mathrm{C}$. The majority of selection experiments where temperature details were provided were done at $25^{\circ} \mathrm{C}$ $(\sim 70 \%)$. The next common temperature was $37^{\circ} \mathrm{C}(\sim 21 \%)$ and least commonly, selections were performed at $4^{\circ} \mathrm{C}(\sim 9 \%)$. To determine whether there was a significant difference between the affinity of aptamers selected at different temperatures, a statistical comparison of the mean $\log _{10}\left(K_{D}\right)$ for each temperature group was performed (Figure 2.22). 


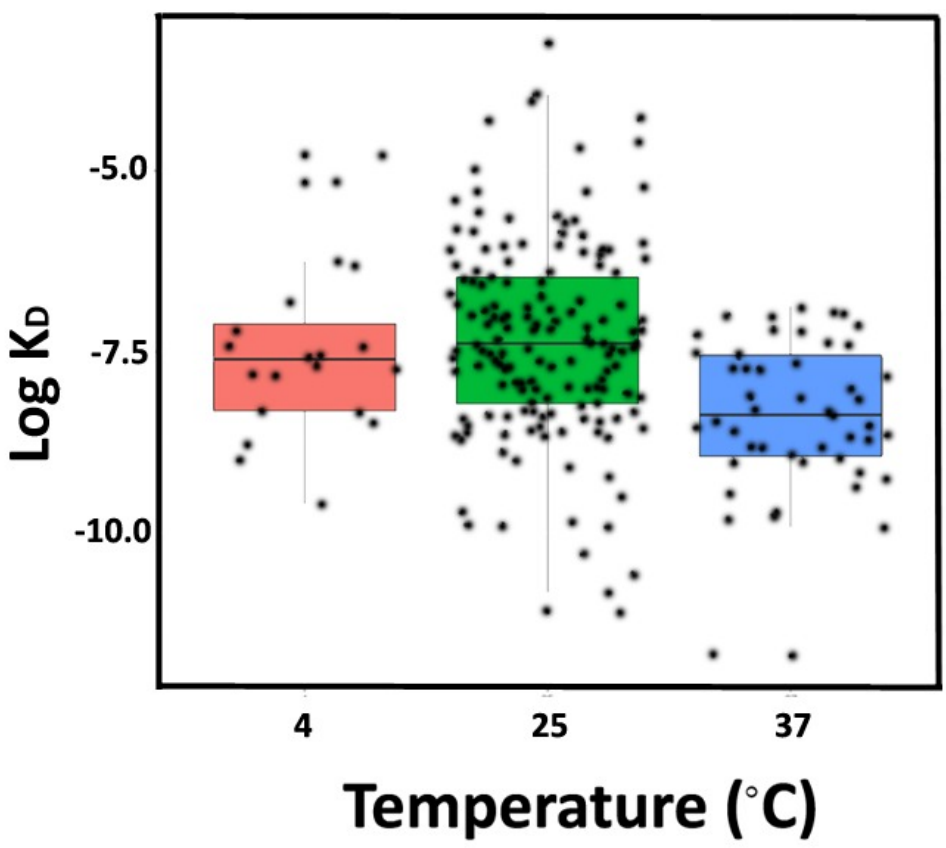

Figure 2.22: Boxplot of mean affinity $\left(\log _{10}\left(K_{D}\right)\right)$ based on selection temperature. Figure reprinted with permission of Springer from McKeague, M.; McConnell, E. M.; Cruz-Toledo, J.; Bernard, E. D.; Pach, A.; Mastronardi, E.; Zhang, X.; Beking, M.; Francis, T.; Giamberardino, A.; Cabecinha, A.; Ruscito, A.; Aranda-Rodriguez, R.; Dumontier, M.; DeRosa, M. C. Analysis of in vitro selection parameters. J. Mol. Evol. 2015, 81 (5), 150. Copyright Springer 2015.

Analysis by a One-Way ANOVA revealed that there was a significant difference between the mean $\log _{10}\left(K_{D}\right)$ for different temperatures $(p<<0.05)$. Significant differences were revealed between the mean $\log _{10}\left(\mathrm{~K}_{\mathrm{D}}\right)$ at $25^{\circ} \mathrm{C}$ and $37^{\circ} \mathrm{C}$ as well as $4^{\circ} \mathrm{C}$ and $37^{\circ} \mathrm{C}(\mathrm{Tukey}$ HSD: $p<<0.05$ and $p=0.037$ respectively). In both instances, the mean $\log _{10}\left(K_{D}\right)$ of the $37^{\circ} \mathrm{C}$ group was lower than the other group, meaning aptamers selected at $37^{\circ} \mathrm{C}$ have higher affinities. There was no significant difference between the mean $\log _{10}\left(K_{D}\right)$ of the $4^{\circ} \mathrm{C}$ and $25^{\circ} \mathrm{C}$ groups $(\mathrm{p}=0.083)$. Aptamers have many biological and in vivo related potential applications, therefore these data suggest that researchers may find it 
advantageous to perform aptamer selections at $37^{\circ} \mathrm{C}$ to optimize the potential affinity of selected aptamers.

\subsubsection{4 pH of the selection buffer}

It is important to consider the $\mathrm{pH}$ of the selection buffer when designing a selection experiment. The $\mathrm{pH}$ of the selection buffer will determine the charge/protonation of both nucleic acids and targets as well as influence the secondary structure and stability of the aptamer-target complex, thus affecting the affinity of aptamer target binding. Examples of the influence of $\mathrm{pH}$ on aptamer affinity have been

shown for the thrombin, ATP and cocaine aptamers. ${ }^{307-309}$ Linear regression analysis was used to investigate whether or not a correlation existed between the selection buffer $\mathrm{pH}$ and aptamer affinity (Figure 2.23). A weakly statistically significant negative correlation $(\mathrm{R}=0.51)$ between the $\mathrm{pH}$ of the selection buffer and $\log _{10}\left(\mathrm{~K}_{\mathrm{D}}\right)$ was revealed suggesting that selections done with buffers at higher $\mathrm{pH}$ values may lead to aptamers with better affinities than those done at lower $\mathrm{pH}$ values. However, the $\mathrm{pH}$ of the buffer should be chosen to best represent the desired application. Performing selections at a slightly basic $\mathrm{pH}$ may not be practical for a physiological application for example. 


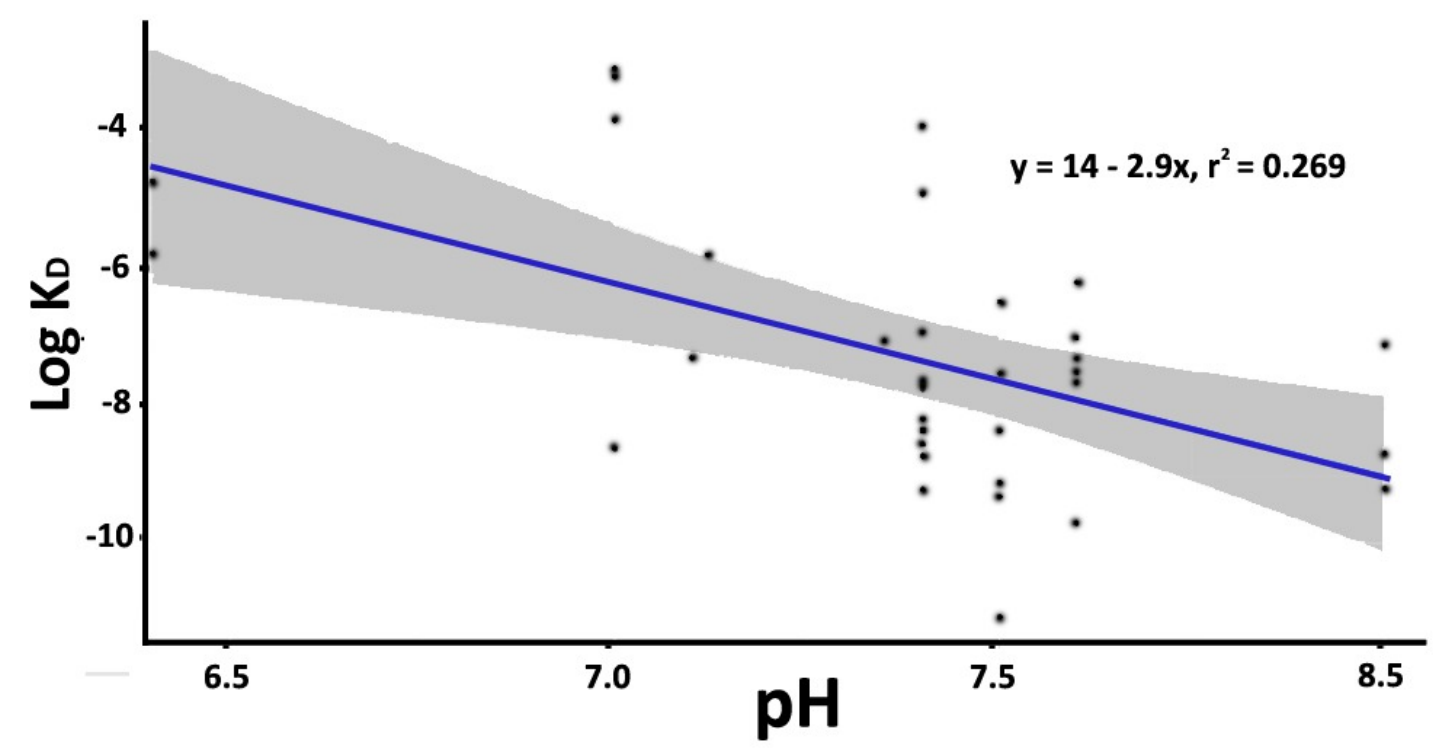

Figure 2.23: Linear regression analysis of the relationship between affinity $\left(\log _{10}\left(K_{D}\right)\right)$ and $\mathrm{pH}$. Figure reprinted with permission of Springer from McKeague, M.; McConnell, E. M.; Cruz-Toledo, J.; Bernard, E. D.; Pach, A.; Mastronardi, E.; Zhang, X.; Beking, M.; Francis, T.; Giamberardino, A.; Cabecinha, A.; Ruscito, A.; Aranda-Rodriguez, R.; Dumontier, M.; DeRosa, M. C. Analysis of in vitro selection parameters. J. Mol. Evol. 2015, 81 (5), 150. Copyright Springer 2015.

\subsubsection{Selection methods}

SELEX technology has advanced significantly in the past 20 years. There have been several adaptations and new methods published that aim to reduce selection time, increase the success rate of selections and to select aptamers with novel structure and functionality. In parallel data analysis methods have improved allowing researchers to go from characterizing fewer than one hundred sequences to billions of sequences post selection. ${ }^{276,311}$ The following sections discuss some of these adaptations and the potential effect of aptamer affinity. 


\subsubsection{Number of selection rounds}

Literature precedence suggests that approximately 5-15 rounds of selection are required for a typical SELEX experiment. ${ }^{283}$ This figure was confirmed by examining the number of rounds performed for the SELEX experiment data contained in the Aptamer Base (Figure 2.24).

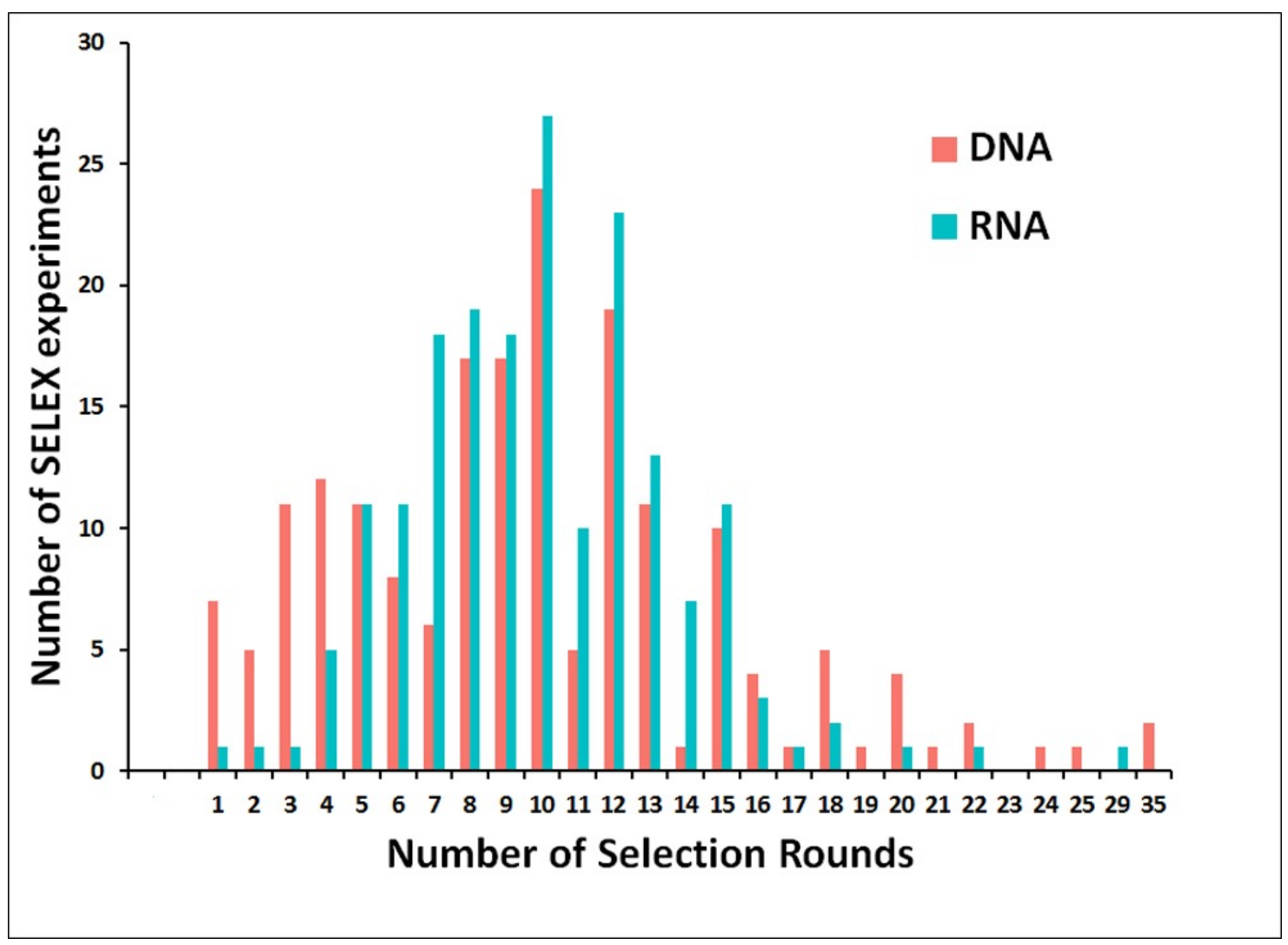

Figure 2.24: The distribution of frequency of SELEX experiments using a particular number of selection rounds is shown. Selection experiments were further divided by nucleic acid type; DNA (pink) and RNA (turquoise). Figure reprinted with permission of Springer from McKeague, M.; McConnell, E. M.; Cruz-Toledo, J.; Bernard, E. D.; Pach, A.; Mastronardi, E.; Zhang, X.; Beking, M.; Francis, T.; Giamberardino, A.; Cabecinha, A.; Ruscito, A.; Aranda-Rodriguez, R.; Dumontier, M.; DeRosa, M. C. Analysis of in vitro selection parameters. J. Mol. Evol. 2015, 81 (5), 150. Copyright Springer 2015. 
The mean number of selection rounds for DNA and RNA was determined to be $10 \pm 6$ and $10 \pm 4$ respectively. Several factors influence the researcher choice of selection rounds. Traditionally, enrichment was used as a metric for the completion of a selection experiment. As technology and methods have improved, reaching a plateau enrichment has become less necessary for successful aptamer selections. Nonetheless, the researcher must strike a balance between a reduced number of selection rounds, which allows for decreased cost, increased throughput and less influence by PCR bias, and performing enough rounds for sufficient enrichment.

Linear regression analysis was used to determine whether or not there was a significant correlation between the number of selection rounds performed and the affinity of selected aptamers (Figure 2.25). 


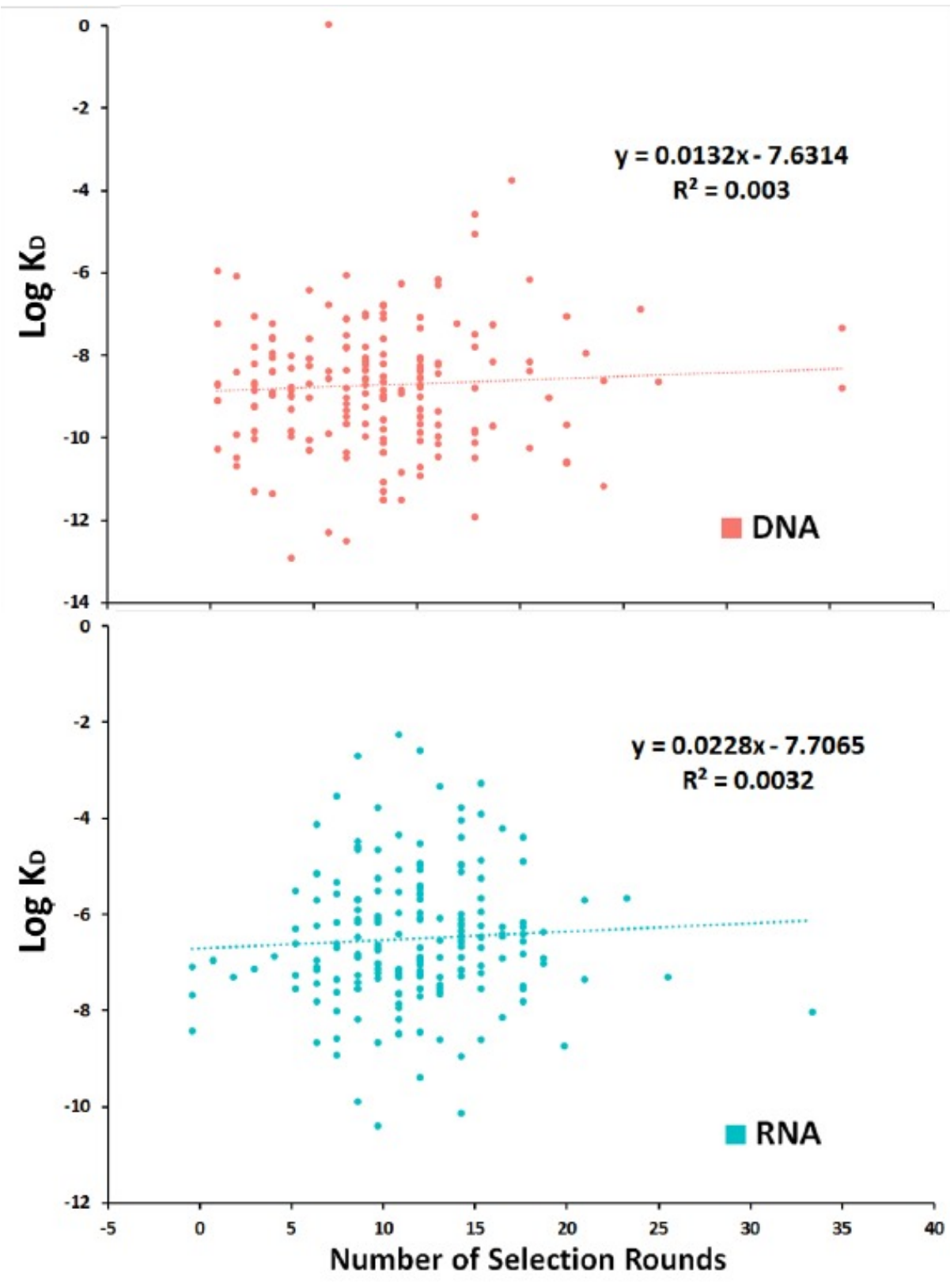

Figure 2.25: Linear regression analysis of number of selection rounds compared to aptamer affinity $\left(\log _{10}\left(K_{D}\right)\right)$. Figure reprinted with permission of Springer from McKeague, M.; McConnell, E. M.; Cruz-Toledo, J.; Bernard, E. D.; Pach, A.; Mastronardi, E.; Zhang, X.; Beking, M.; Francis, T.; Giamberardino, A.; Cabecinha, A.; Ruscito, A.; Aranda-Rodriguez, R.; Dumontier, M.; DeRosa, M. C. Analysis of in vitro selection parameters. J. Mol. Evol. 2015, 81 (5), 150. Copyright Springer 2015. 
There was no significant correlation observed between the number of selection rounds and the affinity $\left(\log _{10}\left(K_{D}\right)\right)$ for either DNA or RNA aptamers. Though this result may seem inconsistent given the expected experimental result of increasing enrichment by round, it is not surprising given that each selection begins by accessing a very limited proportion of the sequence space (Figure 2.26).

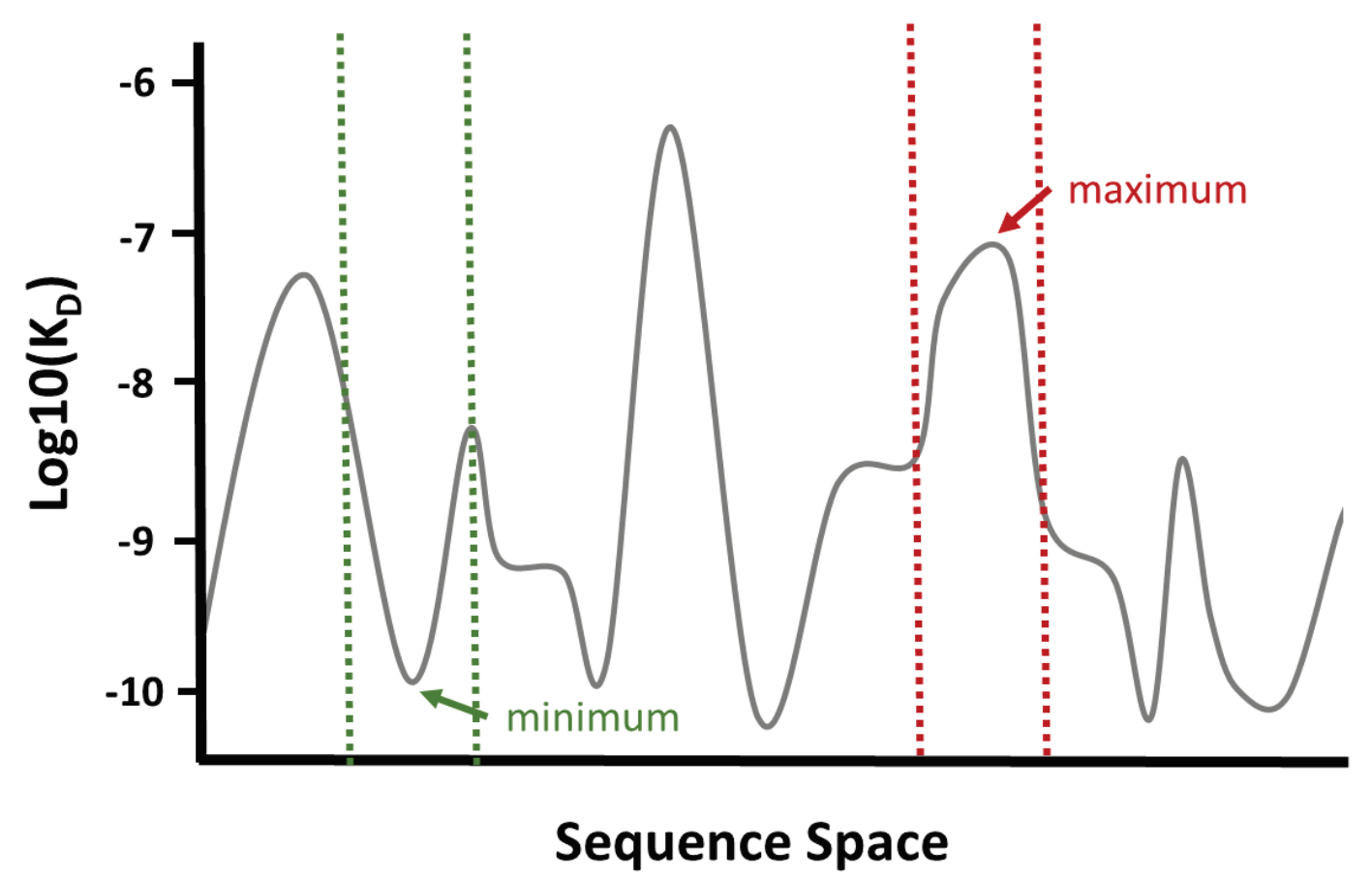

Figure 2.26: Representative minimum (green) and maximum (red) affinity domains in the sequence space of a selection library.

As illustrated in Figure 2.26, each selection library has a defined sequence space. Due to the practical limitations of synthesis technology, only a small fraction of the sequence space is accessed during a particular selection; recall the discussion of Figure 2.9. In Figure 2.26, the fraction of sequence space accessed by a particular selection is represented by either the red or green dashed boundaries. Within those boundaries, either a local minimum (green) or maximum (red) are contained. These peaks represent regions 
of relatively high or low affinity respectively, for a particular target. Therefore, if a selection starts with a library that accesses affinity minima (green boundary) then far fewer selection rounds would be required to reach an enrichment threshold than a selection library that accesses affinity maxima. As such, a true global comparison of affinity by selection round would need to compare only those selections that access equivalent boundaries within the sequence space.

Until enough data exist that the sequence space accessed is represented by a normal distribution, further analysis should examine whether or not significant trends emerge when the data are further subdivided by target type. Though the issue of equivocal sequence space access would still exist, the data would be less confounded by potential differences in target type.

Oftentimes small molecule selections require more selection rounds than larger targets such as proteins to reduce the diversity of the selection library such that high affinity aptamer candidates can be identified due to the unique challenges associated with small molecule target selections. ${ }^{291}$ Historically, low-throughput sequencing was used to analyze selection data. Due to the limitations of this technique, selections required a final library with a relatively low diversity to identify high affinity aptamer candidates. The advent of high-throughput sequencing (HTPS) more recently has allowed to identify highly enriched aptamer candidates from highly diversified libraries, making obtaining a threshold enrichment value less important. Open access software interfaces have been developed to analyze HTPS selection data. ${ }^{312,313}$ As these platforms evolve and the data researchers can garner from these programs expands, the method itself, and the 
information researchers can obtain from that data will drastically streamline aptamer selection methods.

\subsubsection{SELEX methodology}

Finally, the following sections describe the effect of the actual SELEX methodology on aptamer affinity. As aptamer technology changed and evolved several modifications were made to the SELEX method, partitioning method and recovery method. The original SELEX method for the development of aptamers evolved from work done simultaneously by Ellington and Szostak (1990), Robertson and Joyce (1990) and Tuerk and Gold (1990). ${ }^{34-36}$ Here in, SELEX method refers to a method adapted from the original in vitro selection experiments. These adaptations served to improve selected aptamer affinity, streamline selection procedures, or facilitate selection of novel and/or traditionally difficult target types. The partitioning method refers to the way that oligonucleotides that have an affinity to the target are separated from oligonucleotides with little or no affinity to the target. Lastly, the recovery method is the method used to separate binding oligonucleotides from the target prior to PCR amplification. The recovery method can be a physical or chemical method. Typically, researchers change these methods with the intention to improve the partitioning efficiency, to allow for the incorporation of methods to monitor enrichment, or to streamline throughput. ${ }^{314,315}$

Selection methodology data from the Aptamer Base was examined to ascertain whether a particular methodology resulted in higher aptamer affinity. Unfortunately it was difficult to draw meaningful conclusions from these data due to bias toward certain methodologies. There was simply not enough data for certain methods to extrapolate 
generalized trends. Additionally, the limited data within each group was further subdivided by target type. Figure 2.27, Figure 2.28 and Figure 2.29 represent the frequency of SELEX method, partitioning method and recovery method respectively, by target type. In the following figures, for each methodology, the percentage of SELEX experiments performed for a particular target type is shown. The data bias is especially noticeable in these figures where several methods were performed on only one or two target types. Once sufficient data are available for each methodology, it will be interesting to compare the affinity across methodologies for one target type. Until such data are available, more systematic experiments are required to elucidate whether one method results in better affinity aptamers over another for a particular target type. 


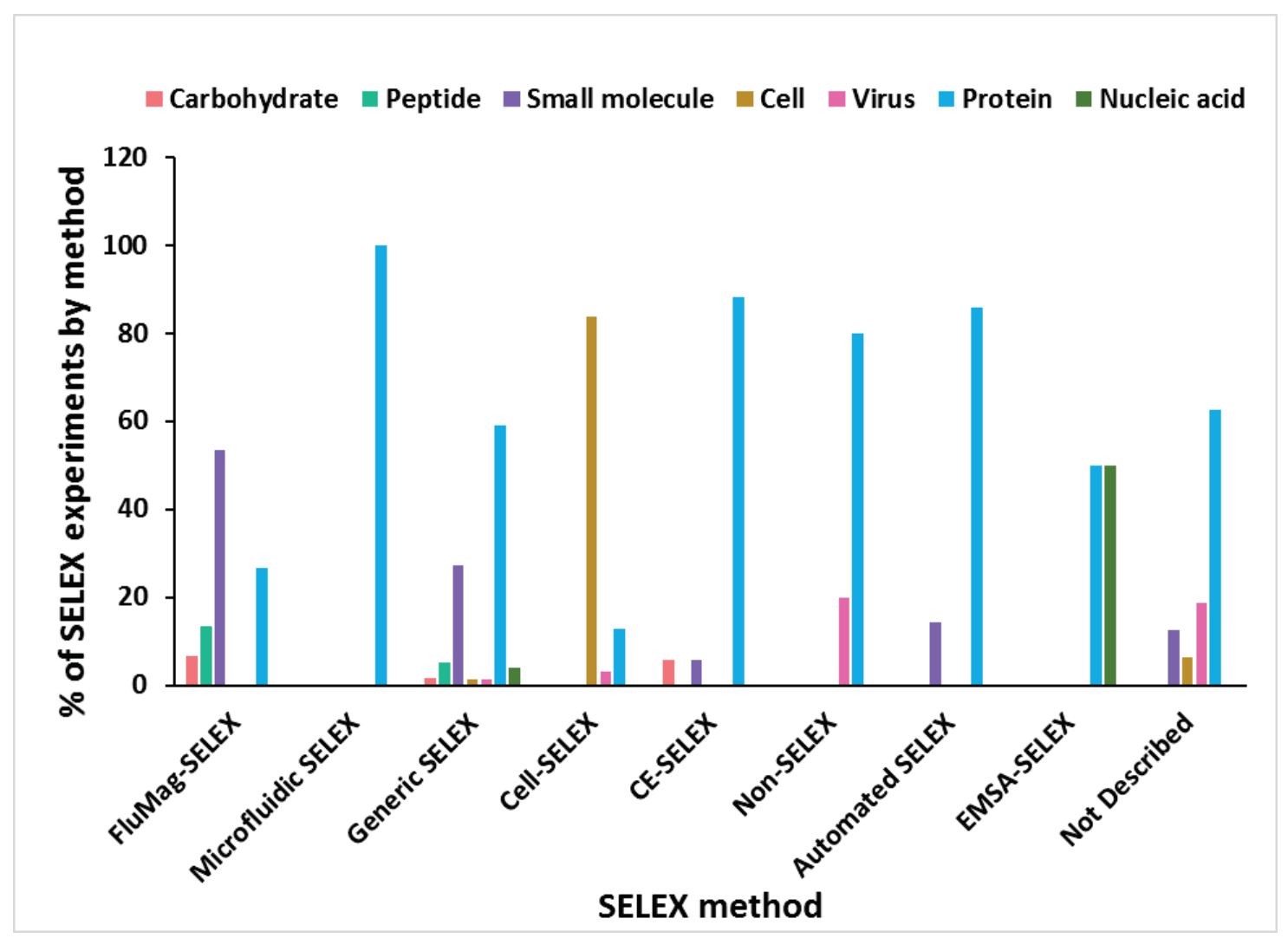

Figure 2.27: Comparison of SELEX method frequency by target type. Figure reprinted with permission of Springer from McKeague, M.; McConnell, E. M.;

Cruz-Toledo, J.; Bernard, E. D.; Pach, A.; Mastronardi, E.; Zhang, X.; Beking, M.; Francis, T.; Giamberardino, A.; Cabecinha, A.; Ruscito, A.; Aranda-Rodriguez, R.; Dumontier, M.; DeRosa, M. C. Analysis of in vitro selection parameters. J. Mol. Evol. 2015, 81 (5), 150. Copyright Springer 2015. 


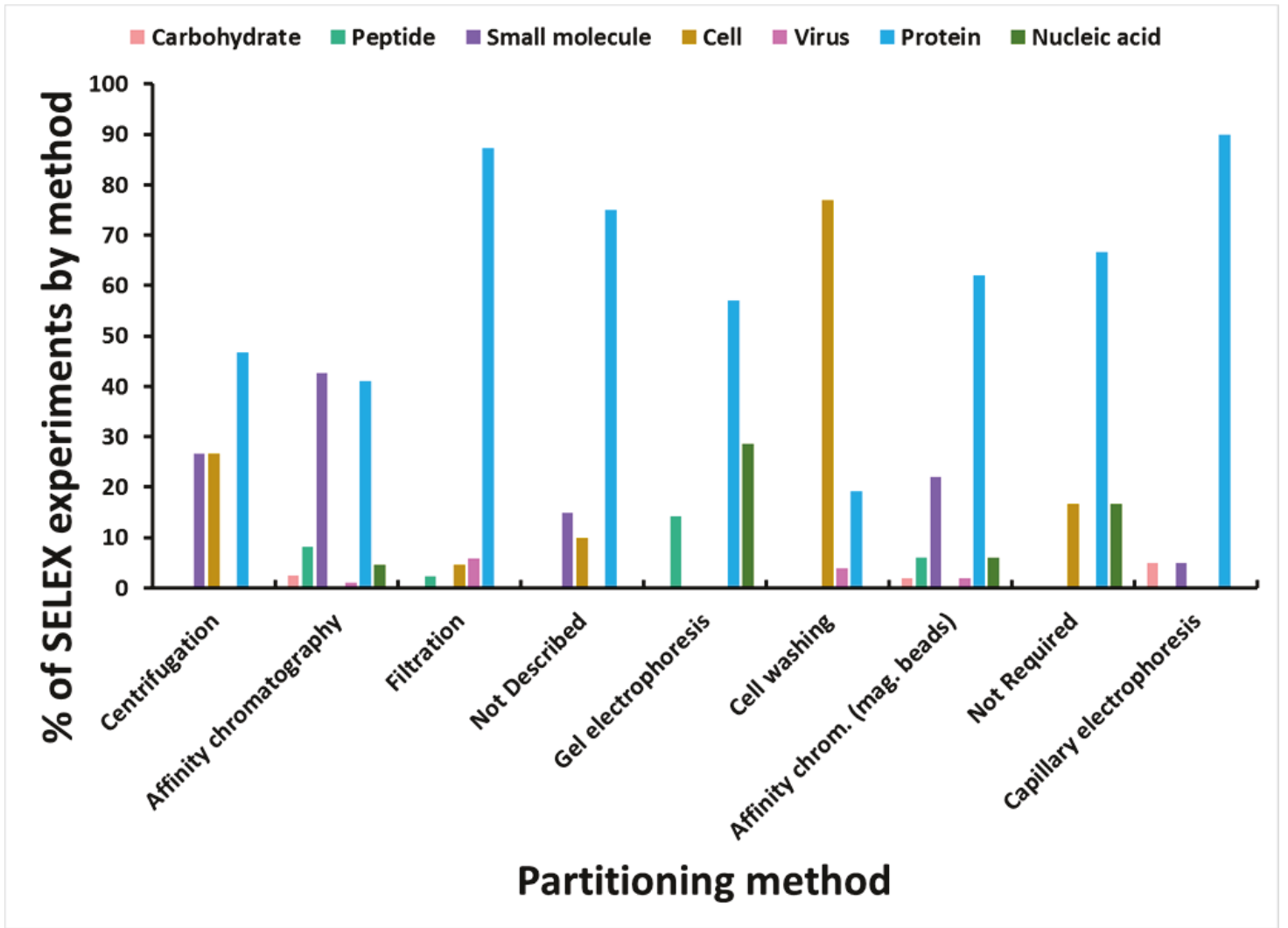

Figure 2.28: Comparison of partitioning method frequency by target type. Figure reprinted with permission of Springer from McKeague, M.; McConnell, E. M.;

Cruz-Toledo, J.; Bernard, E. D.; Pach, A.; Mastronardi, E.; Zhang, X.; Beking, M.; Francis, T.; Giamberardino, A.; Cabecinha, A.; Ruscito, A.; Aranda-Rodriguez, R.; Dumontier, M.; DeRosa, M. C. Analysis of in vitro selection parameters. J. Mol. Evol. 2015, 81 (5), 150. Copyright Springer 2015. 


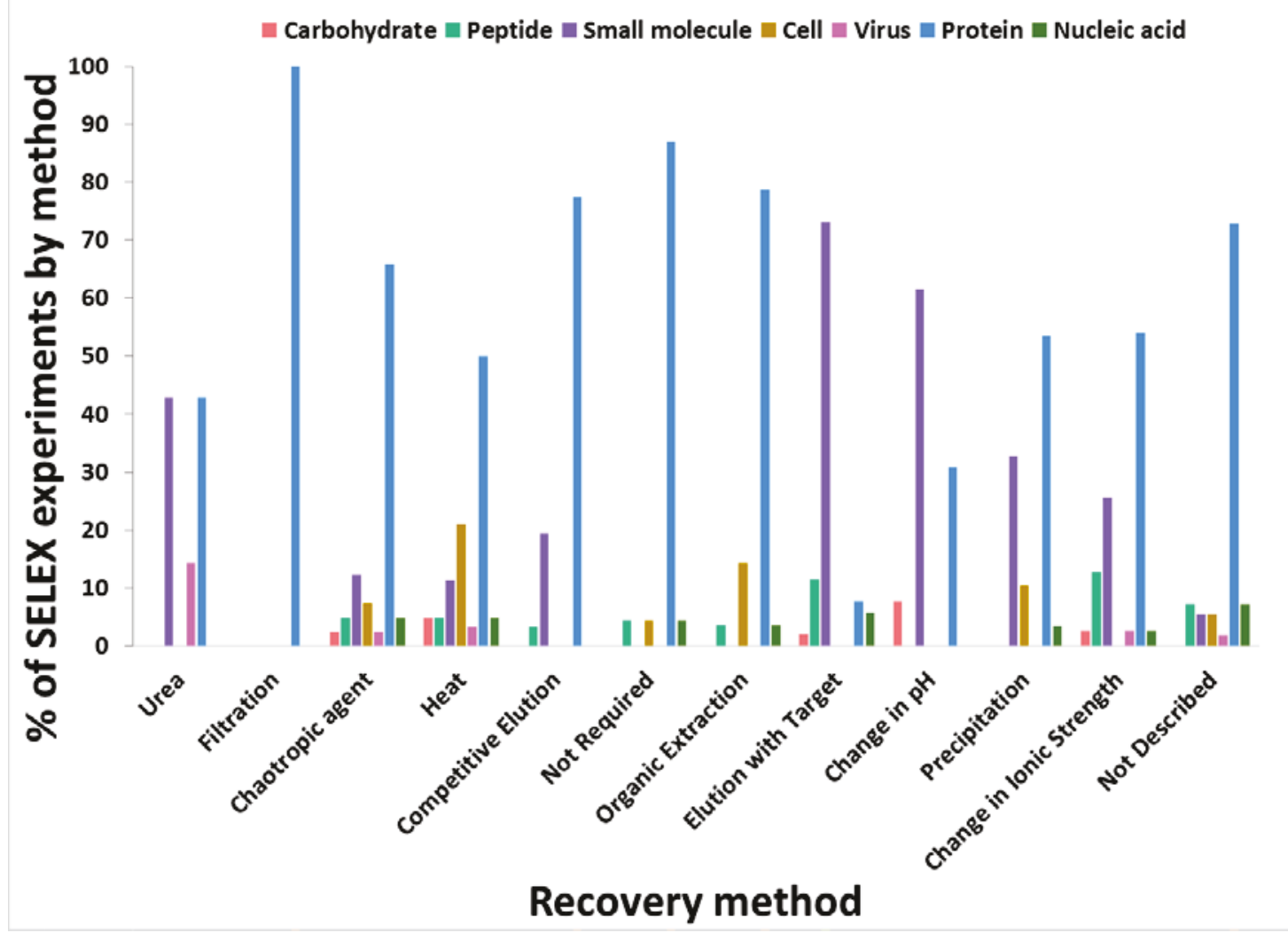

Figure 2.29: Comparison of recovery method frequency by target type. Figure reprinted with permission of Springer from McKeague, M.; McConnell, E. M.; Cruz-Toledo, J.; Bernard, E. D.; Pach, A.; Mastronardi, E.; Zhang, X.; Beking, M.; Francis, T.; Giamberardino, A.; Cabecinha, A.; Ruscito, A.; Aranda-Rodriguez, R.; Dumontier, M.; DeRosa, M. C. Analysis of in vitro selection parameters. J. Mol. Evol. 2015, 81 (5), 150. Copyright Springer 2015.

\subsection{SELEX method}

The most commonly employed selection method was the "generic" method and it was employed for approximately $74 \%$ of the SELEX experiments. The generic method bears the most resemblance to the first generation SELEX experiments in that specialized equipment such as microfluidics or capillary electrophoresis were not applied, and the method was applicable to multiple target types (unlike Cell-SELEX for example). ${ }^{264,315}$ The resultant affinity of each SELEX method is shown in Figure 2.30. 


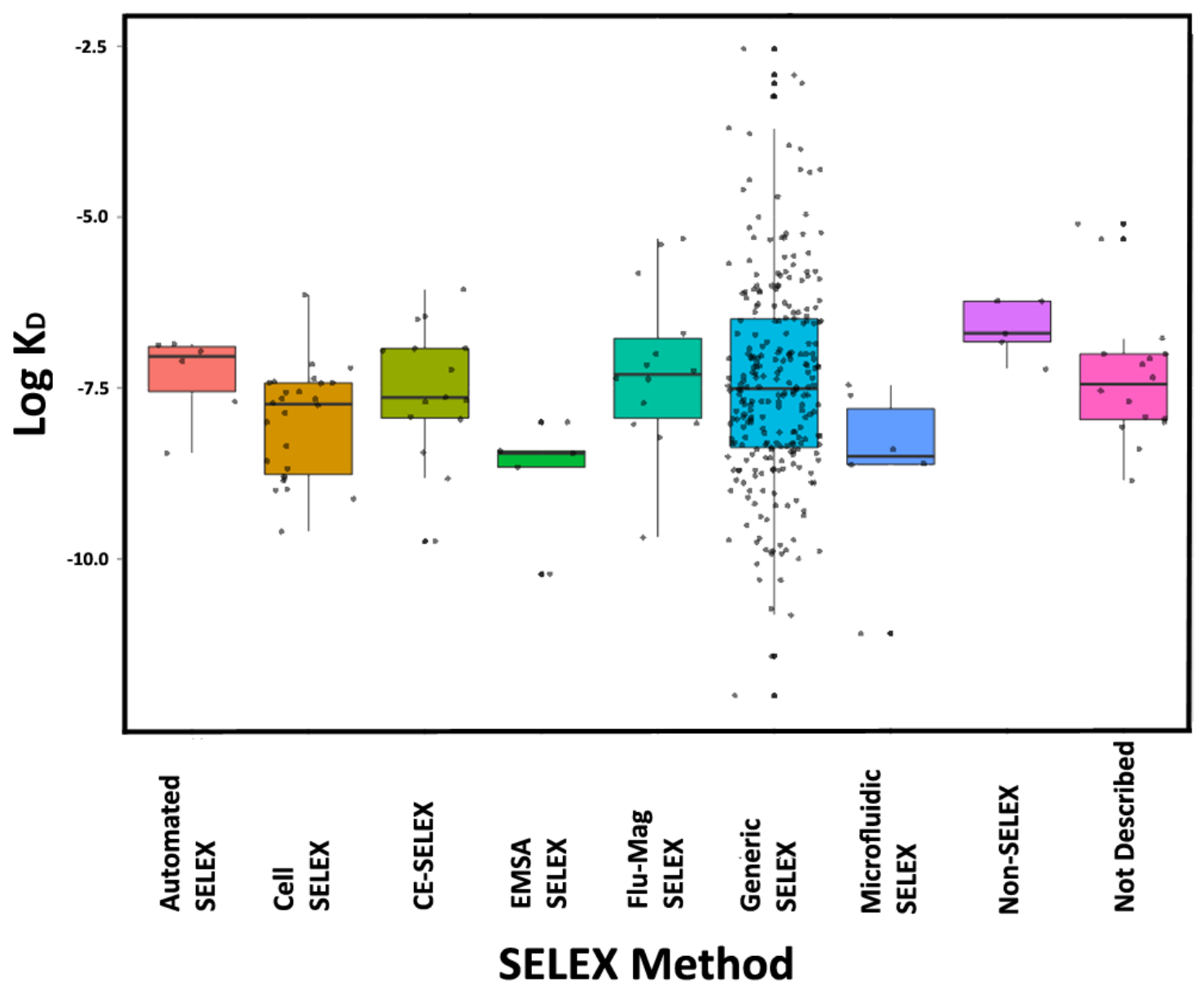

Figure 2.30: Boxplot of affinity $\left(\log _{10}\left(K_{D}\right)\right)$ by SELEX method. Figure reprinted with permission of Springer from McKeague, M.; McConnell, E. M.; Cruz-Toledo, J.; Bernard, E. D.; Pach, A.; Mastronardi, E.; Zhang, X.; Beking, M.; Francis, T.; Giamberardino, A.; Cabecinha, A.; Ruscito, A.; Aranda-Rodriguez, R.; Dumontier, M.; DeRosa, M. C. Analysis of in vitro selection parameters. J. Mol. Evol. 2015, 81 (5), 150. Copyright Springer 2015.

The non-parametric Kruskal-Wallis test was used to evaluate whether there was as significant difference between the mean $\log _{10}\left(\mathrm{~K}_{\mathrm{D}}\right)$ for each SELEX method. This statistical test was chosen due to the bias in sample size and differences in sample variances. There was a significant difference between the mean rank of $\log _{10}\left(K_{D}\right)$ for different SELEX methods (Kruskal-Wallis: $\alpha=0.05, p=0.01$ ) suggesting that the value of the median $\log _{10}\left(\mathrm{~K}_{\mathrm{D}}\right)$ differs between at least two methods. However, before meaningful 
conclusions can be drawn about the relationship between SELEX method and final aptamer affinity, and which methods produce the highest affinity aptamers, more data will be required.

\subsection{Partitioning method}

The most commonly reported partitioning method was affinity chromatography. During this method, the target is chemically immobilized on a solid support matrix (typically agarose-based matrices). Over half of the selection experiments reported using affinity chromatography where $42 \%$ of the selection experiments immobilized their target on a solid support matrix and $12 \%$ specifically immobilized the target on magnetic beads. The second most commonly used partitioning method was filtration, which was reported in $21 \%$ of SELEX experiments. The data were examined to determine whether one partitioning method led to better affinities than another method (Figure 2.31). 


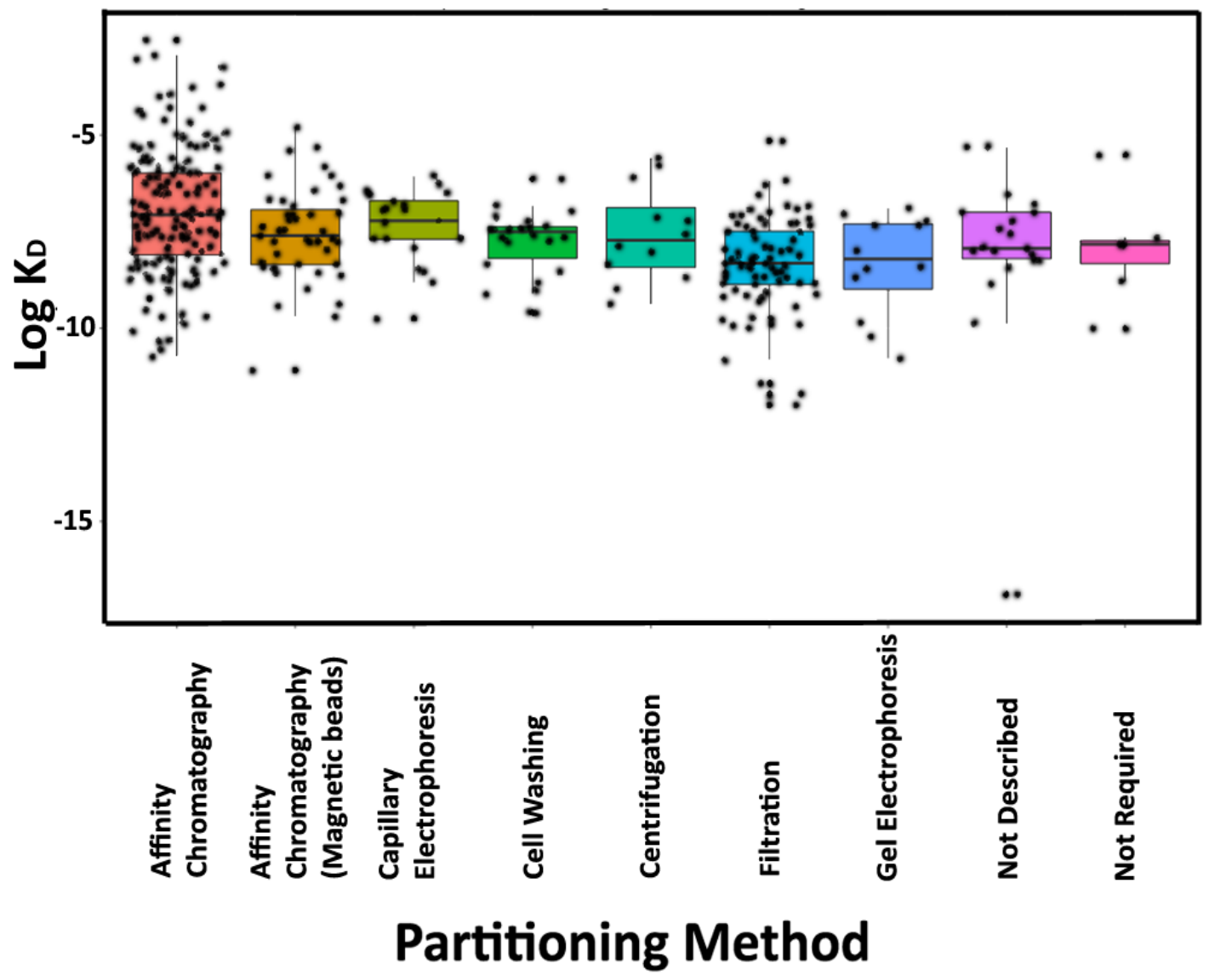

Figure 2.31: Boxplot of affinity by partitioning round. Figure reprinted with permission of Springer from McKeague, M.; McConnell, E. M.; Cruz-Toledo, J.; Bernard, E. D.; Pach, A.; Mastronardi, E.; Zhang, X.; Beking, M.; Francis, T.; Giamberardino, A.; Cabecinha, A.; Ruscito, A.; Aranda-Rodriguez, R.; Dumontier, M.; DeRosa, M. C. Analysis of in vitro selection parameters. J. Mol. Evol. 2015, 81 (5), 150. Copyright Springer 2015.

Again, Kruskal-Wallis was used to evaluate the data. There was a significant difference in the mean rank of the $\log _{10}\left(\mathrm{~K}_{\mathrm{D}}\right)$ value between different partitioning methods $(p<<0.05)$. Since the sample sizes of the affinity chromatography ( $n=160$ for general, and $\mathrm{n}=47$ for magnetic beads) and filtration $(\mathrm{n}=79)$ methods were closer in size, post-hoc analysis by Mann-Whitney U test was performed. A significant difference was observed between the mean rank of $\log _{10}\left(K_{D}\right)$ of both affinity chromatography methods and 
filtration (general: $\mathrm{p}<<0.05$ and magnetic beads: $\mathrm{p}=0.032$ ). This result suggests that filtration as a partitioning method yields aptamers with slightly higher affinity. Again, as more data from novel SELEX experiments are available to analyze, examining trends at the level of target type may yield more interesting trends.

\subsection{Recovery method}

Researchers employ multiple physical and chemical methods for the recovery of nucleic acids from the oligonucleotide/target binding complex. The most commonly used methods in SELEX experiments in order of frequency are heat (16\%), elution with target $(12 \%)$, precipitation $(11 \%)$, chaotropic agent $(11 \%)$ and change in ionic strength $(10 \%)$. The affinity as a function of recovery method is shown in Figure 2.32. 


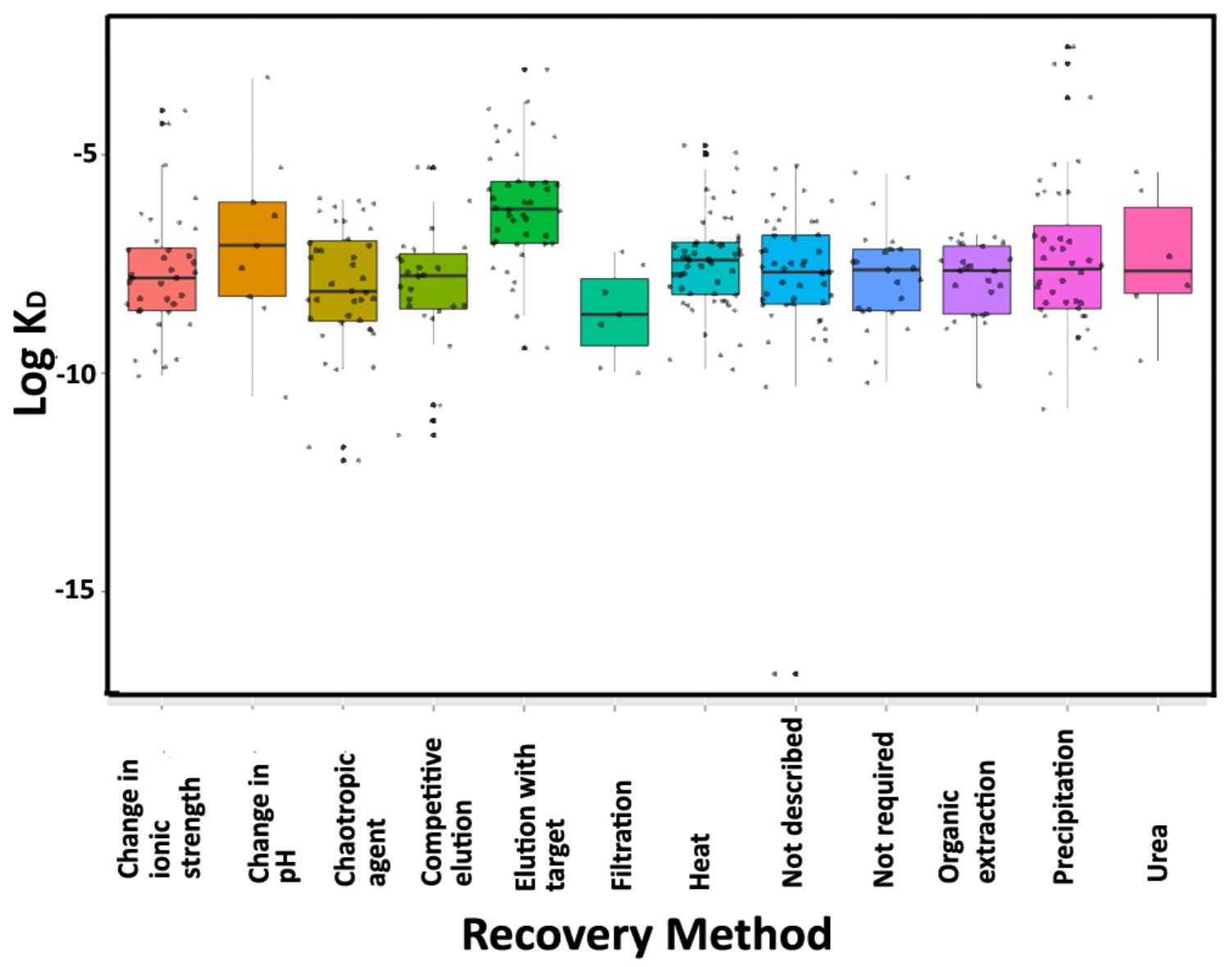

Figure 2.32: Boxplot of affinity by recovery method. Figure reprinted with permission of Springer from McKeague, M.; McConnell, E. M.; Cruz-Toledo, J.; Bernard, E. D.; Pach, A.; Mastronardi, E.; Zhang, X.; Beking, M.; Francis, T.; Giamberardino, A.; Cabecinha, A.; Ruscito, A.; Aranda-Rodriguez, R.; Dumontier, M.; DeRosa, M. C. Analysis of in vitro selection parameters. J. Mol. Evol. 2015, 81 (5), 150. Copyright Springer 2015.

Since there was a greater distribution of recovery method used, the differences between recovery method and aptamer affinity where examined by a One-Way ANOVA. There was a significant difference revealed between the mean $\log _{10}\left(\mathrm{~K}_{\mathrm{D}}\right)$ of different recovery methods $(\alpha=0.05, p<<0.05)$. Upon examination of the recovery method affinity means, elution with target appeared to have a lower affinity compared to all other groups. Therefore, Tukey HSD was used to analyze the differences between the elution with 
target group and all other recovery methods. There was significant difference observed between the mean $\log _{10}\left(K_{D}\right)$ of the elution with target method compared to all other recovery methods ( $\alpha=0.05 ; \mathrm{p}>0.01$ for all pair-wise comparisons), suggesting that the commonly used elution with target recovery method actually produces higher dissociation constants and therefore lower affinity aptamers than all other methods examined. However, this trend is not surprising given that elution with target is commonly used with small molecule targets, which tend to be associated with lower affinity aptamers, due to the target type-associated selection challenges. ${ }^{291}$ Similarly to partitioning method, more significant trends may be revealed by analysis of recovery method at the level of target type.

\subsubsection{Affinity testing methodology}

The buffering agent that the affinity experiment was performed in as well as the method used to assess the affinity an aptamer has for its target were assessed.

\subsubsection{Buffering agent used in affinity experiment}

Finally, the effect of the buffering agent used in affinity experiments on aptamer affinity was examined (Figure 2.33). 


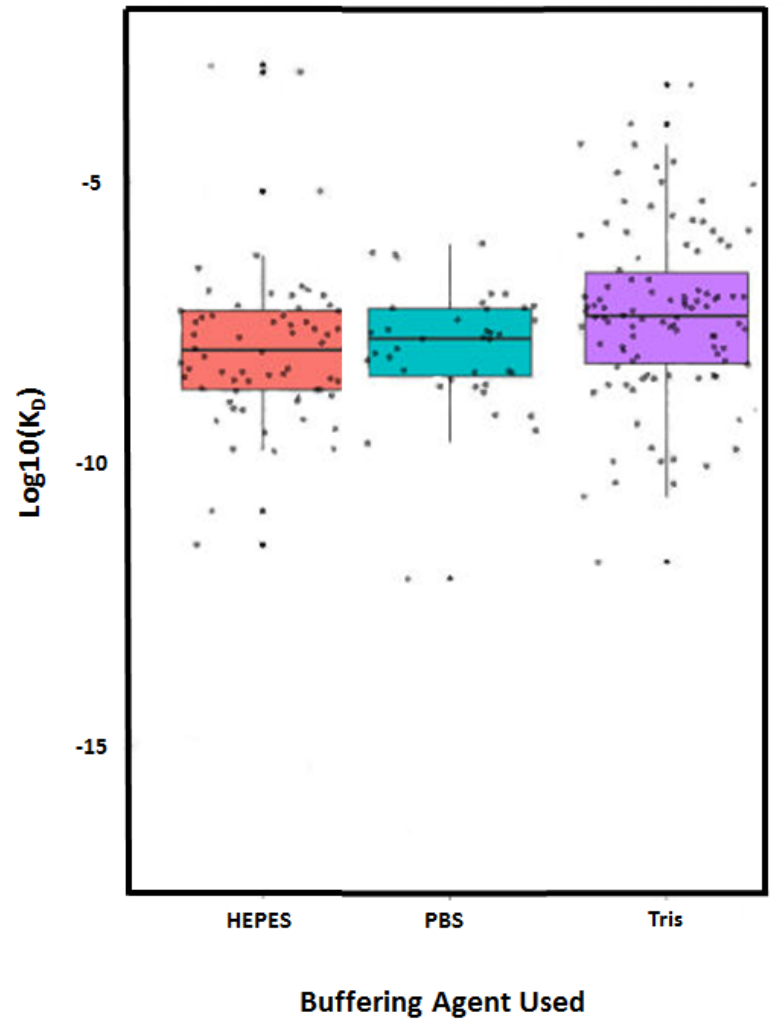

Figure 2.33: Box plot comparison of mean affinity $\left(\log _{10}\left(K_{D}\right)\right)$ of the buffering agent used in affinity experiments. Figure reprinted with permission of Springer from McKeague, M.; McConnell, E. M.; Cruz-Toledo, J.; Bernard, E. D.; Pach, A.; Mastronardi, E.; Zhang, X.; Beking, M.; Francis, T.; Giamberardino, A.; Cabecinha, A.; Ruscito, A.; Aranda-Rodriguez, R.; Dumontier, M.; DeRosa, M. C. Analysis of in vitro selection parameters. J. Mol. Evol. 2015, 81 (5), 150. Copyright Springer 2015.

One-Way ANOVA revealed a significant difference between the mean affinity of different buffering agents $(\mathrm{p}<0.005 ; \alpha=0.05)$. There was not enough data to draw meaningful conclusions about the differences between the groups but preliminary analysis of the mean $\log _{10}\left(\mathrm{~K}_{\mathrm{D}}\right)$ suggests that using either PBS or HEPES led to lower dissociation constants and therefore better affinity. This trend is consistent with what was observed for the buffering agent used in the selection buffer (Figure 2.21). Interestingly, $\sim 34 \%$ of affinity experiments used Tris buffered saline whereas $\sim 23 \%$ used HEPES and 
only $\sim 13 \%$ used phosphate buffered saline. Approximately $31 \%$ of affinity experiments

did not report the buffer that was used during the selection experiments; another example of the importance of complete reporting of experimental details.

\subsubsection{Method of affinity experiment}

Given the tendency of different affinity screening and characterization methods to produce significantly different dissociation constants for the same aptamer/target interaction, the relationship between the affinity method used and the affinity of the aptamer candidate was examined (Figure 2.34).

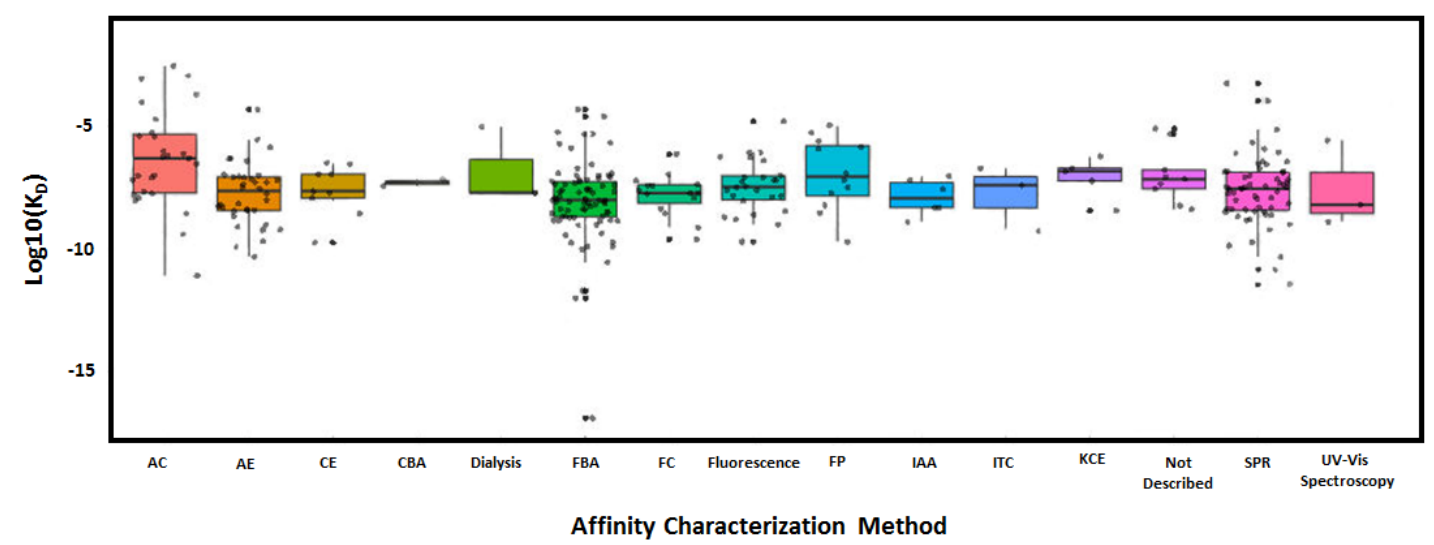

Figure 2.34: Comparison of the mean affinity between different affinity characterization methods. Abbreviations in parentheses: Affinity chromatography (AC), affinity electrophoresis (AE), capillary electrophoresis (CE), cell binding assay (CBA), dialysis, filter binding assay (FBA), flow cytometry (FC), fluorescence, fluorescence polarization (FP), immunoaffinity assay (IAA), isothermal titration calorimetry (ITC), kinetic capillary electrophoresis (KCE), not described, surface plasmon resonance (SPR) and, UV-Visible Spectroscopy. Figure reprinted with permission of Springer from McKeague, M.; McConnell, E. M.; Cruz-Toledo, J.; Bernard, E. D.; Pach, A.; Mastronardi, E.; Zhang, X.; Beking, M.; Francis, T.; Giamberardino, A.; Cabecinha, A.; Ruscito, A.; Aranda-Rodriguez, R.; Dumontier, M.; DeRosa, M. C. Analysis of in vitro selection parameters. J. Mol. Evol. 2015, 81 (5), 150. Copyright Springer 2015. 
Preliminary statistical analysis by One-Way ANOVA revealed a significant difference ( $p=0.017 ; \alpha=0.05$ ) between mean $\log _{10}\left(\mathrm{~K}_{\mathrm{D}}\right)$ of different affinity characterization methods. Post-hoc analysis was not performed due to the large variance in sample sizes for each method.

The most commonly used affinity method was the filter binding assay; approximately $28 \%$ of SELEX experiments used this method to characterize aptamertarget binding. This is not surprising given that this is a commonly used method to evaluate aptamer-protein interactions and over half of the SELEX experiments described were for protein targets. The second most commonly used method, SPR ( 19\%) is a mass dependent technique which limits its applicability to larger targets. However, some work has been done to determine aptamer dissociation constants for small molecule targets by SPR. ${ }^{316-318}$ Affinity electrophoresis, affinity chromatography and fluorescence methods were used in $\sim 13 \%, \sim 10 \%$ and $\sim 8 \%$ of SELEX experiments respectively. The rest of the methods were each used to characterize aptamer affinity in less than 5\% of SELEX experiments.

The success of a SELEX experiment is often measured by the affinity of the selected aptamers. By measuring the dissociation constant of the interaction between an aptamer and its target, researchers can make predictions about how an aptamer will behave in a particular application. There are however, some practical limitations to this approach. Sequencing methods have improved greatly, thus increasing access to the number of potential aptamer candidates compared to traditional sequencing methods. However, affinity characterization methods remain, for the most part low-throughput, 
time consuming and laborious. ${ }^{276}$ For these reasons, typically fewer than ten aptamer candidates are screened for binding before further characterization is performed.

A broad comparison of the dissociation constant produced for the same aptamer by several different affinity methods asserted the necessity of using multiple characterization methods to yield a true dissociation constant estimate. ${ }^{276}$ Additionally, careful consideration of the final application should be given when choosing the affinity characterization method as an aptamer may be successfully evaluated with one method and show little-to-no binding to its target in a different assay. In collaboration with several researchers from the DeRosa (Carleton University: Ottawa, Canada) and De Girolamo (National Research Council of Italy; Bari, Italy) laboratories, a comprehensive workflow (Figure 2.35) for the screening, optimization, characterization and functional validation was developed. ${ }^{276}$

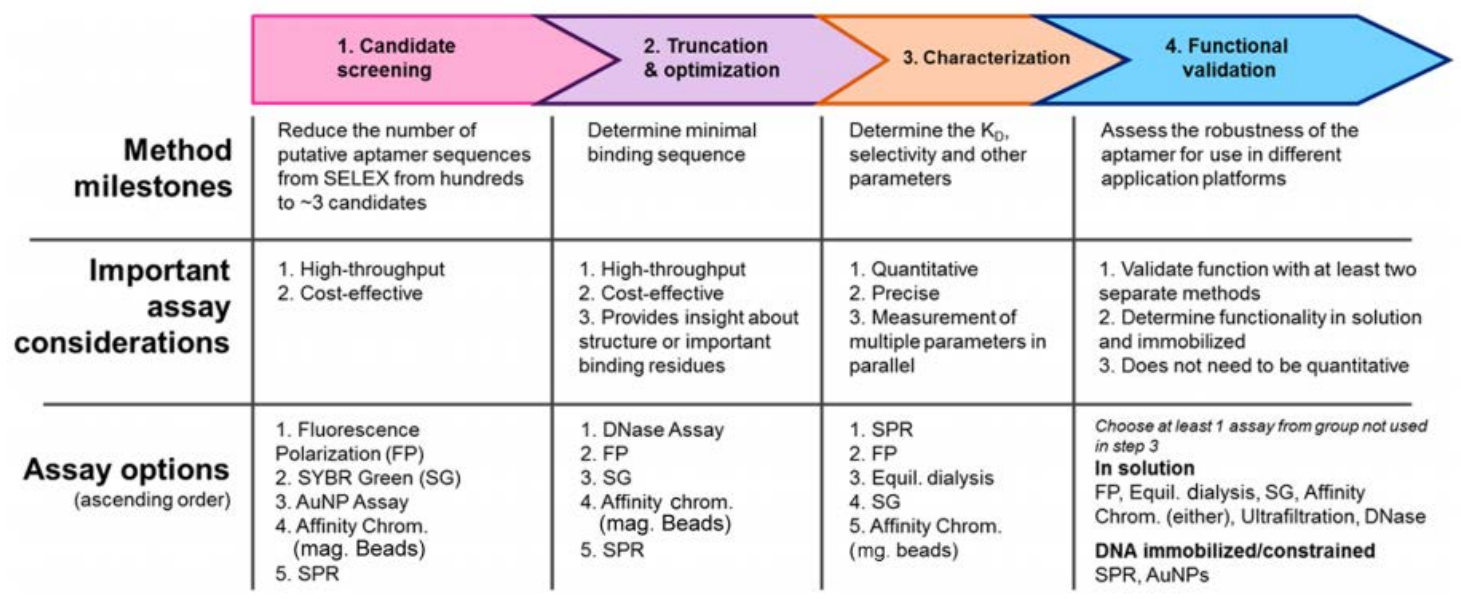

Figure 2.35: Aptamer characterization workflow. Figure reproduced with permission from the American Chemical Society. McKeague, M.; De Girolamo, A.; Valenzano, S.; Pascale, M.; Ruscito, A.; Velu, R.; Frost, N. R.; Hill, K.; Smith, M.; McConnell, E. M.; DeRosa, M. C. Comprehensive analytical comparison of strategies used for small molecules aptamer evaluation. Anal. Chem. 2015, 87 (17), 8608. Copyright 2015 American Chemical Society. 
The workflow summarizes important assay considerations and assay options as they relate to specific aptamer discovery milestones. An important conclusion from this work is that no single method to evaluate aptamer affinity exists that is applicable to all aptamer candidates, which reinforces the importance of multi-method characterization. 


\subsection{Conclusions and future work}

Throughout this chapter, work done to extract data from the Aptamer Base and investigate the effect of the selection parameters, the target, the selection conditions, the selection method, the affinity method and the buffering agent of the affinity experiment on the resulting affinity of selected aptamers was examined. Selection parameters investigated included; nucleic acid type, template length and aptamer base composition. The selection conditions examined include; ionic strength, buffering agent, temperature and $\mathrm{pH}$. From the analysis presented here, target type and selection temperature had the clearest effect on the affinity of the selected aptamers. Interestingly, there was insufficient evidence to suggest that the nucleic acid type, template length, aptamer base composition, or selection and affinity methodologies had a significant effect on the affinity of selected aptamers. Commonly, there were inadequate data to draw meaningful conclusions or make predictions about specific parameters, conditions or methodologies. Additionally, preliminary evidence suggests that the data that were available were confounded by target type. As more selection and affinity experiment data becomes available, further analysis should investigate the described parameters, conditions and methodologies at the level of target type to eliminate the confounding variable of target type dependent counter trends skewing the results. This work was the first example of a comprehensive, statistical analysis of aptamer selection data. The Aptamer Base will be maintained and updated regularly to facilitate future data analysis. 
3 pHAST (pH-Driven Aptamer Switch for Thrombin) Catchand-Release of Target Protein 


\subsection{Statement of Contributions}

Experiments were conceptualized and designed by MC DeRosa and EM McConnell. Initial melting temperature control experiments were performed by MC DeRosa and R Bolzon. pHAST characterization studies were performed by EM McConnell, P Mezin and G. Frahm. pHAST binding experiments were done by EM McConnell. Data analysis was done by EM McConnell, MC DeRosa, G Frahm, and M Johnston.

\subsection{Resulting publications}

This work is published in Bioconjugate Chemistry, ACS Publications (McConnell, EM., Bolzon, R., Mezin, P., Frahm, G., Johnston, M, and DeRosa MC.

Bioconjugate Chemistry. 201627 (6), pp 1493-1499. ${ }^{319}$ The manuscript was prepared by EM McConnell, G Frahm, M Johnston, and MC DeRosa. 


\subsection{Introduction}

\subsubsection{Objective}

The objective of this work was to determine if the $\mathrm{A}^{+}(a n t i) \cdot \mathrm{G}($ syn $)$ base pair could be used to efficiently design an aptamer-based nanoswitch (pHAST) that was conformationally sensitive to changes in $\mathrm{pH}$. Additionally, the ability of the aptamerbased nanoswitch to specifically catch-and-release a target protein in response to changes in $\mathrm{pH}$ was examined.

\subsection{2 pH and the Central Nervous System}

\subsubsection{Regulation of $\mathrm{pH}$ in the CNS}

Many biomolecules have $\mathrm{pH}$-sensitive physicochemical properties that influence their intermolecular interactions, structural transitions and processes (cell migration, apoptosis and proliferation) and therefore $\mathrm{pH}$ homeostasis is highly regulated at the subcellular level. ${ }^{320,321}$ Like in other tissues, the regulation of $\mathrm{pH}$ in tissues of the central nervous system (CNS) is a vital homeostatic function, yet $\mathrm{pH}$ related physiology and neuropathology are poorly understood. ${ }^{322}$ Additionally, the degree to which $\mathrm{pH}$ fluctuates during normal brain function is not clear. ${ }^{322}$ Several active and passive mechanisms, such as ion pump transport and ion channel transport respectively exist to regulate the $\mathrm{pH}$ of cells since intracellular acid is a natural by-product of respiration. ${ }^{323}$ Diversity of acid transport mechanisms between subtypes of neuronal and glial cells of the CNS has been demonstrated, which adds complexity to understanding the regulation of brain $\mathrm{pH} .{ }^{324}$ Another challenge when studying neuronal and glial $\mathrm{pH}$ regulation is the rapid intra- and 
extracellular increases or decreases in $\mathrm{H}^{+}$concentration that occur as a result of electrical activity. ${ }^{324}$

Complementary to the mechanisms that exist to modulate $\mathrm{pH}$ changes in the body, several buffering mechanisms exist. By the reaction of carbon dioxide $\left(\mathrm{CO}_{2}\right)$ with water $\left(\mathrm{H}_{2} \mathrm{O}\right)$ and the consequent formation of carbonic acid $\left(\mathrm{H}_{2} \mathrm{CO}_{3}\right)$, the $\mathrm{CO}_{2} / \mathrm{HCO}_{3}$ - system maintains $\mathrm{pH} .{ }^{322}$ Carbonic acid is dissociated into hydrogen carbonate $\left(\mathrm{HCO}_{3}-\right)$ and hydrogen $\left(\mathrm{H}^{+}\right)$ions. When $\mathrm{pH}$ is low, the equilibrium is shifted away from $\mathrm{H}^{+}$by increasing the $\mathrm{HCO}_{3}$ - concentration and $\mathrm{pH}$ is increased. In contrary, an increase in $\mathrm{CO}_{2}$ causes an equilibrium shifts towards $\mathrm{H}^{+}$production, and the $\mathrm{pH}$ decreases. ${ }^{322}$

Many physiological and behavioural processes such as synaptic plasticity, neurodegeneration, learning, memory and pain result due to the role of $\mathrm{pH}$-sensitive enzymes, receptors and channels, and the neurochemical mechanisms associated with the respective process. ${ }^{322}$ Abnormal fluctuations in $\mathrm{pH}$ have been implicated in multiple disease processes such as Parkinson's disease, Alzheimer's disease, brain cancer and ischemia. $^{325-333}$

\subsubsection{Ischemia associated fluctuations in $\mathrm{pH}$}

The resultant reduction in metabolites such as glucose and oxygen necessary for cellular metabolism due to the thrombosis of a major blood vessel leads to tissue damage and potentially cell death culminating in an ischemic stroke. ${ }^{323}$ In the event of an ischemic stroke, accumulation of $\mathrm{CO}_{2}$ leads to a decrease in intracellular $\mathrm{pH}$ due to poor perfusion of the cell. ${ }^{323}$ Coupled with the increase of $\mathrm{H}^{+}$ions that results from the reduction of available glucose and oxygen for cellular metabolism, the $\mathrm{pH}$ is pushed 
below the maintainable threshold of 6.3-6.4 and cellular damage occurs. ${ }^{323}$ Acidosisinduced cellular damage of neurons, glial cells and microvessels results mainly from free radical formation and changes in $\mathrm{Ca}^{2+}$ ion concentration at $\mathrm{pH}$ values above $6.5 .^{323}$

Currently, tests which evaluate perfusion and diffusion are used to determine the extent of ischemia, however limitations exist in the ability of these methods to understand the dynamic $\mathrm{pH}$ changes that occur during ischemia and predict at-risk tissue. ${ }^{323,334}$ The dynamic $\mathrm{pH}$ changes associated with ischemic stroke have been investigated in several animal models. ${ }^{329,335,336}$ Recently, a clinical trial using $\mathrm{pH}$-weighted magnetic resonance imaging showed that significant $\mathrm{pH}$ changes do occur following ischaemia. ${ }^{337}$ Since the majority of damage from ischaemic stroke occurs within a few hours of the event, there is a need for quick and accurate diagnosis and treatment methods. Given the limitation of current methods, there is a need for the development of novel diagnostic tools and interventions. DNA nanotechnology could be used to develop innovative interventions and improve existing therapies. ${ }^{338,339}$

\subsubsection{DNA nanotechnology}

Deoxyribonucleic acid (DNA) has several structural and functional properties that can be exploited for the design of DNA based nanotechnologies. ${ }^{339,340}$ Additionally, the repeating units making up DNA, adenine $(A)$, cytosine $(C)$, guanine $(\mathrm{G})$ and thymine $(\mathrm{T})$, allow the biopolymer to encode a substantial amount of information. The sequence composition of a single-stranded DNA molecule can be designed such that intra- and intermolecular interactions can be controlled. DNA-based nanomachines, nanodevices and nanoarchitectures have been designed based on the ability of DNA to hydrogen bond 
with its complement strand. Additionally, the ability of DNA to hydrogen bond to other non-nucleic acid molecules has served as the basis for the design of diverse nanotechnologies with applications ranging from sensing to drug delivery. ${ }^{340}$ Functionally, DNA can be exploited for its ability to selectively bind target molecules, provide specific domains to initiate enzymatic cleavage by nicking enzymes and endonucleases, as well as its catalytic properties in the form of DNAzymes. ${ }^{341}$

\subsubsection{DNA switches}

A subclass of rationally designed DNA nanostructures that have proved useful in the design of sensors and electronic devices are DNA switches. ${ }^{342}$ An important structural property of DNA switches is their ability to undergo reversible structural or physical changes in response to external stimuli or signaling molecules. ${ }^{341}$ These stimuli can include molecules such as complementary oligonucleotides, proteins, enzymes and small molecules or environmental stimuli such as temperature, light, ionic concentration and pH..$^{339,341-345}$ The programmable and predictable nature of DNA allows existing nanostructures, such as DNAzymes, G-quadruplexes, i-motifs and aptamers to be

incorporated into the design of DNA switches. ${ }^{5,346,347}$ The stability of these structures can be predictably tailored by controlling the physical properties of the DNA switch (number of base pairs and/or mispairs, sequence composition and use of modified nucleotides) as well as the environment (ionic strength and composition, presence of groove binders, intercalators or other molecular substrates)..$^{341}$ 


\subsection{5 pH exploitable properties of DNA}

$\mathrm{pH}$ is an important environmental stimulus that can have profound effects on molecular interactions, functional activity, and structural transition of biomolecules. This property is employed in natural systems to regulate processes such as the sorting and modification of proteins post-translation, cellular signaling and neurodegeneration. ${ }^{320}$ DNA is a biomolecule that has $\mathrm{pH}$-switchable properties. ${ }^{320}$

The formation of non-canonical DNA base pairs can result from the protonation of $\mathrm{A}$ and $\mathrm{C}$ at acidic $\mathrm{pH}$. Specifically, the following $\mathrm{pH}-$ dependent base pairs can form; $\mathrm{C} \cdot \mathrm{A}+, \mathrm{C} \cdot \mathrm{C}+$, and $\mathrm{A}^{+}($anti $) \cdot \mathrm{G}($ syn $) .{ }^{348}$ The existence of these base pairs can lead to the $\mathrm{pH}$-dependant formation of hairpins, triplexes and i-motif secondary structures which can be exploited for the design of $\mathrm{pH}$-regulated DNA switches. ${ }^{320,347,349,350}$

\subsection{6 pH-switchable nanodevices}

The most common pH-dependent base pair utilised in the design of DNA switches is the $\mathrm{C} \cdot \mathrm{C}+$ base pair that can result in the formation of an i-motif. ${ }^{349}$ Hemiprotonation of $\mathrm{C}$ in slightly acidic $\mathrm{pH}$ can lead to the formation of an i-motif structure in oligonucleotides with stretches of multiple Cs. ${ }^{351}$ I-motifs exist as either intra- or intermolecular four-stranded secondary structures stabilized by $\mathrm{C} \cdot \mathrm{C}+$ base pairing. ${ }^{351,352}$ In this secondary structure two duplexes are stabilized by intercalated $\mathrm{C} \cdot \mathrm{C}+$ base pairs exist. Xu et al., (2007) exploited $\mathrm{pH}$-sensitive base pairing to design a pH-driven nanomolecular device that was able to catch-and-release a target protein and that yielded only water as a waste product. ${ }^{353}$ The DNA duplex could bind to its target protein at neutral $\mathrm{pH}(>7.0)$. When the $\mathrm{pH}$ of the solution was adjusted to $<5.5$, the complementary 
oligonucleotides underwent structural transition to form intramolecular G-quad and intermolecular i-motif conformations, eliminating target binding. A limitation of designing $\mathrm{pH}$-driven DNA switches on the $\mathrm{i}$-motif or $\mathrm{C} \cdot \mathrm{C}+$ base pairs is that the formation of an i-motif requires an elongated stretch of $\mathrm{C}$ in the DNA sequence. Aptamers are selected from random libraries where the base composition should be approximately $25 \%$ for each base. Therefore developing a $\mathrm{pH}$-driven aptamer-based DNA nanomachine by the incorporation of $\mathrm{C} \cdot \mathrm{C}+$ base pairs may not be a universally efficient strategy.

\subsubsection{The incorporation of aptamer technology into functional nanomachines}

An excellent example of existing DNA-based nanoarchitectures being incorporated into DNA switch design is the use of aptamers. Derived by an iterative in vitro selection process termed Systemic Evolution of Ligands by EXponential enrichment (SELEX), aptamers are single-stranded functional nucleic acids that bind to their cognate target molecules (ranging from small molecules to cells) with a high degree of affinity and selectivity. ${ }^{8,34-36}$ Molecular switches capable of the catch-and-release of specific target molecules have been described which incorporated aptamers into functional DNAbased architectures. ${ }^{342,350,353,354}$ Of particular relevance, a nanomachine was designed based on an human $\alpha$-thrombin (henceforth referred to as thrombin) binding aptamer (TBA) that was able to cyclically catch-and-release thrombin. ${ }^{355}$ In the absence of the TBA's partial complement, the TBA existed in the G-quadruplex conformation necessary for the TBA to bind to thrombin. Duplex formation was initiated by the addition of the aptamer's partial complement; this structural change from G-quadruplex to duplex 
blocked the TBA's binding domain, preventing the TBA from binding to thrombin. When the partial complement was removed from the system, the TBA was able to return to the G-quadruplex conformation, the thrombin binding domain was exposed and thrombin binding activity was recovered.

\subsubsection{DNA switches based on the $\mathrm{A}^{+}($anti) $\bullet \mathrm{G}(\mathrm{syn})$ mispair}

Previously, Lee and DeRosa (2010) developed a DNA switch incorporating the $\mathrm{A}^{+}($anti) $\bullet \mathrm{G}($ syn) base pair (shown in Figure 3.1) that showed dramatic structural changes in response to changes in $\mathrm{pH}^{356}$ This was an important proof-of-concept study as it presented evidence that the $\mathrm{A}^{+}($anti $) \cdot \mathrm{G}($ syn $)$ base pair was a viable option in the design of DNA-based, pH-driven molecular switches. Additionally, many existing aptamers are Grich. ${ }^{8,274}$ Further, a common secondary structure for aptamers is the G-quadruplex, made up of G-quartets (shown in Figure)..$^{303}$
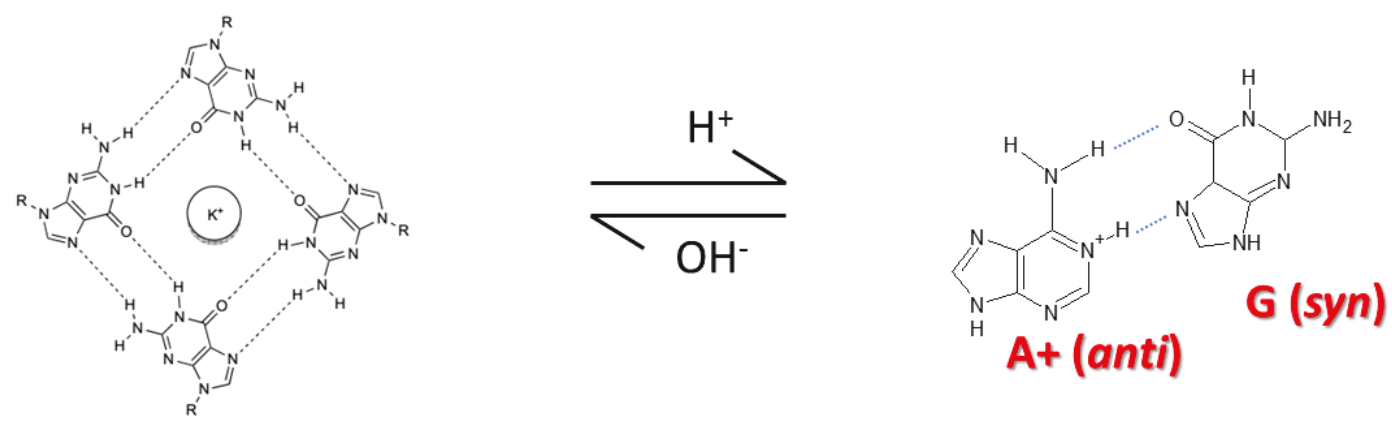

Figure 3.1: The $\mathrm{A}^{+}($anti) $\cdot \mathrm{G}(\mathrm{syn})$ mispair (right) that forms only in acidic conditions could be used to develop pH-driven nanoswitches from aptamer sequences that form G-quadruplex secondary structures from multiple G-quartets (left). Reprinted in part with permission from McConnell, EM., Bolzon, R., Mezin, P., Frahm, G., Johnston, $M$ and DeRosa, MC. pHAST (pH-Driven Aptamer Switch for Thrombin) Catch-and-Release of Target Protein. 2016, 27: 1493-1499. Bioconjugate Chemistry. Copyright 2016 American Chemical Society. 
Therefore, the rational design of aptamer-based, $\mathrm{pH}$-driven molecular switches which exploit the $\mathrm{A}^{+}($anti $) \cdot \mathrm{G}(s y n)$ base pair could be easily done for many existing aptamer sequences. Moreover, the $\mathrm{pH}$ tunable properties of these rationally designed functional nucleic acids would allow for additional control over aptamer-target binding. In the case of an aptamer with a known G-quadruplex secondary structure, the simple addition of an A-rich tail could result in a sequence that exhibits $\mathrm{pH}$-dependent allostery. Further, the allosteric property of the unimolecular switch could be controlled to catch-and-release a target molecule, an especially functional advantage in applications such as controlled delivery. This chapter describes the rational design and characterization of a unimolecular nanomachine, capable of $\mathrm{pH}$-driven catch-and-release of the target molecule, human $\alpha$ thrombin.

\subsection{Materials and methods}

\subsubsection{Reproduction from publication notice}

Sections 3.4.3-3.4.12 were reprinted (adapted) with permission from McConnell, EM., Bolzon, R., Mezin, P., Frahm, G., Johnston, M and DeRosa, MC. pHAST (pHDriven Aptamer Switch for Thrombin) Catch-and-Release of Target Protein. 2016. Bioconjugate Chemistry. DOI: 10.1021/acs.bioconjchem.6b00124. ${ }^{319}$ Copyright 2016 American Chemical Society.

\subsubsection{Chemicals and reagents}

DNA synthesis reagents were purchased from Glean Research (Sterling, VA, USA) and BioAutomation (Irving, TX, USA). Unmodified DNA was synthesized on a MerMade 6 DNA synthesizer (BioAutomation) according to standard phosphoramidite 
chemistry. Polyacrylamide gel electrophoresis (PAGE) was used to purify

oligonucleotides post-synthesis. Cyanine dye-modified oligonucleotides that had been purified post synthesis by high-performance liquid chromatography (HPLC) were purchased from Integrated DNA Technologies (Coralville, IA, USA). Urea, acrylamide/bis-acrylamide (19:1) 40\% solution, TEMED, Tris, boric acid, and EDTA were purchased from BioShop Canada (Burlington, ON, Canada). Glycerol, $\mathrm{HCl}, \mathrm{NaOH}$, $\mathrm{KCl}, \mathrm{NaCl}$, sodium phosphate dibasic $\left(\mathrm{Na}_{2} \mathrm{HPO}_{4}\right)$, monosodium phosphate $\left(\mathrm{NaH}_{2} \mathrm{PO}_{4}\right)$, sodium acetate anhydrous, acetic acid were purchased from Sigma-Aldrich (Oakville, ON, Canada). Human $\alpha$-thrombin was purchased from Haematologic Technologies Inc. (Essex Junction, VT, USA).

\subsubsection{DNA sequence information}

The DNA sequences used in these experiments are shown in Table 3.1. The pHAST was labeled with 5'-Cy5 and 3'-Cy3 for $\mathrm{pH}$ cycling experiments. The Cy3-TBA and Cy3-pHAST were 5'-Cy3 labeled for anisotropy and EMSA studies.

Table 3.1: DNA sequences of the oligonucleotides used in this study. Table reprinted with permission from McConnell, EM., Bolzon, R., Mezin, P., Frahm, G., Johnston, $M$ and DeRosa, MC. pHAST (pH-Driven Aptamer Switch for Thrombin) Catchand-Release of Target Protein. 2016, 27: 1493-1499. Bioconjugate Chemistry. Copyright 2016 American Chemical Society.

\begin{tabular}{|l|l|} 
Sequence & $\mathbf{5}, \mathbf{3}$, \\
\hline $\begin{array}{l}\text { Thrombin Binding } \\
\text { Aptamer (TBA) }\end{array}$ & GGTTGGTGTGGTTGG \\
\hline Cy3-TBA & Cy3-GGTTGGTGTGGTTGG \\
\hline TBA complement & CCAACCACACCAACC \\
\hline
\end{tabular}




\begin{tabular}{|l|l|}
\hline $\begin{array}{l}\text { (Comp) } \\
\text { Partial Complement } 1 \\
\text { (PC1) }\end{array}$ & AAAACCACACCAAAA \\
\hline $\begin{array}{l}\text { Partial Complement } 2 \\
\text { (PC2) }\end{array}$ & CCAAAAACAAAAACC \\
\hline $\begin{array}{l}\text { Partial Complement } 3 \\
\text { (PC3) }\end{array}$ & AAAAAAAAAAAAAAA \\
\hline $\begin{array}{l}\text { pH driven Aptamer } \\
\text { Switch for Thrombin } \\
\text { (pHAST) }\end{array}$ & $\begin{array}{l}\text { Cy5-GGTTGGTGTGGTTGGCTCTAAAAAAAAAAAAAAA- } \\
\text { Cy3 }\end{array}$ \\
\hline \begin{tabular}{l} 
Cy3-pHAST \\
\hline
\end{tabular} & Cy3-GGTTGGTGTGGTTGGCTCTAAAAAAAAAAAAAAA \\
\hline
\end{tabular}

\subsubsection{Structural Analysis by Melting Temperature determination}

Melting temperature data were obtained in either $0.1 \mathrm{M}$ sodium phosphate buffer $(\mathrm{pH} 7.5)$ or $0.1 \mathrm{M}$ sodium acetate buffer $(\mathrm{pH} 5.0)$ containing $140 \mathrm{mM} \mathrm{NaCl}$ and $5.0 \mathrm{mM}$ $\mathrm{KCl}$. Absorbance was measured at $295 \mathrm{~nm}$ (G-quadruplex) and $260 \mathrm{~nm}$ (duplex) over the range of $20^{\circ} \mathrm{C}$ to $80^{\circ} \mathrm{C}$ with a ramp rate of $2^{\circ} \mathrm{C} / \mathrm{min}$. Four ramps (two forward and two reverse) were measured for each sample. Melting temperatures and standard deviation reported were calculated from the average of values obtained from duplicate experiments.

UV-Vis melting studies were performed with the non-fluorophore labelled pHAST (NF-pHAST) in either 0.1 M sodium phosphate buffer (140 mM NaCl, pH 7.5) or $0.1 \mathrm{M}$ sodium acetate buffer $(140 \mathrm{mM} \mathrm{NaCl}, \mathrm{pH} 5.0)$ containing $0.0 \mathrm{mM}, 0.5 \mathrm{mM}$ or 5.0 mM KCl. Absorbance was measured at $295 \mathrm{~nm}$ (G-quadruplex and hairpin) and 260 $\mathrm{nm}$ (hairpin) over a temperature range of $20^{\circ} \mathrm{C}$ to $80^{\circ} \mathrm{C}$; ramp rate was $2^{\circ} \mathrm{C} / \mathrm{min}$. Two forward ramps and two reverse ramps were measured for each sample. $T_{m}$ values and 
standard deviations reported were calculated from values obtained from duplicate experiments.

\subsubsection{Circular dichroism (near U/V) analyses of DNA samples}

DNA samples (either TBA or pHAST) were diluted to $2.5 \mu \mathrm{M}$ in either Buffer 1 (0.1M sodium phosphate, $140 \mathrm{mM} \mathrm{NaCl}, 0.5 \mathrm{mM} \mathrm{KCl}, \mathrm{pH} 7.5)$ or Buffer $2(0.1 \mathrm{M}$ sodium acetate, $140 \mathrm{mM} \mathrm{NaCl}, 0.5 \mathrm{mM} \mathrm{KCl}, \mathrm{pH} 5.0)$ and analyzed with a Jasco 815 spectropolarimeter (Jasco International Co., Ltd., Tokyo, Japan) equipped with a Peltier thermal control unit set to room temperature $\left(20^{\circ} \mathrm{C}\right)$. Samples were measured in $10 \mathrm{~mm}$ quartz cuvettes (Hellma, Mullheim, Germany). The instrument and thermal control unit were controlled with Spectra Manager Software, Version 2 (Jasco International Co.). Each near U/V spectrum for structure analysis represents the average of 5 scans of one sample from 340-220nm with a data pitch of $1 \mathrm{~nm}$ and a response time of $1 \mathrm{~s}$. Spectra were baseline corrected and corrected for buffer signal.

\subsubsection{Thermal stability studies by CD}

Thermal stability studies were carried out by monitoring CD (millidegrees) at $294 \mathrm{~nm}$ between $20^{\circ} \mathrm{C}$ and $80^{\circ} \mathrm{C}$ in increments of $2^{\circ} \mathrm{C}$ per minute. Samples were diluted $(2.5 \mu \mathrm{M})$ in either Buffer 1 or Buffer 2, prepared in $10 \mathrm{~mm}$ quartz cuvettes, and were corrected for buffer signal. Each $T_{m}$ curve is the result of one representative $T_{m}$ scan. Fractional (normalized) change was calculated according to previously published studies, using the following formula:

$$
\mathrm{F}_{(\mathrm{obs})}=\left[\mathrm{E}_{\mathrm{obs}}(\mathrm{T})-\mathrm{E}_{\max }\right] /\left[\mathrm{E}_{20}-\mathrm{E}_{\max }\right]^{*} 100
$$


Where $\mathrm{F}(\mathrm{obs})$ is the relative ellipticity, $\mathrm{E}_{\mathrm{obs}}(\mathrm{T})$ is the ellipticity at $222 \mathrm{~nm}$ at temperature $\mathrm{T}, \mathrm{E}_{\max }$ is the ellipticity at the maximum temperature $\left({ }^{\circ} \mathrm{C}\right)$ used, and $\mathrm{E}_{20}$ is the ellipticity at the initial temperature of $20^{\circ} \mathrm{C} .{ }^{357}$

\subsubsection{Effect of Buffer on pH switching}

pHAST $(0.4 \mu \mathrm{M})$ dissolved in $1 \mathrm{mM}$ sodium phosphate buffer containing 140 $\mathrm{mM} \mathrm{NaCl}$ and either $0.0 \mathrm{mM}, 0.5 \mathrm{mM}$ or $5.0 \mathrm{mM} \mathrm{K}^{+}$was cycled through $\mathrm{pH} 7.0$ and $\mathrm{pH}$ 5.0 ten times. Fluorescence spectra (ex: $460 \mathrm{~nm}$; em: 500-700) at each $\mathrm{pH}$ value were recorded using a Fluorolog-3 (Horiba Jobin-Yvon). Solution $\mathrm{pH}$ was adjusted by the addition of either $2.0 \mathrm{M} \mathrm{HCl}$ or $2.0 \mathrm{M} \mathrm{NaOH}$ and was monitored using $\mathrm{BDH} \mathrm{pH}$ 4.5-10 test strips. Each buffer condition was run in triplicate and involved 20 total measurements at alternating $\mathrm{pH}$ values producing 10 switches. To determine switching efficiency, the ratio of fluorescence intensity of $\mathrm{Cy} 3 / \mathrm{Cy} 5(560 \mathrm{~nm} / 667 \mathrm{~nm})$ was plotted against sequential $\mathrm{pH}$ values.

\subsubsection{Ratiometric analysis of pHAST conformational change due to cyclic changes in solution $\mathbf{p H}$}

The $\mathrm{pH}$ of a $0.4 \mu \mathrm{M}$ pHAST (5'-Cy5-

GGTTGGTGTGGTTGGCTCTAAAAAAAAAAAAAAA-Cy3-3') solution was monitored over multiple cycles of $\mathrm{pH} 7$ and 5 using a Fluorolog-3 fluorescence spectrometer (Horiba Jobin-Yvon, USA). Ratiometric data were collected by measuring the intensity of $\mathrm{Cy} 3$ to $\mathrm{Cy} 5$ emission ( $570 \mathrm{~nm}$ and $667 \mathrm{~nm}$ respectively) upon excitation of $\mathrm{Cy} 3(460 \mathrm{~nm})$. An excitation of $460 \mathrm{~nm}$ for Cy3 was chosen to minimize excitation of Cy5 due to spectral overlap. Solution $\mathrm{pH}$ was monitored using $\mathrm{BDH} \mathrm{pH}$ 4.5-10 test strips 
(VWR, Canada). For the extended pH cycling experiment, cycles 1-10 and 11-35 were performed on separate days. Experiments with thrombin (either $2.0 \mu \mathrm{M}$ or $5.0 \mu \mathrm{M}$ ) were performed as described. Incubation periods (time from $\mathrm{pH}$ change to fluorescence measurement) of $0 \mathrm{~min}, 10$, min and 60 min were investigated in the presence of $2.0 \mu \mathrm{M}$ thrombin.

\subsubsection{Conformational sensitivity to incremental change in solution $\mathrm{pH}$}

The $\mathrm{pH}$ of a solution of Cy3-pHAST $(1.0 \mu \mathrm{M})$ and thrombin $(2.5 \mu \mathrm{M})$ was decreased by $0.5 \mathrm{pH}$ unit increments over the range of $\mathrm{pH} 8.0$ to $\mathrm{pH} 4.0$. Solution $\mathrm{pH}$ was adjusted with $2.0 \mathrm{M} \mathrm{HCl}$ and monitored with $\mathrm{BDH} \mathrm{pH}$ 4.5-10 test strips. The solution was allowed to equilibrate $(15 \mathrm{~min})$ at each $\mathrm{pH}$ point. Aliquots $(25 \mu \mathrm{L})$ were removed at each $\mathrm{pH}$ for EMSA. Gels were imaged using AlphaImager AIC software.

\subsubsection{Cy3-TBA and Cy3-pHAST binding to thrombin measured by fluorescence anisotropy}

The fluorescence anisotropy of 5'-Cy3 labelled TBA (Cy3-TBA) was measured at various concentrations of human $\alpha$-thrombin $(0.5 \mathrm{nM}$ to $5.0 \mu \mathrm{M})$ in binding buffer (20 $\mathrm{mM}$ Tris-acetate; $140 \mathrm{mM} \mathrm{NaCl}, 5.0 \mathrm{mM} \mathrm{KCl}, 1.0 \mathrm{mM} \mathrm{CaCl}_{2}$ and $1.0 \mathrm{mM} \mathrm{MgCl}_{2}$ ) adjusted to either $\mathrm{pH}=7.0$ or $\mathrm{pH}=5.0$ with $2.0 \mathrm{M} \mathrm{HCl}$. The fluorescence anisotropy of the 5'-Cy3 labeled pHAST $(1.0 \mu \mathrm{M})$ at $\mathrm{pH}=7$ and $\mathrm{pH}=5$ was also measured. Samples at $\mathrm{pH}=7.0$ were measured in $1.0 \mathrm{mM}$ sodium phosphate buffer $(140 \mathrm{mM} \mathrm{NaCl}, 0.5 \mathrm{mM}$ $\mathrm{KCl})$ and samples at $\mathrm{pH}=5.0$ were measured in $0.1 \mathrm{mM}$ sodium acetate buffer $(140 \mathrm{mM}$ $\mathrm{NaCl}, 0.5 \mathrm{mM} \mathrm{KCl})$. DNA was heated to $55^{\circ} \mathrm{C}$ and then cooled to room temp before 
mixing. DNA and human $\alpha$-thrombin samples were made up to $2 \mathrm{X}$ concentration, mixed equivolume $(30 \mu \mathrm{L})$ and allowed to incubate at room temperature for $15 \mathrm{~min}$ before anisotropy measurements were taken. Three trials at each $\mathrm{pH}$ condition were run. The two closest data points at each concentration were plotted to eliminate outliers. Represented error was calculated based on the standard deviation between two data points at each concentration. Curves were produced using the four-parameter logistic equation (non-linear regression) in SigmaPlot 13.0. Apparent $K_{D}$ values were fit using the Standard Curve, four-parameter logistic curve in SigmaPlot Version 10.0. The apparent $K_{D}$ values $\left(K_{D} \pm\right.$ Standard Error) shown in Error! Reference source not found. were derived from the fit of the average of the two closest data points at each concentration for each sample.

\subsubsection{Catch-and-release of target protein by non-denaturing EMSA}

The $\mathrm{pH}$ of a solution of Cy3-pHAST $(1.0 \mu \mathrm{M})$ and thrombin $(2.5 \mu \mathrm{M})$ was cyclically changed from $\mathrm{pH} 7$ to 5 over five cycles. Solution $\mathrm{pH}$ was adjusted using either 2.0 $\mathrm{M} \mathrm{NaOH}$ or $\mathrm{HCl}$ and was monitored using $\mathrm{BDH} \mathrm{pH}$ 4.5-10 test strips. At each $\mathrm{pH}$, the sample was allowed to equilibrate at room temperature for $15 \mathrm{~min}$. Aliquots (25 $\mu \mathrm{L}$ ) were removed at each $\mathrm{pH}$ point for non-denaturing EMSA analysis. Gel images were obtained by AlphaImager AIC software. 


\subsubsection{Fluorescence anisotropy of Cy3-pHAST binding to thrombin over multiple pH cycles}

The samples were prepared for anisotropy by first heating $\left(65^{\circ} \mathrm{C}\right)$ and cooling (room temp) the Cy3-pHAST. The Cy3-pHAST and thrombin were made up to $2 \mathrm{X}$ concentration and mixed equal volume $(300 \mu \mathrm{L})$ to yield final concentrations of $1.0 \mu \mathrm{M}$ and $0.5 \mu \mathrm{M}$ respectively. The anisotropy (ex: $460 \mathrm{~nm}$, em: $564 \mathrm{~nm}$ ) of the solution was measured at $\mathrm{pH}=7$. The $\mathrm{pH}$ of the solution was changed to $\mathrm{pH}=5$ by the addition of $2.0 \mathrm{M}$ $\mathrm{HCl}$ and the anisotropy was measured again. The solution was returned to $\mathrm{pH}=7.0$ with 2.0 mM NaOH. $\mathrm{pH}$ was monitored using $\mathrm{BDH} \mathrm{pH}$ 4.5-10 test strips. The anisotropy at each $\mathrm{pH}$ was measured in triplicate over $5 \mathrm{pH}$ cycles. Three trials were performed with and without thrombin. 


\subsection{Results and discussion}

\subsubsection{Design of the pH-driven, aptamer-based unimolecular nanomachine (pHAST)}

The interaction of thrombin with the TBAs selected by either Bock et al. or Tasset et al. are the most commonly investigated in aptamer-based proof-of-concept studies. ${ }^{129,358}$ The aptamer chosen to include as the aptamer domain of the pHAST was the TBA first described by Bock et al. ${ }^{358}$ In addition to its common use in proof-ofconcept studies, the TBA was chosen as its tendency to form a G-quadruplex has been well characterized. ${ }^{359-364}$ The design of pHAST and the pH-dependent conformations it exists in are illustrated in Figure 3.2.
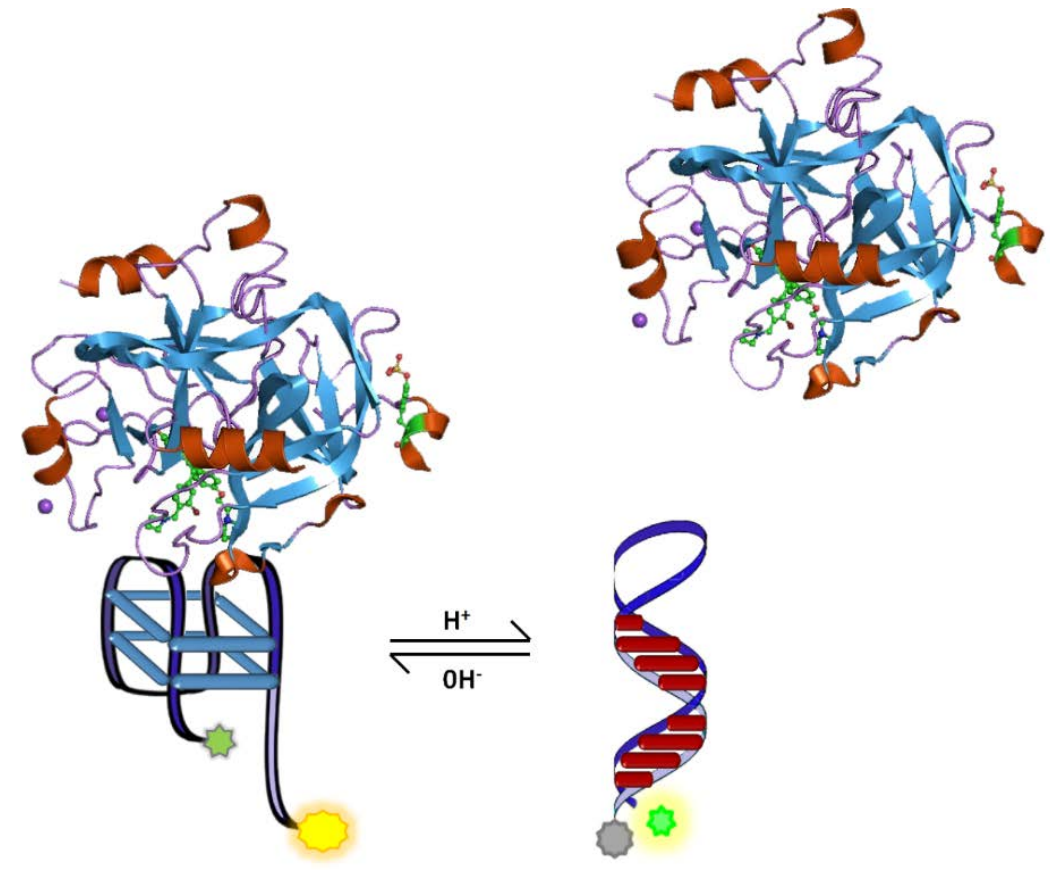

Figure 3.2: Schematic representation of pHAST. The catch-and-release of the target, thrombin, is regulated by the $\mathrm{pH}$-driven confirmation of pHAST. The Cy3/Cy5-pHAST was designed so that at neutral pH it exists in the G-quad 
conformation and the thrombin binding domain is capable of binding to thrombin. Upon acidification, the $\mathrm{Cy} 3 / \mathrm{Cy5}-\mathrm{pHAST}$ experiences a conformational change such that the G-quadruplex is disrupted and the hairpin forms. This conformational change blocks the thrombin binding dopamine and as a result the affinity of the pHAST towards thrombin is eliminated. Dashed blue bars indicate G-quadruplex formation and red bars indicate $\mathrm{A}^{+}($anti) $\cdot \mathrm{G}(\mathrm{syn})$ base pairs. Reprinted with permission from McConnell, EM., Bolzon, R., Mezin, P., Frahm, G., Johnston, M and DeRosa, MC. pHAST (pH-Driven Aptamer Switch for Thrombin) Catch-andRelease of Target Protein. 2016, 27: 1493-1499. Bioconjugate Chemistry. Copyright 2016 American Chemical Society.

The $\mathrm{pH}$-driven $\mathrm{pHAST}$ nanomachine was designed by extending the 3 '-end of the TBA with an adenine-containing partial complement (PC) of the TBA sequence. Addition of the partial complement allowed for the strategic placement of multiple adenines to facilitate $\mathrm{A}^{+}($anti) $\bullet \mathrm{G}(s y n)$ base pairing between the TBA and 3 '-partial complement extension under acidic pH conditions. Förster resonance energy transfer (FRET) was used to monitor the conformation changes of pHAST from a G-quadruplex to a hairpin which resulted from changes of the solution $\mathrm{pH}$. The pHAST was labelled at the 5'- and 3'ends with the FRET pair cyanine dyes $\mathrm{Cy} 5$ and $\mathrm{Cy} 3$ respectively (further represented as Cy3/Cy5-pHAST). As shown in Figure 3.2, due to the presence of the TBA the $\mathrm{Cy} 3 / \mathrm{Cy} 5-\mathrm{pHAST}$ exists mainly in the G-quadruplex conformation at neutral $\mathrm{pH}$. In this conformation the $\mathrm{Cy} 3 / \mathrm{Cy} 5$-pHAST was able to bind to the target protein, human $\alpha$ thrombin. A conformational change from the G-quadruplex to the $\mathrm{A}^{+}(a n t i) \cdot \mathrm{G}(s y n)$ base pair stabilized hairpin occurred upon acidification of the solution. pHAST was designed such that the exploitation of $\mathrm{pH}$-sensitive base pairing could afford tunability of reversible target binding. In theory, the $\mathrm{pH}$-dependent conformational changes described would allow for the binding of the pHAST to the target protein thrombin to be reversibly turned on and off by changes in $\mathrm{pH}$. An important design advantage of the pHAST over 
existing aptamer-based $\mathrm{pH}$-sensitive nanomachines was that it was designed as a unimolecular DNA oligonucleotide. Therefore, cyclic regulation of pHAST would yield only water as waste. This was advantageous compared to bimolecular nanomachines where structure switching is dependent on the presence or absence of complementary sequences. Compared to intermolecularly driven structural transitions, intramolecularly driven structural transitions are more stable and less affected by concentration effects and interfering nucleic acids in cellular environments. ${ }^{320}$

Experimentally, the optimal design of the pHAST was determined by examining the duplex stability of three TBA partial complements (PC) and the TBA by variable temperature UV-Vis spectroscopy. ${ }^{365}$ The PCs (shown in Error! Reference source not found.) were rationally designed to contain terminal (PC1), central (PC2) or both terminal and central (PC3) $\mathrm{A}^{+}($anti $) \cdot \mathrm{G}($ syn $)$ base pairs between the respective PC and the TBA at acidic $\mathrm{pH}$ (Error! Reference source not found.). The location of canonical Watson-Crick base pairing and non-canonical $\mathrm{A}^{+}(a n t i) \cdot \mathrm{G}($ syn $)$ base pairs that would exist in acidic $\mathrm{pH}$ are indicated by solid and dashed lines respectively. 

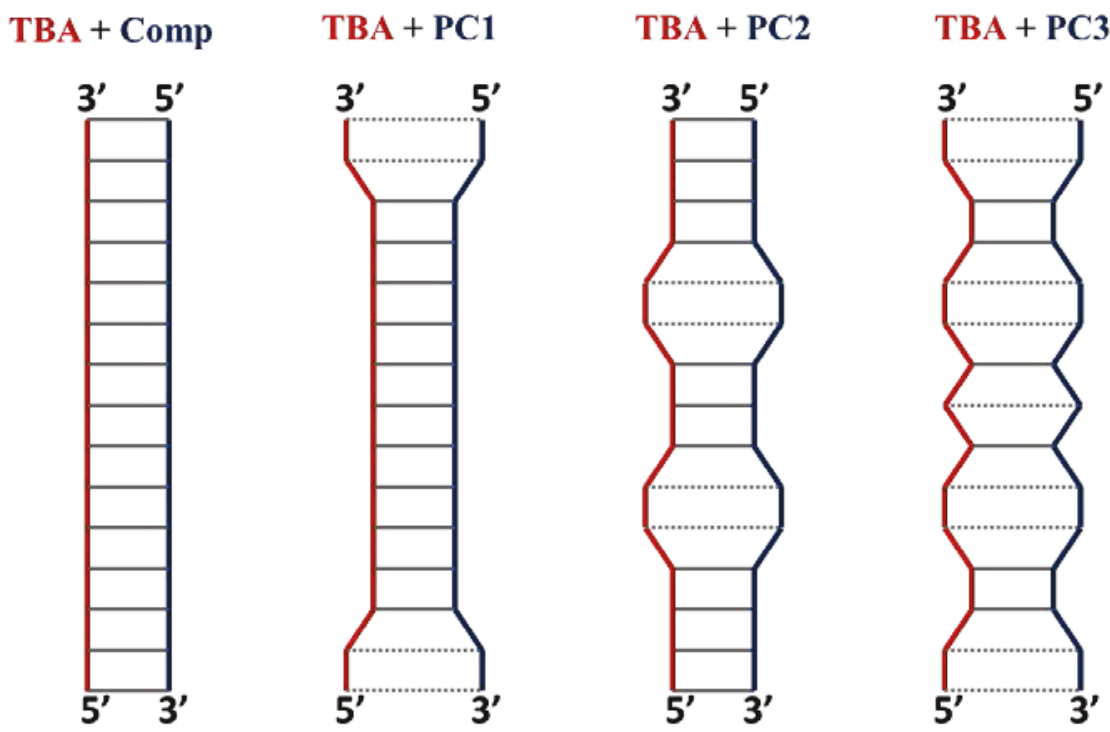

pHAST

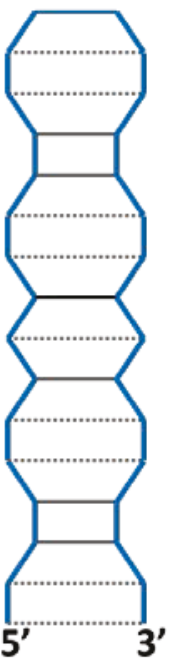

Watson-Crick base pairing
$\cdots \cdots \mathrm{pH}$ induced $\mathrm{A}+\cdot \mathrm{G}$ base pair

Figure 3.3: The location of inserted $\mathrm{A}^{+}($anti) $\cdot \mathrm{G}(\mathrm{syn})$ base pairs in each partially complementary sequence (PC) with respect to the TBA and its fully complementary sequence (Comp) are shown. Abbreviations: TBA (thrombin binding aptamer), Comp (TBA complement). Sequences were designed with terminal (PC1), internal (PC2) or both terminal and internal (PC3) $\mathrm{A}^{+}($anti) $\cdot \mathrm{G}($ syn $)$ base pairs. A 5'-CTCT3' linker was used to create the unimolecular pHAST from TBA+PC3. Reprinted with permission from McConnell, EM., Bolzon, R., Mezin, P., Frahm, G., Johnston, $M$ and DeRosa, MC. pHAST (pH-Driven Aptamer Switch for Thrombin) Catchand-Release of Target Protein. 2016, 27: 1493-1499. Bioconjugate Chemistry. Copyright 2016 American Chemical Society.

Consideration for the design of the PCs comes from previous work by Lee and DeRosa (2010). ${ }^{356}$ This study showed that the presence of $\mathrm{A}^{+}($anti $) \cdot \mathrm{G}(s y n)$ base pairs decreased duplex stability compared to Watson-Crick base pair containing duplex analogues. However, the stability of these duplexes, as represented by the melting temperature $\left(T_{m}\right)$, at $\mathrm{pH} 5$ was consistently higher than at $\mathrm{pH}$ 7. From this knowledge, the most efficient 
pHAST design would then include enough $\mathrm{A}^{+}($anti $) \cdot \mathrm{G}(\mathrm{syn})$ base pairs to favour the hairpin conformation at acidic $\mathrm{pH}$, yet not disrupt G-quad conformation at neutral $\mathrm{pH}$.

Melting temperatures $\left(T_{m}\right)$ were determined for each duplex in neutral (7.5) and acidic (5.0) $\mathrm{pH}$ by measuring the absorbance of the DNA in solution at both $260 \mathrm{~nm}$ and $295 \mathrm{~nm}$. Representative melting curves of the TBA, TBA+Comp, TBA+PC1, TBA+PC2 and TBA $+\mathrm{PC} 3$ are shown in Figure 3.4 - Figure 3.8. Structural information was obtained from the melting curves of each duplex. Generally, hypochromicity observed at $295 \mathrm{~nm}$ with increasing temperature was indicative of G-quadruplex conformation.

Hyperchromicity observed at $260 \mathrm{~nm}$ with increasing temperature was characteristic of duplex conformation.
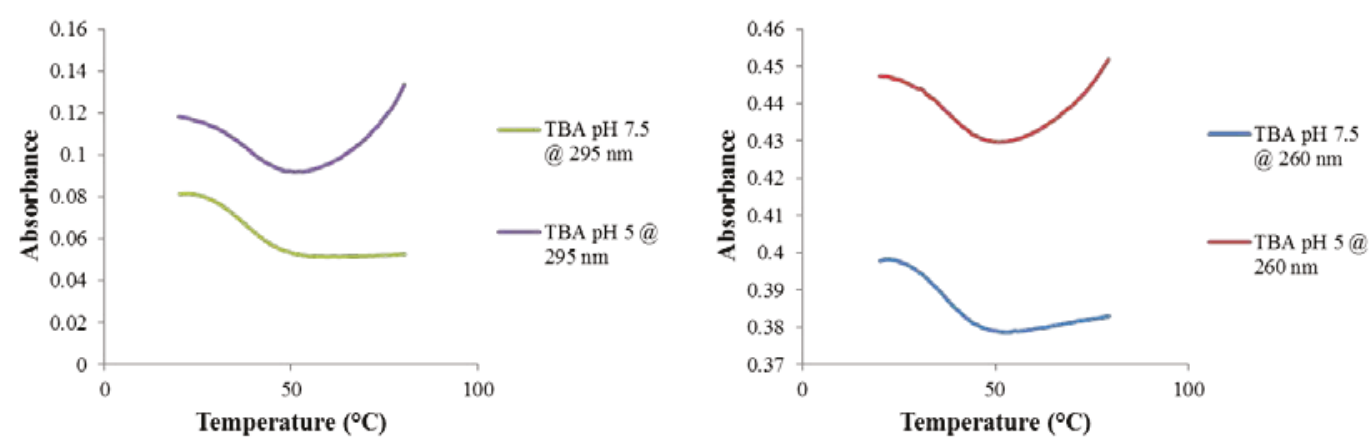

Figure 3.4: Tm profiles of TBA were obtained by UV-Vis thermal denaturation. The absorbance was measured for the TBA in both neutral (pH 7.5) and acidic (pH 5.0) conditions at either $260 \mathrm{~nm}$ or $295 \mathrm{~nm}$. 

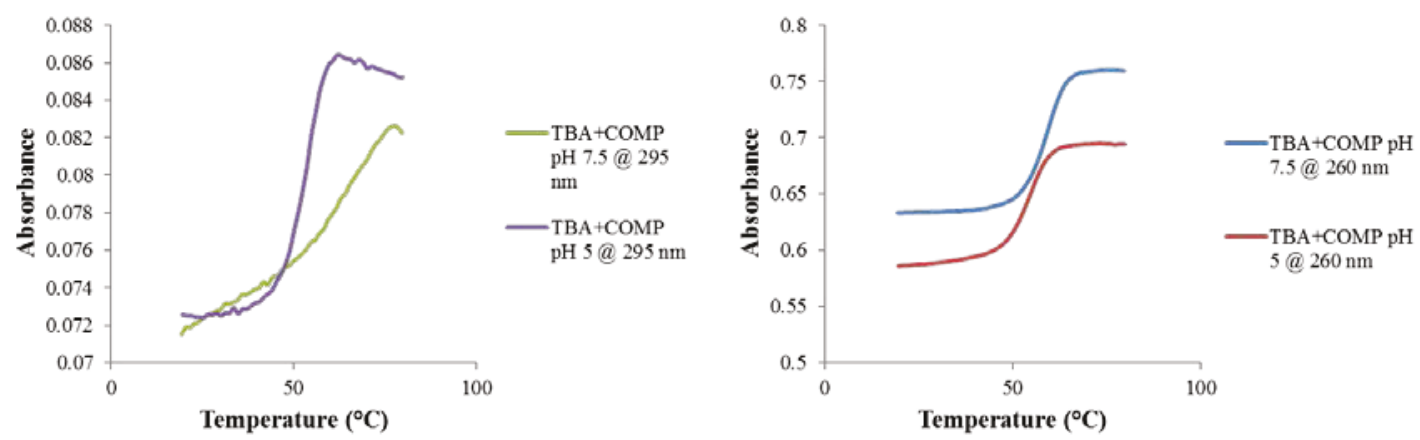

Figure 3.5: Tm profiles of TBA+COMP were obtained by UV-Vis thermal denaturation. The absorbance was measured for the TBA+COMP in both neutral (pH 7.5) and acidic (pH 5.0) conditions at either $260 \mathrm{~nm}$ or $295 \mathrm{~nm}$.
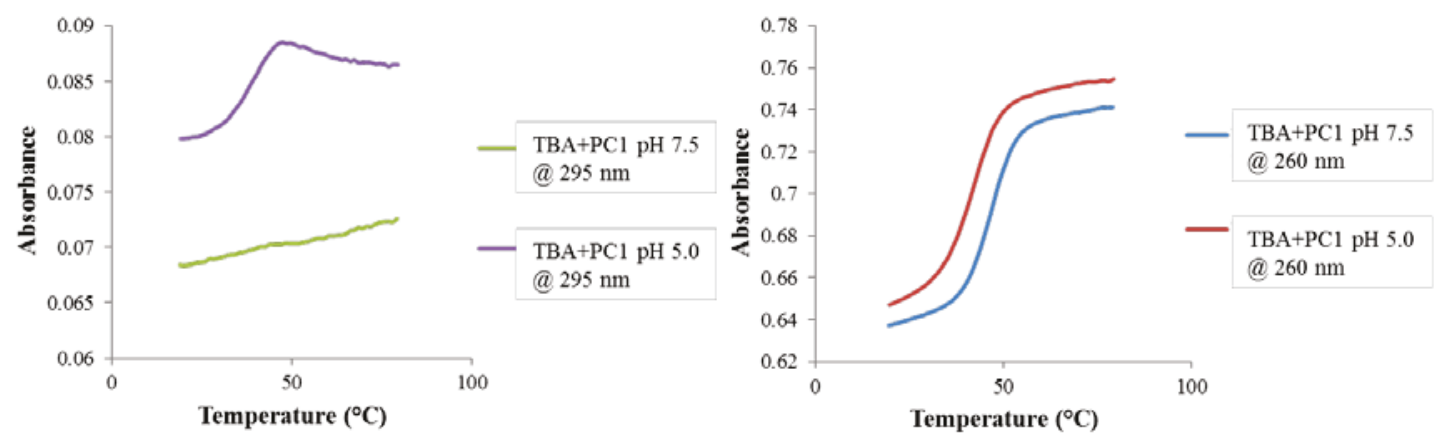

Figure 3.6: Tm profiles of TBA+PC1 were obtained by UV-Vis thermal denaturation. The absorbance was measured for the TBA+PC1 in both neutral (pH 7.5) and acidic (pH 5.0) conditions at either $260 \mathrm{~nm}$ or $295 \mathrm{~nm}$. 

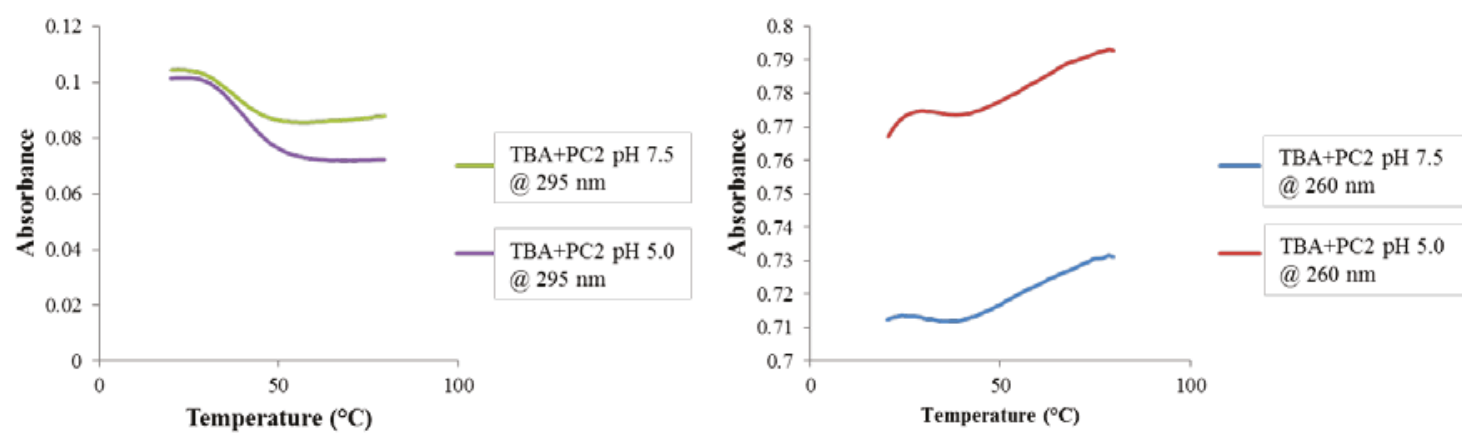

Figure 3.7: Tm profiles of TBA+PC2 were obtained by UV-Vis thermal denaturation. The absorbance was measured for the TBA+PC2 in both neutral (pH 7.5) and acidic (pH 5.0) conditions at either $260 \mathrm{~nm}$ or $295 \mathrm{~nm}$.
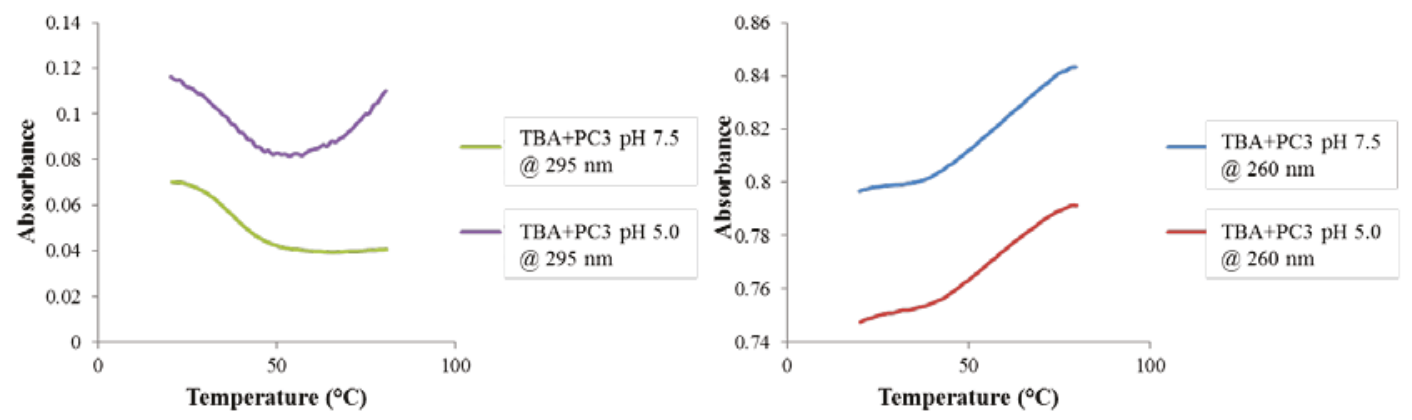

Figure 3.8: Tm profiles of TBA+PC3 were obtained by $\mathrm{UV}-\mathrm{V}$ is thermal denaturation. The absorbance was measured for the TBA+PC3 in both neutral (pH 7.5) and acidic (pH 5.0) conditions at either $260 \mathrm{~nm}$ or $295 \mathrm{~nm}$.

The most useful structural information was taken from the melting curves determined by the absorbance measured at $295 \mathrm{~nm}$ over a temperature gradient that varied from $20^{\circ} \mathrm{C}-80^{\circ} \mathrm{C}$. Over this temperature gradient, a trend of hypochromicity observed in the absorbance measured at 295 was indicative of G-quadruplex conformation. When hyperchromicity was observed, it was likely due to the spectral overlap of DNA absorption at $260 \mathrm{~nm}$ with the g-quartet chromophore. When a Gquadruplex was either not present, or did not constitute a large majority of the sample, a 
small amount of absorption by duplex DNA was observed at $295 \mathrm{~nm}$. This was likely due to the relatively small absorbance at the tail end of the $260 \mathrm{~nm}$ peak. Hyperchromicity was almost always observed at $260 \mathrm{~nm}$ suggesting that some amount of hairpin was present in each sample, with the exception of the TBA sample. Though $\mathrm{A}^{+}($anti $) \cdot \mathrm{G}($ syn $)$ base pairs are destabilizing compared to Watson-Crick base pairs, if enough WatsonCrick base pairs were in close enough proximity, the duplex could still have existed in acidic $\mathrm{pH}^{356}$ Additionally, at the $\mathrm{pH}$ conditions measured, given the $\mathrm{pKa}$ of the N1 (4.2), adenine would have been only partially protonated at acidic $\mathrm{pH}$ and so some DNA may have existed in the G-quad conformation. ${ }^{366}$ Considered together, these factors likely contributed to a conformational equilibrium in which both the G-quadruplex and duplex conformations existed simultaneously. By examining each melting curve at $295 \mathrm{~nm}, \mathrm{PC} 3$ was chosen as the optimal PC for the pHAST design. The TBA+PC1 had 27\% $\mathrm{A}^{+}($anti $) \cdot \mathrm{G}(\mathrm{syn})$ base pair content and was observed to form a stable duplex at both $\mathrm{pH} 5$ and $\mathrm{pH} 7.5$, likely because the terminally located $\mathrm{A} \bullet \mathrm{G}$ mispairs were not destabilizing enough at $\mathrm{pH} 7.5$ to disrupt the duplex. Conversely, centrally located $\mathrm{A} \cdot \mathrm{G}$ mispairs were destabilizing enough that $\mathrm{TBA}+\mathrm{PC} 2$, which also had $27 \% \mathrm{~A}^{+}($anti $) \cdot \mathrm{G}($ syn $)$ base pair content showed G-quadruplex character at $\mathrm{pH} 7.5$, but the $\mathrm{A}^{+}($anti $) \cdot \mathrm{G}($ syn) base pairs were not stabilizing enough at $\mathrm{pH} 5$ to stabilize the duplex. An increase to $60 \%$ $\mathrm{A}+($ anti $) \cdot \mathrm{G}(\mathrm{syn})$ base pair content with both terminal and central $\mathrm{A} \cdot \mathrm{G}$ mispairs was destabilizing enough at $\mathrm{pH} 7.5$ that the G-quadruplex conformation was observed and also stabilizing enough that at $\mathrm{pH} 5$ the $\mathrm{A}^{+}($anti $) \cdot \mathrm{G}($ syn $)$ base pair stabilized duplex was observed, making PC3 the ideal PC candidate for pHAST design. 
From the melting profiles, the $T_{m}$ of each duplex at both neutral and acidic $\mathrm{pH}$ were determined. The $T_{m}$ of each TBA+CD are summarized in Table 3.1.

Table 3.2: The melting temperature $\left(T_{m}\right)$ of sequences described in Table 3.1 were obtained by $\mathrm{UV}-\mathrm{V}$ is spectroscopy. Analysis of the $T_{\mathrm{m}}$ and absorbance profiles at 295 nm showed that TBA+PC1 showed mostly duplex conformation at both $\mathbf{p H}$ conditions, and TBA + PC2 revealed that mostly G-quad conformation existed at both $\mathrm{pH}$ conditions. The absorbance spectra of TBA+PC3 exhibited G-quad character at neutral $\mathrm{pH}$ and duplex character at acidic $\mathrm{pH}$.

\begin{tabular}{|c|c|c|c|c|}
\hline DNA & pH & Wavelength (nm) & $\begin{array}{l}\text { Dominant } \\
\text { Structure }\end{array}$ & $T_{m}\left({ }^{\circ} \mathrm{C}\right)$ \\
\hline \multirow[t]{2}{*}{ TBA } & 7.5 & 295 & G-quad & $35 \pm 4$ \\
\hline & 5.0 & 295 & G-quad & $36 \pm 3$ \\
\hline \multirow[t]{2}{*}{ TBA + Comp } & 7.5 & 260 & Duplex & $62 \pm 4$ \\
\hline & 5.0 & 260 & Duplex & $57 \pm 3$ \\
\hline \multirow[t]{2}{*}{$\mathrm{TBA}+\mathrm{PC} 1$} & 7.5 & 260 & Duplex & $50 \pm 6$ \\
\hline & 5.0 & 260 & Duplex & $47 \pm 6$ \\
\hline \multirow[t]{2}{*}{$\mathrm{TBA}+\mathrm{PC} 2$} & 7.5 & 295 & G-quad & $35 \pm 3$ \\
\hline & 5.0 & 295 & G-quad & $38 \pm 4$ \\
\hline \multirow[t]{2}{*}{$\mathrm{TBA}+\mathrm{PC} 3$} & 7.5 & 295 & G-quad & $35 \pm 3$ \\
\hline & 5.0 & 260 & Duplex & $70 \pm 7$ \\
\hline
\end{tabular}

Abbreviations: TBA (Thrombin Binding Aptamer), Comp (TBA full complement), PC (partial complement).

Differences in the melting temperature data support the trends observed describing the structural information inferred from the shapes of the melting curves. There was no observable difference between the $\mathrm{T}_{\mathrm{m}} \mathrm{s}$ of the TBA at either neutral or acidic $\mathrm{pH}$ which suggested the aptamer maintained its G-quad conformation at both $\mathrm{pHs}$ with comparable 
stability. Likewise, the TBA+COMP likely formed an equally stable duplex in both $\mathrm{pH}$ conditions. The $\mathrm{T}_{\mathrm{m}}$ of the duplex formed between the $\mathrm{TBA}+\mathrm{PC} 1$ at both neutral and acidic $\mathrm{pH}$ were not observably different. The TBA+PC2 formed a G-quadruplex at both neutral and acidic $\mathrm{pH}$ with comparable stability. Consistent with the differences observed in the melting curves, there was an appreciable difference between the $\mathrm{T}_{\mathrm{m}} \mathrm{s}$ of the $\mathrm{TBA}+\mathrm{PC} 3$ at $\mathrm{pH} 7.5$ and $\mathrm{pH} 5$. Additionally, the TBA+PC3 formed a G-quadruplex at neutral $\mathrm{pH}$ and showed a $\mathrm{T}_{\mathrm{m}}$ similar to those reported for the TBA. Interestingly, based on a measureable difference between the $\mathrm{T}_{\mathrm{m}} \mathrm{s}$, the duplex formed by the TBA+PC3 at acidic $\mathrm{pH}$ was more stable than the duplex formed by the TBA+COMP at acidic $\mathrm{pH}$. This could be due to general destabilization of Watson-Crick base pairing by protonation of adenine and cytosine at acid $\mathrm{pH}$.

After variable temperature UV-Vis revealed PC3 to be the most appropriate PC, the final unimolecular pHAST design incorporated a 5'-CTCT-3' linker between the TBA and PC3 (Error! Reference source not found.). The $\mathrm{T}_{\mathrm{m}}$ of the pHAST in various $\mathrm{K}^{+}$ concentrations was measured to confirm pHAST conformational stability and to determine the optimal $\mathrm{K}^{+}$for pHAST stability. The $\mathrm{T}_{\mathrm{m}}$ data are summarized in Table 3.3.

Table 3.3: Thermal denaturation was used to determine the melting temperature of the pHAST in various $\mathrm{KCl}$ concentrations. The $\mathrm{Tm}$ of the $\mathrm{pHAST}$ were consistent with values for TBA+PC3 described in Table 3.2.

\begin{tabular}{|l|l|l|l|l|}
\hline $\mathbf{K C l}(\mathbf{m M})$ & $\mathbf{p H}$ & Wavelength $(\mathbf{n m})$ & Structure & $\mathbf{T} \mathbf{m}\left({ }^{\circ} \mathbf{C}\right)$ \\
\hline 0.0 & 7.5 & 295 & G-quad & $30 \pm 4$ \\
& & & Duplex & $64 \pm 7$ \\
\cline { 2 - 5 } & 7.5 & 260 & Duplex & $68 \pm 11$ \\
\hline
\end{tabular}




\begin{tabular}{|c|c|c|c|c|}
\hline & 5.0 & 295 & G-quad & $26 \pm 7$ \\
\hline & & & Duplex & $67 \pm 7$ \\
\hline & 5.0 & 260 & Duplex & $66 \pm 13$ \\
\hline 0.5 & 7.5 & 295 & G-quad & $29 \pm 4$ \\
\hline & & & Duplex & $66 \pm 6$ \\
\hline & 7.5 & 260 & Duplex & $70 \pm 14$ \\
\hline & 5.0 & 295 & G-quad & $29 \pm 7$ \\
\hline & & & Duplex & $73 \pm 5$ \\
\hline & 5.0 & 260 & Duplex & $60 \pm 11$ \\
\hline 5.0 & 7.5 & 295 & G-quad & $32 \pm 4$ \\
\hline & & & Duplex & $70 \pm 3$ \\
\hline & 7.5 & 260 & Duplex & $69 \pm 9$ \\
\hline & 5.0 & 295 & G-quad & $33 \pm 4$ \\
\hline & & & Duplex & $73 \pm 3$ \\
\hline & 5.0 & 260 & Duplex & $67 \pm 12$ \\
\hline
\end{tabular}

At each $\mathrm{K}^{+}$concentration, there was no appreciable difference between the $\mathrm{T}_{\mathrm{m}} \mathrm{s}$ of the Gquadruplex measured at $295 \mathrm{~nm}$ in neutral and acidic $\mathrm{pH}$ conditions. Likewise at each $\mathrm{K}^{+}$ concentration, neither the $\mathrm{T}_{\mathrm{mS}}$ of the duplex measured at $295 \mathrm{~nm}$ in both acidic and neutral $\mathrm{pH}$, nor the Tms of the duplex measured at $260 \mathrm{~nm}$ in both acidic and neutral $\mathrm{pH}$ were observably different. Additionally, the Tms of the duplexes measured for each $\mathrm{K}+$ concentration at $295 \mathrm{~nm}$ and $260 \mathrm{~nm}$ were not different. Consistent with the TBA+PC3 data, the $T_{m}$ of the G-quadruplex measured at $295 \mathrm{~nm}$ was appreciably different than the $\mathrm{T}_{\mathrm{m}}$ of the duplex measured at $295 \mathrm{~nm}$ and $260 \mathrm{~nm}$ at each $\mathrm{K}^{+}$concentration. Although $\mathrm{K}^{+}$ 
concentration has been shown to influence G-quadruplex stability, no measurable differences in $\mathrm{T}_{\mathrm{m}}$ values of the G-quadruplex in different $\mathrm{K}^{+}$concentrations were observed. This trend was also true for the measured $\mathrm{T}_{\mathrm{mS}}$ of each respective duplex.

\subsubsection{Evaluation of TBA and pHAST stability and structural transition by circular dichroism}

Circular dichroism (CD) was used to confirm the stability of the TBA in neutral and acidic $\mathrm{pH}$. Additionally, it was used to confirm that addition of the 3'-poly A tail did not compromise the stability of the G-quadruplex structure of the TBA. The CD spectra of the TBA at neutral (7.5) and acidic (5.0) $\mathrm{pH}$, measured at $20^{\circ} \mathrm{C}$ are shown in Figure 3.9 A. The relative ellipticities measured at $294 \mathrm{~nm}$ over the temperature range of $20^{\circ} \mathrm{C}$ $80^{\circ} \mathrm{C}$ is shown in Figure 3.9 B.

A

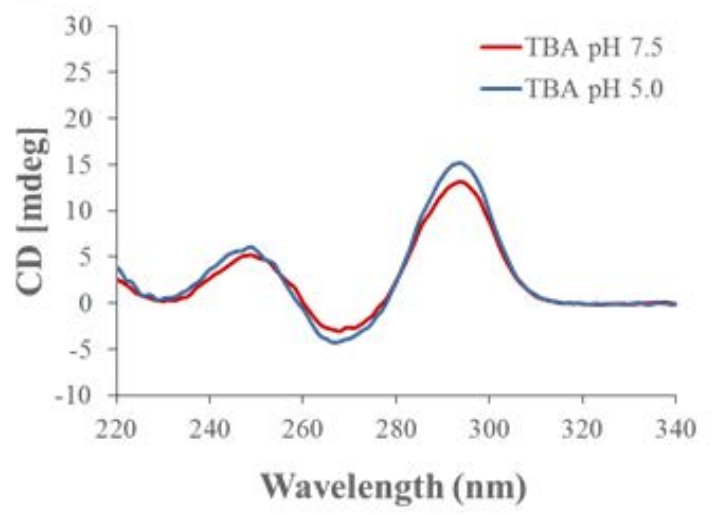

B

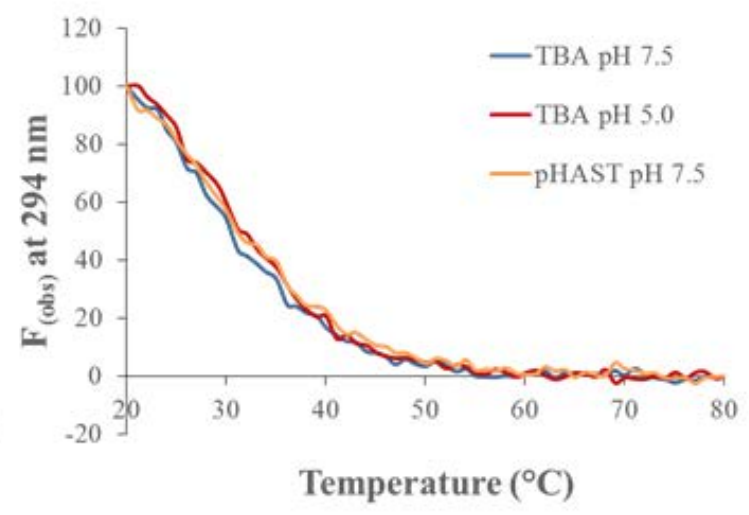

Figure 3.9: The stability of TBA at neutral and acidic pH and the stability of pHAST at neutral pH was confirmed by circular dichroism. (A) CD spectra of TBA (2.5 $\mu M)$ in either $0.1 \mathrm{M}$ sodium phosphate buffer $(140 \mathrm{mM} \mathrm{NaCl}, 0.5 \mathrm{mM} \mathrm{KCl}, \mathrm{pH} 7.5)$ or $0.1 \mathrm{M}$ sodium acetate buffer $(140 \mathrm{mM} \mathrm{NaCl}, 0.5 \mathrm{mM} \mathrm{KCl}, \mathrm{pH} 5.0)$ at $20^{\circ} \mathrm{C}$. Each of the spectrum shown represent the average of 5 scans of a single sample. (B) Relative $\left(F_{(o b s)}\right)$ ellipticity of TBA $(2.5 \mu M)$ in either $0.1 \mathrm{M}$ sodium phosphate buffer 
(140 $\mathrm{mM} \mathrm{NaCl,} 0.5 \mathrm{mM} \mathrm{KCl}$, pH 7.5) or $0.1 \mathrm{M}$ sodium acetate buffer (140 mM NaCl, $0.5 \mathrm{mM} \mathrm{KCl}, \mathrm{pH} 5.0$ ) as a function of temperature at $294 \mathrm{~nm}$ as monitored by farUV circular dichroism with a Jasco 815 spectropolarimeter. Reprinted with permission from McConnell, EM., Bolzon, R., Mezin, P., Frahm, G., Johnston, M and DeRosa, MC. pHAST (pH-Driven Aptamer Switch for Thrombin) Catch-andRelease of Target Protein. 2016, 27: 1493-1499. Bioconjugate Chemistry. Copyright 2016 American Chemical Society.

The TBA has previously been shown to form a chair-type intramolecular G-quadruplex in the presence of potassium as well as other monovalent metal cations. ${ }^{359,367}$ In the CD spectrum, the formation of a G-quadruplex as occurs with the TBA, is represented by the presence of a negative band at $\sim 265 \mathrm{~nm}$ and a positive bands at $\sim 245 \mathrm{~nm}$ and $\sim 295$ nm. ${ }^{359,368-371}$ Consistent with previously reported spectra for the TBA, these bands were observed in this work for the TBA prepared in either the $0.1 \mathrm{M}$ sodium phosphate buffer (140 mM NaCl, $0.5 \mathrm{mM} \mathrm{KCl,} \mathrm{pH} 7.5)$ or $0.1 \mathrm{M}$ sodium acetate buffer ( $140 \mathrm{mM} \mathrm{NaCl}, 0.5$ $\mathrm{mM} \mathrm{KCl}, \mathrm{pH} 5.0$ ) at $20^{\circ} \mathrm{C}$ (Figure 3.9 A). The thermal stability of the TBA at neutral $(\mathrm{pH} 7.5)$ and acidic ( $\mathrm{pH}$ 5.0) as well as pHAST ( $\mathrm{pH}$ 7.5) was examined (Figure 3.9 B). The relative $\left(\mathrm{F}_{(\mathrm{obs})}\right)$ ellipticity of the TBA was measured in both the $0.1 \mathrm{M}$ sodium phosphate buffer $(140 \mathrm{mM} \mathrm{NaCl}, 0.5 \mathrm{mM} \mathrm{KCl}, \mathrm{pH} 7.5)$ and the $0.1 \mathrm{M}$ sodium acetate buffer $(140 \mathrm{mM} \mathrm{NaCl}, 0.5 \mathrm{mM} \mathrm{KCl}, \mathrm{pH} 5.0)$. The thermal melt profiles are shown in Figure 3.9 B. The decrease of relative $\left(\mathrm{F}_{(\mathrm{obs})}\right)$ ellipticity at $294 \mathrm{~nm}$ with increasing temperature is indicative of melting of the TBA. There was no difference in the melting temperature of the TBA in neutral versus acidic $\mathrm{pH}$. To compare the stability of the TBA after the addition of the 3'-poly A tail, the thermal melt profile of $\mathrm{pHAST}$ in neutral $\mathrm{pH}$ was obtained and is shown in Figure 3.9 B. The melting temperature of TBA at neutral $\mathrm{pH}$ (7.5), TBA at acidic $\mathrm{pH}(5.0)$ and $\mathrm{pHAST}$ at neutral $\mathrm{pH}$ (7.5) were determined by fitting the relative $\left(\mathrm{F}_{(\mathrm{obs})}\right)$ ellipticity at $294 \mathrm{~nm}$ from $20^{\circ} \mathrm{C}-80^{\circ} \mathrm{C}$ as $29.7 \pm 0.2^{\circ} \mathrm{C}, 31.1 \pm$ 
$0.2^{\circ} \mathrm{C}$, and $31.1 \pm 0.2{ }^{\circ} \mathrm{C}$ respectively. These values are similar to those reported from the UV-Vis thermal denaturation studies of TBA and pHAST. Given the similarity in the melting temperature of the pHAST in neutral $\mathrm{pH}$ compared to TBA at either $\mathrm{pH}$, it seems the addition of the 3'-poly A tail did not negatively affect the stability of the TBA domain of pHAST.

Thermal melting temperature analysis of data from the UV-Vis thermal denaturation studies supported the hypothesis that the structural conformation of pHAST was dependent on $\mathrm{pH}$. To confirm this observation, the CD spectra of pHAST in Tris, sodium acetate or sodium phosphate buffered solutions with $\mathrm{pH}$ varying from 8.0 to 4.5 were measured and are shown in Figure 3.10.

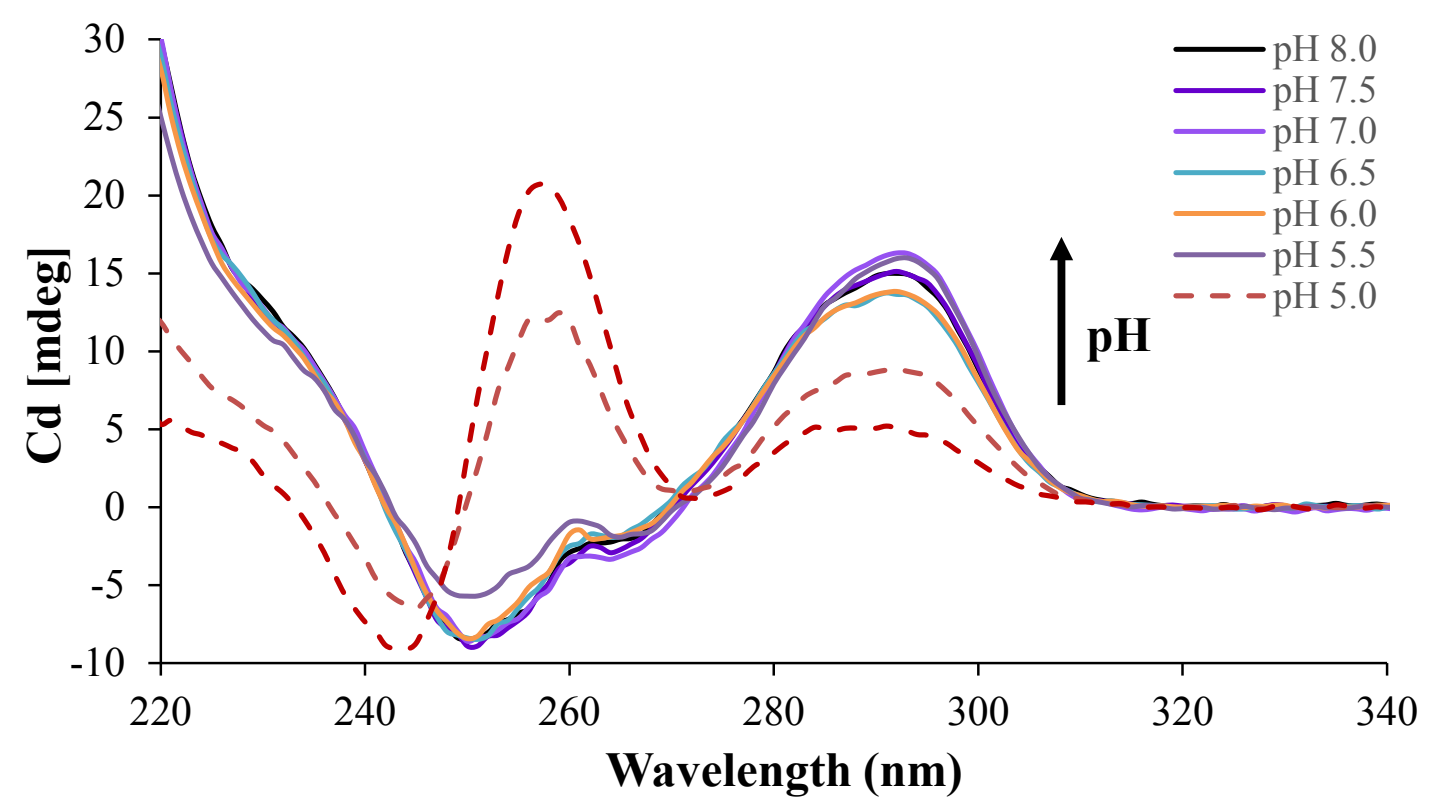

Figure 3.10: The CD spectra of pHAST $(2.5 \mu \mathrm{M})$ at $\mathrm{pHs}$ varying from 8.0-4.5. The significant structural change between $\mathrm{pH}$ values 5.5 and 5.0 is denoted by solid and dashed lines before and after the structural transition respectively. The average of 5 scans of a single sample was used to produce each of the spectra shown. Depending on the $\mathrm{pH}$, the pHAST was prepared in one of three different buffers adjusted to the appropriate $\mathrm{pH}$ value. The buffers were as follows; $10 \mathrm{mM}$ Tris- $\mathrm{HCl}, 0.5 \mathrm{mM} \mathrm{KCl \text {, }}$ 
pH 8.0; 10mM Tris-HCl, 0.5mM KCl, pH 7.5; 10mM Tris-HCl, $0.5 \mathrm{mM} \mathrm{KCl,} \mathrm{pH} \mathrm{7.0;}$ $10 \mathrm{mM}$ sodium phosphate, $0.5 \mathrm{mM} \mathrm{KCl}, \mathrm{pH} 6.5 ; 10 \mathrm{mM}$ sodium phosphate, $0.5 \mathrm{mM}$ $\mathrm{KCl}, \mathrm{pH} 6.0 ; 10 \mathrm{mM}$ sodium acetate, $0.5 \mathrm{mM} \mathrm{KCl}, \mathrm{pH} 5.5 ; 10 \mathrm{mM}$ sodium acetate, $0.5 \mathrm{mM} \mathrm{KCl}, \mathrm{pH} 5.0 ; 10 \mathrm{mM}$ sodium acetate, $0.5 \mathrm{mM} \mathrm{KCl}$, pH 4.5. Reprinted with permission from McConnell, EM., Bolzon, R., Mezin, P., Frahm, G., Johnston, M and DeRosa, MC. pHAST (pH-Driven Aptamer Switch for Thrombin) Catch-andRelease of Target Protein. 2016, 27: 1493-1499. Bioconjugate Chemistry. Copyright 2016 American Chemical Society.

From $\mathrm{pH}$ values ranging from $8.0-5.5$ a positive band was observed at $\sim 295 \mathrm{~nm}$, and negative bands were observed at $\sim 265 \mathrm{~nm}$ and $\sim 250 \mathrm{~nm}$. The broad band at $295 \mathrm{~nm}$ is consistent with the characteristic $295 \mathrm{~nm}$ band of the TBA, but likely also shows overlap of the band characteristic of DNA duplexes at $280 \mathrm{~nm} \cdot{ }^{372}$ A distinct structural transition was observed between $\mathrm{pH} 5.5$ and 5.0. At $\mathrm{pH}$ values below 5.5, strong positive bands were observed at $\sim 260 \mathrm{~nm}$ and $\sim 295 \mathrm{~nm}$, whereas a strong negative band was observed at $\sim 245 \mathrm{~nm}$. The bands at $\sim 245 \mathrm{~nm}$ and perhaps some overlap at $\sim 265 \mathrm{~nm}$ are consistent with the spectrum for a poly[d(A)]•poly[d(T)] duplex. ${ }^{372}$ The spectra suggest there was likely a mix of G-quadruplex and duplex in both the neutral and acidic conditions. Additionally, the blue-shifts of the bands at $\sim 295 \mathrm{~nm}$ and $\sim 265 \mathrm{~nm}$ characteristic of the TBA (Figure 3.9 A) to a broader peak ranging over 280 and $295 \mathrm{~nm}$ and $\sim 250 \mathrm{~nm}$ respectively for $\mathrm{pHAST}$ at $\mathrm{pH}$ values above 5.5 are consistent with the shifts observed when the TBA was bound to its complement. ${ }^{371}$ The broad band at $\sim 295 \mathrm{~nm}$ for values lower than $\mathrm{pH} 5.5$ is likely from residual G-quadruplex structure as well as the expected band at $280 \mathrm{~nm}$ for the poly[d(A)]•poly[d(T)] duplex. ${ }^{372}$ 


\subsubsection{Determination of conformational switching efficiency}

To evaluate conformational switching efficiency the Cy3/Cy5-pHAST was characterized using fluorescence spectroscopy, summarized in Figure 3.11. At neutral $\mathrm{pH}$, the $\mathrm{Cy} 3 / \mathrm{Cy} 5-\mathrm{pHAST}$ existed in the G-quad conformation. In this conformation, the emission of $\mathrm{Cy} 3$ was high compared to the emission of $\mathrm{Cy} 5$ since the dyes were not in close enough proximity for resonance energy transfer to occur. In this case, the expected $\mathrm{Cy} 3 / \mathrm{Cy} 5$ fluorescence intensity ratio was relatively high due to the strong $\mathrm{Cy} 3$ emission observed. Upon acidification of the solution the Cy3/Cy5-pHAST underwent a conformational change to form the $\mathrm{A}^{+}($anti $) \cdot \mathrm{G}(\operatorname{syn})$ base pair stabilized hairpin. In this conformation the FRET pair was in close enough proximity that excitation of Cy3 led to emission of $\mathrm{Cy} 5$ due to resonance energy transfer between the dye pair. Therefore, in this conformation the $\mathrm{Cy} 3 / \mathrm{Cy} 5$ fluorescence intensity ratio was relatively low due to the high emission of $\mathrm{Cy} 5$ and subsequently decreased $\mathrm{Cy} 3$ emission. Therefore, the ratio of the Cy3/Cy5 peak intensity was then interpreted to determine the conformation of Cy3/Cy5pHAST. 


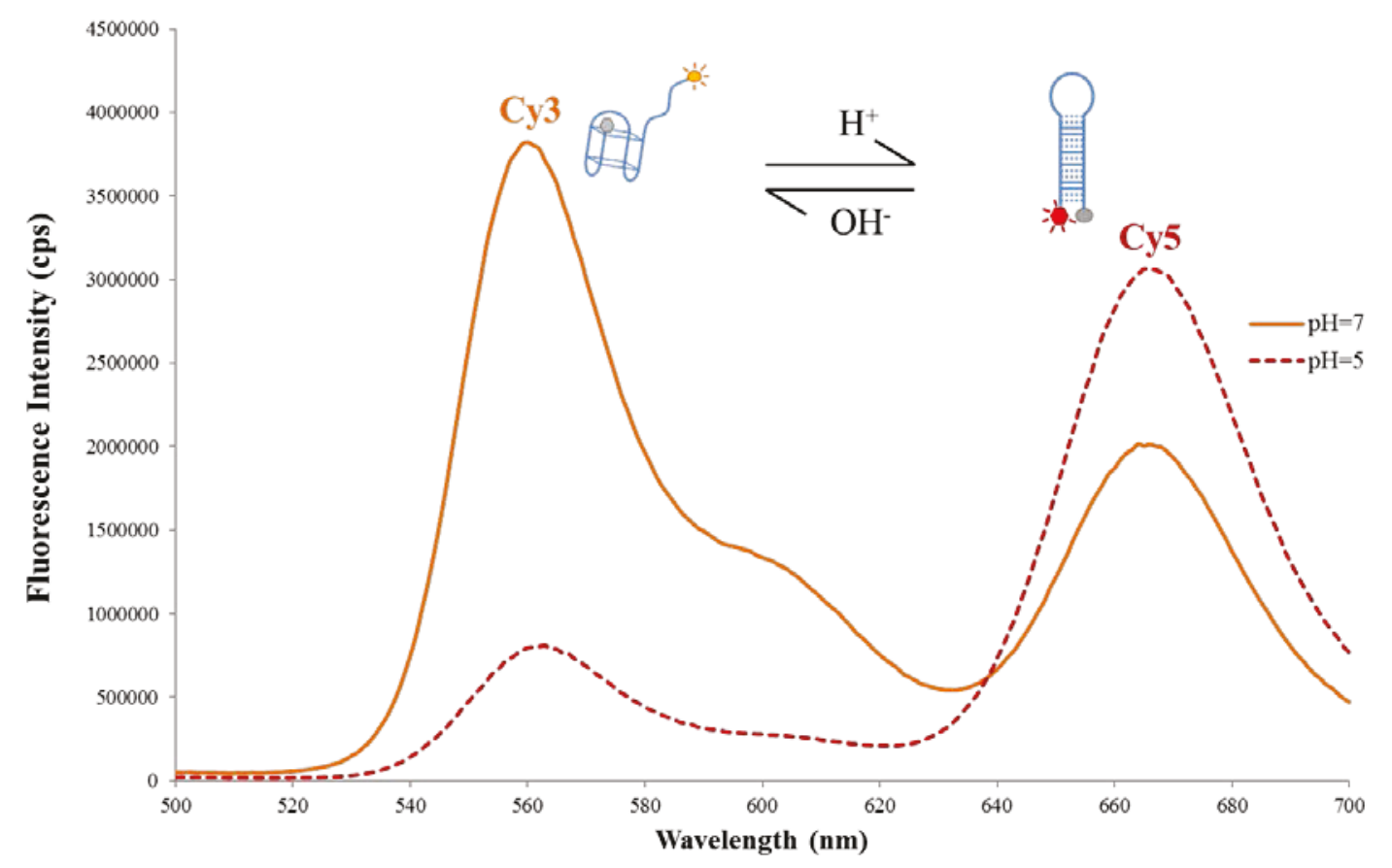

Figure 3.11: Fluorescence spectra of the Cy3/Cy5-pHAST $(0.4 \mu \mathrm{M})$ in neutral vs acidic conditions. The solution $\mathrm{pH}$ was changed from neutral to acidic, $\mathrm{Cy3} / \mathrm{Cy5}-$ pHAST underwent a conformational change from the G-quadruplex conformation to the $\mathrm{A}^{+}($anti) $\cdot \mathrm{G}($ syn $)$ base pair stabilized hairpin. FRET, which most efficiently occurred when the $\mathrm{Cy3} / \mathrm{Cy5}$-pHAST was in the hairpin conformation, was used to monitor the conformation of $\mathrm{Cy3} / \mathrm{Cy5}$-pHAST. The fluorescence intensity values of each of the cyanine dyes were not corrected for the $\mathrm{pH}$ effect on their respective quantum yields. Reprinted with permission from McConnell, EM., Bolzon, R., Mezin, P., Frahm, G., Johnston, M and DeRosa, MC. pHAST (pH-Driven Aptamer Switch for Thrombin) Catch-and-Release of Target Protein. 2016, 27: 1493-1499. Bioconjugate Chemistry. Copyright 2016 American Chemical Society.

Reversibility of the Cy3/Cy5- pHAST was examined by cycling the $\mathrm{pH}$ of the solution from neutral ( $\mathrm{pH}$ 7) to acidic ( $\mathrm{pH}$ 5) over multiple cycles (Figure 3.12). Since the $\mathrm{T}_{\mathrm{m}}$ studies showed no clear effect of $\mathrm{K}^{+}$concentration on structural stability, the ability of the $\mathrm{Cy} 3 / \mathrm{Cy} 5-\mathrm{pHAST}$ to cyclically change conformation based on changes in $\mathrm{pH}$ was examined. The switching efficiency herein represented by the greatest difference in $\mathrm{Cy} 3 / \mathrm{Cy} 5$ ratio at neutral vs acidic $\mathrm{pH}$ was investigated in $1 \mathrm{mM}$ sodium phosphate buffer with $140 \mathrm{mM} \mathrm{NaCl}$ and containing either $0.0 \mathrm{mM} \mathrm{KCl} 0.5 \mathrm{mM}, \mathrm{KCl}$ or $5.0 \mathrm{mM} \mathrm{KCl}$. 


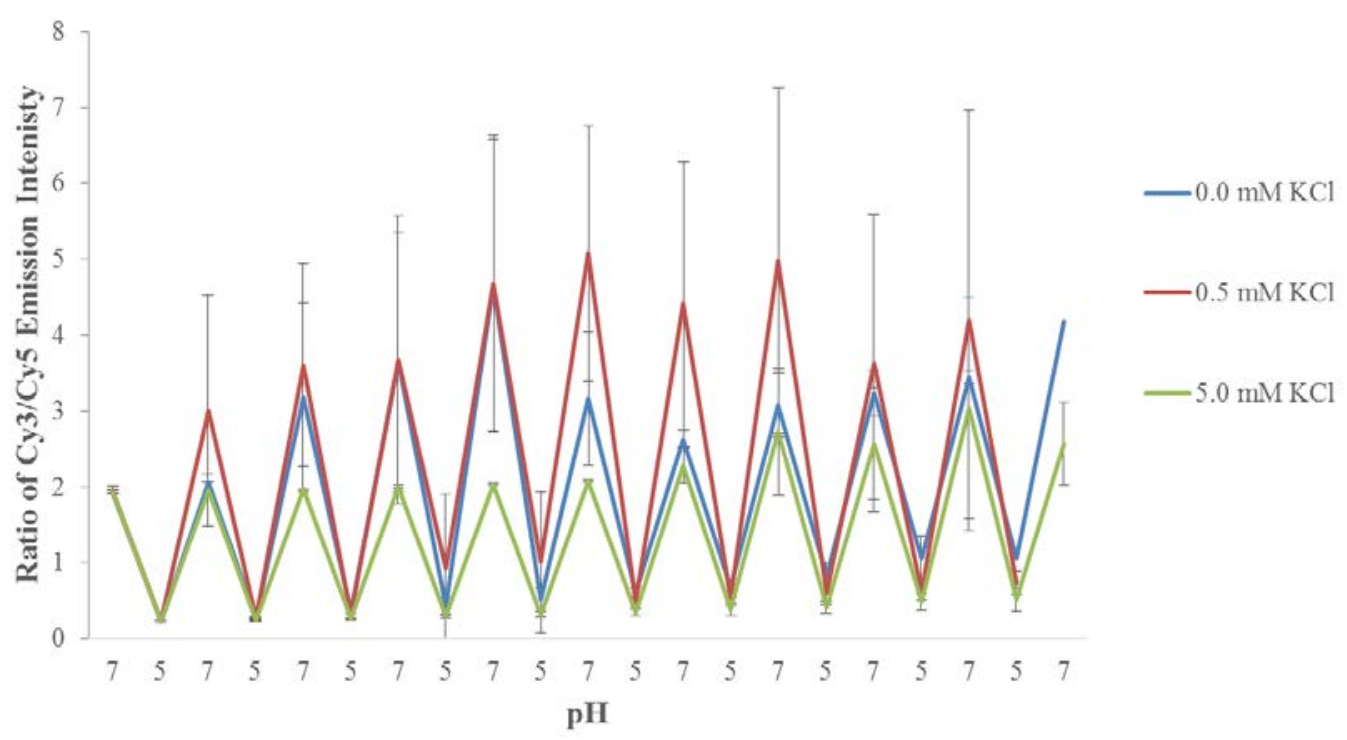

Figure 3.12: The effect of $\mathrm{pH}$ cycling on the conformation of the Cy3/Cy5-pHAST was monitored over 10 switches in each buffer condition. Each buffer contained 140 $\mathrm{mM} \mathrm{NaCl}$ and either $0.0 \mathrm{mM} \mathrm{KCl}, 0.5 \mathrm{mM} \mathrm{KCl}$ or $5.0 \mathrm{mM} \mathrm{KCl}$. The fluorescence intensity ratio of $\mathrm{Cy} 3$ emission over $\mathrm{Cy} 5$ emission was indicative of conformation. At low ratio intensities the $\mathrm{Cy} 3 / \mathrm{Cy5}$-pHAST was in the hairpin conformation. At high ratio intensities the $\mathrm{Cy} 3 / \mathrm{Cy5}$-pHAST was in the G-quad conformation. The fluorescence intensity values of each of the cyanine dyes were not corrected for the pH effect on their respective quantum yields. Reprinted with permission from McConnell, EM., Bolzon, R., Mezin, P., Frahm, G., Johnston, M and DeRosa, MC. pHAST (pH-Driven Aptamer Switch for Thrombin) Catch-and-Release of Target Protein. 2016, 27: 1493-1499. Bioconjugate Chemistry. Copyright 2016 American Chemical Society.

A consistent trend was observed across $\mathrm{K}^{+}$concentrations. At $\mathrm{pH} 7$, the $\mathrm{Cy} 3 / \mathrm{Cy} 5$ ratio intensity was consistently relatively high, and at $\mathrm{pH} 5$ the $\mathrm{Cy} 3 / \mathrm{Cy} 5$ intensity ratio was consistently relatively low. The change in the $\mathrm{Cy} 3 / \mathrm{Cy} 5$ ratio observed between neutral and acidic $\mathrm{pH}$, was maintained across multiple cycles for each $\mathrm{K}^{+}$concentration. The greatest change seemed to occur at the $0.5 \mathrm{mM} \mathrm{KCl}$ concentration, however, there was a large degree of variation within trials at the same concentration and a significant difference was likely not present. Variation between trials could have resulted due to error in reading the $\mathrm{BDH} \mathrm{pH} 4.5-10$ test strips. The $\mathrm{pH}$ of the solution, even \pm 0.5 could 
have greatly affected the $\mathrm{Cy} 3 / \mathrm{Cy} 5$ ratio measured and therefore introduced significant variation. The sensitivity of the switch to changes in $\mathrm{pH}$ was previously demonstrated by circular dichroism (see section 3.5.2). There did not appear to be a significant effect of $\mathrm{K}^{+}$ concentration on switching efficiency. The middle $\mathrm{K}^{+}$ion concentration $(0.5 \mathrm{mM} \mathrm{KCl})$ investigated was chosen for further experiments to allow for the requirement of $\mathrm{K}^{+}$to stabilize the G-quadruplex conformation but also allow for destabilization of the Gquadruplex in acidic conditions.

The ability of the $\mathrm{Cy} 3 / \mathrm{Cy} 5$-pHAST to undergo reversible conformational change was examined by subjecting the sample from trial $2(0.5 \mathrm{mM} \mathrm{KCl}$ condition) of the experiment described in Figure 3.12 to a total of $35 \mathrm{pH}$ cycles (Figure 3.13).

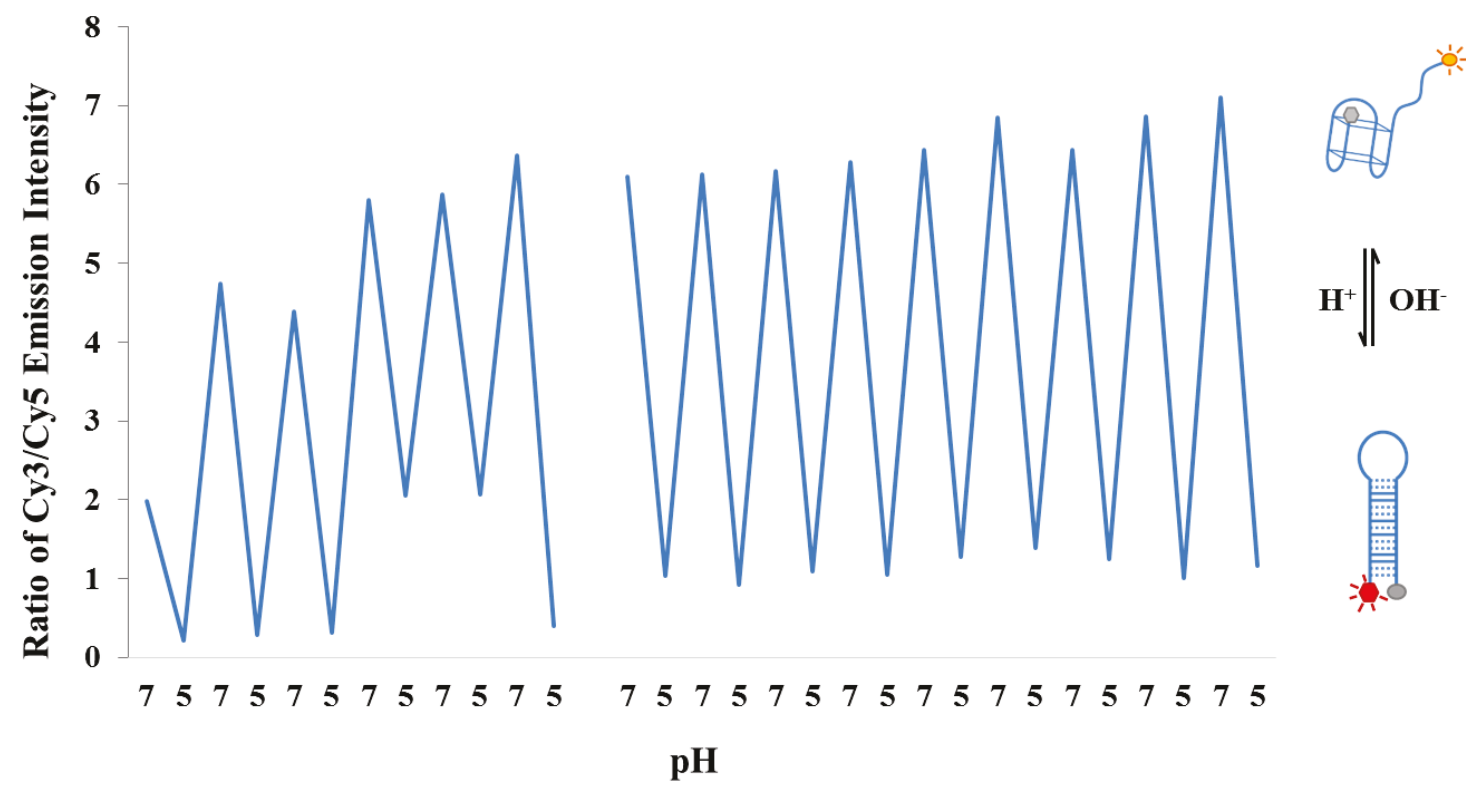

Figure 3.13: Reversible conformational change was shown by extended $\mathrm{pH}$ cycling of the Cy3/Cy5- pHAST in neutral and acidic pH. The ratio of fluorescence emission intensity (cps) of the $\mathrm{Cy} 3 / \mathrm{Cy} 5$ peaks was measured over 69 alternating $\mathrm{pH}$ conditions. A cycle consisted of a neutral and acidic $\mathrm{pH}$ condition. Cycles 1-6 and 27-35 are shown, cycles 7-26 are represented by a break in the $x$-axis. When the $\mathrm{Cy} 3 / \mathrm{Cy} 5$ ratio was high (pH 7), the pHAST was in the G-quadruplex conformation. 
When the Cy3/Cy5 ratio was low (pH 5), the pHAST was in the hairpin conformation. The fluorescence intensity values of each of the cyanine dyes were not corrected for the $\mathrm{pH}$ effect on their respective quantum yields. Reprinted with permission from McConnell, EM., Bolzon, R., Mezin, P., Frahm, G., Johnston, M and DeRosa, MC. pHAST (pH-Driven Aptamer Switch for Thrombin) Catch-andRelease of Target Protein. 2016, 27: 1493-1499. Bioconjugate Chemistry. Copyright 2016 American Chemical Society.

As shown in Figure 3.13, the ratio of $\mathrm{Cy} 3 / \mathrm{Cy} 5$ intensity consistently remained relatively high or low at neutral and acidic $\mathrm{pH}$ respectively, over several $\mathrm{pH}$ cycles. Due to experimental limitation of volume, additional cycles could not be performed.

The individual emission spectra and ratios of fluorescence intensity of the FRET pair Cy3/Cy5 for each sample were monitored over multiple $\mathrm{pH}$ cycles. When examining the emission spectra a consistent trend was observed (Figure 3.14). 

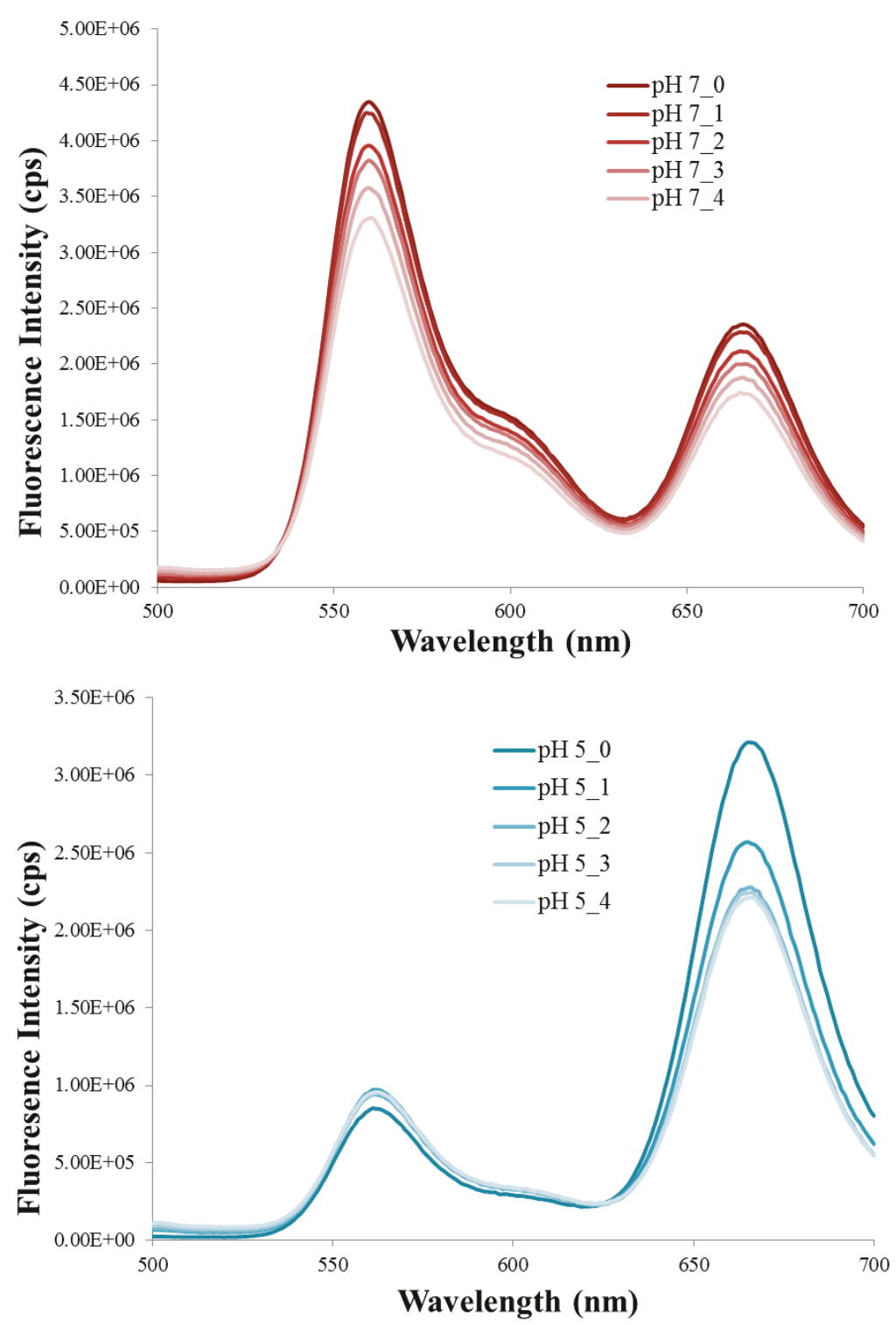

Figure 3.14: Continuous pH cycling over multiple switches led to a sequential decrease in fluorescence emission intensity. This trend is illustrated for all pH 7 (red) and pH 5 (blue) conditions. Traces are named based on the " $\mathrm{pH}$ cycle number". Buffer: $1 \mathrm{mM}$ phosphate buffer with $140 \mathrm{mM} \mathrm{NaCl}$ and $0.5 \mathrm{mM} \mathrm{KCl}$.

A constant decrease in fluorescence intensity is observed with each $\mathrm{pH}$ cycle, as represented by sequentially lighter traces in Figure 3.14. This is likely due to the small decrease in volume and minimal dilution that occur while testing the $\mathrm{pH}$ of the sample 
and adjusting the $\mathrm{pH}$ respectively. Despite this decrease in fluorescence intensity the ratio of $\mathrm{Cy} 3 / \mathrm{Cy} 5$ was maintained over multiple $\mathrm{pH}$ cycles (Figure 3.12) for each $\mathrm{K}^{+}$ concentration investigated.

To evaluate the effect of salt buildup on the system, trial 1 samples from each $\mathrm{K}^{+}$ condition were desalted and then subjected to an additional $5 \mathrm{pH}$ cycles (Figure 3.15 A). Further, the initial (post-desalting) $\mathrm{Cy} 3 / \mathrm{Cy} 5$ ratio of the highest $\mathrm{K}^{+}$concentration (5.0 $\mathrm{mM}$ ) sample was compared to the final (pre-desalting) Cy3/Cy5 ratio value to determine whether or not salt build up had a significant effect on switching. These data are shown in Figure 3.15 B. 


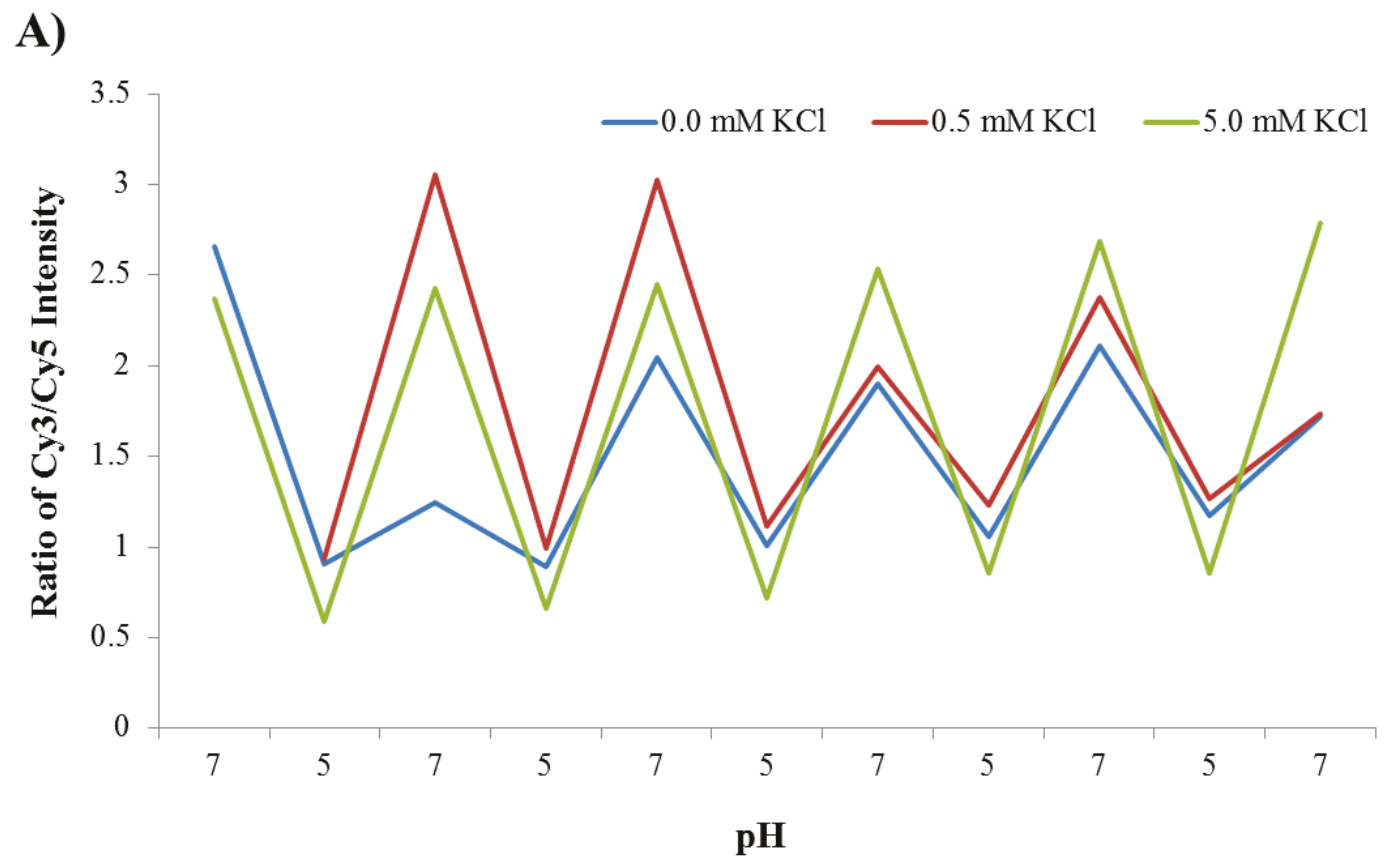

B)

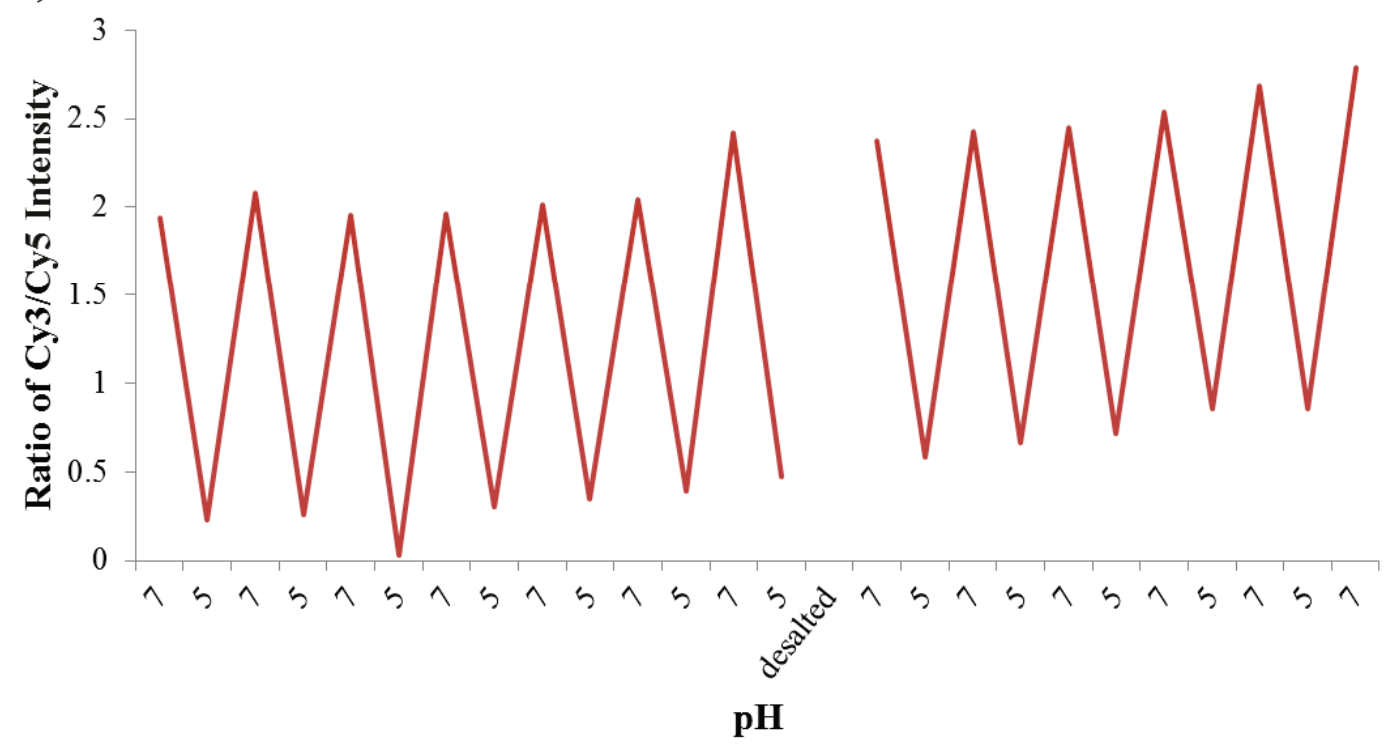

Figure 3.15: A) pH switching ability is maintained at each buffer condition, following desalting. Samples (from trial 1) from each buffer condition were desalted post $\mathrm{pH}$ cycling then subjected to an additional 5 switches. Note: the inconsistency observed for the second $\mathrm{pH}=7$ value was likely caused by an inaccurate $\mathrm{pH}$ measurement. B) Representative profiles from the sample tested in trial 1 of Figure 3.12. (1 mM phosphate buffer, $140 \mathrm{mM} \mathrm{NaCl}$ and $5 \mathrm{mM} \mathrm{KCl}$ ) before and after desalting are shown. Switches 1-7 and 1-5 respectively are depicted. The fluorescence intensity values of each of the cyanine dyes were not corrected for the pH effect on their respective quantum yields. 
In each $\mathrm{K}^{+}$condition, conformational switching was maintained with a Cy3/Cy5 intensity ratio that was consistent with the final cycle ratios pre-desalting, suggesting that excess $\mathrm{Na}^{+}$and $\mathrm{K}^{+}$build up did not hinder conformational change. Combined with the observation that fluorescence intensity decreased with increasing cycle, it is likely that minimal dilution and volume loss had a greater effect on $\mathrm{Cy} 3 / \mathrm{Cy} 5$ ratio than did excess salt buildup, as desalting did not return $\mathrm{Cy} 3$ and $\mathrm{Cy} 5$ intensities closer to their original values. To investigate further the effects of dilution and volume loss, the $\mathrm{Cy} 3 / \mathrm{Cy} 5$ pHAST should be investigated in a flow system where the amount of DNA and the volume remained constant while the $\mathrm{pH}$ was adjusted. Circular dichroism could be used to investigate the effect of increasing salt concentration on conformational switching.

\subsubsection{Evaluation of pHAST binding to thrombin}

The ability of the Cy3-pHAST to bind its target over a range of $\mathrm{pH}(8.0-4.0)$ was evaluated by non-denaturing EMSA. The pH titration curve is shown in Figure 3.16. 


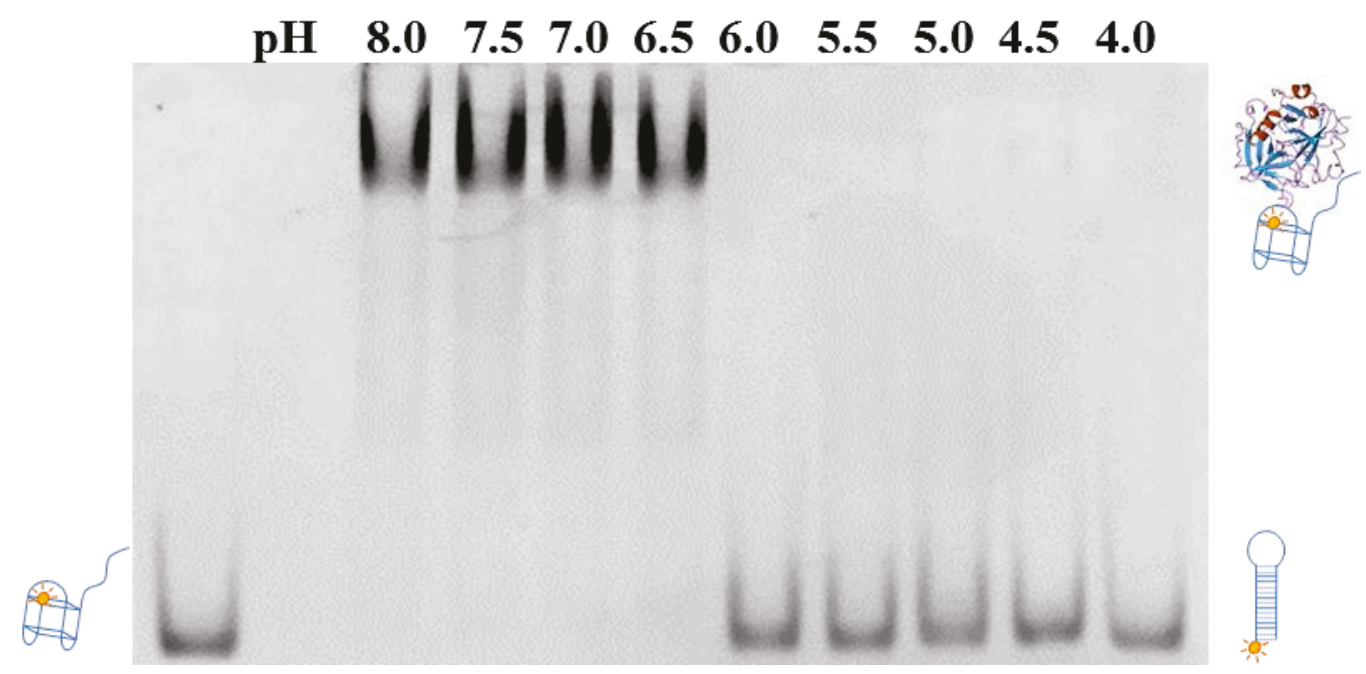

Figure 3.16: Thrombin binding to Cy3-pHAST was disrupted between pH 6.5 and pH 6.0. Cy3-pHAST, in the G-quad conformation, binding to thrombin is shown by the fluorescence (black) in the top bands of lanes 3-6. Unbound Cy3-pHAST, in the hairpin conformation is shown by the fluorescence in the bottom bands in lane 7-11. Lanes: 1. Cy3-pHAST with 0.0 $\mu$ M thrombin at pH 7. Lanes 3-11 contained 1.0 $\mu \mathrm{M}$ Cy3-pHAST incubated with $2.5 \mu \mathrm{M}$ thrombin at $\mathrm{pH}$ 8.0, 7.5, 7.0, 6.5, 6.0, 5.5, 5.0, 4.5, and 4.0 respectively. Reprinted with permission from McConnell, EM., Bolzon, R., Mezin, P., Frahm, G., Johnston, M and DeRosa, MC. pHAST (pH-Driven Aptamer Switch for Thrombin) Catch-and-Release of Target Protein. 2016, 27: 1493-1499. Bioconjugate Chemistry. Copyright 2016 American Chemical Society.

At $\mathrm{pH}$ values between $\mathrm{pH}$ 6.5-8.0, the Cy3-pHAST existed in the G-quad conformation and was able to bind to thrombin. At $\mathrm{pH}$ values below 6.0, a $\mathrm{pH}$ responsive conformation change of the Cy3-pHAST significantly diminished target binding. This result was expected given that adenine is mostly neutral at $\mathrm{pH} 6.0 .{ }^{373}$ At $\mathrm{pH}$ values below 6.0, varying degrees of protonation of the N1 of adenine would have resulted in formation of hairpin stabilizing $\mathrm{A}^{+}($anti) $\bullet \mathrm{G}(s y n)$ base pairs, inducing conformational change and eliminating target binding. Importantly, literature precedence suggests that the disruption of target binding was likely due to conformation change as opposed to lack of affinity of the TBA domain of $\mathrm{pHAST}$ at acidic $\mathrm{pH} .{ }^{307,354}$ 
To confirm that loss of binding was due to conformational change and not loss of affinity of the TBA domain at $\mathrm{pH}$ 5, fluorescence anisotropy was performed (Figure 3.17).

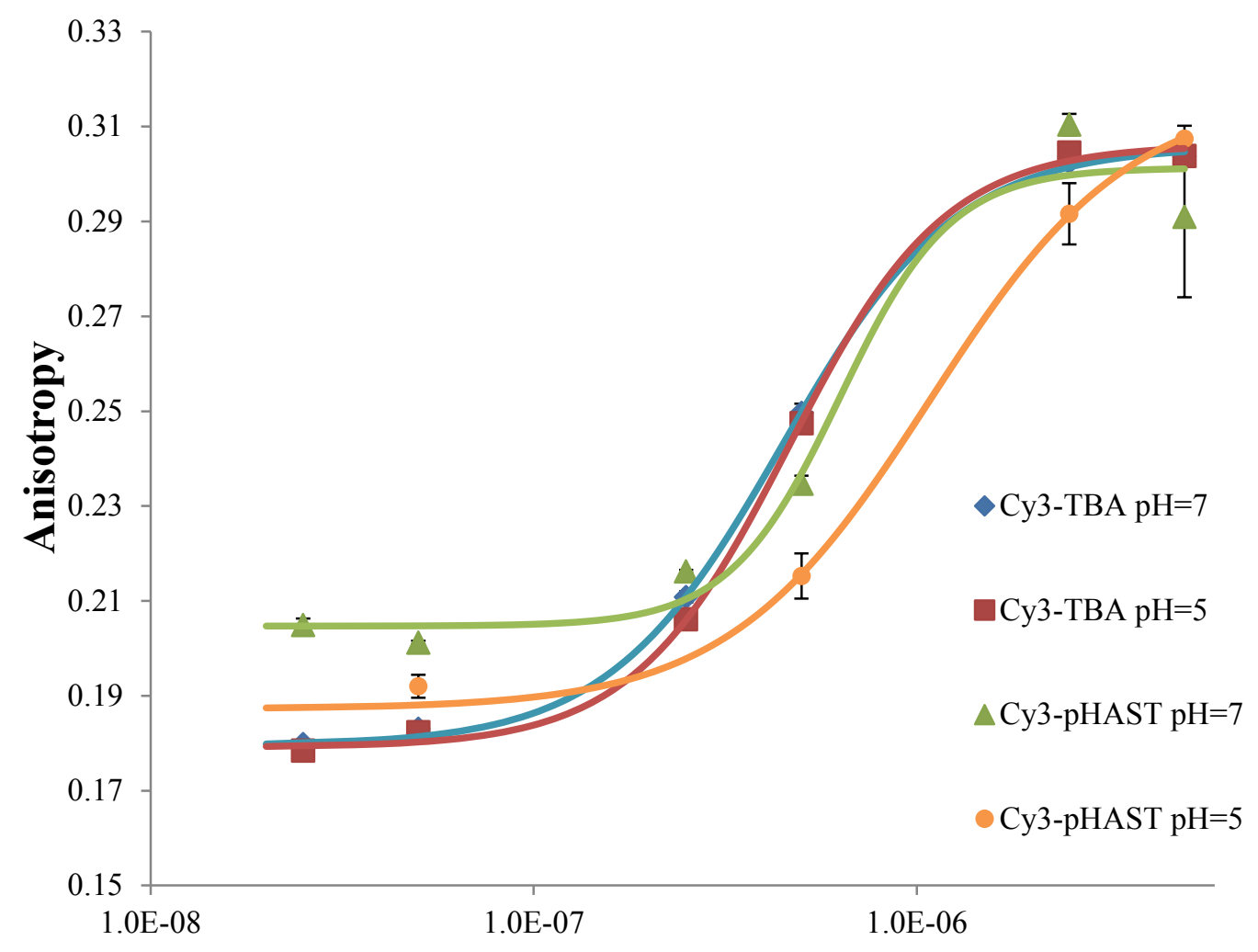

Thrombin Concentration (M)

Figure 3.17: The binding of the Cy3-TBA and Cy3-pHAST to thrombin over a range of thrombin concentrations was evaluated by fluorescence anisotropy. Error bars represent standard deviation. The apparent KDs were; Cy3-TBA (pH 7): $0.45 \pm$ $0.01 \mu \mathrm{M}$, Cy3-TBA (pH 5): $0.46 \pm 0.02 \mu \mathrm{M}$, Cy3-pHAST (pH 7): $0.63 \pm 0.18 \mu \mathrm{M}$, Cy3-pHAST (pH 5): $1.08 \pm 0.18 \mu M$. For each sample, the apparent $K_{D}$ values $\left(K_{D} \pm\right.$ Standard Error) were derived from the fit of the average of the closest two data points at each concentration. Reprinted with permission from McConnell, EM., Bolzon, R., Mezin, P., Frahm, G., Johnston, M and DeRosa, MC. pHAST (pHDriven Aptamer Switch for Thrombin) Catch-and-Release of Target Protein. 2016, 27: 1493-1499. Bioconjugate Chemistry. Copyright 2016 American Chemical Society.

Fluorescence anisotropy confirmed that the TBA was able to bind thrombin at both $\mathrm{pH} 7$ and $\mathrm{pH}$ 5. Further, the Cy3-pHAST at neutral $\mathrm{pH}$ was able to bind thrombin with similar 
affinity to the TBA. Interestingly, the Cy3-pHAST showed some affinity to the thrombin at $\mathrm{pH}$ 5. This is likely due to conformational equilibrium as discussed previously. The curve observed is the resultant average curve given that the Cy3-pHAST that exists in the G-quadruplex conformation at $\mathrm{pH} 5$ is still binding the target well. Since the hairpin conformation is either not binding the thrombin, or non-specifically interacting with thrombin, the resultant curve is shifted towards lower affinity.

To confirm this hypothesis, the Cy3-pHAST samples on which the fluorescence anisotropy was measured were analysed by non-denaturing EMSA (Figure 3.18).

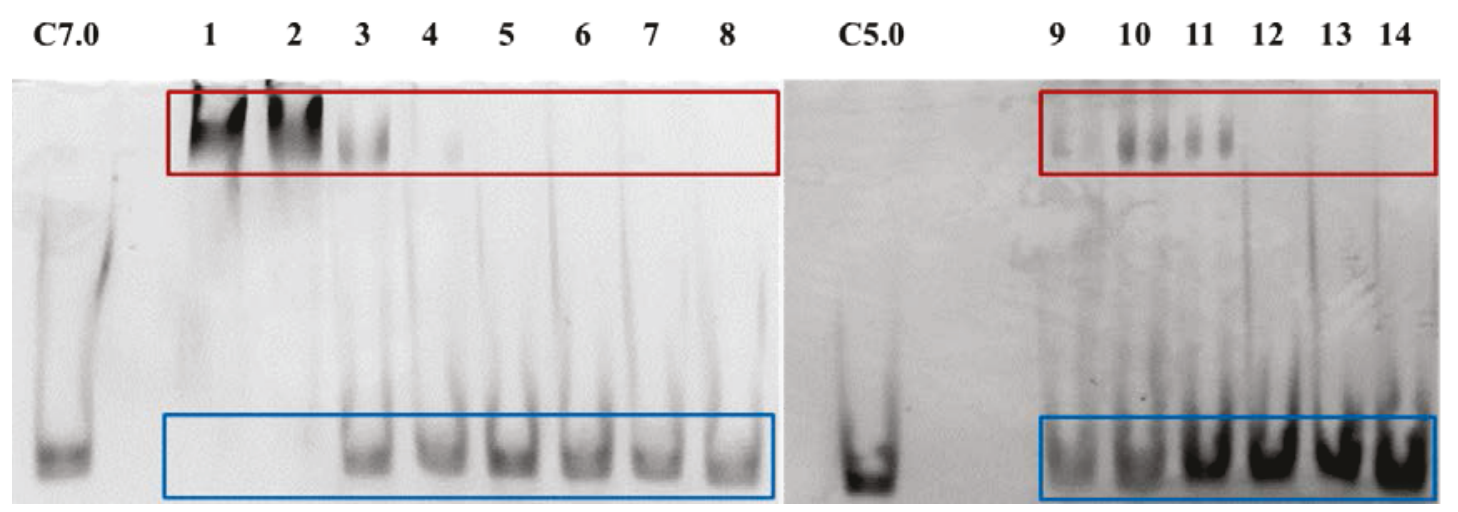

Figure 3.18: Representative gels of full concentration range of thrombin with Cy3pHAST at pH 7.0 (lanes C7.0 and 1-8) and pH 5.0 (lanes C5.0 and 9-14) where $\mathrm{C}$ is the control at a given $\mathrm{pH}$. Cy3-pHAST $(1.0 \mu \mathrm{M})$ was incubated with varying concentrations of thrombin; $0.0 \mu \mathrm{M}$ thrombin (lanes $\mathrm{C}$ 7.0, C5.0), $5.0 \mu \mathrm{M}$ thrombin (lanes 1, 9), $2.5 \mu \mathrm{M}$ (lanes 2, 10), $0.50 \mu \mathrm{M}$ (lanes 3, 11), $0.25 \mu \mathrm{M}$ (lane 4), $50 \mathrm{nM}$ (lanes 5, 12), $25 \mathrm{nM}$ (lane 6), $5.0 \mathrm{nM}$ (lanes 7,13), and $0.5 \mathrm{nM}$ (lanes 8,14).

Consistent with the anisotropy data, binding is observed over a range of thrombin concentrations. At neutral $\mathrm{pH}$, the $\mathrm{Cy} 3-\mathrm{pHAST}$ is observed only in the top band at concentrations $2.5 \mu \mathrm{M}$ thrombin and above. At $0.50 \mu \mathrm{M}$ thrombin, some of the Cy3pHAST bound to thrombin. Below $0.50 \mu \mathrm{M}$ thrombin, Cy3-pHAST is mostly observed in the bottom lane. In support of the anisotropy based hypothesis, some binding is observed 
at pH 5 due to the small amount of Cy3-pHAST in the G-quad conformation (lanes 9-14: top band) as a result of conformational equilibrium. The presence of some pHAST in the G-quadruplex conformation at acidic $\mathrm{pH}$ was further supported by the remaining Gquadruplex signature (at $294 \mathrm{~nm}$ ) in the CD spectra (Figure 3.10). When comparing fluorescence intensity in the bottom bands of the gels at $\mathrm{pH} 7$ and $\mathrm{pH} 5$ conditions, it is evident that affinity of the Cy3-pHAST is significantly diminished at acidic $\mathrm{pH}$ compared to the Cy3-pHAST at neutral $\mathrm{pH}$. Looking at the bottom band of the $\mathrm{pH} 5$ gel, $\mathrm{Cy} 3-$ pHAST in the hairpin conformation (bottom lanes 9-11) is evident at all thrombin concentrations. Unlike the bottom band of the neutral gel in which little to no hairpin is observed at comparable concentrations (bottom lanes 1-3). As thrombin concentration decreased, the bottom band would have also included non-binding Cy3-pHAST in the Gquad conformation at both neutral and acidic conditions (bottom lanes 3-8 and 9-14 respectively).

\subsubsection{Catch-and-Release of thrombin by Cy3-pHAST}

EMSA (Figure 3.19) and fluorescence anisotropy (Figure 3.20) were used to evaluate the ability of Cy3-pHAST to cyclically catch-and-release thrombin. 


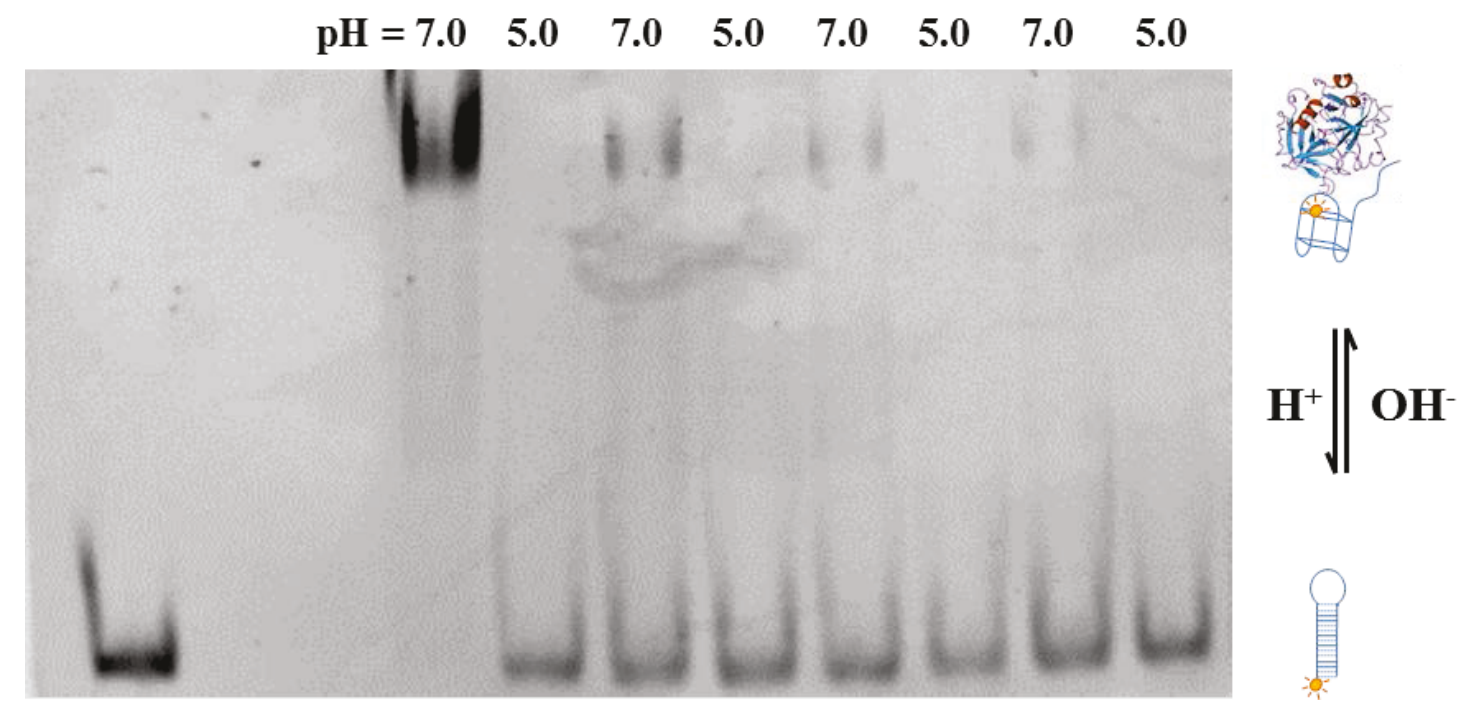

Figure 3.19: pH cycling in the presence of thrombin shows that the Cy3-pHAST can predictably catch-and-release thrombin based on solution $\mathrm{pH}$. Lanes: 1: 1.0 $\mu \mathrm{M}$ Cy3-pHAST control (0.0 $\mu$ M thrombin); 4, 6, 8, 10: $1.0 \mu M$ Cy3-pHASTwith $2.5 \mu \mathrm{M}$ thrombin at pH 7 and 5,7,9,11: $1.0 \mu \mathrm{M}$ Cy3-pHAST with $2.5 \mu \mathrm{M}$ thrombin at pH 5. Reprinted with permission from McConnell, EM., Bolzon, R., Mezin, P., Frahm, G., Johnston, M and DeRosa, MC. pHAST (pH-Driven Aptamer Switch for Thrombin) Catch-and-Release of Target Protein. 2016, 27: 1493-1499. Bioconjugate Chemistry. Copyright 2016 American Chemical Society.

Cy3-pHAST and thrombin were incubated at room temperature and the $\mathrm{pH}$ of the solution was cyclically changed between neutral $(\mathrm{pH} 7)$ and acidic $(\mathrm{pH} 5)$ conditions. At each $\mathrm{pH}$ condition a small aliquot of the sample was removed for evaluation by EMSA. Binding of the Cy3-pHAST at pH 7 was observed by EMSA (see Figure lanes 4, 6, 8, 10). The Cy3-pHAST-thrombin complex can be seen at the top of the lanes. When the $\mathrm{pH}$ was acidic, EMSA revealed that binding was disrupted (see Figure lanes 5, 7, 9, 11), and the DNA was observed in the bottom of the lane. Over multiple cycles, the observed amount of Cy3-pHAST binding to thrombin decreased. 
Fluorescence anisotropy was used to confirm the ability of Cy3-pHAST to catchand-release thrombin (Figure 3.20). The anisotropy was measured in the presence and absence of thrombin while the $\mathrm{pH}$ was cycled from neutral $(\mathrm{pH} 7)$ to acidic $(\mathrm{pH} 5)$. There was a small change in the measured anisotropy of the Cy3-pHAST due to conformational difference in neutral versus acidic $\mathrm{pH}$. However, a much larger difference in the measured anisotropy of the Cy3-pHAST-thrombin complex at $\mathrm{pH} 7$ and the non-binding hairpin at $\mathrm{pH} 5$ were observed. These data support the EMSA work, suggesting that Cy3pHAST was able to cyclically catch-and-release thrombin depending on pH-driven conformational changes. When the Cy3-pHAST was in the G-quadruplex conformation and capable of binding thrombin, a relatively high anisotropy value was observed. On the contrary, when Cy3-pHAST was mostly in the hairpin conformation, a relatively small anisotropy value was observed. The differences in the measured anisotropy values can again be explained by the existing conformational equilibrium. The data presented suggest that when the concentration of thrombin is within the range of the apparent $\mathrm{K}_{\mathrm{D}}$ of Cy3-pHAST for thrombin, the catch-and-release behaviour in neutral and acidic conditions is predictable. 

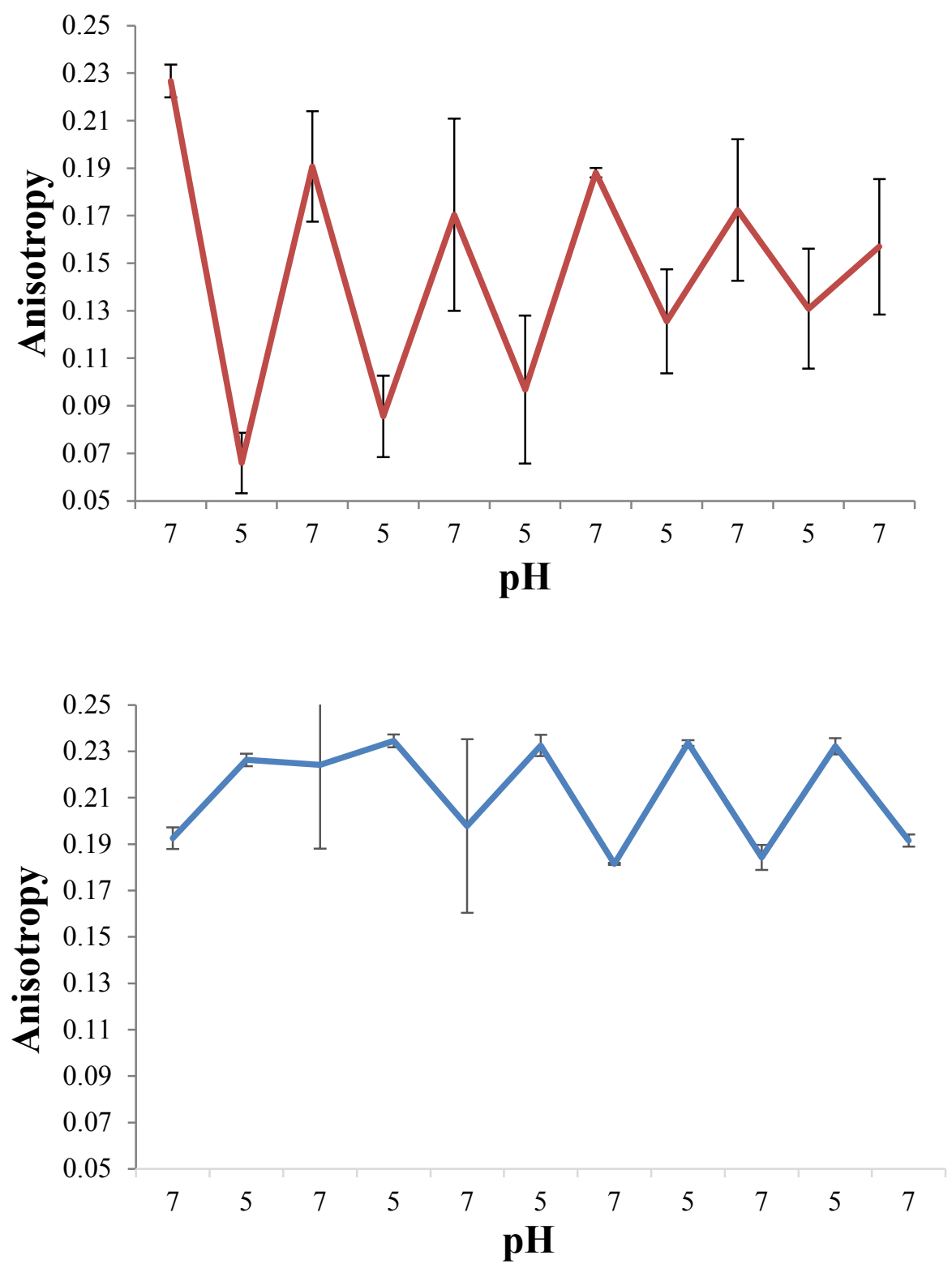

Figure 3.20: Top) The fluorescence anisotropy was measured Cy3-pHAST with thrombin $(0.5 \mu \mathrm{M})$ in $1 \mathrm{mM}$ sodium phosphate buffer $(140 \mathrm{mM} \mathrm{NaCl}, 0.5 \mathrm{mM} \mathrm{KCl})$ during $\mathrm{pH}$ cycling. The large difference in size due to target binding, resulted in different anisotropy values under neutral ( $\mathrm{pH} 7$ ) versus acidic (pH 5) conditions. Error bars represent the standard deviation between three trials. Bottom) The fluorescence anisotropy the Cy3-pHAST in $1 \mathrm{mM}$ sodium phosphate buffer (140 $\mathrm{mM} \mathrm{NaCl}, 0.5 \mathrm{mM} \mathrm{KCl}$ ) was measured while the $\mathrm{pH}$ was cycled to determine the effect of pH-dependent structure on anisotropy values. The change in anisotropy in the absence of thrombin is shown. The change of conformation of the pHAST from G-quadruplex to hairpin resulted in a small change in the measured anisotropy. 
Error bars represent the standard deviation between three trials. Reprinted with permission from McConnell, EM., Bolzon, R., Mezin, P., Frahm, G., Johnston, M and DeRosa, MC. pHAST (pH-Driven Aptamer Switch for Thrombin) Catch-andRelease of Target Protein. 2016, 27: 1493-1499. Bioconjugate Chemistry. Copyright 2016 American Chemical Society.

The data presented so far, validate the design of a $\mathrm{pH}$-driven catch-and-release aptamer system based on the rational inclusion of the $\mathrm{A}^{+}($anti) $\bullet \mathrm{G}(\mathrm{syn})$ base pair into the complementary domain of an aptamer sequence. Though cyclic catch-and-release of thrombin was observed, the property of this system was not fully reversible. This was likely due to the increase in ionic strength over multiple $\mathrm{pH}$ cycles. With increasing ionic strength, the negative charge of Cy3-pHAST and/or thrombin would have been neutralized, effectively eliminating any electrostatic interactions between the DNA and the protein. ${ }^{307}$ Further binding could have been disrupted by physical changes to the protein; thrombin could have become denatured by repeated $\mathrm{pH}$ changes and increasing ionic concentrations, or it could have aggregated. ${ }^{307}$ Future work should examine how optimization of the buffer conditions or modifying the experimental protocol such that fresh thrombin was supplied during the neutral condition might improve the reversibility of the catch-and-release behaviour. 


\subsection{Conclusions and future work}

pHAST, a DNA aptamer-based $\mathrm{pH}$-driven nanomachine, was designed such that it was capable of the controlled catch-and-release of a target protein. The rational inclusion of the $\mathrm{A}^{+}($anti $) \cdot \mathrm{G}($ syn $)$ base pair afforded the $\mathrm{pHAST}$ nanomachine with $\mathrm{pH}$-responsive conformation changes that dictated thrombin binding. This proof-of-concept work demonstrates the potential of the $\mathrm{A}^{+}(a n t i) \cdot \mathrm{G}(s y n)$ base pair to be incorporated into other aptamer based $\mathrm{pH}$-switching systems for various delivery applications. Additionally, this approach could be used to design not only $\mathrm{pH}$-driven nanoswitches but also incorporated into aptamer-based smart material (multifunctional materials exhibiting response to external stimuli) systems such as hydrogels and logic gates. ${ }^{27}$ The addition of $\mathrm{pH}$ controllable functionality would allow for improved responsive control, sensitivity, and selectivity of the material's response. ${ }^{27}$ Further, this proof-of-concept work demonstrates the potential of including the $\mathrm{A}^{+}($anti $) \cdot \mathrm{G}($ syn $)$ base pair to overcome design limitations of current $\mathrm{pH}$-responsive aptamer-based sensors based on the traditionally used $\mathrm{C} \cdot \mathrm{C}+$ mispair (i-motif). ${ }^{348}$ Aptamers are selected from randomized oligonucleotide libraries that typically have equal distributions of each nucleobase. For an effective pH-driven switch to be designed from an existing aptamer using the $\mathrm{C} \cdot \mathrm{C}+$ mispair, that aptamer would need to have elongated stretches of $\mathrm{C}$ in the sequence. Aptamers tend to be $\mathrm{G}$ and $\mathrm{C}$ rich, therefore a combination of the commonly used $\mathrm{C} \cdot \mathrm{C}+$ mispair and the $\mathrm{A}^{+}($anti $) \cdot \mathrm{G}($ syn $)$ base pair investigated in this work would allow for the design of unimolecular $\mathrm{pH}$-driven nanoswitches from most existing aptamers. ${ }^{8}$ More generally, this work could be applied to regulate $\mathrm{pH}$-activated delivery, $\mathrm{pH}$-regulated controlled release, and $\mathrm{pH}$-responsive gating of nanoparticles or surfaces. ${ }^{374}$ 
4 Intra-Accumbens Injection of a Dopamine Aptamer Abates MK-801-Induced Cognitive Dysfunction in a model of Schizophrenia 


\subsection{Statement of Contributions}

Experiments were conceptualized by MC DeRosa, EM McConnell, MR Holahan and D Madularu. Experiments were designed by MC DeRosa and MR Holahan. DNA was prepared by EM McConnell and R. Walsh. Animal preparation and testing were done by D Madularu and MR Holahan with assistance from EM McConnell. Histology and immunohistochemical quantification were done by D Madularu and MR Holahan. Statistical analysis was performed by MR Holahan.

\subsection{Resulting Publications}

This work was published in PLOS One. The manuscript was prepared by MR Holahan, D Madularu, EM McConnell and MC DeRosa (Holahan, MR.; Madularu, D.; McConnell, EM.; Walsh, R.; DeRosa, MC. PLOS One. 2011, 6, e22239). ${ }^{375}$ 


\subsection{Introduction}

\subsubsection{Objectives}

The primary objective of this work was to determine if an aptamer that bound to the small molecule neurotransmitter dopamine would maintain its target binding ability upon direct injection into the nucleus accumbens in an in vivo model of schizophrenia.

\subsubsection{Schizophrenia - the dopamine hypothesis}

Generally, schizophrenia describes a continuum of subtypes characterized by the combination of positive, negative and cognitive symptoms. Positive (psychotic) symptoms may include hallucinations and delusions. ${ }^{376}$ Negative (affective) symptoms include flattened affect, amotivation and social withdrawal. ${ }^{377}$ Deficits in cognitive functioning manifest as impairments in attention, processing speed, executive function and working memory. ${ }^{378}$ Though men and women are equally affected, disease onset occurs differentially with average ages of 18-25 and 25-35 for men and women respectively. ${ }^{379}$ Due to the high morbidity and mortality rates associated with schizophrenia, the burden on health and social care is significant. ${ }^{377}$ This combined with the fact that antipsychotic drugs have remained the standard treatment, despite their disruptive side-effects and ineffectiveness for 1 in 3 patients illustrates the requirement for a better understanding of the underlying neurobiological dysfunction as well as the need to develop alternative treatment strategies. ${ }^{380,381}$

Two main etiological hypotheses based on neurochemical imbalance have been significantly investigated. Work towards understanding the role of dopaminergic and glutamatergic dysfunction in schizophrenia was reviewed recently. ${ }^{377}$ The DA 
hypothesis, the idea that dopamine and dopamine receptor related changes in pre-synaptic and post-synaptic dopaminergic system underlie the neuropathology of schizophrenia has been investigated since the 1950s. ${ }^{377}$ Recently, analysis of dopamine synthesis and release monitored by multiple studies done with Positron Emission Tomography (PET) and molecular imaging with radiotracers further supported that pre-synaptic dopamine synthesis and release are largely elevated in schizophrenic patients compared to matched controls. ${ }^{377}$ Interestingly, dopamine synthesis capacity was consistently elevated in patients that were either acutely psychotic or in patients that were experiencing an acute psychotic relapse compared to either chronic patients or patients in remission. ${ }^{377}$ Additionally, dopamine synthesis capacity was elevated in patients in the prodromal phase of schizophrenia and this elevation was an indication of individuals identified as ultra-high risk that would later develop psychosis. ${ }^{382}$

\subsubsection{The ventral tegmental-nucleus accumbens reward circuit, NMDA receptors, and MK-801}

The cortical regulation of dopamine release is controlled by an intricate network of interconnected glutamatergic, GABAergic and dopaminergic neurons that project between cortical and subcortical tissue (Figure 4.1). The major dopaminergic, glutamatergic and GABAergic connections between the ventral tegmental area (Figure 4.1: VTA) and the nucleus accumbens (Figure 4.1: NAc) implicated in the VTA-NAc reward circuit of the rodent brain are shown in Figure 4.1 as a simplified schematic. ${ }^{383}$ Dopamine release upon reward-related stimuli is modulated by dopaminergic neurons that project from the VTA to the NAc. The inter-relationships between these neuronal 
projections and dopamine release is still not fully understood, however several models have been proposed ${ }^{383-385}$

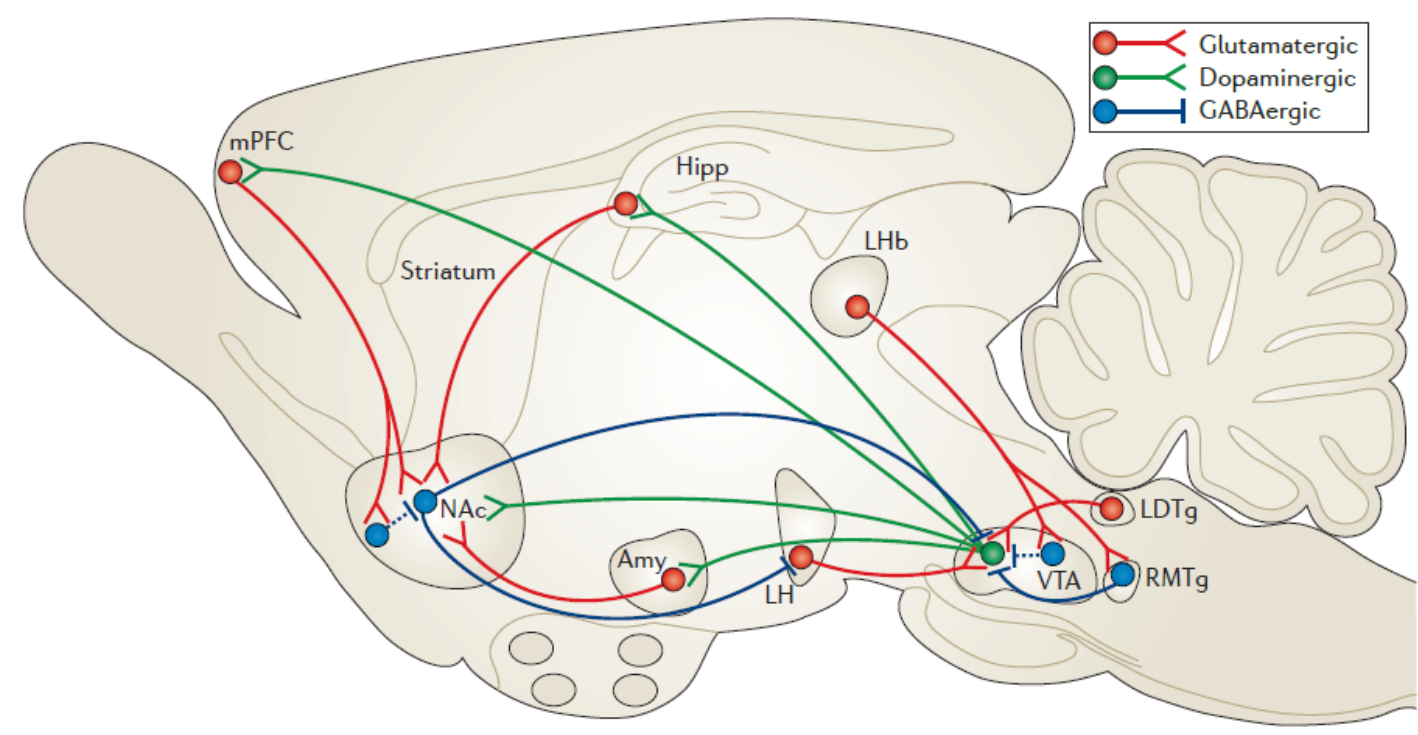

Figure 4.1: Schematic representation of the ventral tegmental area-nucleus accumbens (VTA-NAc) reward circuit. The major dopaminergic (green), glutamatergic (red) and GABAergic (blue) projections between the VTA and NAc in the rodent brain are shown. Other brain structures shown for reference include; the medial prefrontal cortex (mPFC), the striatum, the hippocampus (Hipp), the amygdala (Amy), the lateral dorsal tegmentum (LDTg), the rostromedial tegmentum (RTMg), the lateral habenula (LHb) and the lateral hypothalamus (LH). Reprinted by permission from Macmillan Publishers Ltd: Nature Reviews Neuroscience, (Russo, SJ., and Nestler, EJ. The brain reward circuitry in mood disorder. 2013. Nature Reviews Neuroscience. 14:609-625) ${ }^{383}$ copyright 2013.

Recall that N-methyl-D-aspartate (NMDA) receptors are involved in glutamate mediated synaptic transmission (see section 1.4.6.2.2). ${ }^{250}$ Systemic administration of NMDA receptor antagonists such as MK-801 have been shown to produce behavioural effects in animal models similar to the symptoms observed in patients with schizophrenia. ${ }^{378,386}$ One of the most commonly proposed mechanisms is the MK-801 induced hypofunction of NMDA receptors that leads to the disinhibition of glutamate and dopamine release. ${ }^{387}$ In particular, systemic administration of MK-801 was shown to 
produce hyperlocomotion, ataxia and stereotyped behaviour in rodents. ${ }^{388-390}$ On a cellular level, systemic administration of MK-801 was shown to stimulate the activity of mesolimbic dopaminergic neurons of the VTA which resulted in increased release of dopamine in brain regions such as the NAc. ${ }^{391,392}$ Work done to examine the actions of MK-801 on dopamine release was reviewed by Yan et al., 1997.393

\subsubsection{Justification for the MK-801 model of perseveration}

In particular, cognitive deficits in executive function and working memory prevent individuals with schizophrenia from unlearning behaviour. Cognitive function is affected such that the individual is not able to change, or inhibit their behavioural tendencies once they have learned a task regardless of whether the behavioural tendency they exhibit is contextually appropriate or not. Characteristic of individuals with schizophrenia, this is referred to as perseveration. ${ }^{394,395}$

In this work, perseveration was modeled by behavioural evaluation in an appetitive task following MK-801 administration. The behavioural model was made up of two phases: acquisition and extinction. During the acquisition phase, the animals were placed in an operant conditioning chamber (Figure 4.2) and trained to press a lever two times. Over a period of 5 consecutive days, the animals learned that pressing the left lever twice resulted in a chocolate pellet reward. As additional stimuli, the house light went off and the panel light located above the delivery box changed from red to green. Correct (pressing the left lever) and incorrect (pressing the right lever) pressing as well as nose pokes (as an indication of checking for the chocolate pellet reward) were recorded over a 30 minute period. During the extinction phase, correct lever pressing did not result in a 
chocolate pellet reward. Normal rodents were able to inhibit their behavioural tendencies such that the extinction phase started with high lever pressing, but behavioural frequency was rapidly extinguished.

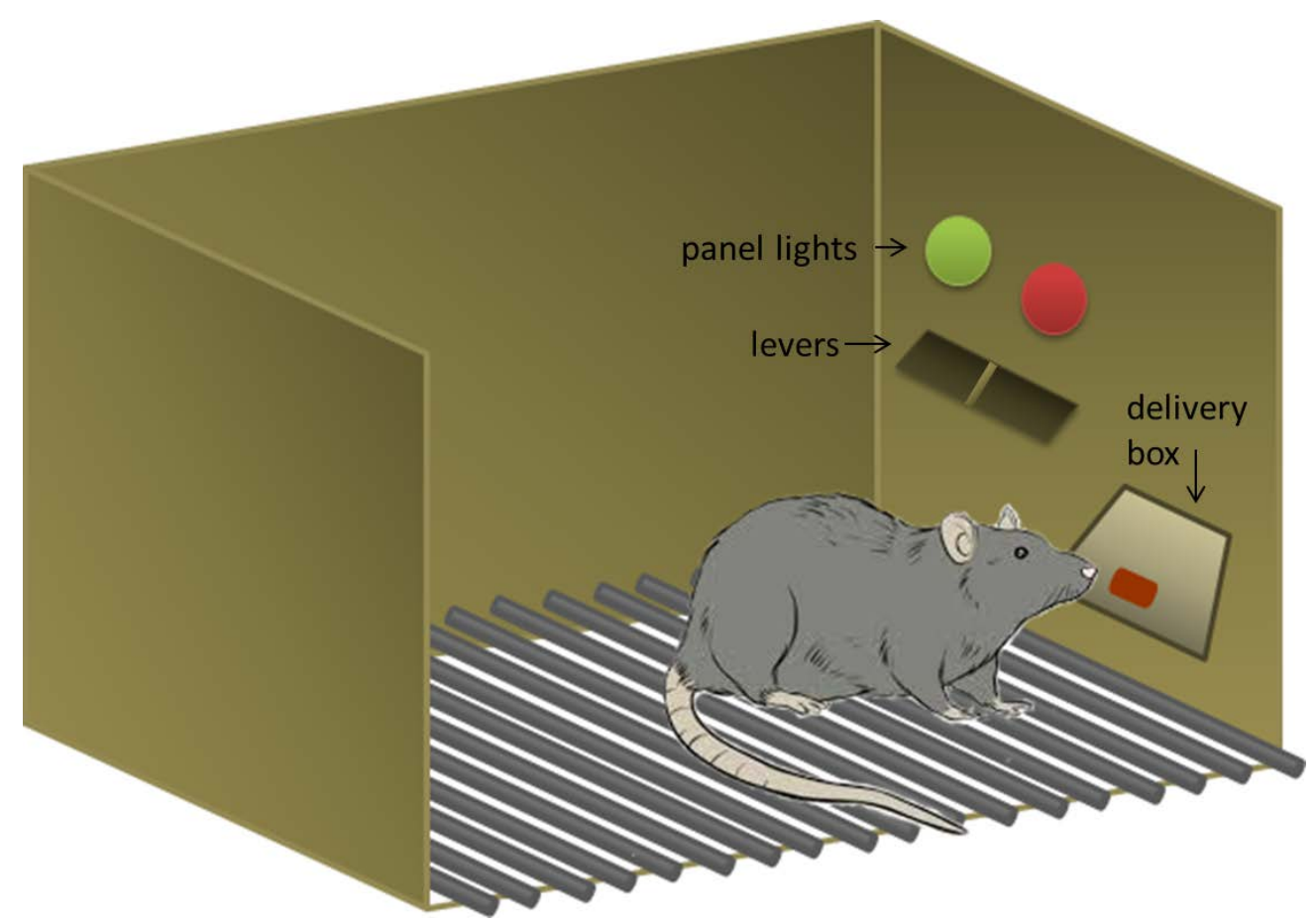

Figure 4.2: Illustration of the operant conditioning chamber. During the acquisition phase the animal is trained to press the lever twice, which shuts off the house light, changes the panel light from red to green and results in the delivery of a chocolate

Previously, Holahan et al., (2010) showed that the administration of a moderate dose $(0.05-0.1 \mathrm{mg} / \mathrm{kg})$ of MK-801 could be used to model perseveration in rodents during the extinction phase of the appetitive test. ${ }^{395}$ As described above, in this work animals were trained during the acquisition phase to press the left lever twice to receive a chocolate pellet reward. Acute MK-801 administration led to significantly elevated lever pressing in $0.1 \mathrm{mg} / \mathrm{kg}$ dose-treated rodents compared to control animals (saline treated). Neither the $0.05 \mathrm{mg} / \mathrm{kg}$ dose nor the $0.15 \mathrm{mg} / \mathrm{kg}$ dose showed a significant increase in correct 
lever pressing compared to saline treated controls. Likely the $0.05 \mathrm{mg} / \mathrm{kg}$ dose was not high enough to affect motor or cognitive behaviour, in contrast the $0.15 \mathrm{mg} / \mathrm{kg}$ dose negatively impacted motor behaviour, which led to decreased lever pressing overall. The effect of MK-801 administration on locomotor activity was assessed using an elevated cross maze. In this study, D1 and D2 receptor antagonists were shown to normalize MK801 induced behaviour. The data from this study was interpreted to suggest that treatment with MK-801 resulted in an over activity of dopamine in the nucleus accumbens (NAc) that manifested as cognitive deficits consistent with perseveration.

\subsubsection{Dopamine-binding aptamers (DBAs)}

Previously, Mannironi et al. (1997) selected an RNA aptamer that binds to dopamine with low micromolar affinity as determined by equilibrium filtration. ${ }^{280}$ The specificity of the aptamer was evaluated by affinity chromatography. Of particular relevance, the aptamer showed some affinity to norepinephrine (58 $\pm 13 \%$ of dopamine elution). Secondary structure analysis was used to predict that the aptamer formed a dopamine binding pocket through the interaction of two stem-loops. The RNA aptamer for dopamine has since been further investigated and multiple aptamer-based sensors have been described. These are discussed in Chapter 6 .

The DNA aptamer used in the experiments described in this chapter was reported by Walsh and DeRosa (2009). ${ }^{396}$ In this study, the DNA homolog of the RNA aptamer described by Mannironi et al. was prepared. ${ }^{280}$ Interestingly, the affinity of the DNA aptamer for dopamine (and norepinephrine) was maintained. Further, mutational analysis suggested the binding site was maintained between nucleic acid types. This work was an 
important example of the retention of aptamer function between RNA and DNA versions of the same aptamer sequence. Previously, only sequences that formed G-quadruplexes were shown to maintain similar functionality. ${ }^{289}$ Before this example, similar binding affinity had not been observed for aptamers forming other secondary structures. ${ }^{60,290}$ Like the RNA aptamer for dopamine, the DNA homolog has also be investigated extensively in sensor design (see Chapter 6).

\subsubsection{The dopamine-binding aptamer hypothesis}

The hypothesis that formed the foundation of this work was the idea that the DNA homolog of the dopamine-binding aptamer could attenuate dopamine-related behaviour by binding directly to synaptic dopamine, thereby rendering the dopamine unable to interact with post-synaptic receptors and autoreceptors. This interaction would effectively normalize synaptic dopamine concentrations until the excess dopamine was removed by naturally occurring mechanisms. In accordance with this hypothesis, following injection of the DNA homolog DBA directly into the nucleus accumbens of rodents, MK-801induced deficits in behaviour (due to elevated synaptic dopamine) would be normalized.

The measure of affinity an aptamer has for its target is represented by the dissociation constant $\left(\mathrm{K}_{\mathrm{D}}\right)$. This value represents a range of concentrations around which equilibrium of aptamer-target binding exists. Importantly, because of the micromolar dissociation constant of the DNA homolog DBA, the aptamer should only bind to dopamine and exert a noticeable effect on behaviour when dopamine concentrations are elevated above basal levels. Further, the DNA homolog DBA should not bind to 
dopamine when concentrations are at basal levels and therefore should not have deleterious effects on normal behaviour.

Though acting by a direct rather than indirect method, the effect of the aptamer on behaviour would be similar to the normalizing effect of D1/D2-like receptor antagonists on MK-801-induced deficits in behaviour. Traditional, D1/D2 dopamine receptor antagonists bind to dopamine receptors and prevent the effects of receptor agonists, thereby indirectly decreasing the effect of dopamine. ${ }^{397}$ Since the aptamer binds directly to dopamine and does not interact with D1/D2-like receptors, treatment with the dopamine binding aptamer was hypothesized to lead to reductions in perseveration that would be similar to that observed after treatment with D1/D2 receptor antagonists. 


\subsection{Materials and Methods}

\subsubsection{DNA and MK-801 preparation}

The DNA homolog DBA (5'-

\section{GTCTCTGTGTGCGCCAGAGACACTGGGGCAGATATG}

GGCCAGCACAGAATGAGGCCC-3') and random oligonucleotide control (5'AGAATCTGTCGGGCTATGTCACTAATACTTTCCAAACGCCCCGTACCGATGCT GAACA-3') were prepared using standard phosphoramidite chemistry on an automated DNA Synthesizer (MerMade, BioAutomation). Phosphoramidites (dA-CE, Ac-dC-CE, dmf-dG-CE and dT-CE), activator solution, cap A and cap B solutions, deblock solution, oxidizer solution and acetonitrile were purchased from Glen Research. $1.0 \mu$ mole synthesis columns (dC(Ac) and $\mathrm{dA}(\mathrm{Bz}): 500 \AA)$ were purchased from BioAutomation. The DNA sequences were purified by $12 \%$ polyacrylamide gel electrophoresis (PAGE). DNA was extracted from the gel by incubation and agitation in water at $37^{\circ} \mathrm{C}$ overnight. Gel pieces were removed by filtration with a $5 \mathrm{~mL}$ syringe (BD) through a PES $0.45 \mu \mathrm{m}$ $30 \mathrm{~mm}$ diameter syringe filter (Mandel Scientific). The filtrate was lyophilized (Labconco) then resuspended in a minimal amount of deionized water and desalted using Amicon Ultra Centrifugal Filter Units (Millipore) with a molecular weight cut off of $3000 \mathrm{~g} / \mathrm{mol}$. Sequence masses were confirmed by ESI-MS (Novatia). Following quantification by UV-Vis spectroscopy (Cary UV), the DNA solution was prepared to the appropriate concentration $(200 \mathrm{nM})$ in $10 \mathrm{mM}$ Tris buffer $(\mathrm{pH}=7.4)$.

MK-801 (Sigma Aldrich, IUPAC name:[5R,10S]-[+]-5-methyl-10,11-dihydro$5 \mathrm{H}$-dibenzo[a,d]cyclohepten-5,10-imine) was prepared the day of testing to $0.10 \mathrm{mg} / \mathrm{kg}$ 
$(\mathrm{pH}=7.4)$ in $0.9 \%$ sterile saline from a stock solution $(1.0 \mathrm{mg} / \mathrm{mL})$ in $0.9 \%$ sterile saline stored frozen $\left(-20^{\circ} \mathrm{C}\right)$. The chosen dosage was based on previous work. ${ }^{394,398-400}$

\subsubsection{Ethics Statement}

Experiments that required animals were designed and performed in accordance with the National Institutes of Health (NIH) Guide for the Use and Care of Laboratory Animals as well as the Canadian Council on Animal Care (CCAC) guidelines. Approval was obtained from the Carleton University Animal Care Committee (AUP ID P09-16).

\subsubsection{Subjects}

Male Long Evans rats $(\mathrm{n}=31)$ purchased from Charles River (St. Constant, Quebec, Canada) were housed in polycarbonate cages $(48 \times 26 \times 20 \mathrm{~cm})$ in groups of two pre-surgery and individually post-surgery. The temperature $\left(21^{\circ} \mathrm{C}\right)$ and lighting (12-hour light/dark cycle, lights on at 0800) of the vivarium in which the rats were housed were controlled. To minimize stress, animals were handled for 5 min each day for a period of 7 days. Pre-surgery and for 10 days post-surgery, animals were fed rat chow (Purina) ad libitum. After an approximately 10 day recovery period the rats' diet was restricted (approximately 8 days) until the animal weighed $85 \%$ of its initial weight (250-300 g).

\subsubsection{Intracranial surgical procedure}

Anesthesia was induced in the animals using $\sim 3 \%$ isoflurane in pure oxygen gas. To prepare the animals for surgery, their scalps were shaved and the exposed tissue was cleaned with $70 \%$ surgical alcohol and iodine solution. The animals were fixed in a stereotaxic apparatus (Stoeling Instruments) using lateral ear bars. Pain and discomfort were minimized by the application of Xylocaine to the tips of the ear bars. Eye dryness 
and damage were prevented by the application of tear gel to the surface of the eyes. According to the following coordinates relative to bregma: antero-posterior $(\mathrm{AP})=-1.7$, latero-medial $(\mathrm{LM})= \pm 1.5$ and dorso-ventral $(\mathrm{DV})=-6.0$, two stainless steel guide cannula (12 mm, $25 \mathrm{Ga})$ were bilaterally implanted in to the nucleus accumbens (Acb).

Dental cement was used to secure each cannula in place and obturators (32 Ga) were inserted. Following cannula insertion, Polysporin and Lidocaine were applied around the insertion site to prevent infection and minimize pain. Additionally at 0,12 and $24 \mathrm{~h}$ postsurgery, Metacam $(0.2 \mathrm{~mL})$ was administered subcutaneously to prevent pain. Cannula placement was confirmed as described in section 4.5.2.1.

\subsubsection{Behavioural testing: Operant Conditioning Procedure}

Two phases of behavioural training were performed after at least 10 days of recovery from surgery. In the first phase, acquisition, animals were trained in operant conditioning chambers with dimensions of $30.5 \mathrm{~cm}$ wide $\times 25.5 \mathrm{~cm}$ deep x $30.5 \mathrm{~cm}$ high (Coulbourn Instruments) that were housed in insulated casings. The chambers were equipped with a house light, two response levers, a panel of three LED lights (red, yellow and green) and a food hopper with pellet dispenser (see Figure 4.2). When the left response lever was pressed, the house light switched off, the red panel light changed to green and one $45 \mathrm{mg}$ chocolate pellet reward (BioServe, New Jersey) was released from the pellet dispenser into the food hopper. Left response lever presses were recorded as correct (eliciting a reward) and right lever presses were recorded as incorrect. Cumulative lever presses were recorded over a period of $30 \mathrm{~min}$ each day for a 5 consecutive days. 
The second phase of operant conditioning was extinction. During the extinction session animals were placed in the operant conditioning chambers described for the acquisition phase. In this phase, correct lever pressing initiated environmental changes (house light switched off and panel light changed from red to green) but no chocolate pellet reward was dispensed into the food hopper. The extinction session occurred three days following the final acquisition day. Cumulative lever presses were recorded over a period of $30 \mathrm{~min}$. A video was taken of the entire session.

\subsubsection{Behavioural testing: Locomotor testing}

Locomotor testing in an elevated $(75 \mathrm{~cm})$, closed wooden cross maze $(60 \times 9 \mathrm{~cm}$ arms) was performed with a subset of rats $(\mathrm{n}=25)$ one week after extinction testing. At the beginning of the test, each animal was placed into the center of the maze and their horizontal activity was monitored for $30 \mathrm{~min}$. An HVS Image 2100 Plus tracking system (HVS Image Ltd, UK) was used to record distance traveled, arm entries and speed.

\subsubsection{Administration}

\subsubsection{Central - intra accumbens injections}

The dopamine binding aptamer, random oligonucleotide control and tris buffer vehicle were injected directly into the nucleus accumbens. Following the removal of the obturators, two stainless-steel injection cannula ( $13 \mathrm{~mm}, 32 \mathrm{Ga})$ that were each connected to $10 \mu \mathrm{L}$ Hamilton syringes by polyethylene tubing were inserted into the guide cannula. The rate at which a $0.5 \mu \mathrm{L}$ injection $(0.25 \mu \mathrm{L} / \mathrm{min})$ was delivered by the syringes was controlled by an injection pump (Braintree Scientific, Inc.). To allow the injection to diffuse into the target nucleus accumbens tissue, the injection cannulai were left in place 
for $60 \mathrm{~s}$ post-injection. Animal stress was minimized by allowing the animals to move freely around their home cage during the injection.

\subsubsection{Peripheral - subcutaneous injections}

Either MK-801 (300 $\mu \mathrm{L}$ of $0.1 \mathrm{mg} / \mathrm{kg})$ or saline were administered via subcutaneous injection immediately following the intra-accumbens injection.

\subsubsection{Administration test groups}

All animals were run tested in the operant conditioning procedure and a subset of animals ( $n=5$ /group) were run through the locomotor testing. Experimental groups were as follows: intra-accumbens injection of tris buffer and subcutaneous saline ( 0 $\mathrm{nM} /$ saline, $\mathrm{n}=5)$; intra-accumbens tris buffer injection and subcutaneous MK-801 (0 $\mathrm{nM} / \mathrm{MK}, \mathrm{n}=7$ ); intra-accumbens injection of $200 \mathrm{nM}$ aptamer and subcutaneous injection of MK-801 (200 nM/MK, n=7); intra-accumbens random oligonucleotide injection and subcutaneous MK-801 (random/MK, n=7); intra-accumbens injection of $200 \mathrm{nM}$ aptamer and subcutaneous saline (200 nM/saline, $\mathrm{n}=5)$.

\subsubsection{Confirmation of cannula placement}

Sections used to verify cannula placement were additionally stained with cresyl violet as follows. Slides were rinsed with distilled water and placed in a $1 \%$ cresyl violet solution for 3 minutes. Excess stain was washed with distilled water, and the slides were immersed in $0.8 \%$ acetic acid solution for 3-5 min. Slides were placed in clarene solution for 15 minutes and then a cover slip was applied using Clarion mounting medium.

Photographs of the brain slices were taken using an Olympus light microscope at $4 \mathrm{X}$ and 10X magnifications. 


\subsubsection{Histology and Immunohistochemisty}

\subsubsection{Preparation of samples for Immunohistochemistry}

Immediately post extinction testing, animals that had been injected subcutaneously with $0.1 \mathrm{mg} / \mathrm{kg}$ MK-801 and either $200 \mathrm{nM}$ aptamer $(\mathrm{n}=3)$ or tris buffer $(\mathrm{n}=3)$ were euthanized to examine the in vivo effects on the nucleus accumbens. Brains were fixed by immersion in 4\% paraformaldehyde (in $0.01 \mathrm{M}$ phosphate buffer, $\mathrm{pH}=$ 7.4) at $4{ }^{\circ} \mathrm{C}$ as previously described. ${ }^{401}$ Tissue was incubated with primary antibodies, either mouse anti-tyrosine hydroxylase (1:1000; Immunostar) or rabbit antiphosphorylated tyrosine hydroxylase (S40, 1:500; Abcam) at room temperature, overnight. Secondary antibody incubation with either goat anti-mouse 488 or goat antirabbit 594 (1:500; Molecular Probes) respectively was performed at room temperature for $2 \mathrm{~h}$. Slices $(30 \mu \mathrm{m})$ were mounted on glass slides and cover-slipped using Fluoromount (Sigma) in preparation for immunohistochemical quantification.

\subsubsection{Immunohistochemical quantification}

An Olympus BX61 microscope (Olympus Canada, ON) was used to obtain digital images of the nucleus accumbens core and shell regions (20X, NA 0.4; InVitro version 3.2.21 Media Cybernetics, MD). Photobleaching was reduced and intensities across subjects were equalized by using the same exposure time. Image-Pro Analyzer (version 6.2.1.491; Media Cybernetics, MD) was used to generate pixel intensity maps for both the $\mathrm{TH}$ and $\mathrm{pTH}$ stained samples. In brief, the generation of five horizontal lines across the entirety of the nucleus accumbens region allowed for the collection of intensity measures starting at the anterior commissure, then the accumbens core and finally the 
shell. This allowed for the generation of an average raw pixel intensity profile. Since core staining at the exposure times used was negligible, intensity measurements in the anterior commissure were used to normalize intensity measurements of the shell. To account for variation in staining or image processing, statistical analyses (t-tests) between groups were made based on normalized intensities of TH intensity, pTH intensity and the ratio of pTH:TH. 


\subsection{Results and Discussion}

\subsubsection{Behavioural data and discussion}

\subsubsection{Behavioural testing: Acquisition}

During the acquisition phase, animals were trained (drug-free) during a $30 \mathrm{~min}$ session for five consecutive days. On each day animals were trained to press the correct lever twice and subsequently receive a chocolate pellet reward. Over the 5 consecutive acquisition sessions the animals learned that correct lever pressing led to a chocolate pellet reward. Examining the cumulative presses $/ 5 \mathrm{~min}$ interval over the 5 consecutive days revealed that maximum pressing for all groups was reached between the third and fourth session (Figure 4.3).

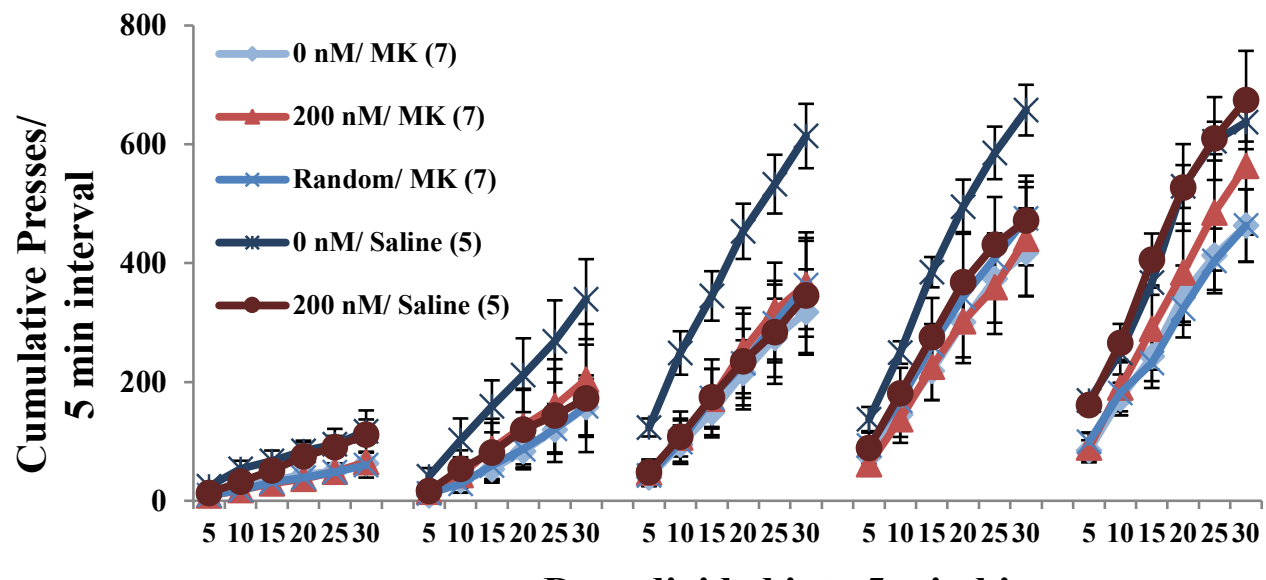

Days divided into 5 min bins

Figure 4.3: During the acquisition phase, the cumulative correct lever presses/5 min interval over the 30-min session, by day reached a maximum between days 4 and 5 . Groups were randomly assigned to be pretreated with intra-accumbens vehicle (0 $\mathrm{nM} / \mathrm{MK} ; \mathrm{n}=7)$, aptamer $(200 \mathrm{nM} / \mathrm{MK} ; \mathrm{n}=7)$, random oligonucleotide control (Random/MK; $n=7)$, saline control ( $\mathrm{nM} / \mathrm{MK}$; $n=5)$ and aptamer/saline control (200 
$n M /$ Saline; $n=5)$. Numbers in parenthesis $=$ animals per group. Data expressed as mean \pm SEM.

To determine whether or not there was a significant difference between the acquisitions (correct lever pressing) of the randomly assigned groups, the mean presses/day for each group were compared and are shown in Figure 4.4.

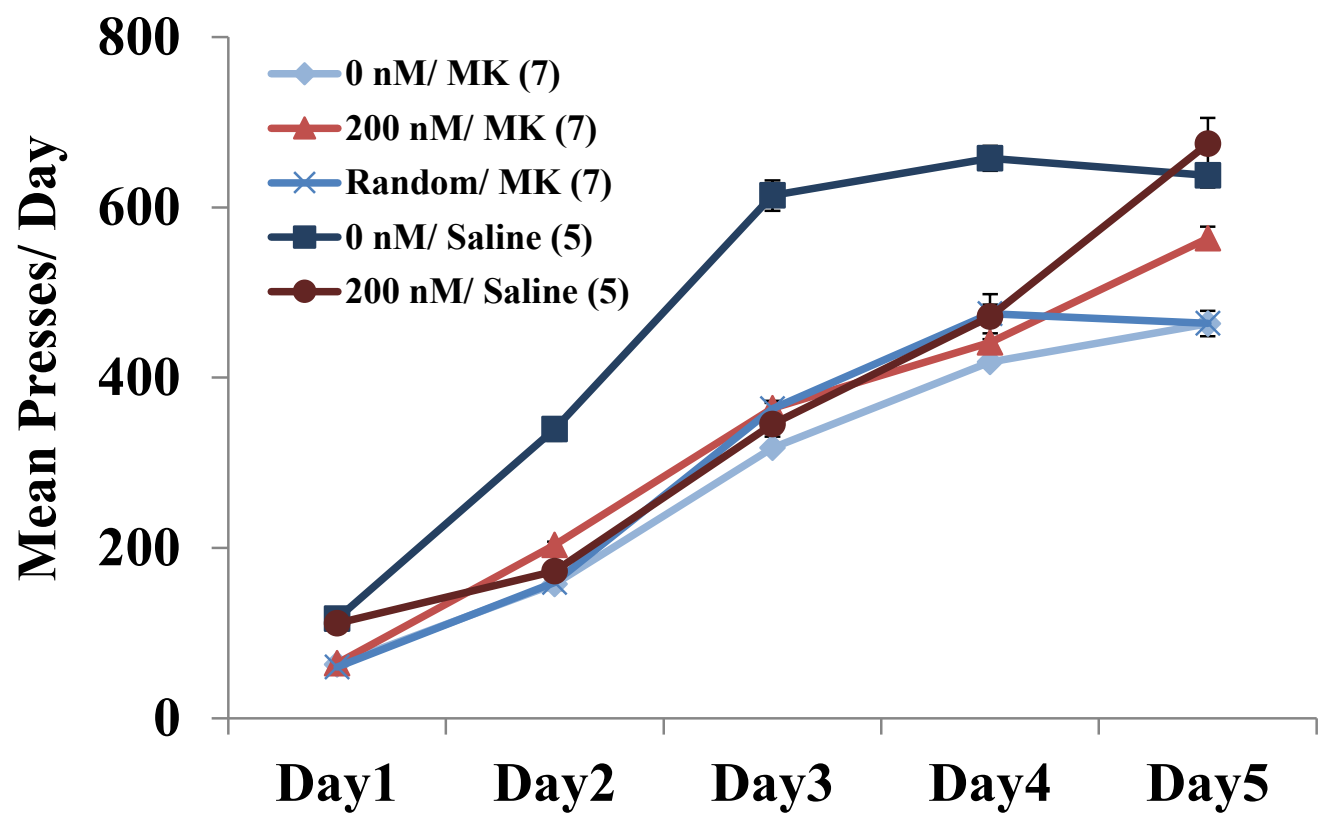

Figure 4.4: Summary of mean correct lever presses by day during the acquisition phase. Groups were randomly assigned to be pretreated with intra-accumbens vehicle (0 nM/MK; $n=7)$, aptamer ( $200 \mathrm{nM} / \mathrm{MK} ; \mathrm{n}=7)$, random oligonucleotide control (Random/MK; $n=7)$, saline control $(0 \mathrm{nM} / \mathrm{MK}$; $n=5)$ and aptamer/saline control (200 nM/Saline; $n=5)$. Two-way ANOVA (day by group) revealed a significant effect of day $(F(4,16)=94.44, p<0.001)$, and no group differences $(F(4,26)$ $=1.57)$ or interaction $(f(16,104)=1.27) . p<0.05$; numbers in parenthesis $=$ animals per group. Data expressed as mean \pm SEM.

To ensure that there was no significant difference between groups prior to treatment, the mean number of correct presses per group was analysed by a two-way ANOVA (day by 
group). There was a main effect of day on the mean number of correct presses observed $(F(4,16)=94.44, p<0.001)$ supporting the observation that correct lever pressing increased by day. As expected, neither a significant difference between groups $(F(4,26)=1.57)$ nor an interaction $(F(16,104)=1.27)$ were observed. The statistical analysis suggests that each group was able to learn the task as expected. Mean cumulative correct presses increased over the acquisition period. This reflects that animals learned to press the lever twice to receive a chocolate pellet and by the last day were pressing the lever upwards of 600 times.

\subsubsection{The efficacy of the DBA was tested in an MK-801 induced model of schizophrenia-like cognitive deficits}

MK-801 has been proposed to increase the firing rates of mesolimbic dopaminergic neurons and therefore increase dopamine output in the striatum and frontal cortex. ${ }^{402-405}$ Administration of MK-801 has been used to produce a rodent model of cognitive deficits associated with schizophrenia wherein, rodents treated with moderate doses of MK-801 (0.05-0.1 mg/kg) showed persistent elevated lever pressing throughout the duration of an entire 30 min extinction session. ${ }^{395}$ In this same study, pre-treatment with D1/D2-like receptor antagonists resulted in normalized extinction behaviour. There are two particularly relevant models of schizophrenia-like cognitive deficits which may explain these observations. Carlsson and colleagues proposed that cortical dopamine output is regulated by complementary "accelerator" and "brake" pathways (Figure 4.5) ${ }^{406,407}$ In this model, the brake pathway is made up of glutamate and GABA synapses, which help to regulate monoamine neurotransmitter (for example dopamine, 
norepinephrine, 5 -HT etc.) synapses. ${ }^{406}$ In the accelerator pathway, glutamate synapses project directly to monoaminergic neurons. When the system is functioning normally, a balance exists between the brake and accelerator pathways wherein the brake is slightly more active. ${ }^{406}$ When these pathways are disrupted, for example by an MK-801 reduction of glutamate function, dopamine (and other monoamine neurotransmitter) release is elevated in brain regions, such as the nucleus accumbens, to which those neurons project. $^{406}$
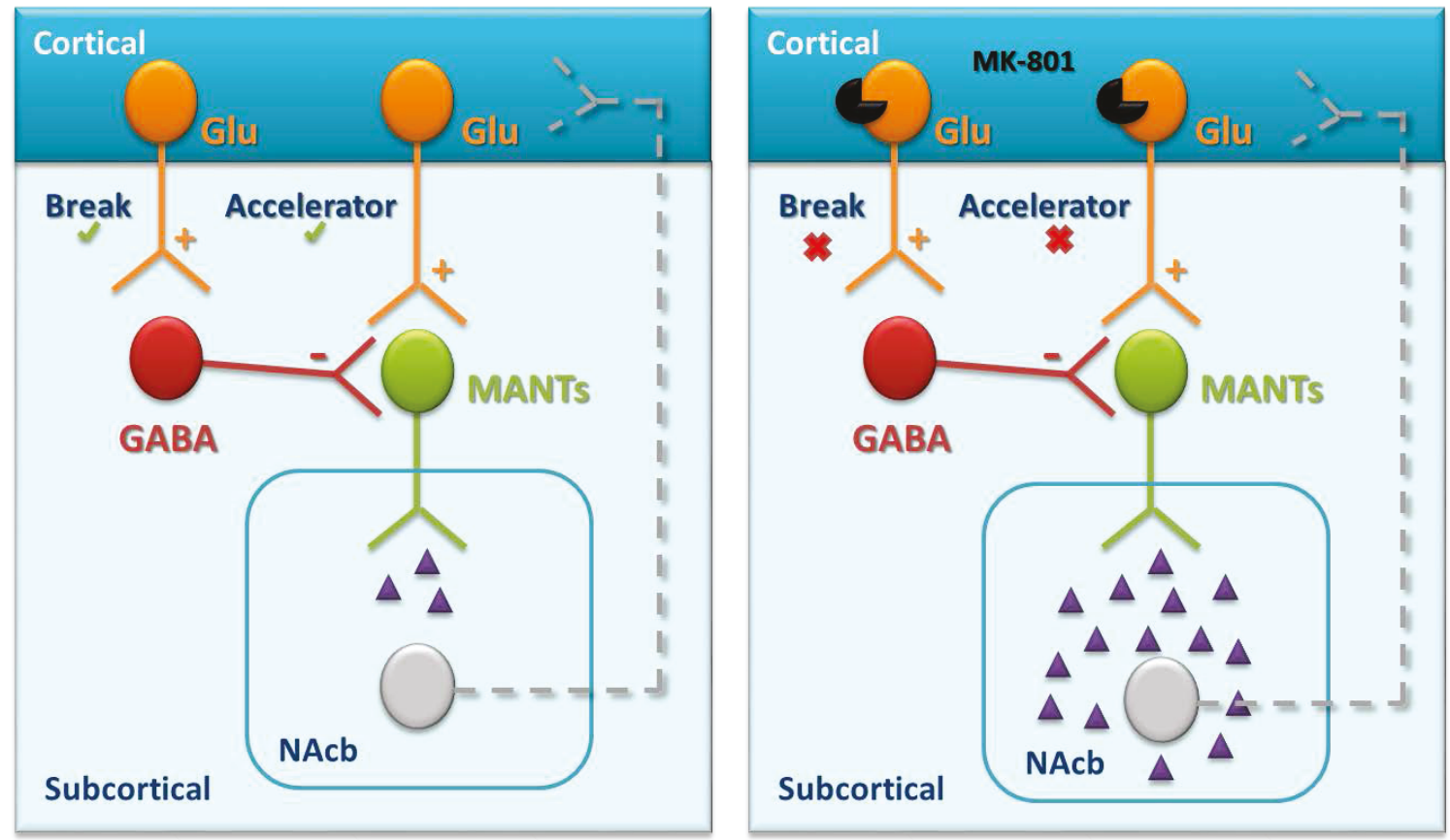

Figure 4.5: Dopamine (purple) release from monoaminergic neurons (MANTs) is mediated by direct glutamatergic (Glu) and indirect glutamatergic/GABAergic (GABA) neuronal pathways. A balance between the accelerator and brake pathways regulates dopamine (DA) and other monoaminergic neurotransmitter release. Typically (left) the brake pathway predominates to regulate normal dopamine release. Failure (right) of these pathways, for example by MK-801 (black) induced hypoglutamatergia, results in over activation of MANTs, and therefore increased dopamine release in terminal projection sites, such as the nucleus accumbens (NAcb). ${ }^{406,407}$ 
Previously, MK-801 administration was shown to reduce neural activation of the infralimbic cortex, and elevated extinction pressing was observed. ${ }^{395}$ From this work, Holahan et al. proposed that MK-801 may reduce cortical glutamatergic control and therefore increase the rates of midbrain dopaminergic activity, which would result in the enhanced dopamine-dependent extinction pressing representative of perseveration.

Alternatively, treatment with MK-801 may have led to the alteration of the fundamental motivational state, which would have intensified the reinforcing properties of the conditioning cues (such as the change in the house-light and the chocolate pellet reward). Previously, MK-801 was shown to increase the effect of food-motivated behavioural responses in both conditioned and unconditioned scenarios. ${ }^{408-410}$ Additionally, Holahan et al., showed that MK-801 treatment led to prolonged progressive ratio responding compared to saline-treated control animals. ${ }^{395}$ In these cases, these enhanced behavioural responses are likely due to MK-801 related elevated dopamine concentrations in the nucleus accumbens (which is consistent with the accelerator/brake model), MK-801 related deficits in signaling processes that are involved in the termination of eating and satiety signaling, or the MK-801 initiation of non-specific perseveration of ongoing behaviours. ${ }^{395,409,411,412}$ Regardless of underlying mechanism, the behavioural changes observed in the extinction testing are most likely due to elevated dopamine concentrations in the nucleus accumbens. For these reasons, the nucleus accumbens was chosen as the site of aptamer injection. 


\subsubsection{Behavioural testing: Extinction}

The ability of the DBA to attenuate behaviour was examined during an extinction session. Following the acquisition phase and a consecutive $48 \mathrm{~h}$ non-test interval, the animals were systemically injected with either MK-801 $(0.1 \mathrm{mg} / \mathrm{kg} / \mathrm{ml})$ or saline and acutely injected $(0.5 \mu \mathrm{L}$ per hemisphere) into the nucleus accumbens with the aptamer, a random oligonucleotide control, or tris buffer vehicle. Fifteen minutes following systemic injections the animals were placed into the operant conditioning chamber for a 30 minute session. The following groups were examined where the intra-accumbens treatment was followed by the systemic treatment: tris buffer and MK-801 ( $0 \mathrm{nM} / \mathrm{MK} ; \mathrm{n}=7), 200 \mathrm{nM}$ aptamer and MK-801 (200 nM/MK; n=7), 200 nM random oligonucleotide control and MK-801 (Random/MK; $n=7)$, tris buffer and saline ( $0 \mathrm{nM} /$ Saline; $\mathrm{n}=5)$, and $200 \mathrm{nM}$ aptamer and saline (200 nM/Saline; $\mathrm{n}=5)$. During the extinction phase, the animals were not rewarded with a chocolate pellet following correct lever pressing. The extinction session was video recorded. 30 -second video samples from approximately 20 min into the 30 min session for a rat directly injected with aptamer and systemically injected with MK-801 (Video 1) and a rat treated with direct injection of tris buffer and systemically injected with MK-801 (Video 2) are available to view online as supporting info for the published article at the following digital object identifiers;

doi:10.1371/journal.pone.0022239.s001, and doi:10.1371/journal.pone.0022239.s002 respectively. ${ }^{375}$ In the videos, the control animal $(0 \mathrm{nM} / \mathrm{MK})$ continues to press the lever repeatedly and check the food delivery box despite not receiving a chocolate pellet reward. This animal is shown to perseverate. On the contrary, the aptamer treated animal (200 nM/MK) was able to extinguish the pressing behaviour, and perseveration was not 
observed. The animal pressed the lever a few times, checked for a reward and then stopped pressing the lever completely. The mean cumulative correct presses over the entire session for each group are summarized in Figure 4.6.

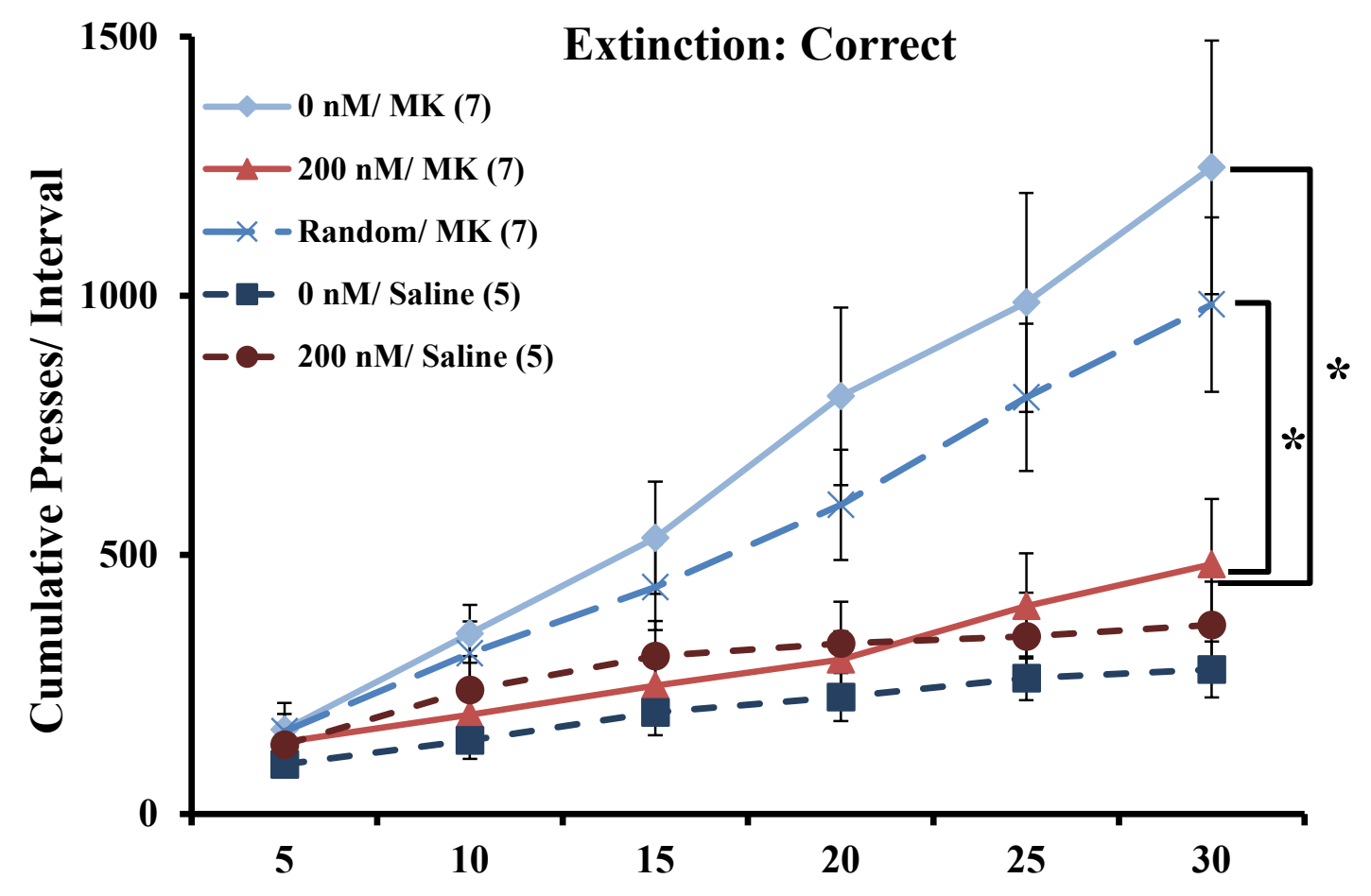

Figure 4.6: Cumulative correct lever presses during the extinction phase ( $30 \mathrm{~min}$ session). Groups pretreated with intra-accumbens vehicle $(0 \mathrm{nM} / \mathrm{MK} ; \mathrm{n}=7)$, aptamer $(200 \mathrm{nM} / \mathrm{MK} ; \mathrm{n}=7)$, random oligonucleotide control (Random/MK; $n=7)$, saline control $(0 \mathrm{nM} / \mathrm{MK} ; \mathrm{n}=5)$ and aptamer/saline control ( $200 \mathrm{nM} / \mathrm{Saline}$; $\mathrm{n}=5)$. Twoway, repeated measures ANOVA (group by 5 min time interval) revealed a significant main effects of group $(F(4,26)=3.84, p<0.05)$ and interval $(F(5,20)=$ $56.74, p<0.001)$. Significant interactions between interval and group were observed $(F(20,130)=6.47, p<0.001) . *, p<0.05$. Numbers in parenthesis $=$ animals per group. Data expressed as mean \pm SEM.

A two-way, repeated measures ANOVA (group by 5-min interval) was used to analyze the mean cumulative correct lever presses during the extinction session. Main effects of group $(F(4,26)=3.84, p<0.05)$ and interval $(F(5,20)=56.74, p<0.001)$ were observed. 
Additionally, a significant interaction between interval and group $(\mathrm{F}(20,130)=6.47$, $\mathrm{p}<0.001)$ was revealed. Comparison between groups by post-hoc analysis using Fisher's Least Significant Difference (LSD) revealed significant differences between the 200 $\mathrm{nM} / \mathrm{MK}$ group and the non-aptamer treated group, $0 \mathrm{nM} / \mathrm{MK}(\mathrm{p}<0.05)$. This suggests that the aptamer had a normalizing effect on extinction pressing behaviour. Additionally, there was a significant difference between the $200 \mathrm{nM} / \mathrm{MK}$ group and the random/MK group $(\mathrm{p}<0.05)$, but no significant difference between the random/MK group and the 0 $\mathrm{nM} / \mathrm{MK}$ group. This random/MK control group was important to include to show that the presence of foreign DNA alone was not responsible for the marked decline in extinction pressing. Comparison of the $200 \mathrm{nM} / \mathrm{MK}$ group to both the $200 \mathrm{nM} /$ saline and 0 $\mathrm{nM} /$ Saline groups showed no significant difference, further supporting that treatment with $200 \mathrm{nM}$ aptamer can normalize extinction pressing behaviour to that of non MK-801 treated controls. The results of this study indicated that extinction was successfully instated following aptamer $(200 \mathrm{nM})$ pre-treatment of MK-801 induced hypoglutamatergic/hyperdopaminergic animals. Consistent with the theory that MK-801 causes over activation of the mesolimbic dopaminergic system and therefore animals fail to achieve extinction behaviour, MK-801-treated control animals showed elevated lever pressing. However, these data suggest that the aptamer was able to successfully bind dopamine within the nucleus accumbens and in doing so, normalized extinction behaviour by dampening the effects of MK- 801 .

Cumulative incorrect lever pressing was also recorded. The mean cumulative incorrect lever pressing, by 5-min interval for the entire 30 min extinction session for each group are shown in Figure 4.7. 


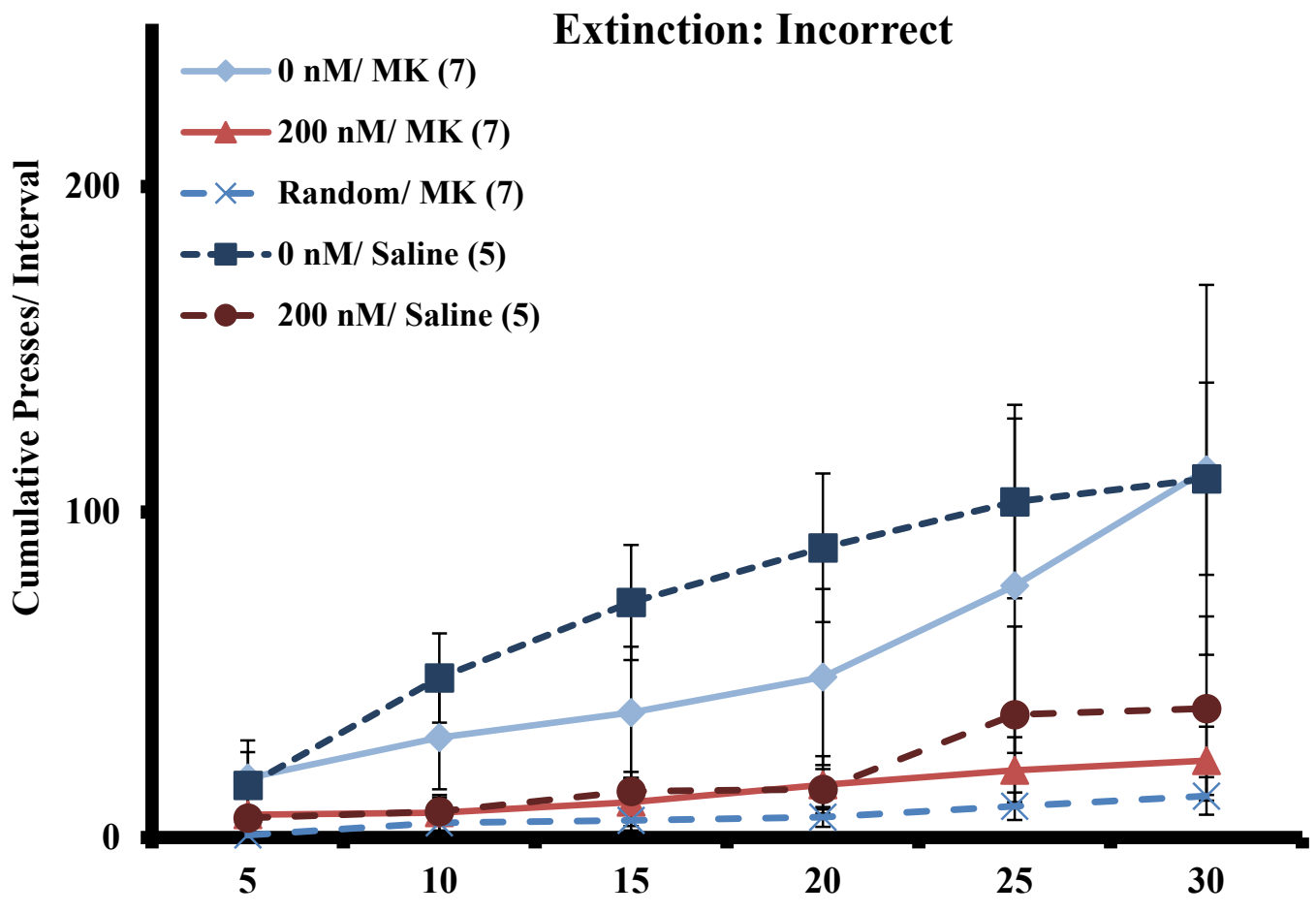

Figure 4.7: Cumulative incorrect lever pressing during the extinction phase. Groups pretreated with intra-accumbens vehicle $(0 \mathrm{nM} / \mathrm{MK} ; \mathrm{n}=7)$, aptamer $(200 \mathrm{nM} / \mathrm{MK}$; $n=7)$, random oligonucleotide control (Random/MK; $n=7)$, saline control (0 $\mathrm{nM} / \mathrm{MK} ; \mathrm{n}=5)$ and aptamer/saline control (200 $\mathrm{nM} / \mathrm{Saline}$; $\mathbf{n}=5)$. A two-way ANOVA (group by 5-min interval) revealed no significant main effect. Numbers in parenthesis $=$ animals per group. Data expressed as mean \pm SEM.

Statistical analysis by two-way ANOVA (group by 5-min interval) revealed no significant differences between groups. This measure was an important control as it provided evidence that the effect of MK-801 was specific to extinction behaviour and the associated correct lever pressing, since MK-801 treated animals showed specifically elevated correct lever pressing and not just elevated lever pressing overall. 
During the extinction session the number of nose pokes, a behaviour indicative of reward seeking was recorded. The mean nose pokes per 5 min interval for each group are summarized in Figure 4.8.

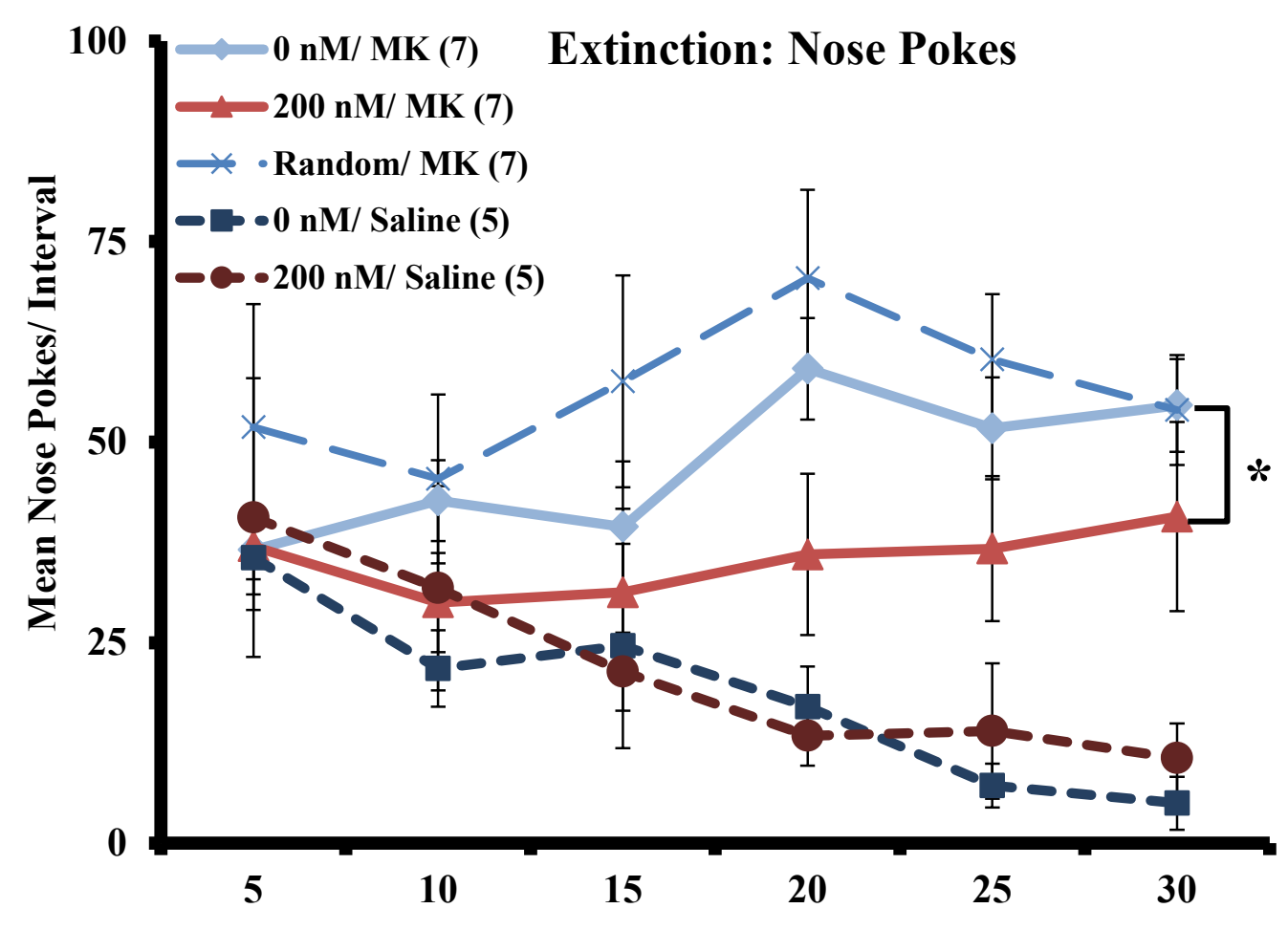

Figure 4.8: Nose pokes during the extinction session as an indication of reward seeking behaviour. Groups pretreated with intra-accumbens vehicle $(0 \mathrm{nM} / \mathrm{MK}$; $\mathrm{n}=7$ ), aptamer (200 nM/MK; $\mathrm{n}=7$ ), random oligonucleotide control (Random/MK; $\mathrm{n}=7)$, saline control $(0 \mathrm{nM} / \mathrm{MK} ; \mathrm{n}=5)$ and aptamer/saline control $(200 \mathrm{nM} /$ Saline; $n=5)$. A two-way ANOVA revealed a significant main group effect $(F(4,26)=6.10$, $\mathbf{p}<0.05)$. Numbers in parenthesis $=$ animals per group. Data expressed as mean \pm SEM.

Animals that were able to extinguish pressing behaviour would be expected to show decreased nose pokes as the extinction session progressed. A two-way (group by 5 min interval), repeated measures ANOVA was performed. Analysis revealed a main effect of group $(\mathrm{F}(4,26)=6.10, \mathrm{p}<0.05)$ and a significant interaction between nose pokes and 
groups $(F(20,130)=1.92, p<0.05)$. Post-hoc analysis by Fisher's LSD revealed a significant decline in mean nose presses between the $200 \mathrm{nM} / \mathrm{MK}$ and Random/MK groups $(\mathrm{p}=0.020)$. A decrease in mean nose pokes of the $200 \mathrm{nM} / \mathrm{MK}$ group compared to the $0 \mathrm{nM} / \mathrm{MK}$ group was observed, but the difference was not significant $(\mathrm{p}=0.171)$. The differences observed between the mean nose pokes of the $200 \mathrm{nM} / \mathrm{MK}$ group and both the saline control groups were not significant $(\mathrm{p}<0.05)$. Since nose pokes are a secondary indication of reward seeking behaviour, the overall decrease in nose poke behaviour of the $200 \mathrm{nM} / \mathrm{MK}$ group compared to the MK-treated controls is perhaps indicative of a decreased tendency of these animals to seek the chocolate pellet reward.

\subsubsection{Behavioural testing: Locomotor activity}

One week following the extinction, a subset of animals was randomly reassigned for locomotor testing. To minimize the conflicting influence of anxiety, the locomotor activity of each animal was testing in an elevated cross maze with closed arms (shown in Figure $10 \mathrm{~A}$ ), as opposed to one with open and closed arms which is typically used to test anxiety. ${ }^{413}$ Following intra-accumbens injection with aptamer, random oligonucleotide control or tris and either MK-801 or saline, the animals were placed in a closed, elevated cross maze. Over a 30 min period distance traveled $(\mathrm{m})$, speed $(\mathrm{m} / \mathrm{s})$ and arm entries data was collected and is shown in Figure 4.9. Arm entries measurements were collected automatically and manually. The number of animals in the $200 \mathrm{nM} / \mathrm{saline}$ group is smaller than the other groups because the data from one animal was lost. 
A

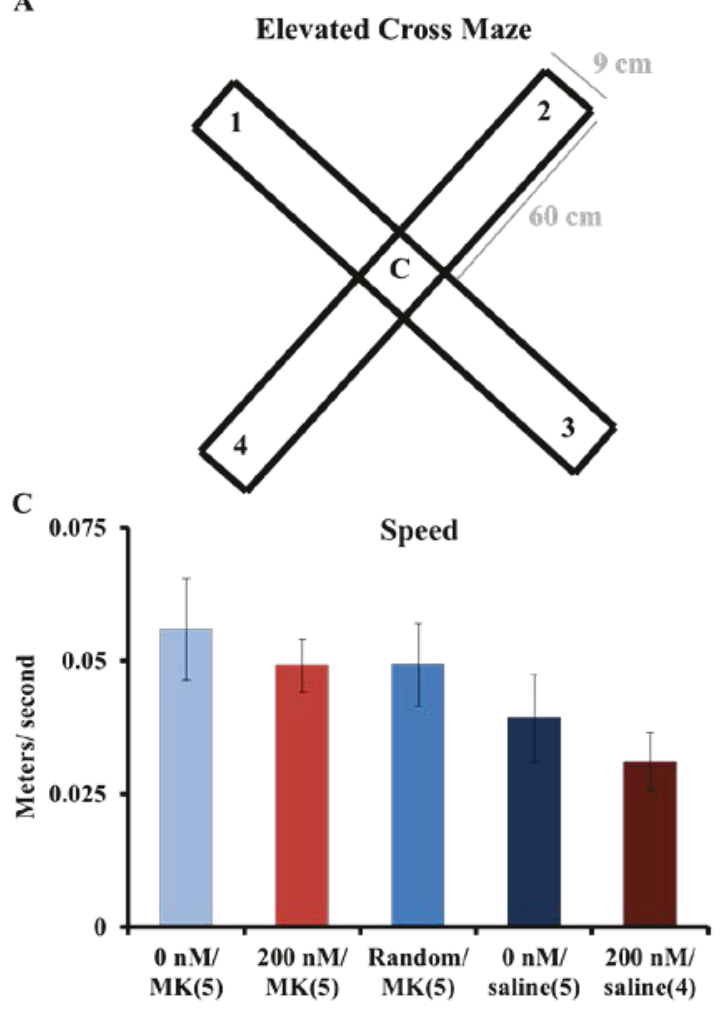

B
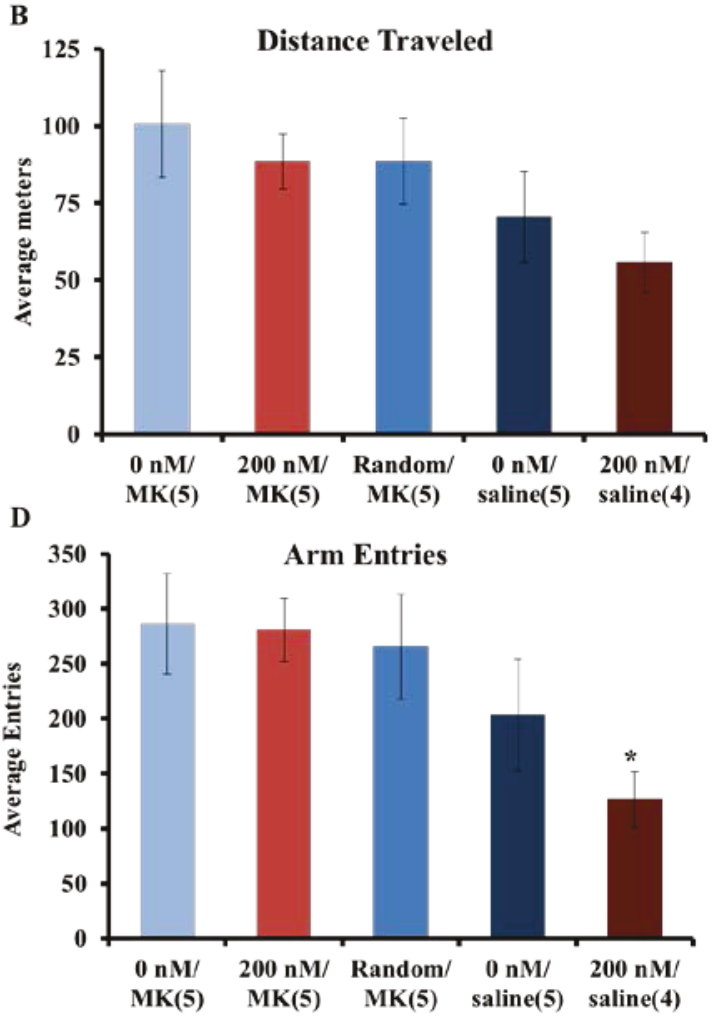

Figure 4.9: Intra-accumbens injection of the DBA did not significantly affect motor behaviour. The locomotor activity of a subset of animals was measured in an elevated cross maze (A) over a 30 min session. Animals were placed in the center of the maze (denoted by $\mathrm{C}$ in Figure $10 \mathrm{~A}$ ) and their locomotor activity was measured as the total distance (meters) travelled (B), the average speed (meters/sec; $C$ ) and the total arm entries (D). Groups were pretreated with intra-accumbens vehicle (0 $\mathrm{nM} / \mathrm{MK} ; \mathrm{n}=5)$, aptamer $(200 \mathrm{nM} / \mathrm{MK} ; \mathrm{n}=5)$, random oligonucleotide control (Random/MK; $n=5)$, vehicle/saline control $(0 \mathrm{nM} / \mathrm{MK} ; \mathrm{n}=5)$ and aptamer/saline control (200 $\mathrm{nM} /$ Saline; $\mathrm{n}=4)$. Number in parenthesis is number of animals per group. Data is presented as mean $\pm \operatorname{SEM} .(*, p<0.05)$

For each dependent measure (distance, speed, arm entries) a separate one-way ANOVA was run. No significant main effects of group were revealed $(F(4,19)=1.53, F(4,19)=$ 1.53 and $F(4,19)=2.31$ for distance, speed and arm entries respectively). The number of arm entries in all MK-801 injected groups were significantly higher than the 200 
$\mathrm{nM} /$ saline treated group $(*, \mathrm{p}<0.05)$. These data are consistent with previous studies which have shown that horizontal locomotor activity is elevated in MK-801-injected animals, regardless of the apparatus used to measure motor behaviour. ${ }^{395,414-416} \mathrm{An}$ important conclusion from this data is that a general lack of locomotor activity was not responsible for the extinction observed in the $200 \mathrm{nM} / \mathrm{MK}$ group since there was no difference in locomotor activity between the MK-801 injected control and experimental groups. There was a significant reduction in the number of arm entries observed for the $200 \mathrm{nM} / \mathrm{saline}$ group compared to the MK-801 treated groups, however the 200 $\mathrm{nM} /$ saline group was not significantly different from the $0 \mathrm{nM} /$ saline group. Therefore, it is unlikely that the observed reduction of arm entries of the $200 \mathrm{nM} /$ saline treated group was due to an effect of the aptamer on locomotor behaviour.

\subsubsection{Behavioural effects as a result of the DBA's affinity for dopamine}

Aptamers interact with their cognate target through some combination of hydrogen bonding, stacking interactions, electrostatic interactions, and van der Waals forces. ${ }^{417}$ The strength of these interactions is quantitatively described as the affinity of the aptamer for its target. The affinity an aptamer has for its target is represented by the dissociation constant $\left(\mathrm{K}_{\mathrm{D}}\right) .{ }^{418}$ The dissociation constant also effectively describes the working concentration range of the aptamer, and therefore the rational choice of an aptamer with a particular dissociation constant would yield noticeable effects from significant target binding only in instances where the target, in this case dopamine was well above basal levels. The DBA was chosen as a good candidate to investigate because it was shown to exhibit $\mathrm{K}_{\mathrm{D}}$ values in the hundreds of nanomolar range for both DA and 
NE. Basal levels of DA in the nucleus accumbens are typically 50 to 70 times less than the $\mathrm{K}_{\mathrm{D}}$ values of the aptamer for DA and NE. ${ }^{419}$ Therefore, the aptamer-DA binding was expected to be very low, producing no noticeable behavioural effects in animals with basal catecholamine levels. In fact, no behavioural effects were observed in non-MK801-treated animals who had received the aptamer pre-treatment. During the extinction testing, the mean cumulative correct lever pressing of the $200 \mathrm{nM} /$ Saline group was not significantly different than the $0 \mathrm{nM} /$ Saline group. Further, the $200 \mathrm{nM} /$ Saline group was not significantly different than the $0 \mathrm{nM} /$ Saline group on any measure of locomotor behaviour (distance travelled, speed, or arm entries).

Dopamine concentrations in the nucleus accumbens were previously shown to increase by $135-145 \%$ in MK-801 treated rats. ${ }^{393}$ Additionally, similar increases in dopamine concentrations were observed in animals presented with cues previously associated with a food reward. ${ }^{420}$ Since similar dopamine concentration increases likely occurred during the extinction experiment described herein, the dopamine concentration (though below the $\mathrm{K}_{\mathrm{D}}$ of the aptamer) in the nucleus accumbens of examined animals may have been elevated enough that aptamer binding resulted in noticeable behavioural effects.

Finally, the ability of the DBA to bind NE should be addressed. Though the aptamer has similar affinity to both DA and NE, both the basal and abnormal concentrations of NE are approximately an order of magnitude lower in the nucleus accumbens than those of DA. ${ }^{393}$ As such, the DBA would preferentially bind to DA and therefore DBA-NE binding would be insignificant. Though DBA-NE complex formation 
is not impossible, due to the relative concentrations of each catecholamine in the nucleus accumbens it is likely that the observed behavioural effects are more likely the result of the DBA binding to DA.

\subsubsection{Histology and Immunohistochemistry data and discussion}

\subsubsection{Confirmation of the cannula placement}

The placement of the cannula and injector into the nucleus accumbens according to the calculated stereotaxic coordinates was confirmed by histological analysis (Figure 4.10). Placement (indicated by the orange arrows) was revealed by staining with cresyl violet. 

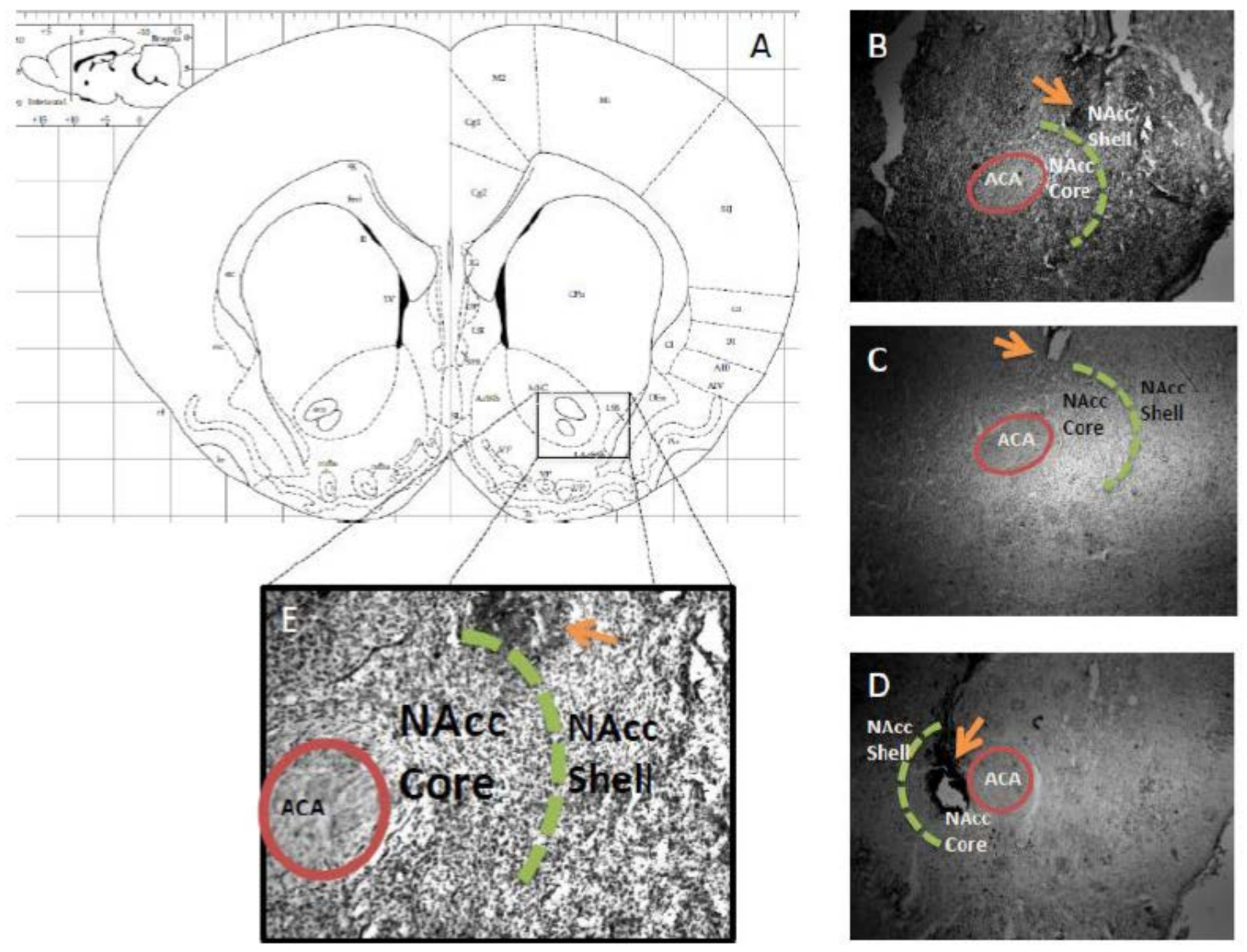

Figure 4.10: Confirmation of cannula placement in the nucleus accumbens of aptamer treated and control animals (reproduced with permission from Dan Madularu's M.Sc. thesis (Carleton University, 2010)). A) Coronal section of a rat brain. The location of the nucleus accumbens is shown in the small square. B-D) The placement of cannula and injector (indicated by orange arrows) in three different animals pre-treated with aptamer is shown. Staining of the brain coronal sections (4X) was done with cresyl violet. E) $10 \mathrm{X}$ magnification of cresyl violet stained brain coronal sections showing placement of cannula and injector. The nucleus accumbens core and shell are delimitated by the green dashed line. Abbreviations: nucleus accumbens (NAcc) and anterior commissure (ACA)

There were no histological differences observed between placement of the cannula in the

nucleus accumbens core compared the nucleus accumbens shell. Cannula placement causes physical damage and it is possible that the placement of the cannula could have caused infection. This infection could have caused subsequent behavioural deficits that 
may have negatively impacted the data but no evidence of infection or behavioural deficits were observed in the control groups.

\subsubsection{Immunohistochemical analysis of aptamer treated animals}

Tyrosine hydroxylase (TH) is an enzyme responsible for catalyzing the hydroxylation of L-tyrosine to L-DOPA, the precursor molecule to the catecholamines; dopamine, norepinephrine and epinephrine. ${ }^{421}$ This conversion is the rate-limiting step in catecholamine synthesis. ${ }^{377}$ The activity of TH is regulated either by medium- to longterm regulation of gene expression or short-term regulation of enzymatic activity. ${ }^{422}$ One of the mechanisms by which $\mathrm{TH}$ is activated is by the phosphorylation by protein kinases of serine residues in the $8,19,31$ and 40 positions. ${ }^{422}$ Specifically there is evidence to suggest that the phosphorylation of Ser40 is primarily responsible the activation of TH and the resultant maintenance of catecholamine tissue levels following catecholamine secretion. $^{422}$

Tyrosine hydroxylase, like phenylalanine hydroxylase and tryptophan hydroxylase, is classified as an iron-containing biopterin-dependent amino acid hydroxylase. These hydroxylases use the cofactor tetrahydrobiopterin $\left(\mathrm{BH}_{4}\right)$ and $\mathrm{O}_{2}$ to hydroxylate the corresponding aromatic amino acid substrates. ${ }^{423} \mathrm{TH}$ is a tetramer where the active site of each subunit is coordinated to $\mathrm{Fe}^{2+}$ and, is catalytically active. ${ }^{422}$ Tyrosine hydroxylase is inactivated when the $\mathrm{Fe}^{2+}$ is oxidized by $\mathrm{O}_{2}$ to $\mathrm{Fe}^{3+} \cdot{ }^{422} \mathrm{TH}$ activity is regulated by two types of feedback inhibition by catechols. In the first mechanism, $\mathrm{TH}$ is inactivated by competitive binding of the catechols over the $\mathrm{BH}_{4}$ to the ferric iron $\left(\mathrm{Fe}^{3+}\right)$ of the active site. ${ }^{422}$ This inhibits the necessary cofactor interaction, 
which renders the enzyme inactive. ${ }^{422}$ Therefore, this mechanism is able to act as a sensor to regulate catechol synthesis. In the second feedback inhibition mechanism catechols bind, nearly irreversibly with the ferric iron $\left(\mathrm{Fe}^{3+}\right)$ at the $\mathrm{TH}$ catalytically active site. ${ }^{422}$ When TH is phosphorylated, the conformation of the enzyme is changed such that the affinity of the catalytically active site for catechols is greatly reduced. ${ }^{422}$ The enzyme is reactivated when the catechol dissociates and ferric iron is reduced by $\mathrm{BH}_{4}$ to ferrous iron $\left(\mathrm{Fe}^{2+}\right) \cdot{ }^{422}$

Fifteen minutes following the extinction test, animals that had been injected with either aptamer $(200 \mathrm{nM} / \mathrm{MK} ; \mathrm{n}=3)$ or vehicle $(0 \mathrm{nM} / \mathrm{MK} ; \mathrm{n}=3)$ were euthanized. Their brains were removed for immunohistochemical analysis. The levels of $\mathrm{TH}$ and $\mathrm{pTH}$ in the nucleus accumbens of aptamer treated and control animals were examined by immunohistochemical staining (Figure 4.11). Immuno detection was based on detection of phosphorylated Ser40 TH. 

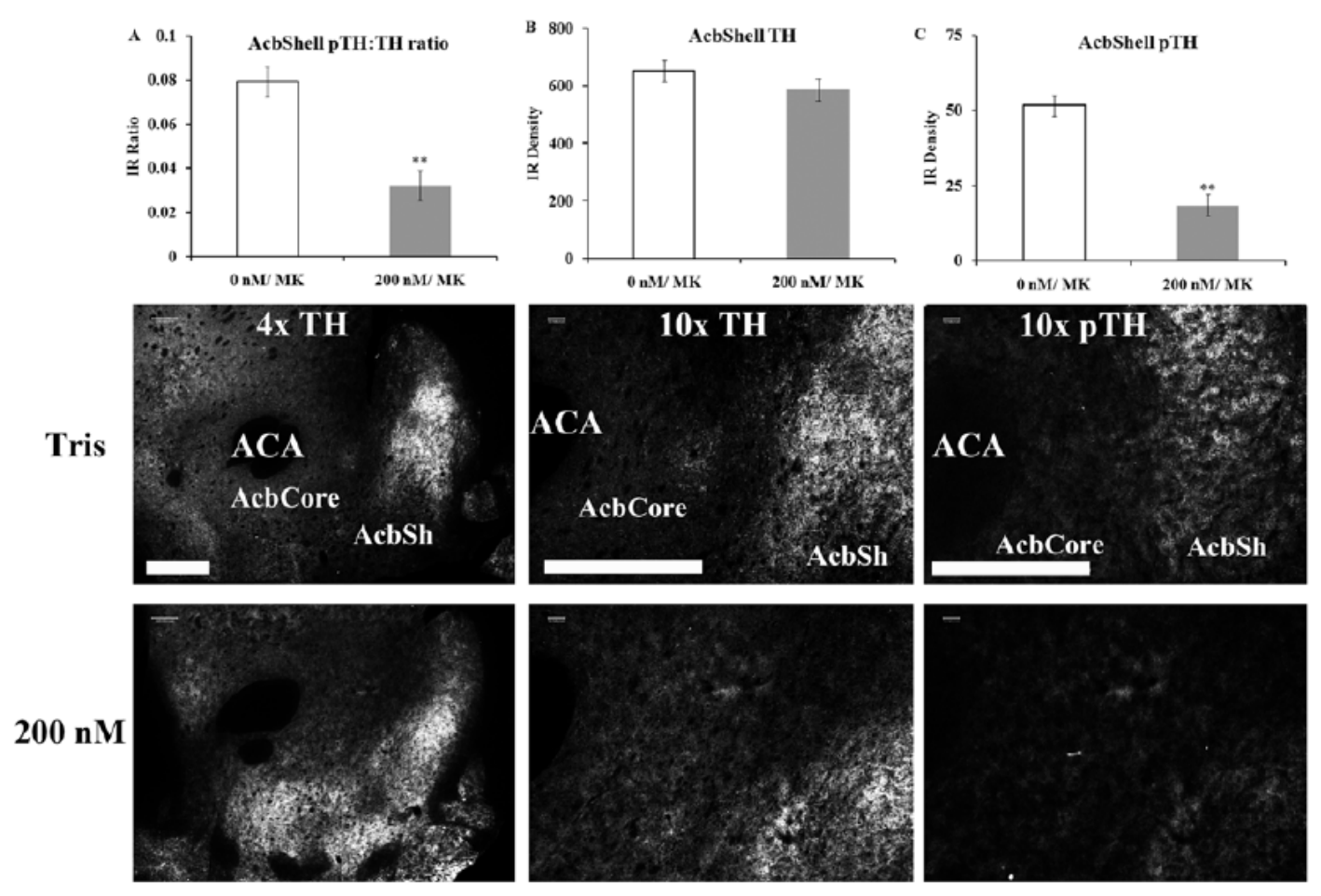

Figure 4.11: Representative images of reduced pTH staining in the nucleus accumbens of aptamer-treated rats shown by histological analysis. A) The ratio of phosphorylated tyrosine hydroxylase (pTH) to tyrosine hydroxylase (TH) in the nucleus accumbens shell region was quantified. The ratio was significantly lower in the $200 \mathrm{nM} / \mathrm{MK}$ group $(* * \mathrm{p}<0.01)$. B) TH staining in the nucleus accumbens shell region was quantified and similar levels were observed between the $0 \mathrm{nM} / / \mathrm{MK}$ and $200 \mathrm{nM} / \mathrm{MK}$ groups. C) pTH quantification in the nucleus accumbens shell region revealed lower levels of staining in the $200 \mathrm{nM} / \mathrm{MK}$ group than the $0 \mathrm{nM} / \mathrm{MK}$ group $\left({ }^{* *} \mathbf{p}<0.01\right)$. The sample size (n) was $3 /$ group. Scale bars represent $500 \mu \mathrm{m}$. The magnification shown at the top of the $0 \mathrm{nM}$ (Tris) images was the same for the 200 $\mathrm{nM}$ images. In the top panel the data is represented as mean $\pm \mathrm{SEM}$.

Student's t-test was used to compare the normalized intensities (TH intensity, pTH intensity and the ratio of pTH:TH) between groups. Intensities were normalized to account for variations in either staining or imaging processing. The ratio of $\mathrm{pTH}$ :TH (inactive to active enzyme) staining in the nucleus accumbens shell was significantly reduced (Figure 4.11; panel A: $<<0.01)$ in the aptamer-treated animals $(200 \mathrm{nM} / \mathrm{MK})$ compared to controls $(t(4)=7.02, p<0.01)$; therefore the staining of each enzyme state 
was investigated independently. Importantly, there was no significant difference between the levels of TH in the aptamer-treated ( $200 \mathrm{nM} / \mathrm{MK})$ group compared to the control group (0 nM/MK) (Figure 4.11: panel B) This preliminary evidence suggests that aptamer treatment did not damage dopaminergic terminals such that the ability of the cells to synthesize dopamine was compromised. Significantly reduced levels of pTH were observed in the aptamer-treated animals (200nM/MK) compared to the vehicle-treated controls $(0 \mathrm{nM} / \mathrm{MK})$ (Figure 4.11: panel C: $\mathrm{t}(4)=9.25, \mathrm{p}<0.01)$. This reduction of $\mathrm{pTH}$ in aptamer-treated animals suggests that in addition to binding to dopamine/norepinephrine and preventing the catecholamine's direct effects, it may also in some way be limiting the subsequent synthesis of DA/NE. In this experiment the observed reduction in pTH staining was likely indicative in a reduction of DA synthesis. This marked reduction may have been caused by free extracellular dopamine, albeit a low concentration not bound to the aptamer, exerting a regulatory effect via post-synaptic autoreceptors. ${ }^{424}$ Work done with low concentrations of dopamine agonists supports this hypothesis; treatment with low concentrations of apomorphine was shown to inhibit $\mathrm{TH}$ activity. ${ }^{424}$ In this experiment, the primary effect of the aptamer would have been to bind (for an unknown time) to extracellular dopamine/norepinephrine in the synaptic cleft thus reducing the catecholamine's effect on the postsynaptic receptor. Secondarily, aptamer binding led to reduced $\mathrm{pTH}$ and subsequently reduced catecholamine synthesis. These immunohistochemical conclusions are consistent with the behavioural data presented herein. 


\subsection{Conclusions and future work}

The results of the experiments described in this chapter present significant evidence that the DBA should be investigated further for its potential as a novel therapeutic modality for dopamine-related behavioural abnormalities. Administration of the aptamer in animals that were treated with MK-801 was shown to normalize extinction pressing behaviour. Additionally, neither devastating behavioural consequences nor neuronal damage occurred as a result of acute aptamer administration into the nucleus accumbens. Since the mechanism of action is relatively general (DBA binds directly to DA) compared to traditional therapeutics (that act on the receptors), the efficacy of the DBA is not limited to models of schizophrenia, but should be investigated in other hyperdopaminergic maladies as well. More generally, this was the first example of using an aptamer to modulate animal behaviour, providing convincing evidence that aptamers may be effective tools for investigating other CNS related diseases.

Currently, the use of aptamers to study mental health disease is limited by the challenge of delivery to the brain. The development of strategies to deliver aptamers to the brain is necessary to allow for the full characterization of the pharmacokinetics profile of a potential aptamer therapeutic. A continuation of this work (described in Chapter 5) involved investigating strategies to both bypass the BBB via targeted deliver and efficiently deliver aptamer-based technologies to the brain. 
5 In vivo use of a multi-DNA aptamer-based payload/targeting system to study dopamine dysregulation in in the central nervous system 


\subsection{Statement of Contributions}

The experiments described herein were conceptualized by EM McConnell, MC DeRosa, K Ventura and MR Holahan. Liposome and DNA preparation and characterization were performed mainly by EM McConnell and MC DeRosa. TEM imaging was done by Jianqun Wang (Manager of the Nano Imaging Facility, Carleton University). Nanoparticle tracking analysis was done by Ray Eby (Particle Metrix). Animal care and habituation activity were performed by the Department of Neuroscience (Carleton University) animal care staff, K Ventura and MR Holahan. Animal studies were performed by K Ventura, EM McConnell, Z Dwyer and MR Holahan. RT-qPCR reagent preparation, experimental analyses and data analysis were done by $\mathrm{Z}$ Dwyer, $\mathrm{K}$ Ventura, M Sieczkos (Research Associate, Department of Neuroscience, Carleton University), and EM McConnell. Histochemical analysis was done by K Ventura and MR Holahan. Statistical analyses were performed by K Ventura and MR Holahan.

\subsection{Resulting Publications}

This work was submitted to Molecular Therapy - Nucleic Acids (2016MTNA000317) April 18 $8^{\text {th }}, 2016$ and is currently under revisions. The manuscript was prepared by EM McConnell, K Ventura, MR Holahan and MC DeRosa. (Erin M. McConnell, Katelyn Ventura, Zachary Dwyer, Matthew R. Holahan and Maria C. DeRosa, In vivo use of a multi-DNA aptamer-based payload/targeting system to study dopamine dysregulation in in the central nervous system). 


\subsection{Introduction}

\subsubsection{Objectives}

The objectives of the work described in this chapter were to provide proof-ofconcept for the development of a multi-DNA aptamer payload/targeting system for delivery of an aptamer across the blood brain barrier.

\subsubsection{Aptamers as therapeutics}

Aptamers are small DNA or RNA oligonucleotides that are typically smaller than 100 bases in length which are selected by an iterative in vitro process termed $\underline{\text { Systemic }}$ Evolution of Ligands by EXponential enrichment ( $\underline{\text { SELEX) }} .{ }^{34-36}$ Aptamers are capable of binding diverse targets including small molecules to whole cells with high selectivity and affinity. ${ }^{8}$ Aptamers form unique three-dimensional secondary structures that along with the ability of nucleic acids to contribute to hydrogen bonding, $\pi-\pi$ stacking and shape complementarity facilitates the interaction of the aptamer with its cognate target. ${ }^{262}$ Aptamers have many potential analytical, diagnostic and therapeutic applications due to their chemical stability, biostability, and ease of modification. ${ }^{26,28,32}$ The comparison of aptamers to antibodies, and the advantages of aptamers over antibodies has been

extensively reviewed and discussed in Chapter $1 .{ }^{26,28}$ Specifically, the high specificity and affinity, little to no immunogenicity, relative low cost, and ease of synthesis make aptamers optimal therapeutic and detection candidates. ${ }^{46,425,426}$ Moreover, the complementarity of nucleic acids naturally provides each aptamer with its own antidote. This is a particularly important advantage of aptamers over traditional therapeutics and is especially useful in therapies, such as anticoagulation, that are associated with high risk 
complications. ${ }^{132}$ For more information about the potential of aptamers as molecular recognition elements for diagnostic and therapeutic applications within the central nervous system see Chapter $1{ }^{1}$

\subsubsection{Intra-accumbens injection of a dopamine binding aptamer abates MK-801- induced cognitive dysfunction in a model of Schizophrenia}

In the late 1990s Mannironi et al., reported the selection of an RNA aptamer for dopamine (DA) that exhibited moderate binding affinity. ${ }^{280}$ In recent years, the development of the DNA homolog of the RNA aptamer sequence was described by Walsh and DeRosa. ${ }^{284}$ Compared to the original RNA aptamer, the DNA homolog showed improved binding affinity and stability for the target dopamine and additionally had similarly high affinity for norepinephrine. ${ }^{284}$ As described in Chapter 4, the acute injection of the DNA homolog aptamer into the nucleus accumbens of animals treated with MK-801, which caused an increase in dopamine-dependent behavioural perseveration, was able to reduce the behavioral perseveration to control levels. ${ }^{375}$ The proposed mechanism of action of this aptamer was that when injected directly into the brain, the DNA homolog aptamer (henceforth referred to as the dopamine binding aptamer; DBA) was able to bind to DA and norepinephrine, effectively preventing those neurotransmitters from activating postsynaptic targets. ${ }^{375}$ While this work was the first example in which a targeted intra-cranial injection of an aptamer was shown to attenuate behaviour and therefore shows validity of this approach in basic research as well as preclinical investigations, the challenge of attenuating behaviour by the delivery of the aptamer across the blood-brain barrier (BBB) following systemic administration 
remained a significant challenge. To further this work, it was necessary to design a delivery vehicle capable of delivering aptamer payload across the BBB and subsequently investigating the aptamer's effects on neurobehavioral function in an animal model of behaviour. The development of the multi-DNA aptamer payload/targeting system may present novel therapeutic potential for central nervous system related maladies as well as present novel diagnostic and analytical research applications.

\subsubsection{The transferrin receptor aptamer and transferrin receptor mediated transcytosis}

The work described in this chapter made use of the transport-mediating function of the transferrin receptor aptamer (TRA) to deliver DBA-loaded liposomes across the

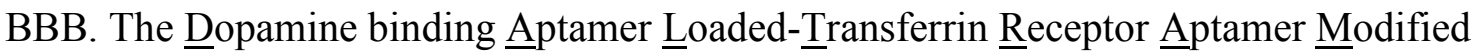
liposome formulation shall further be referred to as DAL-TRAM. The TRA which was conjugated to the surface of the DAL-TRAM was selected to bind to the extracellular domain of the mouse transferrin receptor. ${ }^{53}$ Recall that its ability to induce endocytosis across mouse fibroblast (Ltk- cells) was shown as discussed in section 1.4.6.1. ${ }^{53}$ Additionally, the TRA was also successfully used as a targeting moiety to deliver flurbiprofen encapsulated micelles across an in vitro murine model of the BBB (for this and the previous example see section 1.4.6.1). ${ }^{242}$ Known components of the BBB and the blood cerebrospinal fluid barrier (BCSFB), transferrin receptors are highly expressed on endothelial cells and are known to be involved in transport of their ligand, transferrin across the BBB and BCSFB ${ }^{49,50,427}$ Receptor mediated transcytosis across the BBB exploiting the initiating role of transferrin and the transferrin receptor has been used as a 
strategy to specifically deliver cargo to the brain. In these instances, either transferrin, or an anti-transferrin receptor antibody was conjugated to the surface of a nanoparticle, such as an immunoliposome, or directly to another antibody to induce receptor mediated transcytosis. ${ }^{428-432}$ Of particular relevance, oligonucleotide cargo was delivered to the brain by this strategy. ${ }^{433}$ Previously, aptamers have been conjugated to the surface of liposomes to facilitate the specific delivery of their payload, such as anti-cancer drugs, and have been shown to improve specific cellular uptake and toxicity. ${ }^{434}$ However, aptamer-conjugated nanoparticles have not been used to deliver an aptamer payload to the brain.

\subsubsection{Design of the "Molecular Trojan Horse" inspired dopamine binding aptamer loaded-transferrin receptor aptamer modified liposomes (DAL-TRAM)}

An effective approach to deliver nucleic acid payload to the brain involves the use of a nucleic acid-loaded vehicle, referred to as a "Molecular Trojan Horse", to carry a nucleic acid payload across the BBB via the exploitation of receptor mediated endocytosis. ${ }^{429,435-438}$ For the past forty years, liposomes, which are spherical vesicles consisting of one or more lipid bilayers enclosing aqueous compartments, have been widely investigated as carriers to improve the specific delivery of therapeutic payloads, including nucleic acids to sites in the body. ${ }^{439-442}$ The specific delivery of nucleic acid payload to the brain has been attempted by modifying liposomes with a recognition element. Typically monoclonal antibodies which are capable of targeting transferrin receptors are used to increase access of the liposome to the brain via receptor or absorptive-mediated transcytosis across the BBB. ${ }^{436}$ This chapter describes the synthesis 
and use of an aptamer-based modification of the Molecular Trojan Horse design, where both the targeting agent and the payload were aptamers. In addition to the potential that aptamers hold for diagnostic and therapeutic applications in the CNS, the practical advantages that aptamers have over the traditionally used antibodies in terms of cost and ease of synthesis motivated this design.

\subsubsection{Cocaine induced hyperlocomotion as a model for addiction}

A cocaine-induced hyperlocomotion model was used to assess the ability of systemically administered DAL-TRAM to cross the BBB and alter neurobehavioral function. Cocaine has been shown to be a potent activator of the dopamine-reward system, the noradrenergic system and the serotonergic systems. ${ }^{443}$ Though dopamine is significantly involved in motor behaviour, cocaine-induced hyperlocomotion was chosen as an appropriate model to test the DAL-TRAM as the DBA was previously shown to have no effect on locomotion following direct intra-accumbens injection (see Chapter 4). ${ }^{375}$ The proposed mechanism of action of the DBA is shown in Figure 5.1. Under normal conditions, dopamine is synthesized and stored in presynaptic neurons. Upon release into the synaptic cleft, signal transduction occurs when dopamine binds to dopamine receptors (for example D1 and D2) on the post synaptic neuronal membrane. Cocaine competitively binds to and inhibits the dopamine transporter on pre-synaptic neurons thereby increasing dopamine release into the synaptic cleft. ${ }^{444}$ Additionally, cocaine has been shown to increase extracellular dopamine concentrations by promoting pre-synaptic vesicle release. ${ }^{444}$ Increased synaptic dopamine concentrations lead to increased post-synaptic signal transduction which manifests behaviourally as 
hyperlocomotion. When the aptamer is present, it binds to excess synaptic dopamine, which prevents binding of the excess dopamine to post-synaptic receptors, and signal transduction is reduced.

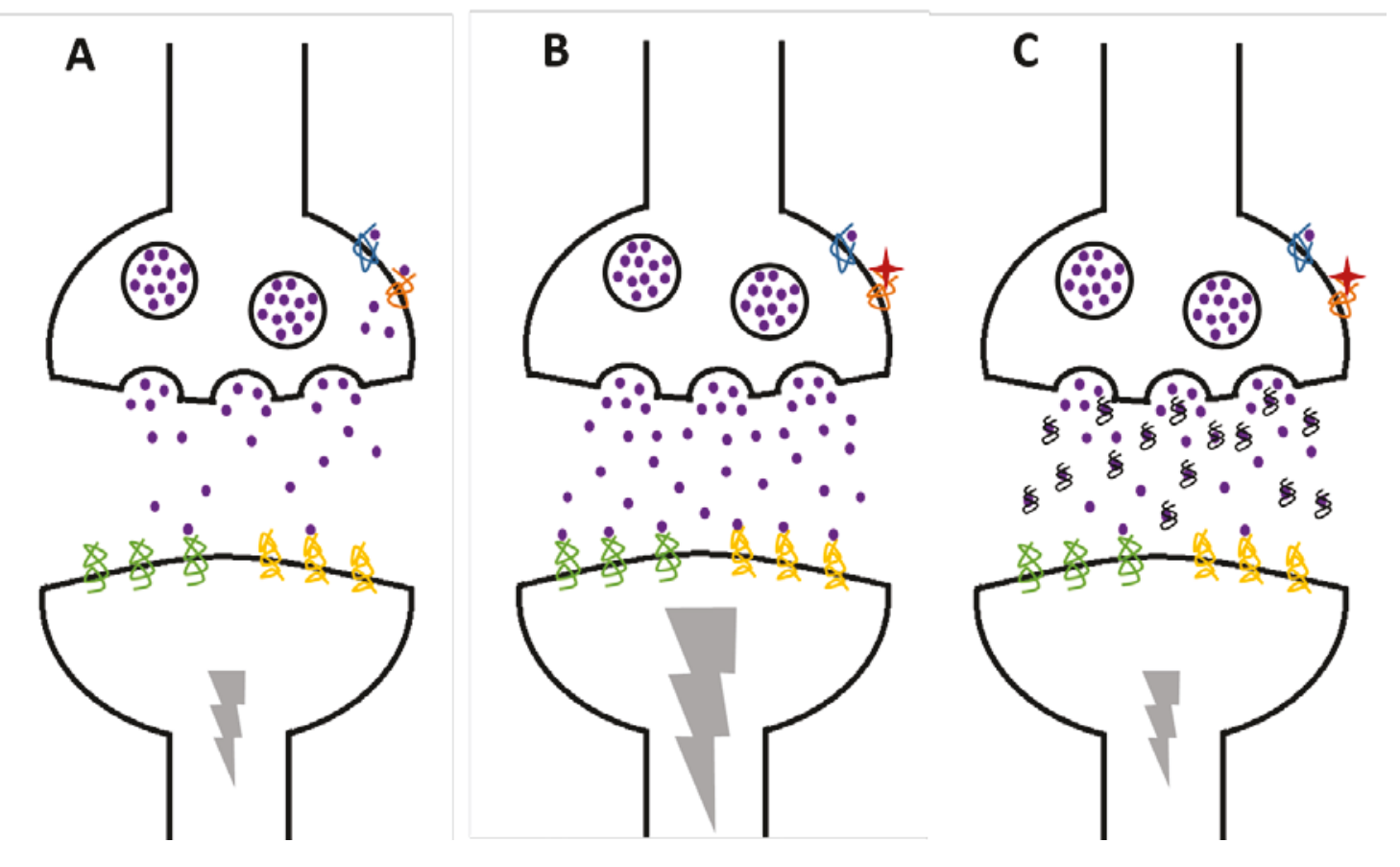

Figure 5.1: Schematic representation of the mechanism of action of the DBA in hyperdopaminergic conditions. A) Under normal conditions, dopamine (purple) is released from the pre-synaptic neuron into the synaptic cleft and is free to bind to post-synaptic dopamine receptors (green and yellow) and pre-synaptic dopamine autoreceptors (blue) and transporters (orange). B) Cocaine (red) increases the synaptic concentration of dopamine which intensifies signal transduction (grey). C) The DBA (black hairpin) binds to excess dopamine, normalizing signal transduction.

Cocaine addiction is a particularly interesting topic of assessment as treatment strategies for cocaine addiction, particularly the prevention of craving and relapse, are limited. ${ }^{445}$ Additionally, the effectiveness of existing treatment strategies remains questionable. ${ }^{445}$ Therefore, cocaine was chosen as it is not only a well-characterized drug for experimental purposes in the laboratory but it also has clinical relevancy. 


\subsection{Experimental Methods}

\subsubsection{Chemicals and reagents - liposome and cocaine solution preparation}

DNA synthesis reagents and modifiers were obtained from Glen Research

(Sterling, VA, USA). Columns for DNA synthesis were purchased from BioAutomation (Irving, TX, USA). Ammonium persulfate, Boric Acid, EDTA, HEPES, TEMED, Tris and Urea were purchased from BioShop Canada (Burlington, ON, Canada). Mini extruder, $100 \mathrm{~nm}$ polycarbonate membranes and filter supports, 1-palmitoyl-2-oleoyl-snglycero-3-phosphocholine (16:0-18:1 PC, POPC), dimethyldioctadecylamonium bromide salt (18:0 DDAB), 1,2-distearoyl-sn-glycero-3-phosphoethanolamine-N-

[methoxy(polyethylene glycol)-2000] (ammonium salt) (18:0 DSPE-PEG 2000 PE), 1,2distearoyl-sn-glycero-3-phosphoethanolamine-N-[malemide(polyethylene glycol)-2000] (ammonium salt) DSPE-PEG 2000-malemide, and Liss Rhod PE (1,2-dipalmitoyl-snglycero-3-phosphoethanolamine-N-(lissamine rhodamine B sulfonyl) (ammonium salt)) were purchased from Avanti Polar Lipids, Inc. (Alabaster, AL). Dialysis cassettes (SlideA-Lyser G2) were purchased from Thermo Fisher Scientific Canada (Nepean, ON, Canada). Saline solution $(0.9 \% \mathrm{NaCl})$ and cocaine (ecgonine methyl ester benzoate $\mathrm{HCl})$ were purchased from Sigma-Aldrich Canada (Oakville, ON, Canada).

\subsubsection{Oligonucleotide Preparation}

DNA used in these experiments were prepared using standard phosphoramidite chemistry performed on a MerMade 6 DNA synthesizer (BioAutomation, Irving, TX, USA). The following oligonucleotides (Table 5.1) were prepared; 
Table 5.1: Sequences of oligonucleotides

\begin{tabular}{|l|l|l|}
\hline Oligonucleotide & Sequence $\left(\mathbf{5}^{\prime} \rightarrow \mathbf{3}^{\prime}\right)$ & Notes \\
\hline $\begin{array}{l}\text { Unmodified } \\
\text { Dopamine Binding } \\
\text { Aptamer (DBA) }\end{array}$ & $\begin{array}{l}\text { GTCTCTGTGTGCGCCAGAGACACTGGGGC } \\
\text { AGATATGGGCCAGCACAGAATGAGGCCC }\end{array}$ & \\
\hline $\begin{array}{l}\text { 5'-thiol (Thiol- } \\
\text { modifier C6 S-S) } \\
\text { modified TRA }\end{array}$ & $\begin{array}{l}\text { GAATTCCGCGTGTGCACACGGTCACAGTT } \\
\text { AGTATCGCTACGTTCTTTGGTAGTCCGTT } \\
\text { CGGAT }\end{array}$ & \\
\hline $\begin{array}{l}\text { Unmodified } \\
\text { Substituted DBA } \\
\text { control (Sub) }\end{array}$ & $\begin{array}{l}\text { GTCTCTGTGCCAAACAGAGACACTGGGG } \\
\text { CAGATAGGGCCGCACAGAATCCGGCC }\end{array}$ & $\begin{array}{l}\text { base } \\
\text { substitutions } \\
\text { corresponding to } \\
\text { the DBA made }\end{array}$ \\
$\begin{array}{l}\text { C } \\
\text { to eliminate } \\
\text { binding are } \\
\text { underlined }\end{array}$ \\
\hline $\begin{array}{l}\text { Random } \\
\text { Oligonucleotide } \\
\text { (ROL) }\end{array}$ & $\begin{array}{l}\text { AGAATCTGTCGGGCTATGTCACTAATACT } \\
\text { TTCAACGCCCCGTACCGATGCTGAACA }\end{array}$ & \\
\hline
\end{tabular}

Polyacrylamide gel electrophoresis was used to purify DNA prior to molecular weight confirmation by ESI-MS.

\subsubsection{Liposome preparation}

The following were dissolved in chloroform and combined in a $10 \mathrm{~mL}$ round bottom flask: POPC (19.2 $\mu \mathrm{mol})$, DDAB (0.2 $\mu \mathrm{mol})$, DSPE-PEG 2000 (0.4 $\mu \mathrm{mol})$, Liss Rhod PE (0.2 $\mu \mathrm{mol})$ and DSPE-PEG 2000-malemide (30 nmol). For the fluorescent imaging experiment (see section 5.4.6) Liss Rhod PE was substituted with 16:0 NBD PE $(0.2 \mu \mathrm{mol})$. The flask was capped with a septum. While shaking and under a steady flow of argon, the chloroform was evaporated off to produce a thin uniform lipid film. The lipid film was hydrated $(0.2 \mathrm{~mL}$ of $50 \mathrm{mM}$ tris- $\mathrm{HCl} \mathrm{pH=7.0)}$ and then vortexed for 30 
min. The flask was then stored under argon and sonicated in a bath sonicator at room temperature for 10 minutes. $38 \mathrm{nmol}$ of either DBA, Sub or ROL were added to the flask in a volume of $0.2 \mathrm{~mL}(50 \mathrm{mM}$ Tris- $\mathrm{HCl})$. To encapsulate the DNA $0.6 \mathrm{~mL}$ of $67 \%$ ethanol (to yield a final concentration of $40 \%$ ethanol) was slowly added to the flask. Samples were again stored under argon before being subjected to 10 freeze/thaw cycles ( $5 \mathrm{~min}$ in an ethanol/dry ice bath followed by $2 \mathrm{~min}$ in a $40^{\circ} \mathrm{C}$ water bath). Samples were extruded by being subjected to 25 passes through $100 \mathrm{~nm}$ polycarbonate membranes contained in a mini-extruder. The samples were dialysed using Slide-A-Lyser cassettes (20 000 Molecular Weight Cut Off) into $50 \mathrm{mM}$ HEPES buffer ( $\mathrm{pH}=7.0$ ) overnight to remove the ethanol. Nuclease digestion was used to remove DNA that was nonspecifically interacting with the exterior of the liposome. Following nuclease digestion, to remove digested DNA, samples were dialysed into $50 \mathrm{mM}$ HEPES buffer $(\mathrm{pH}=7.0)$ overnight. Cleavage of the disulfide bond protecting the thiol modified transferrin receptor aptamer (TRA) was performed in $50 \mathrm{mM}$ Tris- $\mathrm{HCl}(\mathrm{pH}=8.4)$ containing 100 mM DTT. The TRA was purified using biospin columns then buffered exchanged into 50 mM HEPES (pH=7.0) containing $7 \mathrm{mM}$ EDTA. TRA was added to the liposome suspension and the reaction was allowed to proceed for 2 hours at room temperature with gentle shaking. To remove EDTA and unreacted TRA, the samples were then dialysed overnight into $50 \mathrm{mM}$ HEPES buffer $(\mathrm{pH}=7.0)$. The liposome concentration was characterized via UV-Vis spectroscopy to maintain consistency between separate sample preparations. To determine the approximate concentration of the DAL-TRAM stock preparation, the absorbance of the rhodamine peak $(570 \mathrm{~nm})$ of a 1 in 100 dilution of the stock preparation was measured. Absorbance values of the rhodamine peak for the 1 in 
100 dilution of $\sim 0.05$ and $\sim 0.10$ were assigned as $1 \mathrm{X}$ and $2 \mathrm{X}$ concentrations respectively. The homogeneity of the liposome sample was characterized by TEM microscopy. Aptamer encapsulation and liposome stability over time were confirmed by gel electrophoresis, nanoparticle tracking analysis and TEM imaging.

\subsubsection{Transmission Electron Microscopy (TEM) imaging}

TEM imaging and liposome size measurement were obtained and performed by Jianqun Wang and EM McConnell. Carbon film on 300 mesh copper grids (CF300-Cu) were purchased from Electron Microscopy Sciences (Hatfield, USA) and used as purchased. Kimwipes (Kimberly-Clark) were purchased from Fisher Scientific Company (Ottawa, Canada).

Samples were prepared for TEM imaging by depositing 5-10 $\mu \mathrm{L}$ of the sample solution onto the copper grid. Excess solution was whisked away with a Kimwipes (after $10 \mathrm{~min}$ ) and the copper grid was left to dry overnight. An FEI Tecnai G2 F20 Transmission Electron microscope (Hillsboro, USA) equipped with a Gatan ORIUS TEM CCD Camera (Pleasanton, USA) imaging system at either $120 \mathrm{kV}$ or $200 \mathrm{kV}$ was used to examine liposomes deposited on the grid. The Cal Line tool of the Gatan Microscopy Suite Digital Micrograph software (Version 2.32.888.0, Gatan, Inc.) was used to determine the size of individual liposomes.

\subsubsection{Nanoparticle tracking analysis}

Ray Eby (Particle Metrix Inc.: Mebane, USA) performed nanoparticle tracking analysis (NTA) using the ZetaView (Particle Metrix Inc.: software version 8.02.30.01). For the measurements, liposomes were diluted in deionized water according to the 
manufacture's specifications. Measurement mode 'size distribution 1 cycle' was used. The laser wavelength was $520 \mathrm{~nm}$ and the analysis parameters were max size (500), min size (10) and min brightness (15). The number of traced particles was 202. The conductivity and temperature were $27.32 \mu \mathrm{S} / \mathrm{cm}$ and $23.36^{\circ} \mathrm{C}$ respectively.

\subsubsection{Fluorescent detection of DAL-TRAM in the Brain}

Mice ( $\mathrm{n}=3)$ were injected (0.15 mL i.p.) of equimolar Rhodamine-labeled DALTRAM (TRA-positive liposome) and NBD-labeled DAL (TRA-negative liposome) to provide an initial, qualitative assessment of DAL-TRAM entry into the brain. The extinction coefficients for rhodamine $\left(95000 \mathrm{M}^{-1} \mathrm{~cm}^{-1}\right.$ at $\left.560 \mathrm{~nm}\right)$ and NBD $\left(13000 \mathrm{M}^{-1}\right.$ $\mathrm{cm}^{-1}$ at $458 \mathrm{~nm}$ ) were used to estimate the concentration of the liposome preparation. ${ }^{446}$ Excitation and emission wavelengths for Liss Rhod PE and NBD PE were $560 \mathrm{~nm} / 583 \mathrm{~nm}$ and $460 \mathrm{~nm} / 535 \mathrm{~nm}$ respectively. For comparison, imaging experiments were done at $1 \mathrm{X}$ concentration as described in section 5.4.3. After $10 \mathrm{~min}$, mice were rapidly decapitated. Tissue was not perfused. Brains were removed and immersion fixed in $4 \%$ paraformaldehyde ( $0.1 \mathrm{M}$ phosphate buffer $\mathrm{pH} 7.4)$. Tissue was stored at $4^{\circ} \mathrm{C}(24$ hours $)$ and then stored in $30 \%$ sucrose/ $0.1 \mathrm{M}$ phosphate ( $\mathrm{pH} 7.4$ ) at $4^{\circ} \mathrm{C}$ (minimum 72 hours).

Brains were sectioned $(35 \mu \mathrm{m})$ through the nucleus accumbens (anterior posterior plane of the Paxinos and Watson atlas: $2.20-1.0 \mathrm{~mm}$ from bregma) using a cryostat and then were mounted on glass slides and cover slipped with Fluoromount. An Olympus BX61 microscope (Olympus Canada Inc., Richmond Hill, Canada) was used to obtain digital images of the nucleus accumbens (20x). Fluorescent imaging was performed with 
red $(594 \mathrm{~nm})$ and green $(488 \mathrm{~nm})$ filters. Background fluorescence was minimized by setting the exposure time to the same value for both fluorescent channels.

\subsubsection{RT-qPCR detection of DBA delivered to the brain by DAL-TRAM}

Thirty min following treatment, mice were rapidly decapitated without anesthesia. Brains from the following groups; DAL-TRAM $+10 \mathrm{mg} / \mathrm{mL}$ cocaine $(\mathrm{n}=5)$ and DALTRAM + saline $(n=4)$ were extracted within 30 seconds of sacrifice. Tissue punches from nucleus accumbens were sterilely collected then flash frozen on dry ice within 60 seconds. Prior to extraction, samples were stored at $-80^{\circ} \mathrm{C}$. DAL-TRAM treated animals received liposome solution that was twice as concentrated $(2 \mathrm{X})$ as the solution administered for the fluorescent imaging and behavioural testing. To extract the target oligonucleotide from the brain punches, tissue was homogenized in Trizol then extracted using the Invitrogen Purelink RNA micro kit (Fisher Scientific Inc., Ottawa, Canada) as per to the manufacturer's instructions. Sample extract were stored at $-80^{\circ} \mathrm{C}$ until use.

The presence of the target oligonucleotide, DBA, in tissue extract was determined by standard RT-qPCR. The sequences of the DBA-forward and reverse primers are shown in Table 5.2.

Table 5.2: Forward and reverse primers for RT-qPCR amplification of the DBA and Sub aptamer sequences

\begin{tabular}{|l|l|}
\hline Primer & Sequence $\left(\mathbf{5}^{\prime} \rightarrow \mathbf{3}^{\prime}\right)$ \\
\hline DBA forward & $\begin{array}{l}\text { CTAGACTAGAAGCTGAGCTGCTAGACTAGAAGCTGA } \\
\text { GCTGGTCTCTGTGTGCGCCAGA }\end{array}$ \\
\hline DBA reverse & $\begin{array}{l}\text { ACGTTACGTTATGACATGACACGTTACGTTATGACA } \\
\text { TGACGGGCCTCATTCTGTGCTG }\end{array}$ \\
\hline
\end{tabular}

aPrimer regions are bolded 
PCR reactions were prepared using $20 \mathrm{uL}$ reactions $(5 \mathrm{uL}$ of extracted DNA, $2.66 \mathrm{uL}$ of combined primers ( $2 \mu \mathrm{M}$ total concentration), $2.34 \mathrm{uL}$ of Milli-Q Ultrapure $\mathrm{H}_{2} \mathrm{O}$, and 10 uL of BioRad Green Supermix Master Mix). The reaction was performed using the following thermal profile: $95^{\circ} \mathrm{C}$ for 3 mins; 40 two-step cycles $\left[95^{\circ} \mathrm{C}\right.$ for $10 \mathrm{sec} ; 58^{\circ} \mathrm{C}$ for $30 \mathrm{sec}] 95^{\circ} \mathrm{C}$ for $10 \mathrm{sec}$ on a Bio-Rad CFX Connect Real Time System (Bio-Rad, Mississauga, Canada). Once completed a melt curve from $60^{\circ} \mathrm{C}$ to $95^{\circ} \mathrm{C}$ was run in increments of $0.5^{\circ} \mathrm{C}$. Melt curve and quantification data were used as the primary detection method and electrophoretic analysis was run to confirm findings

The resultant DNA product was run on a standard $2 \%$ agarose gel stained with 0.01\% SYBR Safe DNA stain (Thermo Fisher Scientific, Nepean, ON, Canada). Sample $(17 \mu \mathrm{L})$ and $0.25 \%$ bromothymol blue loading buffer $(3 \mu \mathrm{L})$ were combined in each well. Invitrogen TrackIt 50 bp DNA ladder (5 $\mu$ L; Thermo Fisher Scientific, Nepean, ON, Canada) was run as a control. Gels were run $(120 \mathrm{~V}, 60 \mathrm{~min})$ then imaged using a Gel Doc (BioRad, Saint-Laurent, QC, Canada). Analyses were performed using Quantity One 4.4.0 software (BioRad, Saint-Laurent, QC, Canada).

\subsubsection{Behavioural testing}

\subsubsection{Subjects}

Male CD-1 mice (32 - 38 days old) were purchased from Charles River (St. Constant, Canada) and housed individually in $27 \times 21 \times 14 \mathrm{~cm}$ clear, polycarbonate cages. Vivarium temperature $\left(19-22^{\circ} \mathrm{C}\right)$ and humidity $(50-60 \%)$ were controlled in a fixed range. The lighting remained on a constant cycle (12 hour dark/ light cycle, lights on at 08:00). Purina mice chow and water were available ad libitum. 


\subsubsection{Ethics statement}

All animal procedures were approved by the Carleton University Animal Care Committee in accordance with guidelines set by the Canadian Council on Animal Care (CCAC). Approval was obtained from the Carleton University Animal Care Committee (AUP ID 102061).

\subsubsection{Apparatus}

To assess locomotor activity, animals were placed in enclosed $48 \times 26 \times 20 \mathrm{~cm}$ clear, polycarbonate cages in a windowless room. Horizontal movement of each mouse was recorded by 16 sensors (TSE-Systems, Inc., Chesterfield, MO) distributed $2.5 \mathrm{~cm}$ apart along the bottom of the locomotor box. A break in the infrared sensor led to the detection of horizontal movement which was then counted. Activity counts were collected on a computer running Fusion HC software (AccuScan Instruments Inc., Columbus, $\mathrm{OH})$.

\subsubsection{Experiment I and II: Examining the efficiency of DAL-TRAM in reducing cocaine-induced hyperlocomotion and the specificity of the DAL-TRAM components}

\subsection{Drugs}

DAL-TRAM and control variations of the multi-DNA aptamer payload/targeting system were prepared as described under liposome preparation (see section 5.3.3). Mice $(\mathrm{n}=77)$ were assigned to the following conditions: DAL-TRAM $+10 \mathrm{mg} / \mathrm{mL}$ cocaine $(\mathrm{n}=$ 8), DAL-TRAM $+5 \mathrm{mg} / \mathrm{mL}$ cocaine $(\mathrm{n}=8), \mathrm{DAL}-\mathrm{TRAM}+1 \mathrm{mg} / \mathrm{mL}$ cocaine $(\mathrm{n}=7)$;

DAL-TRAM+saline $(n=8)$; TRAM + saline $(n=8)$; saline + saline $(n=6)$, Sub- 
TRAM $+10 \mathrm{mg} / \mathrm{mL}$ cocaine $(\mathrm{n}=8) ; \mathrm{DAL}+10 \mathrm{mg} / \mathrm{mL}$ cocaine $(\mathrm{n}=9) ; \mathrm{ROL}-\mathrm{TRAM}+10$ $\mathrm{mg} / \mathrm{mL}$ cocaine $(\mathrm{n}=7)$; and TRAM $+10 \mathrm{mg} / \mathrm{mL}$ cocaine $(\mathrm{n}=8)$. Cocaine hydrochloride (10 mg/mL; Sigma-Aldrich, USA) was dissolved in $0.9 \% \mathrm{NaCl} .5 \mathrm{mg} / \mathrm{mL}$ and $1 \mathrm{mg} / \mathrm{mL}$ solutions were prepared from the $10 \mathrm{mg} / \mathrm{mL}$ stock solution. All cocaine solutions were prepared within $24 \mathrm{~h}$ of use.

\subsection{Behavioral Procedure}

For at least 7 days prior to any experimental manipulations the mice were acclimated to the laboratory housing conditions. Once the animals were acclimatized all mice were habituated to the locomotor apparatus. Mice were placed in the locomotor cages for 30 min on each of 3 consecutive days. All habituation trials took place between 08:00 and 10:00 am. On the test day, DAL-TRAM or control variations of the multiDNA aptamer payload/targeting system were injected ( $0.1 \mathrm{~mL}$ i.p.) $5 \mathrm{~min}$ prior to a cocaine or saline injection ( $0.1 \mathrm{~mL}$ i.p.). Animals were placed in the locomotor apparatus and locomotor testing commenced five minutes after the mice received the cocaine/saline injection. Locomotor activity was recorded over a $30 \mathrm{~min}$ period and processed as the total activity count per $5 \mathrm{~min}$ time bin.

\subsection{Statistical analysis}

IBM SPSS Statistics (Version 22; Armonk, NY, USA) was used to run a One-way ANOVA was to investigate group effects. Either Fisher's least significant difference (LSD) or Tukey's honest significant difference (HSD) post-hoc analyses were performed to compare groups. 


\subsubsection{Experiment III A: Chronic administration of DAL-TRAM in saline treated animals}

\subsection{Drugs}

DAL-TRAM and TRAM were prepared as described under liposome preparation (see section 5.3.3). Mice were assigned to one the following groups; DAL-TRAM $(n=5)$, $\operatorname{TRAM}(\mathrm{n}=5)$ or no injection $(\mathrm{n}=5)$.

\subsection{Behavioral Procedure}

For at least 7 days prior to any experimental manipulations the mice were acclimated to the laboratory housing conditions. Once the animals were acclimatized all mice were habituated to the locomotor apparatus. Mice were placed in the locomotor cages for $30 \mathrm{~min}$ on each of 3 consecutive days. All habituation trials took place between 08:00 and 10:00 am.

Following the habituation phase, mice were assigned to receive an i.p. injection $(0.1 \mathrm{~mL})$ of either DAL-TRAM or TRAM, or received no injection $5 \mathrm{~min}$ prior to placement in the locomotor apparatus on each of 6 test days. On each of the 6 test days, the locomotor activity was recorded over a $30 \mathrm{~min}$ period and processed as the total activity count per 5 min time bin.

\subsection{Statistical analysis}

IBM SPSS Statistics (Version 22; Armonk, NY, USA) was used to run a One-way ANOVA to investigate group effects. Post-hoc analyses were not performed. 


\subsubsection{Experiment III B: Fluorescent histochemical staining by Fluoro-Jade B}

Mice from experiment III B were sacrificed by rapid decapitation to perform an initial assessment of the toxicity of the DAL-TRAM and TRAM in a chronic model of administration. Brains were extracted, were post fixed in a solution of $30 \%$ sucrose in $4 \%$ paraformaldehyde, were flash frozen, and then were sliced into $35 \mu \mathrm{m}$ coronal sections using a cryostat. Sections were mounted frozen and were allowed to dry. Once dry, mounted sections were washed 6 times $(10 \mathrm{~min})$ in $0.1 \mathrm{M}$ phosphate buffer $(\mathrm{pH} 7.4)$. Tissue was then immersed consecutively in 100\% ethanol ( 3 mins), $70 \%$ ethanol (2 mins), $30 \%$ ethanol (2 mins), and then distilled $\mathrm{H}_{2} \mathrm{O}$ (2 mins). Sections were incubated in filtered $0.06 \% \mathrm{KMnO}_{4}(15 \mathrm{~min})$ then rinsed with distilled $\mathrm{H}_{2} \mathrm{O}(2 \mathrm{~min})$. Finally, sections were incubated in $0.001 \%$ Fluoro-Jade B (EDM Millipore, Etobicoke, Canada) dissolved in $0.1 \%$ acetic acid solution $(30 \mathrm{~min})$ and then were briefly rinsed in distilled $\mathrm{H}_{2} \mathrm{O}$. Post Fluoro-Jade B incubation, sections were dried using a drier (high; $2 \mathrm{~min}$ ) to remove excess water from the slides. Sections were then placed in Clearene Solvent (2 min; Leica Biosystems, Concord, Canada) then were cover slipped using DPX Mountant for histology (Sigma-Aldrich Canada, Oakville, Canada). 


\subsection{Results and Discussion}

\subsubsection{Design and synthesis of dopamine aptamer-loaded, transferrin receptor aptamer-modified (DAL-TRAM) liposomes}

A persistent challenge in the development of new therapeutics targeted to the brain is delivery across the blood-brain barrier (BBB) and blood-cerebrospinal fluid barrier (BCSFB). The natural function of these barriers is to protect the brain and therefore passage across these barriers is well regulated. Only a small class of drugs are able to cross the BBB. These molecules are lipid soluble and less than $400 \mathrm{Da}$ in size. Large molecule therapeutics such as non-viral gene medicines, recombinant proteins, and siRNAs are unable to permeate the BBB. ${ }^{437}$ Aptamers are relatively large $(\sim 10-15$ $\mathrm{KDa}$ ), charged, polar molecules and therefore are no exception. Recently, RT-qPCR has shown that low levels of systemically administered aptamer has been detected in the brain, and some work has been done to specifically select aptamers that cross the BBB. ${ }^{50,51}$ Nevertheless, the development of vehicles for efficient BBB passage remain an important goal. Work done by Shi et al. 2001, in which a monoclonal antibody for the transferrin receptor was conjugated to PEGylated liposomes loaded with plasmid DNA served as the inspiration for the design of the DAL-TRAM. ${ }^{435}$ The antibody-conjugated PEGylated liposomes, termed "Molecular Trojan Horses" when administered peripherally were shown to transverse the BBB to deliver the oligonucleotide payload. In this work, the DBA was chosen as the oligonucleotide payload and to mediate BBB passage, an aptamer that binds to the transferrin receptor (TRA) was conjugated to the 
surface of the liposome. The design of the DAL-TRAM is shown schematically in Figure 5.2 .
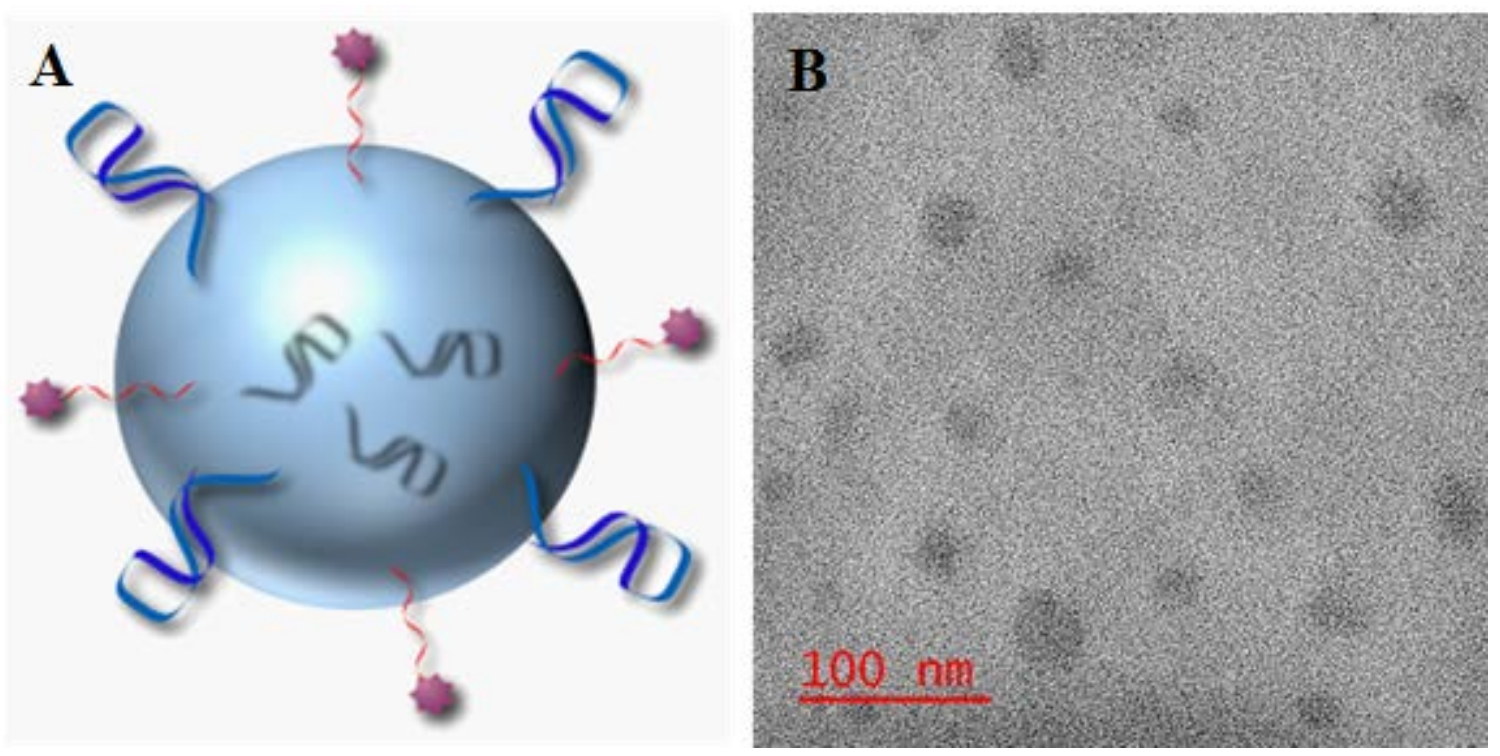

Figure 5.2: A) Schematic of the multi-DNA aptamer payload/targeting system. The lipid vesicle (blue sphere) is loaded with payload aptamer (black ribbons), and surface modified with a rhodamine-label (pink stars) and transferrin receptor aptamer (TRA: blue ribbons). B) Representative transmission electron microscope image of aptamer loaded-TRAM liposomes.

The DNA payload encapsulated inside the TRAM delivery vehicles was either a dopamine binding aptamer (DBA), non-binding mutated dopamine aptamer (Sub), random oligonucleotide (ROL) or a control oligonucleotide (details are described in Table 5.3).

Table 5.3: Aptamer sequences used in the multi-aptamer targeting/payload system design

\begin{tabular}{|l|l|}
\hline Aptamer & Sequence $\left(\mathbf{5}^{\prime} \rightarrow \mathbf{3}^{\prime}\right)$ \\
\hline $\begin{array}{l}\text { Dopamine binding } \\
\text { aptamer (DBA) }\end{array}$ & $\begin{array}{l}\text { GTCTCTGTGTGCGCCAGAGACACTGGGGCAGATA } \\
\text { TGGGCCAGCACAGAATGAGGCCC }\end{array}$ \\
\hline $\begin{array}{l}\text { Non-binding base } \\
\text { substituted DBA } \\
\text { (Sub) }^{\mathbf{a}}\end{array}$ & $\begin{array}{l}\text { GTCTCTGTGCCAAACAGAGACACTGGGGCAGATA } \\
\text { TGGGCCEGCACAGAATCCGGCCC }\end{array}$ \\
\hline
\end{tabular}




\section{Random AGAATCTGTCGGGCTATGTCACTAATACTTTCCAAA oligonucleotide $\quad$ CGCCCCGTACCGATGCTGAACA \\ (ROL) \\ Transferrin receptor aptamer (TRA) \\ GAATTCCGCGTGTGCACACGGTCACAGTTAGTATCG CTACGTTCTTTGGTAGTCCGTTCGGGAT}

\section{Control Unpublished sequence \\ Oligonucleotide}

${ }^{a}$ Base substitutions are underlined. Primer regions are in bold.

As a substitute to the monoclonal antibodies for the transferrin receptor used in the Molecular Trojan Horse design, the liposome was surface modified with a thiol modified transferrin receptor aptamer (TRA) via conjugation of the thiol-TRA to the surface of the liposome through a thioether bond to maleimide-modified lipid. ${ }^{53}$ To monitor the central distribution of the peripherally injected DAL-TRAM, rhodamine-modified lipid was included in the design to provide a fluorescent label for characterization by fluorescent microscopy. In addition to DAL-TRAM, multiple control variations of the multi-DNA aptamer payload/targeting system were also synthesized and are described in Table 5.4.

Table 5.4: Aptamer and, multi-aptamer payload/targeting system and control variation abbreviations

\begin{tabular}{|c|c|}
\hline Abbreviation & Aptamer details \\
\hline DBA & Dopamine binding aptamer \\
\hline Sub & $\begin{array}{l}\text { Non-binding/base substituted dopamine binding aptamer. The } \\
\text { sequence is identical to DBA with the exception of specific point } \\
\text { mutations, the presence of which eliminates dopamine-aptamer } \\
\text { binding } 284\end{array}$ \\
\hline ROL & $\begin{array}{l}\text { Random oligonucleotide of comparable length to the DBA } \\
\text { previously shown not to affect dopamine-related behavior }{ }^{375}\end{array}$ \\
\hline Control Oligo & $\begin{array}{l}\text { The control oligonucleotide is an aptamer of similar size to the } \\
\text { DBA that was used during the liposome synthesis phase as a } \\
\text { complex secondary structure analog. This control oligo was not }\end{array}$ \\
\hline
\end{tabular}


evaluated in the behavioral testing.

\begin{tabular}{|c|c|}
\hline Abbreviation & Component details \\
\hline DAL-TRAM & $\begin{array}{l}\text { dopamine aptamer loaded-transferrin receptor aptamer modified } \\
\text { liposome (TRA-positive liposome) }\end{array}$ \\
\hline DAL & Dopamine aptamer loaded liposome (TRA-negative liposome) \\
\hline TRAM & $\begin{array}{l}\text { Transferrin receptor aptamer modified liposome (no } \\
\text { oligonucleotide payload) }\end{array}$ \\
\hline Sub-TRAM & $\begin{array}{l}\text { Non-binding/base substituted dopamine binding aptamer loaded - } \\
\text { transferrin receptor aptamer modified liposome }\end{array}$ \\
\hline ROL-TRAM & $\begin{array}{l}\text { Random oligonucleotide loaded-transferrin receptor aptamer } \\
\text { modified liposome }\end{array}$ \\
\hline
\end{tabular}

The synthesis of DAL-TRAM and control variations of the multi-DNA aptamer payload/targeting system is described in Figure 5.3. Small, unilamellar liposomes were prepared as previously described. ${ }^{436,447}$ However, some modifications were made to incorporate the conjugation of a DNA aptamer targeting moiety (TRA) in place of a monoclonal antibody as well as the encapsulation of a DNA aptamer payload. Due to their well characterized and demonstrated biodegradability and biocompatibility, liposomes were chosen as the delivery vehicle for the multi-DNA aptamer based payload/targeting system. ${ }^{441}$ Additionally, PEG inclusion has shown to increase circulation time yielding a cost effective, easily mass produced, effective delivery modality and therefore PEGylated liposomes were chosen. ${ }^{439}$ Liposomes present an ideal complement to aptamers for the development of targeted drug delivery systems due to their relative ease of synthesis, stability, and batch-to-batch reproducibility. Liposomebased drug delivery systems are being extensively investigated. In fact, the U.S. Food and 
Drug Administration has approved the clinical use of several liposome-based drug delivery system to treat disease. ${ }^{434}$

In the first step of synthesis the lipids were dissolved in chloroform and combined in a round bottom flask (step 1). DSPE-PEG 2000 and DSPE-PEG-2000-malemide were included to provide biostability and a mechanism through which to anchor the TRA. ${ }^{448}$ Liss Rhod PE was used to fluorescently modify the liposome for imaging purposes. To produce a thin lipid film (step 2), the round bottom flask was capped with a septum and dried under a steady stream of argon while being gently agitated by vortexing (lowest setting). The lipid film was hydrated (step 3) by the addition of aqueous buffer which lead to the formation of a mixture of multilamellar and giant unilamellar liposomes. ${ }^{449}$ Sonication (step 3) of the liposome solution served to decrease liposome size and lamellarity. ${ }^{450,451}$ The DNA aptamer payload was encapsulated by the addition of ethanol. Ethanol both condenses DNA and destabilizes the permeability of the liposomal membrane, leading to the efficient encapsulation of the aptamer payload (step 4). ${ }^{447}$ Subjecting the liposome formulation to several freeze-thaw cycles (step 4) and extrusion through a $100 \mathrm{~nm}$ polycarbonate membrane (step 5) further ensured the liposomes were small unilamellar vesicles. ${ }^{450}$ Finally, through the reaction of the thiol modified TRA to the maleimide of the DSPE-PEG-2000-maleimide phospholipid, the TRA was conjugated to surface of the liposome via a thioether bond (step 6). Following synthesis, the liposomes were characterized by transmission electron microscopy (TEM) imaging (step 7) and then prepared for the animal studies (step 8). 
1. POPC, DDAB, DSPE-

PEG-2000, Liss Rhod PE,

DSPE-PEG-2000-malemide

were dissolved in

cholorform and combined

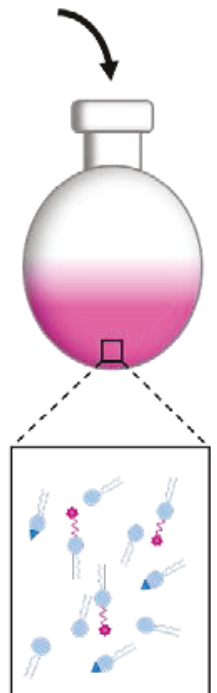

5. The sample was extruded through 100 $\mathrm{nm}$ polycarbonate membranes to filter out larger vesicles
2. The solution was dried under a steady stream of argon to produce a thin lipid film
3. The lipid film was hydrated and the solution was sonicated to induce liposome formation
4. The payload DNA was encapsulated by addition of ethanol and multiple freeze-thaw cycles

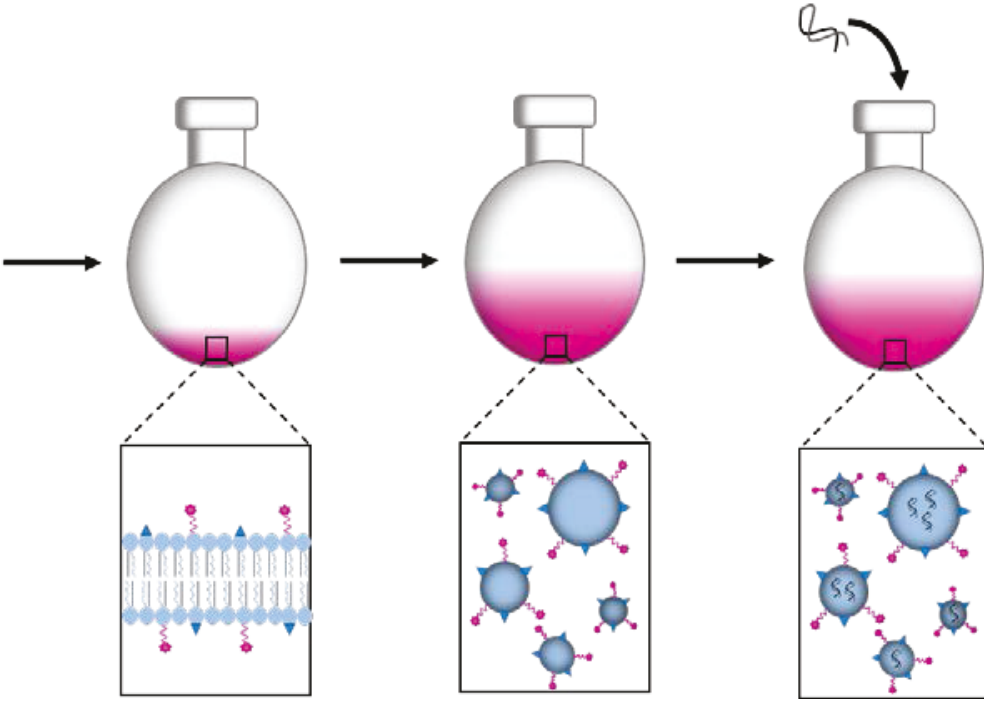

6. The thiol-modified targeting DNA (TRA) was conjugated to the surface of the liposome via formation of a thioether bond

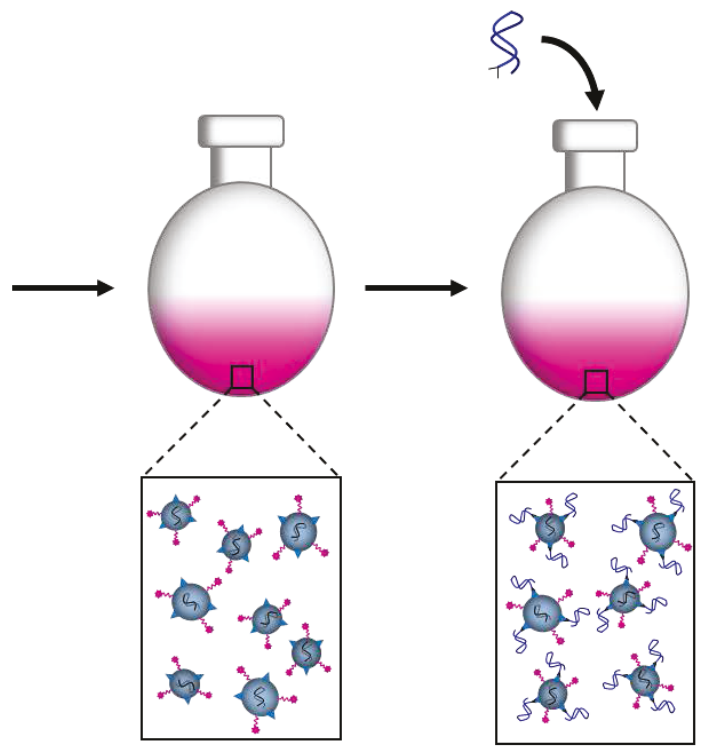

7. TEM imaging was used to confirm successful synthesis

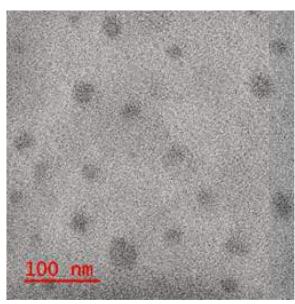

8. Liposomes were I.P. injected into mice for imaging, RT-PCR and behavioural studies
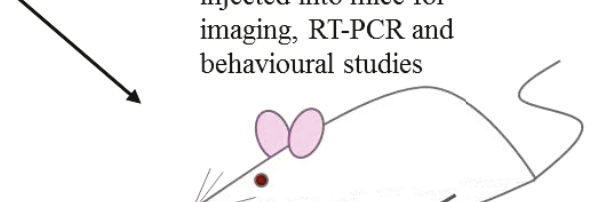

Figure 5.3: Schematic representation of the synthesis of DAL-TRAM and control variations of the multi-DNA aptamer payload/targeting system. 
The encapsulation of the DNA aptamer payload into the liposome was

investigated by gel electrophoresis. Liposomes were synthesized as described in the section 5.3.3 however, the rhodamine labelled phospholipid and surface modification of TRA were not included. Specifically, the rhodamine labelled lipid was excluded for the liposome preparation so the rhodamine fluorescence would not interfere with the fluorescence of the ethidium bromide staining used to identify the encapsulated DNA. A small aliquot of the liposome formulation from each phase of synthesis was run on a nondenaturing polyacrylamide gel (Figure 5.4) to determine the encapsulation of a control oligonucleotide into the liposome.

\section{$\begin{array}{lllllllllllllll}1 & 2 & 3 & 4 & 5 & 6 & 7 & 8 & 9 & 10 & 11 & 12 & 13 & 14 & 15\end{array}$}

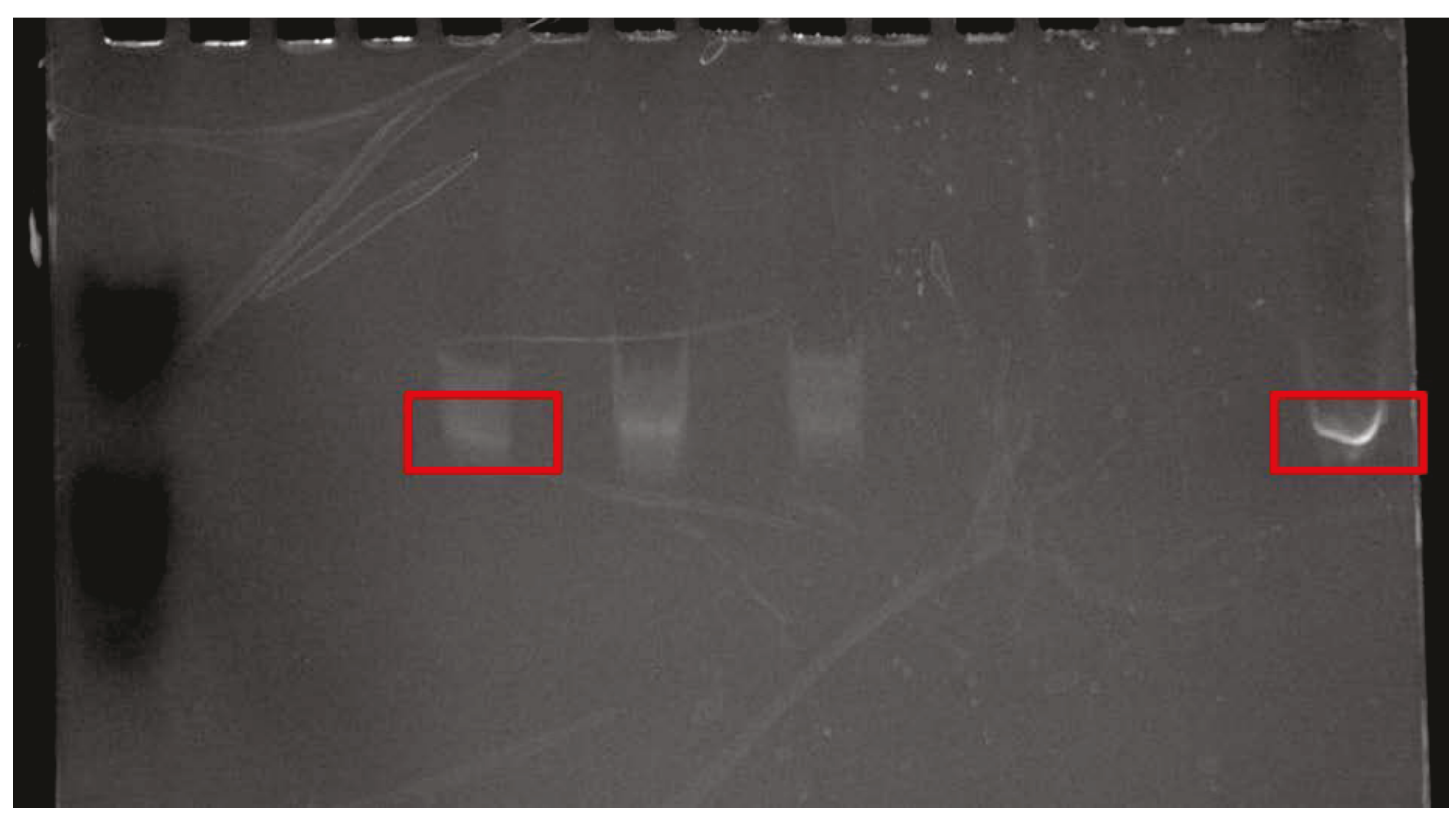

Figure 5.4: Encapsulation of the DNA payload was confirmed by non-denaturing PAGE analysis. Lanes: Loading dye (1), DNA (control oligo) (5), after extrusion (7), after dialysis 1 (9), after nuclease digestion (11), after dialysis 2 (13) and after lysis (15). DNA was imaged by ethidium bromide staining. 
Upon comparison of the bands in lane 5 (control) and lane 15 (after lysis), the control oligonucleotide was identified in the final liposome preparation (Figure 5.4: see red boxes around the bands in lanes 5 and 15). DNA was encapsulated into the liposome before extrusion however encapsulation is not $100 \%$ efficient. Therefore nonencapsulated DNA may have non-specifically interacted with the exterior of the liposomes. In addition, DNA may have been released during the freeze-thaw and extrusion steps as larger vesicles were disrupted. The presence of the oligonucleotide after extrusion (lane 7) and after the first dialysis step (lane 9) can be explained by the lingering presence of non-encapsulated DNA. When non-encapsulated DNA was removed from the preparation by nuclease digestion, no DNA was observed (see lanes 11 and 13). Lysis of the final liposome preparation led to the liberation of encapsulated DNA, observed as a band in lane 15 .

The encapsulation of the aptamer payload into the liposomal vehicle afforded protection to the unmodified nucleic acid payload, allowing the aptamer to be delivered to the brain intact and in its natural state. Alternative strategies that have been investigated to increase nucleic acid circulation time include modifications to the nucleic acid backbone, 5'- and 3'- end modifications and the inclusion of modified nucleotides. ${ }^{32,42}$ However, the secondary structure of the aptamer as well as the affinity of the aptamer to its target can be disrupted when these modifications are made post aptamer selection. Another advantage of encapsulation of the aptamer payload into the targeting liposome was that the aptamer payload was effectively concentrated in the cellular target by the TRA-mediate specific delivery of the liposomal vehicle. For these reasons, 
delivery of the unmodified aptamer payload by encapsulation within the protective liposomal vehicle was investigated.

Following synthetic preparation, lamellarity, and monodispersity of the DALTRAM formulation was examined by TEM (Figure 5.2 B, Figure 5.5 and Figure 5.6).
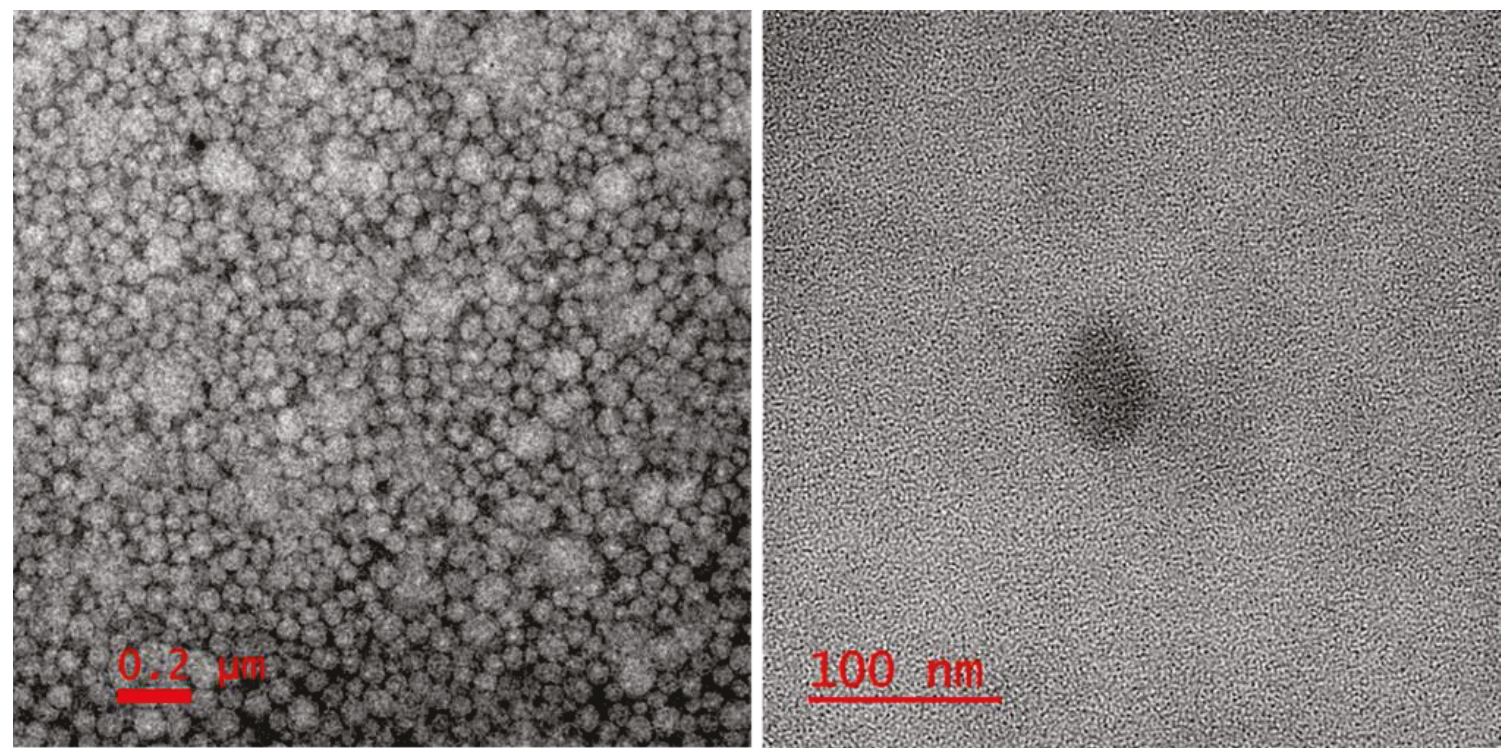

Figure 5.5: The homogeneity of the DAL-TRAM was characterized by transmission electron microscopy (TEM). TEM images of the liposome targeting system at $0.2 \mu \mathrm{m}$ (left) and $100 \mathrm{~nm}$ (right) scale. Images were obtained at $120 \mathrm{kV}$.

TEM images were obtained as described in section 5.3.4. Mostly small unilamellar vesicles, interspersed with some larger vesicles and possibly aggregates (Figure 5.5: left) were revealed upon imaging of the liposome sample deposited on the copper grid on the $0.2 \mu \mathrm{m}$ scale. Closer examination on smaller scale $(100 \mathrm{~nm})$ revealed the formation of small unilamellar liposomes, smaller than $100 \mathrm{~nm}$ (Figure 5.5: right). 


\section{A)}
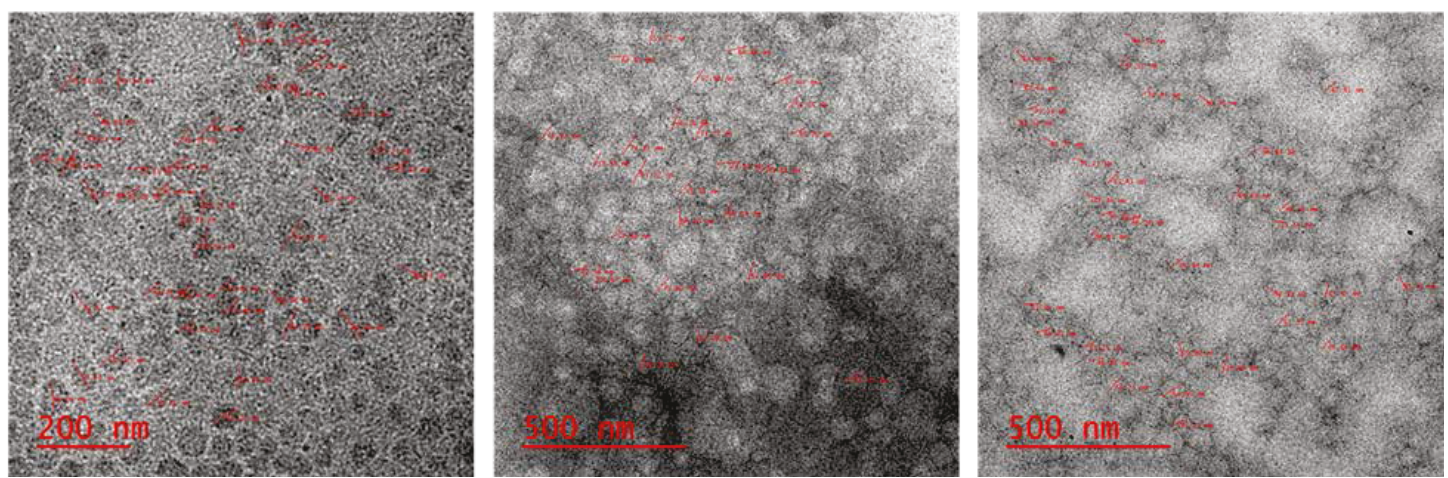

B)

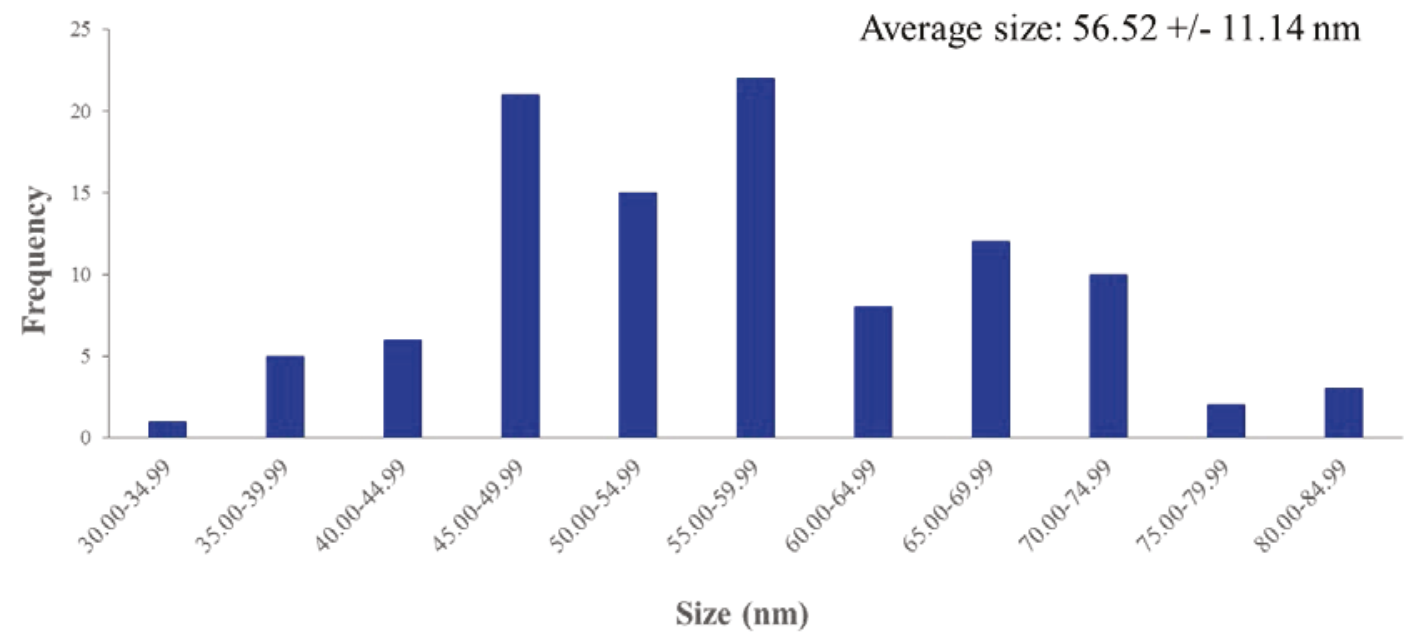

Figure 5.6: TEM was used to determine the measurement (A) and size distribution histogram (B) of the liposome targeting system. TEM images (A) were obtained at $120 \mathrm{kV}$.

The sizes of 105 liposomes over a total of three images (Figure 5.6 $\mathrm{A}$ ) were determined using the Cal Line tool of Gatan Microscopy Suite Digital Micrograph software (Version 2.32.888.0, Gatan, Inc.). To determine the size distribution of the liposome targeting system, liposomes were assigned to a bin $(\sim 5 \mathrm{~nm})$ according to their measured size. The size distribution histogram (Figure 5.6 B) was plotted by determining the frequency (number of liposomes) assigned to each size bin. From this data, the final loaded-TRAM 
formulations were determined to consist of mostly small unilamellar liposomes with an average diameter of $56.5 \pm 11.1 \mathrm{~nm}$.

Further, TEM and nanoparticle tracking analysis (NTA) was used to assess the stability of the liposome targeting system after 5 and 2 months respectively (Figure 5.7).
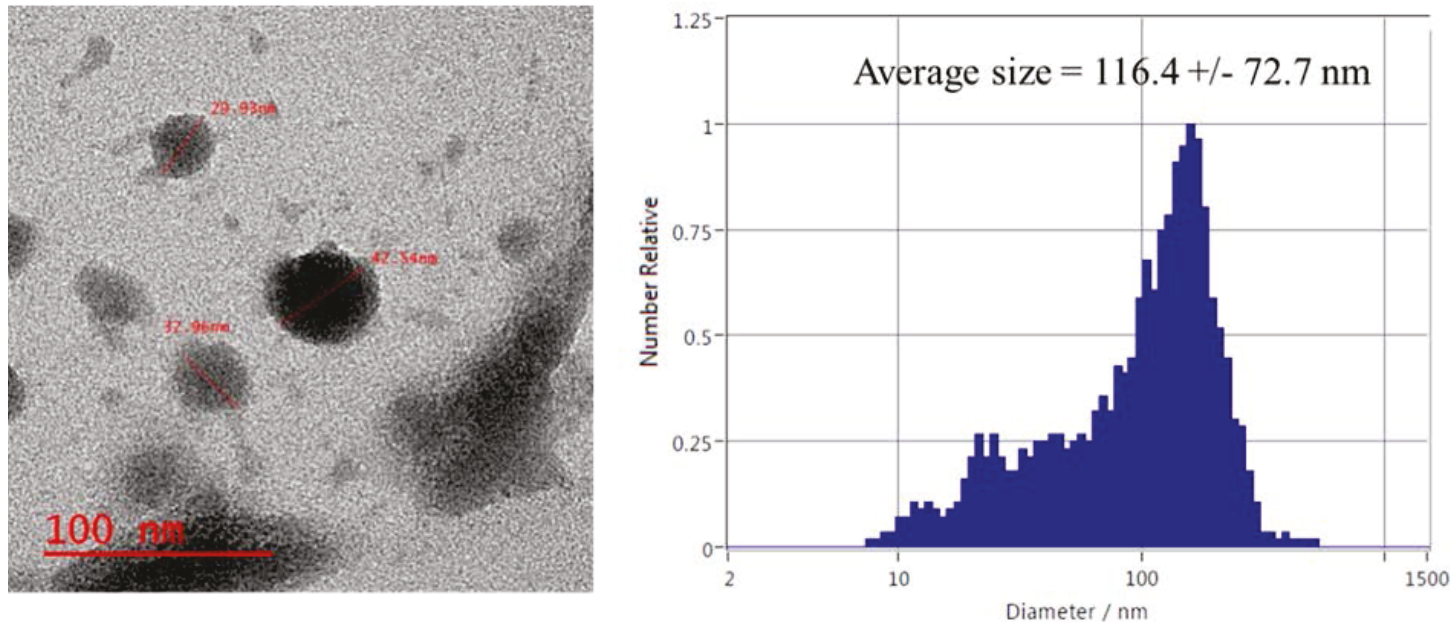

Figure 5.7: Stability of the liposome formulation over time was assessed by TEM (left) and by nanoparticle tracking analysis (right) after 5 and 2 months respectively. The TEM image was obtained at $200 \mathrm{kV}$.

TEM imaging and NTA measurement were used to examine the stability of the liposome targeting system over time. TEM images were obtained as described in section 5.4.4 after five months of being stored under argon at room temperature. TEM imaging revealed stable liposomes (Figure 5.7, left) consistent in size with those previously observed, though some aggregation and vesicle collapse were seen. After 2 months of storage under argon at room temperature, NTA was performed. The average particle size determined at a concentration of $58.2 \times 10^{6}$ particles $/ \mathrm{mL}$ was $116.4 \pm 72.7 \mathrm{~nm}$. The increase in size can be explained by the difference in techniques; TEM measures the diameter of dry particles and the diameter determined by NTA represents that of hydrated particles. Additionally, a 
larger observed diameter would have also resulted from the presence of aggregated particles. Therefore intact liposomes were observed by NTA after 2 months, and by TEM imaging after 5 months however some aggregation was observed in the TEM images which was supported by the larger diameter observed by NTA. Regardless, for all further experiments loaded-TRAMs were prepared and used within 4 days.

\subsubsection{Detection of DAL-TRAM in the brain by fluorescent microscopy}

Fluorescence microscopy was used to qualitatively assess the distribution of peripherally administered DAL-TRAM in brain tissue. Mice $(\mathrm{n}=3)$ were injected with $0.15 \mathrm{~mL}$ of equimolar DAL-TRAM (rhodamine labelled) and DAL (NDB labelled) liposomes combined in HEPES buffer. Coronal sections through the nucleus accumbens were obtained and representative images are shown in Figure 5.8.

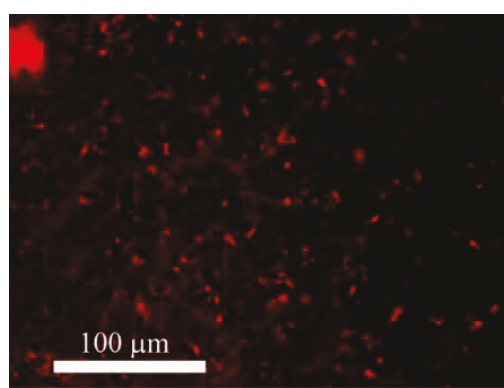

DAL-TRAM

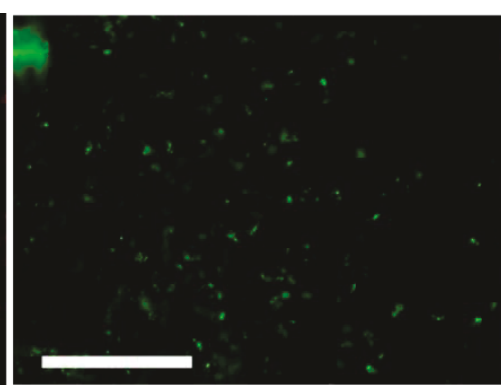

DAL

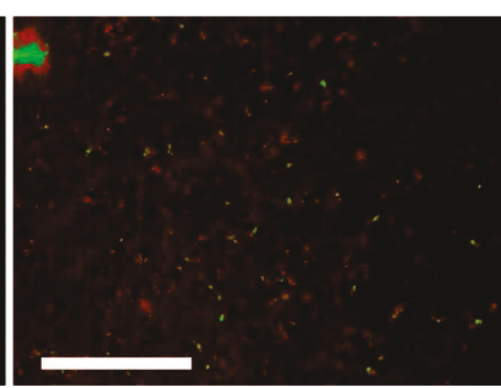

Overlay

Figure 5.8: Fluorescence microscopy was used to determine the distribution of rhodamine fluorescence in a coronal section ( $35 \mu \mathrm{m}$ thickness) of the nucleus accumbens. Mice were injected with a solution containing TRA-positive liposomes (DAL-TRAM: rhodamine (red) labelled) and TRA-negative liposomes (DAL: NBD (green) labelled) then euthanized $10 \mathrm{~min}$ later. Digital fluorescence images were obtained at 20X magnification. The scale bar for the DAL and overlay images are as indicated in DAL-TRAM image.

The distribution of red or green fluorescence based on the presence or absence of the

TRA respectively was very clearly different. In the TRA-negative liposome (DAL) 
treated group, green fluorescence was observed mostly in dense clusters, likely around the capillaries. In contrast, the observed distribution of red fluorescence in the TRApositive liposomes (DAL-TRAM) treated group was much more diffuse than the DAL treated group. In the representative image of the TRA-positive (DAL-TRAM) treated group, red fluorescence was observed in dense clusters (likely capillaries) as well as diffusely surrounding cell bodies (black spheres) and in the interstitial space (surrounding area). The differences in the observed distribution patterns likely occurred because the TRA-negative DAL were mostly trapped in the capillary and unable to traverse the BBB, where as the TRA-positive DAL-TRAM were able to cross the BBB. Overlay of the DAL-TRAM and DAL images further illustrates the more diffuse distribution of DALTRAM (red) compared to DAL (green) based fluorescence.

\subsubsection{Identification of systemically delivered DBA in brain tissue through amplification by RT-qPCR}

RT-qPCR was used to confirm the presence of the DBA in brain tissue from the nucleus accumbens. Chapter 4 describes experiments in which direct intra-accumbens injection of the DBA was shown to normalize MK-801 induced extinction deficit. Therefore, tissue punches from the nucleus accumbens post systemic injection of DALTRAM were examined for the presence of the aptamer payload, DBA. RT-qPCR was used to assess the delivery of the DBA, by DAL-TRAM to the brain. To compensate for the relatively small RT-qPCR target size, the 5'-ends of the DBA forward and reverse primers were extended with 40 base tails. This strategy was previously shown to successfully amplify aptamer from brain tissue. ${ }^{51}$ To validate the method, samples were 
first extracted from DAL-TRAM + saline treated animals $(n=2)$ and amplification of the DBA in these samples was compared to amplification in a non-template control reaction. The DBA was successfully and specifically amplified RT-qPCR (Figure 5.9 A). The presence and size of the amplification product was confirmed by gel electrophoretic analysis (Figure 5.9 B).
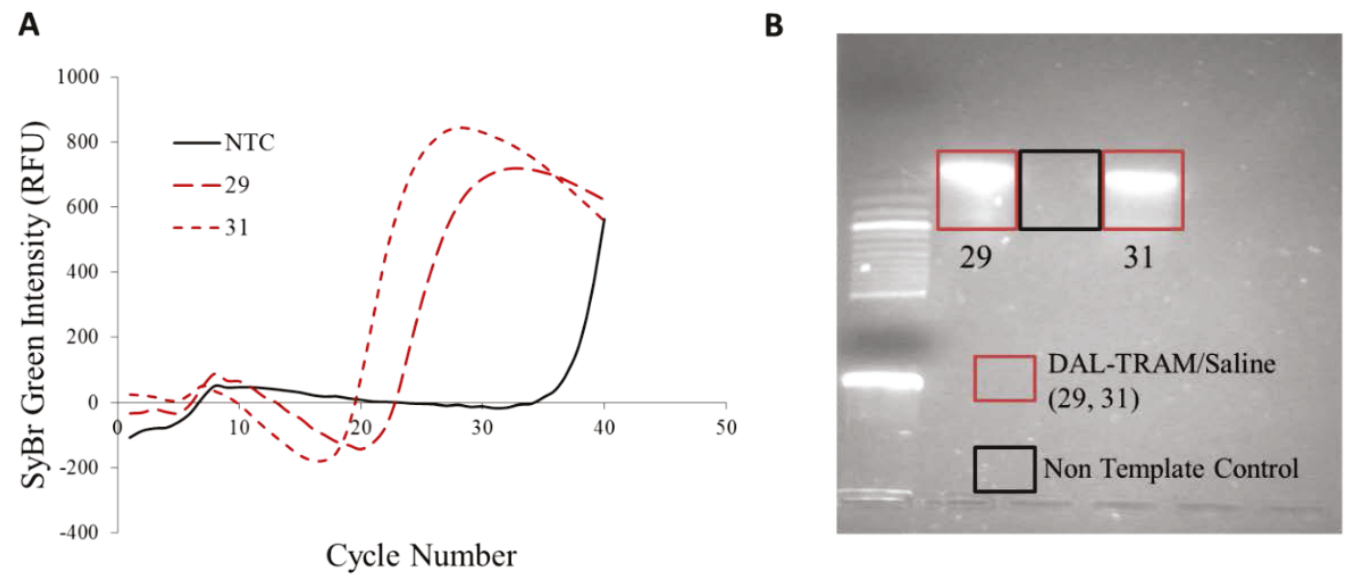

Cycle Number

Figure 5.9: Specific amplification by RT-qPCR of DBA delivered to the brain tissue by DAL-TRAM in saline treated animals compared to a non-template control (NTC). The amplification curves of the DBA from animals 29 and 31 are shown in (A). Amplification of the desired product was confirmed by gel electrophoresis (B). Lanes from left to right: 50 bp DNA ladder (1), animal 29 (2), non-template control (NTC) (3) and animal 31 (4).

Importantly, amplification of the desired product (DBA) was observed by RT-qPCR (Figure 5.9 A) and amplification was not observed in the non-template control reaction. Analysis of the RT-qPCR product by gel electrophoresis further supported the specific amplification of DBA. The expected length of the PCR product was $137 \mathrm{bp}$. This includes the full length of the DBA and the 40 base primer extensions at the 5 '-end and 3'-end. The migration of the amplification product (Figure 5.9: lanes 2 and 4) was comparable to the $150 \mathrm{bp}$ band of the DNA ladder (Figure 5.9: lane 1). Additionally, no 
amplification product was observed in the NTC band (Figure 5.9: lane 3). Therefore, sufficient evidence was provided by RT-qPCR and gel electrophoresis that amplification of DBA from brain tissue extract by RT-qPCR with DBA specific elongated primers was a viable approach for the detection of DBA in brain tissue.

To ensure that the amplification of DBA from brain tissue extract was reproducible and not affected by cocaine administration, tissue samples from animals treated with DAL-TRAM and either cocaine or saline were examined. Tissue extract was collected from animals that were treated with either DAL-TRAM+saline $(n=3)$, or DALTRAM $+10 \mathrm{mg} / \mathrm{mL}$ cocaine $(\mathrm{n}=5)$. As expected, amplification of the DBA was observed in the extract of tissue samples from the nucleus accumbens of animals treated with either cocaine or saline. A representative amplification and melt curve for an animal from each treatment condition are shown in Figure 5.10.
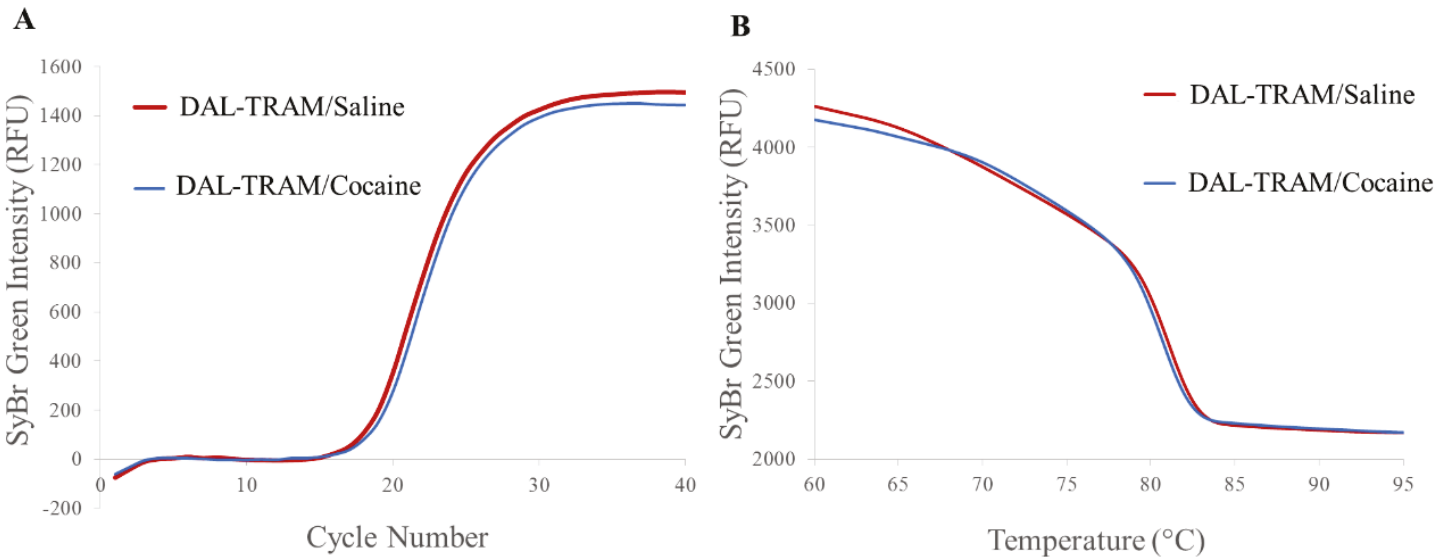

Figure 5.10: Representative amplification (A) and melt curves (B) for the RT-qPCR product of the DBA delivered to the brain via DAL-TRAM after oligonucleotide isolation from nucleus accumbens tissue.

DBA was identified by RT-qPCR in both saline and cocaine treated animals. No significant differences in either the average quantitation cycle $(\mathrm{Cq})$ or the melting 
temperature $\left(\mathrm{T}_{\mathrm{m}}\right)$ between the cocaine and saline treated animals was observed. The average $\mathrm{Cq}$ for amplification of the DBA from DAL-TRAM treated animals was $19.3 \pm$ 1.5 and $18.7 \pm 1.4$ for saline and cocaine treated animals respectively. The average $T_{m}$ of the RT-qPCR amplification product was $81{ }^{\circ} \mathrm{C}$ and $80.9 \pm 0.2{ }^{\circ} \mathrm{C}$ for saline and cocaine treated animals respectively. The observed melting temperature is consistent with the basic melting temperature of $81.2^{\circ} \mathrm{C}$ that was predicted by Oligo Calc for the expected RT-qPCR product. ${ }^{452}$

Additionally, the delivery of Sub to the brain via systemically administered SubTRAM was evaluated by RT-qPCR. Tissue was extracted and prepared as described in section 5.4.7 from animals treated with Sub-TRAM $+10 \mathrm{mg} / \mathrm{mL}$ cocaine $(\mathrm{n}=5)$. Given the similarity of the Sub sequence to the DBA (see Table 5.3), the DBA forward and reverse primers were used to amplify Sub. Representative amplification and melt curves are shown in Figure 5.11.
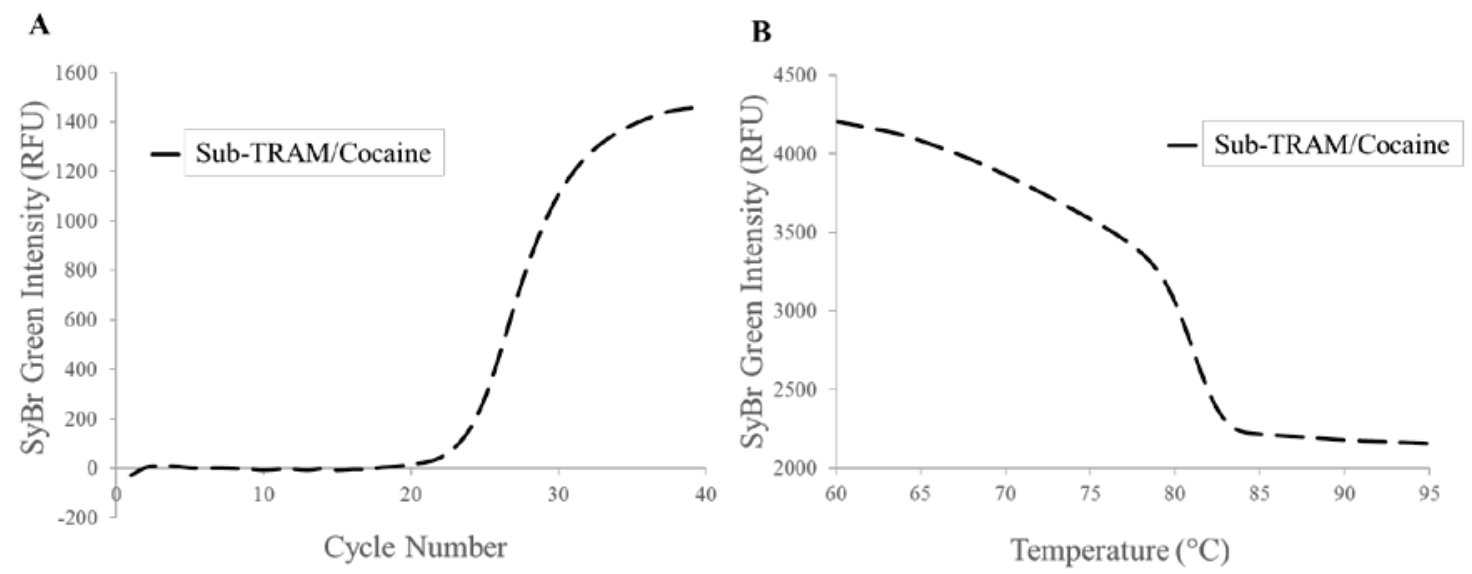

Figure 5.11: Representative amplification (A) and melt (B) curves of the Sub sequence from nucleus accumbens tissue after the systemic injection of Sub-TRAM are shown. 
The Cq values for the amplification of the Sub control oligonucleotide (Sub-TRAM; $\mathrm{n}=2$ ) were 24.4 and 23.9. It is important to note that the base substitutions in the non-binding substitute sequence (Sub) fall within the RT-qPCR primer regions, and therefore the initial amplification of Sub by the DBA forward and reverse primers would not have been as efficient as the amplification of the DBA (see Table 5.3). These differences in amplification efficiency are reflected in the difference between the Cq values of the DBA and Sub by RT-qPCR. The $\mathrm{T}_{\mathrm{m}}$ for the two substituted samples were 81.0 and $81.5^{\circ} \mathrm{C}$. The average quantitation cycle was lower for the Sub sequence compared to the DBA (18.9 \pm 1.4) suggesting more cycles were required to amplify the target DNA. However the $T_{m}$ of the Sub amplification product was the same as the DBA product, suggesting the products were similar. Therefore both sequences were successfully delivered to the brain and the behavioural differences observed cannot be attributed to lack of delivery of the non-binding Sub control sequence. Of the delivered oligonucleotides, only the DBA which specifically interacted with dopamine, led to a significant behavioural change.

\subsubsection{Behavioural and histochemical assessment of systemically administered DAL-TRAM}

The efficacy of systemically administered DAL-TRAM was assessed in three different experiments. The first experiment examined the efficacy of systemically administered DAL-TRAM in attenuating the hyperlocomotion exhibited in cocainetreated animals. The second experiment investigated the specific functional role of the DBA in mitigating cocaine-induced behavioural changes compared to control variations of the multi-DNA aptamer payload/targeting system. Finally, the third experiment 
assessed the effect of chronic systemic administration of DAL-TRAM on motor behaviour and neuronal degeneration.

\subsubsection{Experiment I: Examining the efficiency of DAL-TRAM in reducing cocaine-induced hyperlocomotion}

The purpose of the first part of the experiment was to determine the effect of systemically administered DAL-TRAM on hyperlocomotion induced by different cocaine dosages. Mice $(n=64)$ were assigned to one of the following groups depending on the cocaine dosage; $10 \mathrm{mg} / \mathrm{mL}$ cocaine ((DAL-TRAM+cocaine $(\mathrm{n}=8), \mathrm{DAL}-\mathrm{TRAM}+$ saline $(\mathrm{n}=5)$, TRAM+cocaine $(\mathrm{n}=8)) ; 5 \mathrm{mg} / \mathrm{mL}$ cocaine $((\mathrm{DAL}-\mathrm{TRAM}+$ cocaine $(\mathrm{n}=8)$, DALTRAM + saline $(n=8)$, TRAM + cocaine $(n=8))$ and $1 \mathrm{mg} / \mathrm{mL}$ cocaine $((D A L-$ TRAM+cocaine $(n=7)$, DAL-TRAM+saline $(n=5)$, TRAM+cocaine $(n=7))$. Mice received an i.p. injection $(0.1 \mathrm{~mL})$ of the pre-treatment (DAL-TRAM or TRAM) followed $5 \mathrm{~min}$ later by an i.p. injection $(0.1 \mathrm{~mL})$ of either cocaine or saline. Five min following the second injection, animals were placed in the locomotor apparatus and their horizontal activity was monitored over a 30 min session. Figure 5.12, Figure 5.13 and Figure 5.14 show the motor behaviour, divided into $5 \mathrm{~min}$ bins, for the entire $30 \mathrm{~min}$ session at each cocaine dosage. In each case, the DAL-TRAM+X $\mathrm{mg} / \mathrm{mL}$ cocaine was compared to both TRAM- X mg/mL cocaine and DAL-TRAM+saline where $\mathrm{X}$ represents $10 \mathrm{mg} / \mathrm{mL}, 5 \mathrm{mg} / \mathrm{mL}$ and $1 \mathrm{mg} / \mathrm{mL}$ cocaine. 


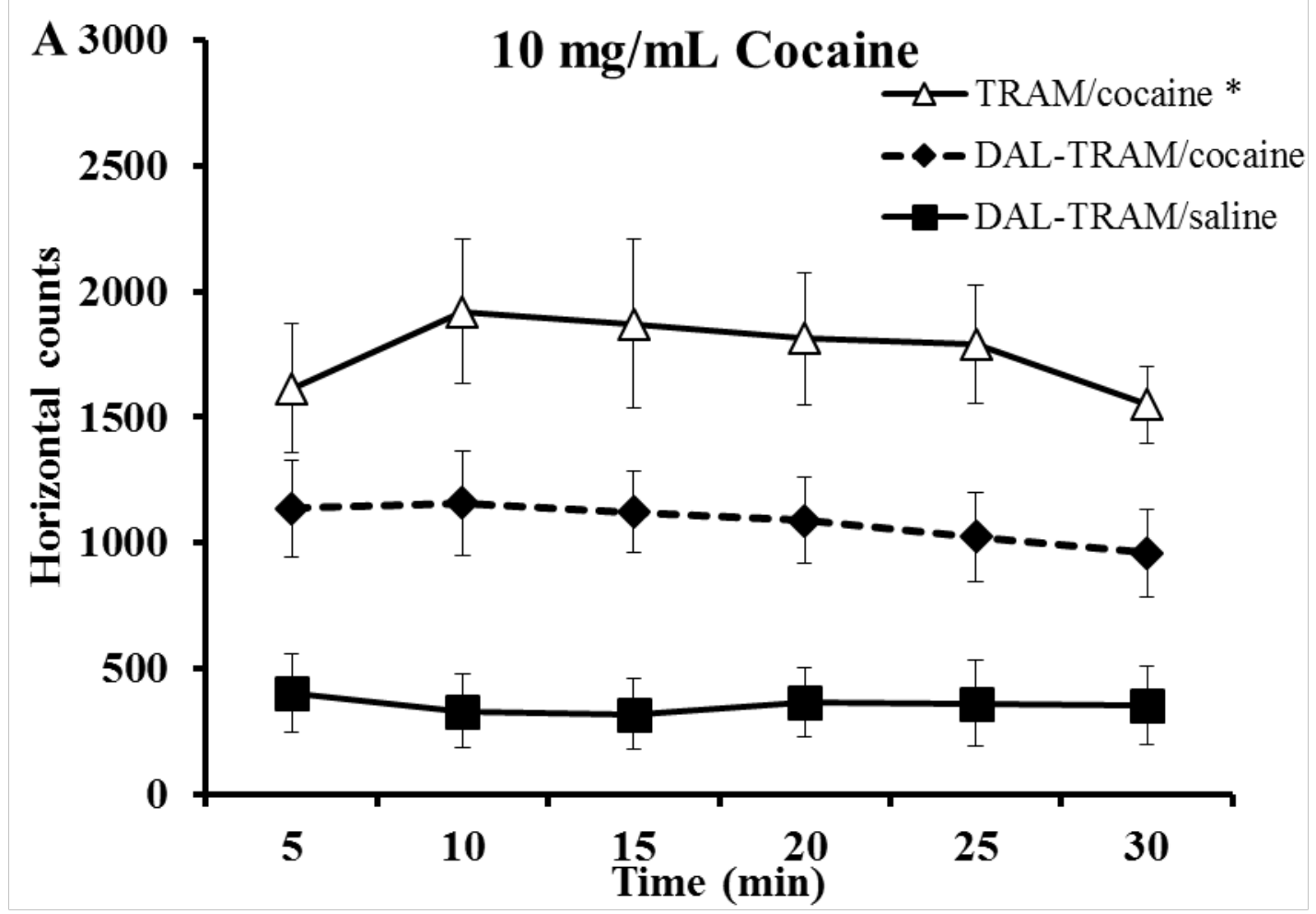

Figure 5.12: Horizontal locomotor activity of animals treated with $10 \mathrm{mg} / \mathrm{mL}$ cocaine. There was a significant decrease in locomotor behaviour of the DALTRAM treated animals compared to animals treated with TRAM. The horizontal locomotor activities over a 30 min session for the following groups; DAL-TRAM+10 $\mathrm{mg} / \mathrm{mL}$ cocaine $(n=8), T R A M+10 \mathrm{mg} / \mathrm{mL}$ cocaine $(n=8)$, and DAL-TRAM+saline $(n=5)$ are shown. Error bars represent "mean \pm SEM".

A main effect of treatment $(\mathrm{F}(2,18)=10.90, \mathrm{p}<0.001)$ was revealed by statistical analyses by a two-Way ANOVA (treatment condition by time) however, neither a main effect of time $(\mathrm{F}(5,10)=1.14)$ nor an interaction $(\mathrm{F}(10,90)<1.0)$ were revealed. Post-hoc analyses by Tukey's honest significant difference (HSD) examining the main effect of treatment condition revealed that there was a significant difference between the activity levels of both the DAL-TRAM $+10 \mathrm{mg} / \mathrm{mL}$ cocaine and DAL-TRAM+saline groups compared to the significantly more active TRAM $+10 \mathrm{mg} / \mathrm{mL}$ cocaine group $(* \mathrm{p}<0.05)$. 
Additionally, there was no significant difference between the DAL-TRAM $+10 \mathrm{mg} / \mathrm{mL}$ cocaine and DAL-TRAM+saline groups $(\mathrm{p}=0.07)$.

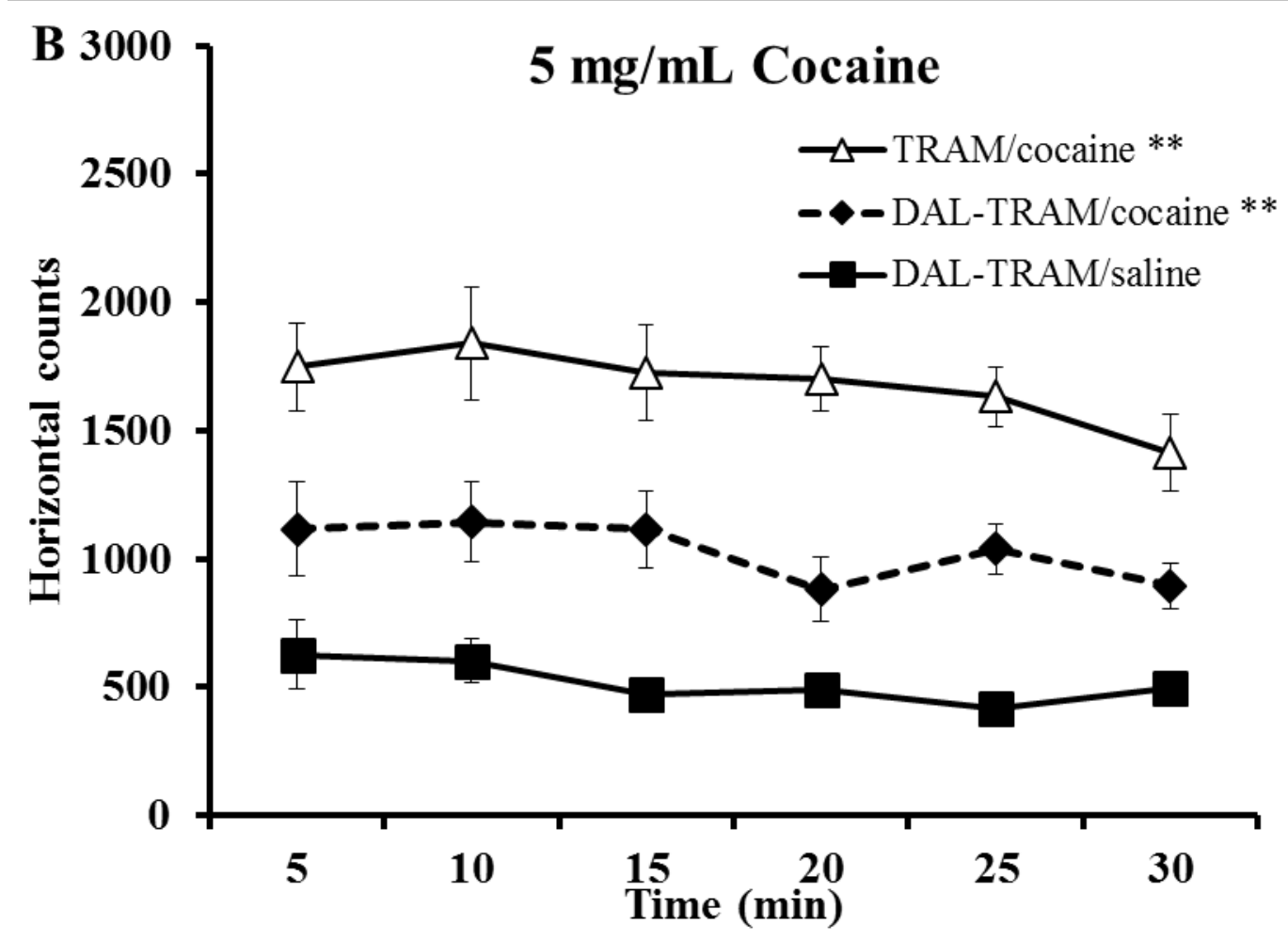

Figure 5.13: Horizontal locomotor activity of animals treated with $5 \mathrm{mg} / \mathrm{mL}$ cocaine. A significant decrease in locomotor behaviour of the DAL-TRAM treated animals compared to animals treated with TRAM was observed. The horizontal locomotor activities over a 30 min session of animals from the following groups; DALTRAM $+5 \mathrm{mg} / \mathrm{mL}$ cocaine $(\mathrm{n}=8)$, TRAM $+5 \mathrm{mg} / \mathrm{mL}$ cocaine $(\mathrm{n}=8)$, and DALTRAM+saline $(n=8)$ are shown. Error bars represent "mean \pm SEM".

A two-Way ANOVA (treatment condition by time) analyses revealed a significant main effect of treatment $(F(2,21)=28.25, p<0.001)$ and a significant main effect of time $(F(5,10)=4.46, p<0.001)$. However, no significant interaction $(F(10,105)=1.0)$ was revealed. Post-hoc analyses by Tukey's HSD on the main effect of treatment condition revealed a statistically significant difference in the activity level of both the DALTRAM $+5 \mathrm{mg} / \mathrm{mL}$ cocaine and DAL-TRAM+saline groups compared to the more active 
TRAM $+5 \mathrm{mg} / \mathrm{mL}$ cocaine group $(* * \mathrm{p}<0.01)$. Additionally, the difference in activity counts between the DAL-TRAM $+5 \mathrm{mg} / \mathrm{mL}$ cocaine group and the DAL-TRAM+saline group $(* * \mathrm{p}<0.01)$ was significant.

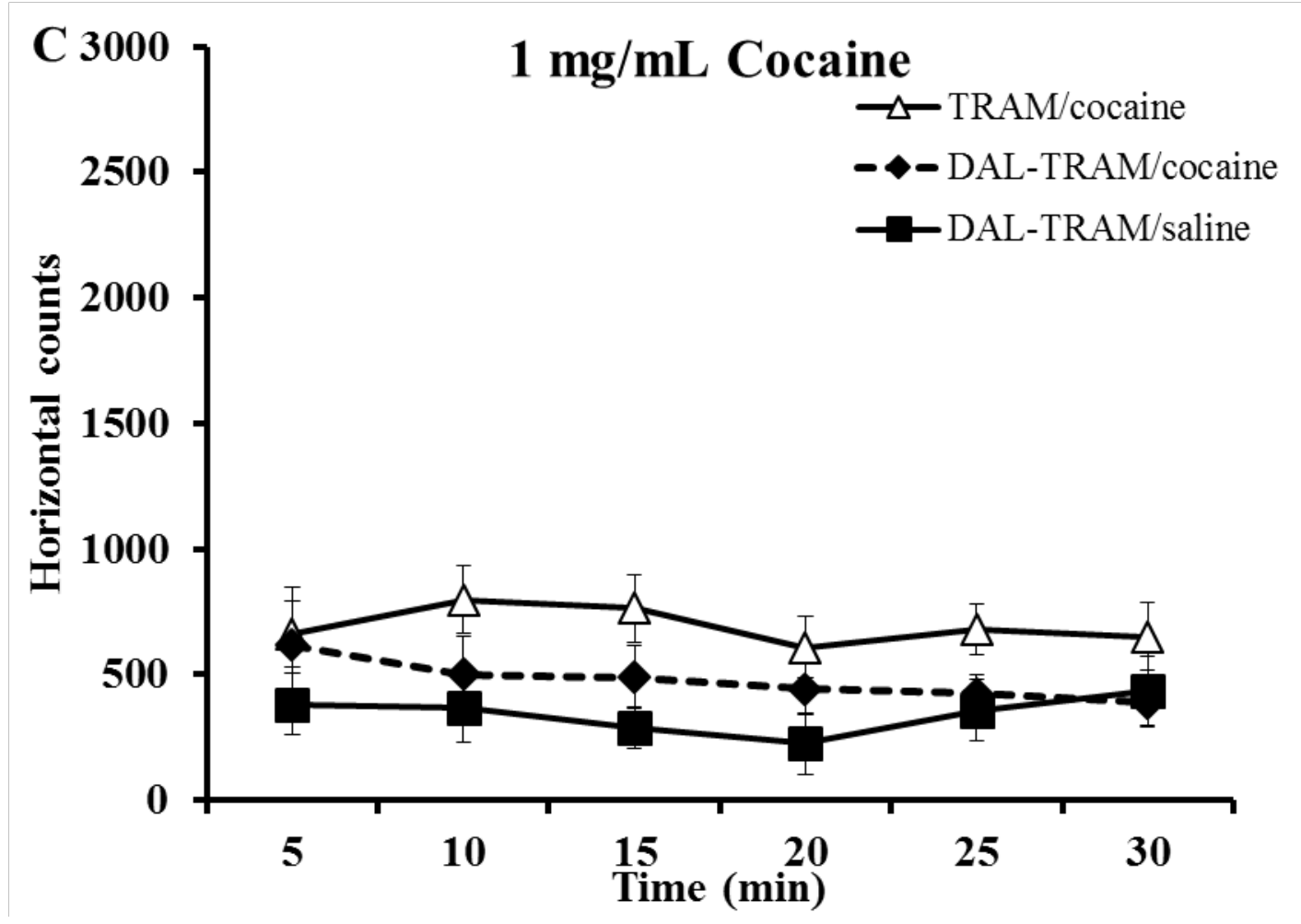

Figure 5.14: Horizontal locomotor activity of animals treated with $1 \mathrm{mg} / \mathrm{mL}$ cocaine. No significant effect on locomotor behaviour after treatment with either DALTRAM or TRAM was observed. The horizontal locomotor activities over a $30 \mathrm{~min}$ session of animals from the following groups; DAL-TRAM $+1 \mathrm{mg} / \mathrm{mL}$ cocaine $(\mathrm{n}=7)$, TRAM+1 $\mathrm{mg} / \mathrm{mL}$ cocaine $(\mathrm{n}=7)$, and DAL-TRAM+saline $(\mathrm{n}=5)$ are shown. Error bars represent "mean \pm SEM".

Analyses by a two-way ANOVA (treatment condition by time) revealed neither a main effect of treatment $(F(2,16)=2.20)$, nor a main effect of time $(F(5,10)=1.58)$.

Additionally, a significant interaction $(\mathrm{F}(10,80)<1.0)$ was not observed. Therefore, no further post-hoc analyses were performed. These data are consistent with observations previously published in which similar cocaine dosages $(10 \mathrm{mg} / \mathrm{kg}, 5 \mathrm{mg} / \mathrm{kg}$ and 2.5 
$\mathrm{mg} / \mathrm{kg}$ ) were administered to induce hyperlocomotion. ${ }^{397}$ In this study, hyperlocomotion was observed in the $10 \mathrm{mg} / \mathrm{kg}$ and $5 \mathrm{mg} / \mathrm{kg}$ dosage groups, but no significant difference in locomotion was observed for the $2.5 \mathrm{mg} / \mathrm{mL}$ group. Further, treatment with D1 and D2 receptor antagonists (SCH23390, raclopride, haloperidol, clozapine) significantly decreased motor behaviour in animals treated with $10 \mathrm{mg} / \mathrm{kg}$ of cocaine. Therefore to compare the therapeutic efficacy of the DAL-TRAM to traditional D1/D2 receptor antagonists, further experiments were conducted at $10 \mathrm{mg} / \mathrm{mL}$ cocaine.

In the second part of the experiment, the DAL-TRAM+cocaine experimental groups from the first half of the experiment were compared to several control groups. Mice $(n=53)$ were assigned to one of 7 conditions where i.p. injections were as follows pretreatment+treatment; Sub-TRAM+10 mg/mL cocaine $(\mathrm{n}=8)$; DAL-TRAM+10 $\mathrm{mg} / \mathrm{mL}$ cocaine $(\mathrm{n}=8)$; DAL-TRAM $+5 \mathrm{mg} / \mathrm{mL}$ cocaine $(\mathrm{n}=8)$ : DAL-TRAM $+1 \mathrm{mg} / \mathrm{mL}$ cocaine $(\mathrm{n}=7)$; DAL-TRAM+saline $(\mathrm{n}=8)$; TRAM + saline $(\mathrm{n}=8)$; saline + saline $(\mathrm{n}=6)$.

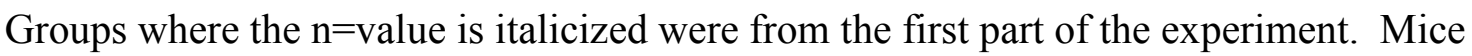
received the first injection of either loaded-TRAM (loaded with DBA or Sub) or emptyTRAM and then 5 min later received the second injection (cocaine or saline). Five minutes following the second injection animals were placed into the locomotor apparatus and their horizontal motor activity was monitored over a 30 min session. The total horizontal activity of each group is shown in Figure 5.15. 


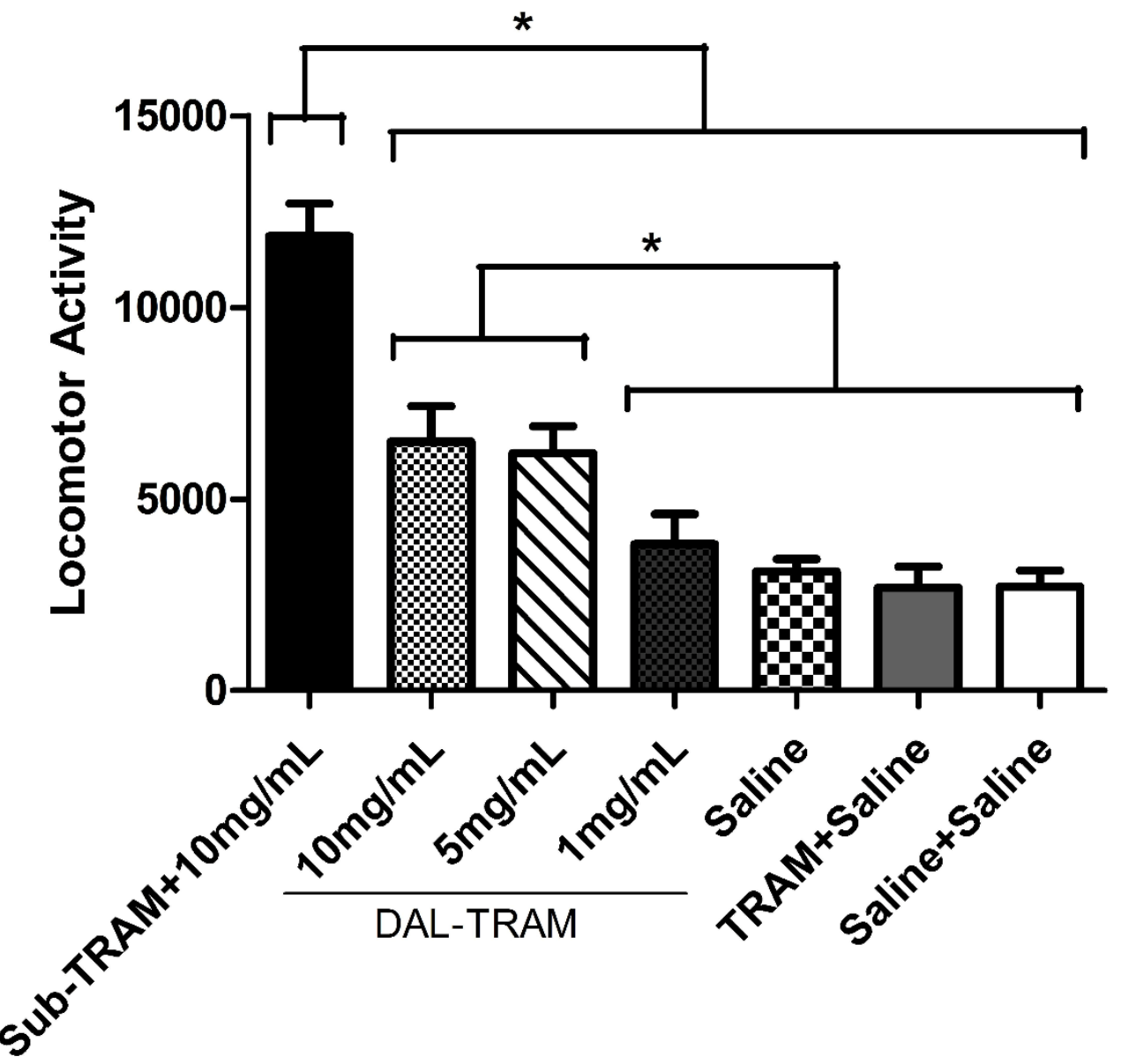

Treatment

Figure 5.15: Total horizontal locomotor activity of DAL-TRAM and control treated animals over a 30 min session. A significant effect of group $(F(6,46)=22.71$, p<0.001) was revealed by On-Way ANOVA analysis. Post-hoc analyses by Fisher's LSD revealed significant differences between treatment conditions as indicated (*p $<0.05)$. Groups sizes were as follows; Sub-TRAM+10 mg/mL cocaine $(\mathrm{n}=8)$, DALTRAM+10 $\mathrm{mg} / \mathrm{mL}$ cocaine $(n=8)$, DAL-TRAM+5 $\mathrm{mg} / \mathrm{mL}$ cocaine $(\mathrm{n}=8)$, DALTRAM+1 mg/mL cocaine $(n=7)$, DAL-TRAM+saline $(n=8)$, TRAM+saline $(n=8)$ and saline+saline $(n=6)$. Error bars represent "mean + SEM".

A significant group effect $(F(6,46)=22.71, p<0.001$; Figure 5.15) was revealed by analyses with One-way ANOVA on the total number of activity counts during the $30 \mathrm{~min}$ session. Post-hoc analyses by Fisher's LSD revealed that the Sub-TRAM $+10 \mathrm{mg} / \mathrm{mL}$ 
cocaine group was more active than all other groups $(\mathrm{p}<0.05)$. The DAL-TRAM +10 $\mathrm{mg} / \mathrm{mL}$ cocaine and DAL-TRAM $+5 \mathrm{mg} / \mathrm{mL}$ cocaine groups were more active than the DAL-TRAM $+1 \mathrm{mg} / \mathrm{mL}$ cocaine group and the 3 saline-treated control groups $(\mathrm{p}<0.05)$. Taken together, these results suggest that the specific affinity of the DBA for dopamine was responsible for the observed differences in total locomotor activity between the experimental (DAL-TRAM) and control groups. This finding complements the RT-qPCR data which showed that both the DBA and Sub sequences were present in brain tissue. However, as observed in the behavioural data, only the DBA was able to bind to dopamine resulting in the attenuation of hyperlocomotion in cocaine-treated animals. The experimental evidence presented supports the conclusion that the DAL-TRAM was effective in decreasing cocaine-induced hyperlocomotion, however it is important to note that locomotor behaviour was not normalized to the locomotor activity levels of the saline controls. Future work should examine optimizing the multi-DNA aptamer payload/targeting system to increase the therapeutic effect.

The observed reduction of locomotor activity in DAL-TRAM treated animals is comparable to the effect of traditional D1/D2 antagonists, which prevent the action of dopamine by binding to dopamine receptors and blocking the action of receptor agonists. ${ }^{397}$ However, a different mechanism of action has been proposed for the DBA (see Chapter 4). Briefly, the DBA is hypothesized to bind directly to synaptic DA based on the affinity of the aptamer for free dopamine. The interaction of the DBA with DA would effectively decrease the available concentration of DA in the synaptic cleft that is able to act on post-synaptic dopamine receptors and autoreceptors. This effective decrease in synaptic dopamine which manifests behaviourally as nearly or fully 
normalized locomotor behaviour which is consistent with what was observed in this experiment and the experiments described in Chapter 4 respectively.

\subsubsection{Experiment II: Variations of the multi-aptamer payload/targeting system (DAL-TRAM) were examined to determine the specific role of each} component of the system

Several control variations of the DAL-TRAM system were tested and compared to the efficacy of DAL-TRAM in vivo to determine the possible interfering effects of the non-active components of the multi-DNA aptamer payload/targeting system on cocaine induced hyperlocomotion. The following experimental $(10 \mathrm{mg} / \mathrm{mL}$ cocaine treated $)$ and control (saline treated) groups were examined: saline/saline $(n=6)$, TRAM + saline $(n=8)$, DAL-TRAM+saline ( $n=8)$, DAL-TRAM+cocaine $(n=8)$, DAL+cocaine $(n=9)$, ROLTRAM+cocaine $(n=7)$, TRAM+cocaine $(n=8)$, and Sub-TRAM+cocaine $(n=8)$. As was done in Experiment I, mice were given the first injection (DAL-TRAM, DAL-TRAM variation, or saline) followed 5 minutes later by the second injection (cocaine or saline). Following another 5 min delay, mice were placed into the locomotor apparatus and their total horizontal activity over the $30 \mathrm{~min}$ session was monitored (Figure 5.16). 


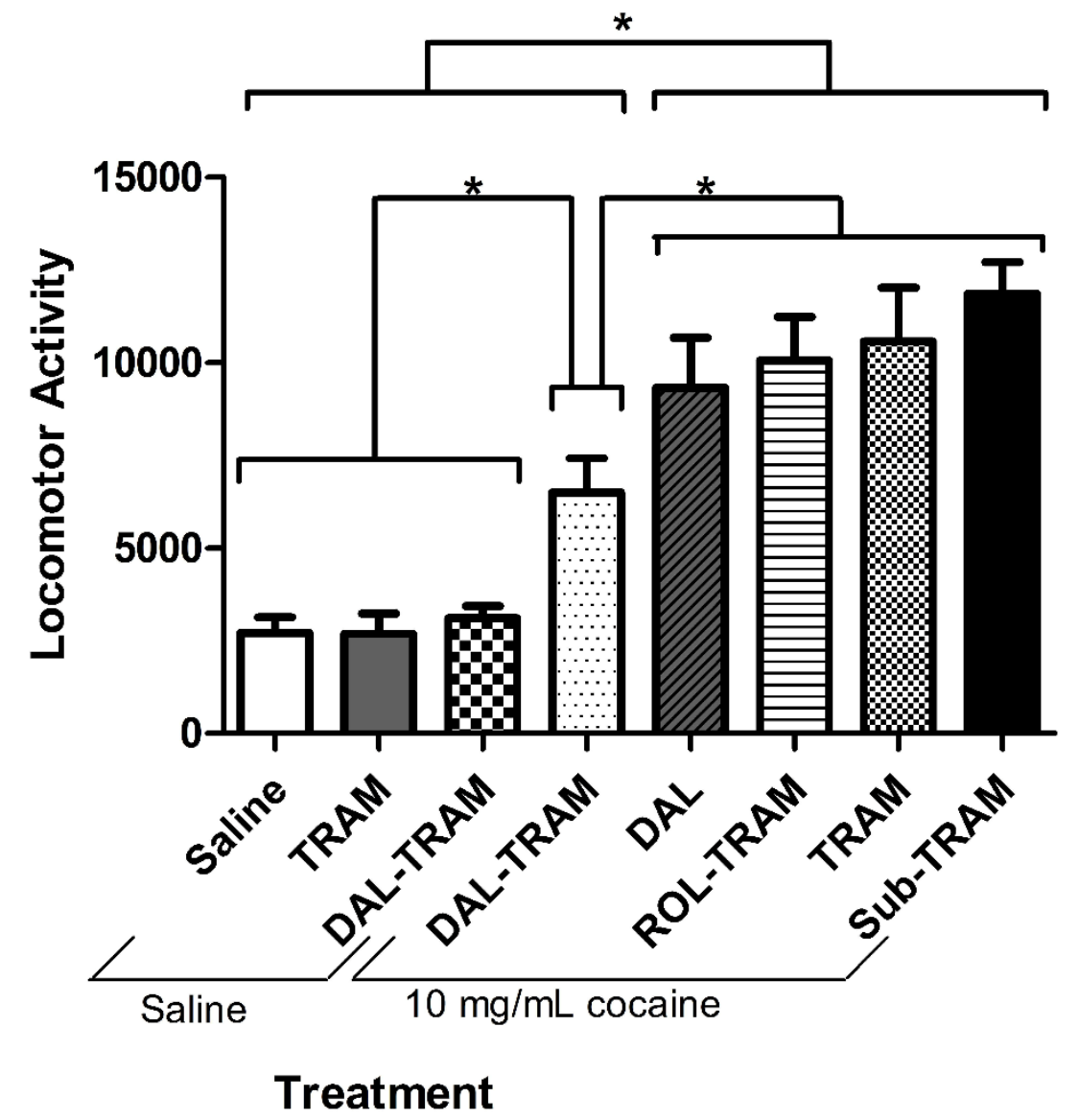

Figure 5.16: The efficacy of multiple control variations of the multi-DNA aptamer payload/targeting system in decreasing cocaine $(10 \mathrm{mg} / \mathrm{mL})$ induced hyperlocomotion was examined. The total horizontal activity over a 30 min session is shown. A significant group effect $(F(7,54)=14.22, p<0.001)$ was revealed through analysis by a One-way ANOVA. Fisher's LSD post-hoc analyses revealed a significant difference between groups $(* p<0.05)$. Examined groups were as follows; saline+saline $(n=6)$, TRAM+saline $(n=8)$, DAL-TRAM+saline $(n=8)$, DALTRAM+10 $\mathrm{mg} / \mathrm{mL}$ cocaine $(n=8), D A L+10 \mathrm{mg} / \mathrm{mL}$ cocaine $(n=9), R O L-T R A M+10$ $\mathrm{mg} / \mathrm{mL}$ cocaine $(n=7)$, TRAM $+10 \mathrm{mg} / \mathrm{mL}$ cocaine $(n=8)$, and $S u b-T R A M+10 \mathrm{mg} / \mathrm{mL}$ cocaine $(n=8)$. Error bars represent "mean + SEM"

A significant group effect $(F(7,54)=14.22, p<0.001$; Figure 5.16) was revealed through analysis by One way ANOVA on the total number of activity counts during the $30 \mathrm{~min}$ session. Post-hoc analyses by Fisher's LSD revealed a significant difference between the 
DAL-TRAM $+10 \mathrm{mg} / \mathrm{mL}$ cocaine group and all other control and variation groups $(\mathrm{p}<0.05)$. The total horizontal activity of the DAL $+10 \mathrm{mg} / \mathrm{mL}$ cocaine, ROL-TRAM +10 $\mathrm{mg} / \mathrm{mL}$ cocaine, TRAM $+10 \mathrm{mg} / \mathrm{mL}$ cocaine and Sub-TRAM $+10 \mathrm{mg} / \mathrm{mL}$ cocaine groups was significantly elevated compared to both the aptamer (DAL-TRAM+cocaine; $\mathrm{p}<0.05$ ) and saline control groups $(\mathrm{p}<0.001)$. Taken together, these data suggest that the TRA mediated delivery of the DBA payload to the brain mediated the observed decrease in hyperlocomotion.

Specifically, a liposome with no oligonucleotide payload (TRAM) was administered to determine whether or not hyperlocomotion was affected by the TRA conjugated liposome. Neither locomotion in the absence of cocaine nor a reduction in hyperlocomotion in cocaine treated animals was observed following treatment with TRAM suggesting that the TRAM vehicle did not have a non-specific deleterious effect on locomotion. To examine the essential role of the TRAM in delivering the aptamer payload (DBA) to the brain animals were administered with DAL, DBA loaded liposome without transferrin aptamer. No reduction in hyperlocomotion was observed when the DAL was administered. Therefore, systemic administration of the DBA via DAL (without the TRAM component of the system) was ineffective in delivering the DBA to the brain. Previously, the direct injection of a random oligonucleotide into the nucleus accumbens showed no effect on locomotor behaviour (see chapter 4). To confirm that neither a random oligonucleotide (ROL-TRAM) nor a non-binding variant of the DBA (Sub-TRAM) had non-specific effects on hyperlocomotion, these control variants were examined. No reduction in hyperlocomotion was observed in either case. These observations confirmed that DBA was essential to the system, and simply the presence of 
foreign DNA alone, did not lead to a significant decrease in locomotor behaviour in cocaine treated animals. Importantly, the singular role of the DBA in decreasing locomotor behaviour was confirmed by the significant decrease in locomotor behaviour observed between the DAL-TRAM/cocaine treated animals compared to all other control variations (the transferrin-negative liposomes (DAL+cocaine), random oligonucleotide (ROL-TRAM+cocaine), non-binding DBA variant (Sub-TRAM+cocaine) and the liposome with no oligonucleotide payload (TRAM+cocaine)). Additionally, acute treatment with the DAL-TRAM in saline treated animals was examined to determine whether the DAL-TRAM had any effect on locomotor behaviour in normal animals. Compared to the saline+saline control group, treatment with DAL-TRAM did not produce significant motor deficits in saline treated animals. This finding is consistent with the work described in Chapter 4 and is an important advantage of the DBA over traditional D1/D2 antagonists, which often cause disruptive extrapyramidal side effects. $^{453}$

\subsubsection{Experiment III: The effect of chronic administration of DAL-TRAM was examined by behavioural and histochemical methods}

\subsubsection{Experiment III A: Chronic administration of DAL-TRAM in saline treated animals}

Preliminary evidence from the first and second experiments suggested that motor deficits were not produced by acute injection of the DAL-TRAM in saline treated animals. Therefore the purpose of the third behavioural experiment was to examine whether or not chronic injection of either the DAL-TRAM/saline or the TRAM/saline 
would produce motor deficits. Mice $(\mathrm{n}=15)$ were treated for 6 days with DAL-TRAM $(\mathrm{n}=$ 5), TRAM $(n=5)$ or no injection $(n=5)$. The horizontal locomotor activity for each group was recorded over a 30 min session on each of the 6 test days (Figure 5.17 A). The total horizontal movement recorded during the final day is shown in Figure 5.17 B.
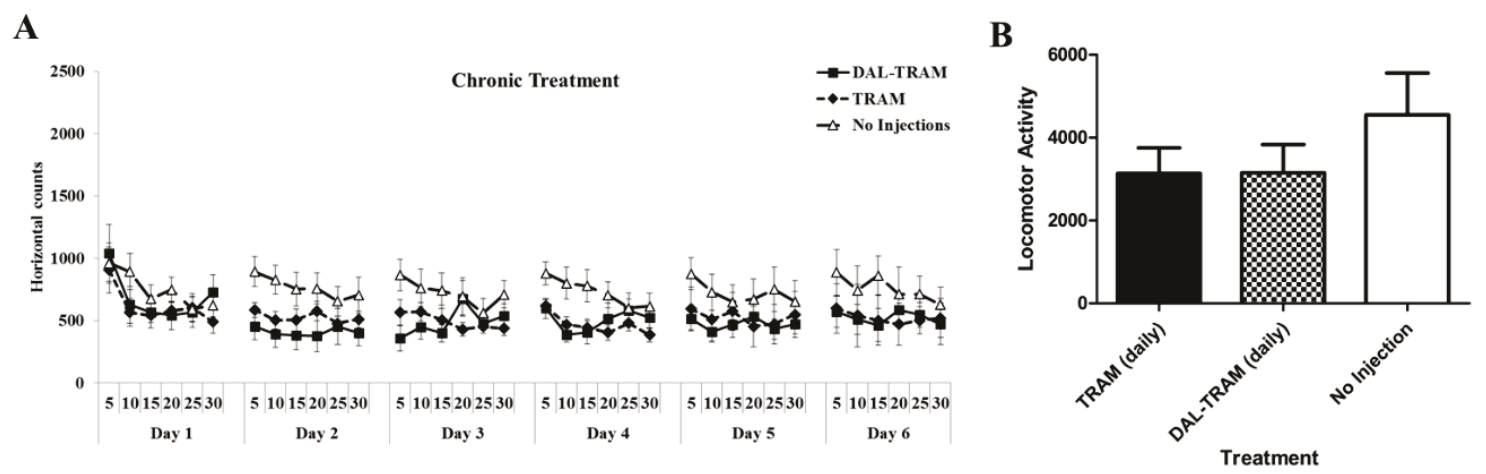

Figure 5.17: A) No significant effect on locomotor activity was observed after chronic administration of either DAL-TRAM or TRAM. The horizontal locomotor activity of the following groups was monitored over a $30 \mathrm{~min}$ session once a day for 6 days; DAL-TRAM $(n=5)$, TRAM $(n=5)$, and no injections $(n=5)$. Horizontal activity is divided into $5 \mathrm{~min}$ bins (shown on the $\mathrm{x}$-axis) for each $30 \mathrm{~min}$ session. Error bars represent "mean \pm SEM". B) Total horizontal activity recorded during the final $30 \mathrm{~min}$ session of the experiment (day 6) is shown. One-way ANOVA revealed no significant group effect $(F(3,24)=0.949, p=0.433)$. The following groups were examined; TRAM $(n=5)$, DAL-TRAM $(n=5)$ and no injection $(n=5)$. Error bars represent "mean + SEM".

To first examine whether there was an effect of chronic administration of either DALTRAM or TRAM on locomotor behaviour, a three-way ANOVA (treatment condition by day by 5 min time bin) was performed. No main effect of treatment condition $(\mathrm{F}(2,12)=$ 1.35), no main effect of day $(F(5,10)=1.71)$ and no interaction between treatment condition and day $(\mathrm{F}(10,60)<1.0)$ were revealed. There was a main effect of 5-min time bin $(F(5,10)=14.18, p<0.001)$ and a significant interaction between 5-min time bin and treatment condition $(\mathrm{F}(10,60)=2.99, \mathrm{p}<0.01)$. Lastly, a significant interaction between 
day and 5 -min time bin $(F(25,50)=2.00, \mathrm{p}<0.01)$ as well as a significant three-way interaction between treatment condition, day and 5-min time bin $(F(50,300)=1.65, p<$ 0.01) was observed. However, since there was no main effect of treatment condition, no further analyses were performed on this data.

To further analyze whether chronic administration of either the DAL-TRAM or TRAM led to motor deficits, a one-way ANOVA on the total locomotor activity was performed (Figure 5.17 B). No main effect of group $(F(3,24)=0.949, p=0.433)$ was revealed. Together, these data suggest that horizontal locomotor activity was not affected by the chronic administration of DAL-TRAM or TRAM.

\subsubsection{Experiment III B: Fluorescent histochemical investigation of neuronal degeneration by Fluoro-Jade B Staining}

Whether or not cellular degeneration occurred in animals that had been chronically treated with either DAL-TRAM or TRAM was investigated. Fluoro-Jade B, known to stain the cell bodies, the axons, the axon terminals and the dendrites of degenerating neurons with high affinity and minimal background staining regardless of the source or mechanism of injury was used to examine brain tissue from DAL-TRAM or TRAM treated animals after chronic administration. ${ }^{454,455}$ To examine whether chronic systemic administration of either DAL-TRAM or TRAM caused damage at the cellular level, mice were euthanized and brain tissue was processed for Fluoro-Jade B staining 48 hours after the last locomotor assessment of experiment III A. ${ }^{454}$ Representative images of tissue samples from the DAL-TRAM, TRAM and no injection groups were compared to control tissue (Figure 5.18) to identify cellular damage. In the positive control image, 
physical damage was caused by implantation of a chronic, indwelling cannula and as a result of the damage extensive Fluoro-Jade B staining near the cannula tip and extending outward was revealed. ${ }^{456}$ This pattern of fluorescence was consistent with damaged neurons (Figure 5.18: white arrows) and reactive astrocytes or gliosis. ${ }^{456}$ In comparison, appearing similarly stained to the negative, no injection control (D), Fluoro-Jade B stained tissue from both the chronic administration of the DAL-TRAM (B) and the TRAM (C) groups showed no evidence of cellular degeneration.
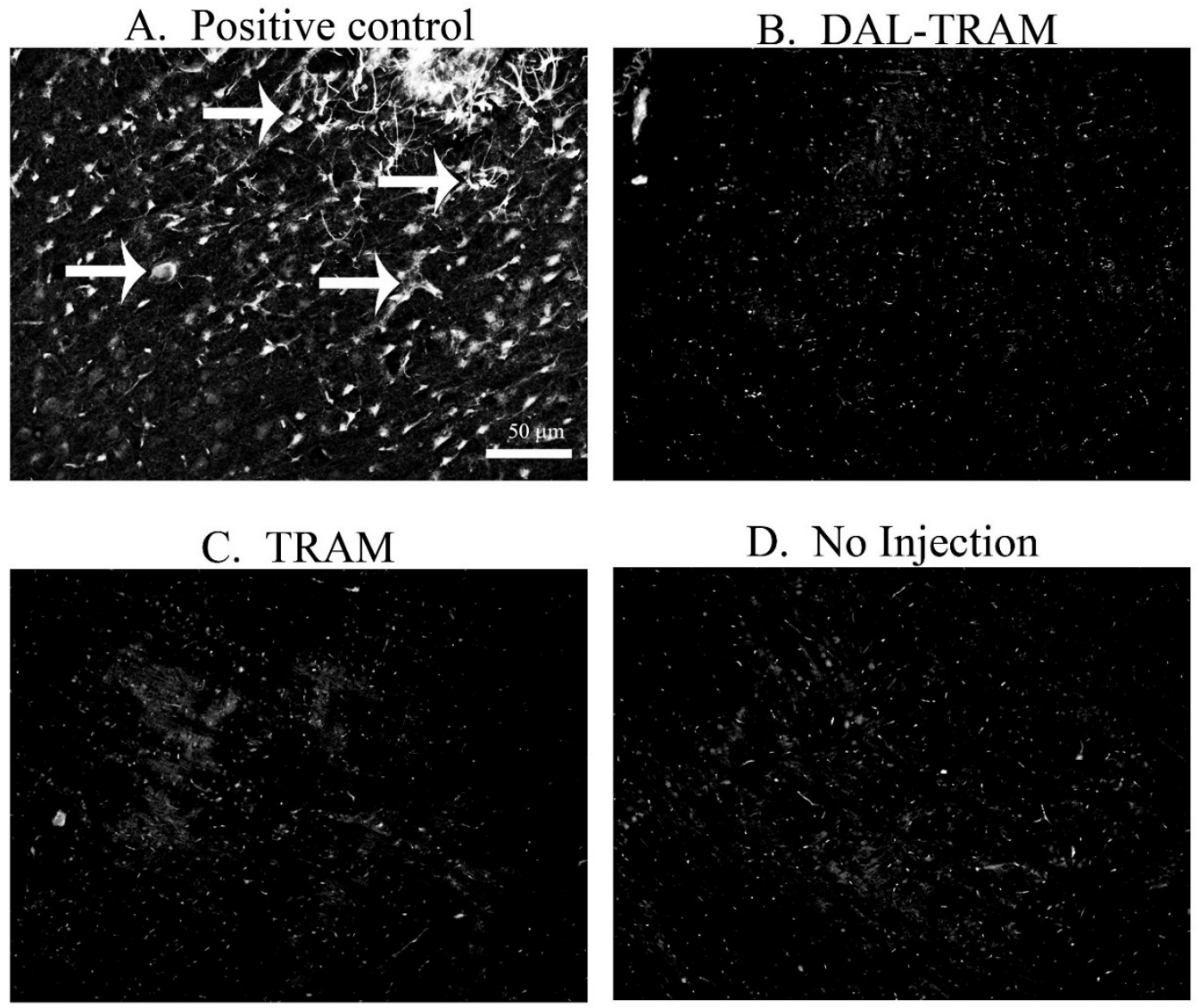

Figure 5.18: No indication of neuronal degeneration in animals that had been chronically treated with DAL-TRAM was revealed by Fluoro-Jade B staining. (A) Regions of neural tissue damage are indicated by white arrows. Magnification is at 20X. 
No sign of cellular damage after repeated (chronic) systemic injections of either DALTRAM or TRAM was observed in the nucleus accumbens, indicating that cellular viability was maintained after chronic treatment with either DAL-TRAM or TRAM. Early indications of inflammation and cellular damage should be more thoroughly investigated in future experiments. 


\subsection{Conclusions and Future Work}

The data presented in this chapter have shown that the multi-aptamer payload/targeting system described was able to effectively decrease cocaine-induced hyperlocomotion without causing motor side effects or neuronal degeneration. The application of this multi-DNA aptamer payload/targeting system to investigate dopaminerelated pathology is not limited to this model of cocaine addiction but could also be used to investigate cognitive deficits associated with schizophrenia as well. ${ }^{375}$ In fact, given the success of the DBA in normalizing schizophrenic-like behaviour after acute intraaccumbens injection (Chapter 4), it is possible that delivery of the DBA to the brain via the systemic administration of DAL-TRAM could also normalize schizophrenic-like behaviour. ${ }^{375}$ Importantly, the impact of the multi-DNA aptamer payload/targeting system should be acknowledged as to the authors' knowledge these data presented here demonstrate the first example of a systemically administered multi-aptamer payload/targeting system to delivery aptamer payload across the BBB and attenuate behaviour in an animal model of mental illness-associated behaviours.

Additionally, the potential applications of the multi-DNA aptamer payload/targeting system are only limited by the availability of aptamers for specific targets within the CNS. Several aptamers have been selected for CNS-related targets, and many of these aptamers could be combined to develop novel multi-aptamer payload/targeting systems. ${ }^{1}$ The potential applications of the payload aptamer are also diverse; currently applications of aptamers within the CNS include but are not limited to diagnostics, therapeutics, contrast and imaging agents, receptor modulators etc. ${ }^{1}$ 
Furthermore, the inclusion of multiple aptamers (for different targets or with different affinities) or modified aptamers such as aptazymes and SOMAmers could broaden the target diversity and increase the functionally responsive range of the aptamer payload for its cognate target(s), as well as add additional elements of biostability, structural stability, and functionality to the multi-aptamer payload/targeting system. ${ }^{457,458}$

Future work should investigate modifications to the multi-DNA aptamer payload/targeting system described in order to optimize it for more generalized CNS treatment approaches. An example of such a modification would be to change to the TRA targeting moiety of the multi-DNA aptamer payload/targeting system. Recently, increased structural stability and biological activity in comparison to the originally reported TRA (investigated in this work) were reported for truncated minimers as well as rationally designed full length mutants. ${ }^{240}$ Moreover, aptamer modifications to increase in vivo stability and circulation time of the aptamer post liposome release should also be examined. Finally, the properties of the payload/targeting liposomal system, such as in vivo stability and payload release from the liposome vehicle should be optimized to enhance the therapeutic efficacy of the multi-DNA aptamer payload/targeting system. 
6 The selection of dopamine-binding aptamers for central nervous system related applications 


\subsection{Statement of Contributions}

The selection of DNA aptamers for dopamine was performed by EM McConnell, with guidance from R. Walsh and MC DeRosa. The selection described in this section was performed by EM McConnell in 2008-2009 as an undergraduate student prior to the commencement of EM McConnell's Ph.D. work. A brief summary of this work is necessary to include as it is contextually important. The affinity and specificity of the aptamers for dopamine were examined by EM McConnell with assistance from C. Bradley and Y. Miguel. Conceptual design of experiments for the determination of $\mathrm{K}_{\mathrm{D}}$ and specificity was by EM McConnell and MC DeRosa. The gold nanoparticle sensor work was done by EM McConnell and JP Callahan. Animal related work was conceptualized by EM. McConnell, K. Ventura, MR. Holahan and MC. DeRosa. Animal related work was done by EM. McConnell, K. Ventura, Z. Dwyer, T. Wilson, M. Sieczkos and MR. Holahan.

\subsection{Resulting Publications}

None of the data presented in this chapter are published. The manuscript of this work is under preparation. 


\subsection{Introduction}

\subsubsection{Objectives}

This chapter describes the selection of novel dopamine binding DNA aptamers for the neurotransmitter target dopamine. The secondary structure, affinity and specificity of one minimal aptamer, DopaA20min were characterized by multiple methods. The development of an aptamer-based gold nanoparticle sensor for the detection of dopamine is also described. Finally, the effect of peripherally delivered DopaA20min on hyperlocomotion was examined in an animal model.

\subsubsection{Dopamine in the CNS}

The monoamine neurotransmitter dopamine is involved in multiple complex processes within the CNS including normal motor function, learning and memory, emotion, motivation, and reward. ${ }^{384,459}$ Dopamine-related abnormal functioning has been linked to psychiatric and neurodegenerative diseases and disorders such as Parkinson's, schizophrenia, addiction, Huntington's, Tourette syndrome, bipolar disorder and attention deficit hyperactivity disorder ${ }^{169,459-464}$ Clearly, DA plays a critical role in complex CNS functioning. Therefore the selection of novel DNA aptamers may provide a valuable tool for dopamine-related maladies.

\subsubsection{Existing dopamine binding aptamers (DBAs)}

Two commonly used and well characterized aptamers have been selected for dopamine. The first aptamer selected for dopamine was an RNA aptamer described by Mannironi et al., (1997) and was one of the first aptamers selected for a small molecule target. $^{280,291}$ The tertiary structure of the aptamer was predicted to be two stem loop 
motifs that come together to form a binding domain in which a single dopamine molecule was able to interact. Equilibrium filtration was used to estimate the dissociation constant of this interaction as $1.6 \pm 0.2 \mu \mathrm{M}$. This prediction was supported by minimization and mutational analysis studies used to identify nucleotides implicated in dopamine-aptamer binding. The specificity of the aptamer was evaluated by testing its binding affinity against similar derivative molecules and other small molecule neurotransmitters using affinity chromatography. Some affinity of the aptamer for norepinephrine (NE) and L3,4-dihydroxyphenylalanine (L-DOPA) was shown with relative elution values of $53 \pm 13$ and $30 \pm 13$ percent of dopamine elution respectively. The final reported aptamer sequence was a minimal 57 nucleotide sequence (henceforth referred to as the RNA DBA)

An interesting feature of the sequence of the RNA DBA was that the nucleobases important for binding of the dopamine to the RNA aptamer were not specific to RNA. For this reason, the ability of the DNA homolog of the RNA DBA sequence to bind dopamine was investigated. ${ }^{284}$ The DNA homolog was shown to not only retain its dopamine binding functionality but also to have an improved affinity for both dopamine and NE. Of note, the DNA homolog showed slightly higher affinity for NE $(0.4 \mu \mathrm{M})$ than dopamine $(0.7 \mu \mathrm{M})$. The differences observed in the measured affinity could be due to either the affinity method used or the structural differences between DNA and RNA. ${ }^{276,284}$ In fact, increased sensitivity and specificity of the DNA aptamer over the RNA aptamer was observed when the two aptamers were compared in an enzyme-linked aptamer assay (ELAA). ${ }^{465}$ The authors proposed that the secondary structure and 
resultant tertiary interaction of the dopamine and DNA homolog was similar to that described for the RNA aptamer. This claim was supported by mutational analysis and later confirmed by the use of circular dichroism. ${ }^{284,466}$ It is important to highlight that this is one of only a few examples where the aptamer sequence was transcribed from one nucleic acid type to another and similar structure and functionality were maintained. ${ }^{467}$

\subsubsection{Dopamine binding aptamer (DBA) based detection assays}

Multiple sensing-based applications have been developed using either the DNA or RNA dopamine binding aptamers. Most commonly, electrochemical biosensors were developed. Additionally, enzyme-linked and gold nanoparticle based assays were also described. Depending on the method the detection range spanned from picomolar to micromolar concentrations, providing functionality within physiologically relevant conditions. This work is summarized in Table 6.1, Table 6.2, and Table 6.3.

Table 6.1: RNA DBA ${ }^{280}$ based dopamine detection assays

\begin{tabular}{l|l|l|}
$\begin{array}{l}\text { Detection } \\
\text { matrix }\end{array}$ & Detection method and sensor design & \multicolumn{1}{l}{ Reference } \\
\hline Human serum & $\begin{array}{l}\text { Competitive enzyme-linked apt assay } \\
\text { (DR: } 50 \mathrm{pM}-0.1 \mu \mathrm{M} \text {; LOD: } 1.0 \mathrm{pM})\end{array}$ & $\begin{array}{l}\text { (Park and } \\
\text { Paeng, 2011) }\end{array}$ \\
\hline $\begin{array}{l}\text { PBS spiked with } \\
\text { relevant } \\
\text { competitors }\end{array}$ & $\begin{array}{l}\text { Specific label-free electrochemical biosensor } \\
\text { (DR: } 100 \mathrm{nM}-5 \mu \mathrm{M} \text {; LOD: } 0.1 \mu \mathrm{M})\end{array}$ & $\begin{array}{l}\text { (Farjami et al., } \\
2013)^{469}\end{array}$ \\
\hline $\begin{array}{l}\text { Tris buffer }(\mathrm{pH} \\
7) \text { spiked with } \\
\text { relevant } \\
\text { competitors }\end{array}$ & $\begin{array}{l}\text { Specific electrochemical biosensor } \\
\text { (DR: } 100 \mathrm{nM}-5 \mu \mathrm{M} \text {; LOD: } 50 \mathrm{nM})\end{array}$ & $\begin{array}{l}\text { (Jiao et al., } \\
2013)^{470}\end{array}$ \\
\hline
\end{tabular}




\begin{tabular}{|l|l|l|}
\hline PBS (pH 7.4) & $\begin{array}{l}\text { Optimization of biosensor reported by Farjami et } \\
\text { al., } 2013^{469} \\
\left(\mathrm{~K}_{\mathrm{D}}: 0.12 \pm 0.01 \mu \mathrm{M} \text {; DR: 0.1 -2 } \mu \mathrm{M} \text {; LOD: } 0.1\right. \\
\mu \mathrm{M})\end{array}$ & $\begin{array}{l}\text { (Álvarez-Martos } \\
\text { et al., 2015) }\end{array}$ \\
\hline Fetal calf serum & $\begin{array}{l}\text { Specific label-free aptasensor and confirmation of } \\
\text { the dissociation constant } \\
\left(\mathrm{K}_{\mathrm{D}}: 1.03 \pm 0.09 \mu \mathrm{M} \text {; DR: 0.1-1 } \mu \mathrm{M} \text {; LOD: } 62\right. \\
\mathrm{nM})\end{array}$ & $\begin{array}{l}\text { (Álvarez-Martos } \\
\text { et al., 2016) }\end{array}$ \\
\hline
\end{tabular}

Abbreviations: aptamer (apt), detection/dynamic range (DR), limit of detection (LOD), dissociation constant $\left(\mathrm{K}_{\mathrm{D}}\right)$

Table 6.2: Sensors developed based on both the RNA ${ }^{280}$ and DNA ${ }^{284}$ dopaminebinding aptamers (DBAs)

\begin{tabular}{|c|c|c|}
\hline $\begin{array}{l}\text { Detection } \\
\text { matrix }\end{array}$ & Detection method and sensor design & Reference \\
\hline Human serum & $\begin{array}{l}\text { Comparison of sensitivity and specificity of both } \\
\text { aptamers in competitive ELAA } \\
\text { (RNA: DR: } 1.0 \times 10^{-4} \mathrm{~mol} / \mathrm{L}-1.0 \times 10^{-7} \mathrm{~mol} / \mathrm{L} \text {; } \\
\text { LOD: } 6.3 \times 10^{-8} \mathrm{~mol} / \mathrm{L} \text {; DNA: DR: } 5.0 \times 10^{-6} \\
\mathrm{~mol} / \mathrm{L} \text { to } 1.0 \times 10^{-10} \mathrm{~mol} / \mathrm{L} \text { LOD: } 3.2 \times 10^{-12} \\
\mathrm{~mol} / \mathrm{L} \text { ) }\end{array}$ & $\begin{array}{l}\text { (Kim et al., } \\
2014)^{465}\end{array}$ \\
\hline
\end{tabular}

Abbreviations: detection/dynamic range (DR), limit of detection (LOD), enzyme-linked aptamer assay (ELAA)

Table 6.3: Dopamine detection assays based on the DNA dopamine-binding aptamer $^{284}$

\begin{tabular}{|c|c|c|}
\hline $\begin{array}{l}\text { Detection } \\
\text { matrix }\end{array}$ & Detection method and sensor design & Reference \\
\hline Aqueous & $\begin{array}{l}\text { Apt AuNP based colorimetric biosensor (DR: } \\
0.54-5.4 \mu \mathrm{M} \text {; LOD: } 0.36 \mu \mathrm{M} \text { ) }\end{array}$ & $\begin{array}{l}\text { (Zheng et al., } \\
\text { 2011) }\end{array}$ \\
\hline Human serum & $\begin{array}{l}\text { Label-free electrochemical aptasensor } \\
\text { (DR: 0.007-90 nM; LOD: } 0.00198 \mathrm{nM} \text { ) }\end{array}$ & $\begin{array}{l}\text { (Liu et al., } \\
2012)^{473}\end{array}$ \\
\hline $\begin{array}{l}\text { DA release } \\
\text { from } \mathrm{PC} 12 \text { cells } \\
\text { under hypoxic }\end{array}$ & $\begin{array}{l}\text { Specific label-free nanowire-transistor biosensor } \\
\left(\mathrm{K}_{\mathrm{D}}: 120 \pm 10 \mathrm{pM} \text {; DR: } 10^{-11}-10^{-8} \mathrm{~mol} / \mathrm{L} \text {;LOD: }\right.\end{array}$ & $\begin{array}{l}\text { (Li et al., } \\
2013)^{474}\end{array}$ \\
\hline
\end{tabular}




\begin{tabular}{|c|c|c|}
\hline conditions & $\left.10^{-11} \mathrm{~mol} / \mathrm{L}\right)$ & \\
\hline Human serum & $\begin{array}{l}\text { Label-free electrochemical aptasensor } \\
\text { (DR: 5-150 nM; LOD: } 1.0 \mathrm{nM} \text { ) }\end{array}$ & $\begin{array}{l}\text { (Zhou et al., } \\
2014)^{475}\end{array}$ \\
\hline Human serum & $\begin{array}{l}\text { Personal glucose meter-based apt biosensor } \\
\text { (DR: 0.08-100 } \mu \mathrm{M} \text {; LOD: } 0.03 \mu \mathrm{M} \text { ) }\end{array}$ & $\begin{array}{l}\text { (Hun et al., } \\
2015)^{476}\end{array}$ \\
\hline PBS (pH 7.4) & $\begin{array}{l}\text { Apt- } \operatorname{In}_{2} \mathrm{O}_{3} \text { biosensor } \\
\left.\text { (DR: } 10^{-11}-10^{-7} \mathrm{M}\right)\end{array}$ & $\begin{array}{l}\text { (Kim et al., } \\
2015)^{477}\end{array}$ \\
\hline PBS & $\begin{array}{l}\text { Detection of DA by SERS using an apt } \\
\text { immobilized by a thiol linker to noble metal on a } \\
\text { fused silica chip } \\
\text { (DR and LOD: not reported) }\end{array}$ & $\begin{array}{l}\text { (Peters et al., } \\
2015)^{478}\end{array}$ \\
\hline $\begin{array}{l}\text { Tris buffer }(\mathrm{pH} \\
7.4)\end{array}$ & $\begin{array}{l}\text { Electrochemical detection based on apt- } \\
\text { functionalized glassy carbon electrode } \\
\text { (DR: } 100-1000 \mu \mathrm{M} \text {; LOD: } 100 \mu \mathrm{M} \text { ) }\end{array}$ & $\begin{array}{l}\text { (Walsh et al., } \\
2015)^{479}\end{array}$ \\
\hline $\begin{array}{l}\text { Swine feed and } \\
\text { chicken liver }\end{array}$ & $\begin{array}{l}\text { Apt-AuNP based fluorescence sensor } \\
\text { (DR: } 26-2.9 \times 10^{3} \mathrm{nM} \text {; LOD: } 2 \mathrm{nM} \text { ) }\end{array}$ & $\begin{array}{l}\text { (Xu et al., } \\
2015)^{480}\end{array}$ \\
\hline PBS (pH 7.0) & $\begin{array}{l}\text { Apt-based fiber optic biosensor } \\
\text { (DR: } 0.5-10 \mu \mathrm{M})\end{array}$ & $\begin{array}{l}\text { (Zibaii et al., } \\
2015)^{481}\end{array}$ \\
\hline PBS (pH 7.4) & $\begin{array}{l}\text { Chemiluminescence-based detection and } \\
\text { dissociation constant determination }\left(\mathrm{K}_{\mathrm{D}}: 0.710\right. \\
\mu \mathrm{M} \text {; DR: } 1 \mathrm{pM} \text { to } 1 \mathrm{nM} \text {; LOD: } 0.9 \mathrm{pM})\end{array}$ & $\begin{array}{l}\text { (Gao et al., } \\
2016)^{482}\end{array}$ \\
\hline Human serum & $\begin{array}{l}\text { Label-free electrochemical aptasensor } \\
\text { (DR: } 5 \text { - } 75 \mu \mathrm{M} \text {; LOD: } 3.36 \mu \mathrm{M} \text { ) }\end{array}$ & $\begin{array}{l}\text { (Jarczewska et } \\
\text { al., 2016) }\end{array}$ \\
\hline PBS & $\begin{array}{l}\text { Apt-covered AuNP-based electrochemical } \\
\text { aptasensor } \\
\text { (DR: } 5 \mathrm{nM}-500 \mathrm{nM} \text {; LOD: } 1.8 \mathrm{nM} \text {. Good } \\
\text { recovery of DA }(\sim 95-107 \%) \text { in serum) }\end{array}$ & $\begin{array}{l}\text { (Liu et al., } \\
2016)^{484}\end{array}$ \\
\hline $\begin{array}{l}\text { TRIS buffer } \\
(\mathrm{pH} \mathrm{7.4)}\end{array}$ & $\begin{array}{l}\text { Label-free fluorescent aptasensor } \\
\text { (DR: 0.03-0.21 } \mu \mathrm{M} \text {; LOD: } 19 \mathrm{nM} \text {. Good recovery }\end{array}$ & $\begin{array}{l}\text { (Huang et al., } \\
2016)^{485}\end{array}$ \\
\hline
\end{tabular}




$$
\text { of DA }(\sim 101-103 \%) \text { in Fetal Bovine Serum) }
$$

Abbreviations: aptamer (apt), detection/dynamic range (DR), limit of detection (LOD), enzyme-linked aptamer assay (ELAA), gold nanoparticle (AuNP), dissociation constant $\left(\mathrm{K}_{\mathrm{D}}\right)$, dopamine-binding aptamer (DBA)

\subsubsection{Development of an aptamer-gold nanoparticle (Apt-AuNP) based sensor for}

\section{the detection of dopamine}

Many of the sensors developed for the detection of dopamine use methods such as electrochemistry, fluorescence spectroscopy or UV-Visible spectroscopy. These techniques are time consuming and require trained personnel to prepare samples as well as collect and analyze the obtained data. The development of a colourimetric sensor for the detection of dopamine could provide a simple, cost-effective alternative detection method that could be quickly performed and interpreted without complex equipment or highly qualified personnel. A few colourimetric methods have been reported for the detection of dopamine, however in some cases low sensitivity, selectivity or ease of use have limited their applications. ${ }^{466}$ Specifically, the progress in detecting dopamine with gold nanoparticles (AuNP) by optical and electrochemical methods was recently reviewed. $^{486,487}$

Aptamers are commonly paired with gold nanoparticle technology to develop sensors. Apt-AuNP based sensors have been used to detect targets such as proteins and small molecules. ${ }^{466,488}$ Previously, Zheng et al., (2011) reported the development of an aptamer-based colourimetric biosensor based on unmodified gold nanoparticles. This biosensor used the DNA homolog DBA and had a good detection range $(0.54 \mu \mathrm{M}-5.4$ $\mu \mathrm{M})$ and limit $(0.36 \mu \mathrm{M})$. The interaction between DNA and AuNPs is well known and 
has been extensively investigated. ${ }^{488}$ The mechanism by which aptamers interacts with the surface of AuNPs in Apt-AuNP based sensors has been previously described. ${ }^{466}$ The sensor design that was investigated in this work is represented schematically in Figure

\section{1.}

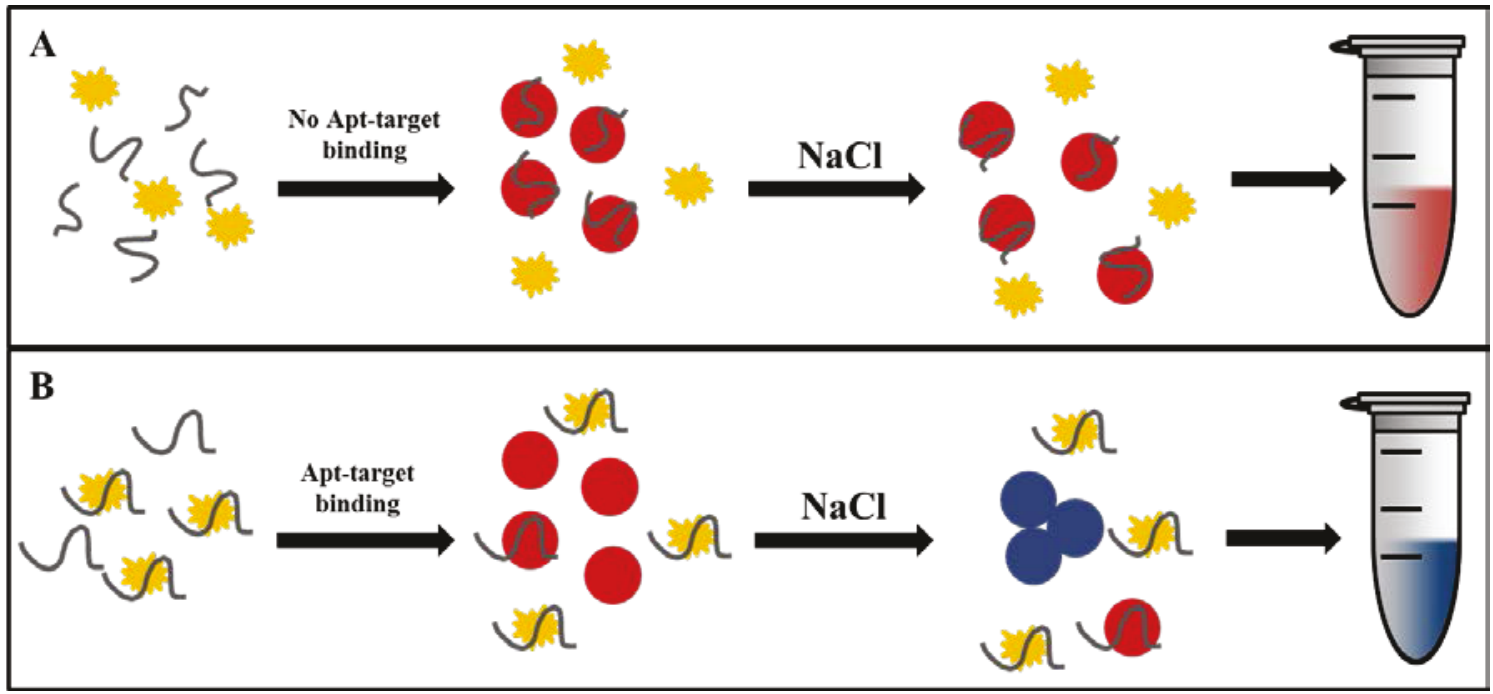

Figure 6.1: Schematic representation of the DopaA20min aptamer-AuNP based sensor. A) When no aptamer (apt)-target binding occurs, the test solution remains red upon addition of $\mathrm{NaCl}$. B) When apt-target binding occurs, the addition of $\mathrm{NaCl}$ led to a noticeable colour change of the test solution from red to blue. DNA (aptamer or random control) is represented by grey lines, the target is yellow, AuNP are red, and aggregated AuNP are shown as blue.

Briefly, when the aptamer is free in solution, a structural equilibrium likely exists where some aptamer is in a random coil and some is in the secondary structure necessary for target binding. ${ }^{466}$ When the DNA is in the random coil it can non-specifically interact with the surface of the nanoparticle. When target is present, a shift in the structural equilibrium towards the stable secondary structure due to target binding occurs, which results in a decreased amount of random coil DNA capable of protecting the AuNPs. Therefore, when aptamer-target binding does not occur (Figure 6.1 A), the AuNPs are 
protected from salt induced aggregation and the solution remains coloured red. On the contrary, when aptamer-target binding occurs (Figure 6.1 B), the AuNPs are no longer protected from salt induced aggregation and the solution turns blue in colour. This distinct colour change allows for the colourimetric detection of dopamine in solution.

\subsubsection{Expanding the functionality of DBA from simple detection}

In addition to dopamine detection under in vitro physiological conditions and in biological samples, the DNA homolog DBA has also been investigated for its ability to attenuate behaviour in live animals (see Chapter 4 and Chapter 5). The DNA homolog DBA has also been used to improve the functionality of existing DNAzyme technology. In this work, a DNAzyme and aptamer hybrid was designed to include either one or two DNA homolog DBA domains. ${ }^{284,489,490}$ The addition of this domain to the DNAzyme, allowed for the concentration of the substrate, dopamine, by increasing the proximity of the substrate molecule to the DNAzyme domain, thereby enhancing the catalytic activity 20 -fold over the separate DNAzyme and DBA molecules. ${ }^{489}$ In this way the DNAzymeaptamer hybrid was able to act like a nucleic acid based synthetic enzyme by bringing the active site (hemin/G-quadruplex DNAzyme) in close proximity to the substrate binding site (DBA aptamer). This was an important study as it shows that aptamers can be combined with DNAzymes to design and tailor the functionality of synthetic enzymes.

\subsubsection{Justification for the selection of novel dopamine binding aptamers}

The first DBA aptamer was selected from an RNA library that was 114 nucleotides long (random region: 80 bases). The affinity of the examined full length candidate was $2.8 \mu \mathrm{M}$. A minimal aptamer was identified through secondary selections 
and its affinity and selectivity were evaluated as described in section 6.3.3. Finally a further minimized 57 base aptamer was reported as the minimal aptamer (RNA DBA). Since DNA is an intrinsically more stable molecule, Walsh and DeRosa (2009) developed and characterized the DNA homolog of the minimal RNA DBA aptamer. ${ }^{284}$ The DNA homolog proved to be an equally successful aptamer exhibiting a slightly better dissociation constant of $0.7 \mu \mathrm{M}$ compared to the $1.6 \pm 0.2 \mu \mathrm{M}$ of the original RNA aptamer. ${ }^{280,284}$ Further, both aptamers exhibited some affinity to norepinephrine with the DNA homolog having a slightly better affinity for norepinephrine $(0.4 \mu \mathrm{M})$ than dopamine. $^{284}$

Therefore, a novel selection was carried out to select DBAs with better affinity and selectivity than either existing aptamer. Selection conditions considered to optimize selection experiment success were the use of a DNA library and long library template length. DNA was chosen as the nucleic acid type to increase stability of the selected aptamers. The template length of the DNA pool was purposely kept long (96 bases with a random region of 60 bases) in order to access complex secondary structures. Additionally, the selection was motivated by the desire to select aptamers with different dissociation constants than the previously reported aptamers for the purpose of investigating the effect of varying aptamer affinity on aptamer success in animal models. 


\subsection{Materials and Methods}

\subsubsection{Selection of a DNA aptamer that binds to dopamine}

\subsubsection{Chemicals and reagents}

Acrylamide, agarose, ammonium acetate, ammonium persulfate, EDTA, LB Broth, TEMED, Tris, N,N'-methylenebisacrylamide, urea, and X-Gal were purchased from BioShop Canada (Burlington, ON, Canada). Reagents for PCR (15 mM MgCl 2,10 mM dNTP mix, and Taq DNA polymerase) were obtained from BioShop Canada. C-52 sephadex, dopamine hydrochloride, formamide, $\mathrm{G}-50$ sephadex, $\mathrm{KCl}, \mathrm{KIO}_{4}$, $\mathrm{L}$-ascorbic acid, Magnesium acetate tetrahydrate, $\mathrm{NaCl}$, and Triton $\mathrm{X}-100$ were purchased from Sigma-Aldrich (Oakville, ON, Canada). Dopamine-agarose column was obtained from MP Biomedicals, Inc. (Santa Ana, CA, USA). HCl was purchased from Anachemia. $\mathrm{NaOH}$ was obtained from VWR (Mississauga, ON, Canada). Anhydrous ethyl alcohol was purchased from Commercial Alcohols, Inc. (Brampton, ON, Canada). LB-Agar Ampicillin plates were kindly provided by Derek Hodgson of Health Canada. DNA synthesis reagents and phosphoramidites were purchased from Glen Research (Sterling, VA, USA) and BioAutomation (Irving, TX, USA).

\subsubsection{Oligonucleotide library preparation}

The initial DNA library was prepared on a MerMade 6 DNA synthesizer (BioAutomation, Irving, TX, USA) using standard phosphoramidite chemistry. Synthesized DNA was cleaved from the synthesis columns in ammonium hydroxide at $55^{\circ} \mathrm{C}$ overnight. The DNA library was then purified using an Agilent 1200 series HPLC (Agilent Technologies: Santa Clara, CA, USA). The template of the random ssDNA pool 
was 5'-ATACCAGCTTATTCAATT-N60-AGATAGTAAGTGCAATCT-3', where N60 represents the number of randomized nucleotides. This template was originally described by Stoltenburg, et al., (2005). ${ }^{315}$ The forward and reverse PCR primers were as follows; 5'-ATACCAGCTTATTCAATT-3' and 5 '-AGATTGCACTTACTATCT-3'. During the selection the forward primer was 5'-modified with 6-fluorescein phosphoramidite, and the reverse primer was 5'-modified with poly-dA20-HEG to allow for efficient separation of the double stranded PCR product and isolation of the fluorescently labelled selection pool. For cloning, the PCR primers were used without 5'-modifications. For the purposes of screening aptamer candidates after cloning and sequencing, DNA was purchased from Alpha DNA (Montreal, QC, Canada).

\subsubsection{SELEX}

Selection was performed as previously described with some modifications. ${ }^{280,315}$ The DNA library was dissolved in SELEX column buffer (SCB: $50 \mathrm{mM}$ Tris-HCl pH 7.4, $5 \mathrm{mM} \mathrm{MgCl} 2,0.5 \mathrm{M} \mathrm{NaCl}$ and $0.02 \% \mathrm{~L}$-ascorbic acid), heated to $90^{\circ} \mathrm{C}$ for $10 \mathrm{~min}$, cooled to $4^{\circ} \mathrm{C}(15 \mathrm{~min})$ then let stand for $5 \mathrm{~min}$ at room temperature. The selection column was prepared by loading dopamine-agarose into an EconoColumn (BioRad, Saint-Laurent, QC, Canada). The column volume of the first selection round was $1 \mathrm{~mL}$ and subsequent rounds were $0.2 \mathrm{~mL}$. Before each selection round began the column was washed with 50 vol. of SCB. To begin each selection round, the fluorescence of the DNA library was measured before it was loaded onto the dopamine-agarose column and allowed to equilibrate with gentle agitation for 1 hour. To remove non-specifically interacting DNA following the 1 hour incubation period, the column was washed with 
SCB and the DNA coming off was monitored by UV-Vis and/or fluorescence spectroscopy. When the amount of non-specific DNA was indiscernible, dopaminebinding DNA was eluted with 0.1 M dopamine solution prepared in SCB. During the third round of selection, a second wash with $90^{\circ} \mathrm{C}, 7.4 \mathrm{M}$ urea dissolved in SCB was introduced. The purpose of this was wash was to elute strongly binding DNA that had not been eluted by the $0.1 \mathrm{M}$ dopamine wash. In the fourth selection round, since the $0.1 \mathrm{M}$ dopamine was no longer efficient at eluting bound DNA, the library was eluted solely with 7.4 $\mathrm{M}$ urea. Immediately following elution, the fractions were concentrated using YM-3 centrifugal filter units (Millipore, USA) and the fluorescence of the post-selection pool was measured using a Cary Eclipse Fluorescence Spectrophotometer (Varian, USA) using a $40 \mu \mathrm{L}$ fluorescence cell (Varian, USA). The DNA library was then dried using a Savant DNA120 SpeedVac Concentrator (ThermoElectron Corporation, USA) in preparation for PCR. Before the $1^{\text {st }}$ and $4^{\text {th }}$ rounds of selection, the library underwent negative selection against an agarose column prior to the positive selection on the dopamine-agarose column.

\subsubsection{Amplification of the DNA library by polymerase chain reaction (PCR)}

PCR reactions were prepared to a total volume of $\sim 100 \mu \mathrm{L}$ by combining $50 \mu \mathrm{L}$ of FluMag PCR buffer (0.2 M Tris-HCl pH 9, $0.1 \mathrm{M} \mathrm{KCl}, 2 \%$ triton X-100), $3.8 \mu \mathrm{L}$ of 15 $\mathrm{mM} \mathrm{MgCl} 2,2 \mu \mathrm{L}$ of dNTP mix, $0.5 \mu \mathrm{L}$ of the either the unmodified or fluorescently labeled forward primer (Flu primer), $0.5 \mu \mathrm{L}$ of either the unmodified or the poly $\mathrm{A}$ elongated reverse primer (poly A primer), $1 \mathrm{uL}$ TAQ DNA polymerase, and $41 \mu \mathrm{L}$ deionized $\mathrm{H}_{2} \mathrm{O}$ to the DNA pool. Positive and negative control reactions were prepared 
by substituting $1 \mu \mathrm{L}$ of control pool or deionized $\mathrm{H}_{2} \mathrm{O}$ respectively in place of the respective selection round DNA pool. PCR was run on a Mastercycler epgradient (Eppendorf, Hauppauge, NY, USA) using the following thermal profile: $94^{\circ} \mathrm{C}$ for $10 \mathrm{~min}$ then either 30 or 45 cycles of $94.0^{\circ} \mathrm{C}(1 \mathrm{~min})$, followed by $47.0^{\circ} \mathrm{C}(1 \mathrm{~min})$ then $72.0^{\circ} \mathrm{C}(1$ min). After the final cycle was complete, the reactions were held at $72.0^{\circ} \mathrm{C}(10 \mathrm{~min})$, then cooled to and held $4^{\circ} \mathrm{C}$.

\subsubsection{Single-stranded DNA library purification by polyacrylamide gel electrophoresis (PAGE)}

After PCR, the ssDNA pool was purified and isolated by polyacrylamide gel electrophoresis (PAGE) on 12\% denaturing gels using an SE 600 Chroma Standard Vertical Electrophoresis Unit (Hoefer, USA) with an FB100 power supply (Fisher Scientific, Ottawa, Canada). Briefly, polyacrylamide gels were prepared by combining 31.5 g of urea, $23.5 \mathrm{~mL}$ of acrylamide stock (5.3 M acrylamide and $130 \mathrm{mM} \mathrm{N}, \mathrm{N}$ 'methylenebisacrylamide), $15 \mathrm{~mL}$ of $5 \mathrm{X}$ TBE ( $0.445 \mathrm{M}$ Tris, $0.445 \mathrm{M}$ boric acid and $0.010 \mathrm{M}$ EDTA), and $14 \mathrm{~mL}$ of deionized $\mathrm{H}_{2} \mathrm{O}$ while heating the solution to $37^{\circ} \mathrm{C}$ with stirring. The solution was filtered by gravity and allowed to cool to room temperature before the addition of $450 \mu \mathrm{L}$ of $10 \%$ ammonium persulfate and $35 \mu \mathrm{L}$ of TEMED. Gels were poured between vertical plates (SE6102; Fisher Scientific, Ottawa, Canada) and allowed to set for 30 minutes. Samples were dissolved in equal volume formamide and $10 \mathrm{mM}$ tris buffer ( $\mathrm{pH} 7.4)$, heated to $55^{\circ} \mathrm{C}$ then loaded onto the gel. Gels were run at a constant amperage of $25 \mathrm{~mA}$ for $\sim 2$ hours to allow for adequate separation of the double stranded PCR product into the single strands. Gels were imaged using an AlphaImager 
EC (Alpha Innotech: Fisher Scientific, Ottawa, Canada) using both the Epi UV and 302 $\mathrm{nm}$ settings. The desired bands were cut from the gel and suspended in elution buffer $\left(0.1 \% \mathrm{SDS}, 0.5 \mathrm{M}\right.$ ammonium acetate, $10 \mathrm{mM}$ magnesium acetate) at $37^{\circ} \mathrm{C}$ overnight to extract the DNA from the gel. The extracted DNA was filtered to remove the gel pieces then desalted and dried prior to the next round of selection.

\subsubsection{Cloning}

The DNA library from the final selection round was cloned using a StrataClone PCR Cloning Kit (Agilent Technologies, USA) as per the manufacturer's instructions. The cloning kit included a StrataClone Vector Mix amp/kan, StrataClone Cloning Buffer, StrataClone control insert ( $5 \mathrm{ng} / \mu \mathrm{L})$, StrataClone SoloPack Competent cells, and pUC18 Control Plasmid ( $0.1 \mathrm{ng} / \mu \mathrm{L}$ in TE buffer). The colonies were grown on LB-ampicillin agar plates $(1.0 \% \mathrm{NaCl}, 1.0 \%$ tryptone, $0.5 \%$ yeast extract, $2 \%$ agar, and $100 \mu \mathrm{g} / \mu \mathrm{L})$ at $37^{\circ} \mathrm{C}$ overnight. Blue/white screening was used to identify colonies to sequence.

\subsubsection{Sequencing}

Colonies identified as having aptamer present by blue/white screening were sent to MCLAB (Molecular Cloning Laboratories: San Francisco, CA, USA) for oligonucleotide sequencing. DNA was sequences with special consideration that the sequences likely contained hairpin secondary structure. Sequences analysis was done using the MCLAB 96-Well Pairwise Alignment Tool. 


\subsubsection{Screening of aptamer candidates by fluorescence anisotropy}

To screen the aptamer candidates identified by cloning and sequencing, an apparent dissociation constant for each candidate was measured by fluorescence anisotropy. Measurements were obtained on a Fluoro-Log 3 (Horiba Jobin Yvon: Edison, NJ, USA). Samples were prepared by combining a constant concentration of dopamine $\left(1.0 \times 10^{-7} \mathrm{~mol} / \mathrm{L}\right)$ with DNA concentrations ranging from $10^{-11}$ to $10^{-7} \mathrm{~mol} / \mathrm{L} .{ }^{491}$ The apparent dissociation constant was determined based on a linear model between theoretical and experimental anisotropy. The best fit was obtained by optimizing the residual values between the theoretical and experimental anisotropy values using the 'Solver' application in Microsoft Excel. ${ }^{284,492}$

\subsubsection{Identification of minimal aptamers from DopaA20}

The DopaA20 sequence was arbitrarily truncated and the affinities of these sequences were screened by fluorescence anisotropy. The best minimal aptamer was 5'AGCGGGAGGATATGCTCTGCTGTTGGATAGGTGTATATGTGGAGATAGTAAGTGCAATCT-3' and shall henceforth be referred to as DopaA20min.

\subsubsection{Determination of aptamer stability by thermal denaturation}

\subsubsection{Secondary Structure prediction}

The secondary structure of the DopaA20min aptamer was predicted using RNAstructure "Fold" which predicts structures based on the lowest free energy. ${ }^{493}$ The software was developed by the Mathews Group and is available free-of-charge at the following web address: http://rna.urmc.rochester.edu/RNAstructureWeb/Servers/Fold/Fold.html. QGRS Mapper 
and imGQfinder were used to analyze DopaA20min for the presence of G-

quadruplexes. ${ }^{494,495}$ QGRS Mapper and imGQfinder are available online at the following web addresses respectively: http://bioinformatics.ramapo.edu/QGRS/index.php and http://imgqfinder.niifhm.ru/. The following user-defined parameters were used when analyzing DopaA20min with imGQfinder; Quad type (G), Number of tetrads (2), Maximum loop length (8), and Number of defects (1).

\subsubsection{Investigation of secondary structure by thermal denaturation}

Two samples were prepared for melting temperature analyses; DopaA20min+DA and DopaA20min with no DA. Samples were prepared in SCB containing $5 \mathrm{mM} \mathrm{KCl}$. The aptamer and DA were prepared in a 1:5 ratio such that the final concentration of the DNA was $1.91 \times 10^{-6} \mathrm{~mol} / \mathrm{L}$ and the final dopamine concentration was $9.55 \times 10^{-6} \mathrm{~mol} / \mathrm{L}$. The absorbance value at $295 \mathrm{~nm}$ was measured over the range of $20^{\circ} \mathrm{C}$ to $80^{\circ} \mathrm{C}$ with a ramp rate of $5^{\circ} \mathrm{C} /$ min using a Cary 300 Bio UV-Visible Spectrophotometer (Agilent: Santa Clara, CA, USA) equipped with a Cary Temperature Controller (Agilent: Santa Clara, CA, USA) running the Cary Win UV Thermal application software (Version 3.00 (1.82)) . Three ramps were obtained (two reverse and one forward) for each sample.

\subsubsection{Determination of the affinity of DopaA20min for dopamine}

The apparent dissociation constant of DopaA20min was determined by fluorescence anisotropy. Equivolumes of dopamine solution $\left(1.0 \times 10^{-7} \mathrm{~mol} / \mathrm{L}\right)$ and DopaA20min solutions (concentrations ranged from micromolar to low nanomolar) prepared in SCB were combined, mixed and the anisotropy was measured. Measurements 
were obtained on a Fluoro-Log 3 equipped with polarizers (Horiba Jobin Yvon: Edison, NJ, USA) according to the following equation ${ }^{496}$;

$$
r=\frac{\left(\frac{I_{\mathrm{VV} * I_{\mathrm{HH}}}}{I_{\mathrm{VH}} * I_{\mathrm{HV}}}\right)-1}{\left(\frac{I_{\mathrm{VV}} * I_{\mathrm{HH}}}{I_{\mathrm{VH}} * I_{\mathrm{HV}}}\right)+2}
$$

Equation 1: Formula for the calculation of anisotropy (r) by FluorEssence software where I represents intensity, the first subscript refers to the position of the excitation polarizer and the second subscript refers to the position of the emission polarizer. Further, vertically oriented polarizers $(\mathrm{V})$ and horizontally oriented polarizers $(\mathrm{H})$ are at $0^{\circ}$ and $90^{\circ}$ respectively with respect to normal.

The formula described in Equation 1 is based on the following formula ${ }^{496}$;

$$
r=\frac{I_{\mathrm{VV}}-I_{\mathrm{VH}}}{I_{\mathrm{Vv}}+2 I_{\mathrm{VH}}}
$$

Equation 2: The formula for anisotropy (r) is shown where I represents intensity and the first and second subscripts represent the position of the excitation and emission polarizers respectively.

However, Equation 1 is corrected for the grating factor $(\mathrm{G})$ of the instrument. The equation for $\mathrm{G}$ factor used in the FluorEssence software calculations is shown below ${ }^{496}$;

$$
G\left(\lambda_{\mathrm{EM}}\right)=\frac{I_{\mathrm{HV}}}{I_{\mathrm{HH}}}
$$

Equation 3: The formula for $G$ factor at a specific emission wavelength $\left(\lambda_{\mathrm{EM}}\right)$ where $I$ is intensity and the first and second subscript refer to the position of the excitation and emission polarizers respectively.

For each anisotropy measurement, excitation and emission wavelengths of $285 \mathrm{~nm}$ and

$317 \mathrm{~nm}$ respectively were used. Slit widths were set at $4 \mathrm{~nm}$. Six measurements were obtained for each DNA concentration and the average anisotropy value was plotted against the DNA concentration. The standard curve analysis (four parameter logistic 
curve) feature of SigmaPlot (Version 10.0: Systat Software, Inc.) was used to fit the anisotropy data based on the following equation;

$$
y=\min +(\max -\min ) /\left(1+\left(\frac{x}{E C 50}\right)^{\text {Hillslope }}\right)
$$

Equation 4: Four parameter logistic curve where $y=$ anisotropy, $x=$ DNA concentration, $\min =$ minimum anisotropy value that can be obtained, $\max =$ maximum anisotropy value that can be obtained, EC50 is the point of inflection of the curve, Hillslope $=$ corresponds to the steepness of the curve at the inflection point

The predicted EC 50 value was taken as an estimation of the apparent $\mathrm{K}_{\mathrm{D} .}{ }^{497}$ The reported apparent dissociation constant represents the average of four trials and the error represents the standard deviation between those predicted values.

\subsubsection{Development of an Aptamer-AuNP sensor for the detection of dopamine}

\subsubsection{Chemicals and reagents}

The phosphoramidites and reagents required for DNA synthesis were purchased from Glen Research (Sterling, VA, USA). The CPG columns and acetonitrile required for DNA synthesis were obtained from BioAutomation (Irving, TX, USA). Ultra High Purity 5.0 argon was obtained from Praxair. Ammonium persulfate, boric acid, EDTA, TEMED, Tris and urea were obtained from BioShop Canada (Burlington, ON, Canada). Sodium chloride, sodium citrate and gold (III) chloride trihydrate $\left(\mathrm{HAuCl}_{4}\right)$ were purchased from Sigma-Aldrich (Oakville, ON, Canada). The water used in these experiments was Millipore Milli-Q deionized water $(18 \mathrm{M} \Omega \mathrm{cm})$. 


\subsubsection{Oligonucleotide synthesis}

DNA was prepared on a MerMade 6 DNA synthesizer (BioAutomation, Irving, TX, USA) using standard phosphoramidite chemistry. The sequence of the random control was:

AGAATCTGTCGGGCTATGTCACTAATACTTTCCAAACGCCCCGTACCGATGCTGAACA. Upon completion of oligonucleotide synthesis the DNA was purified by polyacrylamide gel electrophoresis and the mass was confirmed by ESI-MS.

\subsubsection{Gold nanoparticle (AuNP) synthesis}

The synthesis of AuNP was based on methods described by Turkevich et al., (1951). ${ }^{498}$ Before synthesis, all glassware was prepared by cleaning with Aqua Regia (3:1 $\mathrm{HCl}: \mathrm{HNO}_{3}$ ). Stock solutions of $50 \mathrm{mM} \mathrm{HAuCl}_{4}$ and $38.8 \mathrm{mM}$ sodium citrate were prepared. In a $200 \mathrm{~mL}$ Erlenmeyer flask, $98 \mathrm{~mL}$ of deionized $\mathrm{H}_{2} \mathrm{O}$ was combined with 2 $\mathrm{mL}$ of the $20 \mathrm{mM} \mathrm{HAuCl}_{4}$ stock solution and heated (with stirring) until the solution was vigorously boiling. Once boiling, $10 \mathrm{~mL}$ of the $38.8 \mathrm{mM}$ sodium citrate stock solution was added to the Erlenmeyer flask. Nanoparticle formation was indicated by a distinct colour change of the solution from yellow to clear to purple to red. Once the red colour was observed the solution was allowed to boil for an additional $15 \mathrm{~min}$. The Erlenmeyer flask was then removed from the heat and the AuNP solution was allowed to cool to room temperature while stirring. Before use, the AuNP solution was quantified according to the absorbance at $520 \mathrm{~nm}\left(\right.$ extinction coefficient $\left.2.7 \times 10^{8} \mathrm{M}^{-1} \mathrm{~cm}^{-1}\right) .{ }^{466}$ 


\subsubsection{Aptamer-AuNP (Apt-AuNP) assay protocol}

The Apt-AuNP assay was performed as described previously, with some modifications. ${ }^{466}$ Stock solutions of $0.25 \mathrm{M} \mathrm{NaCl}, 10.5 \mathrm{M}$ AuNPs, dopamine (DA) and norepinephrine solutions ranging from $3.6 \times 10^{-10} \mathrm{~mol} / \mathrm{L}$ to $3.6 \times 10^{-5} \mathrm{~mol} / \mathrm{L}$, and methionine concentrations ranging from $1.0 \times 10^{-9} \mathrm{~mol} / \mathrm{L}$ to $1.0 \times 10^{-4} \mathrm{~mol} / \mathrm{L}$ were prepared in deionized water. DNA was prepared to $10 \mu \mathrm{M}$ in deionized water, heated to $55^{\circ} \mathrm{C}$ for 10 mins then allowed to cool to room temperature before use. The DNA used in the experiment was either the DopaA20min aptamer or random DNA. To begin, $243 \mu \mathrm{L}$ of target (DA, NE, or methionine) solution were combined with $6 \mu \mathrm{L}$ of DNA $(10 \mu \mathrm{M})$ in a $1.5 \mathrm{~mL}$ Eppendorf tube. 8 samples were prepared, six tubes contained a different DA concentration and DNA, one tube contained $243 \mu \mathrm{L}$ of deionized water in place of DA and DNA (No target control), and one tube contained $249 \mu \mathrm{L}$ of deionized in place of DA and DNA (No DNA control). The solutions were mixed and allowed to incubate at room temperature for $10 \mathrm{~min}$. Following incubation, $135 \mu \mathrm{L}$ of AuNP solution $(10.5 \mathrm{nM})$ was added to each of the Eppendorf tubes. The solutions were mixed and allowed to let stand for $5 \mathrm{~min}$ then photographed. Aggregation was induced by adding $72 \mu \mathrm{L}$ of $\mathrm{NaCl}(0.25$ M). The solutions were mixed, allowed to incubated at room temperature for $5 \mathrm{~min}$ and then photographed before the absorbance spectra were measured on a Cary 300 Bio UVVisible Spectrophotometer (Agilent, CA, USA) running the Cary Win UV Scan application software (Version 3.00 (1.82)). Data analysis was performed using Microsoft Excel. The mean and error (standard deviation) were calculated using the "average" and "stdeva" functions. 
The Apt-AuNP assays carried out to obtain the sensor data (target: DA) were performed as described. To determine the specificity of DopaA20min using the AptAuNP assay, single concentration measurements were performed using norepinephrine (NE), ascorbic acid (AA), tyramine (Try) or serotonin (ST) solutions prepared at $3.6 \times$ $10^{-6} \mathrm{~mol} / \mathrm{L}$ and AuNPs diluted to $11 \mathrm{nM}$. For the characterization of the Apt-AuNP sensor response to NE after an extended aptamer-target incubation period, the first incubation period was extended from 10 min to 85 min. To determine the Apt-AuNP sensor response to DA in artificial cerebral spinal fluid (ACSF) the DA solutions were prepared in ACSF and ranged in concentration from $3.6 \times 10^{-9} \mathrm{~mol} / \mathrm{L}$ to $3.6 \times 10^{-3} \mathrm{~mol} / \mathrm{L}$. ACSF was prepared by mixing two stock solutions (A and B) together in a 1:1 ratio before use as described previously. ${ }^{499}$ Stock solution A contained $0.3 \mathrm{M} \mathrm{NaCl}, 0.006 \mathrm{M} \mathrm{KCl}, 0.003$ $\mathrm{M} \mathrm{CaCl}_{2} \cdot 2 \mathrm{H}_{2} \mathrm{O}$ and $0.002 \mathrm{M} \mathrm{MgCl}_{2} \bullet 6 \mathrm{H}_{2} \mathrm{O}$. Stock solution B contained $0.002 \mathrm{M}$ of $\mathrm{Na}_{2} \mathrm{HPO}_{4} \bullet 7 \mathrm{H}_{2} \mathrm{O}$ and $0.0004 \mathrm{M} \mathrm{NaH}_{2} \mathrm{PO}_{4} \bullet \mathrm{H}_{2} 0$. The stock solutions of DA prepared in ACSF were diluted $100 \mathrm{X}$ and $243 \mu \mathrm{L}$ of the $100 \mathrm{X}$ diluted solutions were used in the assay.

\subsubsection{Determination of the apparent $K_{D}$ from the Apt-AuNP assay}

The standard curves analysis (four parameter logistic curve) feature of SigmaPlot 10.0 was used to fit the absorbance ratio data based on the Equation 4 (section 6.3.3). From this, an EC 50 value was obtained which was interpreted as the apparent $\mathrm{K}_{\mathrm{D}}$. The presented value represents the fit and error predicted by SigmaPlot for the average of 3 trials. 


\subsubsection{Transmission electron microscopy (TEM) imaging}

TEM imaging was performed with assistance from Jianqun Wang. Carbon film on 300 mesh copper grids $(\mathrm{CF} 300-\mathrm{Cu})$ were purchased from Electron Microscopy Sciences (Hatfield, USA) and used as purchased. Kimwipes (Kimberly-Clark) were purchased from Fisher Scientific Company (Ottawa, Canada).

To prepare samples for TEM imaging, 5-10 $\mu \mathrm{L}$ of the sample solution was deposited onto the copper grid. Excess solution was whisked away with a Kimwipe (after $10 \mathrm{~min}$ ) and the copper grid was left to dry overnight. An FEI Tecnai G2 F20 Transmission Electron microscope (Hillsboro, USA) equipped with a Gatan ORIUS TEM CCD Camera (Pleasanton, USA) imaging system at $200 \mathrm{kV}$ was used to examine the

degree of AuNP aggregation. Images were resized using the Gatan Microscopy Suite Digital Micrograph software (Version 2.32.888.0, Gatan, Inc.).

\subsubsection{Investigation of the efficacy of DopaA20min in reducing cocaine-induced hyperlocomotion animal model}

\subsubsection{Ethics Statement}

All animal procedures were approved by the Carleton University Animal Care Committee in accordance with guidelines set by the Canadian Council on Animal Care (CCAC). Approval was obtained from the Carleton University Animal Care Committee (AUP ID 102061). 


\subsubsection{Subjects}

CD-1 mice (male: 32-38 days old) were obtained from Charles River (St.

Constant, Canada). Animals were housed individually in clear, polycarbonate cages with the following dimensions: $27 \times 21 \times 14 \mathrm{~cm}$. The vivarium temperature $\left(19-22^{\circ} \mathrm{C}\right)$, humidity $(50-60 \%)$, and lighting (12 hour dark/light cycle with lights on at 08:00) were controlled. Animals were fed Purina mice chow and given water ad libitum.

\subsubsection{Drugs}

DA20m-TRAM, Sub-TRAM and DAL-TRAM were prepared as described in Chapter 5, section 5.3.3. Mice $(n=40)$ were assigned to one of the following groups; SubTRAM $+10 \mathrm{mg} / \mathrm{mL}$ cocaine $(\mathrm{n}=7)$, DA20m-TRAM $+10 \mathrm{mg} / \mathrm{mL}$ cocaine $(\mathrm{n}=8)$, DALTRAM + $10 \mathrm{mg} / \mathrm{mL}$ cocaine $(\mathrm{n}=8), \mathrm{DA} 20 \mathrm{~m}-\mathrm{TRAM}+$ saline $(\mathrm{n}=9)$ and DAL-TRAM + saline $(\mathrm{n}=8)$. Cocaine hydrochloride $(10 \mathrm{mg} / \mathrm{mL}$; Sigma-Aldrich, USA) was prepared in $0.9 \% \mathrm{NaCl}$ and used within $24 \mathrm{~h}$.

\subsubsection{Locomotor apparatus}

Horizontal locomotor activity was assessed in clear polycarbonate cages (48 x 26 x $20 \mathrm{~cm}$ ) housed in a windowless room. Sixteen sensors (TSE-Systems, Inc., Chesterfield, MO) distributed $2.5 \mathrm{~cm}$ apart along the bottom of a locomotor box ere used to asses the horizontal movement of each animal. Horizontal movement was detected as a break in the infrared sensors. These activity counts were measured by a computer running Fusion HC software (AccuScan Instruments Inc., Columbus, OH). 


\subsubsection{Behavioural Procedure}

Animals (male CD-1 mice; 32-38 days old) were acclimated to the laboratory housing conditions for at least 7 days prior to experimental manipulations. Postacclimation, animals were habituated to the locomotor apparatus. To do so, mice were pleased in the locomotor apparatus for a $30 \mathrm{~min}$ period on each of three consecutive days. Habituation trials were performed between 08:00 $\mathrm{h}$ and 10:00 $\mathrm{h}$.

To evaluate the efficacy of TRAM delivered DopaA20min, animals were first injected i.p (0.1 mL) with DA20m-TRAM, Sub-TRAM or DAL-TRAM followed 5 min later with an i.p. injection $(0.1 \mathrm{~mL})$ of either $10 \mathrm{mg} / \mathrm{mL}$ cocaine or saline. Five minutes following the last injection, animals were placed in the locomotor apparatus and their locomotor activity was recorded in 5-min bins over an entire $30 \mathrm{~min}$ period.

\subsubsection{Statistical analysis}

One-way ANOVA (to investigate group effects) and post hoc analyses (to compare groups) were performed using IBM SPSS Statistics (Version 22; Armon, NY, USA). 


\subsection{Results and Discussion}

\subsubsection{Selection of DNA aptamers for dopamine}

The selection of aptamers for small molecule targets is known to be especially challenging, the reasons for which have been previously reviewed. ${ }^{6}$ One of the biggest challenges with small molecule targets is that they simply do not contain the same amount of functional groups or exhibit the same structural complexity as larger targets. ${ }^{6}$ Generally these properties make selecting aptamers with high affinities difficult. Despite these challenges, small molecule aptamers have been selected and widely used in medicinal, agricultural and analytical applications.

Novel aptamers that bind to dopamine were selected by affinity chromatography using a dopamine-agarose column over four rounds of selection. Careful consideration was given to the design of the ssDNA library template. A template previously used by Stoltenburg et al., (2005) with a 60-nucleotide random region was chosen to balance the limitations of practical synthesis but also maximize the potential structural complexity of the sequences (see Chapter 2 section 2.5.1.2). Though evidence from the analysis of Aptamer Base data suggests choosing a longer template would not necessarily improve the probability of selecting higher affinity aptamers, some evidence has been presented that successful small molecule selections require larger template lengths. ${ }^{8,297}$ The ssDNA library was subjected to negative selections against an agarose column before the first and fourth rounds of selection. Non-binding sequences were partitioned from binding sequences by washing non-binding sequences through the column. Binding DNA that remained interacting with the dopamine-agarose column was removed by either $0.1 \mathrm{M}$ 
dopamine, $90^{\circ} \mathrm{C}$ urea $(7.4 \mathrm{M})$, or both. The ssDNA library was interacting so strongly with the dopamine-agarose column that it was necessary to use $90^{\circ} \mathrm{C}$ heated urea to elute binding sequences from the column by the final round of selection. At this point, neither $0.1 \mathrm{M}$ dopamine nor $60^{\circ} \mathrm{C}$ urea $(7.4 \mathrm{M})$ was sufficient. Additionally, it was becoming increasingly difficult to recover the bound DNA from the dopamine. For these reasons, after the fourth round of selection the ssDNA library was cloned and sequenced. The aptamer candidates were screened for affinity by fluorescence anisotropy and had apparent dissociation constants ranging from $\sim 0.1-40 \mu \mathrm{M}$. The selection was deemed successful as this range of values (micromolar) was consistent with the mean dissociation constant reported for aptamers binding to small molecule targets in the literature (see Chapter 2 section 2.5.2) ${ }^{8,500}$ Based on the aptamer incidence post sequencing and the affinity screening one aptamer candidate, DopaA20, was chosen for further analysis. Truncation of the DopaA20 aptamer identified one particularly promising aptamer candidate, DopaA20min which was used in all further analyses.

\subsubsection{Physical characterization of DopaA20min}

\subsubsection{Secondary structure analysis}

RNAstructure was used to predict the secondary structure of DopaA20min. ${ }^{493}$ The predicted structure is shown in Figure 6.2. It is similar to the predicted structures of the RNA aptamer and DNA homolog in that is has 3 stem loops, however the predicted middle and 3'-stem loops were predicted to be much less stable (have fewer base pairs). ${ }^{280,396}$ 


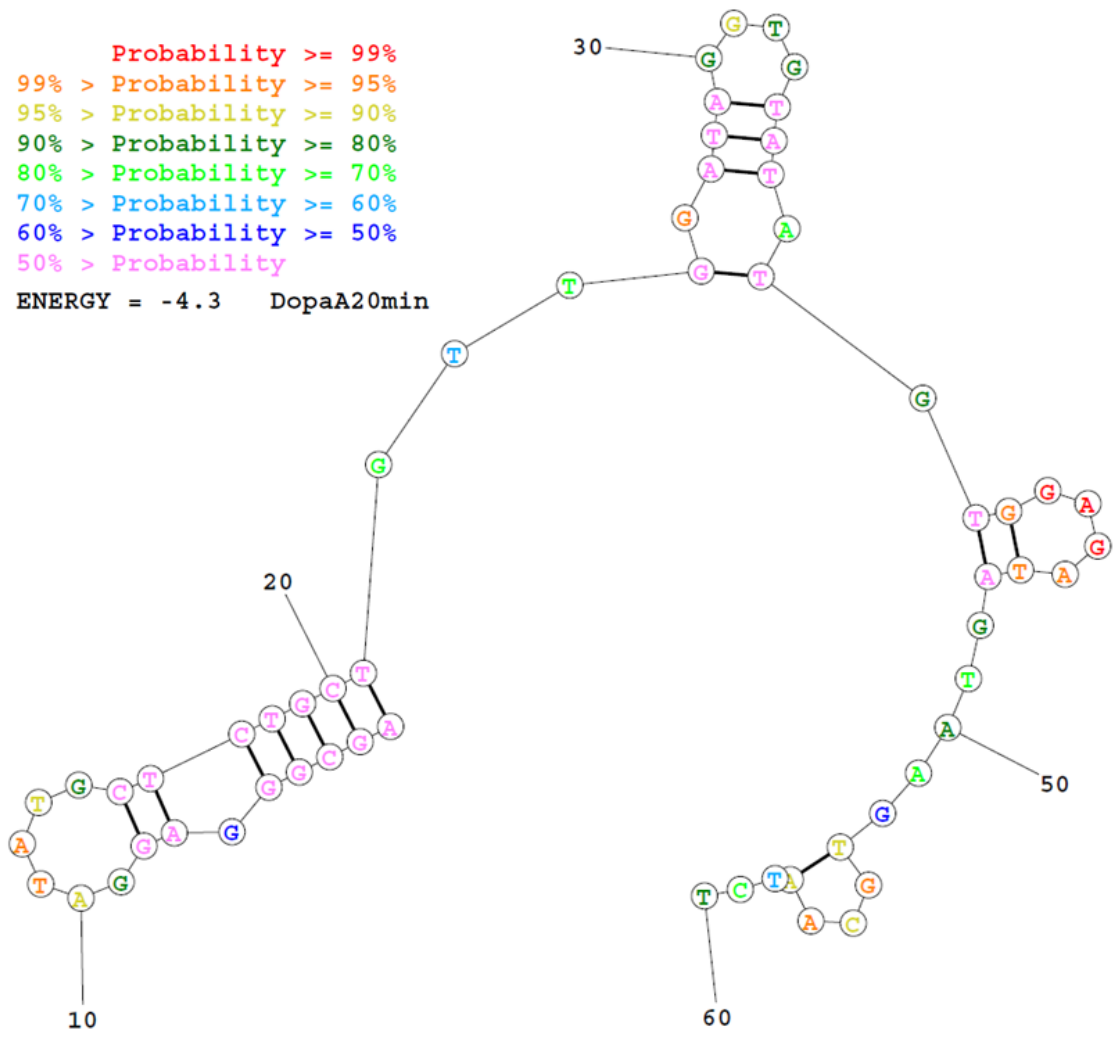

Figure 6.2: The secondary structure of DopaA20min was predicted by RNAstructure Fold. ${ }^{493}$ The colour-coded index represents the probability of the base pair in percent.

As discussed in Chapter 2 (see section 2.5.1.3) aptamers tend to be $\mathrm{G}$ and $\mathrm{C}$ rich. ${ }^{274}$ The G-quadruplex is also a very common aptamer secondary structure. ${ }^{303-305}$ RNAstructure is limited in its prediction of secondary structures because it cannot predict G-quadruplexes. The base composition of the sequence was examined to determine whether the DopaA20min aptamer may form a G-quadruplex. The percent composition of each nucleobase is shown in Figure 6.3. 


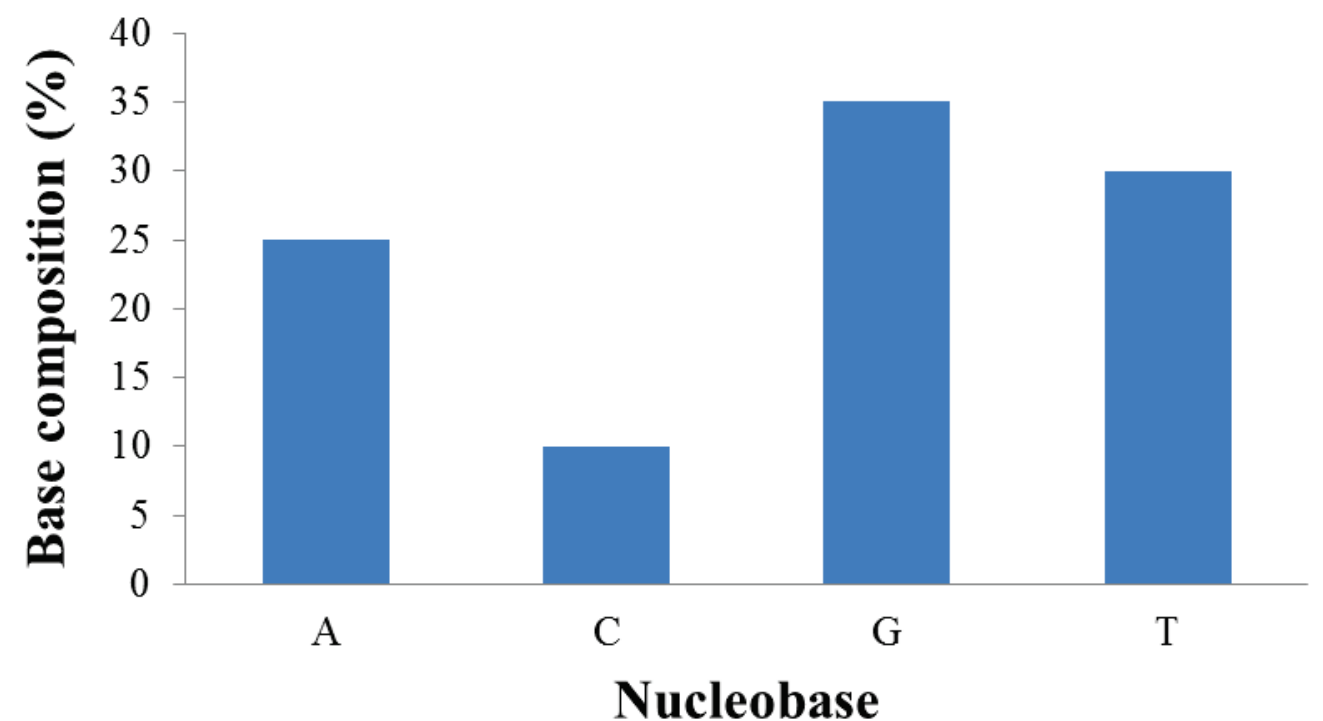

\section{Figure 6.3: Base composition of DopaA20min}

Aptamer libraries are prepared with a central random region, therefore the initial library should have an equal percent composition of $25 \%$ for each base (A, G, C, T/U). As the selection progresses and certain sequences dominate the selection pool the percent composition of the pool can shift away from equal representation. In the DopaA20min aptamer, the percent composition of $\mathrm{C}$ was significantly decreased. In contrast, the percent composition of $\mathrm{G}$ and $\mathrm{T}$ were increased. This presented strong evidence that the DopaA20min aptamer may form a G-quadruplex secondary structure. $G$ is required to form the foundational G-quartets of a G-quadruplex, and $\mathrm{T}$ is the most stabilizing loop nucleotide. ${ }^{364,501}$ Further, evidence suggests that G-quadruplexes can exist with bulges, mismatches in the tetrads and unequal loop lengths. ${ }^{502-504}$ There is also evidence that when a G-quadruplex exists, A-tetrads and T-tetrads can also be present. ${ }^{505,506}$ For these reasons, the DopaA20min sequence was analyzed using two different types of Gquadruplex prediction software. QGRS Mapper predicts perfect G-quadruplexes in an 
oligonucleotide sequence and provides a corresponding G-score which represents the likelihood of the G-quadruplex existing. ${ }^{494,507}$ imGQfinder was used to predict imperfect G-quadruplexes by allowing the user to define the number of G-quartets required, the maximum loop length, and the number of defects (substitutions) allowed. ${ }^{495}$ The Gquadruplexes predicted by both QGRS Mapper and imGQfinder are presented in Table

\section{4 and Table 6.5}

Table 6.4: Possible G-Quadruplexes predicted by QGRS Mapper

\begin{tabular}{|c|c|c|c|}
\hline Position & Length & QGRS Mapper Predicted Sequence & G-Score \\
\hline 4 & 28 & 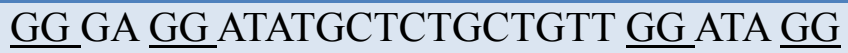 & 8 \\
\hline 5 & 27 & GG A GG ATATGCTCTGCTGTT GG ATA GG & 7 \\
\hline
\end{tabular}

Table 6.5: Possible G-Quadruplexes predicted by imGQfinder

\begin{tabular}{|c|c|c|}
\hline Position & Length & imGQfinder Predicted Sequence \\
\hline 4 & 23 & 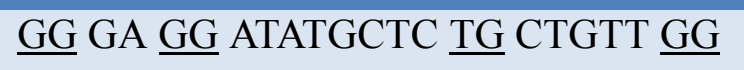 \\
\hline 5 & 22 & 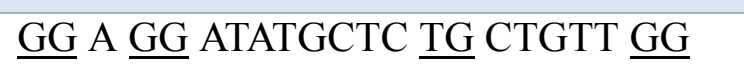 \\
\hline 8 & 24 & 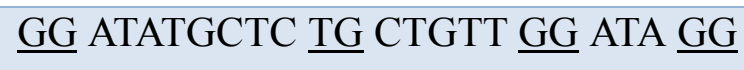 \\
\hline 25 & 18 & 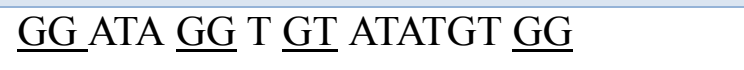 \\
\hline 25 & 18 & 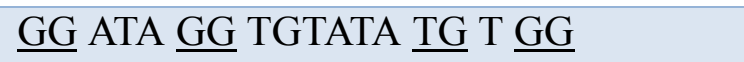 \\
\hline
\end{tabular}

Analysis with the QGRS Mapper software predicted two perfect G-quadruplexes. The Gscore presented represents the likelihood that the predicted G-quadruplex will form. The G-score calculation is based on the tendency of shorter loops to be more common than longer loops, that the loops are typically relatively equal in size, and that the stability of the G-quadruplex increases with increasing number of G-quartets. ${ }^{494}$ A higher G-score 
suggests a more stable quadruplex. With these parameters in mind, the analysis of the DopaA20min sequence for imperfect G-quadruplexes with smaller loops was performed using imGQfinder. Five possible imperfect G-quadruplexes were predicted that ranged in size from 18-23 nucleotides.

To determine whether or not DopaA20min formed a stable G-quadruplex secondary structure the sequence was investigated using thermal denaturation. The melting curves obtained by measuring the absorbance at $295 \mathrm{~nm}$ are shown in Figure 6.4.

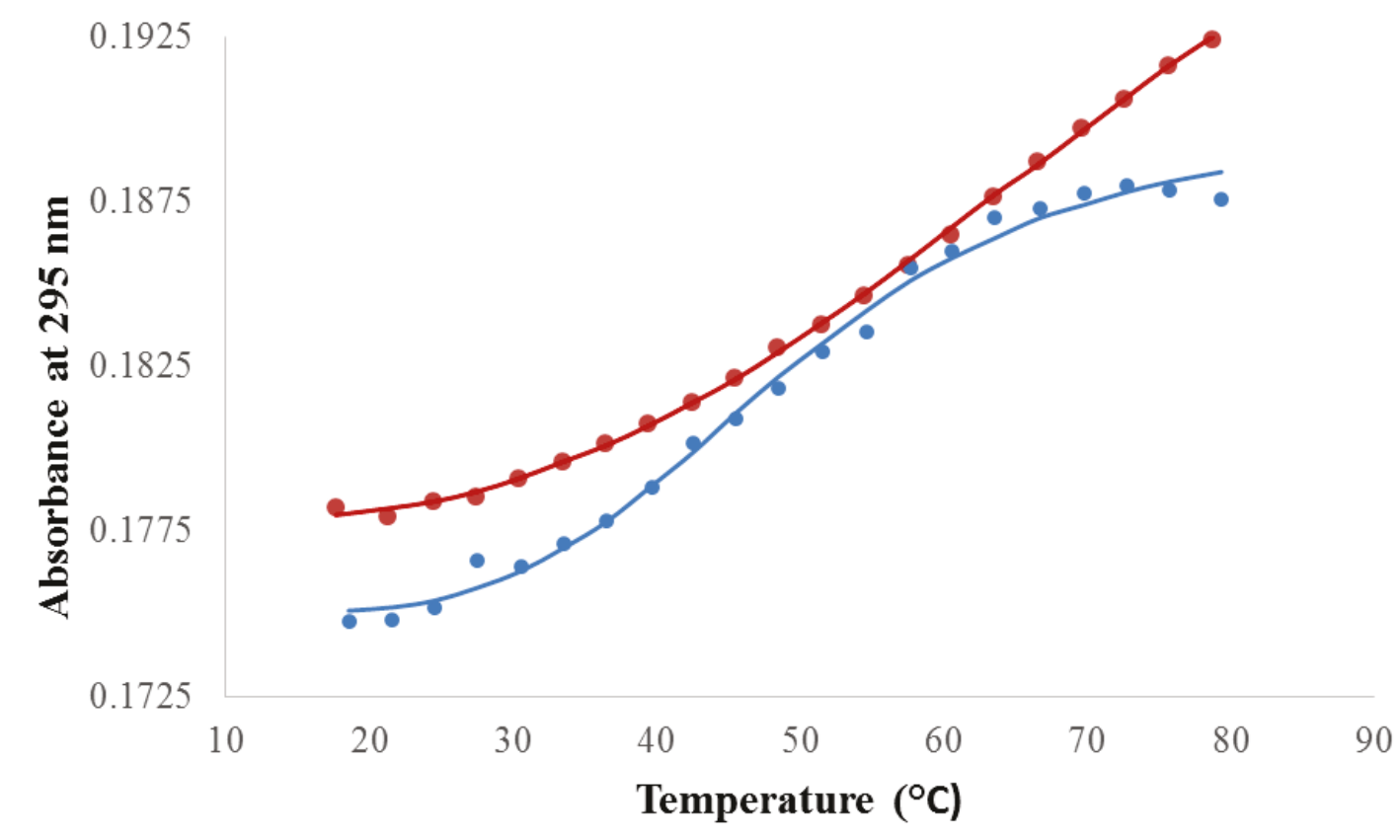

Figure 6.4: Melting temperature (Tm) analysis of DopaA20min in the presence (blue) and absence (red) of dopamine. Data points represent the average of either two (DopaA20min + DA) or three (DopaA20min) temperature ramps. The fit of the average values was predicted by SigmaPlot 10 and is represented by a solid line.

The absorbance of DopaA20min (red) and DopaA20min with dopamine (blue) were measured at $295 \mathrm{~nm}$ over the temperature range of $20^{\circ} \mathrm{C}$ to $80^{\circ} \mathrm{C}$. For each condition, three temperature ramps (two reverse and one forward) were measured. For the 
DopaA20min control (red) the curve shown is for the average of all three ramps. The curve shown for the DopaA20min with dopamine (blue) represents the average of the two reverse ramps. In the case of DNA, the forward and reverse temperature ramps should be superimposable however, this is not always the case when the melting temperature is measured in the presence of target. ${ }^{508}$ For these data, a G-quadruplex secondary structure either with or without dopamine present seems unlikely. Generally, the presence of a Gquadruplex is indicated by hypochromicity with increasing temperature at $295 \mathrm{~nm} .{ }^{508} \mathrm{In}$ this case, hyperchromicity was observed with increasing temperature suggesting the Gquartet chromophore was not present. From these data the melting temperature of DopaA20min and DopaA20min with dopamine were estimated as $80 \pm 4^{\circ} \mathrm{C}$ and $50 \pm 1{ }^{\circ} \mathrm{C}$ respectively, however the error between ramps was so high that the temperature difference observed with and without target cannot be described as significant. Future work should investigate measuring the melting temperature while changing the experimental parameters such as the temperature ramp rate, the DNA concentration, the target concentration and the metal cation concentrations of the buffer. ${ }^{508}$ Another approach would be to use circular dichroism to obtain information about the secondary structure and melting temperature of DopaA20min with and without dopamine. ${ }^{46,502,503}$

The preliminary evidence presented in this section suggests that the secondary structure of DopaA20min is more likely similar to the secondary structure of the RNA and DNA homolog DBAs in which the aptamer interacts with dopamine by forming a binding domain between two stem loops, and does not likely form a G-quadruplex. It is possible the aptamer forms an entirely different secondary structure than either of those 
proposed in this section. In fact recently, an aptamer made of modified nucleic acids was described that forms an unprecedented prism-like secondary structure. ${ }^{165}$

\subsubsection{Evaluation of the affinity of DopaA20min for dopamine}

\subsubsection{Fluorescence anisotropy}

The affinity of an aptamer for its cognate target is described by the dissociation constant $\left(\mathrm{K}_{\mathrm{D}}\right)$. The apparent $\mathrm{K}_{\mathrm{D}}$ of the binding interaction between DopaA20min and dopamine was determined by fluorescence anisotropy (Figure 6.5).

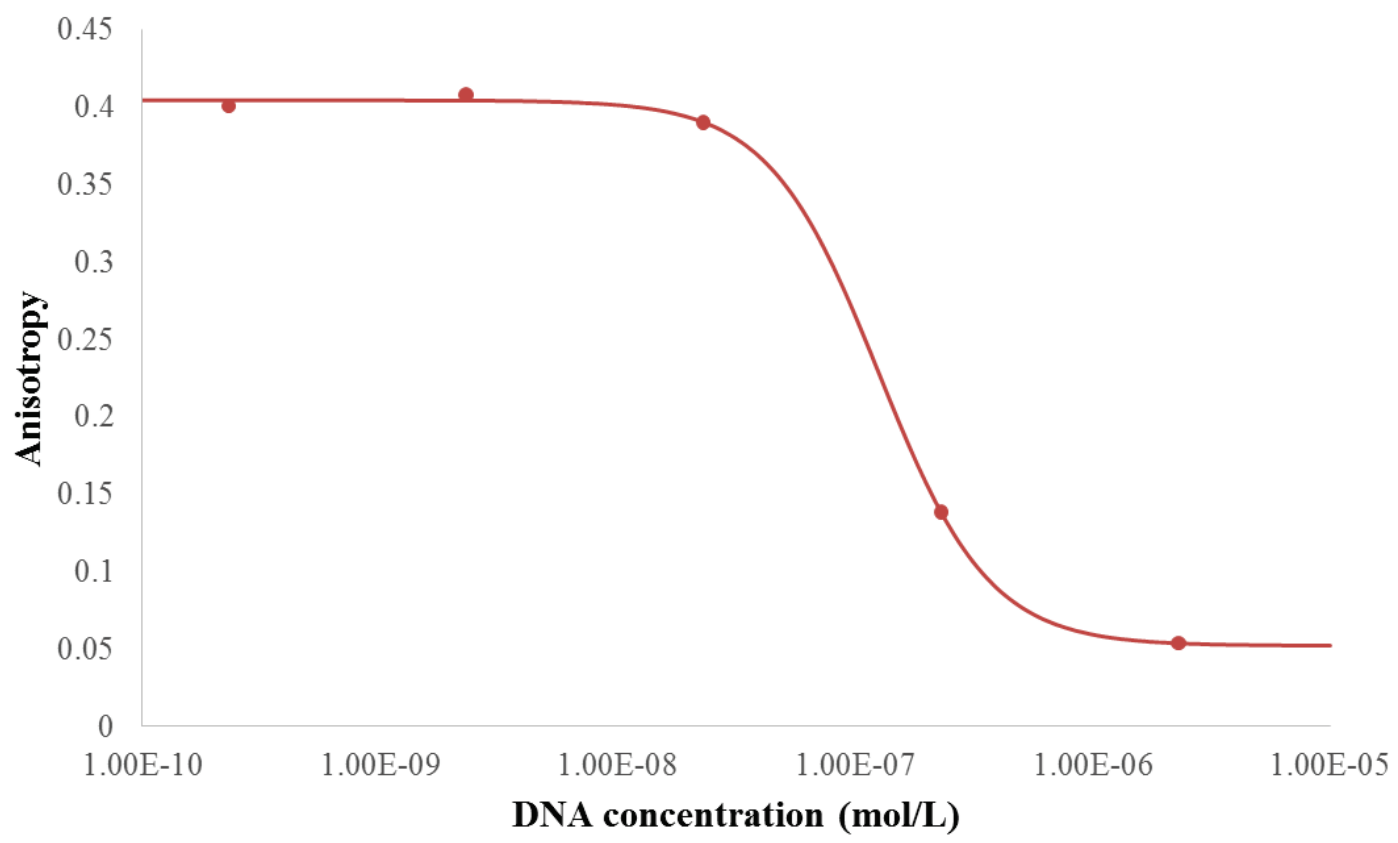

Figure 6.5: The apparent dissociation constant of DopaA20min was determined by fluorescence anisotropy. The dopamine concentration was held constant $\left(1.0 \times 10^{-7}\right.$ $\mathrm{mol} / \mathrm{L}$ ) and the concentration of the aptamer was varied. Anisotropy values were measured with an excitation and emission wavelength of $285 \mathrm{~nm}$ and $317 \mathrm{~nm}$ respectively. Data points represent a single trial and the corresponding fit.

To determine the apparent $\mathrm{K}_{\mathrm{D}}$ of DopaA20min, equivolumes of a constant concentration of dopamine and various concentrations of aptamer over the $10^{-10} \mathrm{~mol} / \mathrm{L}$ to $10^{-6} \mathrm{~mol} / \mathrm{L}$ 
range were prepared in SCB then mixed together immediately prior to measuring the anisotropy of the sample. The apparent $\mathrm{K}_{\mathrm{D}}$ of DopaA20min was determined to be $1.1 \pm$ $0.8 \times 10^{-7} \mathrm{~mol} / \mathrm{L}$ where the value is the average of four trials and the error is the standard deviation between those trials. The apparent $\mathrm{K}_{\mathrm{D}}$ of the DopaA20min is significantly better than the reported dissociation constants of both the RNA DBA aptamer $(1.6 \pm 0.2$ $\mu \mathrm{M})$ and the DNA homolog DBA $(0.7 \mu \mathrm{M})$. Therefore the first objective of the novel selection was satisfied; a DBA with better affinity than either existing aptamer was identified.

Fluorescence anisotropy is a commonly employed technique used to measure the affinity of aptamers for protein targets but has also been used to determine the apparent dissociation constant of small molecule-aptamer interactions. ${ }^{276,396,418,509}$ In this experiment, the fluorescence properties of dopamine were exploited. The anisotropy value measured at each aptamer concentration was dependent on the rotation of the fluorescent dopamine target in solution. Dopamine that is free in solution rotates differently than dopamine that is aptamer-bound. This difference resulted in a change in the anisotropy value measured. Generally anisotropy values are relatively large for the aptamer-target complex in solution and relatively small for the free aptamer/target in solution. However, the opposite trend has also been observed; in fact relatively larger anisotropy values for free dopamine than for aptamer-bound dopamine were observed for the DNA homolog DBA. ${ }^{396,418}$ The cause of the change in anisotropy due to aptamertarget interaction is multifactorial and has not been well characterized. ${ }^{510}$ 
The judicious choice of the affinity characterization method should be based on the intended application. The dissociation constant determined for an aptamer-target interaction is largely dependent on the affinity characterization method used. ${ }^{276}$ Indeed an aptamer (A08) selected to bind to the small molecule ochratoxin A was shown to have an apparent $\mathrm{K}_{\mathrm{D}}$ in the nanomolar range by both affinity chromatography and the $\mathrm{SyBr}$ Green assay, but was described as non-binding in fluorescence polarization and SPR methods. ${ }^{276}$ The fluorescence anisotropy method was chosen because it allowed for the measurement of the aptamer's affinity for the target in free solution without modification or immobilization of either the aptamer or the target. This was especially important as the intended applications for the DopaA20min aptamer were the development of a DopaA20min-based gold nanoparticle sensor and the investigation of the effect of DopaA20min administration on cocaine-induced hyperlocomotion. In both applications, the aptamer would be required to bind to dopamine in free solution.

\subsubsection{Development of an aptamer-gold nanoparticle (Apt-AuNP) based sensor for the detection of dopamine}

\subsubsection{Design and optimization of the Apt-AuNP sensor}

The objective of the work described in this section was to develop an Apt-AuNP based colourimetric sensor for the detection of dopamine based on the novel DopaA20min aptamer. Briefly, the first step of the test involved the incubation of the aptamer $(6 \mu \mathrm{L}$ of $10 \mu \mathrm{M}$ DNA) with its cognate target $(243 \mu \mathrm{L})$, in this case DopaA20min aptamer was incubated with dopamine. In this assay, various dopamine concentrations ranging from $10^{-5}$ to $10^{-10} \mathrm{~mol} / \mathrm{L}$ were prepared in deionized water and 
examined. After allowing the aptamer and target to interact for a 10 min incubation period, AuNPs ( $135 \mu \mathrm{L}$ of $10.5 \mathrm{nM}$ stock) were added to the solution. The addition of $\mathrm{NaCl}(72 \mu \mathrm{L}$ of $0.25 \mathrm{M}$ stock) to the test solution facilitated the colourimetric detection of the target. The concentrations of the DNA and $\mathrm{NaCl}$ used in this assay are consistent with those described by Zheng et al., 2012. ${ }^{466}$ Aptamer that was not interacting with the target was able to interact with the surface of the AuNPs during a 5 min incubation. When no target (or low amounts) was present as in Figure 6.1 A, the colour of the solution remained red due to the non-specific interaction of the aptamer and the AuNP surface. Upon the addition of $\mathrm{NaCl}$ to the solution containing target (Figure 6.1 B), AuNPs that were not protected by non-binding aptamer were forced to aggregate. This aggregation resulted in a noticeable colour change of the solution from red to blue that was directly dependent on the amount of target, in this case dopamine, that was present. A representative set of samples from this assay performed using the DopaA20min aptamer and dopamine are shown in Figure 6.6.
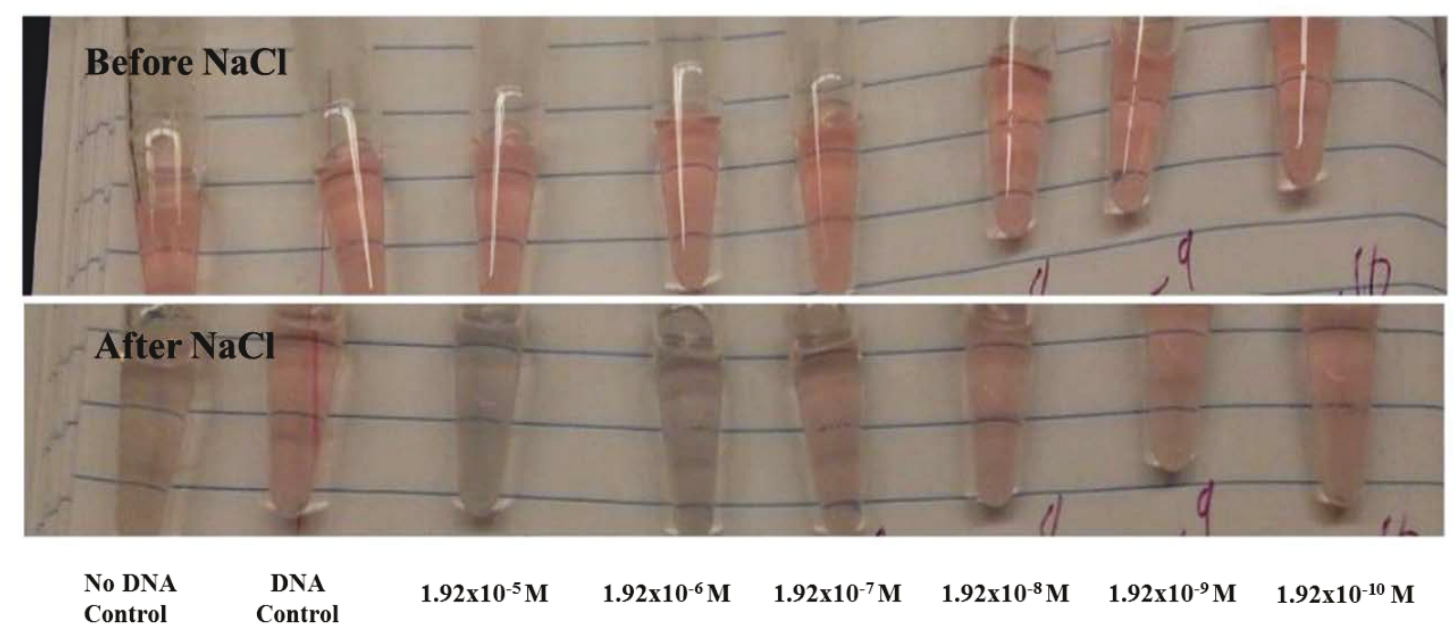

Figure 6.6: Representative images of the aptamer-AuNP assay before and after the addition of $\mathrm{NaCl}$. Top panel: before the addition of $\mathrm{NaCl}$, all of the samples (with 
and without dopamine) look similarly red. Bottom panel: following $\mathrm{NaCl}$ addition, the presence of higher dopamine concentrations resulted in more significant aggregation. Visually aggregation was represented by a colour change from red to blue. $\mathrm{M}: \mathrm{mol} / \mathrm{L}$

Before the addition of $\mathrm{NaCl}$ (Figure 6.6: top panel) to the test solution, all of the dopamine concentrations appeared similarly red in colour. Importantly, in the presence of the aptamer, aggregation of the AuNPs was not caused by the presence of the target dopamine alone. Following the addition of $\mathrm{NaCl}$ (Figure 6.6: bottom panel) aggregation was observed due to the interaction of the aptamer with dopamine, and thereby the lack of protection of the AuNPs by the DNA. In the case of little (or no) dopamine, the solutions remained red in colour (see Figure 6.6: bottom panel, dopamine concentrations $1.92 \times 10^{-8}$ to $1.92 \times 10^{-10} \mathrm{~mol} / \mathrm{L}$ ). This is consistent with the idea that free DopaA20min was able to nonspecifically interact with the surface of the AuNP, protecting the AuNP from salt induced aggregation. When the dopamine concentration was high and therefore the DopaA20min aptamer was interacting specifically with dopamine as opposed to nonspecifically with the AuNP, or there was no DNA to protect the AuNPs aggregation was observed. Depending on the degree of aggregation either a blue (relatively high - see Figure 6.6: bottom panel: $1.92 \times 10^{-5} \mathrm{~mol} / \mathrm{L}$ ) or purple (intermediate amount - see Figure 6.6: $\left.1.92 \times 10^{-6} \mathrm{~mol} / \mathrm{L}\right)$ were observed. To quantify this change and detect more subtle differences the UV-Visible absorbance of the samples were measured (Figure 6.7). 


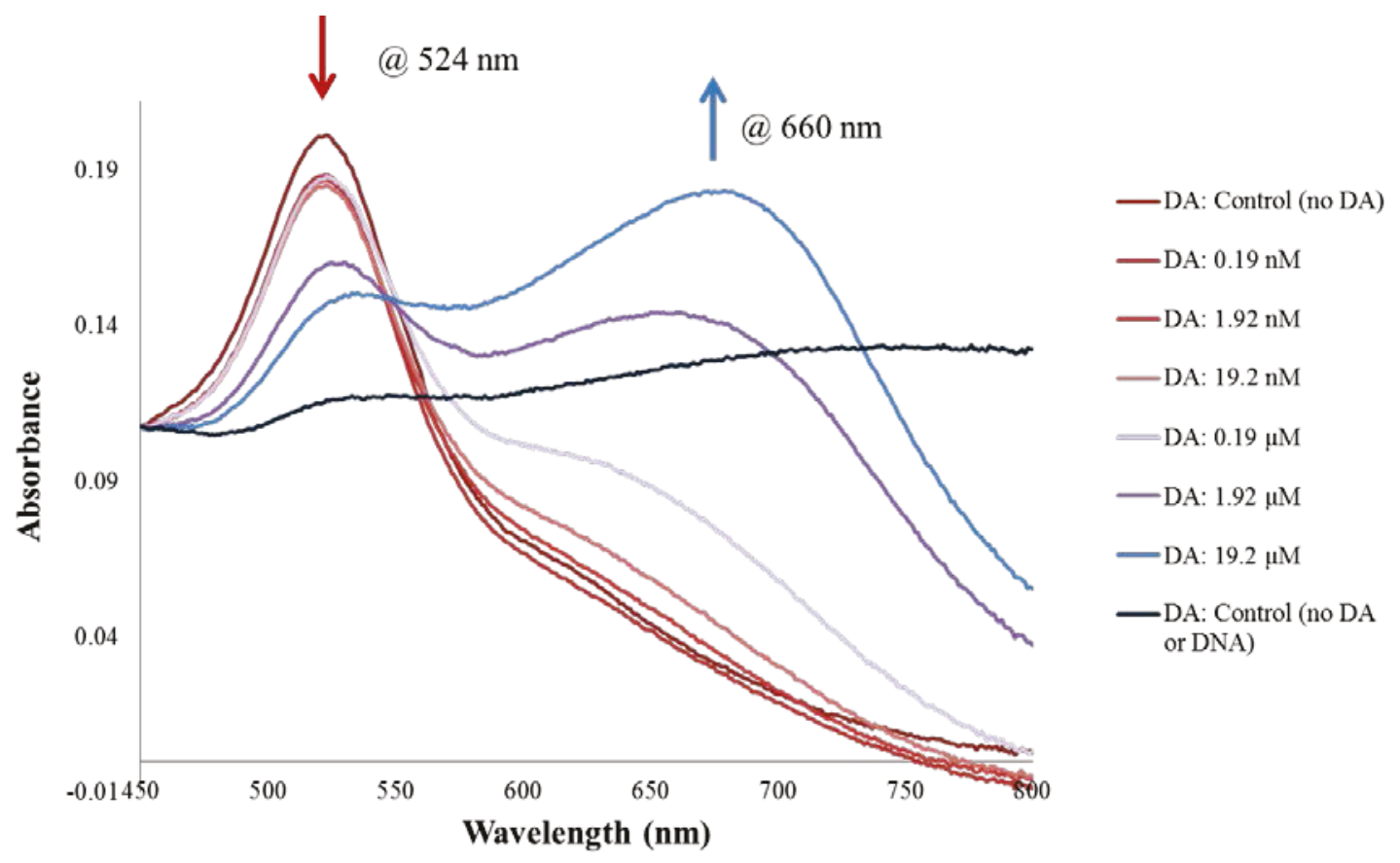

Figure 6.7: Representative UV-Visible absorbance spectra of the DopaA20min aptamer-AuNP based sensor in response to various dopamine concentrations ranging from $1.92 \times 10^{-5} \mathrm{M}$ to $1.92 \times 10^{-10} \mathrm{M}$. The "DA: control (no DA)" was run with the appropriate amount of aptamer but water instead of dopamine (DA). The "DA: Control (no DA or DNA)" was run with deionized water in place of DA and DNA. Spectra were corrected to the DA: Control (no DNA) absorbance at $450 \mathrm{~nm}$.

Due to the chemical, physical and optical properties of the AuNPs used in this assay, the absorbance of the AuNPs in water was at $2520 \mathrm{~nm} .{ }^{511}$ When $\mathrm{NaCl}$ was added to the AuNP solution (DA: Control (no DA or DNA)", the absorption band at $524 \mathrm{~nm}$ decreased and a new band at $660 \mathrm{~nm}$ appeared due to the aggregation of the AuNPs in solution. When the aptamer was present but there was no dopamine in solution (DA: Control (no DA), the absorption band was maintained at $524 \mathrm{~nm}$, therefore the aptamer was able to non-specifically interact with the surface of the AuNPs and protect them from salt induced aggregation. In the presence of increasing dopamine concentrations (DA: 0.19 $\mathrm{nM}-19.2 \mu \mathrm{M})$, the absorption band at $524 \mathrm{~nm}$ decreased while the absorption band at 
$660 \mathrm{~nm}$ increased. This change in the spectra with increasing dopamine concentration reflects an increase in AuNP aggregation. The absorption band changes from $524 \mathrm{~nm}$ (red) when particles are dispersed in solution to $660 \mathrm{~nm}$ (blue) upon aggregation due to the plasmonic properties of AuNPs. ${ }^{486,488}$

Transmission electron microscopy was used to examine the AuNPs in the presence and absence of dopamine (Figure 6.8).

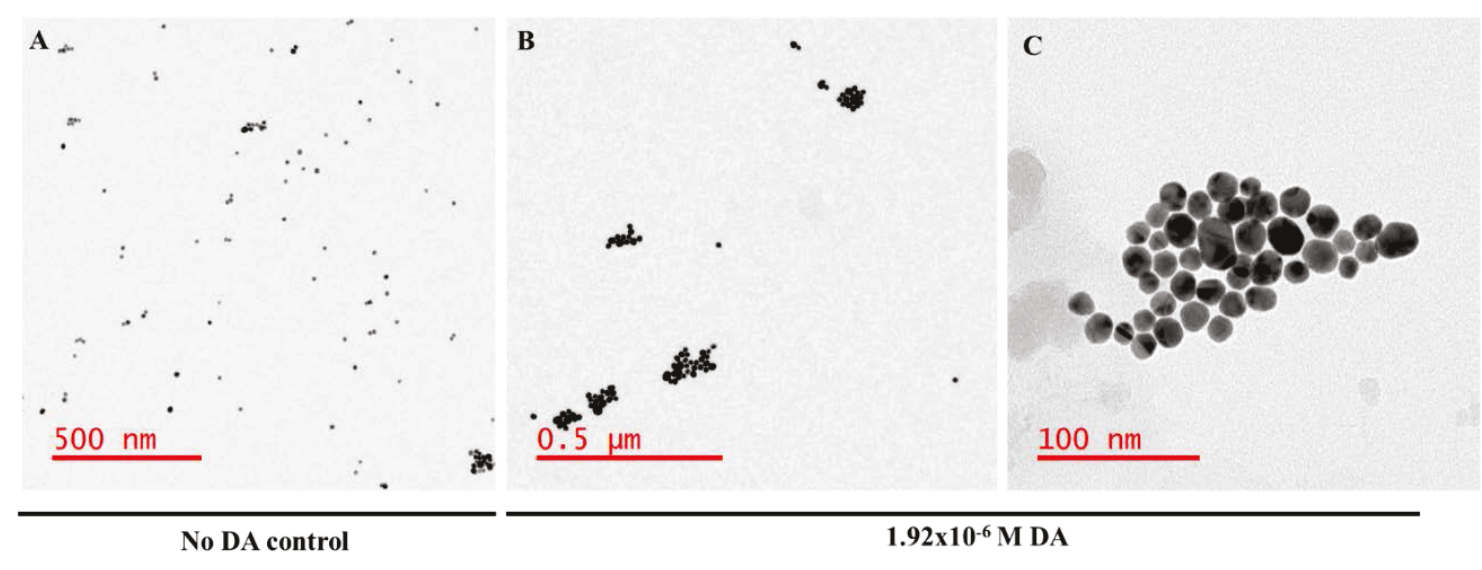

Figure 6.8: Transmission electron microscopy images of the aptamer-AuNP sensor in the absence (A) and presence (B,C) of dopamine. $A)$ When no dopamine (DA) is present, the DopaA20min aptamer remained nonspecifically interacting with the surface of the AuNP, thereby having a protective effect against salt-induced aggregation. B) When DA was present, the aptamer specifically bound to DA, leaving the unprotected AuNP susceptible to salt induced aggregation.

In the absence of dopamine, the AuNPs were protected by the aptamer and the particles were dispersed (Figure 6.8 - left image). Addition of dopamine led to aptamer-target binding, and therefore decreased AuNP protection. In this case, salt-induced aggregation was observed (Figure 6.8 - middle and right images). In contrast to the multiple single particles observed when the AuNPs were protected by aptamer, multiple aggregates and 
only very few single particles were observed when aptamer-dopamine binding prevented the interaction of DNA with the AuNP surface.

\subsubsection{Determination of the linear dynamic range and detection limit of dopamine of the Apt-AuNP sensor in deionized water}

The response of the sensor was evaluated by examining the ratio of the absorbance measured at $660 \mathrm{~nm}$ and $524 \mathrm{~nm}\left(\mathrm{~A}_{660 /} \mathrm{A}_{524}\right.$; Figure 6.9). The sensor response to increasing dopamine (blue) is shown in Figure 6.9 A. To ensure that the Apt-AuNP sensor was sensitive and selective to dopamine, the $\mathrm{A}_{660 /} \mathrm{A}_{524}$ ratio of the Apt-AuNP sensor to methionine (green) was also measured. Additionally, the $\mathrm{A}_{660 /} \mathrm{A}_{524}$ ratio of random DNA-AuNP to dopamine (red) was evaluated.
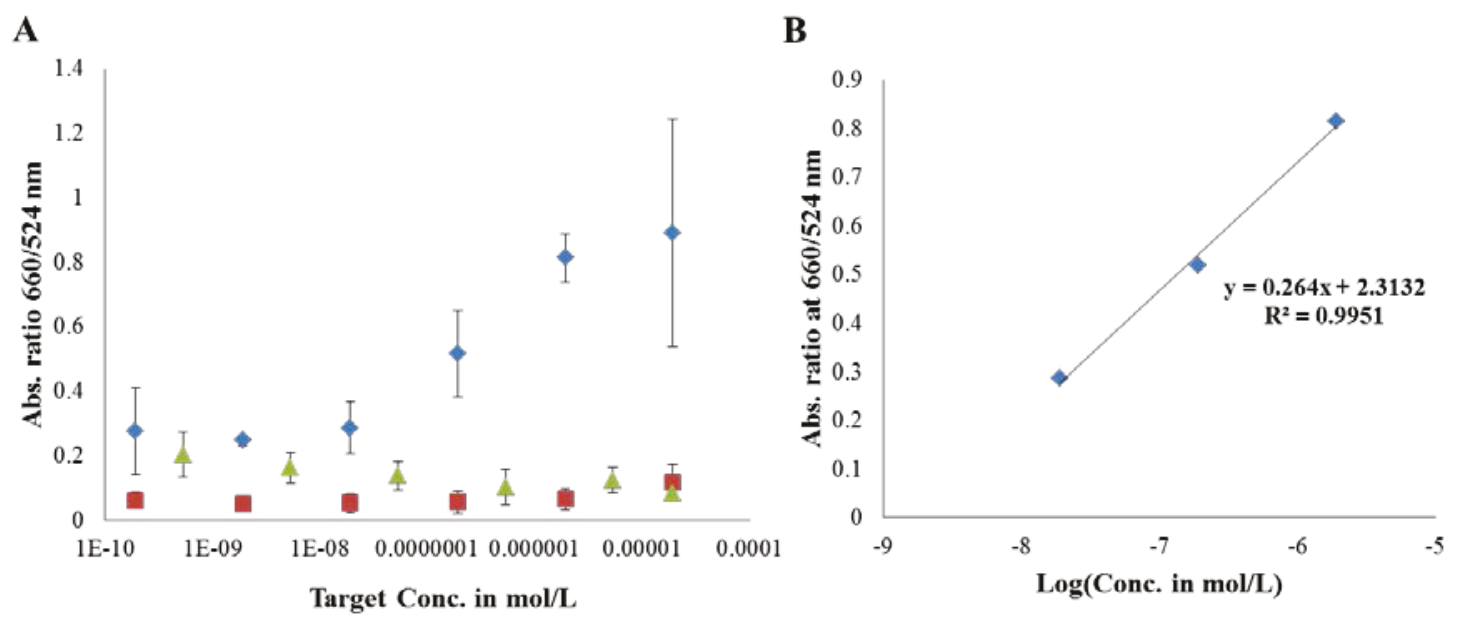

Figure 6.9: A) The average $(\mathrm{n}=3)$ absorbance ratios at $660 / 524 \mathrm{~nm}$ of the DopaA20min aptamer-AuNP sensor in response to varying DA (blue), and methionine (green) concentrations. The response of random DNA (red) to increasing $D A$ concentrations was also investigated. Error bars represent "mean $\pm S D$ " of $n=2$ or 3. B) The linear dynamic range of the sensor in response to $D A$ was determined to be $1.92 \times 10^{-8} \mathrm{~mol} / \mathrm{L}$ to $1.92 \times 10^{-6} \mathrm{~mol} / \mathrm{L}$. The limit of detection $(3 \sigma)$ was $9.98 \times$ $10^{-9} \mathrm{~mol} / \mathrm{L}$. 
Importantly, when the aptamer was replaced with random DNA, there was no $\mathrm{A}_{660} / \mathrm{A}_{524}$ ratio trend observed with increasing dopamine concentration. Further, when the AptAuNP sensor was evaluated with increasing methionine concentrations no trend in $\mathrm{A}_{660} / \mathrm{A}_{524}$ ratio was observed. Methionine was chosen as a control since it is a small molecule with functional groups similar to dopamine, but is structurally unrelated. The Apt-AuNP sensor showed a selective response to dopamine where the $\mathrm{A}_{660} / \mathrm{A}_{524}$ ratio increased with increasing dopamine. Physically, this suggests that with increasing dopamine the amount of AuNPs dispersed in solution decreased and the number of aggregated particles increased. There was a linear correlation $\left(\mathrm{R}^{2}=0.9951\right)$ between the $\mathrm{A}_{660} / \mathrm{A}_{524}$ ratio and dopamine concentration ranging between $1.92 \times 10^{-8} \mathrm{~mol} / \mathrm{L}$ to $1.92 \times$ $10^{-6} \mathrm{~mol} / \mathrm{L}$ with a corresponding detection limit $(\mathrm{LOD}=3 \sigma)$ of $9.98 \times 10^{-9} \mathrm{~mol} / \mathrm{L}$. These parameters are a marked improvement over the DNA homolog DBA-AuNP sensor described previously. The detection range of the Apt-AuNP sensor is bigger than the DNA homolog DBA-AuNP sensor $(0.54 \mu \mathrm{M}-5.4 \mu \mathrm{M})$ and the limit of detection of the Apt-AuNP sensor is two orders of magnitude lower than the limit of detection for the DNA homolog DBA-AuNP sensor $(0.36 \mu \mathrm{M})$. In fact, the detection range and LOD of the described Apt-AuNP were comparable to that reported for a variation on the DBAAuNP that used fluorescence resonance energy transfer between rhodamine B and AuNPs to increase detection range and lower the LOD to $2.6 \times 10^{-8} \mathrm{~mol} / \mathrm{L}$ to $2.9 \times 10^{-6}$ $\mathrm{mol} / \mathrm{L}$ and $2 \times 10^{-9} \mathrm{~mol} / \mathrm{L}$ respectively. ${ }^{480}$ Since the DNA homolog DBA-AuNP sensor protocol was easily adapted to produce the Apt-AuNP described herein it stands to reason that the detection range and LOD of the Apt-AuNP could be significantly increased by incorporating rhodamine $\mathrm{B}$ into the sensor design. 


\subsubsection{Characterization of the affinity of DopaA20min for dopamine by the Apt- AuNP assay}

The data shown in Figure 6.9 was also be used to determine the apparent $\mathrm{K}_{\mathrm{D}}$ of the DopaA20min-dopamine interaction. The fit of the average $A_{660 /} A_{524}$ ratio $(n=3)$ for dopamine concentrations ranging between $1.92 \times 10^{-10} \mathrm{~mol} / \mathrm{L}$ to $1.92 \times 10^{-6} \mathrm{~mol} / \mathrm{L}$ was analyzed using the standard curve analysis (four parameter logistic curve) function of SigmaPlot. From the fit, the apparent $\mathrm{K}_{\mathrm{D}}$ was determined to be $2.8 \pm 0.4 \times 10^{-7} \mathrm{~mol} / \mathrm{L}$. This is slightly higher (lower affinity) than was determined by fluorescence anisotropy. The difference could be due to the method (fluorescence versus absorbance) or to the fact that measuring the apparent $\mathrm{K}_{\mathrm{D}}$ by the Apt-AuNP assay provides an indirect indication of affinity (the measurable change is due to the aggregation of the AuNPs) as compared to the direct approach of fluorescence anisotropy where the observed change is directly due to the aptamer-target binding interaction.

\subsubsection{The selectivity of DopaA20min was determined by Apt-AuNP assay}

The Apt-AuNP assay was used to screen the DopaA20min aptamer for selectivity against similar neurotransmitters and common interferents. To assess the selectivity of the DopaA20min aptamer, the $\mathrm{A}_{660 /} \mathrm{A}_{524}$ ratio at a single concentration $(1.92 \mu \mathrm{M})$ was measured for each target (Figure 6.10 A). Visually, binding (as evident by a colour change from red to blue) was observed for dopamine and norepinephrine (Figure 6.10 B). This was confirmed by the increased $\mathrm{A}_{660 /} \mathrm{A}_{524}$ ratio compared to the "No Target" blank (Figure 6.10 A). Additionally, some affinity to both tyramine and serotonin was observed, however no affinity for ascorbic acid was seen. 
A

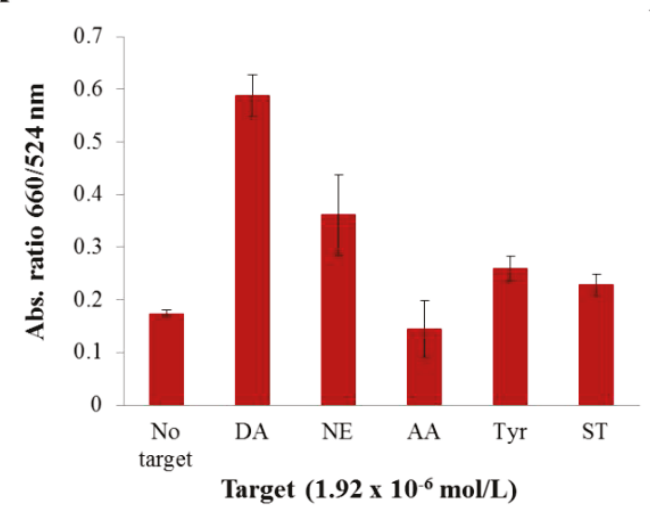

B
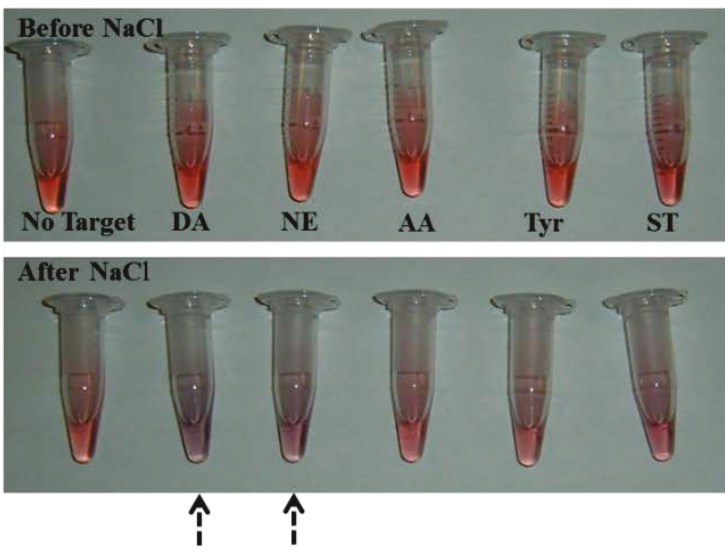

C

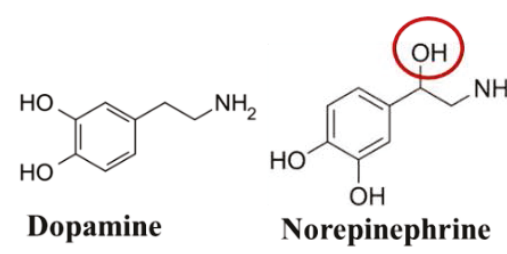

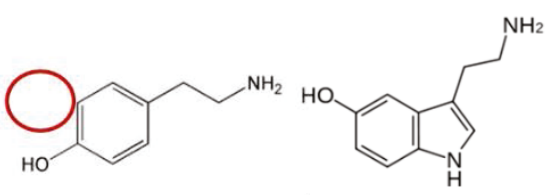

Tyramine

Serotonin

Figure 6.10: A) The AuNP assay was used to determine the specificity of the DopaA20min aptamer. Error represents "mean \pm SD" of $n=2$. B) At the target concentration investigated $\left(1.92 \times 10^{-6} \mathrm{~mol} / \mathrm{L}\right)$, only $\mathrm{NE}$ elicited a noticeable degree of aggregation. $\mathrm{C}$ ) The following targets were investigated due to their structural similarity to dopamine (DA); norepinephrine (NE), ascorbic acid (AA), tyramine (Tyr) and serotonin (ST). Red circles represent a single difference of the addition or subtraction of an hydroxyl group compared to DA.

The affinity of the DopaA20min aptamer for DA $>\mathrm{NE}>\mathrm{Tyr}>\mathrm{ST}$ is not surprising given the structural similarity of the targets tested (see Figure 6.10 C). Additionally, counter selections against these targets were not performed during the selection of the aptamer.

Further, during their initial assessments both the RNA and DNA homolog DBAs showed some affinity for norepinephrine and minimal affinity for tyramine. ${ }^{280,396}$ The RNA aptamer showed $58 \pm 13 \% \mathrm{NE}$ relative elution to dopamine when assessed by column chromatography, whereas the DNA homolog aptamer exhibited a slightly better dissociation constant for NE as determined by fluorescence anisotropy. In the initial assessment, neither DBA were evaluated for their affinity to serotonin. However, 
serotonin was detected by the DBA in an electrochemical study where the DBA was functionalized to a glassy carbon electrode. ${ }^{479}$

The affinity of DopaA20 min for NE, Tyr and ST could also be exploited as the starting point for selecting specific aptamers for those neurotransmitters. Currently, aptamers have been specifically selected for NE (commercially by Base Pair Technologies) and Tyr with dissociation constants in the high nanomolar to midmicromolar range. ${ }^{512}$ Aptamers have not been selected for serotonin. To yield aptamers with higher affinities for NE and Tyr or to select aptamers that are specific for ST, the DopaA20min could be partially mutated to develop a new selection pool. ${ }^{513}$ Selections using this mutagenized pool should yield higher affinity aptamers and the judicious inclusion of counter selections against competing neurotransmitters could produce highly selective aptamers. In the same way, aptamers with better selectivity for dopamine could be selected as well.

Since the DopaA20min aptamer exhibited the greatest secondary affinity for NE, the response of the Apt-AuNP sensor to increasing NE concentrations was evaluated (Figure 6.11 A) 
A

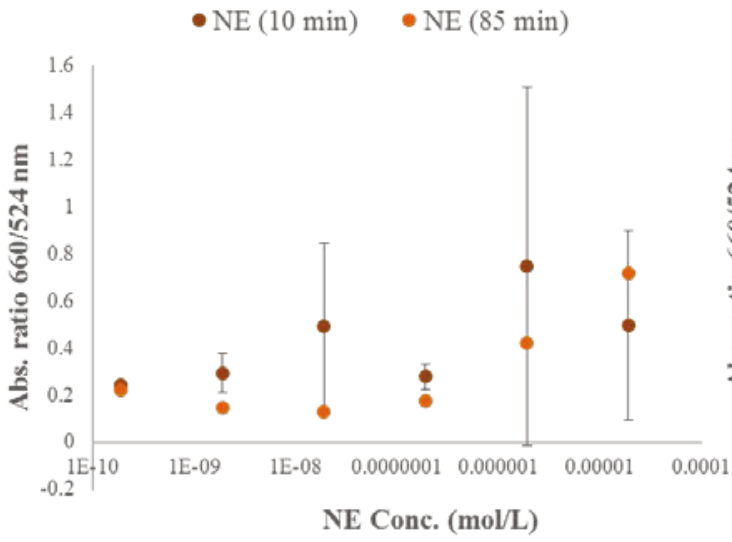

B

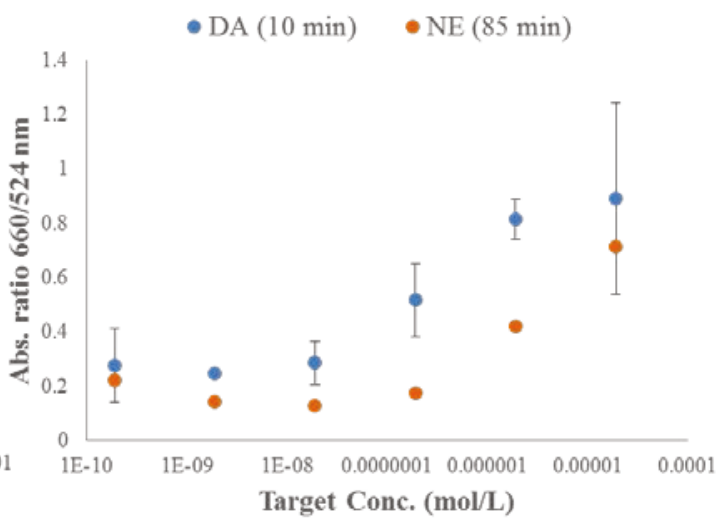

Figure 6.11: The response of the Aptamer-AuNP sensor to increasing concentrations of norepinephrine (NE) was evaluated. A) Increasing the second incubation time from 10 min (brown; $n=2$ ) to 85 min (orange; $n=1$ ) produced a more consistent response curve. B) Compared to the Aptamer-AuNP sensor response to increasing concentrations of dopamine (DA; blue, $n=3$ ), the sensor's response to $\mathrm{NE}$ after an 85 min incubation (dark orange; $n=1$ ) was less sensitive even with a longer incubation period. Error bars represent "mean \pm SD".

Following a 10 min incubation period, the Apt-AuNP sensor did exhibit a generally increasing $\mathrm{A}_{660 /} \mathrm{A}_{524}$ ratio with increasing NE concentration, however the response was largely varied between two trials. Different target-aptamer incubation times ranging from 3 min-30 min have been investigated for other aptamer-based AuNP assays to optimize the response of the sensor. ${ }^{511,514-516}$ Since there was literature precedence that the $\mathrm{A}_{\text {aggregated }} / \mathrm{A}_{\text {dispersed }}$ nanoparticle absorbance ratio could be maximized by increasing the aptamer-target incubation time, the incubation time was increased to $85 \mathrm{~min}^{514}$ With the increased incubation period, the increase in $\mathrm{A}_{660 /} \mathrm{A}_{524}$ ratio was much more consistent with increasing NE concentration. The trends in $\mathrm{A}_{660 /} \mathrm{A}_{524}$ ratios for the 10 min and 85 min incubation times are shown in Figure 6.11 A. For reference, in Figure 6.11 B the Apt-AuNP sensor response to increasing NE concentrations after an 85 min incubation period was plotted against the Apt-AuNP sensor response to increasing dopamine 
concentrations after a 10 min incubation period. Even after an increased incubation period, the Apt-AuNP response to NE is decreased compared to dopamine at equal concentrations. Taken together these data suggest that the DopaA20min aptamer has a lower affinity for NE compared to dopamine. Therefore the second objective of the novel selection was satisfied: the DopaA20min is relatively more selective against norepinephrine than the DNA homolog DBA. Based on the degree of aggregation observed in the specificity testing, the affinities for tyramine and serotonin are likely substantially lower. Furthermore, the aptamer seems to exhibit different binding kinetics for dopamine compared to NE. The binding on-rate is likely much faster for dopamine than for NE. These are parameters that could be exploited to optimize the selectivity of the Apt-AuNP sensor. For example, the Apt-AuNP sensor could be made more selective by decreasing the aptamer-target incubation time. Given the increased affinity of the aptamer for dopamine and the preliminary indication of faster binding kinetics, this would allow for the selective, reliable detection of dopamine. In fact, Yang et al., (2011) optimized their testing parameters such that ochratoxin A was detected by an Apt-AuNP

sensor within 5 minutes. ${ }^{516}$ In future work, these parameters should be considered in the design of other DopaA20min-based sensors to optimize selectivity and sensor response time.

\subsubsection{Detection of dopamine in artificial cerebral spinal fluid}

The Apt-AuNP response to increasing dopamine in artificial cerebral spinal fluid (ACSF) was evaluated. ASCF was chosen to mimic biologically relevant conditions. To test the response of the Apt-AuNP sensor to increasing dopamine concentrations in 
ACSF, dopamine solutions were prepared ranging from $3.6 \times 10^{-9} \mathrm{~mol} / \mathrm{L}$ to $3.6 \times 10^{-3}$ $\mathrm{mol} / \mathrm{L}$. The samples were then diluted 100X in the Apt-AuNP assay so that the actual dopamine concentrations measured in the test were the same as those measured in water $\left(1.92 \times 10-{ }^{11} \mathrm{~mol} / \mathrm{L}\right.$ to $\left.1.92 \times 10^{-9} \mathrm{~mol} / \mathrm{L}\right)$. The Apt-AuNP response $\left(\mathrm{A}_{660} / \mathrm{A}_{524}\right.$ ratio $)$ measured for increasing dopamine concentrations in 100X diluted ACSF is shown in

Figure 6.12.

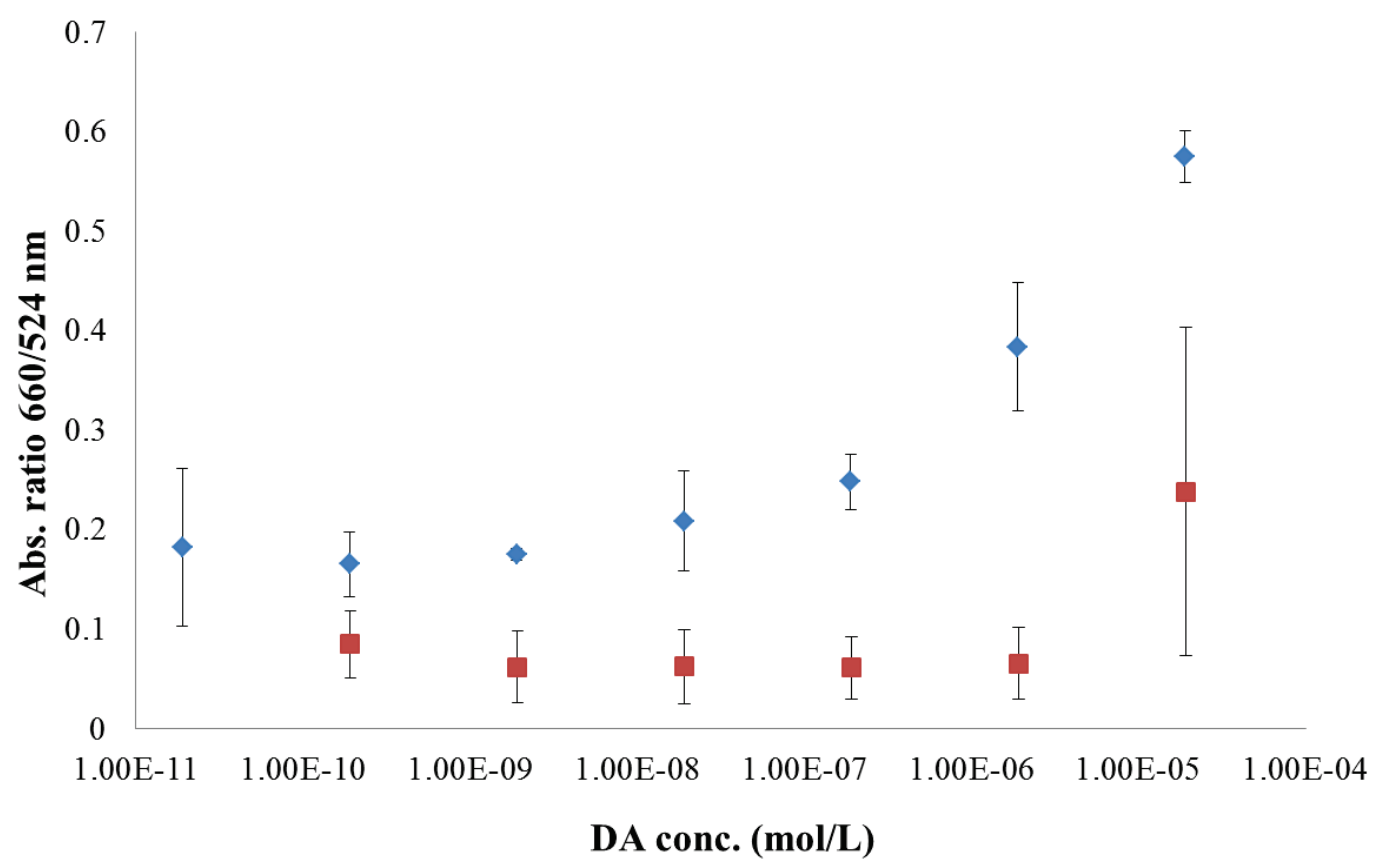

Figure 6.12: Detection of dopamine (DA) in 100X diluted artificial cerebral spinal fluid (ACSF) by aptamer (blue; $n=3$ ) AuNP assay. As a control the response of random DNA (red; $n=2)$ in the presence of increasing DA was monitored. Error bars represent "mean \pm SD".

In ACSF, with increasing dopamine concentration the $\mathrm{A}_{660 /} \mathrm{A}_{524}$ ratio attributed to DopaA20min-DA binding increased. This is shown in Figure 6.12 where the response of DopaA20min (blue) is compared to the response of a random DNA (red) in place of the aptamer. In ACSF the Apt-AuNP sensor was able to detect DA levels above $1.92 \times 10^{-6}$ mol/L. Though the sensitivity of the Apt-AuNP decreased in ACSF, the fact that the 
DopaA20min can be used to detect DNA in this biological matrix is an important first step. Further optimization of the experimental parameters such as DNA concentration, salt concentration and incubation time may improve the Apt-AuNP response to dopamine in ACSF. Additionally, modifications of the method for example adding rhodamine B to the assay may increase the sensitivity significantly.

Typically methods such as microdialysis, HPLC and electrochemistry are used to detect small molecule neurotransmitters and their metabolites in CSF. ${ }^{517-519}$ The concentration of dopamine in the CSF of healthy controls ranges from the 10s of picomolar to low nanomolar. ${ }^{520}$ In individuals with Parkinson's disease, multiple systems atrophy, pure autonomic failure and other synucleinopathies the levels of dopamine in the CSF can be even lower. ${ }^{520}$ This makes dopamine hard to detect in CSF as it is predicted to exist in levels that are often times below the limit of detection of the technique used. ${ }^{520}$ An Apt-AuNP based sensor that could detect dopamine in CSF at these levels could provide a relatively simple, cost effective alternative to traditional methods. Moreover, elevated levels of dopamine in CSF compared to healthy controls have been reported in early stage/asymptomatic HIV-infected individuals (low nanomolar to low micromolar), Menkes disease (nanomolar with similarly elevated NE), depression (mid nanomolar), and schizophrenia treated with neuroleptics (nanomolar) ${ }^{521-524}$ Finally, the ability to detect dopamine in CSF could be generally useful in understanding the pathological mechanisms of monoamine neurotransmitter disorders. These disorders, characterized by abnormalities in the biosynthesis, degradation and transport of dopamine, norepinephrine, epinephrine and serotonin effect individuals from the infant to the elderly population. 


\subsubsection{Investigation of DopaA20min in a model of cocaine induced hyperlocomotion}

The efficacy of peripherally delivered DopaA20min in reducing cocaine-induced

hyperlocomotion was examined in an in vivo animal model. DopaA20min was delivered to the brain of mice via encapsulation in a TRAM vehicle (described in Chapter 5).

Briefly, the DopaA20min aptamer (abbreviated as DA20m for this work) was encapsulated into transferrin receptor aptamer-modified liposomes (TRAM). The DA20m-TRAM were delivered peripherally by IP injection. Delivery of the DA20m aptamer to the brain was facilitated by the specific binding interaction of the transferrin receptor aptamer (TRA) to the transferrin receptor. This interaction triggered receptor mediated endocytosis, the mechanism by which the DA20m-TRAM was taken across the BBB. In this experiment, mice $(n=40)$ were assigned to one of the following groups; SubTRAM $+10 \mathrm{mg} / \mathrm{mL}$ cocaine $(\mathrm{n}=7)$, DA20m-TRAM $+10 \mathrm{mg} / \mathrm{mL}$ cocaine $(\mathrm{n}=8)$, DALTRAM + $10 \mathrm{mg} / \mathrm{mL}$ cocaine $(\mathrm{n}=8)$, DA20m-TRAM + saline $(\mathrm{n}=9)$ and DAL-TRAM + saline $(\mathrm{n}=8)$. Mice received an i.p. injection $(0.1 \mathrm{~mL})$ of the pretreatment (Sub-TRAM, DA20m-TRAM or DAL-TRAM) and then 5 min later were given an i.p. injection (0.1 $\mathrm{mL}$ ) or either $10 \mathrm{mg} / \mathrm{mL}$ cocaine or saline. Animals were placed in the locomotor testing apparatus $5 \mathrm{~min}$ after receiving their second injection. The horizontal activity of each animal was recorded in 5 min bin over an entire 30 min session. The mean + SEM cumulative locomotor activity for each treatment group are shown in Figure 6.13. 


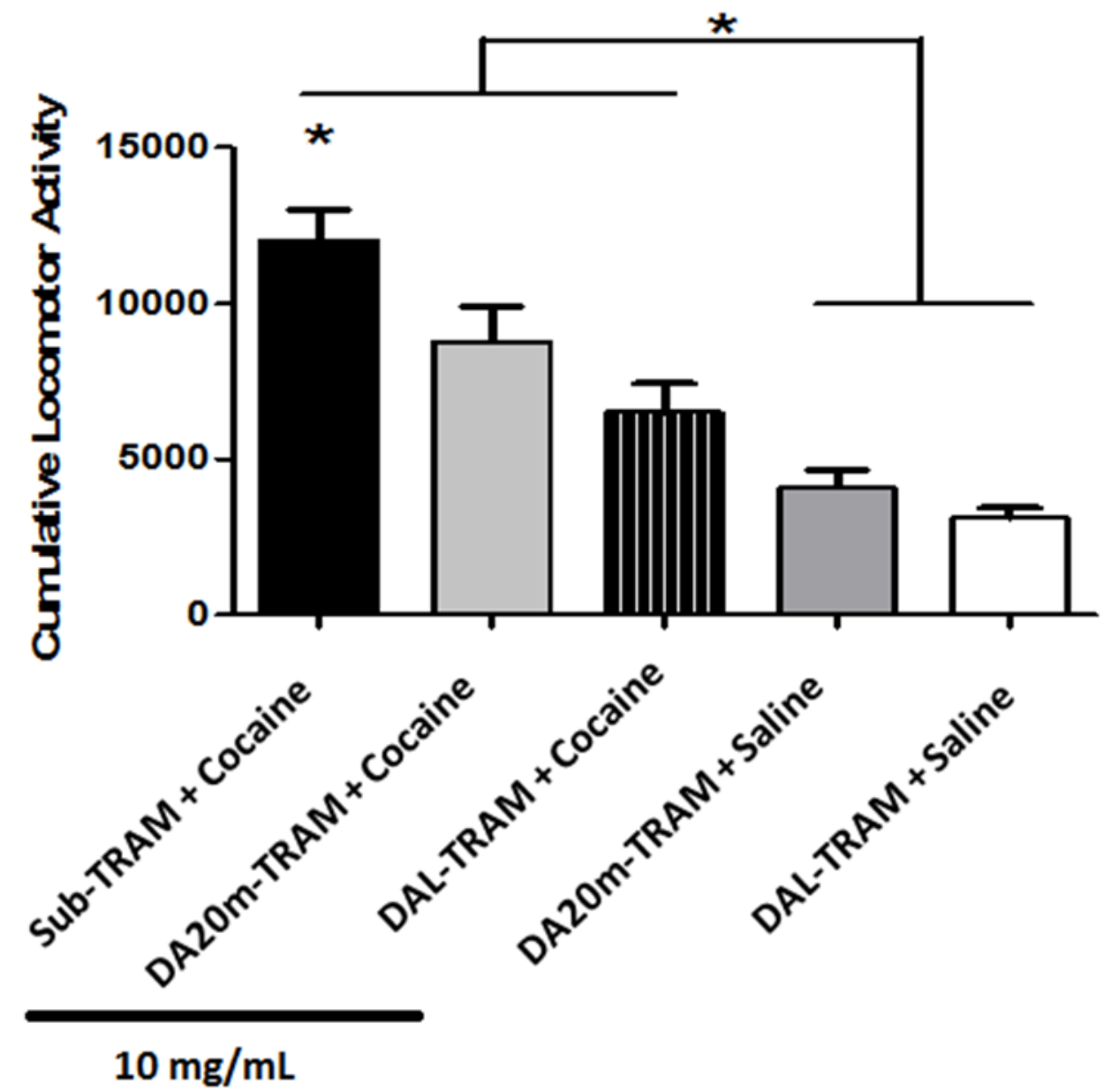

Treatment

Figure 6.13: Cumulative horizontal locomotor activity of DA20m-TRAM compared to controls over an entire 30 min session. One-way ANOVA analysis revealed a significant effect of group $(F(4,34)=20.12, p<0.001)$. Significant differences between treatment conditions were revealed by Post-hoc analysis by Fisher's LSD.

Significance is indicated by $(* \mathbf{p}<0.05)$. Group sizes were as follows; Sub-TRAM +10 $\mathrm{mg} / \mathrm{mL}$ cocaine $(n=7)$, DA20m-TRAM $+10 \mathrm{mg} / \mathrm{mL}$ cocaine $(n=8)$, DAL-TRAM + 10 $\mathrm{mg} / \mathrm{mL}$ cocaine $(n=8)$, DA20m-TRAM + saline $(n=9)$ and DAL-TRAM + saline $(n=8)$. Error bars represent "mean + SEM".

Analyses by One-way ANOVA revealed a significant effect of group $(\mathrm{F}(4,34)=20.12$,

$\mathrm{p}<0.001$; Figure 6.13) on the cumulative locomotor activity over the entire $30 \mathrm{~min}$

session. Post-hoc analyses by Fisher's LSD revealed significant differences between 
treatment groups. The cumulative locomotor activity of both the DA20m-TRAM +10 $\mathrm{mg} / \mathrm{mL}$ cocaine treated group and the DAL-TRAM $+10 \mathrm{mg} / \mathrm{mL}$ cocaine treated group were significantly lower than the Sub-TRAM $+10 \mathrm{mg} / \mathrm{mL}$ cocaine treated control group $(p=0.009$ and $p<0.001$ respectively). Further, the difference between the DA20m-TRAM $+10 \mathrm{mg} / \mathrm{mL}$ cocaine and the DAL-TRAM $+10 \mathrm{mg} / \mathrm{mL}$ cocaine treated groups was not significant $(\mathrm{p}=0.054)$. Consistent with the DAL-TRAM data presented in Chapter 5, the cumulative locomotor activity of DA20m-TRAM $+10 \mathrm{mg} / \mathrm{mL}$ cocaine treated animals was still significantly higher than DA20m-TRAM + saline treated controls $(\mathrm{p}<0.001)$. Importantly, there was no difference between DA20m-TRAM + saline and DAL-TRAM + saline treated control groups. These data can be interpreted to suggest that the DopaA20min aptamer is at least as effective in reducing cocaine-induced hyperlocomotion as the DNA homolog dopamine binding aptamer evaluated in Chapter 5. Further, treatment with DA20m-TRAM does not have deleterious effects on locomotion. Given the difference in affinity of the DopaA20min aptamer compared to DNA homolog dopamine binding aptamer for norepinephrine, it may be interesting to use this aptamer to investigate the specific effects of DA versus NE in animal models of dopamine-related disease. ${ }^{284}$ More generally, these data support the use of DopaA20min in animal models.

The mechanism of action by which the DopaA20min aptamer reduces cocaineinduced hyperlocomotion is the same as that proposed for the DNA-homolog dopamine binding aptamer examined in Chapter 5. Typically, behaviours associated with increased dopamine are reversed or reduced by treatment with D1/D2 antagonists. These drugs indirectly prevent dopamine-related effects by binding to dopamine receptors whereby 
the dopamine binding aptamers presented herein prevent the actions of dopamine by binding directly to dopamine. ${ }^{397}$ Therefore in this experiment, the effective reduction in dopamine concentration created by dopamine-aptamer binding manifested behaviourally as reduced locomotor behaviour. Future work should investigate increasing either the dosage of the aptamer or the DA20m-TRAM, or both should be optimized to maximize the reduction in hyperlocomotion observed. Further, the targeting component of the delivery vehicle (TRAM) could be replaced by a better aptamer to increase delivery. ${ }^{240}$ 


\subsection{Conclusion}

This chapter described the selection, characterization and use of a dopaminebinding aptamer, DopaA20min. The aptamer showed preferential affinity for dopamine over similar neurotransmitters (norepinephrine, tyramine and serotonin) and had an apparent dissociation constant of $1.1 \pm 0.8 \times 10^{-7} \mathrm{~mol} / \mathrm{L}$. An aptamer-gold nanoparticle based sensor (Apt-AuNP) was developed that was able to detect dopamine within the linear detection range of $1.92 \times 10^{-8} \mathrm{~mol} / \mathrm{L}$ to $1.92 \times 10^{-6} \mathrm{~mol} / \mathrm{L}$ and had a detection limit $(\mathrm{LOD}=3 \sigma)$ of $9.98 \times 10^{-9} \mathrm{~mol} / \mathrm{L}$. Finally, the effect of peripherally delivered DopaA20min on cocaine-induced hyperlocomotion was examined. Animals treated with DopaA20min showed significantly reduced locomotor activity compared to animals treated with a non-dopamine binding oligonucleotide control. The work presented here demonstrates the varied potential applications of DopaA20min. Future work should focus on the use of DopaA20min in other sensor designs to fully optimize the specificity, working range and detection capabilities of the aptamer. Additionally, future work should investigate the effect of peripherally delivered DopaA20min in other dopamine-related maladies and behaviours. 
7 Future directions and contributions to knowledge 


\subsection{The use of aptamers for nervous system related applications}

The nervous system (central and peripheral) remains an important biological and medical arena in which the potential of aptamers has not yet been fully investigated. The research presented herein supports the promising trajectory of aptamer research for central nervous system (CNS) related targets. Since aptamers were first described in the 1990s, their effectiveness as molecular recognition probes has been widely demonstrated for medical, biological and environmental applications. An approach that should be considered for extending the use of aptamers for applications in the nervous system is optimizing selection experiments. By rationally designing the selection experiment to optimize aptamer-target binding for the intended application, further aptamer technology will be significantly improved. The analysis of data from the Aptamer Base (Chapter 2) may provide researchers the information required to optimize their selection experiments.

One of the greatest advantages of aptamers is their ease of synthesis and chemical modification. This allows aptamers to be simply incorporated into existing technology to add an additional element of recognition and specificity. An example of this approach is the development of an aptamer-based nanomachine (pHAST) for the catch-and-release of a target protein presented in Chapter 3. This example illustrates the potential of how existing diagnostic and therapeutic technologies used to study nervous system related maladies could be improved by a simple aptamer modification. Another excellent example of where aptamers could be used to improve existing technology are the multiple imaging strategies currently used to evaluate dementia. ${ }^{525}$ Several of these approaches could be easily modified to include aptamer technology, for example by 
adding specificity and functionality to nanoprobes and nanoparticles used for imaging and treating gliomas. ${ }^{56}$ Additionally, aptamers could be used to increase the effectiveness of current photodynamic therapy approaches. ${ }^{231}$ In both instances aptamers could improve the selectivity of the technology and decrease off-target effects.

An important CNS-related target subset that should be further explored in selection experiments are small molecules. The work described in Chapter 4, Chapter 5 and Chapter 6 describes the characterization and use of dopamine binding aptamers to reverse and decrease specific behavioural disturbances associated with dopamine overactivity. Importantly, aptamers offer an alternative direct approach to modulating neurotransmitter function; where as traditionally receptors were targeted to indirectly modulate function, aptamers allow for the direct targeting of neurotransmitters. The work presented in Chapter 4, Chapter 5 and Chapter 6 shows how a small molecule neurotransmitter-binding aptamer can modulate behaviour, but aptamers could also be used to achieve a better understanding of neurotransmitter function. Another important application of newly selected aptamers would be their incorporation into existing and novel sensing platforms for the detection of their target molecule. An example of this was described in Chapter 6 where an aptamer-gold nanoparticle based sensor for the detection of dopamine was described. Other small molecule neurotransmitters that should be studied include glutamate, serotonin and GABA due to their roles in neurodegenerative diseases, neuropsychiatric diseases, and anxiety and affective disorders. ${ }^{527-529}$ Along with interesting small molecule targets, several protein targets of interest related to AD, ALS, HD and PD have also been described ${ }^{530}$ Specifically new aptamer selections should focus on the targets Tau, superoxide dismutase 1, and Huntingtin as well as TDP-43 for 
their role in AD, ALS, and HD respectively. The aforementioned misfolded proteins exhibit a propensity to form toxic aggregates. The selection of aptamers for these targets could identify diagnostic and therapeutic agents capable of preventing aggregation or degrading existing aggregates. Finally, optimized selection methods could also be used to simultaneously select aptamers and identify novel biomarkers for nervous system related targets. ${ }^{531}$

If current challenges of CNS related aptamer work, such as specific delivery of aptamers across the blood-brain and blood-cerebrospinal fluid barriers, can be overcome, the application of aptamer technology will be limited only to the availability of aptamers for a specific target. Along with the work presented in Chapter 5, several strategies have been investigated to transverse or circumvent the BBB. ${ }^{526}$ Further investigation should examine the ability of novel vehicles as well as novel incorporation of aptamers into existing vehicles for their ability to cross the BBB. Additionally, aptamer-based chimeric conjugate systems may prove to be an efficient delivery approach.

The work described in the chapters of this thesis has contributed to the foundation necessary for the improvement of noninvasive diagnostics, therapeutics, targeted drug delivery and imaging modalities. In summary, aptamer-based technologies present novel therapeutic and unique diagnostic value to basic and applied medical research for applications in nervous system related maladies. 


\section{References}

(1) McConnell, E. M.; Holahan, M. R.; DeRosa, M. C. Nucleic Acid Ther. 2014, 24 (6), 388.

(2) Crick, F. Nature 1970, 227 (5258), 561.

(3) Gilbert, W. Nature 1986, 319 (6055), 618.

(4) Deigan, K. E.; Ferré-D'Amaré, A. R. Acc. Chem. Res. 2011, 44 (12), 1329.

(5) Teller, C.; Willner, I. Curr. Opin. Biotechnol. 2010, 21 (4), 376.

(6) McKeague, M.; Derosa, M. C. J. Nucleic Acids 2012, 2012, 748913.

(7) Ye, M.; Hu, J.; Peng, M.; Liu, J.; Liu, J.; Liu, H.; Zhao, X.; Tan, W. Int. J. Mol. Sci. 2012, 13 (3), 3341.

(8) McKeague, M.; McConnell, E. M.; Cruz-Toledo, J.; Bernard, E. D.; Pach, A.; Mastronardi, E.; Zhang, X.; Beking, M.; Francis, T.; Giamberardino, A.; Cabecinha, A.; Ruscito, A.; Aranda-Rodriguez, R.; Dumontier, M.; DeRosa, M. C. J. Mol. Evol. 2015, 81 (5), 150.

(9) Famulok, M.; Hartig, J. S.; Mayer, G. Chem. Rev. 2007, 107 (9), 3715.

(10) Winkler, W.; Nahvi, A.; Breaker, R. R. Nature 2002, 419 (6910), 952.

(11) Winkler, W. C.; Cohen-Chalamish, S.; Breaker, R. R. Proc. Natl. Acad. Sci. U. S. A. 2002, $99(25), 15908$.

(12) Sudarsan, N.; Wickiser, J. K.; Nakamura, S.; Ebert, M. S.; Breaker, R. R. Genes Dev. 2003, 17 (21), 2688.

(13) Mironov, A. S.; Gusarov, I.; Rafikov, R.; Lopez, L. E.; Shatalin, K.; Kreneva, R. A.; Perumov, D. A.; Nudler, E. Cell 2002, 111 (5), 747.

(14) McDaniel, B. A. M.; Grundy, F. J.; Artsimovitch, I.; Henkin, T. M. Proc. Natl. Acad. Sci. U. S. A. 2003, 100 (6), 3083.

(15) Mandal, M.; Breaker, R. R. Nat. Struct. Mol. Biol. 2004, 11 (1), 29.

(16) Mandal, M.; Lee, M.; Barrick, J. E.; Weinberg, Z.; Emilsson, G. M.; Ruzzo, W. L.; Breaker, R. R. Science 2004, 306 (5694), 275.

(17) Mandal, M.; Boese, B.; Barrick, J. E.; Winkler, W. C.; Breaker, R. R. Cell 2003, $113(5), 577$.

(18) Epshtein, V.; Mironov, A. S.; Nudler, E. Proc. Natl. Acad. Sci. U. S. A. 2003, 100 (9), 5052.

(19) Rodionov, D. A.; Vitreschak, A. G.; Mironov, A. A.; Gelfand, M. S. J. Biol. Chem. 2002, 277 (50), 48949.

(20) Grate, D.; Wilson, C. Bioorg. Med. Chem. 2001, 9 (10), 2565.

(21) Grate, D.; Wilson, C. Proc. Natl. Acad. Sci. U. S. A. 1999, 96 (11), 6131.

(22) Werstuck, G.; Green, M. R. Science 1998, 282 (5387), 296.

(23) Hanson, S.; Berthelot, K.; Fink, B.; McCarthy, J. E. G.; Suess, B. Mol. Microbiol. 
2003, 49 (6), 1627.

(24) Suess, B.; Hanson, S.; Berens, C.; Fink, B.; Schroeder, R.; Hillen, W. Nucleic Acids Res. 2003, 31 (7), 1853.

(25) Suess, B.; Fink, B.; Berens, C.; Stentz, R.; Hillen, W. Nucleic Acids Res. 2004, 32 (4), 1610.

(26) Ruigrok, V. J. B.; Levisson, M.; Eppink, M. H. M.; Smidt, H.; van der Oost, J. Biochem. J. 2011, 436, 1.

(27) Mastronardi, E.; Foster, A.; Zhang, X.; DeRosa, M. C. Sensors (Basel). 2014, 14 (2), 3156.

(28) Keefe, A. D.; Pai, S.; Ellington, A. Nat. Rev. Drug Discov. 2010, 9 (7), 537.

(29) Szatkowska, P.; Koba, M.; Ko, P.; Szablewski, M. 2013, 400.

(30) Binz, H. K.; Plückthun, A. Curr. Opin. Biotechnol. 2005, 16 (4), 459.

(31) Tolle, F.; Mayer, G. Chem. Sci. 2013, 4 (1), 60.

(32) Mayer, G. Angew. Chemie Int. Ed. 2009, 48 (15), 2672.

(33) Dong, Y.; Xu, Y.; Yong, W.; Chu, X.; Wang, D. Crit. Rev. Food Sci. Nutr. 2014, 54 (12), 1548.

(34) Tuerk, C.; Gold, L. Science 1990, 249 (4968), 505.

(35) Ellington, A. D.; Szostak, J. W. Nature 1990, 346 (6287), 818.

(36) Robertson, D. L.; Joyce, G. F. Nature 1990, 344 (6265), 467.

(37) Nutiu, R.; Li, Y. Angew. Chem. Int. Ed. Engl. 2005, 44 (7), 1061.

(38) Darmostuk, M.; Rimpelova, S.; Gbelcova, H.; Ruml, T. Biotechnol. Adv. 2015, 33 (6 Pt 2), 1141.

(39) Bruno, J. Pharmaceuticals 2013, 6 (3), 340.

(40) Healy, J. M.; Lewis, S. D.; Kurz, M.; Boomer, R. M.; Thompson, K. M.; Wilson, C.; McCauley, T. G. Pharm. Res. 2004, 21 (12), 2234.

(41) Hicke, B. J.; Stephens, A. W.; Gould, T.; Chang, Y.-F.; Lynott, C. K.; Heil, J.; Borkowski, S.; Hilger, C.-S.; Cook, G.; Warren, S.; Schmidt, P. G. J. Nucl. Med. 2006, 47 (4), 668.

(42) Bruno, J. G. Molecules 2015, 20 (4), 6866.

(43) The Government of Canada. Mapping Connections an Understanding of Neurological Conditions; 2014.

(44) Wang, T.; Ray, J. Protein Cell 2012, 3 (10), 739.

(45) Lee, J. H.; Yigit, M. V; Mazumdar, D.; Lu, Y. Adv. Drug Deliv. Rev. 2010, 62 (6), 592.

(46) Bunka, D. H. J.; Platonova, O.; Stockley, P. G. Curr. Opin. Pharmacol. 2010, 10 (5), 557 . 
(47) Engelhardt, S.; Patkar, S.; Ogunshola, O. O. Br. J. Pharmacol. 2014, 171 (5), 1210 .

(48) Alyautdin, R.; Khalin, I.; Nafeeza, M. I.; Haron, M. H.; Kuznetsov, D. Int. J. Nanomedicine 2014, 9, 795.

(49) de Lange, E. C. M. Fluids Barriers CNS 2013, 10 (1), 12.

(50) Cheng, C.; Chen, Y. H.; Lennox, K. a; Behlke, M. a; Davidson, B. L. Mol. Ther. Nucleic Acids 2013, 2 (November 2012), e67.

(51) Perschbacher, K.; Smestad, J. a; Peters, J. P.; Standiford, M. M.; Denic, A.; Wootla, B.; Warrington, A. E.; Rodriguez, M.; Maher, L. J. Nucleic Acid Ther. 2015, 25 (1), 11.

(52) Hwang, S. R.; Kim, K. Arch. Pharm. Res. 2014, 37 (1), 24.

(53) Chen, C. B.; Dellamaggiore, K. R.; Ouellette, C. P.; Sedano, C. D.; Lizadjohry, M.; Chernis, G. a; Gonzales, M.; Baltasar, F. E.; Fan, A. L.; Myerowitz, R.; Neufeld, E. F. Proc. Natl. Acad. Sci. U. S. A. 2008, 105 (41), 15908.

(54) Kaeser, P. S.; Regehr, W. G. Annu. Rev. Physiol. 2014, 76, 333.

(55) Bruno, J. G.; Carrillo, M. P.; Phillips, T.; King, B. In Vitro Cell. Dev. Biol. Anim. 2008, 44 (3-4), 63.

(56) Chen, S.-J.; Huang, Y.-F.; Huang, C.-C.; Lee, K.-H.; Lin, Z.-H.; Chang, H.-T. Biosens. Bioelectron. 2008, 23 (11), 1749.

(57) Bruno, J. G.; Richarte, A. M.; Phillips, T. Microchem. J. 2014, 115, 32.

(58) Zhu, J.; Wang, J.; Su, Z.-C.; Li, Q.; Cheng, M.-S.; Zhang, J.-H. Arch. Pharm. Res. 2008, 31 (9), 1120.

(59) Mendonsa, S. D.; Bowser, M. T. J. Am. Chem. Soc. 2005, 127 (26), 9382.

(60) Huizenga, D. E.; Szostak, J. W. Biochemistry 1995, 34 (2), 656.

(61) Wang, J.; Zhang, P.; Li, J. Y.; Chen, L. Q.; Huang, C. Z.; Li, Y. F. Analyst 2010, 135 (11), 2826.

(62) Tokunaga, T.; Namiki, S.; Yamada, K.; Imaishi, T.; Nonaka, H.; Hirose, K.; Sando, S. J. Am. Chem. Soc. 2012, 134 (23), 9561.

(63) Busl, K. M.; Bleck, T. P. Neurotherapeutics 2012, 9 (1), 139.

(64) Čapek, P.; Dickerson, T. J. Toxins (Basel). 2010, 2 (1), 24.

(65) Tok, J. B.-H.; Fischer, N. O. Chem. Commun. (Camb). 2008, No. 16, 1883.

(66) Fan, M.; McBurnett, S. R.; Andrews, C. J.; Allman, A. M.; Bruno, J. G.; Kiel, J. L. J. Biomol. Tech. 2008, 19 (5), 311.

(67) Bruno, J. G.; Richarte, A. M.; Carrillo, M. P.; Edge, A. Biosens. Bioelectron. 2012, $31(1), 240$

(68) Wei, F.; Ho, C.-M. Anal. Bioanal. Chem. 2009, 393 (8), 1943.

(69) Wei, F.; Bai, B.; Ho, C.-M. Biosens. Bioelectron. 2011, 30 (1), 174. 
(70) Janardhanan, P.; Mello, C. M.; Singh, B. R.; Lou, J.; Marks, J. D.; Cai, S. Talanta 2013, 117, 273.

(71) Bogomolova, A.; Aldissi, M. Biosens. Bioelectron. 2015, 66, 290.

(72) Fetter, L.; Richards, J.; Daniel, J.; Roon, L.; Rowland, T. J.; Bonham, A. J. Chem. Commun. 2015, 51, 8 .

(73) Chang, T.-W.; Blank, M.; Janardhanan, P.; Singh, B. R.; Mello, C.; Blind, M.; Cai, S. Biochem. Biophys. Res. Commun. 2010, 396 (4), 854.

(74) Elshafey, R.; Siaj, M.; Zourob, M. Biosens. Bioelectron. 2015, 68, 295.

(75) Eissa, S.; Siaj, M.; Zourob, M. Biosens. Bioelectron. 2015, 69, 148.

(76) Gao, S.; Hu, B.; Zheng, X.; Cao, Y.; Liu, D.; Sun, M.; Jiao, B.; Wang, L. Biosens. Bioelectron. 2016, 79, 938.

(77) Handy, S. M.; Yakes, B. J.; DeGrasse, J. a; Campbell, K.; Elliott, C. T.; Kanyuck, K. M.; Degrasse, S. L. Toxicon 2013, 61, 30.

(78) Campbell, K.; Rawn, D. F. K.; Niedzwiadek, B.; Elliott, C. T. Food Addit. Contam. Part A. Chem. Anal. Control. Expo. Risk Assess. 2011, 28 (6), 711.

(79) Burrell, S.; Crum, S.; Foley, B.; Turner, A. D. TrAC - Trends Anal. Chem. 2016, 75, 10.

(80) Ye, F.; Zheng, Y.; Wang, X.; Tan, X.; Zhang, T.; Xin, W.; Wang, J.; Huang, Y.; Fan, Q.; Wang, J. PLoS One 2014, 9 (8), 2.

(81) Bruno, J. G.; Phillips, T.; Montez, T. J. Bionanoscience 2015, 9, 270.

(82) Gutiérrez, J. M.; Burnouf, T.; Harrison, R. A.; Calvete, J. J.; Kuch, U.; Warrell, D. A.; Williams, D. J. Bull. World Health Organ. 2014, 92 (7), 526.

(83) Hesselberth, J. R.; Miller, D.; Robertus, J.; Ellington, A. D. J. Biol. Chem. 2000, 275 (7), 4937.

(84) Tang, J.; Xie, J.; Guo, L.; Yan, Y.; Shao, N. Front. Chem. China 2007, 2 (4), 431.

(85) Tang, J.; Xie, J.; Shao, N.; Yan, Y. Electrophoresis 2006, 27 (7), 1303.

(86) Lamont, E. a; He, L.; Warriner, K.; Labuza, T. P.; Sreevatsan, S. Analyst 2011, 136 (19), 3884.

(87) Kirby, R.; Cho, E. J.; Gehrke, B.; Bayer, T.; Sok, Y.; Neikirk, D. P.; Mcdevitt, J. T.; Ellington, A. D.; Park, Y. S. Anal. Chem. 2004, 76 (14), 4066.

(88) Fan, S.; Wu, F.; Martiniuk, F.; Hale, M. L.; Ellington, A. D.; Tchou-Wong, K. M. World J. Gastroenterol. 2008, 14 (41), 6360.

(89) Huang, Y.; Chen, X.; Wu, S.; Duan, N.; Yu, Y.; Wang, Z. Microchim. Acta 2015, $182(5-6), 1035$.

(90) He, L.; Lamont, E.; Veeregowda, B.; Sreevatsan, S.; Haynes, C. L.; DiezGonzalez, F.; Labuza, T. P. Chem. Sci. 2011, 2 (8), 1579.

(91) Haes, A. J.; Giordano, B. C.; Collins, G. E. Anal. Chem. 2006, 78 (11), 3758. 
(92) Cho, E. J.; Collett, J. R.; Szafranska, A. E.; Ellington, A. D. Anal. Chim. Acta 2006, 564 (1), 82 .

(93) Ding, S.; Gao, C.; Gu, L. Q. Anal. Chem. 2009, 81 (16), 6649.

(94) Wang, B.; Guo, C.; Chen, G.; Park, B.; Xu, B. Chem. Commun. 2011, 48 (11), 1644.

(95) Wang, B.; Lou, Z.; Park, B.; Kwon, Y.; Zhang, H.; Xu, B. Phys. Chem. Chem. Phys. 2015, 17 (1), 307.

(96) Wang, B.; Park, B.; Kwon, Y.; Xu, B. Appl. Phys. Lett. 2014, 104 (19), 193702.

(97) Wang, B.; Xu, B. Phys. Rev. E - Stat. Nonlinear, Soft Matter Phys. 2014, 89 (2), 1.

(98) Wang, B.; Guo, C.; Zhang, M.; Park, B.; Xu, B. J. Phys. Chem. B 2012, 116 (17), 5316.

(99) Förster, C.; Zydek, M.; Rothkegel, M.; Wu, Z.; Gallin, C.; Geßner, R.; Lisdat, F.; Fürste, J. P. Biochem. Biophys. Res. Commun. 2012, 419 (1), 60.

(100) Förster, C.; Oberthuer, D.; Gao, J.; Eichert, A.; Quast, F. G.; Betzel, C.; Nitsche, A.; Erdmann, V. A.; Fürste, J. P. Acta Crystallogr. Sect. F. Struct. Biol. Cryst. Commun. 2009, 65 (Pt 9), 881.

(101) Nastasijevic, B.; Wright, B. R.; Smestad, J.; Warrington, A. E.; Rodriguez, M.; Maher, L. J. PLoS One 2012, 7 (6), e39595.

(102) Burmeister, P. E.; Wang, C.; Killough, J. R.; Lewis, S. D.; Horwitz, L. R.; Ferguson, A.; Thompson, K. M.; Pendergrast, P. S.; McCauley, T. G.; Kurz, M.; Diener, J.; Cload, S. T.; Wilson, C.; Keefe, A. D. Oligonucleotides 2006, 16 (4), 337.

(103) Wang, J.; Takeuchi, H.; Sonobe, Y.; Jin, S.; Mizuno, T.; Miyakawa, S.; Fujiwara, M.; Nakamura, Y.; Kato, T.; Muramatsu, H.; Muramatsu, T.; Suzumura, A. Proc. Natl. Acad. Sci. U. S. A. 2008, 105 (10), 3915.

(104) Gravel, M.; Robert, F.; Kottis, V.; Gallouzi, I. E.; Pelletier, J.; Braun, P. E. J. Neurosci. Res. 2009, 87 (5), 1069.

(105) Ishiguro, A.; Akiyama, T.; Adachi, H.; Inoue, J. I.; Nakamura, Y. Arthritis Rheum. 2011, 63 (2), 455.

(106) Prodeus, A.; Cydzik, M.; Abdul-Wahid, A.; Huang, E.; Khatri, I.; Gorczynski, R.; Gariépy, J. Mol. Ther. Nucleic Acids 2014, 3 (April), e190.

(107) Vorobjeva, M. a; Krasitskaya, V. V; Fokina, A. a; Timoshenko, V. V; Nevinsky, G. a; Venyaminova, A. G.; Frank, L. a. Anal. Chem. 2014, 86 (5), 2590.

(108) O’Connor, K. C.; Lopez-Amaya, C.; Gagne, D.; Lovato, L.; Moore-Odom, N. H.; Kennedy, J.; Krupp, L.; Tenembaum, S.; Ness, J.; Belman, A.; Boyko, A.; Bykova, O.; Mah, J. K.; Stoian, C. A.; Waubant, E.; Kremenchutzky, M.; Ruggieri, M.; Bardini, M. R.; Rensel, M.; Hahn, J.; Weinstock-Guttman, B.; Yeh, E. A.; Farrell, K.; Freedman, M. S.; Iivanainen, M.; Bhan, V.; Dilenge, M.; Hancock, M. A.; Gano, D.; Fattahie, R.; Kopel, L.; Fournier, A. E.; Moscarello, M.; Banwell, B.; 
Bar-Or, A. J. Neuroimmunol. 2010, 223 (1-2), 92.

(109) Mostafa, G. A.; El-Khashab, H. Y.; AL-Ayadhi, L. Y. J. Neuroimmunol. 2015, $280,16$.

(110) Toledano, M.; Weinshenker, B. G.; Solomon, A. J. Curr. Neurol. Neurosci. Rep. 2015, 15 (8), 57.

(111) Rozenblum, G. T.; Kaufman, T.; Vitullo, A. D. Mol. Ther. Acids 2014, 3 (9), e192.

(112) Tyler, J. Y.; Xu, X.-M.; Cheng, J.-X. Nanoscale 2013, 5, 8821.

(113) Smestad, J.; Maher, L. J. Nucleic Acids Res. 2013, 41 (2), 1329.

(114) Perschbacher, K.; Smestad, J. a; Peters, J. P.; Standiford, M. M.; Denic, A.; Wootla, B.; Warrington, A. E.; Rodriguez, M.; Maher, L. J. Nucleic Acid Ther. 2015, 25 (1), 11.

(115) Wang, Y.; Khaing, Z. Z.; Li, N.; Hall, B.; Schmidt, C. E.; Ellington, A. D. PLoS One 2010, 5 (3), e9726.

(116) Li, W.; Wang, K.; Zhao, M.; Yang, X.; Chen, M.; Lan, X. Thromb. Res. 2014, 134 (4), 769.

(117) Mayer, G.; Rohrbach, F.; Pötzsch, B.; Müller, J. Hamostaseologie 2011, 31 (4), 258.

(118) Hu, P.-P.; Zhang, K.-H. Blood Coagul. Fibrinolysis 2015, 26 (1), 1.

(119) Kalladka, D.; Muir, K. W. Stem Cells Cloning 2014, 7, 31.

(120) Blake, C. M.; Wang, H.; Laskowitz, D. T.; Sullenger, B. A. Oligonucleotides 2011, 21 (1), 11.

(121) Bjerregaard, N.; Bøtkjær, K. A.; Helsen, N.; Andreasen, P. A.; Dupont, D. M. Thromb. Haemost. 2015, 114 (1), 139.

(122) Rusconi, C. P.; Scardino, E.; Layzer, J.; Pitoc, G. a; Ortel, T. L.; Monroe, D.; Sullenger, B. a. Nature 2002, 419 (6902), 90.

(123) Lincoff, A. M.; Mehran, R.; Povsic, T. J.; Zelenkofske, S. L.; Huang, Z.; Armstrong, P. W.; Steg, P. G.; Bode, C.; Cohen, M. G.; Buller, C.; Laanmets, P.; Valgimigli, M.; Marandi, T.; Fridrich, V.; Cantor, W. J.; Merkely, B.; LopezSendon, J.; Cornel, J. H.; Kasprzak, J. D.; Aschermann, M.; Guetta, V.; Morais, J.; Sinnaeve, P. R.; Huber, K.; Stables, R.; Sellers, M. A.; Borgman, M.; Glenn, L.; Levinson, A. I.; Lopes, R. D.; Hasselblad, V.; Becker, R. C.; Alexander, J. H. Lancet 2015, 387 (10016), 349.

(124) Bae, O. N. Arch. Pharm. Res. 2012, 35 (10), 1693.

(125) Markus, H. S.; McCollum, C.; Imray, C.; Goulder, M. a; Gilbert, J.; King, A. Stroke. 2011, 42 (8), 2149.

(126) Diener, J. L.; Daniel Lagassé, H. A.; Duerschmied, D.; Merhi, Y.; Tanguay, J.-F.; Hutabarat, R.; Gilbert, J.; Wagner, D. D.; Schaub, R. J. Thromb. Haemost. 2009, 7 (7), 1155. 
(127) Deng, B.; Lin, Y.; Wang, C.; Li, F.; Wang, Z.; Zhang, H.; Li, X.-F.; Le, X. C. Anal. Chim. Acta 2014, 837, 1.

(128) Bock, L. C.; Griffin, L. C.; Latham, J. a; Vermaas, E. H.; Toole, J. J. Nature 1992, $355,564$.

(129) Tasset, D. M.; Kubik, M. F.; Steiner, W. J. Mol. Biol. 1997, 272 (5), 688.

(130) Lancellotti, S.; De Cristofaro, R. Cardiovasc. Hematol. Agents Med. Chem. 2009, $7,19$.

(131) Tan, X.; Dey, S. K.; Telmer, C.; Zhang, X.; Armitage, B. A.; Bruchez, M. P. ChemBioChem 2014, 15 (2), 205.

(132) Soule, E. E.; Bompiani, K. M.; Woodruff, R. S.; Sullenger, B. A. Nucleic Acid Ther. 2015, 26 (1), 1.

(133) Vater, A.; Jarosch, F.; Buchner, K.; Klussmann, S. Nucleic Acids Res. 2003, 31 (21), e130.

(134) Goadsby, P. J.; Edvinsson, L.; Ekman, R. Ann. Neurol. 1990, 28 (2), 183.

(135) Lassen, L. H.; Haderslev, P. A.; Jacobsen, V. B.; Iversen, H. K.; Sperling, B.; Olesen, J. Cephalalgia 2002, 22 (1), 54.

(136) Hoehlig, K.; Johnson, K. W.; Pryazhnikov, E.; Maasch, C.; Clemens-Smith, A.; Purschke, W. G.; Vauleon, S.; Buchner, K.; Jarosch, F.; Khiroug, L.; Vater, A.; Klussmann, S. Br. J. Pharmacol. 2015, 172 (12), 3086.

(137) Vater, A.; Klussmann, S. Drug Discov. Today 2015, 20 (1), 147.

(138) Denekas, T.; Tröltzsch, M.; Vater, A.; Klussmann, S.; Messlinger, K. Br. J. Pharmacol. 2006, 148 (4), 536.

(139) Juhl, L.; Edvinsson, L.; Olesen, J.; Jansen-Olesen, I. Eur. J. Pharmacol. 2007, 567 $(1-2), 117$.

(140) Amiri, H.; Saeidi, K.; Borhani, P.; Manafirad, A.; Ghavami, M.; Zerbi, V. ACS Chem. Neurosci. 2013, 4 (11), 1417.

(141) Yang, Y.; Ren, X.; Schluesener, H. J.; Zhang, Z. Curr. Med. Chem. 2011, 18 (27), 4159.

(142) Tannenberg, R. K.; Shamaileh, H. Al; Lauridsen, L. H.; Kanwar, J. R.; Dodd, P. R.; Veedu, R. N. Curr. Alzheimer Res. 2013, 10 (4), 442.

(143) Lee, J. H.; Shin, S. K.; Jiang, Y.; Choi, W. H.; Hong, C.; Kim, D.-E.; Lee, M. J. Sci. Rep. 2015, 5, 10757.

(144) Rahimi, F.; Murakami, K.; Summers, J. L.; Chen, C.-H. B.; Bitan, G. PLoS One 2009, 4 (11), e7694.

(145) Ylera, F.; Lurz, R.; Erdmann, V. a; Fürste, J. P. Biochem. Biophys. Res. Commun. 2002, 290 (5), 1583.

(146) Takahashi, T.; Tada, K.; Mihara, H. Mol. Biosyst. 2009, 5 (9), 986.

(147) Mathew, A.; Aravind, A.; Brahatheeswaran, D.; Fukuda, T.; Nagaoka, Y.; 
Hasumura, T.; Iwai, S.; Morimoto, H.; Yoshida, Y.; Maekawa, T.; Venugopal, K.; Kumar, D. S. Bionanoscience 2012, 2 (2), 83.

(148) Yang, F.; Lim, G. P.; Begum, A. N.; Ubeda, O. J.; Simmons, M. R.; Ambegaokar, S. S.; Chen, P. P.; Kayed, R.; Glabe, C. G.; Frautschy, S. A.; Cole, G. M. J. Biol. Chem. 2005, 280 (7), 5892.

(149) Bunka, D. H. J.; Mantle, B. J.; Morten, I. J.; Tennent, G. a; Radford, S. E.; Stockley, P. G. J. Biol. Chem. 2007, 282 (47), 34500.

(150) Rentmeister, A.; Bill, A.; Wahle, T.; Walter, J.; Famulok, M. RNA 2006, 12 (9), 1650 .

(151) Liang, H.; Shi, Y.; Kou, Z.; Peng, Y.; Chen, W.; Li, X.; Li, S.; Wang, Y.; Wang, F.; Zhang, X. PLoS One 2015, 10 (10), 1.

(152) Ozawa, D.; Nakamura, T.; Koike, M.; Hirano, K.; Miki, Y.; Beppu, M. Biol. Pharm. Bull. 2013, 36 (10), 1587.

(153) Ireson, C. R.; Kelland, L. R. Mol. Cancer Ther. 2006, 5 (12), 2957.

(154) Blennow, K.; Dubois, B.; Fagan, A. M.; Lewczuk, P.; de Leon, M. J.; Hampel, H. Alzheimers. Dement. 2015, 11 (1), 58.

(155) Ritter, A.; Cummings, J. Front. Neurol. 2015, 6 (Aug), 186.

(156) Baird, A. L.; Westwood, S.; Lovestone, S. Front. Neurol. 2015, 6 (NOV), 236.

(157) Kim, S.; Lee, H. J. Anal. Chem. 2015, 87 (14), 7235.

(158) Kiddle, S. J.; Sattlecker, M.; Proitsi, P.; Simmons, A.; Westman, E.; Bazenet, C.; Nelson, S. K.; Williams, S.; Hodges, A.; Johnston, C.; Soininen, H.; Kłoszewska, I.; Mecocci, P.; Tsolaki, M.; Vellas, B.; Newhouse, S.; Lovestone, S.; Dobson, R. J. B. J. Alzheimers. Dis. 2014, 38 (3), 515.

(159) Woodbury, N.; Greving, M. Banques d'aptameres non aleatoires et leurs procedes de creation. WO 2007109067 A2, 2007.

(160) Brody, E. N.; Gold, L.; Lawn, R. M.; Walker, J. J.; Zichi, D. Expert Rev. Mol. Diagn. 2010, 10 (8), 1013.

(161) Gold, L.; Ayers, D.; Bertino, J.; Bock, C.; Bock, A.; Brody, E. N.; Carter, J.; Dalby, A. B.; Eaton, B. E.; Fitzwater, T.; Flather, D.; Forbes, A.; Foreman, T.; Fowler, C.; Gawande, B.; Goss, M.; Gunn, M.; Gupta, S.; Halladay, D.; Heil, J.; Heilig, J.; Hicke, B.; Husar, G.; Janjic, N.; Jarvis, T.; Jennings, S.; Katilius, E.; Keeney, T. R.; Kim, N.; Koch, T. H.; Kraemer, S.; Kroiss, L.; Le, N.; Levine, D.; Lindsey, W.; Lollo, B.; Mayfield, W.; Mehan, M.; Mehler, R.; Nelson, S. K.; Nelson, M.; Nieuwlandt, D.; Nikrad, M.; Ochsner, U.; Ostroff, R. M.; Otis, M.; Parker, T.; Pietrasiewicz, S.; Resnicow, D. I.; Rohloff, J.; Sanders, G.; Sattin, S.; Schneider, D.; Singer, B.; Stanton, M.; Sterkel, A.; Stewart, A.; Stratford, S.; Vaught, J. D.; Vrkljan, M.; Walker, J. J.; Watrobka, M.; Waugh, S.; Weiss, A.; Wilcox, S. K.; Wolfson, A.; Wolk, S. K.; Zhang, C.; Zichi, D. PLoS One 2010, 5 (12), e15004.

(162) Lollo, B.; Steele, F.; Gold, L. Proteomics 2014, 14 (6), 638. 
(163) Nelson, S. K.; Sterling, D.; Cudkowicz, M. E.; Sattlecker, M.; Kiddle, S.; Dobson, R.; Baird, G.; Saccomano, N.; Williams, S. Discovery of neurological biomarker signatures using SOMAscan ${ }^{T M}$ Multiplex Proteomic Technology; 2011.

(164) Gupta, S.; Hirota, M.; Waugh, S. M.; Murakami, I.; Suzuki, T.; Muraguchi, M.; Shibamori, M.; Ishikawa, Y.; Jarvis, T. C.; Carter, J. D.; Zhang, C.; Gawande, B.; Vrkljan, M.; Janjic, N.; Schneider, D. J. J. Biol. Chem. 2014, 289 (12), 8706.

(165) Jarvis, T. C.; Davies, D. R.; Hisaminato, A.; Resnicow, D. I.; Gupta, S.; Waugh, S. M.; Nagabukuro, A.; Wadatsu, T.; Hishigaki, H.; Gawande, B.; Zhang, C.; Wolk, S. K.; Mayfield, W. S.; Nakaishi, Y.; Burgin, A. B.; Stewart, L. J.; Edwards, T. E.; Gelinas, A. D.; Schneider, D. J.; Janjic, N. Structure 2015, 23 (7), 1293.

(166) Baird, G. S.; Nelson, S. K.; Keeney, T. R.; Stewart, A.; Williams, S.; Kraemer, S.; Peskind, E. R.; Montine, T. J. Am. J. Pathol. 2012, 180 (2), 446.

(167) Farrar, C. T.; William, C. M.; Hudry, E.; Hashimoto, T.; Hyman, B. T. PLoS One 2014, 9 (2), e89901.

(168) Simao, T.; Ng, A.; Fatehi, D.; Corluka, S.; Abulrob, A.; Zourob, M. J. Biomed. Nanotechnol. 2015, 11 (12), 2264.

(169) Bisaglia, M.; Filograna, R.; Beltramini, M.; Bubacco, L. Ageing Res. Rev. 2014, 13, 107.

(170) Tsukakoshi, K.; Harada, R.; Sode, K.; Ikebukuro, K. Biotechnol. Lett. 2010, 32 (5), 643.

(171) Tsukakoshi, K.; Abe, K.; Sode, K.; Ikebukuro, K. Anal. Chem. 2012, 84 (13), 5542.

(172) Aguzzi, A.; Falsig, J. Nat. Neurosci. 2012, 15 (7), 936.

(173) Boese, A. S.; Majer, A.; Saba, R.; Booth, S. a. Expert Opin. Drug Discov. 2013, 8 (10), 1265.

(174) Gilch, S.; Schätzl, H. M. Cell. Mol. Life Sci. 2009, 66 (15), 2445.

(175) Praseetha P, K.; Thampy, A. S.; Venugopalan, P.; Chavali, M. S. Nano Biomed. Eng. 2012, 4 (2), 50.

(176) Yang, Y.; Yang, D.; Schluesener, H. J.; Zhang, Z. Biomol. Eng. 2007, 24 (6), 583.

(177) Ogasawara, D.; Hasegawa, H.; Kaneko, K.; Sode, K.; Ikebukuro, K. Prion 2007, 1 (4), 248.

(178) Murakami, K.; Nishikawa, F.; Noda, K.; Yokoyama, T.; Nishikawa, S. Prion 2008, 2 (June), 73.

(179) Mashima, T.; Matsugami, A.; Nishikawa, F.; Nishikawa, S.; Katahira, M. Nucleic Acids Res. 2009, 37 (18), 6249.

(180) Medic, Š.; Podbevšek, P.; Plavec, J. Croat. Chem. Acta 2014, 87 (4), 321.

(181) Mashima, T.; Nishikawa, F.; Kamatari, Y. O.; Fujiwara, H.; Saimura, M.; Nagata, T.; Kodaki, T.; Nishikawa, S.; Kuwata, K.; Katahira, M. Nucleic Acids Res. 2013, $41(2), 1355$. 
(182) Hayashi, T.; Oshima, H.; Mashima, T.; Nagata, T.; Katahira, M.; Kinoshita, M. Nucleic Acids Res. 2014, 42 (11), 6861.

(183) Katahira, M. In Long Noncoding RNAs: Structures and Functions; Kurokawa, R., Ed.; Springer Japan: Tokyo, 2015; pp 47-56.

(184) Bibby, D. F.; Gill, A. C.; Kirby, L.; Farquhar, C. F.; Bruce, M. E.; Garson, J. a. J. Virol. Methods 2008, 151 (1), 107.

(185) Rhie, A.; Kirby, L.; Sayer, N.; Wellesley, R.; Disterer, P.; Sylvester, I.; Gill, A.; Hope, J.; James, W.; Tahiri-Alaoui, A. J. Biol. Chem. 2003, 278 (41), 39697.

(186) Sayer, N. M.; Cubin, M.; Rhie, A.; Bullock, M.; Tahiri-Alaoui, A.; James, W. J. Biol. Chem. 2004, 279 (13), 13102.

(187) Takemura, K.; Wang, P.; Vorberg, I.; Surewicz, W.; Priola, S. A.; Kanthasamy, A.; Pottathil, R.; Chen, S. G.; Sreevatsan, S. Exp. Biol. Med. 2006, 231 (2), 204.

(188) Kouassi, G. K.; Wang, P.; Sreevatan, S.; Irudayaraj, J. Biotechnol. Prog. 2007, 23 (5), 1239.

(189) Xiao, S. J.; Hu, P. P.; Li, Y. F.; Huang, C. Z.; Huang, T.; Xiao, G. F. Talanta 2009, 79 (5), 1283.

(190) Hossain, M. T.; Shibata, T.; Kabashima, T.; Kai, M. Anal. Sci. 2010, 26 (6), 645.

(191) Xiao, S. J.; Hu, P. P.; Wu, X. D.; Zou, Y. L.; Chen, L. Q.; Peng, L.; Ling, J.; Zhen, S. J.; Zhan, L.; Li, Y. F.; Huang, C. Z. Anal. Chem. 2010, 82 (23), 9736.

(192) Xiao, S. J.; Hu, P. P.; Xiao, G. F.; Yi, W.; Liu, Y.; Huang, C. Z. J. Phys. Chem. B 2012, $116(32), 9565$.

(193) Zhan, L.; Peng, L.; Yu, Y.; Zhen, S. J.; Huang, C. Z. Analyst 2012, 137 (21), 4968.

(194) Hu, P. P.; Zheng, L. L.; Zhan, L.; Li, J. Y.; Zhen, S. J.; Liu, H.; Luo, L. F.; Xiao, G. F.; Huang, C. Z. Anal. Chim. Acta 2013, 787, 239.

(195) Miodek, a; Poturnayová, a; Snejdárková, M.; Hianik, T.; Korri-Youssoufi, H. Anal. Bioanal. Chem. 2013, 405 (8), 2505.

(196) Miodek, A.; Castillo, G.; Hianik, T.; Korri-Youssoufi, H. Anal. Chem. 2013, 85 (16), 7704.

(197) Xiao, S. J.; Hu, P. P.; Chen, L. Q.; Zhen, S. J.; Peng, L.; Li, Y. F.; Huang, C. Z. PLoS One 2013, 8 (2), e53935.

(198) Zhan, L.; Liang, L. J.; Zhen, S. J.; Li, C. M.; Huang, C. Z. Analyst 2013, 138 (3), 825.

(199) Zhen, S. J.; Zhuang, H. L.; Wang, J.; Huang, C. Z. Anal. Methods 2013, 5 (24), 6904.

(200) Zhou, Y. W.; Li, C. M.; Liu, Y.; Huang, C. Z. Analyst 2013, 138 (3), 873.

(201) Zhuang, H. L.; Zhen, S. J.; Wang, J.; Huang, C. Z. Anal. Methods 2013, 5 (1), 208.

(202) Miodek, a; Castillo, G.; Hianik, T.; Korri-Youssoufi, H. Biosens. Bioelectron. 2014, 56, 104. 
(203) Zhang, H.-J.; Lu, Y.-H.; Long, Y.-J.; Wang, Q.-L.; Huang, X.-X.; Zhu, R.; Wang, X.-L.; Liang, L.-P.; Teng, P.; Zheng, H.-Z. Anal. Methods 2014, 6 (9), 2982.

(204) Hu, P. P.; Liu, H.; Zhan, L.; Zheng, L. L.; Huang, C. Z. Talanta 2015, 139, 35.

(205) Yu, P.; Zhang, X.; Zhou, J.; Xiong, E.; Li, X.; Chen, J. Sci. Rep. 2015, 5 (October), 16015.

(206) Chen, L. Q.; Xiao, S. J.; Hu, P. P.; Peng, L.; Ma, J.; Luo, L. F.; Li, Y. F.; Huang, C. Z. Anal. Chem. 2012, 84 (7), 3099.

(207) Mashima, T.; Nishikawa, F.; Kamatari, Y. O.; Fujiwara, H.; Saimura, M.; Nagata, T.; Kodaki, T.; Nishikawa, S.; Kuwata, K.; Katahira, M. Nucleic Acids Res. 2013, $41(2), 1355$.

(208) Blank, M.; Weinschenk, T.; Priemer, M.; Schluesener, H. J. Biol. Chem. 2001, 276 (19), 16464.

(209) Cerchia, L.; Ducongé, F.; Pestourie, C.; Boulay, J.; Aissouni, Y.; Gombert, K.; Tavitian, B.; de Franciscis, V.; Libri, D. PLoS Biol. 2005, 3 (4), e123.

(210) Daniels, D. a; Chen, H.; Hicke, B. J.; Swiderek, K. M.; Gold, L. Proc. Natl. Acad. Sci. U. S. A. 2003, 100 (26), 15416.

(211) Hicke, B. J.; Marion, C.; Chang, Y. F.; Gould, T.; Lynott, C. K.; Parma, D.; Schmidt, P. G.; Warren, S. J. Biol. Chem. 2001, 276 (52), 48644.

(212) Delač, M.; Motaln, H.; Ulrich, H.; Lah, T. T. Cytom. Part A 2015, 87 (9), 806.

(213) de Franciscis, V.; Esposito, C. L.; Catuogno, S.; Cellai, L.; Cerchia, L. CNS Neurol. Disord. Drug Targets 2009, 8 (5), 393.

(214) Belter, A.; Gudanis, D.; Rolle, K.; Piwecka, M.; Gdaniec, Z.; Naskre??tBarciszewska, M. Z.; Barciszewski, J. PLoS One 2014, 9 (11), 1.

(215) Bayrac, A. T.; Sefah, K.; Parekh, P.; Bayrac, C.; Gulbakan, B.; Oktem, H. A.; Tan, W. ACS Chem. Neurosci. 2011, 2 (3), 175.

(216) Cerchia, L.; Esposito, C. L.; Jacobs, A. H.; Tavitian, B.; de Franciscis, V. PLoS One 2009, 4 (11), e7971.

(217) Kang, D.; Wang, J.; Zhang, W.; Song, Y.; Li, X.; Zou, Y.; Zhu, M.; Zhu, Z.; Chen, F.; Yang, C. J. PLoS One 2012, 7 (10), e42731.

(218) Wu, X.; Liang, H.; Tan, Y.; Yuan, C.; Li, S.; Li, X.; Li, G.; Shi, Y.; Zhang, X. PLoS One 2014, 9 (6), e90752.

(219) Kim, Y.; Wu, Q.; Hamerlik, P.; Hitomi, M.; Sloan, A. E.; Barnett, G. H.; Weil, R. J.; Leahy, P.; Hjelmeland, A. B.; Rich, J. N. Cancer Res. 2013, 73 (15), 4923.

(220) Da Rocha Gomes, S.; Miguel, J.; Azéma, L.; Eimer, S.; Ries, C.; Dausse, E.; Loiseau, H.; Allard, M.; Toulmé, J.-J. Bioconjug. Chem. 2012, 23 (11), 2192.

(221) Aptekar, S.; Arora, M.; Lawrence, C. L.; Lea, R. W.; Ashton, K.; Dawson, T.; Alder, J. E.; Shaw, L. PLoS One 2015, 10 (8), 1.

(222) Wu, Q.; Wu, L.; Wang, Y.; Zhu, Z.; Song, Y.; Tan, Y.; Wang, X.-F.; Li, J.; Kang, 
D.; Yang, C. J. Biosens. Bioelectron. 2016, 80, 1.

(223) Cheng, Y.; Morshed, R. a; Auffinger, B.; Tobias, A. L.; Lesniak, M. S. Adv. Drug Deliv. Rev. 2014, 66, 42.

(224) Hernández-Pedro, N. Y.; Rangel-López, E.; Magaña-Maldonado, R.; de la Cruz, V. P.; del Angel, A. S.; Pineda, B.; Sotelo, J. Biomed Res. Int. 2013, 2013, 351031.

(225) Li, J.; Zhu, J.-J. Analyst 2013, 138 (9), 2506.

(226) Li, Z.; Huang, P.; He, R.; Lin, J.; Yang, S.; Zhang, X.; Ren, Q.; Cui, D. Mater. Lett. 2010, 64 (3), 375.

(227) Kim, B.; Yang, J.; Hwang, M.; Choi, J.; Kim, H.-O.; Jang, E.; Lee, J. H.; Ryu, S.H.; Suh, J.-S.; Huh, Y.-M.; Haam, S. Nanoscale Res. Lett. 2013, 8 (1), 399.

(228) Nair, B. G.; Nagaoka, Y.; Morimoto, H.; Yoshida, Y.; Maekawa, T.; Kumar, D. S. Nanotechnology 2010, 21 (45), 455102.

(229) Liu, Y.; Kuan, C.-T.; Mi, J.; Zhang, X.; Clary, B. M.; Bigner, D. D.; Sullenger, B. A. Biol. Chem. 2009, 390 (2), 137.

(230) Camorani, S.; Crescenzi, E.; Colecchia, D.; Carpentieri, A.; Amoresano, A.; Fedele, M.; Chiariello, M.; Cerchia, L. Oncotarget 2015, 6 (35), 37570.

(231) Bechet, D.; Mordon, S. R.; Guillemin, F.; Barberi-Heyob, M. a. Cancer Treat. Rev. 2014, 40 (2), 229.

(232) Kruspe, S.; Meyer, C.; Hahn, U. Mol. Ther. Acids 2014, 3 (1), e143.

(233) Kudo, M.; Jono, H.; Shinriki, S.; Yano, S.; Nakamura, H.; Makino, K.; Hide, T.; Muta, D.; Ueda, M.; Ota, K.; Ando, Y.; Kuratsu, J.-I. J. Neurosurg. 2009, 111 (2), 219.

(234) Guo, J.; Gao, X.; Su, L.; Xia, H.; Gu, G.; Pang, Z.; Jiang, X.; Yao, L.; Chen, J.; Chen, H. Biomaterials 2011, 32 (31), 8010.

(235) Gao, H.; Qian, J.; Yang, Z.; Pang, Z.; Xi, Z.; Cao, S.; Wang, Y.; Pan, S.; Zhang, S.; Wang, W.; Jiang, X.; Zhang, Q. Biomaterials 2012, 33 (26), 6264.

(236) Chen, H.; Zheng, X.; Di, B.; Wang, D.; Zhang, Y.; Xia, H.; Mao, Q. J. Biotechnol. 2013, 168 (4), 362.

(237) Huse, J. T.; Holland, E. C. Nat. Rev. Cancer 2010, 10 (5), 319.

(238) Gao, H.; Qian, J.; Cao, S.; Yang, Z.; Pang, Z.; Pan, S.; Fan, L.; Xi, Z.; Jiang, X.; Zhang, Q. Biomaterials 2012, 33 (20), 5115.

(239) Li, J.; Feng, L.; Fan, L.; Zha, Y.; Guo, L.; Zhang, Q.; Chen, J.; Pang, Z.; Wang, Y.; Jiang, X.; Yang, V. C.; Wen, L. Biomaterials 2011, 32 (21), 4943.

(240) Porciani, D.; Signore, G.; Marchetti, L.; Mereghetti, P.; Nifosì, R.; Beltram, F. Mol. Ther. Nucleic Acids 2014, 3, e144.

(241) Hu, J.; Al-Waili, D.; Hassan, A.; Fan, G.-C.; Xin, M.; Hao, J. Neuroscience 2016, $329,30$.

(242) Mu, C.; Dave, N.; Hu, J.; Desai, P.; Pauletti, G.; Bai, S.; Hao, J. J. Microencapsul. 
2013, 30 (7), 701.

(243) Wilner, S. E.; Wengerter, B.; Maier, K.; de Lourdes Borba Magalhães, M.; Del Amo, D. S.; Pai, S.; Opazo, F.; Rizzoli, S. O.; Yan, A.; Levy, M. Mol. Ther. Nucleic Acids 2012, 1 (November 2011), e21.

(244) de Castro, M. A. G.; Rammner, B.; Opazo, F. Methods Mol. Biol. 2016, 1380, 197.

(245) Huang, Z.; Pei, W.; Jayaseelan, S.; Shi, H.; Niu, L. Biochemistry 2007, 46 (44), 12648.

(246) Du, M.; Ulrich, H.; Zhao, X.; Aronowski, J.; Jayaraman, V. Neuropharmacology 2007, $53(2), 242$.

(247) Liu, Y.; Sun, Q.-A.; Chen, Q.; Lee, T. H.; Huang, Y.; Wetsel, W. C.; Michelotti, G. a; Sullenger, B. a; Zhang, X. J. Neurochem. 2009, 108 (1), 147.

(248) Ferretti, V.; Perri, V.; Cristofoli, A.; Vetere, G.; Fragapane, P.; Oliverio, A.; Teule, M. A.; Mele, A. Brain Struct. Funct. 2014, 220 (5), 2653.

(249) Lee, H.-K.; Takamiya, K.; He, K.; Song, L.; Huganir, R. L. J. Neurophysiol. 2010, $103(1), 479$.

(250) Lee, G.; MacLean, D. M.; Ulrich, H.; Zhao, X.; Aronowski, J.; Jayaraman, V. ACS Chem. Neurosci. 2014, 5 (7), 559.

(251) Ulrich, H.; Ippolito, J. E.; Pagán, O. R.; Eterović, V. a; Hann, R. M.; Shi, H.; Lis, J. T.; Eldefrawi, M. E.; Hess, G. P. Proc. Natl. Acad. Sci. U. S. A. 1998, 95 (24), 14051.

(252) Chatzidaki, A.; Millar, N. S. Biochem. Pharmacol. 2015, 97 (4), 408.

(253) Hess, G. P.; Ulrich, H.; Breitinger, H.-G.; Niu, L.; Gameiro, A. M.; Grewer, C.; Srivastava, S.; Ippolito, J. E.; Lee, S. M.; Jayaraman, V.; Coombs, S. E. Proc. Natl. Acad. Sci. 2000, 97 (25), 13895.

(254) Sivaprakasam, K.; Pagán, O. R.; Hess, G. P. J. Membr. Biol. 2010, 233 (1-3), 1.

(255) Cui, Y.; Ulrich, H.; Hess, G. P. J. Membr. Biol. 2004, 202 (3), 137.

(256) Krivoshein, A. V; Hess, G. P. Biochemistry 2004, 43 (2), 481.

(257) Cui, Y.; Rajasethupathy, P.; Hess, G. P. Biochemistry 2004, 43 (51), 16442.

(258) Huang, Y. Z.; Hernandez, F. J.; Gu, B.; Stockdale, K. R.; Nanapaneni, K.; Scheetz, T. E.; Behlke, M. a; Peek, A. S.; Bair, T.; Giangrande, P. H.; McNamara, J. O. Mol. Pharmacol. 2012, 82 (4), 623.

(259) Lennarz, S.; Alich, T. C.; Kelly, T.; Blind, M.; Beck, H.; Mayer, G. Angew. Chemie - Int. Ed. 2015, 54 (18), 5369.

(260) Ito-Ishida, A.; Kakegawa, W.; Yuzaki, M. Eur. J. Neurosci. 2006, 24 (6), 1617.

(261) Cruz-Toledo, J.; McKeague, M.; Zhang, X.; Giamberardino, A.; McConnell, E.; Francis, T.; DeRosa, M. C.; Dumontier, M. Database (Oxford). 2012, 2012, bas006.

(262) Hermann, T.; Patel, D. J. Science 2000, 287 (5454), 820. 
(263) Rozenblum, G. T.; Lopez, V. G.; Vitullo, A. D.; Radrizzani, M. Expert Opin. Drug Discov. 2016, 11 (2), 127.

(264) Berezovski, M.; Musheev, M.; Drabovich, A.; Krylov, S. N. J. Am. Chem. Soc. 2006, 128 (5), 1410.

(265) Berezovski, M.; Berezovski, M.; Musheev, M.; Musheev, M.; Drabovich, A.; Drabovich, A.; Jitkova, J.; Jitkova, J.; Krylov, S.; Krylov, S. Nat Protoc 2006, 1 (3), 1359.

(266) Ashley, J.; Ji, K.; Li, S. F. Y. Electrophoresis 2012, 33 (17), 2783.

(267) Cho, E. J.; Lee, J.-W.; Ellington, A. D. Annu. Rev. Anal. Chem. (Palo Alto. Calif). 2009, 2, 241.

(268) Gold, L.; Janjic, N.; Jarvis, T.; Schneider, D.; Walker, J. J.; Wilcox, S. K.; Zichi, D. Cold Spring Harb. Perspect. Biol. 2012, 4 (3), a003582.

(269) Hall, B.; Micheletti, J. M.; Satya, P.; Ogle, K.; Pollard, J.; Ellington, A. D. In Current Protocols in Molecular Biology; John Wiley \& Sons, Inc.: Hoboken, NJ, USA, 2009.

(270) Jhaveri, S.; Ellington, A. In Current Protocols in Nucleic Acid Chemistry; John Wiley \& Sons, Inc.: Hoboken, NJ, USA, 2002; pp 9.5.1-9.5.14.

(271) Sefah, K.; Shangguan, D.; Xiong, X.; O’Donoghue, M. B.; Tan, W. Nat. Protoc. 2010, 5 (6), 1169.

(272) Zhu, G.; Ye, M.; Donovan, M. J.; Song, E.; Zhao, Z.; Tan, W. Chem. Commun. 2012, 48 (85), 10472.

(273) Ciesiolka, J.; Illangasekare, M.; Majerfeld, I.; Nickles, T.; Welch, M.; Yarus, M.; Zinnen, S. In Combinatorial Chemistry; Enzymology, B. T.-M. in, Ed.; Academic Press, 1996; Vol. Volume 267, pp 315-335.

(274) Lee, J. F.; Hesselberth, J. R.; Meyers, L. A.; Ellington, A. D. Nucleic Acids Res. 2004, 32 (Database issue), D95.

(275) Ponomarenko, J. V; Orlova, G. V; Frolov, A. S.; Gelfand, M. S.; Ponomarenko, M. P. Nucleic Acids Res. 2002, 30 (1), 195.

(276) McKeague, M.; De Girolamo, A.; Valenzano, S.; Pascale, M.; Ruscito, A.; Velu, R.; Frost, N. R.; Hill, K.; Smith, M.; McConnell, E. M.; DeRosa, M. C. Anal. Chem. 2015, 87 (17), 8608.

(277) Jagannathan, V.; Roulet, E.; Delorenzi, M.; Bucher, P. Nucleic Acids Res. 2006, 34 (Database issue), D90.

(278) Thodima, V.; Pirooznia, M.; Deng, Y. BMC Bioinformatics 2006, 7 Suppl 2, S6.

(279) Cruz-Toledo, J.; McKeague, M.; Zhang, X.; Giamberardino, A.; McConnell, E.; Francis, T.; DeRosa, M. C.; Dumontier, M. Database (Oxford). 2012, 2012, bas006.

(280) Mannironi, C.; Di Nardo, A.; Fruscoloni, P.; Tocchini-Valentini, G. P. Biochemistry 1997, 36 (32), 9726. 
(281) Pan, W.; Clawson, G. a. Molecules 2009, 14 (4), 1353.

(282) Sabeti, P. C.; Unrau, P. J.; Bartel, D. P. Chem. Biol. 1997, 4 (10), 767.

(283) Silverman, S. K. In Functional Nucleic Acids for Analytical Applications; Springer New York: New York, NY, 2009; pp 47-108.

(284) Walsh, R.; DeRosa, M. C. Biochem. Biophys. Res. Commun. 2009, 388 (4), 732.

(285) Travers, A.; Muskhelishvili, G. FEBS J. 2015, 282 (12), 2279.

(286) Bercy, M.; Bockelmann, U. Nucleic Acids Res. 2015, 43 (20), 9928.

(287) Antao, V. P.; Tinoco, I. Nucleic Acids Res. 1992, 20 (4), 819.

(288) Ellington, A.; Szostak, J. Nature 1992, 355, 850.

(289) Lauhon, C. T.; Szostak, J. W. J. Am. Chem. Soc. 1995, 117 (4), 1246.

(290) Dieckmann, T.; Butcher, S. E.; Sassanfar, M.; Szostak, J. W.; Feigon, J. J. Mol. Biol. 1997, 273 (2), 467.

(291) McKeague, M.; Derosa, M. C. J. Nucleic Acids 2012, 2012.

(292) Keefe, A. D.; Cload, S. T. Curr. Opin. Chem. Biol. 2008, 12 (4), 448.

(293) Tolle, F.; Brändle, G. M.; Matzner, D.; Mayer, G. Angew. Chem. Int. Ed. Engl. 2015, 54 (37), 10971.

(294) Lozupone, C.; Changayil, S.; Majerfeld, I.; Yarus, M. RNA 2003, 9 (11), 1315.

(295) Li, Y.; Geyer, C. R.; Sen, D. Biochemistry 1996, 35 (21), 6911.

(296) Velez, T. E.; Singh, J.; Xiao, Y.; Allen, E. C.; Wong, O. Y.; Chandra, M.; Kwon, S. C.; Silverman, S. K. ACS Comb. Sci. 2012, 14 (12), 680.

(297) Legiewicz, M.; Lozupone, C.; Knight, R.; Yarus, M. RNA 2005, 11 (11), 1701.

(298) Winkler, W. C.; Breaker, R. R. ChemBioChem 2003, 4 (10), 1024.

(299) Kulbachinskiy, A. V. Biochemistry. (Mosc). 2007, 72 (13), 1505.

(300) Luo, X.; McKeague, M.; Pitre, S.; Dumontier, M.; Green, J.; Golshani, A.; Derosa, M. C.; Dehne, F. RNA 2010, 16 (11), 2252.

(301) Hamm, J. Nucleic Acids Res. 1996, 24 (12), 2220.

(302) Hamm, J.; Huber, J.; Lührmann, R. Proc. Natl. Acad. Sci. U. S. A. 1997, 94 (24), 12839.

(303) Tucker, W. O.; Tanner, K. T. S. and J. A. Current Pharmaceutical Design. 2012, pp 2014-2026.

(304) Gatto, B.; Palumbo, M.; Sissi, C. Curr. Med. Chem. 2009, 16 (10), 1248.

(305) Avino, A.; Fabrega, C.; Tintore, M.; Eritja, R. Curr. Pharm. Des. 2012, 18, 2036.

(306) Carothers, J. M.; Goler, J. A.; Kapoor, Y.; Lara, L.; Keasling, J. D. Nucleic Acids Res. 2010, 38 (8), 2736.

(307) Hianik, T.; Ostatná, V.; Sonlajtnerova, M.; Grman, I. Bioelectrochemistry 2007, 70 (1), 127. 
(308) Deng, Q.; German, I.; Buchanan, D.; Kennedy, R. T. Anal. Chem. 2001, 73 (22), 5415.

(309) Neves, M. A. D.; Reinstein, O.; Saad, M.; Johnson, P. E. Biophys. Chem. 2010, $153(1), 9$.

(310) Catherine, A. T.; Shishido, S. N.; Robbins-Welty, G. A.; Diegelman-Parente, A. FEBS Open Bio 2014, 4, 788.

(311) Wu, J.; Zhu, Y.; Xue, F.; Mei, Z.; Yao, L.; Wang, X.; Zheng, L.; Liu, J.; Liu, G.; Peng, C.; Chen, W. Mikrochim. Acta 2014, 181 (5-6), 479.

(312) Alam, K. K.; Chang, J. L.; Burke, D. H. Mol. Ther. Acids 2015, 4 (August 2014), e230.

(313) Hoinka, J.; Berezhnoy, A.; Dao, P.; Sauna, Z. E.; Gilboa, E.; Przytycka, T. M. Nucleic Acids Res. 2015, 43 (12), 5699.

(314) Carothers, J. M.; Oestreich, S. C.; Szostak, J. W. J. Am. Chem. Soc. 2006, 128 (24), 7929.

(315) Stoltenburg, R.; Reinemann, C.; Strehlitz, B. Anal. Bioanal. Chem. 2005, 383 (1), 83.

(316) Win, M. N.; Klein, J. S.; Smolke, C. D. Nucleic Acids Res. 2006, 34 (19), 5670.

(317) Spiga, F. M.; Maietta, P.; Guiducci, C. ACS Comb. Sci. 2015, 17 (5), 326.

(318) González-Fernández, E.; De-los-Santos-Álvarez, N.; Miranda-Ordieres, A. J.; Lobo-Castañón, M. J. Talanta 2012, 99, 767.

(319) McConnell, E. M.; Bolzon, R.; Mezin, P.; Frahm, G.; Johnston, M.; DeRosa, M. C. Bioconjug. Chem. 2016, 27 (6), 1493.

(320) Saha, S.; Krishnan, Y. In DNA Conjugates and Sensors; The Royal Society of Chemistry, 2012; pp 166-189.

(321) Srivastava, J.; Barber, D. L.; Jacobson, M. P. Physiology (Bethesda). 2007, 22, 30.

(322) Magnotta, V. A.; Heo, H.-Y. Y.; Dlouhy, B. J.; Dahdaleh, N. S.; Follmer, R. L.; Thedens, D. R.; Welsh, M. J.; Wemmie, J. A. Proc. Natl. Acad. Sci. U. S. A. 2012, $109(21), 8270$.

(323) Orlowski, P.; Chappell, M.; Park, C. S.; Grau, V.; Payne, S. Interface Focus 2011, $1(3), 408$.

(324) Chesler, M. Physiol. Rev. 2003, 83 (4), 1183.

(325) Jabre, A.; Bao, Y.; Spatz, E. L. Surg. Neurol. 2000, 54 (1), 55.

(326) Rossi, D. J.; Brady, J. D.; Mohr, C. Nat. Neurosci. 2007, 10 (11), 1377.

(327) LaManna, J. C.; McCracken, K. A. Anal Biochem 1984, 142 (1), 117.

(328) Hoffman, T. L.; Lamanna, J. C.; Pundik, S.; Selman, W. R.; Whittingham, T. S.; Ratcheson, R. A.; Lust, W. D. J Neurosurg. 1994, pp 567-573.

(329) Zhou, J.; Zhang, L.; Tian, Y. Anal. Chem. 2016, 88 (4), 2113. 
(330) Preece, P.; Cairns, N. J. Mol. Brain Res. 2003, 118 (1-2), 60.

(331) Urcelay, E.; Ibarreta, D.; Parrilla, R.; Ayuso, M. S.; Martín-Requero, a. Neurobiol. Dis. 2001, 8 (2), 289.

(332) Nedergaard, M. Acta Neurol. Scand. 1988, 77 (2), 81.

(333) Neri, D.; Supuran, C. T. Nat. Rev. Drug Discov. 2011, 10 (10), 767.

(334) Copen, W. A. Curr. Treat. Options Cardiovasc. Med. 2015, 17 (3), 368.

(335) Nathaniel, T. I.; Williams-Hernandez, A.; Hunter, L. A.; Liddy, C.; Peffley, D. M.; Umesiri, F. E.; Imeh-Nathaniel, A. Brain Res. Bull. 2015, 114, 1.

(336) Zhao, F.; Zhang, L.; Zhu, A.; Shi, G.; Tian, Y. Chem. Commun. 2016, 52, 3717.

(337) Harston, G. W. J.; Tee, Y. K.; Blockley, N.; Okell, T. W.; Thandeswaran, S.; Shaya, G.; Sheerin, F.; Cellerini, M.; Payne, S.; Jezzard, P.; Chappell, M.; Kennedy, J. Brain 2015, 138 (Pt 1), 36.

(338) Angell, C.; Xie, S.; Zhang, L.; Chen, Y. Small 2016, 12 (9), 1117.

(339) Bath, J.; Turberfield, A. J. Nat. Nanotechnol. 2007, 2 (5), 275.

(340) Lu, C.-H.; Willner, B.; Willner, I. ACS Nano 2013, 7 (10), 8320.

(341) Wang, F.; Liu, X.; Willner, I. Angew. Chem. Int. Ed. Engl. 2015, 54 (4), 1098.

(342) Tang, Y.; Ge, B.; Sen, D.; Yu, H.-Z. Chem. Soc. Rev. 2014, 43 (2), 518.

(343) Giannetti, A.; Tombelli, S.; Baldini, F. Anal. Bioanal. Chem. 2013, 405 (19), 6181.

(344) Vallée-Bélisle, A.; Plaxco, K. W. Curr. Opin. Struct. Biol. 2010, 20 (4), 518.

(345) Del Grosso, E.; Dallaire, A.-M.; Vallée-Bélisle, A.; Ricci, F. Nano Lett. 2015, 15 (12), 8407.

(346) Jones, M. R.; Seeman, N. C.; Mirkin, C. A. Science 2015, 347 (6224), 1260901.

(347) Idili, A.; Vallée-Bélisle, A.; Ricci, F. J. Am. Chem. Soc. 2014, 136 (16), 5836.

(348) Jissy, a K.; Datta, A. J. Phys. Chem. B 2010, 114 (46), 15311.

(349) Dong, Y.; Yang, Z.; Liu, D. Acc. Chem. Res. 2014, 47 (6), 1853.

(350) Porchetta, A.; Idili, A.; Vallée-Bélisle, A.; Ricci, F. Nano Lett. 2015, 15 (7), 4467.

(351) Day, H. A.; Pavlou, P.; Waller, Z. A. E. Bioorganic Med. Chem. 2014, 22 (16), 4407.

(352) Snoussi, K.; Nonin-Lecomte, S.; Leroy, J.-L. J. Mol. Biol. 2001, 309 (1), 139.

(353) Xu, Y.; Hirao, Y.; Nishimura, Y.; Sugiyama, H. Bioorganic Med. Chem. 2007, 15 (3), 1275.

(354) Shastri, A.; McGregor, L. M.; Liu, Y.; Harris, V.; Nan, H.; Mujica, M.; Vasquez, Y.; Bhattacharya, A.; Ma, Y.; Aizenberg, M.; Kuksenok, O.; Balazs, A. C.; Aizenberg, J.; He, X. Nat. Chem. 2015, 7 (5), 447.

(355) Dittmer, W. U.; Reuter, A.; Simmel, F. C. Angew. Chemie Int. Ed. 2004, 43 (27), 3550. 
(356) Lee, J. A.; DeRosa, M. C. Chem. Commun. (Camb). 2010, 46 (3), 418.

(357) Digel, I.; Maggakis-Kelemen, C.; Zerlin, K. F.; Linder, P.; Kasischke, N.; Kayser, P.; Porst, D.; Temiz Artmann, a; Artmann, G. M. Biophys. J. 2006, 91 (8), 3014.

(358) Bock, L. C.; Griffin, L. C.; Latham, J. A.; Vermaas, E. H.; Toole, J. J. Nature 1992, 355 (6360), 564.

(359) Nagatoishi, S.; Tanaka, Y.; Tsumoto, K. Biochem. Biophys. Res. Commun. 2007, $352(3), 812$.

(360) Macaya, R. F.; Schultze, P.; Smith, F. W.; Roe, J. a; Feigon, J. Proc. Natl. Acad. Sci. U. S. A. 1993, 90 (8), 3745.

(361) Mao, X. A.; Gmeiner, W. H. Biophys. Chem. 2005, 113, 155.

(362) Russo Krauss, I.; Merlino, A.; Randazzo, A.; Novellino, E.; Mazzarella, L.; Sica, F. Nucleic Acids Res. 2012, 40 (16), 8119.

(363) Zhao, D.; Dong, X.; Jiang, N.; Zhang, D.; Liu, C. Nucleic Acids Res. 2014, 42 (18), 11612.

(364) Nagatoishi, S.; Isono, N.; Tsumoto, K.; Sugimoto, N. Biochimie 2011, 93 (8), 1231.

(365) Małgowska, M.; Gudanis, D.; Teubert, A.; Dominiak, G.; Gdaniec, Z. BioTechnologia 2012, 93 (4), 381.

(366) Kapinos, L. E.; Operschall, B. P.; Larsen, E.; Sigel, H. Chem. - A Eur. J. 2011, 17 (29), 8156.

(367) Schultze, P.; Macaya, R. F.; Feigon, J. J. Mol. Biol. 1994, 235 (5), 1532.

(368) Kankia, B. I.; Marky, L. A. J. Am. Chem. Soc. 2001, 123 (44), 10799.

(369) Smirnov, I.; Shafer, R. H. J. Mol. Biol. 2000, 296 (1), 1.

(370) Smirnov, I. V.; Kotch, F. W.; Pickering, I. J.; Davis, J. T.; Shafer, R. H. Biochemistry 2002, 41 (40), 12133.

(371) Kumar, N.; Maiti, S. Biochem. Biophys. Res. Commun. 2004, 319 (3), 759.

(372) Vorlíčková, M.; Kejnovská, I.; Bednářová, K.; Renčiuk, D.; Kypr, J. Chirality 2012, 24 (9), 691.

(373) Cameselle, J.; Meireles Ribeiro, J.; Sillero, A. Biochem. Educ. 1986, 14 (3), 131.

(374) Ozalp, V. C.; Eyidogan, F.; Oktem, H. A. Pharmaceuticals 2011, 4 (12), 1137.

(375) Holahan, M. R.; Madularu, D.; McConnell, E. M.; Walsh, R.; DeRosa, M. C. PLoS One 2011, 6 (7), e22239.

(376) Lodge, D. J.; Grace, A. a. Trends Pharmacol. Sci. 2011, 32 (9), 507.

(377) Howes, O.; Mccutcheon, R.; Stone, J. J. Psychopharmacol. 2015, 29 (2), 97.

(378) Neill, J. C.; Barnes, S.; Cook, S.; Grayson, B.; Idris, N. F.; McLean, S. L.;

Snigdha, S.; Rajagopal, L.; Harte, M. K. Pharmacol. Ther. 2010, 128 (3), 419.

(379) Langlois, K. a; Samokhvalov, a V; Rehm, J.; Spence, S. T.; Connor Gorber, S. K. 
Health state descriptions for Canadians: Mental illnesses.; 2011.

(380) Howes, O. D.; Vergunst, F.; Gee, S.; McGuire, P.; Kapur, S.; Taylor, D. Br. J. Psychiatry 2012, 201 (6), 481.

(381) Emsley, R.; Kilian, S.; Phahladira, L. Curr. Opin. Psychiatry 2016, 29 (3), 224.

(382) Howes, O. D.; Bose, S. K.; Turkheimer, F.; Valli, I.; Egerton, A.; Valmaggia, L. R.; Murray, R. M.; McGuire, P. Am. J. Psychiatry 2011, 168 (11), 1311.

(383) Russo, S.; Nestler, E. Nat. Rev. Neurosci. 2013, 625 (September), 609.

(384) Bissonette, G. B.; Roesch, M. R. Genes, Brain Behav. 2015, n/a.

(385) Grace, A. a. Neuropharmacology 2012, 62 (3), 1342.

(386) Meltzer, H. Y.; Horiguchi, M.; Massey, B. W. Psychopharmacology (Berl). 2011, $213(2-3), 289$.

(387) Cohen, S. M.; Tsien, R. W.; Goff, D. C.; Halassa, M. M. Schizophr. Res. 2015, 167 (1-3), 98.

(388) Koek, W.; Woods, J. H.; Winger, G. D. J. Pharmacol. Exp. Ther. 1988, 245 (3), 969.

(389) Liljequist, S.; Ossowska, K.; Grabowska-Andén, M.; Andén, N. E. Eur. J. Pharmacol. 1991, 195 (1), 55.

(390) Tiedtke, P. I.; Bischoff, C.; Schmidt, W. J. J. Neural Transm. - Gen. Sect. 1990, 81 (3), 173.

(391) Bubser, M.; Keseberg, U.; Notz, P. K.; Schmidt, W. J. Eur. J. Pharmacol. 1992, $229(1), 75$.

(392) Löscher, W.; Hönack, D. Eur. J. Pharmacol. 1993, 242 (1), 99.

(393) Yan, Q. S.; Reith, M. E. a; Jobe, P. C.; Dailey, J. W. Brain Res. 1997, 765 (1), 149.

(394) van der Meulen, J. a J.; Bilbija, L.; Joosten, R. N. J. M. a; de Bruin, J. P. C.;

Feenstra, M. G. P. Neuroreport 2003, 14 (17), 2225.

(395) Holahan, M. R.; Clarke, M. J.; Hines, D. D. Psychopharmacology (Berl). 2010, $212(3), 393$.

(396) Walsh, R.; DeRosa, M. C. Biochem. Biophys. Res. Commun. 2009, 388 (4), 732.

(397) O’Neill, M. F.; Shaw, G. Psychopharmacology (Berl). 1999, 145 (3), 237.

(398) Pitts, R. C.; Buda, D. R.; Keith, J. R.; Cerutti, D. T.; Galizio, M. Psychopharmacology (Berl). 2006, 189 (2), 135.

(399) Wozniak, D. F.; Olney, J. W.; Kettinger, L.; Price, M.; Miller, J. P. Psychopharmacology (Berl). 1990, 101 (1), 47.

(400) Port, R. L.; Seybold, K. S. Physiol. Behav. 1998, 64 (3), 391.

(401) Holahan, M. R.; Honegger, K. S.; Tabatadze, N.; Routtenberg, A. Learn. Mem. 2007, 14 (6), 407.

(402) Ali, S. F.; Newport, G. D.; Bracha, H. S. Neurotoxicol. Teratol. 1994, 16 (4), 335. 
(403) Padilla-de La Torre, M.; Franco-Pérez, J.; Santamaría, A.; Galvan, S.; González, E.; Paz, C. Ann. N. Y. Acad. Sci. 2008, 1139, 259.

(404) Zhang, J.; Chiodo, L. A.; Freeman, A. S. Brain Res. 1992, 590 (1-2), 153.

(405) Murase, S.; Mathé, J. M.; Grenhoff, J.; Svensson, T. H. J. Neural Transm. Gen. Sect. 1993, 91 (1), 13.

(406) Carlsson, A.; Waters, N.; Holm-Waters, S.; Tedroff, J.; Nilsson, M.; Carlsson, M. L. Annu. Rev. Pharmacol. Toxicol. 2001, 41 (1), 237.

(407) Carlsson, A.; Waters, N.; Carlsson, M. L. Biol. Psychiatry 1999, 46 (10), 1388.

(408) Covasa, M.; Hung, C.; Ritter, R. C.; Burns, G. A. 2004, 1462.

(409) Burns, G. A.; Ritter, R. C. Pharmacol. Biochem. Behav. 1997, 56 (1), 145.

(410) Yonghui, L.; Xigeng, Z.; Yunjing, B.; Xiaoyan, Y.; Nan, S. J. Psychopharmacol. 2006, $20(1), 40$.

(411) Ahn, S.; Phillips, A. G. Psychopharmacology (Berl). 2007, 191 (3), 641.

(412) Hironaka, N.; Ikeda, K.; Sora, I.; Uhl, G. R.; Niki, H. Ann. N. Y. Acad. Sci. 2004, $1025,140$.

(413) Walf, A. a; Frye, C. a. Nat. Protoc. 2007, 2 (2), 322.

(414) Danysz, W.; Essmann, U.; Bresink, I.; Wilke, R. Pharmacol. Biochem. Behav. 1994, 48 (1), 111.

(415) Löscher, W.; Hönack, D. Eur. J. Pharmacol. 1992, 215 (2-3), 199.

(416) Murschall, A.; Hauber, W. Psychopharmacology (Berl). 2005, 182 (2), 290.

(417) Matsugami, A.; Kobayashi, S.; Ouhashi, K.; Uesugi, S.; Yamamoto, R.; Taira, K.; Nishikawa, S.; Kumar, P. K. R.; Katahira, M. Structure 2003, 11 (5), 533.

(418) Jing, M.; Bowser, M. T. Anal. Chim. Acta 2011, 686 (1-2), 9.

(419) Willuhn, I.; Wanat, M. J.; Clark, J. J.; Phillips, P. E. M. In Brain Imaging in Behavioral Neuroscience; 2010; pp 29-71.

(420) Bassareo, V.; Di Chiara, G. Eur. J. Neurosci. 1999, 11 (12), 4389.

(421) Liew, F. F.; Hasegawa, T.; Fukuda, M.; Nakata, E.; Morii, T. Bioorganic Med. Chem. 2011, 19 (15), 4473.

(422) Dunkley, P. R.; Bobrovskaya, L.; Graham, M. E.; Von Nagy-Felsobuki, E. I.; Dickson, P. W. J. Neurochem. 2004, 91 (5), 1025.

(423) Fitzpatrick, P. F. Annu. Rev. Biochem. 1999, 68 (2), 355.

(424) Goldstein, M. Ann. N. Y. Acad. Sci. 1984, 430, 1.

(425) Bouchard, P. R.; Hutabarat, R. M.; Thompson, K. M. Annu. Rev. Pharmacol. Toxicol. 2010, 50, 237.

(426) Famulok, M.; Mayer, G. ChemBioChem 2005, 6 (1), 19.

(427) Deane, R.; Zheng, W.; Zlokovic, B. V. J Neurochem 2004, 88 (4), 813. 
(428) Dufès, C.; Al Robaian, M.; Somani, S. Ther. Deliv. 2013, 4 (5), 629.

(429) Pardridge, W. M. Curr. Opin. Pharmacol. 2006, 6 (5), 494.

(430) Clark, A. J.; Davis, M. E. Proc. Natl. Acad. Sci. U. S. A. 2015, 112 (40), 12486.

(431) Sehlin, D.; Fang, X. T.; Cato, L.; Antoni, G.; Lannfelt, L.; Syvänen, S. Nat. Commun. 2016, 7, 10759.

(432) Cabezón, I.; Manich, G.; Martín-Venegas, R.; Camins, A.; Pelegrí, C.; Vilaplana, J. Mol. Pharm. 2015, 12 (11), 4137.

(433) Ko, Y. T. J. Drug Target. 2013, 21 (9), 866.

(434) Jiang, F.; Liu, B.; Lu, J.; Li, F.; Li, D.; Liang, C.; Dang, L.; Liu, J.; He, B.; Badshah, S. A.; Lu, C.; He, X.; Guo, B.; Zhang, X. B.; Tan, W.; Lu, A.; Zhang, G. Int. J. Mol. Sci. 2015, 16 (10), 23784.

(435) Shi, N.; Boado, R. J.; Pardridge, W. M. Pharm. Res. 2001, 18 (8), 1091.

(436) Shi, N.; Pardridge, W. M. Proc. Natl. Acad. Sci. U. S. A. 2000, 97 (13), 7567.

(437) Pardridge, W. M. Methods Enzymol. 2003, 373, 507.

(438) Pardridge, W. M. J Control Release 2007, 122 (3), 345.

(439) Allen, T. M.; Cullis, P. R. Adv. Drug Deliv. Rev. 2013, 65 (1), 36.

(440) Nogueira, E.; Gomes, A. C.; Preto, A.; Cavaco-Paulo, A. Colloids Surfaces B Biointerfaces 2015, 136, 514.

(441) Simão, A. M. S.; Bolean, M.; Cury, T. A. C.; Stabeli, R. G.; Itri, R.; Ciancaglini, P. Biophys. Rev. 2015, 7 (4), 391.

(442) Shim, M. S.; Kwon, Y. J. FEBS J. 2010, 277 (23), 4814.

(443) Beninger, R. J.; Banasikowski, T. J. Neurotox. Res. 2008, 14 (1), 57.

(444) Venton, B. J. J. Neurosci. 2006, 26 (12), 3206.

(445) Levy, D.; Shabat-Simon, M.; Shalev, U.; Barnea-Ygael, N.; Cooper, A.; Zangen, A. J. Neurosci. 2007, 27 (51), 14179.

(446) Hermanson, G. T. In Bioconjugate Techniques; Elsevier, 1996; pp 297-416.

(447) Skjørringe, T.; Gjetting, T.; Jensen, T. G. J. Control. Release 2009, 139 (2), 140.

(448) Immordino, M. L.; Dosio, F.; Cattel, L. Int. J. Nanomedicine 2006, 1 (3), 297.

(449) Jesorka, A.; Orwar, O. Annu. Rev. Anal. Chem. (Palo Alto. Calif). 2008, 1, 801.

(450) Patil, Y. P.; Jadhav, S. Chem. Phys. Lipids 2014, 177, 8.

(451) Wagner, A.; Vorauer-Uhl, K. J. Drug Deliv. 2011, 2011, 1.

(452) Kibbe, W. A. Nucleic Acids Res. 2007, 35 (Web Server issue), W43.

(453) Werner, F.-M.; Coveñas, R. Expert Opin. Drug Saf. 2014, 13 (8), 1031.

(454) Schmued, L. C.; Hopkins, K. J. Brain Res. 2000, 874 (2), 123.

(455) Kuroda, K. O.; Ornthanalai, V. G.; Kato, T.; Murphy, N. P. 
Neuropsychopharmacology 2010, 35 (3), 641.

(456) Wartman, B. C.; Gabel, J.; Holahan, M. R. PLoS One 2014, 9 (10), e108711.

(457) Rohloff, J. C.; Gelinas, A. D.; Jarvis, T. C.; Ochsner, U. A.; Schneider, D. J.; Gold, L.; Janjic, N. Mol. Ther. Nucleic Acids 2014, 3 (August), e201.

(458) Famulok, M.; Hartig, J. S.; Mayer, G. Chem. Rev. 2007, 107 (9), 3715.

(459) Abraham, A. D.; Neve, K. a; Lattal, K. M. Neurobiol. Learn. Mem. 2014, 108, 65.

(460) Seeman, M. V; Seeman, P. Prog. Neuropsychopharmacol. Biol. Psychiatry 2014, $48,155$.

(461) Trifilieff, P.; Martinez, D. Neuropharmacology 2014, 76 Pt B, 498.

(462) Buse, J.; Schoenefeld, K.; Münchau, A.; Roessner, V. Neurosci. Biobehav. Rev. 2013, 37 (6), 1069.

(463) Vaughan, R. A.; Foster, J. D. Trends Pharmacol. Sci. 2013, 34 (9), 489.

(464) André, V. M.; Cepeda, C.; Levine, M. S. CNS Neurosci. Ther. 2010, 16 (3), 163.

(465) Kim, E.; Paeng, I. R. J. Immunoassay Immunochem. 2014, 35 (1), 83.

(466) Zheng, Y.; Wang, Y.; Yang, X. Sensors Actuators B Chem. 2011, 156 (1), 95.

(467) Magbanua, E.; Zivkovic, T.; Hansen, B.; Beschorner, N.; Meyer, C.; Lorenzen, I.; Grötzinger, J.; Hauber, J.; Torda, A. E.; Mayer, G.; Rose-John, S.; Hahn, U. RNA Biol. 2013, 10 (2), 216.

(468) Park, H.; Paeng, I. R. Anal. Chim. Acta 2011, 685 (1), 65.

(469) Farjami, E.; Campos, R.; Nielsen, J. S.; Gothelf, K. V; Kjems, J.; Ferapontova, E. E. Anal. Chem. 2013, 85 (1), 121.

(470) Jiao, L.; Lu, G.; Zhang, Q.; Yuan, Y.; Wang, J.; Zhang, C.; Li, P. Micro Nano Lett. 2013, $8(12), 903$.

(471) Álvarez-Martos, I.; Campos, R.; Ferapontova, E. E. Analyst 2015, 140 (12), 4089.

(472) Álvarez-Martos, I.; Ferapontova, E. E. Anal. Chem. 2016, 88 (7), 3608.

(473) Liu, S.; Xing, X.; Yu, J.; Lian, W.; Li, J.; Cui, M.; Huang, J. Biosens. Bioelectron. 2012, $36(1), 186$.

(474) Li, B.-R.; Hsieh, Y.-J.; Chen, Y.-X.; Chung, Y.-T.; Pan, C.-Y.; Chen, Y.-T. J. Am. Chem. Soc. 2013, 135 (43), 16034.

(475) Zhou, J. W.; Wang, W. Y.; Yu, P.; Xiong, E. H.; Zhang, X. H.; Chen, J. H. Rsc Adv. 2014, 4 (94), 52250.

(476) Hun, X.; Xu, Y.; Xie, G.; Luo, X. Sensors Actuators, B Chem. 2015, 209, 596.

(477) Kim, J.; Rim, Y. S.; Chen, H.; Cao, H. H.; Nakatsuka, N.; Al, K. I. M. E. T. ACS Nano 2015, 9 (4), 4572.

(478) Peters, R. F.; Gutierrez-Rivera, L.; Dew, S. K.; Stepanova, M. J. Vis. Exp. 2015, No. $97,1$. 
(479) Walsh, R.; Ho, U.; Wang, X. L.; DeRosa, M. C. Can. J. Chem. 2015, 93 (5), 572.

(480) Xu, J.; Li, Y.; Wang, L.; Huang, Y.; Liu, D.; Sun, R.; Luo, J.; Sun, C. Dye. Pigment. 2015, 123, 55.

(481) Zibaii, M. I.; Latifi, H.; Asadollahi, A.; Bayat, A. H.; Haghparast, A. Proc. SPIE 2015, 9634, 2.

(482) Gao, L.; Ju, L.; Cui, H. RSC Adv. 2016, 6 (7), 5305.

(483) Jarczewska, M.; Sheelam, S. R.; Ziółkowski, R.; Górski, Ł. J. Electrochem. Soc. 2016, 163 (3), B26.

(484) Liu, L.; Xia, N.; Meng, J.-J.; Zhou, B.-B.; Li, S.-J. J. Electroanal. Chem. 2016, $775,58$.

(485) Huang, H.; Shi, S.; Gao, X.; Gao, R.; Zhu, Y.; Wu, X.; Zang, R.; Yao, T. Biosens. Bioelectron. 2016, 79, 198.

(486) Yusoff, N.; Pandikumar, A.; Ramaraj, R.; Lim, H. N.; Huang, N. M. Microchim. Acta 2015, 182 (13-14), 2091.

(487) Wen, D.; Liu, W.; Herrmann, A.-K.; Haubold, D.; Holzschuh, M.; Simon, F.; Eychmüller, A. Small 2016, 12 (18), 2439.

(488) Samanta, A.; Medintz, I. L. Nanoscale 2016, 8 (17), 9037.

(489) Golub, E.; Albada, H. B.; Liao, W.-C.; Biniuri, Y.; Willner, I. J. Am. Chem. Soc. 2016, 138 (1), 164.

(490) Sen, D.; Poon, L. C. H. Crit. Rev. Biochem. Mol. Biol. 2011, 46 (May), 1.

(491) Luedtke, N. W.; Tor, Y. Biopolymers 2003, 70 (1), 103.

(492) Nenov, I. P.; Fylstra, D. H. In Reliable Computing; 2003; Vol. 9, pp 143-159.

(493) Mathews, D. H. Curr. Protoc. Bioinformatics 2014, 46, 12.6.1.

(494) Kikin, O.; D’Antonio, L.; Bagga, P. S. Nucleic Acids Res. 2006, 34 (Web Server issue), W676.

(495) Varizhuk, A.; Ischenko, D.; Smirnov, I.; Tatarinova, O.; Severov, V.; Novikov, R.; Tsvetkov, V.; Naumov, V.; Kaluzhny, D.; Pozmogova, G. bioRxiv 2014.

(496) Polarizers with FluorEssence Operation Manual; HORIBA Jobin Yvon Inc.: Edison, New Jersey, USA, 2007.

(497) Müller, J.; Freitag, D.; Mayer, G.; Pötzsch, B. J. Thromb. Haemost. 2008, 6 (12), 2105.

(498) Turkevich, J.; Stevenson, P. C.; Hillier, J. Discuss. Faraday Soc. 1951, 11 (c), 55.

(499) Preparation of Artificial CSF

http://www.alzet.com/products/guide_to_use/cfs_preparation.html.

(500) Wilson, D. S.; Szostak, J. W. Annu. Rev. Biochem. 1999, 51, 435.

(501) Smirnov, I.; Shafer, R. H. Biochemistry 2000, 39 (6), 1462.

(502) Guédin, A.; Gros, J.; Alberti, P.; Mergny, J. L. Nucleic Acids Res. 2010, 38 (21), 
7858.

(503) Tomaško, M.; Vorlíčková, M.; Sagi, J. Biochimie 2009, 91 (2), 171.

(504) Mukundan, V. T.; Phan, A. T. J. Am. Chem. Soc. 2013, 135 (13), 5017.

(505) Cáceres, C.; Wright, G.; Gouyette, C.; Parkinson, G.; Subirana, J. a. Nucleic Acids Res. 2004, 32 (3), 1097.

(506) Gavathiotis, E.; Searle, M. S. Org. Biomol. Chem. 2003, 1 (10), 1650.

(507) Tradigo, G.; Mannella, L.; Veltri, P. Proc. - IEEE Symp. Comput. Med. Syst. 2014, 243.

(508) Guédin, A.; Lacroix, L.; Mergny, J. Fox, K. R., Ed.; Methods in Molecular Biology; Humana Press: Totowa, NJ, 2010; Vol. 613, pp 25-35.

(509) Ruta, J.; Perrier, S.; Ravelet, C.; Fize, J.; Peyrin, E. Anal. Chem. 2009, 81 (17), 7468 .

(510) Zhang, D.; Lu, M.; Wang, H. 2011, 9188.

(511) McKeague, M.; Foster, A.; Miguel, Y.; Giamberardino, A.; Verdin, C.; Chan, J. Y. S.; DeRosa, M. C. RSC Adv. 2013, 3 (46), 24415.

(512) Valenzano, S.; De Girolamo, A.; DeRosa, M. C.; McKeague, M.; Schena, R.; Catucci, L.; Pascale, M. ACS Comb. Sci. 2016, 18 (6), 302.

(513) Huang, Z.; Szostak, J. W. RNA 2003, 9, 1456.

(514) Wei, H.; Li, B.; Li, J.; Wang, E.; Dong, S. Chem. Commun. (Camb). 2007, No. 36, 3735 .

(515) Liu, X.; Zhou, Z.; Zhang, L.; Tan, Z.; Shen, G.; Yu, R. Chinese J. Chem. 2009, 27 (10), 1855.

(516) Yang, C.; Wang, Y.; Marty, J.-L.; Yang, X. Biosens. Bioelectron. 2011, 26, 2724.

(517) Yoshitake, T.; Kehr, J.; Todoroki, K.; Nohta, H.; Yamaguchi, M. Biomed. Chromatogr. 2006, 20 (3), 267.

(518) Venton, B. J.; Wightman, R. M. Anal. Chem. 2003, 75 (19), 414 A.

(519) Van Staden, J. F.; Van Staden, R. I. S. Talanta 2012, 102, 34.

(520) Goldstein, D. S.; Holmes, C.; Sharabi, Y. Brain 2012, 135 (6), 1900.

(521) Scheller, C.; Arendt, G.; Nolting, T.; Antke, C.; Sopper, S.; Maschke, M.; Obermann, M.; Angerer, a.; Husstedt, I. W.; Meisner, F.; Neuen-Jacob, E.; Müller, H. W.; Carey, P.; Ter Meulen, V.; Riederer, P.; Koutsilieri, E. J. Neural Transm. 2010, 117 (6), 699.

(522) Kaler, S. G.; Goldstein, D. S.; Holmes, C.; Salerno, J. a.; Gahl, W. a. Ann. Neurol. 1993, $33(2), 171$.

(523) Gjerris, A.; Werdelin, L.; Rafaelsen, O. J.; Alling, C.; Christensen, N. J. J. Affect. Disord. 1987, 13 (3), 279.

(524) Gattaz, W. F.; Riederer, P.; Reynolds, G. P.; Gattaz, D.; Beckmann, H. Psychiatry 
Res. 1983, 8 (4), 243.

(525) Bertelson, J. a; Ajtai, B. Neurol. Clin. 2014, 32 (1), 59.

(526) Gao, X.; Li, C. Small 2014, 10 (3), 426.

(527) Schaeffer, E.; Duplantier, A. In Neurodegenerative Diseases; Dominguez, C., Ed.; Springer Berlin Heidelberg: Berlin, Heidelberg, 2010; pp 91-147.

(528) Brady, R. O.; McCarthy, J. M.; Prescot, A. P.; Jensen, J. E.; Cooper, A. J.; Cohen, B. M.; Renshaw, P. F.; Ongür, D. Bipolar Disord. 2013, 15 (4), 434.

(529) Murphy, D. L.; Moya, P. R.; Fox, M. A.; Rubenstein, L. M.; Wendland, J. R.; Timpano, K. R. Philos. Trans. R. Soc. Lond. B. Biol. Sci. 2013, 368 (1615), 20120435.

(530) Marciniuk, K.; Taschuk, R.; Napper, S. Clin. Dev. Immunol. 2013, 2013, 473706.

(531) Berezovski, M. V; Lechmann, M.; Musheev, M. U.; Mak, T. W.; Krylov, S. N. J. Am. Chem. Soc. 2008, 130 (28), 9137.

(532) Stoltenburg, R.; Schubert, T.; Strehlitz, B. PLoS One 2015, 10 (7), 1.

(533) Hybarger, G.; Bynum, J.; Williams, R. F.; Valdes, J. J.; Chambers, J. P. Anal. Bioanal. Chem. 2006, 384 (1), 191.

(534) Lou, X.; Qian, J.; Xiao, Y.; Viel, L.; Gerdon, A. E.; Lagally, E. T.; Atzberger, P.; Tarasow, T. M.; Heeger, A. J.; Soh, H. T. Proc. Natl. Acad. Sci. U. S. A. 2009, 106 (9), 2989.

(535) Huang, C. J.; Lin, H. I.; Shiesh, S. C.; Lee, G. Bin. Biosens. Bioelectron. 2010, 25 (7), 1761.

(536) Shangguan, D.; Bing, T.; Zhang, N. In Aptamers Selected by Cell-SELEX for Theranostics; Springer Berlin Heidelberg: Berlin, Heidelberg, 2015; pp 13-33.

(537) Li, Q.; Zhao, X.; Liu, H.; Qu, F. J. Chromatogr. A 2014, 1364, 289.

(538) Mendonsa, S. D.; Bowser, M. T. J. Am. Chem. Soc. 2004, 126 (1), 20.

(539) Eaton, R. M.; Shallcross, J. A.; Mael, L. E.; Mears, K. S.; Minkoff, L.; Scoville, D. J.; Whelan, R. J. Anal. Bioanal. Chem. 2015, 6965.

(540) Berezovski, M.; Musheev, M.; Drabovich, A.; Krylov, S. N. J. Am. Chem. Soc. 2006, $128(5), 1410$.

(541) Hünniger, T.; Wessels, H.; Fischer, C.; Paschke-Kratzin, A.; Fischer, M. Anal. Chem. 2014, 86 (21), 10940.

(542) Cox, J. C.; Rudolph, P.; Ellington, A. D. Biotechnol. Prog. 1998, 14 (6), 845.

(543) Goertz, P. W.; Cox, J. C.; Ellington, A. D. JALA - J. Assoc. Lab. Autom. 2004, 9 (3), 150.

(544) Eulberg, D.; Buchner, K.; Maasch, C.; Klussmann, S. Nucleic Acids Res. 2005, 33 (4), 1.

(545) Beinoraviciūte-Kellner, R.; Lipps, G.; Krauss, G. FEBS Lett. 2005, 579 (20), 4535. 
(546) Li, S. H.; Li, H.; Yang, X. Q.; Wang, W.; Huang, A. X.; Li, J.; Qin, X. L.; Li, F.; Lu, G. Y.; Ding, H. M.; Su, X. T.; Hou, L. B.; Xia, W.; Shi, M.; Zhang, H. W.; Zhao, Q.; Dong, J.; Ge, X. F.; Sun, L. Q.; Bai, C. J.; Wang, C. N.; Shen, X. L.; Fang, T.; Wang, F. S.; Zhang, H. Q.; Shao, N. S. Oncotarget 2015, 6 (12), 10045.

(547) McKeague, M.; Bradley, C. R.; Girolamo, A. De; Visconti, A.; Miller, J. D.; DeRosa, M. C. Int. J. Mol. Sci. 2010, 11 (12), 4864.

(548) Gaillard, C.; Strauss, F. BMC Biochem. 2000, $1,1$.

(549) Hu, J. M.; Easley, C. J. Analyst 2011, 136 (17), 3461.

(550) Quang, N. N.; Pestourie, C.; Cibiel, A.; Ducongé, F. Methods 2016, 97, 35.

(551) Cruz-Aguado, J. A.; Penner, G. J. Agric. Food Chem. 2008, 56 (22), 10456.

(552) Hall, B.; Arshad, S.; Seo, K.; Bowman, C.; Corley, M.; Jhaveri, S. D.; Ellington, A. D. Curr. Protoc. Mol. Biol. 2009, Chapter 24, Unit 24.3.

(553) Flinders, J.; DeFina, S. C.; Brackett, D. M.; Baugh, C.; Wilson, C.; Dieckmann, T. ChemBioChem 2004, 5 (1), 62.

(554) Rotherham, L. S.; Maserumule, C.; Dheda, K.; Theron, J.; Khati, M. PLoS One 2012, 7 (10), 1 .

(555) Potty, A. S. R.; Kourentzi, K.; Fang, H.; Jackson, G. W.; Zhang, X.; Legge, G. B.; Willson, R. C. Biopolymers 2009, 91 (2), 145.

(556) Drabovich, A. P.; Berezovski, M.; Okhonin, V.; Krylov, S. N. Anal. Chem. 2006, $78(9), 3171$.

(557) Bao, J.; Krylova, S. M.; Reinstein, O.; Johnson, P. E.; Krylov, S. N. Anal. Chem. 2011, 83 (22), 8387. 
9 Appendix 2.1: Additional information for Chapter 3 "A comprehensive analysis of SELEX and aptamer data from the Aptamer Base" 


\subsection{SELEX Methods used to select aptamers}

Table 9.1: SELEX methods used to select aptamers

\begin{tabular}{|c|c|c|}
\hline Selex Method & Description & Reference \\
\hline FluMag-SELEX & $\begin{array}{l}\text { Aptamers are selected from a } \\
\text { library modified with fluorescein } \\
\text { instead of radiolabels. In addition, } \\
\text { the target is immobilized on } \\
\text { magnetic beads as opposed to } \\
\text { agarose. }\end{array}$ & 315,532 \\
\hline Microfluidic SELEX & $\begin{array}{l}\text { Aptamers are selected on a } \\
\text { miniature, automated SELEX } \\
\text { platform based on microfluidic } \\
\text { technologies }\end{array}$ & $533-535$ \\
\hline Generic SELEX & $\begin{array}{l}\text { Aptamers selection experiments } \\
\text { that do not fit into another defined } \\
\text { category because neither } \\
\text { specialized equipment or } \\
\text { significantly adapted methods were } \\
\text { used compared to the original } \\
\text { selection experiments }\end{array}$ & $34-36,288$ \\
\hline Cell-SELEX & $\begin{array}{l}\text { Methods were adapted specifically } \\
\text { for the selection of aptamers that } \\
\text { have affinity for whole cells. }\end{array}$ & $210,271,536$ \\
\hline $\begin{array}{l}\text { CE-SELEX (capillary } \\
\text { electrophoresis } \\
\text { SELEX) }\end{array}$ & $\begin{array}{l}\text { In this method, partitioning of } \\
\text { bound and non-specific binding } \\
\text { oligonucleotides is performed using } \\
\text { capillary electrophoresis. }\end{array}$ & $84,537-539$ \\
\hline Non-SELEX & $\begin{array}{l}\text { Aptamers are selected after } \\
\text { multiple steps of partitioning but } \\
\text { without amplification }\end{array}$ & $265,266,540$ \\
\hline Automated SELEX & $\begin{array}{l}\text { Aptamers are selected within an } \\
\text { automated system, decreasing } \\
\text { labour and processing time. }\end{array}$ & $541-544$ \\
\hline EMSA-SELEX & During the partitioning stage, & 545,546 \\
\hline
\end{tabular}




\begin{tabular}{|l|l|l|}
\hline & $\begin{array}{l}\text { binding sequences are separated } \\
\text { from non-binding sequences by } \\
\text { EMSA. }\end{array}$ & \\
\hline Not Described & $\begin{array}{l}\text { For these experiments the authors } \\
\text { did not describe the selection } \\
\text { method used }\end{array}$ \\
\hline
\end{tabular}

\subsection{Partitioning methods used in SELEX experiments}

Table 9.2: Partitioning methods used in SELEX experiments

\begin{tabular}{|l|l|}
\hline Partitioning method & $\begin{array}{l}\text { description } \\
\text { binding sequences are separated by non-binders by } \\
\text { isolating the aptamer-target complex using centrifugation }\end{array}$ \\
\hline $\begin{array}{l}\text { Affinity } \\
\text { chromatography }\end{array}$ & $\begin{array}{l}\text { Binding sequences are separated from non-binders by } \\
\text { column chromatography or other form of solid support } \\
\text { matrix }\end{array}$ \\
\hline Filtration & $\begin{array}{l}\text { Binders are separated from binding sequences by either } \\
\text { size exclusion or target based retention to the filter (for } \\
\text { example nitrocellulose) }\end{array}$ \\
\hline Not Described & $\begin{array}{l}\text { Partitioning details were not described in the } \\
\text { experimental details provided by the authors }\end{array}$ \\
\hline Gel electrophoresis & $\begin{array}{l}\text { Binding sequences are separated from non-binding } \\
\text { sequences by differential gel migration }\end{array}$ \\
\hline Cell washing & $\begin{array}{l}\text { Non-binding sequences are washed away from adherent } \\
\text { cells while binding sequences remain and are isolated for } \\
\text { the next round of selection }\end{array}$ \\
\hline $\begin{array}{l}\text { Affinity } \\
\text { chromatography } \\
\text { (magnetic beads) }\end{array}$ & $\begin{array}{l}\text { magnetic beads are used as the solid support matrix. The } \\
\text { target is immobilized on the bead surface and binding } \\
\text { sequences are partitioned by physical separation when the } \\
\text { magnetic beads are drawn to a magnet and the non- } \\
\text { binding sequences remain in the supernatant. }\end{array}$ \\
\hline Not Required & \begin{tabular}{l} 
This was selected with a SELEX method did not require \\
\hline
\end{tabular} \\
\hline
\end{tabular}


a partitioning step.

Capillary

electrophoresis
Binding sequences are separated from non-binding sequences based on electrokinetic separation

\subsection{Recovery methods used in SELEX experiments}

Table 9.3: Recovery methods used in SELEX experiments

\begin{tabular}{|c|c|}
\hline Recovery method & description \\
\hline Urea & $\begin{array}{l}\text { The aptamer-target complex is separated by the specific } \\
\text { chaotropic agent urea }\end{array}$ \\
\hline Filtration & $\begin{array}{l}\text { The aptamer-target complex is separated by multiple } \\
\text { filtration steps or differential affinity of the binding } \\
\text { sequences and target to the filter }\end{array}$ \\
\hline Chaotropic agent & $\begin{array}{l}\text { Separation of the aptamer-target complex is induced by } \\
\text { disruption of hydrogen bonding between the binding } \\
\text { sequences and the target }\end{array}$ \\
\hline Heat & $\begin{array}{l}\text { The aptamer-target complex is separated by physical } \\
\text { denaturation at high temperatures }\end{array}$ \\
\hline Competitive elution & $\begin{array}{l}\text { The aptamer-target complex is disrupted when the } \\
\text { binding sequences competitively interact with another } \\
\text { target }\end{array}$ \\
\hline Not required & $\begin{array}{l}\text { The recovery step was not necessary in these SELEX } \\
\text { experiments }\end{array}$ \\
\hline Organic extraction & $\begin{array}{l}\text { An organic solvent is used to separate the aptamer-target } \\
\text { complex. For example, phenol chloroform is used to } \\
\text { separate protein from DNA as the protein is extracted } \\
\text { from the aqueous phase into the organic layer. }\end{array}$ \\
\hline Elution with target & $\begin{array}{l}\text { When the aptamer-target complex is immobilized on a } \\
\text { column (such as with affinity chromatography), a higher } \\
\text { concentration of the target free in solution is used to }\end{array}$ \\
\hline
\end{tabular}




\begin{tabular}{|l|l|}
\hline Change in pH & $\begin{array}{l}\text { remove binding sequences from the target immobilized to } \\
\text { the column. }\end{array}$ \\
\hline Precipitation & $\begin{array}{l}\text { Hydrogen bonding between binding sequences and the } \\
\text { target is disrupted by changes in pH. Commonly } \\
\mathrm{NaOH}_{(\mathrm{Aq})} \text { is used. }\end{array}$ \\
\hline $\begin{array}{l}\text { Change in ionic } \\
\text { strength }\end{array}$ & $\begin{array}{l}\text { The aptamer-target complex is separated by ethanol } \\
\text { precipitation in which the DNA is precipitated out. }\end{array}$ \\
\hline $\begin{array}{l}\text { Cations are often required to stabilize the aptamer } \\
\text { secondary structure and the aptamer-target complex } \\
\text { tertiary structure. When the cations are removed, } \\
\text { separation of the binding DNA from the target occurs. } \\
\text { Recovery method details were not provided by the } \\
\text { authors. }\end{array}$ \\
\hline
\end{tabular}

\subsection{Affinity methods used to determine the dissociation constant of selected aptamers}

Table 9.4: Affinity methods used to determine the dissociation constant of selected aptamers

\begin{tabular}{|l|l|l|}
\hline $\begin{array}{l}\text { Affinity Method } \\
\text { chromatography }\end{array}$ & $\begin{array}{l}\text { Description } \\
\text { In this method, either the target or the } \\
\text { aptamer is immobilized to a solid support } \\
\text { matrix. Then either the aptamer or target } \\
\text { respectively is incubated with the solid } \\
\text { support matrix. The matrix is then separated } \\
\text { from the mobile phase and the amount of } \\
\text { free target or aptamer is measured to } \\
\text { determine affinity. Immobilization of either } \\
\text { the aptamer or target can have an effect on } \\
\text { affinity. }\end{array}$ \\
\hline $\begin{array}{l}\text { Affinity } \\
\text { electrophoresis }\end{array}$ & $\begin{array}{l}\text { The tendency of the aptamer, target and } \\
\text { aptamer-target complex to migrate } \\
\text { differently in a gel matrix is exploited to } \\
\text { determine the affinity. A common example is }\end{array}$ & \\
\hline
\end{tabular}




\begin{tabular}{|c|c|c|}
\hline & $\begin{array}{l}\text { the EMSA (electrophoretic mobility shift } \\
\text { assay) }\end{array}$ & \\
\hline $\begin{array}{l}\text { Capillary } \\
\text { electrophoresis }\end{array}$ & $\begin{array}{l}\text { An electric field is applied to separate the } \\
\text { aptamer, the target and the aptamer-target } \\
\text { complex. Components are then measured to } \\
\text { determine affinity. }\end{array}$ & 549 \\
\hline $\begin{array}{l}\text { Cell binding } \\
\text { assay }\end{array}$ & $\begin{array}{l}\text { In a cell binding assay, the amount of } \\
\text { aptamer that interacts with the cell is } \\
\text { measured and used to determine affinity. }\end{array}$ & 550 \\
\hline Dialysis & $\begin{array}{l}\text { In this method, the aptamer and target are } \\
\text { allowed to equilibrate between two } \\
\text { compartment chambers separated by a semi- } \\
\text { permeable membrane. The distribution of } \\
\text { either the aptamer or target is monitored and } \\
\text { the affinity can be determined. Fluorescence } \\
\text { or absorbance of either the aptamer or target } \\
\text { are commonly used to measure changes. }\end{array}$ & 551 \\
\hline $\begin{array}{l}\text { Filter binding } \\
\text { assay }\end{array}$ & $\begin{array}{l}\text { In this method, the aptamer-target complex is } \\
\text { separated from the individual components by } \\
\text { filtration. Affinity is determined by } \\
\text { measuring the amount bound of either } \\
\text { aptamer or target, depending which allows } \\
\text { for a quantifiable signal. }\end{array}$ & 552 \\
\hline Flow cytometry & $\begin{array}{l}\text { The affinity of aptamers for larger targets } \\
\text { like cells can be determined by binding } \\
\text { dependent fluorescent labelling of the } \\
\text { aptamer-target complex and determining the } \\
\text { amount bound by fluorescence detection }\end{array}$ & 271 \\
\hline $\begin{array}{l}\text { Fluorescence } \\
\text { intensity }\end{array}$ & $\begin{array}{l}\text { In this method, either the aptamer or target } \\
\text { needs to be intrinsically fluorescent, either } \\
\text { the aptamer or target needs to be } \\
\text { fluorescently labeled, or a fluorescent dye } \\
\text { (such as SyBr Green I) is used. Upon } \\
\text { aptamer-target binding, fluorescence } \\
\text { intensity is either increased or quenched. }\end{array}$ & 553 \\
\hline $\begin{array}{l}\text { Fluorescence } \\
\text { polarization }\end{array}$ & $\begin{array}{l}\text { In this method, affinity is determined by a } \\
\text { change in polarization of the aptamer-target } \\
\text { complex compared to either the aptamer or }\end{array}$ & 276,551 \\
\hline
\end{tabular}




\begin{tabular}{|c|c|c|}
\hline & $\begin{array}{l}\text { target alone. Either the aptamer or target } \\
\text { need to be intrinsically fluorescent or } \\
\text { fluorescently labelled. Since the change in } \\
\text { polarization is related to a change in size of } \\
\text { the aptamer-target complex this method is } \\
\text { more effective with larger targets. }\end{array}$ & \\
\hline $\begin{array}{l}\text { Immunoaffinity } \\
\text { assay }\end{array}$ & $\begin{array}{l}\text { In these assays, both an aptamer and } \\
\text { antibody or antibodies are used together to } \\
\text { determine the affinity of the aptamer, for } \\
\text { example in a competitive assay. }\end{array}$ & 554 \\
\hline $\begin{array}{l}\text { Isothermal } \\
\text { titration } \\
\text { calorimetry }\end{array}$ & $\begin{array}{l}\text { This method is based on the exothermic } \\
\text { properties of the aptamer-target complex } \\
\text { formation. It allows determination of } \\
\text { stoichiometry and thermodynamic properties } \\
\text { in addition to affinity. This method is } \\
\text { particularly useful for aptamers that } \\
\text { experience a substantial conformational } \\
\text { change upon target binding. }\end{array}$ & 555 \\
\hline $\begin{array}{l}\text { Kinetic capillary } \\
\text { electrophoresis }\end{array}$ & $\begin{array}{l}\text { Similar to capillary electrophoresis except } \\
\text { that the equilibrium distribution of the } \\
\text { mixture can be measured and used to } \\
\text { determine aptamer affinity. }\end{array}$ & 556,557 \\
\hline Not described & $\begin{array}{l}\text { Details about the affinity method used to } \\
\text { characterize aptamer-target binding was not } \\
\text { described by the authors }\end{array}$ & \\
\hline $\begin{array}{l}\text { Surface plasmon } \\
\text { resonance }\end{array}$ & $\begin{array}{l}\text { In this method, either the aptamer or the } \\
\text { target is immobilized to the surface of a chip. } \\
\text { The affinity is determined by flowing the } \\
\text { target or aptamer respectively over the chip. } \\
\text { Binding is characterized by a difference in } \\
\text { the measured refractive index. This method } \\
\text { is mass dependent, therefore better results } \\
\text { are achieved with larger targets. }\end{array}$ & 316 \\
\hline $\begin{array}{l}\text { UV-Visible } \\
\text { Spectroscopy }\end{array}$ & $\begin{array}{l}\text { In this method, a change in either the } \\
\text { absorption intensity or absorption } \\
\text { wavelength of either the aptamer or target } \\
\text { upon binding is observed. }\end{array}$ & 418,508 \\
\hline
\end{tabular}




\title{
9.5 The Freebase Aptamer Base User Guide
}

\author{
Jose Cruz-Toledo \\ Maureen McKeague \\ Matthew Chan \\ Michel Dumontier
}

\subsubsection{Background}

The advent of modern molecular biology has fueled an exponential increase in the amount of data available: protein interaction experiments, in vitro selected aptamers, microarrays, DNA and protein sequencing and the solving of numerous protein structures are just some of the types of high-throughput data now available online. However, the vast majority of this information is still only available in the textual unstructured format of web pages or peer reviewed articles, which only contain rendering instructions for computers to display and thus for humans to consume. Moreover, there is an increasing necessity to enable researchers to seamlessly combine the immense amount of data generated from different experimental techniques so as to enable the generation of biologically meaningful assertions regarding the contents of the data. If we consider the data generated by sequencing projects alone, where scientists wish to integrate the information about the predicted or experimentally proven genes from different sequencing projects, they are sure to find numerous obstacles in this particular task. The complications arise when scientists try to incorporate annotations of the functional roles of genes, or quality information, such as the evidence on which the annotations are based from different sources. Most sequence annotations currently do not meet any stringent classification criteria that would facilitate this sort of task and therefore, make such a task daunting and practically impossible for scientists that are not familiarized with information management.

Freebase (available at: http://www.freebase.com) is a free openly licensed community built database that contains structured information on millions of topics in hundreds of categories and enables anyone to contribute, create and share information with others. Freebase provides an interface that allows non-programmers to fill in structured data about any piece of general information, and to categorize and connect data items in a meaningful and semantically coherent manner. Freebase shares with Wikipedia the principle that a community of sufficiently curious individuals will not only create good quality data but also constantly maintain and curate the millions of resources created by others. However the technological backend of Freebase is very different from any other open shared database. Freebase runs on a database infrastructure that utilizes a non-hierarchical graph model to store its data. Unlike conventional relational databases where data is stored in tables, Freebase defines its data structure as a set of nodes and links that establish relationships between the nodes. A major benefit of having this type of non-hierarchical data structure is that in Freebase one can model very complex relationships between individual 
elements that would be otherwise impossible to do in relational database systems such as MediaWiki ${ }^{1}$, the one that runs Wikipedia. One of the main consequences of Freebase's use of a different underlying structure is that entries added to this dataset function as dynamic information hubs which can be easily extensible by users, rather than static isolated information silos as found in other databases.

The structured information stored in Freebase enables the maintenance of a high level of consistency between all entries that are added to its database, thus making it possible for users to mine information from Freebase and re-use for the creation of other applications. Freebase allows users to perform conventional keyword based searches but the novel data modeling strategies used in Freebase enable users to pose complex questions along the lines of: "What are all the names of the spouses of the children of all U.S. republican presidents?", "What are the dissociation constants for all RNA aptamers that bind to cyclic AMP?" or "What are all the PubMed identifiers for peptide aptamers that are reported in Freebase?". The developers of Freebase have also made their data easily accessible for other data providers/creators, therefore information about certain topics can be extracted and incorporated into other related data sources in a rather simple manner.

Here we present a user guide for the Freebase Aptamer Base (available at: http://aptamer.freebase.com) which has been tailored to contain user contributed annotations from scientific literature about RNA, DNA or peptide aptamer SELEX experiments. The structured data contained in the Aptamer Base includes a variety of information that not only has a very wide span but also leverages other pre-existing topics in Freebase for the annotation of Aptamers.

This guide has been created to supplement the existing Freebase Documentation (found here: http://www.freebase.com/help/faq) in aiding users in the addition of content for the Aptamer Base as extracted directly from scientific literature, through a practical demonstration.

We will commence this tutorial by exploring some of the basics of Freebase. First let's get familiarized with some jargon used by Freebase. Freebase is essentially composed of four basic elements: Bases, Topics, Types and Properties. Bases are a collection of related assertions created by users. For example, the Chemistry commons (or Base) holds over 14,000 community contributed facts (Topics + Types) about chemistry related entities. Topics are used to represent real world things in Freebase (i.e. the Eiffel Tower, Theodore Roosevelt or The Simpson's episode seventeen from season two entitled "Old Money"). The modeling scheme used by Freebase allows Topics to hold any number of Types, therefore the Theodore Roosevelt topic has as type: Person, US President, Nobel Prize Winner, Family Member, etc. Finally, Properties are values that relate to a particular Type. For example, the Water Topic belongs to the Chemistry Base, has as Type Chemical Compound and has as Property an average molar mass of 18.0153 $\mathrm{g} / \mathrm{mol}$.

\footnotetext{
${ }^{1}$ http://mediawiki.org
} 
The Aptamer Base's primary objective is to enable the accurate annotation of the results of interaction experiments that describe the creation of aptamers as directly extracted from scientific literature. The data model used by the Aptamer Base is composed at its core, by a generic type relation map as illustrated in Figure 1.

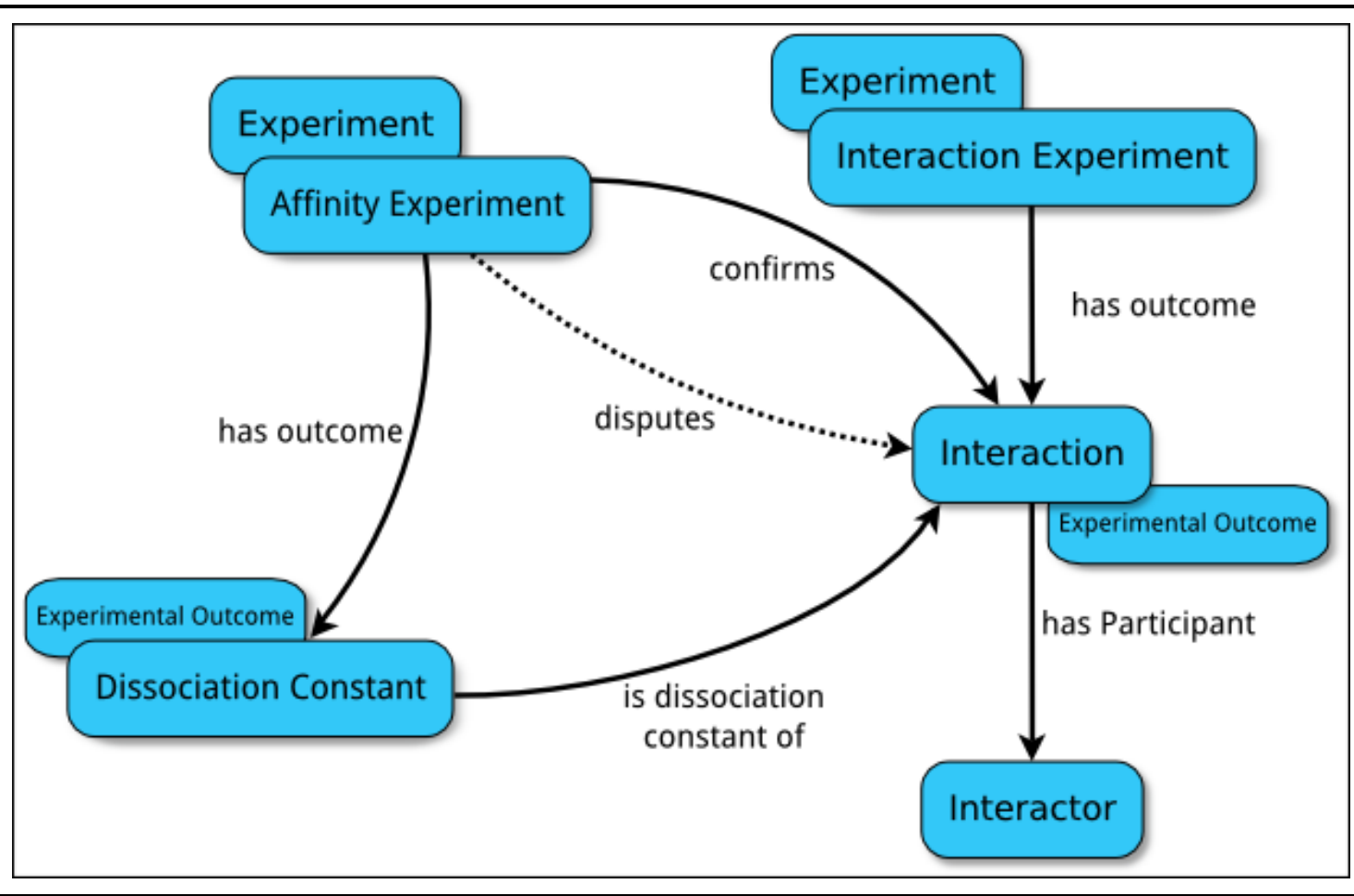

Figure 1: Basic type relation map used by the Aptamer Base. The Interaction Experiment type relates to the Interaction type through a "has outcome" relation that is associated with the Experiment type. Each Interaction type has at least two interactors (Aptamer and Aptamer Target respectively) as participants. The Affinity Experiment type, also makes use of the "has outcome" relation to link to the dissociation constant and either "confirms" or "disputes" an interaction. Full resolution image found here.

We created the Interaction Experiment type so as to represent the results and details of experimental procedures that elucidate biomolecular interactions. Our Interaction Experiment type has been modelled so that it may report one or more Interactions (Figure 1). An Interaction is a biochemical process in which some set of attractive forces act between at least two Interactors e.g: a biomolecule (Aptamer) and a ligand (Aptamer Target) therefore, each Interaction can be uniquely characterized by: the number of members that compose it, the Interaction Experiment that elucidated the Interaction and the Affinity Experiment(s) that confirm or dispute it. When an Interaction has only two participants it should be further typed as a Pairwise Interaction (Figure 2). Similarly, if the interaction has more than two members it should be typed as a Collective Interaction. 
An Affinity Experiment makes reference to the experimental process that confirms or disputes an Interaction. If a given Affinity Experiment confirms an Interaction, then a Dissociation Constant must be reported as an outcome of the latter (Figure 2). Consequently, in our modelling scheme Dissociation Constants are related to an Interaction through an Affinity Experiment that confirms it.

Freebase's modeling nature will regard any two Topic entries with the same name as being the same. Therefore we will need to make sure that any entries that we incorporate into the Aptamer Base follow a systematic naming scheme, such that users can easily identify the source and main characteristics of the topics incorporated into the Aptamer Base from both the name and Types that the incorporated Topics hold. Our proposed topic naming scheme is illustrated in Figure 2.

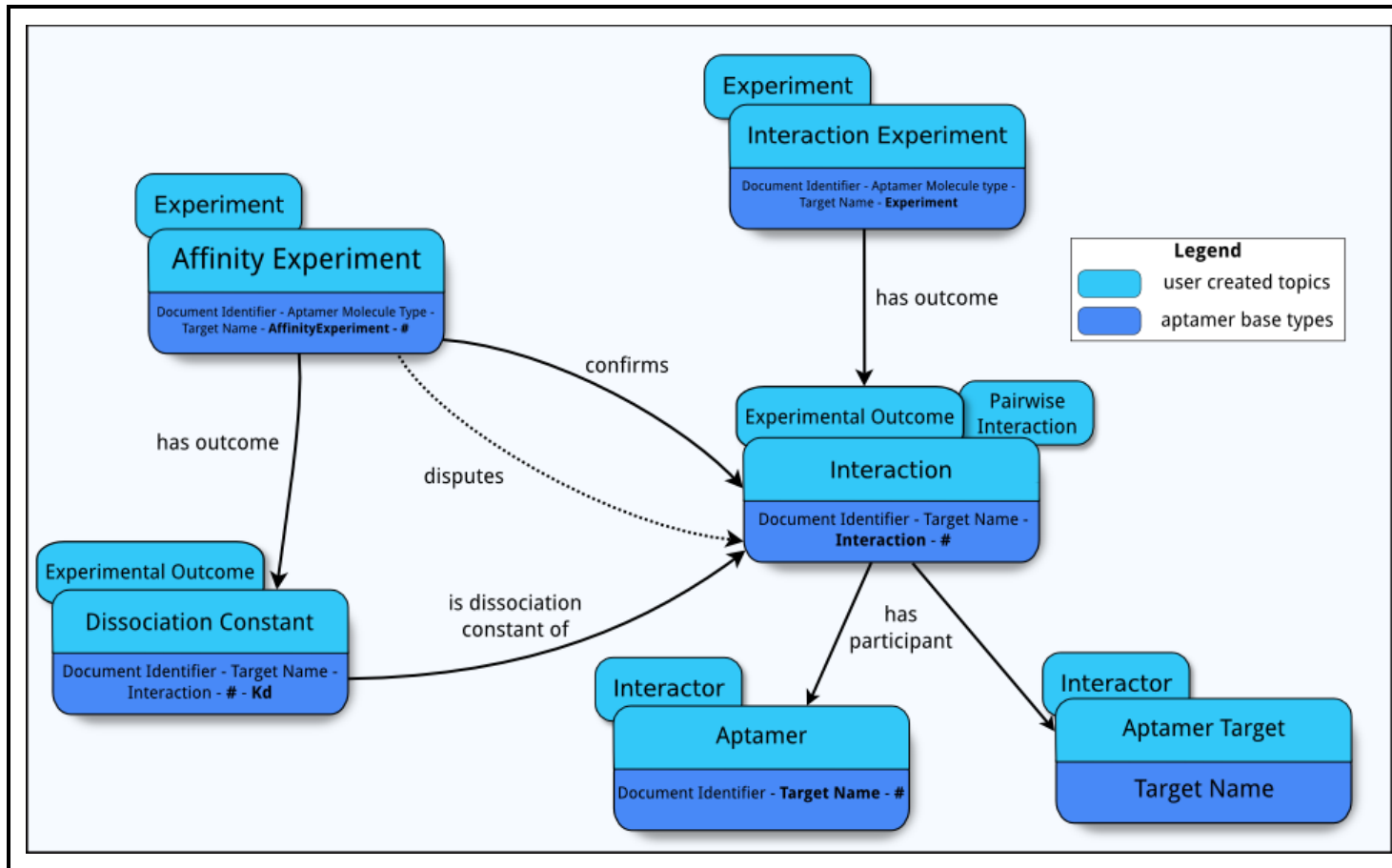

Figure 2: Illustration of topic naming scheme used by the Aptamer Base. Words in bold represent non variable sections of the name. Dark blue rectangles show topics with their proposed naming scheme. Light blue ellipses depict the corresponding types. Full resolution image available here.

Our naming scheme will require the following pieces of information about the experiment, aptamer and aptamer target to accurately describe topic names:

\section{Document Identifier:}

Currently most of the scientific literature that refers to aptamer experiments can be found through Pubmed, a free database of citations, abstracts and some full text articles on life sciences and biomedical topics indexed by MEDLINE. Entries in Pubmed are identified through a unique number (i.e. 20693539). However, if it were the case that the reported 
aptamer experiment cannot be found in Pubmed, we suggest the usage of the document's Digital Object Identifier (i.e. 10.1093/nar/gkq615).

Aptamer Molecule Type:

This refers to the biological nature of the aptamer that you wish to represent namely:

RNA, DNA or Peptide.

Target Name:

This string describes the common name of the chemical compound that was found to be the aptamer target or ligand. For example: AMP, Cocaine, GMP, etc.

\subsubsection{Modeling of Minimal Aptamers}

In order to accurately model a minimal aptamer we will consider minimal aptamers as being part of a separate Interaction that is reported by the Interaction Experiment. Consider for example Figure 3. 


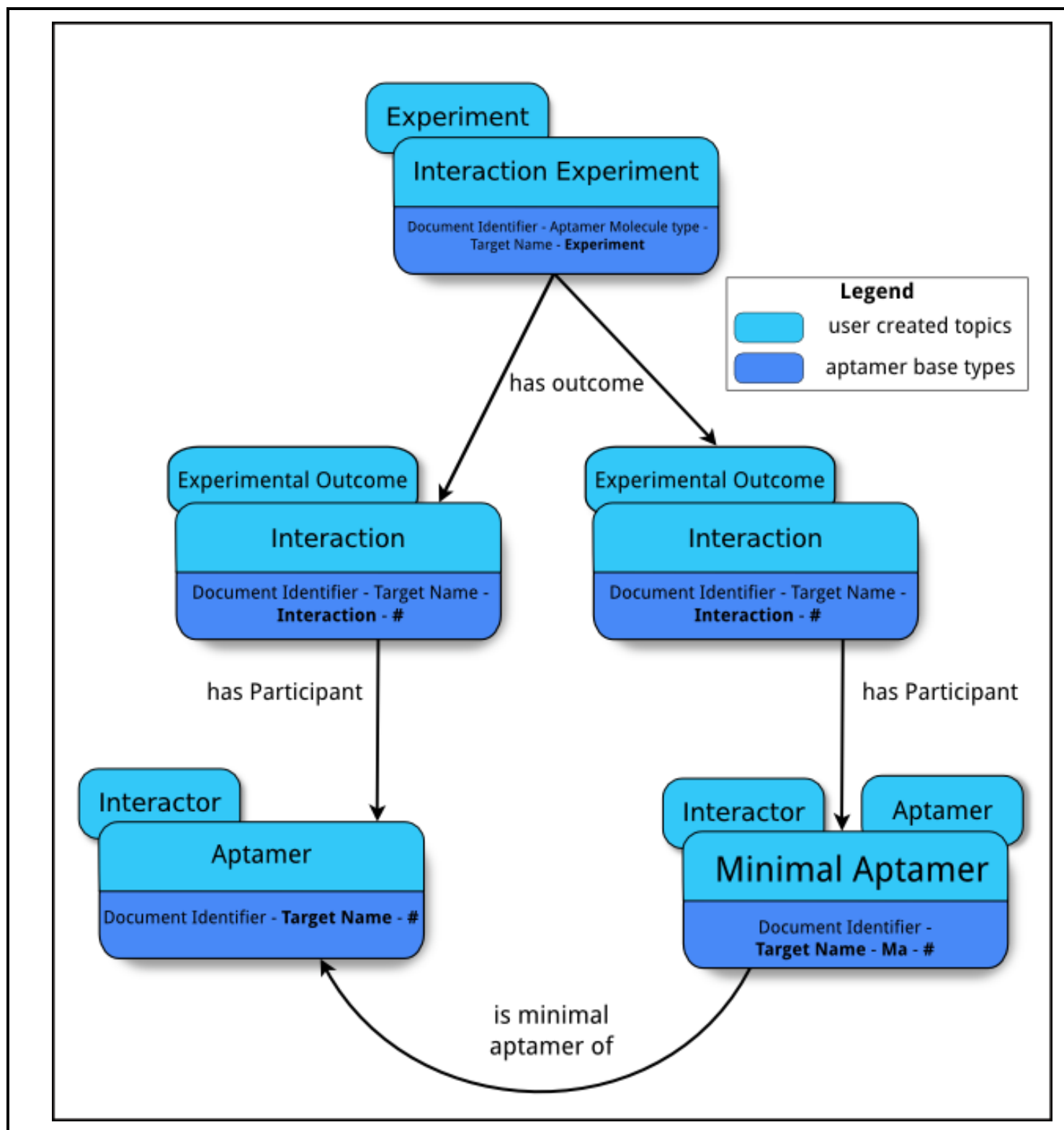

Figure 3: Abridged schematic representation of a minimal aptamer in the Aptamer Base. An Aptamer is typed as a Minimal Aptamer which uses the "is minimal aptamer of" relation to link the minimal aptamer back to its parent aptamer. Full resolution image available here.

A minimal aptamer is an aptamer that is derived from another aptamer. Given that minimal aptamers usually have different dissociation constant values, we create a new Interaction topic to describe a minimal aptamer. A minimal aptamer is typed as being both an Aptamer and a Minimal Aptamer, by doing so the minimal aptamer topic that you will create will get the properties from the Aptamer type and the additional property from the Minimal Aptamer type that links to the aptamer from which it derived, through the use of the "is minimal aptamer of" property. 


\subsubsection{Extracting Information from scientific literature}

In this section we will proceed to exemplify how to create a set of topics that will accurately describe a particular SELEX experiment as described by a journal article. Before commencing the addition of data please make sure that you have created a user in Freebase. To do so simply visit https://www.freebase.com/signin/register and follow the instructions found therein. You can join the Aptamer Base by clicking on the "Join Base" button found here.

Go to the Aptamer Base and sign-in with your Freebase username and password and also have a copy of Koizumi, M. and R.R. Breaker: "Molecular Recognition of cAMP by an RNA Aptamer". Biochemistry, 2000. 39(30): 8983-92. (http://www.ncbi.nlm.nih.gov/pubmed/10913311)

Koizumi and Breaker describe the extraction of two classes (or collections) of RNA aptamers that bind to the second messenger adenosine 3',5'-cyclic monophosphate (cAMP) from a random sequence pool using in vitro selection. In this article the authors report on two classes of aptamers formed by 33 and 31 nucleotides respectively, the aptamers found displayed a dissociation constant for $\left(\mathrm{K}_{\mathrm{d}}\right) \mathrm{cAMP}$ of $\sim 10 \mu \mathrm{M}$. The information that we will be extracting from this paper will follow the modeling scheme used in the Aptamer Base (Figure 4). 


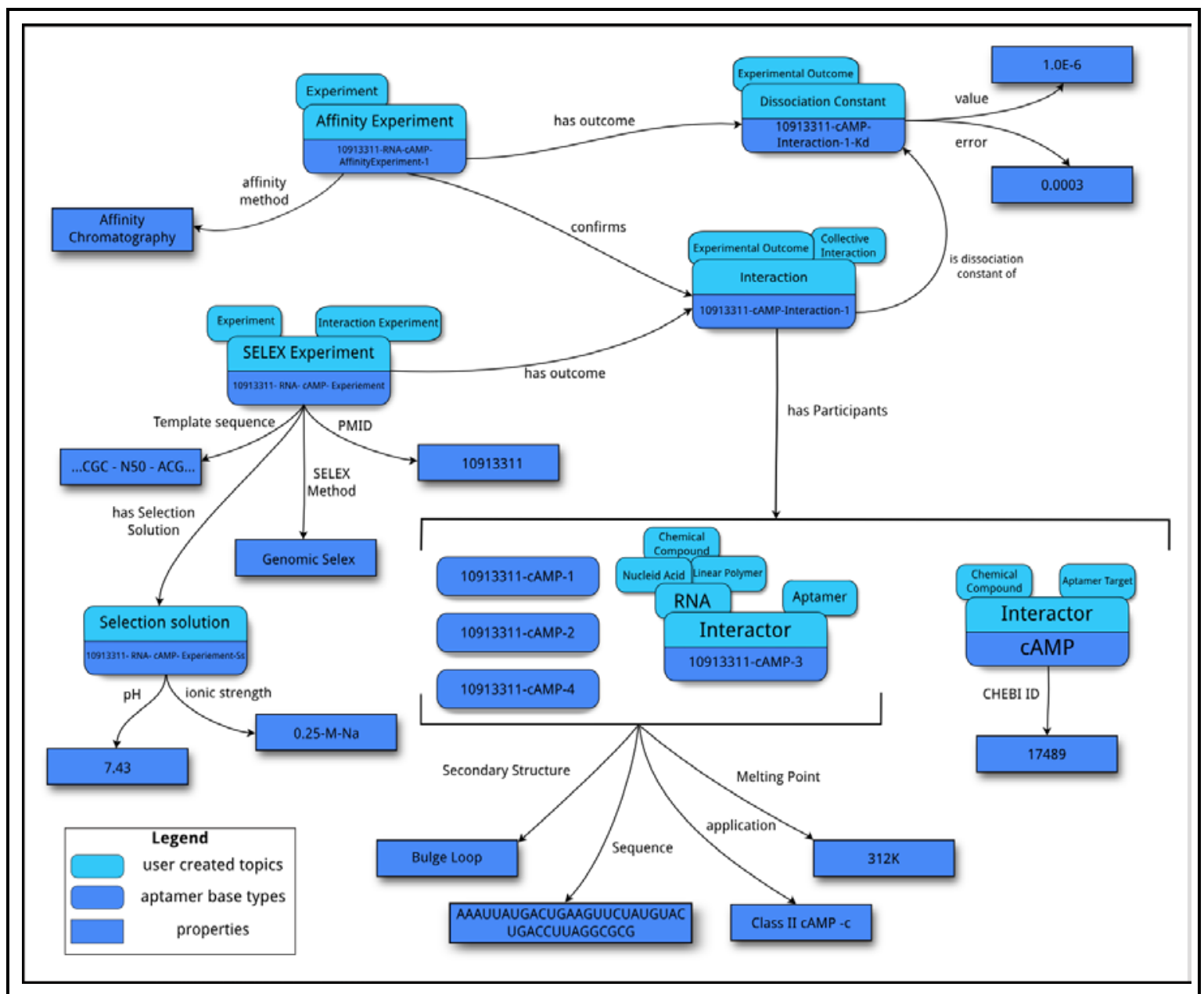

Figure 4: Schematic representation of the modeling scheme used in the Aptamer Base. Four RNA aptamers that bind to cAMP with a dissociation constant of $1.0 \mathrm{nM}$. Note that not all Interactors were fully typed in the figure so as to save space. Full resolution image available here.

In order facilitate the realization of the data model used by the Aptamer Base we recommend the following approach:

\section{Step I: Gather necessary information for assigning names}

We notice that Koizumi and Breaker describe in Pubmed:10913311 a total of four aptamers that bind to cAMP with a particular dissociation constant $(\mathrm{Kd})$ of $1 \mathrm{E}-6$; in other words, the authors performed an Interaction Experiment that produced as an outcome an Interaction involving four RNA aptamers and 1 aptamer target. Additionally the authors performed an Affinity Experiment that elucidated a Dissociation Constant between these interactors. Consequently we have the three key bits of information that are required to follow our naming scheme (Figure 2):

\section{Document Identifier:}

$$
10913311
$$


Aptamer Molecule Type: $\quad$ RNA

Target Name: $\quad$ cAMP

\section{Step II: Create a SELEX Experiment Topic}

We note that Koizumi and Breaker utilize the SELEX method for the creation of the described aptamers, therefore we need to create a topic of type SELEX Experiment, which will automatically include the Experiment and Interaction Experiment types. To create a new SELEX Experiment topic, we can start by searching for the SELEX Experiment Type in Freebase (be sure to select the "SELEX Experiment" Type described in the Aptamer Base from the drop down menu) or by simply clicking here. You will be presented with a list of all topics that have SELEX Experiment as type. To add a new topic simply click on the Add it! Link at the bottom of the list, you will be presented with a form as the one shown in Figure 6.

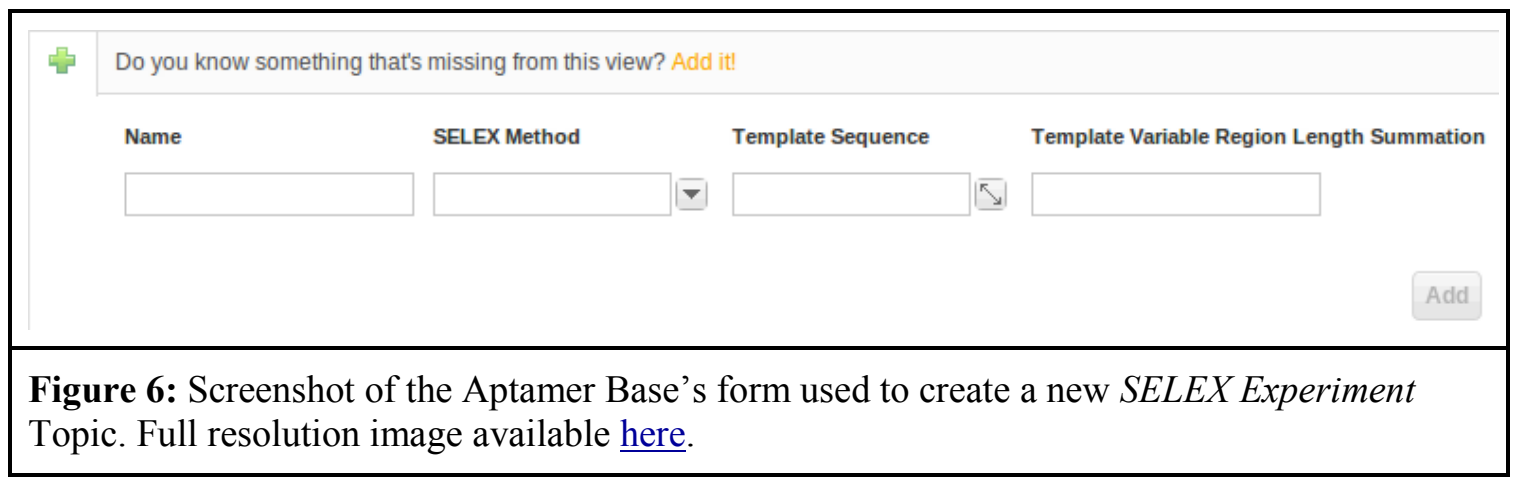

Fill out the required information about the SELEX Experiment topic that is described in this article. Namely, 10913311-RNA-cAMP-Experiment for the topic name, Genomic SELEX for the SELEX method and we fill out the template sequence. When we start filling out the form depicted in Figure 6 we will notice that Freebase first does a search on its entire database to make sure that the topic that you are creating does not already exist. This unique feature allows the community to further extend pre-existing entries by incorporating information that the original author of the topic missed or never thought to add. Once finished we click on the Add button.

\section{Step III: Incorporate details about the SELEX Experiment}

Once the topic has been added you can click on its name to see the topic's details. Now click on the "Edit this topic" button to further annotate this entry. At creation time (Step II) we had already filled out the SELEX method and template sequence properties. Now we can proceed to incorporate additional details.

The "template sequence" property is to be filled with the primer sequences written in the 5'->3' direction and including the length of the variable aptamer region. In our example the template sequence was: "GGAAGAGAUGGCGAC-N50-CGGUAAGCUUGGCAC". Notice that the variable region was specified using the letter " $N$ " immediately followed by the length i.e. 50, surrounded by dashes. 
The "template variable region length summation" is a property that refers to the summation of the variable length region described by template sequences. In this case there is only one variable region of length 50 in our sequence, therefore we use 50 as the value of this property. We can also proceed to incorporate details about the experiment type, namely its Pubmed identifier: 10913311. (Note that if the PubMed Id is provided for the experiment type then there is no need to fill the Bibliographic Reference property.)

The "number of selection rounds needed" refers to the maximum number of rounds used to obtain the selected aptamers. For example, if 15 selection rounds were completed, but all the aptamers were cloned and sequences after round 13, then only 13 rounds were needed. If 8 rounds were completed, some aptamers were cloned from round 4 and some from round 8 , then a total of 8 rounds were needed.

Finally can fill the "has outcome" property by following our naming convention Figure 5, namely 10913311-cAMP-Interaction-1.

\section{Step IV: Add details about the Selection Solution}

From the previous step use the "has selection solution" property to create a new topic of type "Selection Solution" (remember to add "Selection Solution as a type) to describe the details about the solution used in the SELEX experiment to do the selection of potential aptamer sequences between each amplification round. Use the following naming scheme to name Selection Solutions:

\section{Document Identifier-Aptamer Molecule Type-Target Name-Experiment-Ss 10913311-RNA-cAMP-Experiment-Ss}

The "ionic strength" property refers to the concentration of any metal cations used throughout the experiment (in this case typically sodium, magnesium, calcium or potassium). In SELEX experiments this value will be taken from the total concentration of each cation used in the selection buffer, column buffer, or other buffer used in the selection experiment. The total ion concentration (ionic strength) will affect important properties of the solution and the aptamer interaction. For this particular property we recommend using the following pattern:

$$
\text { VALUE - M - CATION NAME -> 0.5-M-Na }
$$

Finally fill in the "pH" property for this Solution Selection topic

\section{Step V: Add details about the reported Interaction(s)}

Now click on the Interaction topic that we had just created in Step III or click here. To incorporate more details. From the article written by Koizumi and Breaker we note that they describe only one interaction between 4 RNA aptamers and cAMP as the aptamer target, 
therefore we can immediately add the Collective Interaction to this topic by clicking on the "Add a type" link. Conversely, if it were the case that a reported interaction would have only two participants then we would type it as a Pairwise Interaction. You will notice that when adding either the Pairwise Interaction or Collective Interaction types to the experimental outcome you will also get the Interaction type incorporated.

Fill in the "has participant" property provided by the Interaction type. Here you will give unique names to four aptamers and one aptamer target (Figure 4).

\section{Step VI: Add details about each of the Interactors}

We can now proceed to add more details about each of the Interactors (i.e.: 10913311-cAMP-1, 10913311-cAMP-2, ..., cAMP) that participate in this paper's reported Interaction. For each of the four aptamers described in this paper, we need incorporate the additional types namely the RNA and Aptamer types and fill the corresponding properties. Also note that the aptamer type has a "has minimal aptamer" property that should be used if the aptamer had a minimal aptamer derived from it by following the naming scheme from figure 3 .

Finally we need to add details about the Cyclic AMP interactor. We need to add the Aptamer Target and fill the CHEBI identifier property for this entry.

\section{Step VII: Include details about the Affinity Experiment}

Now we need to add details about the affinity experiment that was performed by Koizumi and Breaker which elucidated a nanomolar dissociation constant for the reported interaction by making use of equilibrium filtration analysis.

To create a new Affinity Experiment topic, we can start by searching for the Affinity Experiment type in Freebase (be sure to select the "Affinity Experiment" type described in the Aptamer Base from the drop down menu) or by simply clicking here. You will be presented with a list of all topics that have Affinity Experiment as type. To add a new topic simply click on the Add it! link at the bottom of the list. Remember to make use of the Affinity Experiment naming scheme shown in figures 2 and 4 namely, 10913311-RNA-cAMP-AffinityExperiment-1.

You will need to provide details about the Affinity Experiment such as the affinity method used, the bibliographic reference (PubMed Id) and also you must provide a link to the interaction that it confirms or disputes. This particular Affinity Experiment also produced as an outcome a dissociation constant thus, fill the "has outcome" property of the experiment with the corresponding naming scheme Figure 2 (i.e. 10913311-RNA-cAMP-AffinityExperiment-1).

To add information further details about the Dissociation Constant click on the created topic or click here. You will notice that this topic does not yet have the dissociation constant type added to it. We therefore commence by adding Dissociation Constant as the type. The authors report a dissociation constant of $1 \times 10^{-6}$, we can use the "value" property to input this information. Note that currently there are some known issues with Freebase and very small or very large numbers, 
which may be rounded to undesirable values. That is why we have incorporated the "temporary string value" and "temporary string value range", which should be used if you experience problems with the "value" an "value range properties". You will finally need to fill in the "is dissociation constant of" property with the corresponding interaction, i.e.: 10913311-cAMPInteraction-1.

THANK YOU!!

:)

\subsubsection{Glossary}

\section{- $\quad$ Freebase}

Freebase is a (available at: http://www.freebase.com/) tool which allows searching through large datasets. It has the ability to create relationships between data and also to filter through the data using those relationships. The user interface for Freebase provides a very simple way to create and access data.

\section{- Base}

A base is a collection of topics created by the community. For example, the Aptamer base contains data related to Aptamers and interactions between molecules. Other examples include the World War Two Base (http://worldwartwo.freebase.com/) or The Computer Science Base (http://computerscience.freebase.com/)

\section{- Aptamer Base}

The Aptamer Base is a Base in Freebase that is related specifically to aptamers and molecular interactions. Types which have been defined within the Aptamer base include RNA, DNA, molecular interactions, and interaction experiments.

\section{- $\quad$ Topic}

A topic is a collection of data on Freebase. Topics represent specific objects or events in the real world and have properties which are defined by a Type. For example, a Topic could be about a calculus book. Its properties would be that it is 500 pages long, and is on the subject of calculus. Topics often will have more than one Type associated with them.

\section{- Type}

A type is a collection of properties which Topics can follow. Properties specified by a Type have a data type, but do not have values. Types are usually less specific than topics. For example, a Type could be a book, with properties such as number of pages and subject.

\section{- $\quad$ Property}


Properties in Freebase define a "has a" relationship between a topic and the value of the property. For example, Calculus book \{topic\} has 3500 \{value\} pages \{property\}.

\section{- Enumeration}

A property which has been restricted to accept only from a list of predefined values.

\subsubsection{References}

1. Koizumi, M. and R.R. Breaker: Molecular Recognition of cAMP by an RNA Aptamer. Biochemistry, 2000. 39(30): 8983-92. 